Fall 1965

\title{
1965 Miracle Yearbook
}

Cedarville College

Follow this and additional works at: https://digitalcommons.cedarville.edu/yearbooks

Part of the Higher Education Commons, Organizational Communication Commons, and the Public Relations and Advertising Commons

\section{Recommended Citation}

Cedarville College, "1965 Miracle Yearbook" (1965). Yearbooks. 45.

https://digitalcommons.cedarville.edu/yearbooks/45

This Book is brought to you for free and open access by DigitalCommons@Cedarville, a service of the Centennial Library. It has been accepted for inclusion in Yearbooks by an authorized administrator of DigitalCommons@Cedarville. For more information, please contact digitalcommons@cedarville.edu. 
Public Relatións Dept. Copy \#1

Ar thot Remove

Dr geremiah 


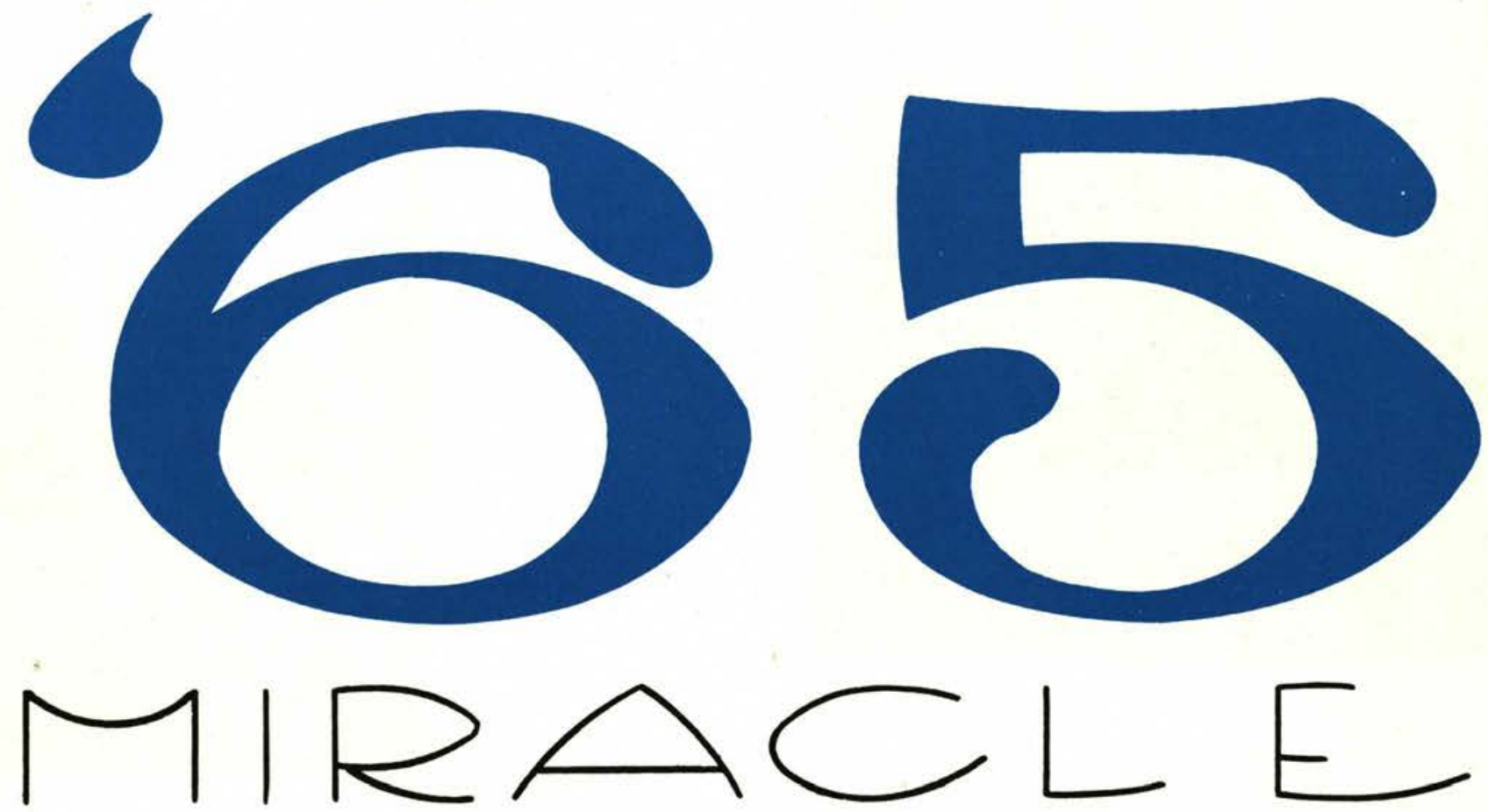

Paul S. Brower editor

Dennis Pearce .............. business manager

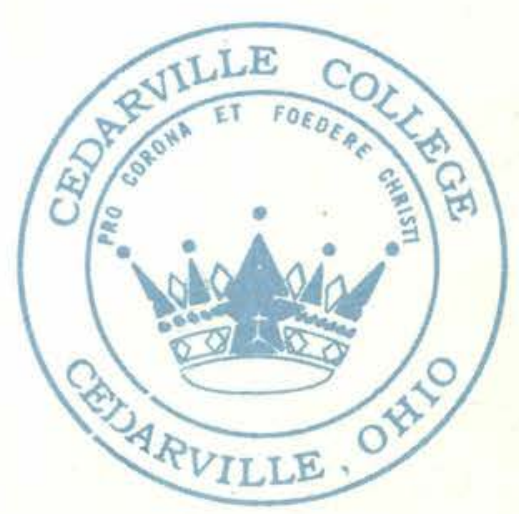

Edward E. Spencer advisor 


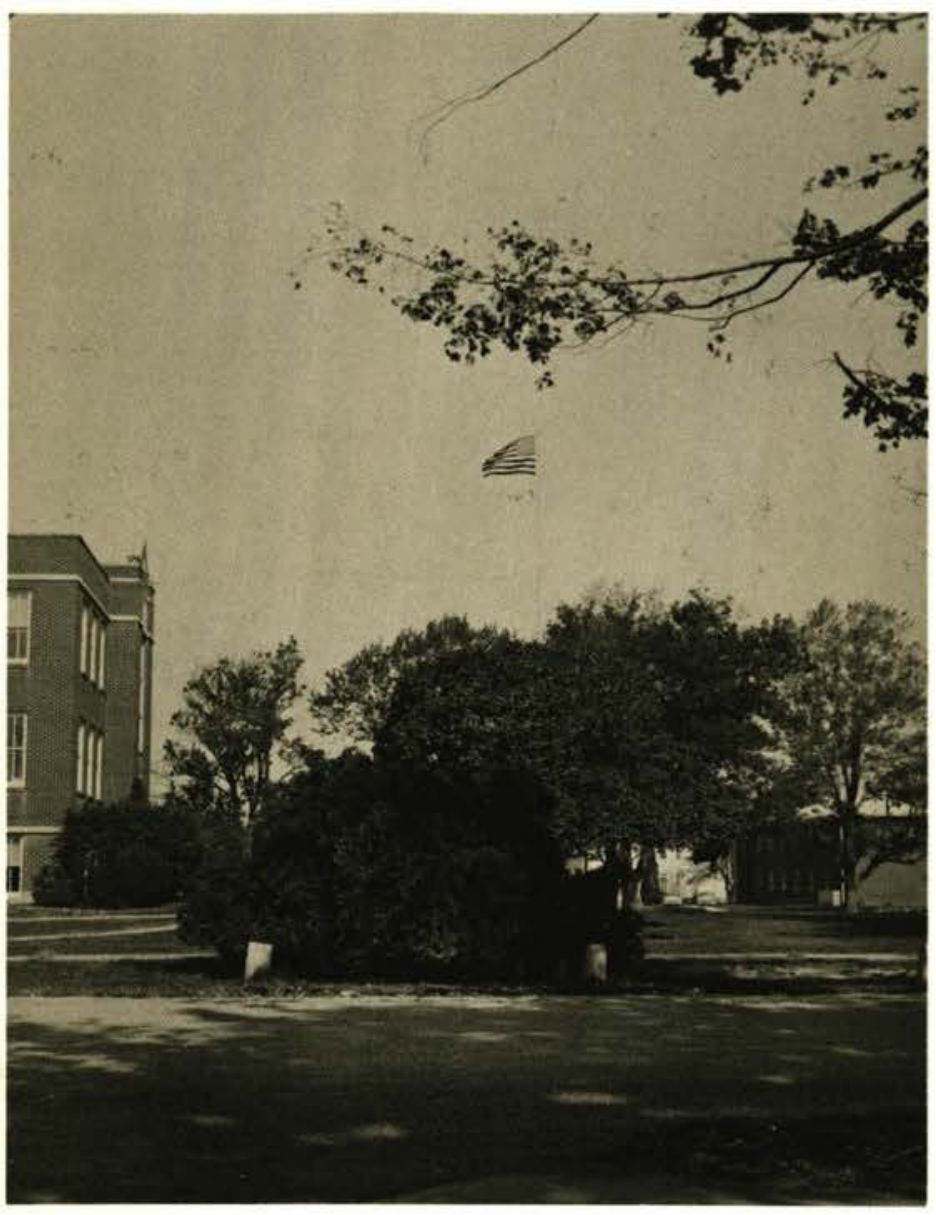

FALL ...
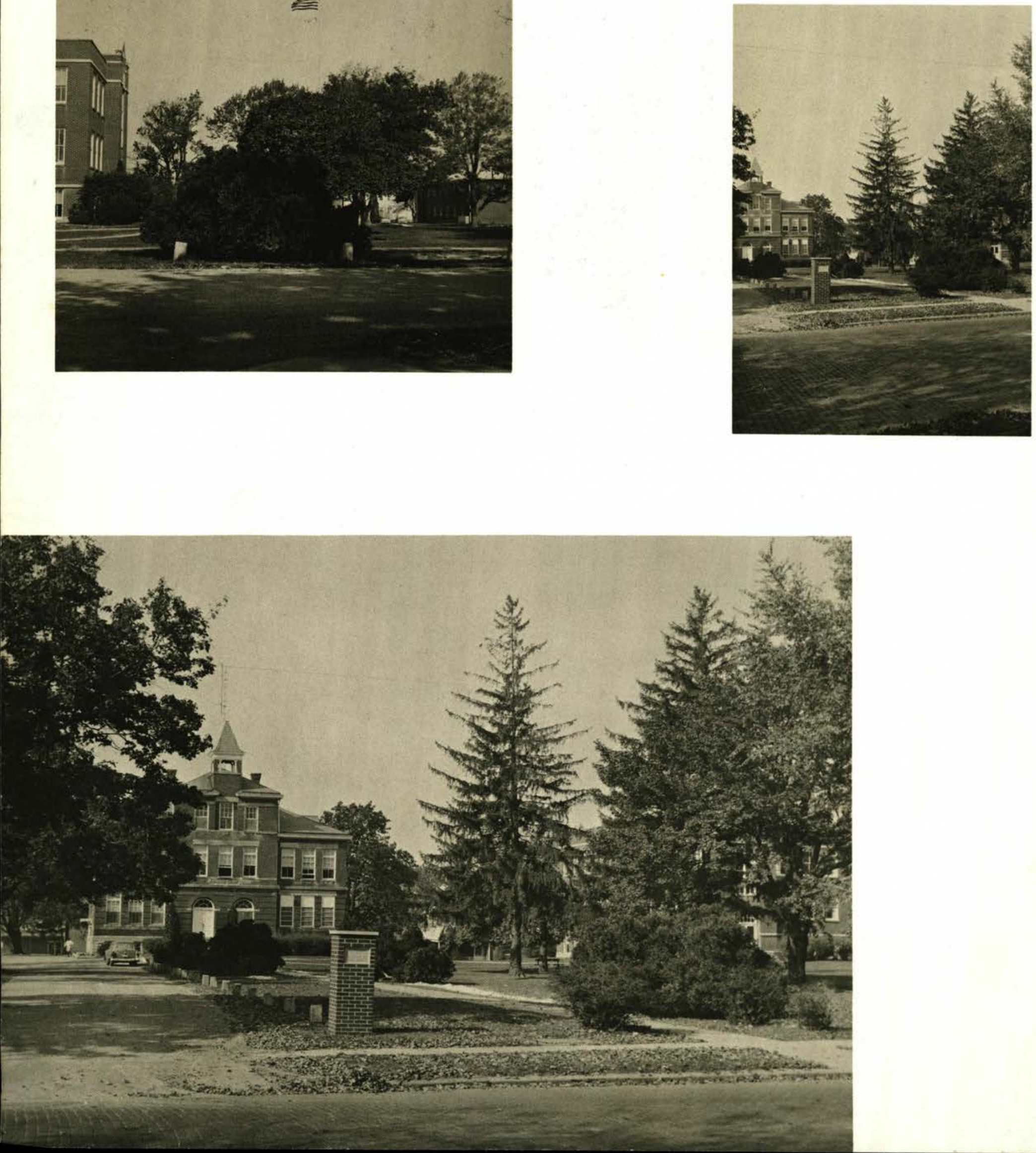


\section{WINTER . .}

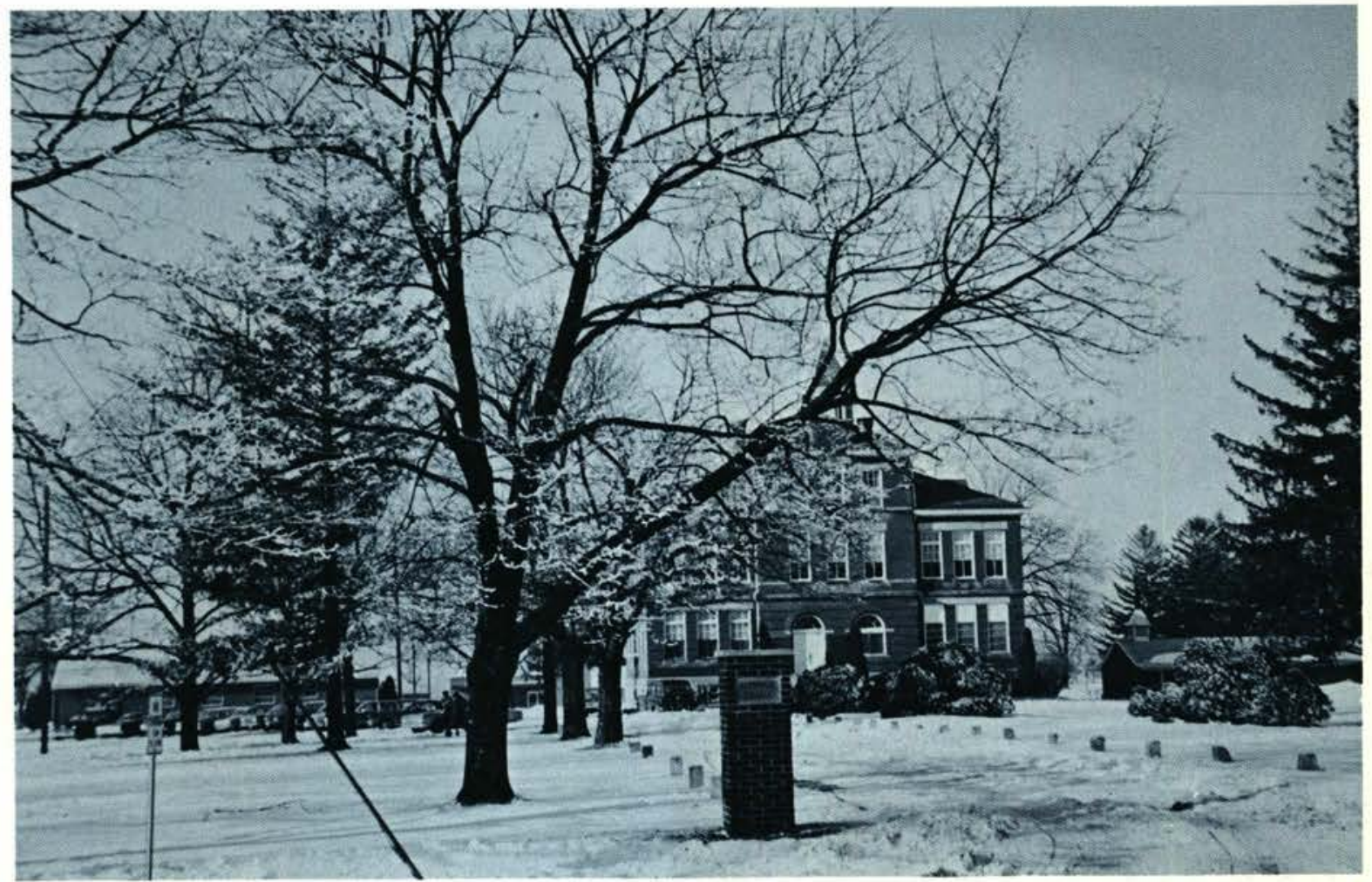

SPRING . . .

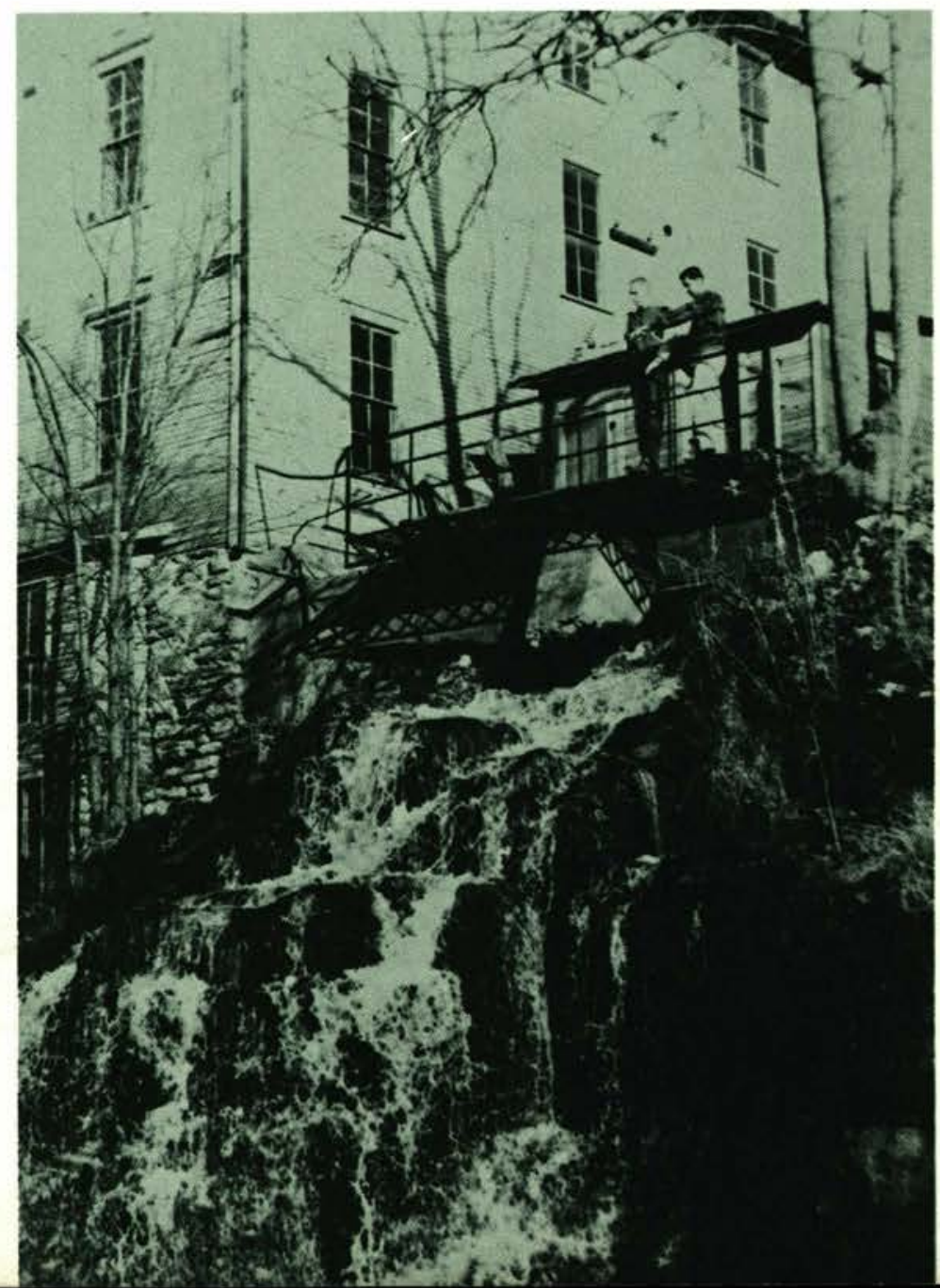




\section{$\theta \theta+\theta$}

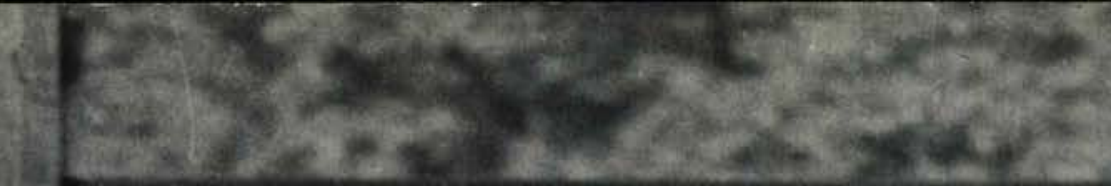

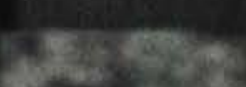

邹
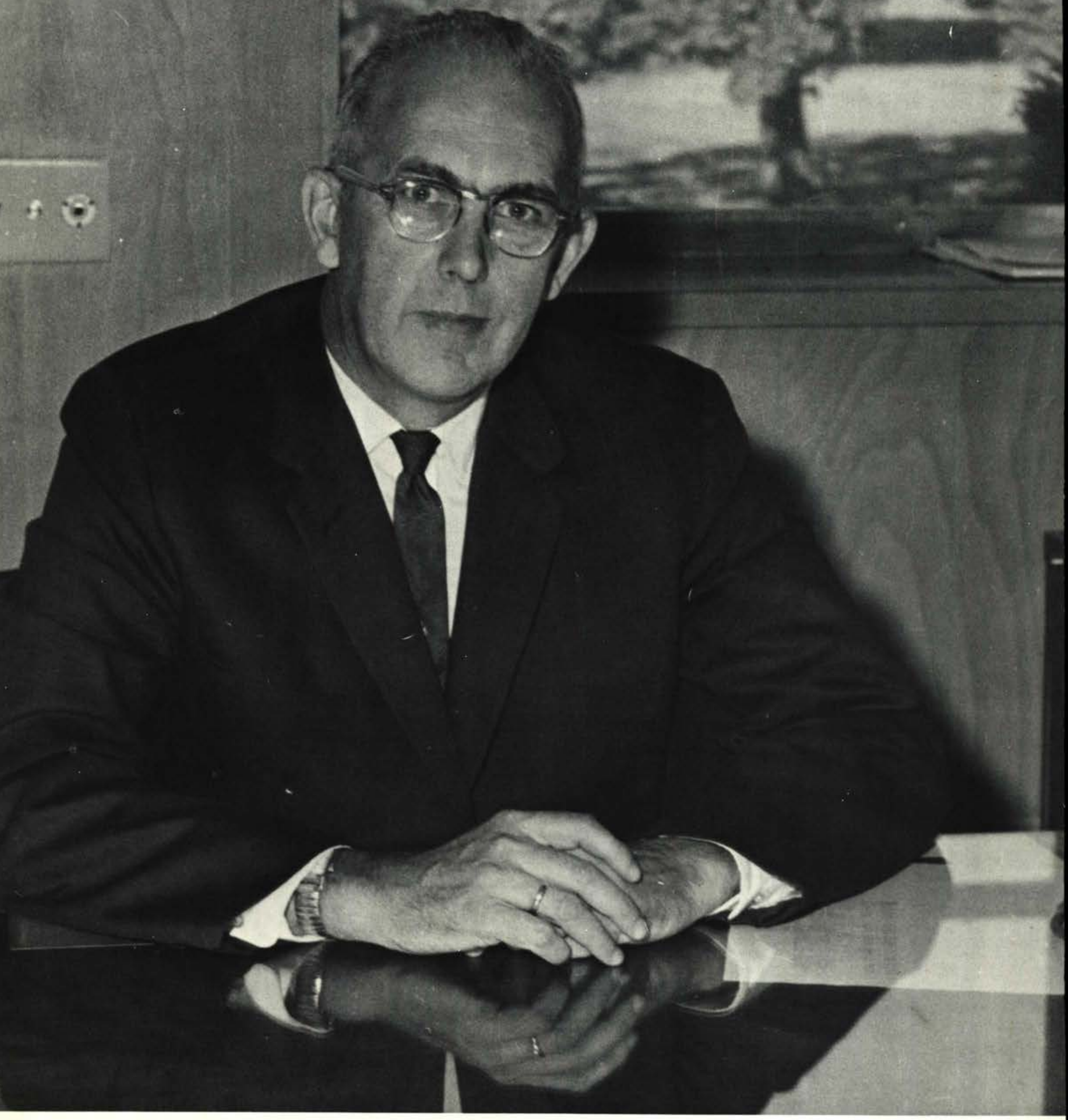

DEDICATION 
"An institution is the lengthened shadow of one man." Ralph Waldo Emerson

As the man, so the institution. One of the most important reasons for the success and growth of Cedarville College is the influence and personality of its president-Dr. James T. Jeremiah. Eleven years ago he came to us by faith, prepared to meet the challenge of a newly-organized Baptist college. As an able administrator, he has successfully led the school through many times when progress seemed impossible and then forward into many new endeavors made possible only through careful planning and confident faith. His performance in the role of decision-maker has been one of the chief determinants of the bright future now taking shape for Cedarville College.

As a representative of the college, he is faithfully presenting to the public, and the churches in particular, the aims, growth, and needs of the school. He continues to win the admiration of many around the conference table or in the audience as he presents to them a dynamic institution created and working "for the Word of God and the testimony of Jesus Christ."

As a friend to all, he knows the faculty and many of the students personally. His pleasant greeting and warm personality are known to all. There is something attractive about this man dedicated first of all to the Lord and then to the task as President of Cedarville College.

It is to you, President James T. Jeremiah, that we, the students of Cedarville College, dedicate the MIRACLE of 1965. 

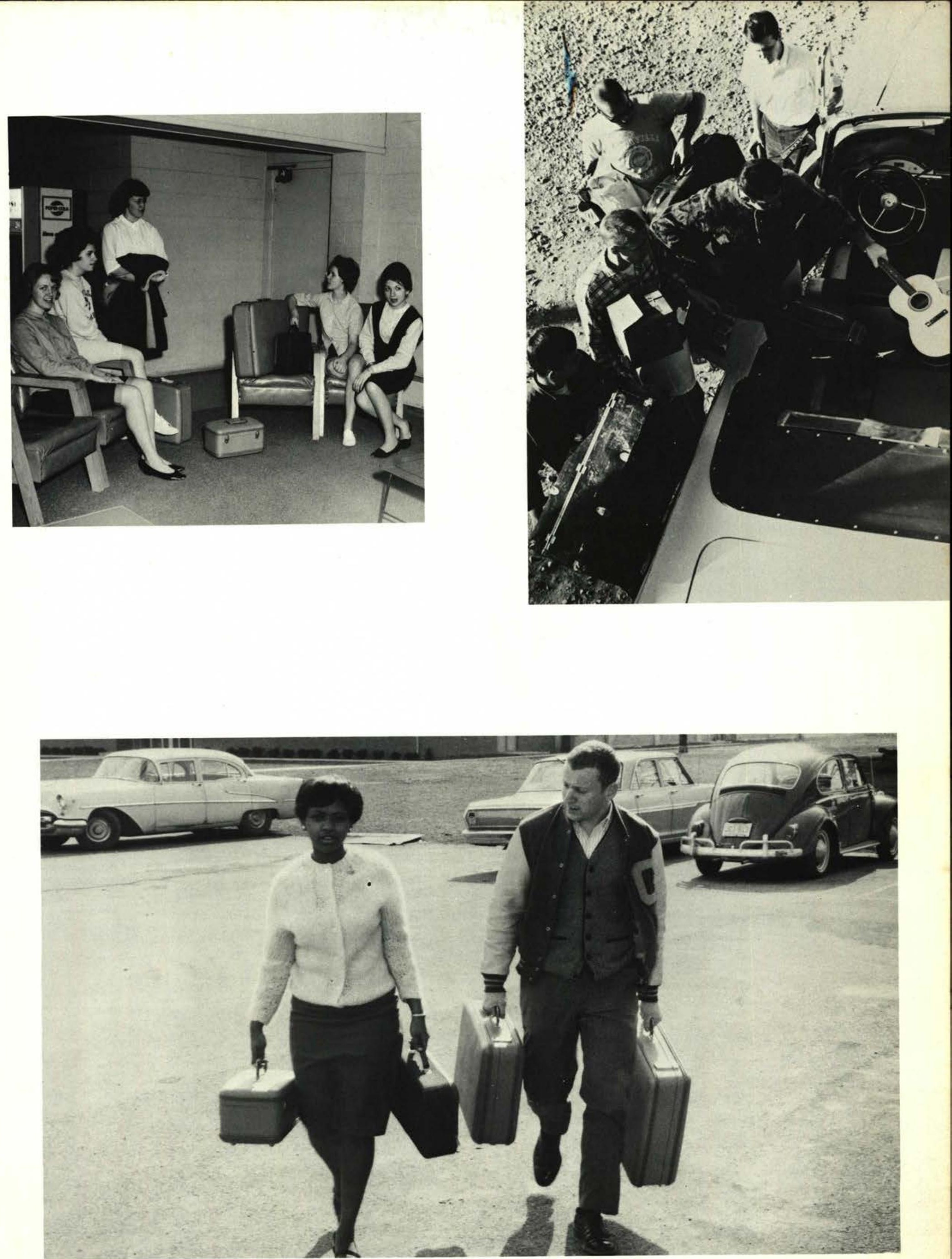


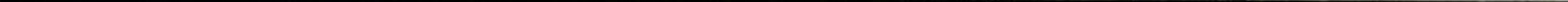



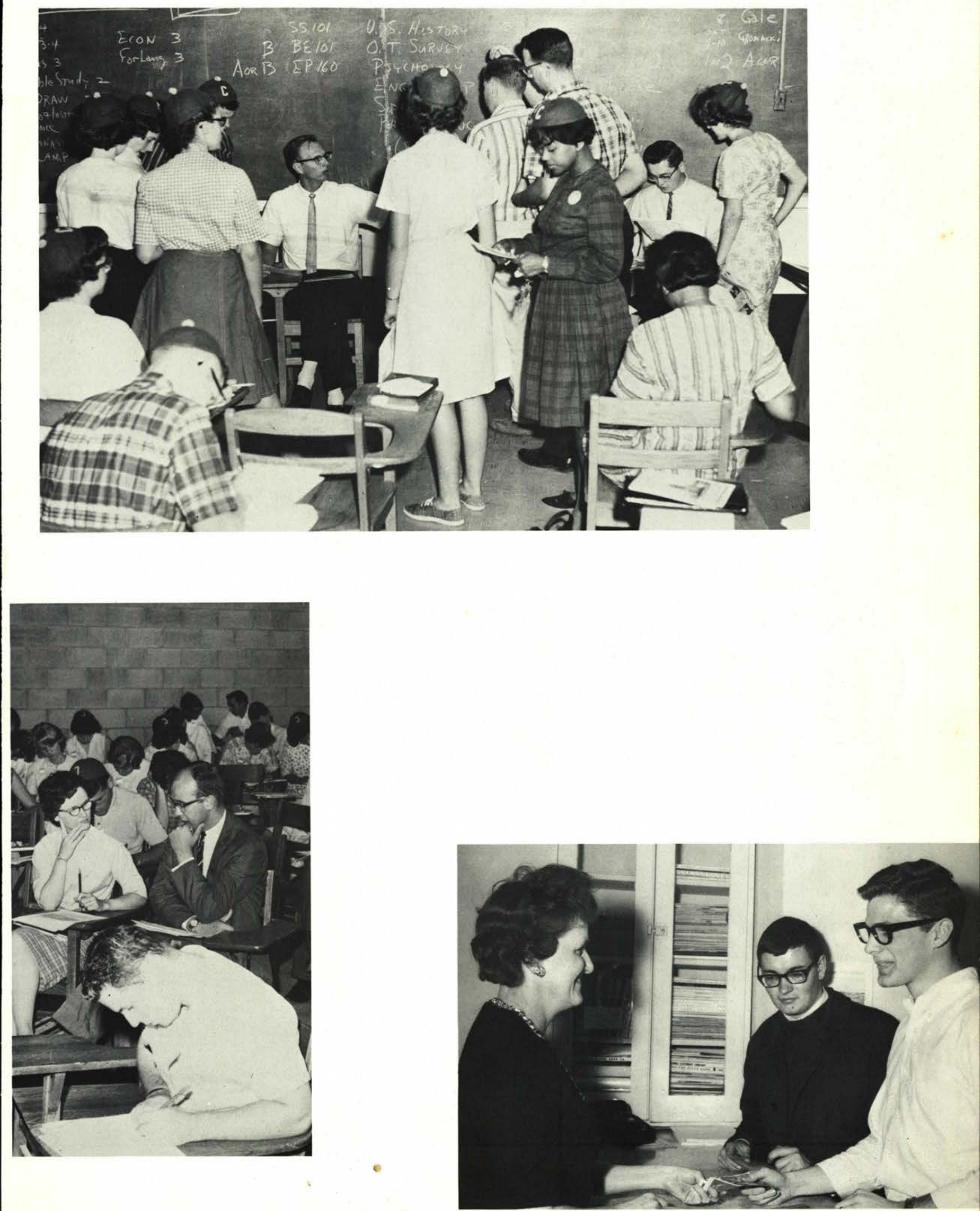


\section{Senior Sneak}
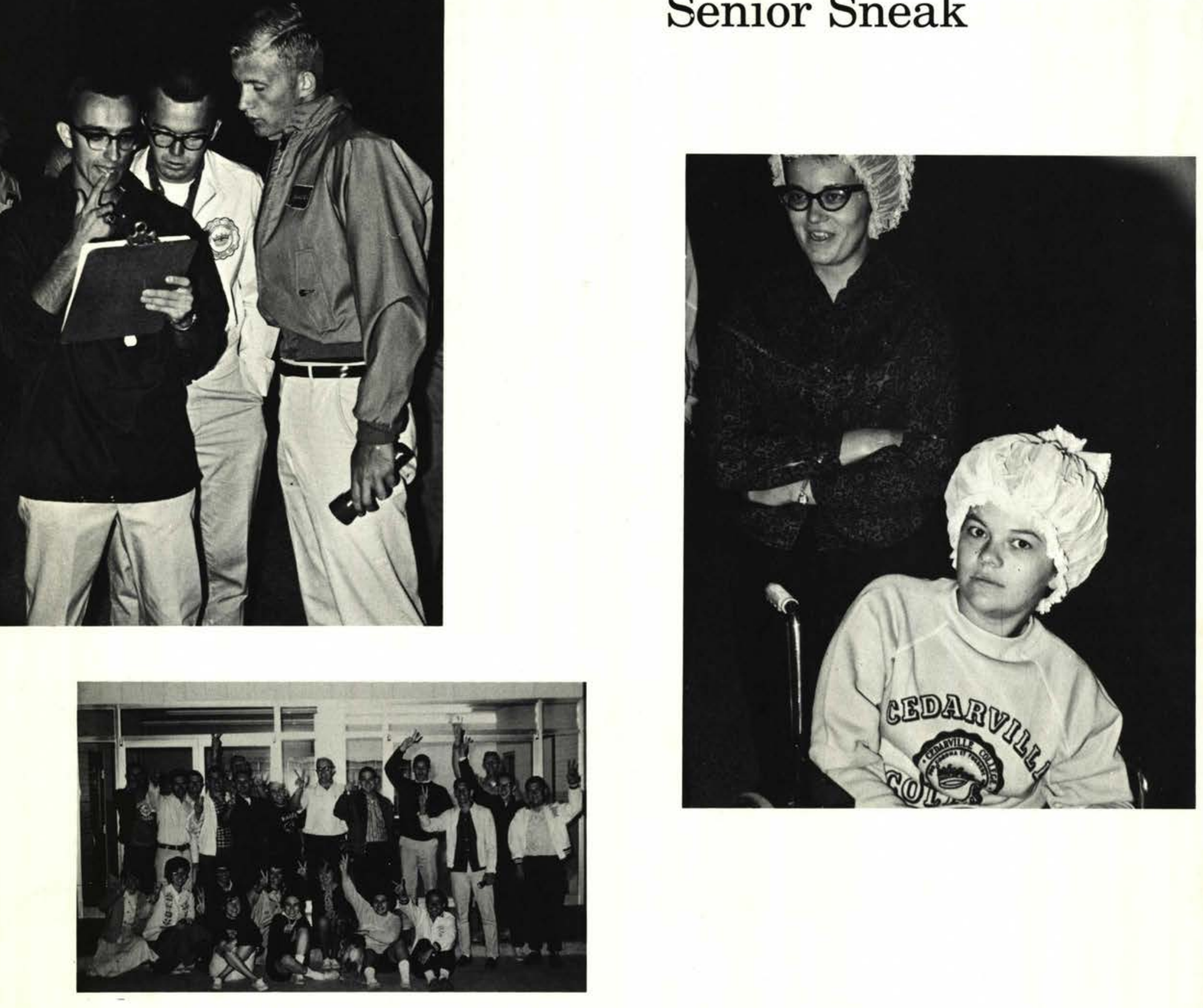


\section{Challenged by Word ... by Prayer}

In a world of challenges, a no more effective one than to live a truly dedicated Christian life could be given to Christian college students. Those accepting this challenge from Rev. Donald Tyler during the Fall Bible Conference know what it is to have real joy in serving Jesus.

Highlighting the week-long conference, the Fall Day of Prayer was a special blessing to all. The day,

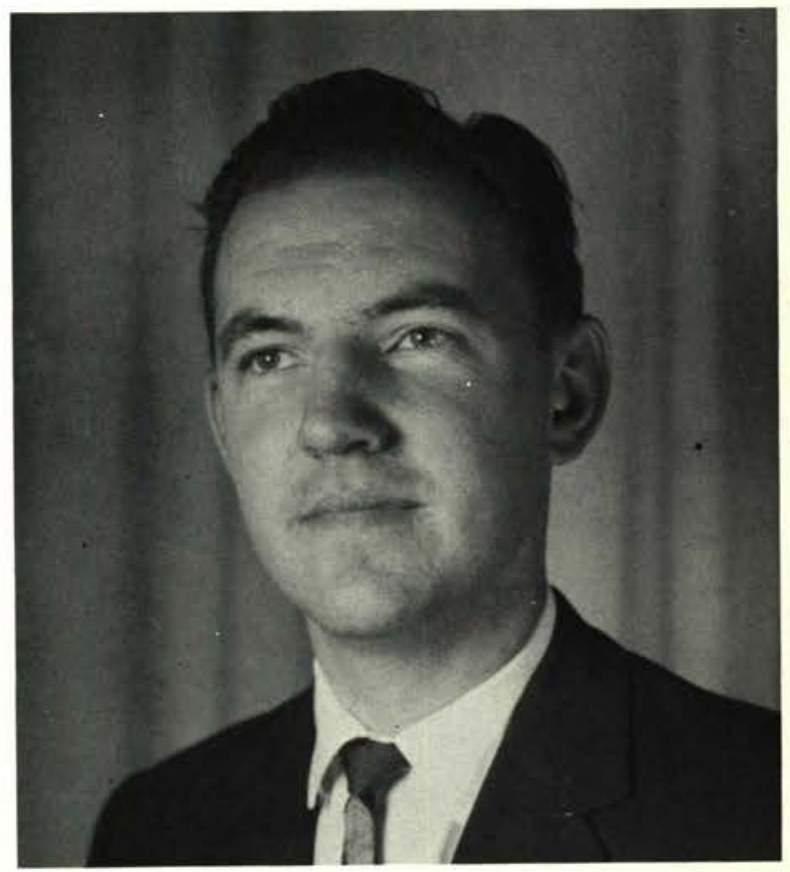
set aside for prayer, provided the needed time apart with the Lord before plunging into the activities of the year.

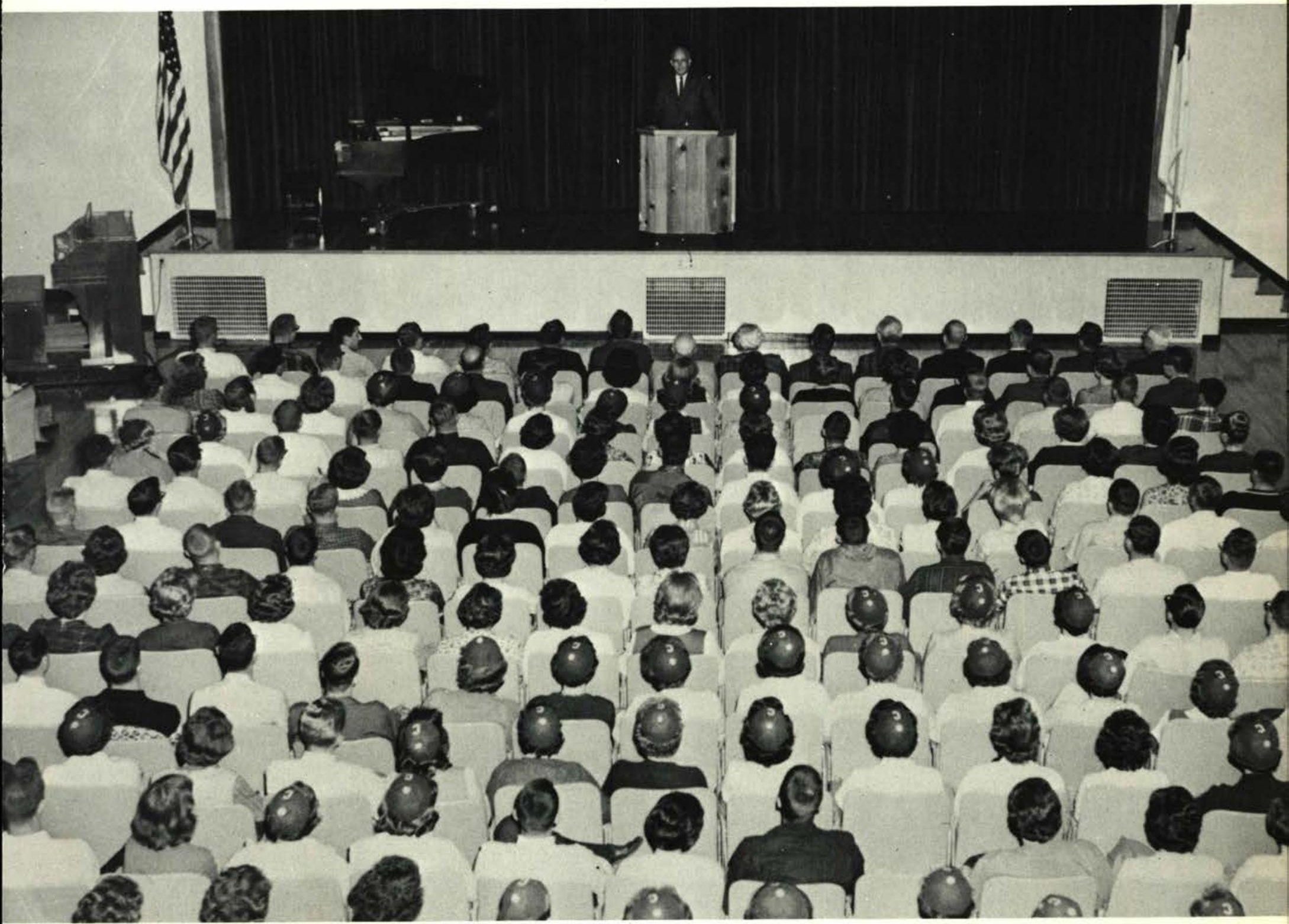


Welcome to
V.C.
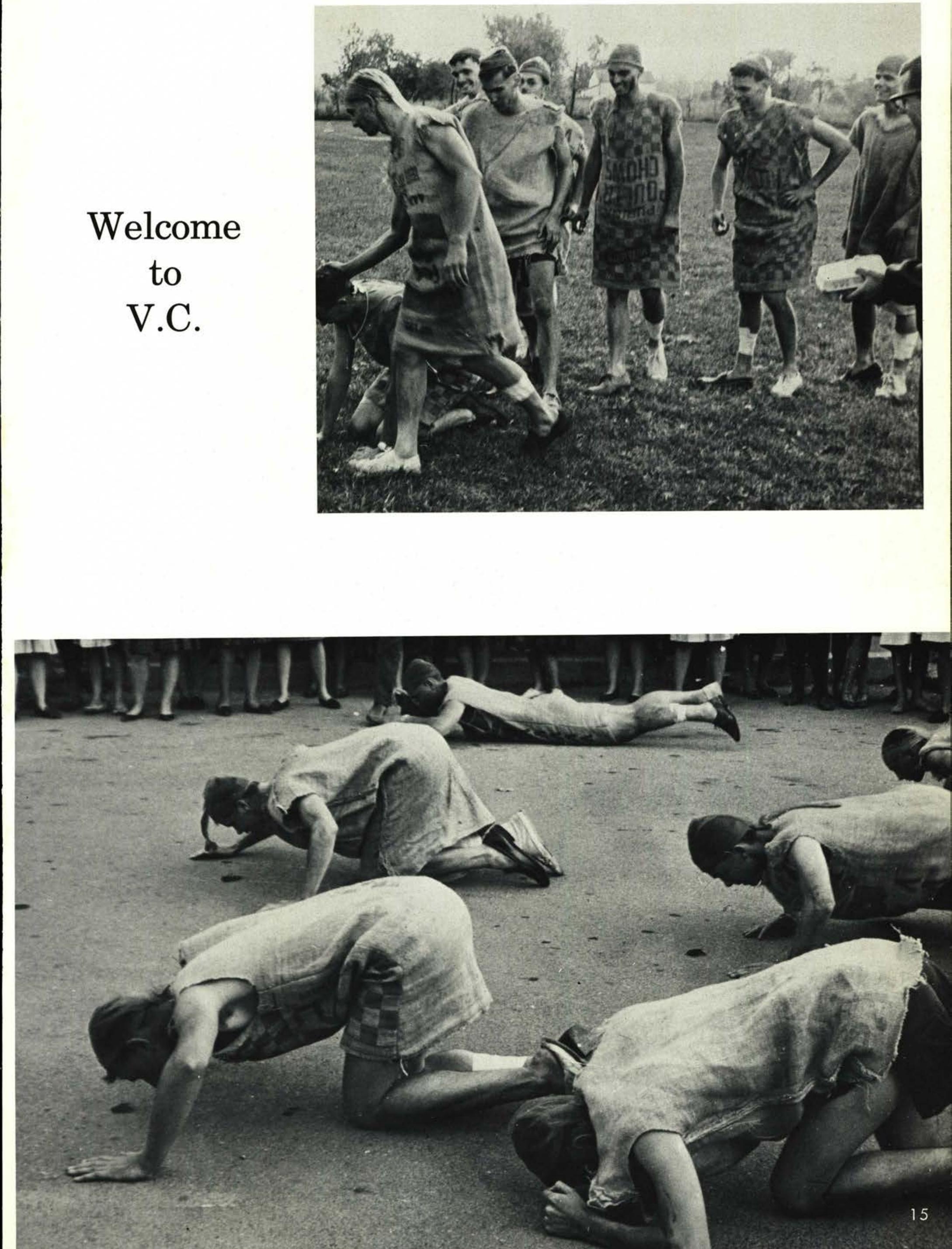


\section{Homecoming Highlights}
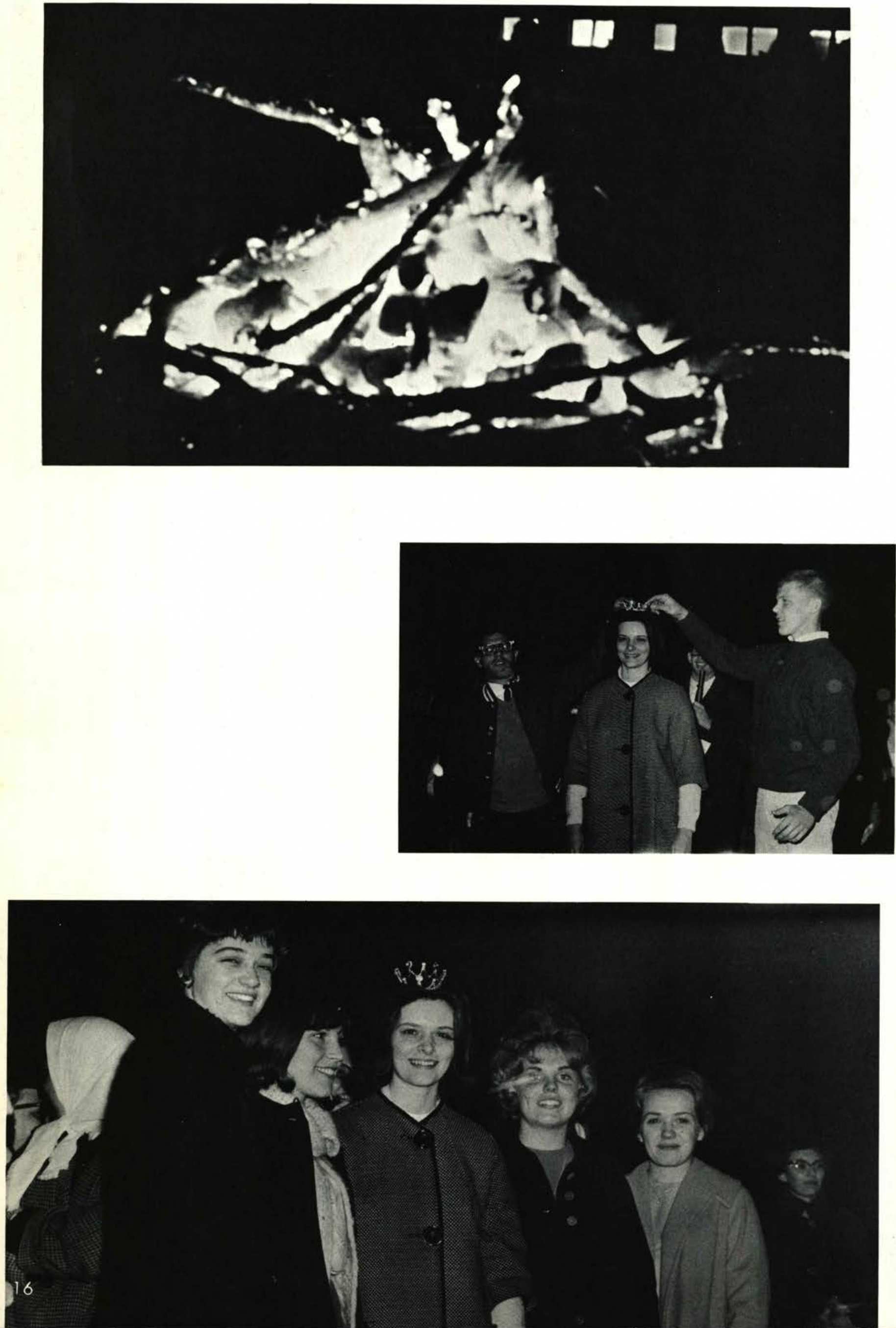


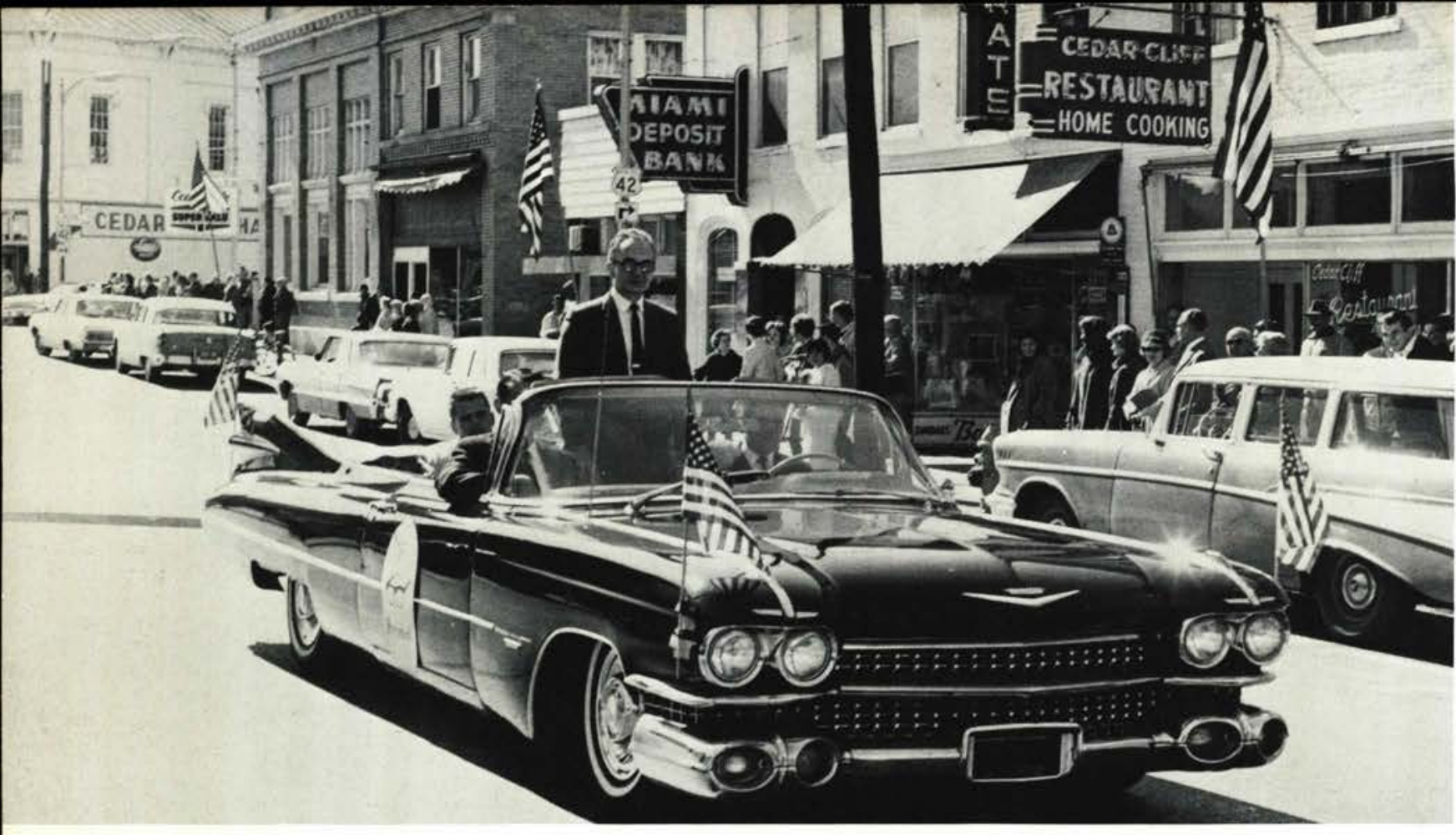

Conservative Club ...
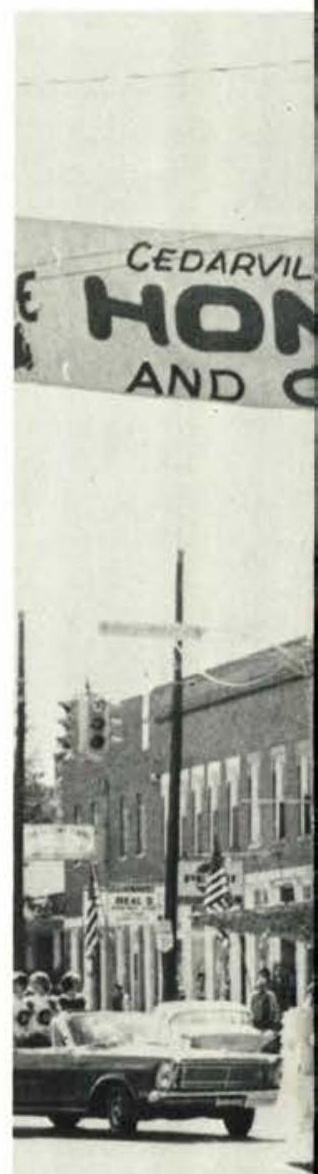

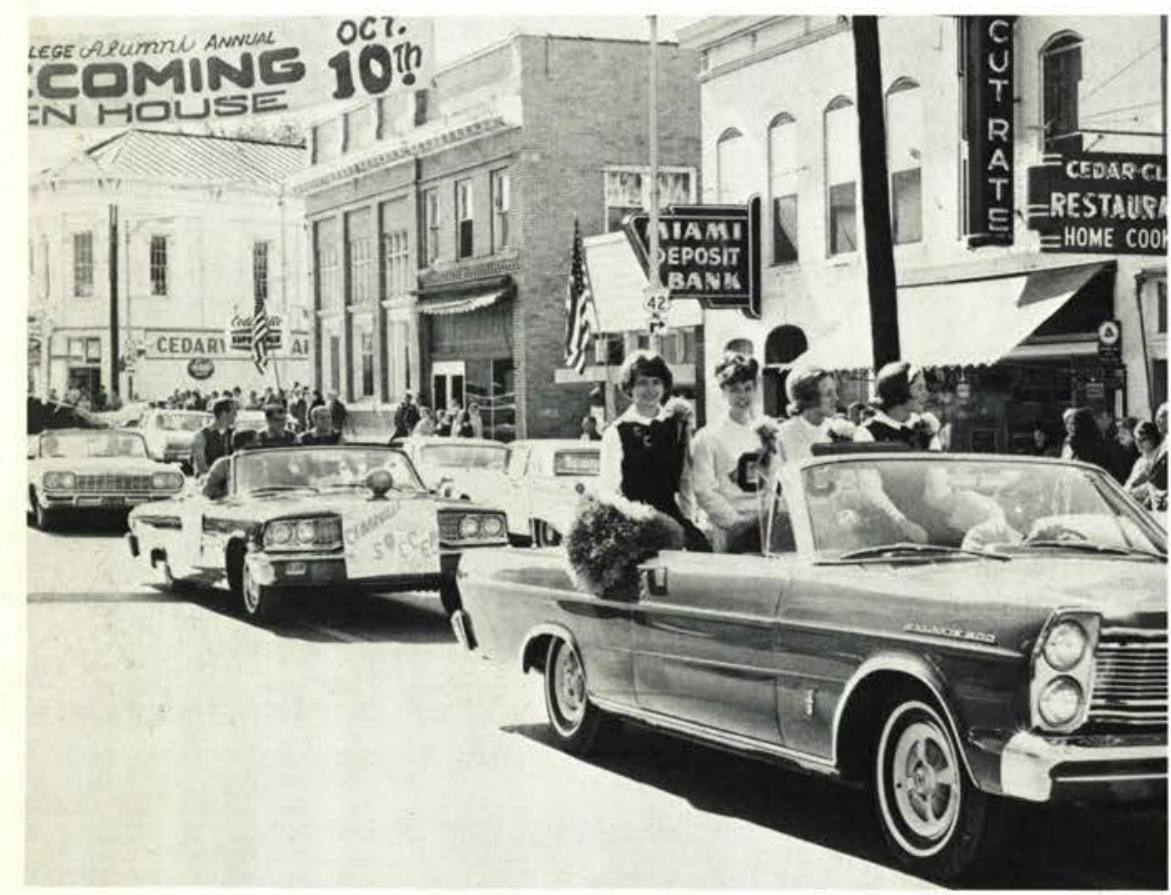

Cheerleaders ... 


\section{.. . on parade}

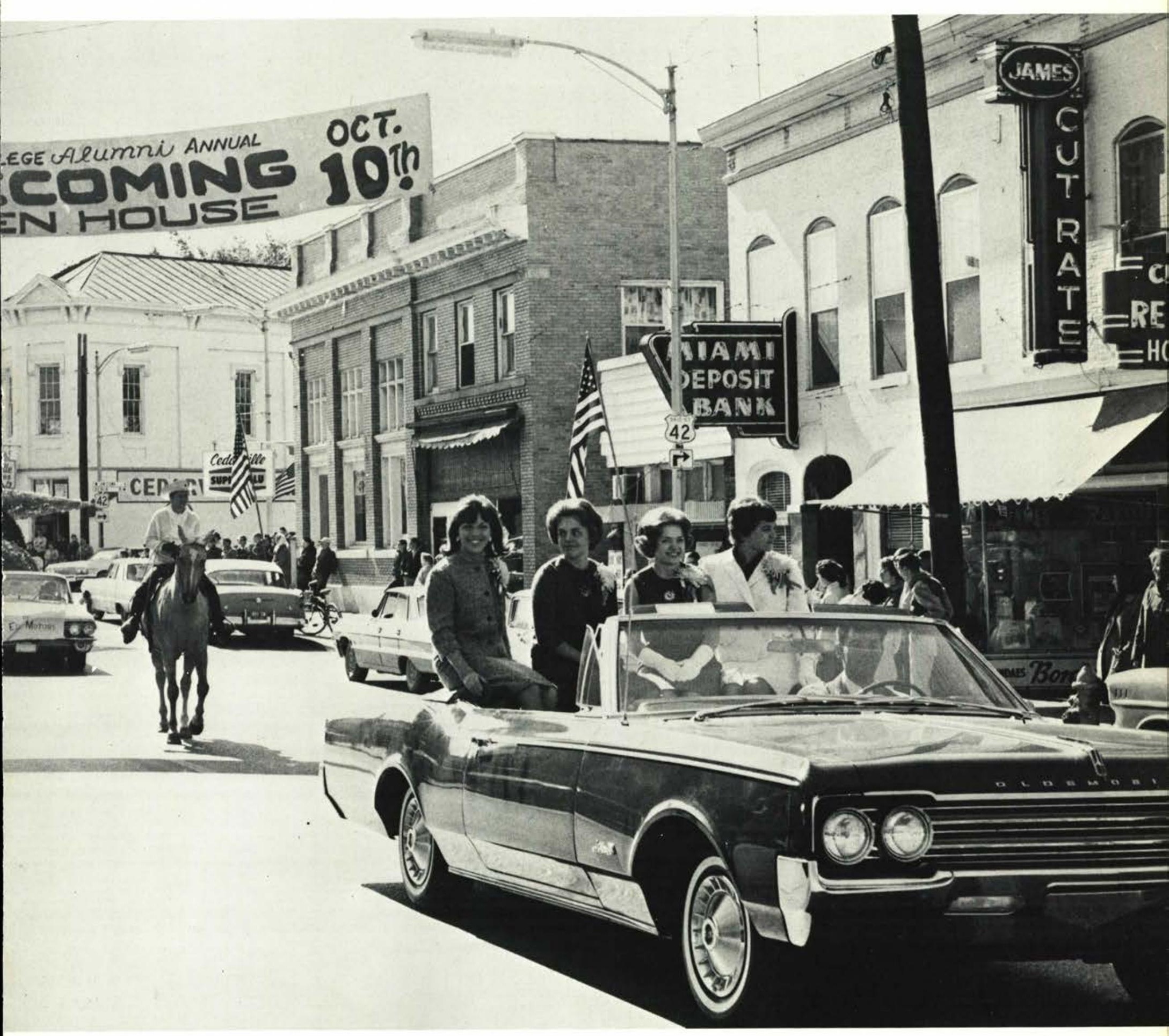

Queen's Court ... 


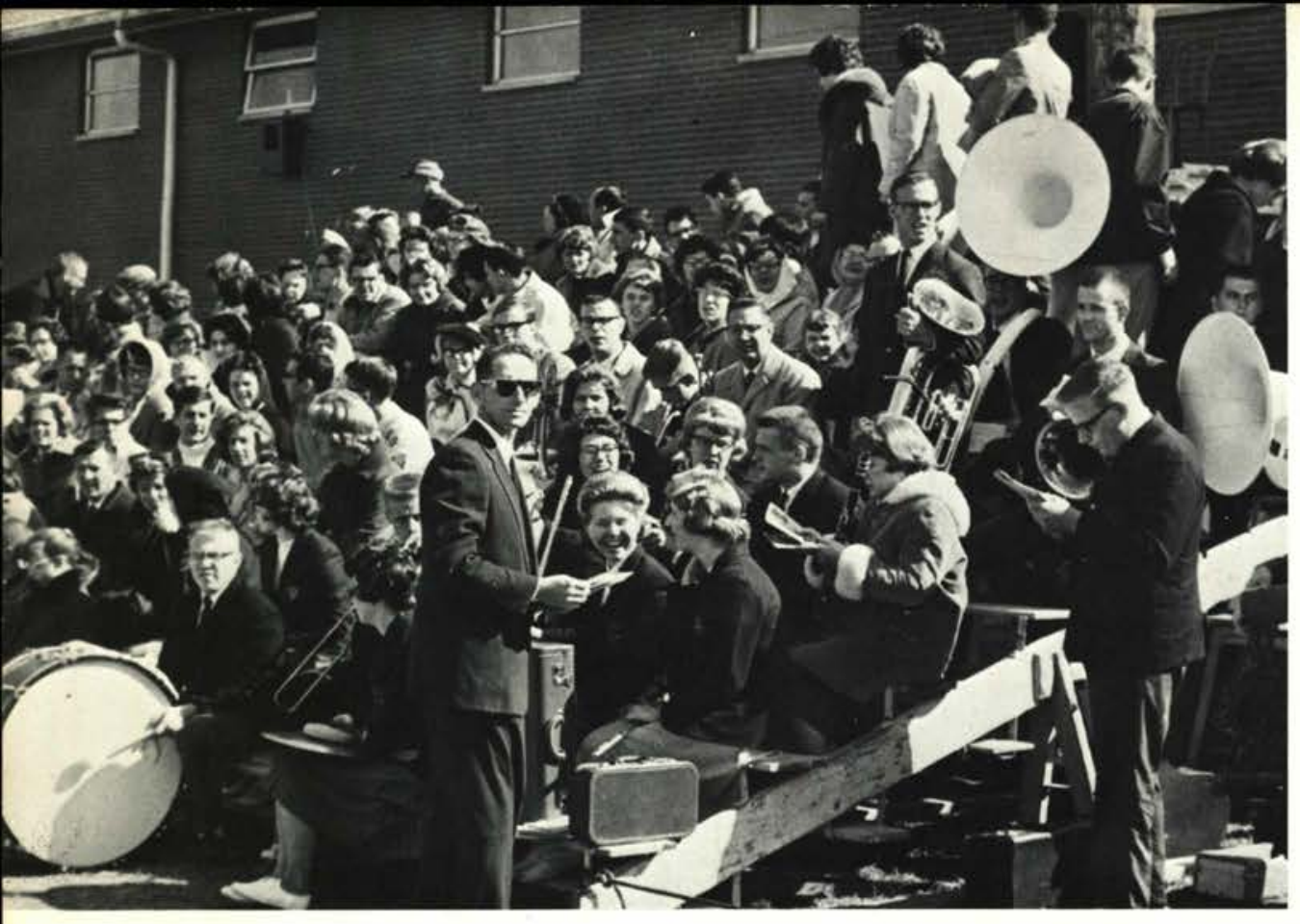

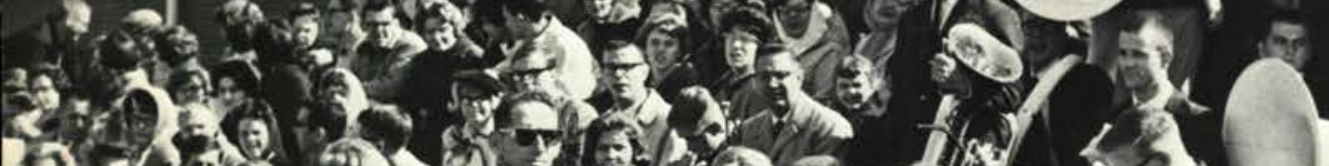
M 5 की
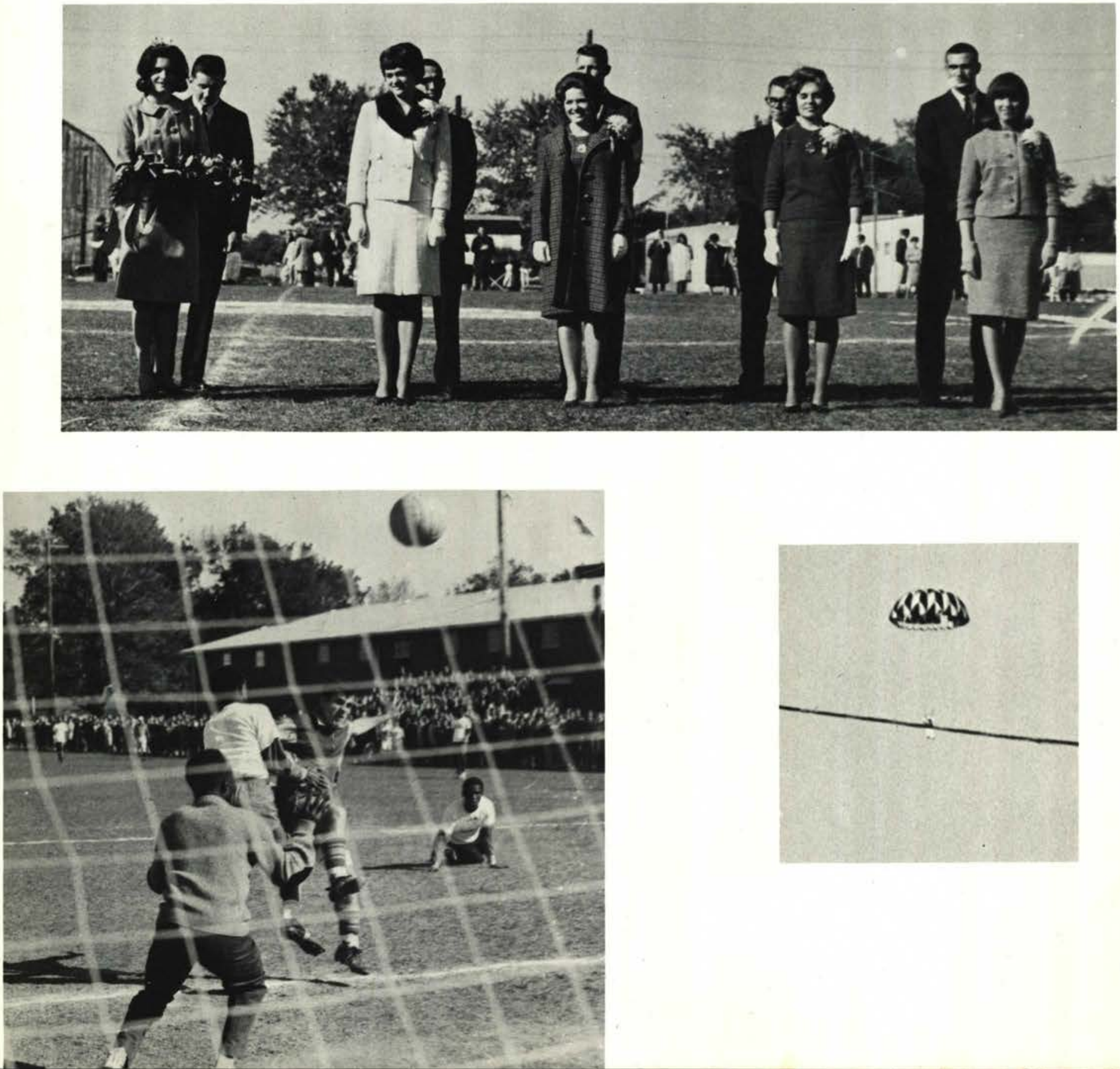


\section{Soccer's \\ Second Season}

University of Dayton

Philadelphia College of the Bible

Fenn

Wilmington

Indiana Tech

Wilberforce

Central State (Homecoming)

Bowling Green State University

Miami

Antioch

Denison University
WE THEY

1

$0 \quad 4$

$1 \quad 0$

14

$3 \quad 0$

$2 \quad 0$

$4 \quad 2$

$4 \quad 3$

12

$0 \quad 0$

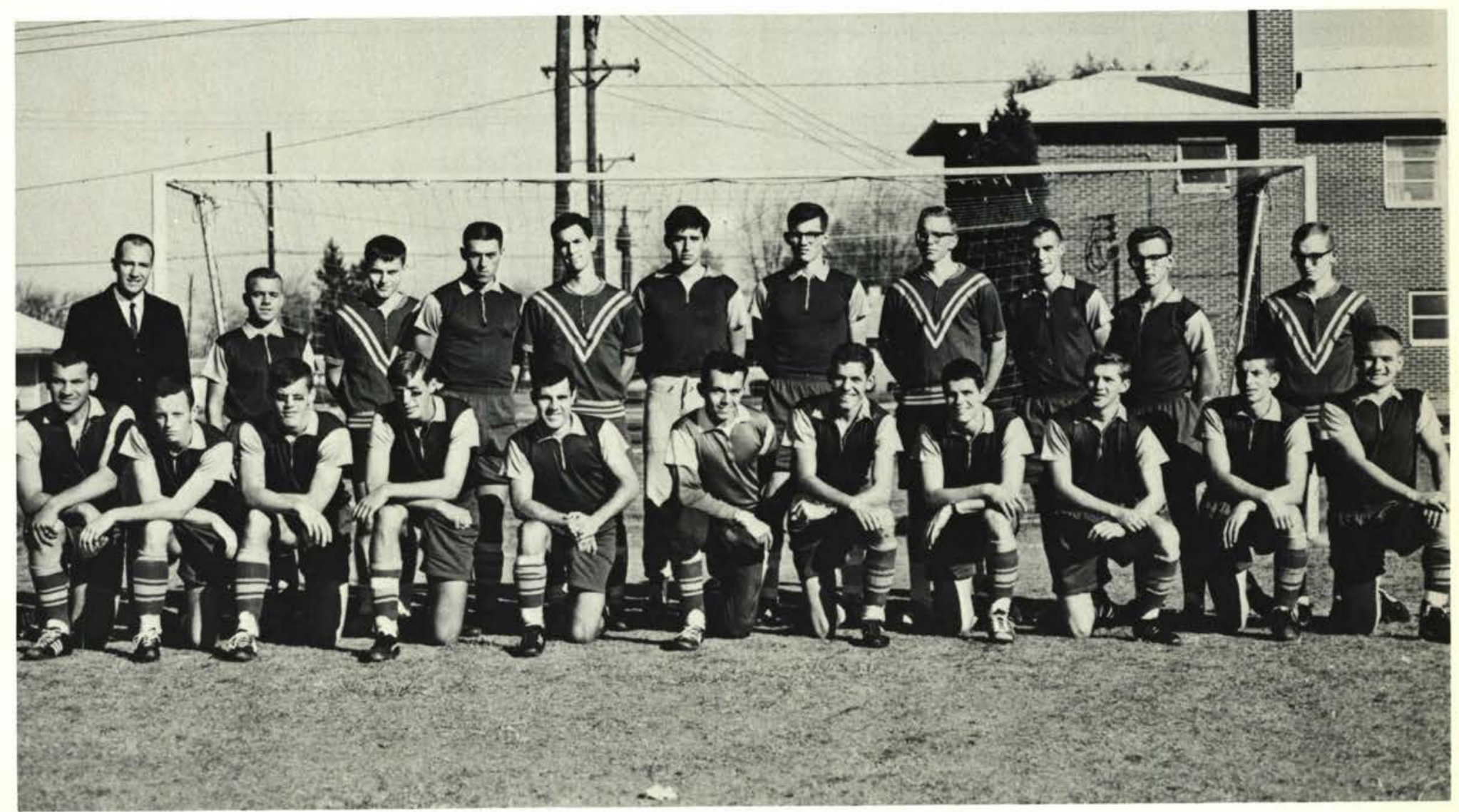

Standing: L. Moody, Coach; D. Rockwell, J. Hess, J. Shelton, D. Leach, M. Woodend, L. Maidment, J. French, M. Kniffen, P. Carlson, M. Hamilton. Kneeling: J. Barker, J. Hay, J. Stowell, co-captain; C. Stillwell, T. Rotondi, co-captain; M. Pereira, M. Ziegler, R. Ross, M. Trimble, J. Engleman, T. Duff. 

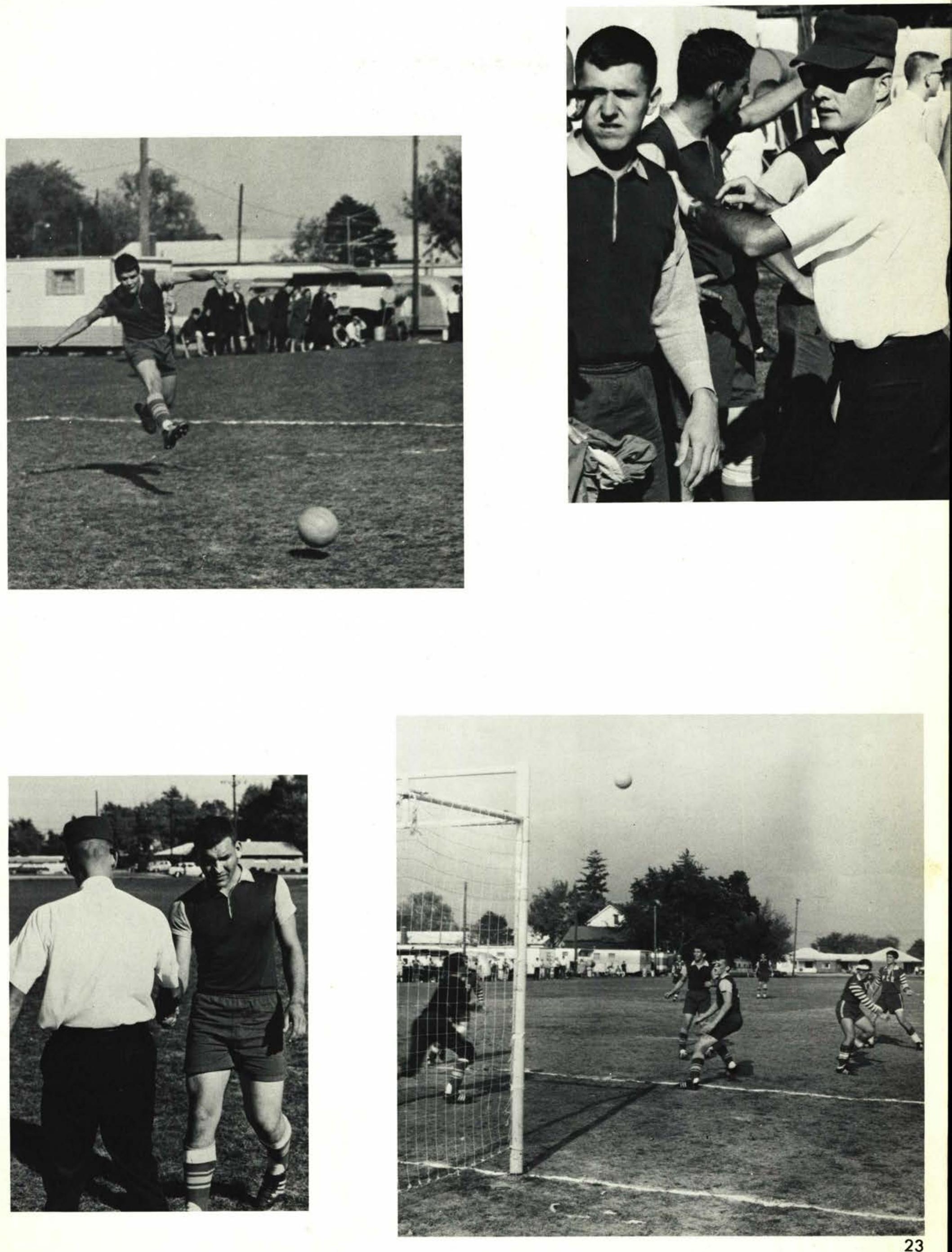

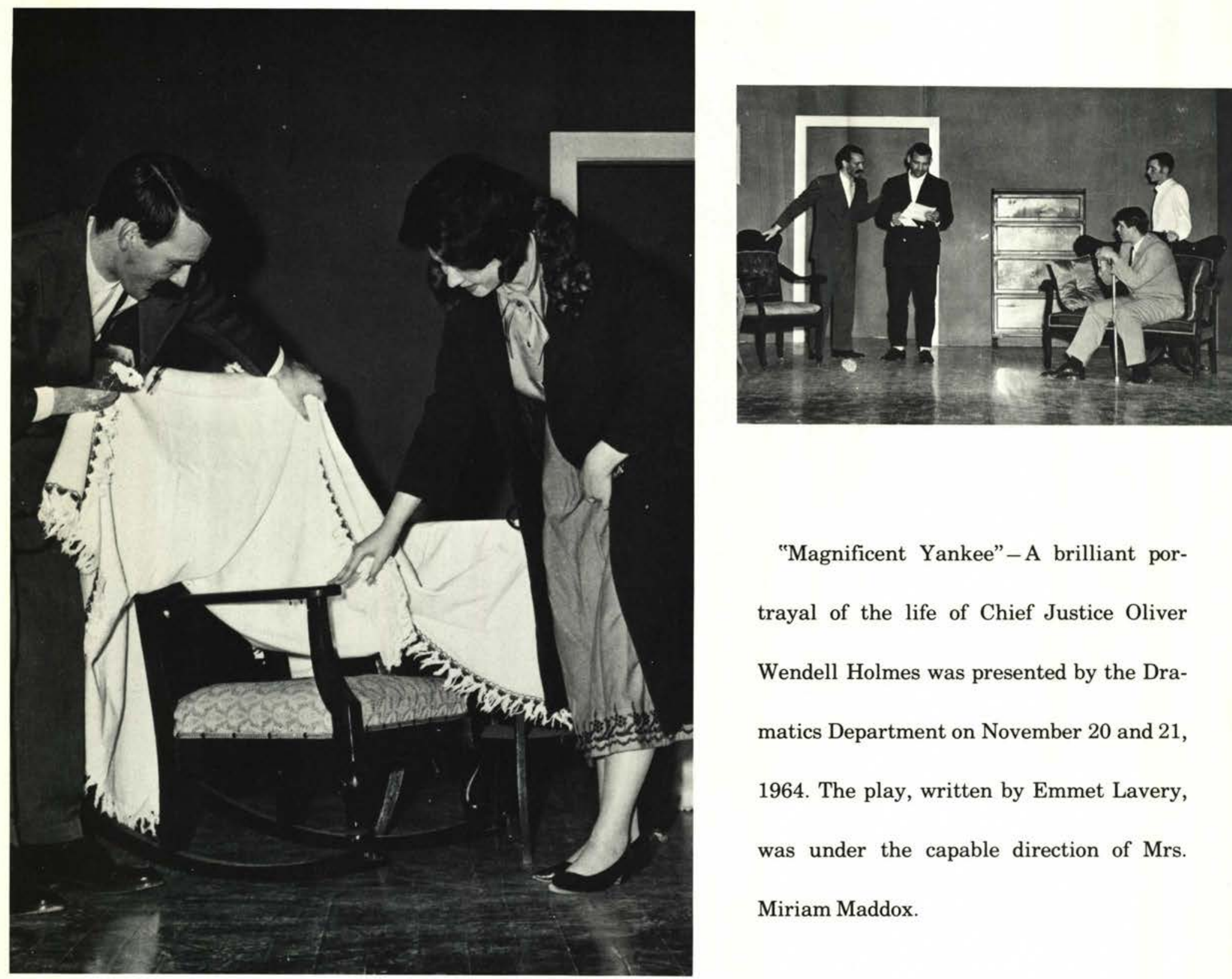

\section{"Magnificent Yankee"-A brilliant por-} trayal of the life of Chief Justice Oliver Wendell Holmes was presented by the Dramatics Department on November 20 and 21, 1964. The play, written by Emmet Lavery, was under the capable direction of Mrs. Miriam Maddox.
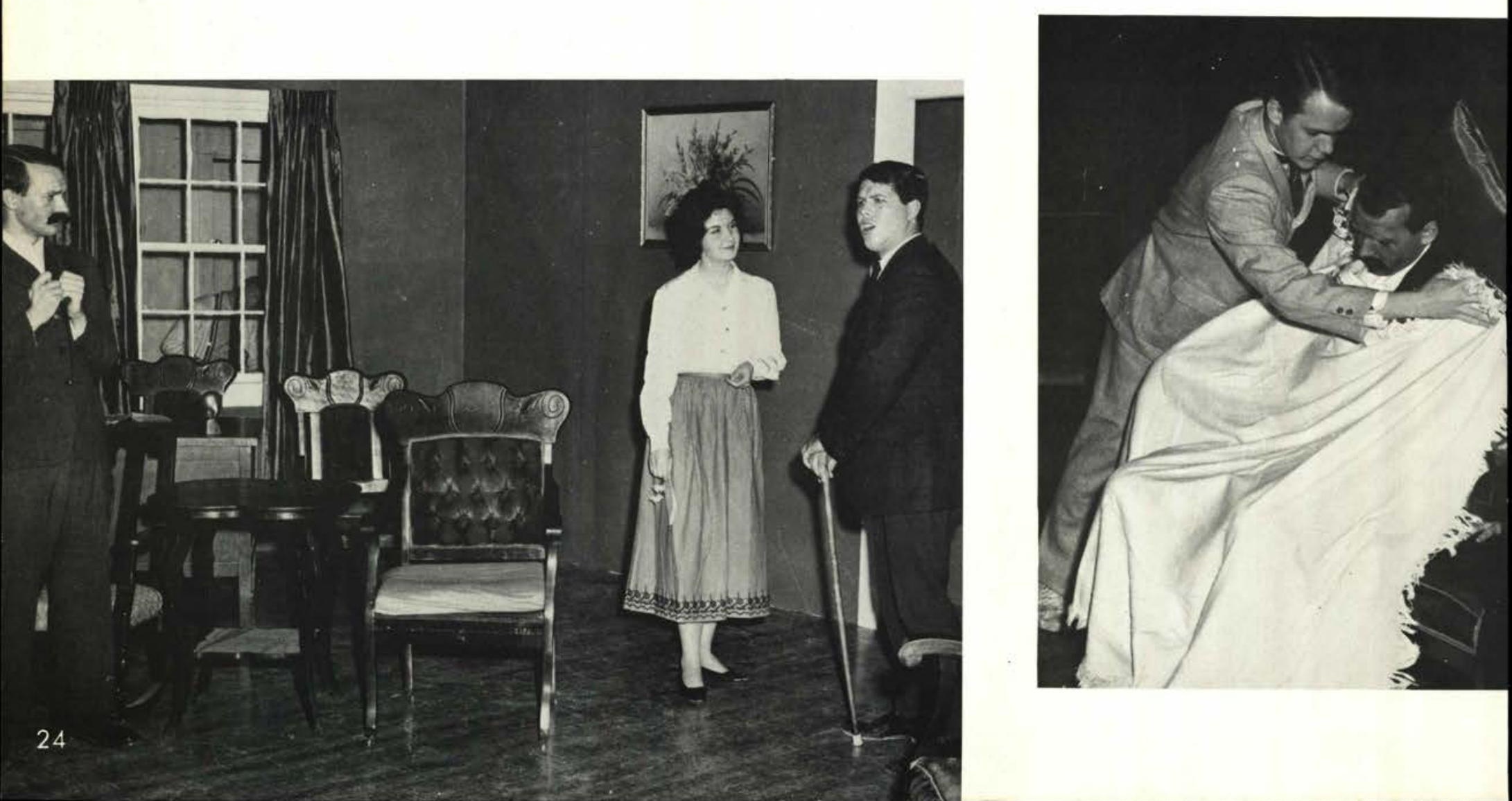


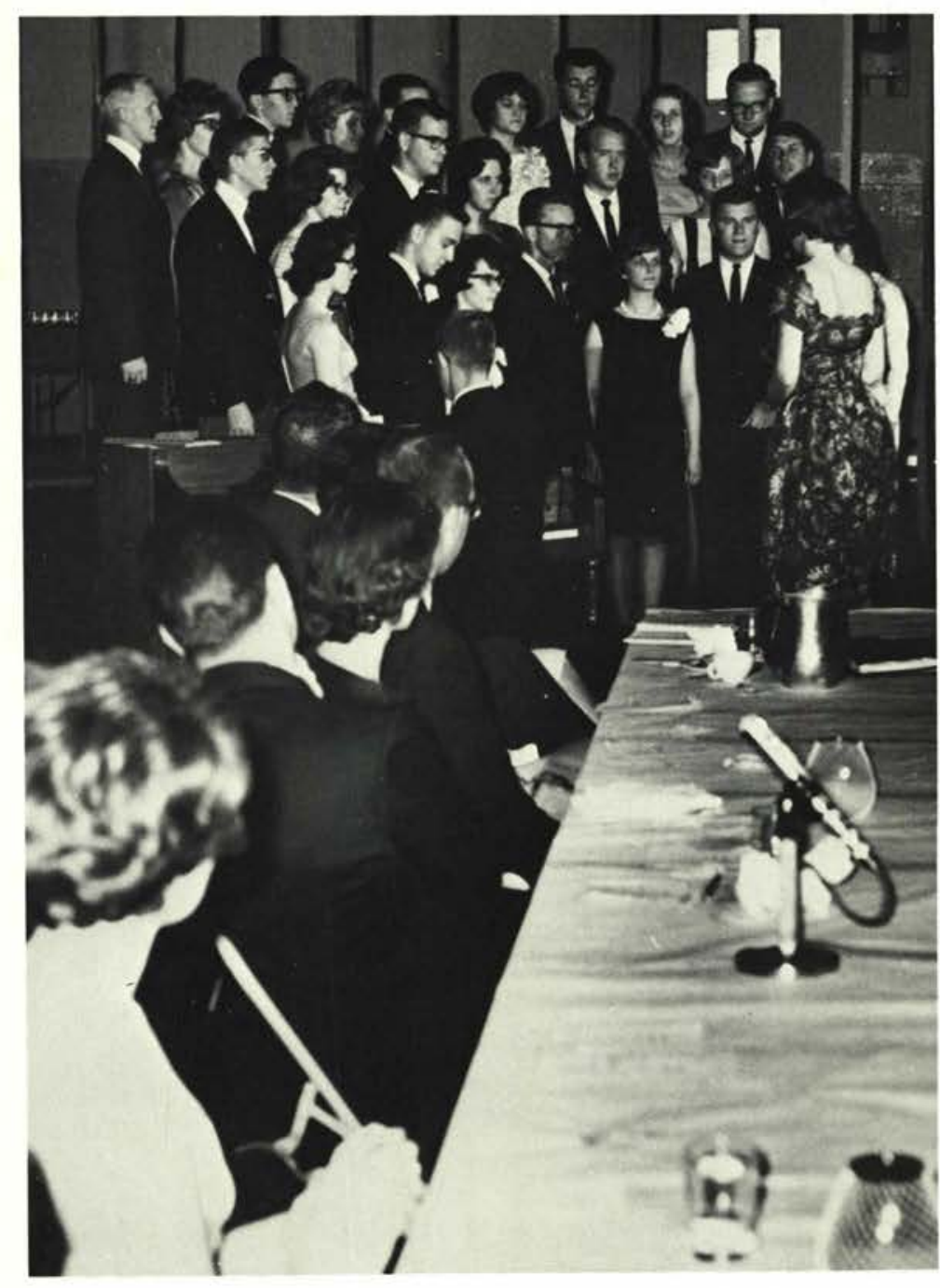

\section{Alpha Chi \\ Presents ...}

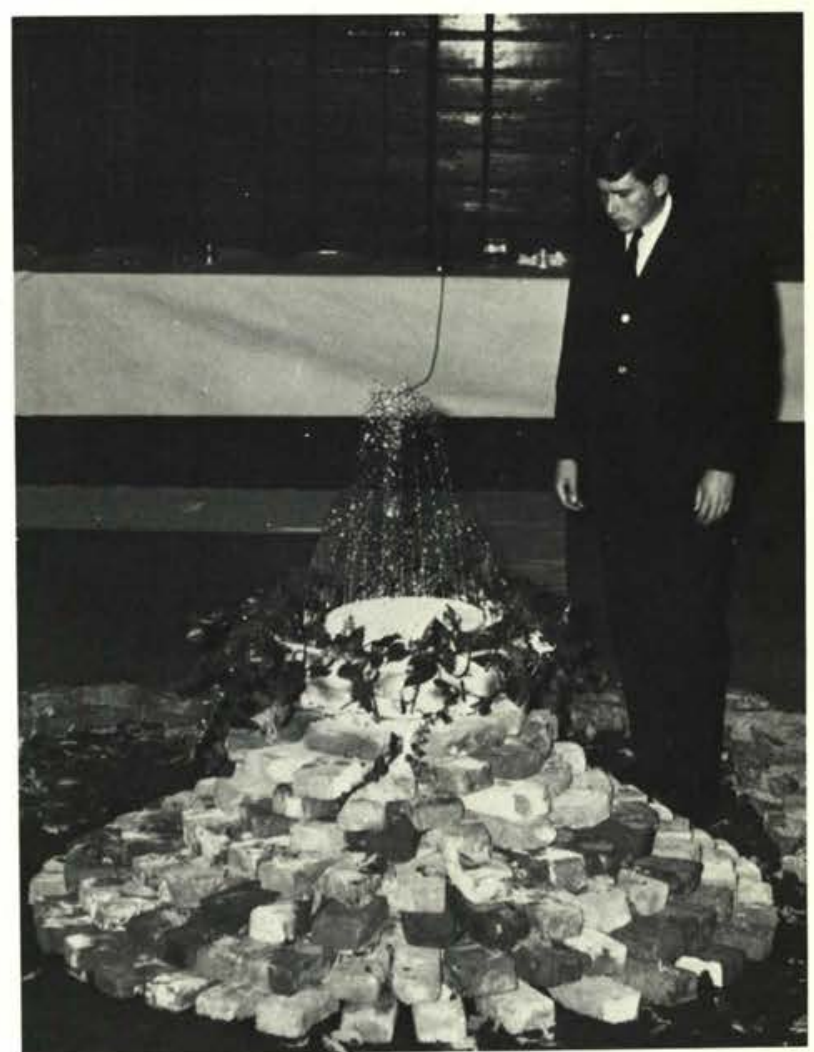

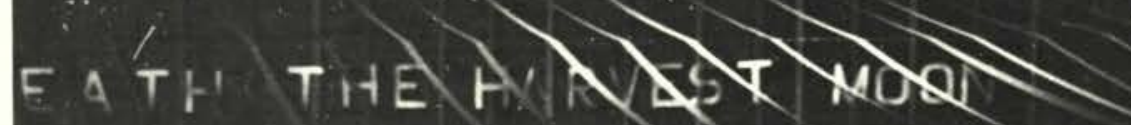
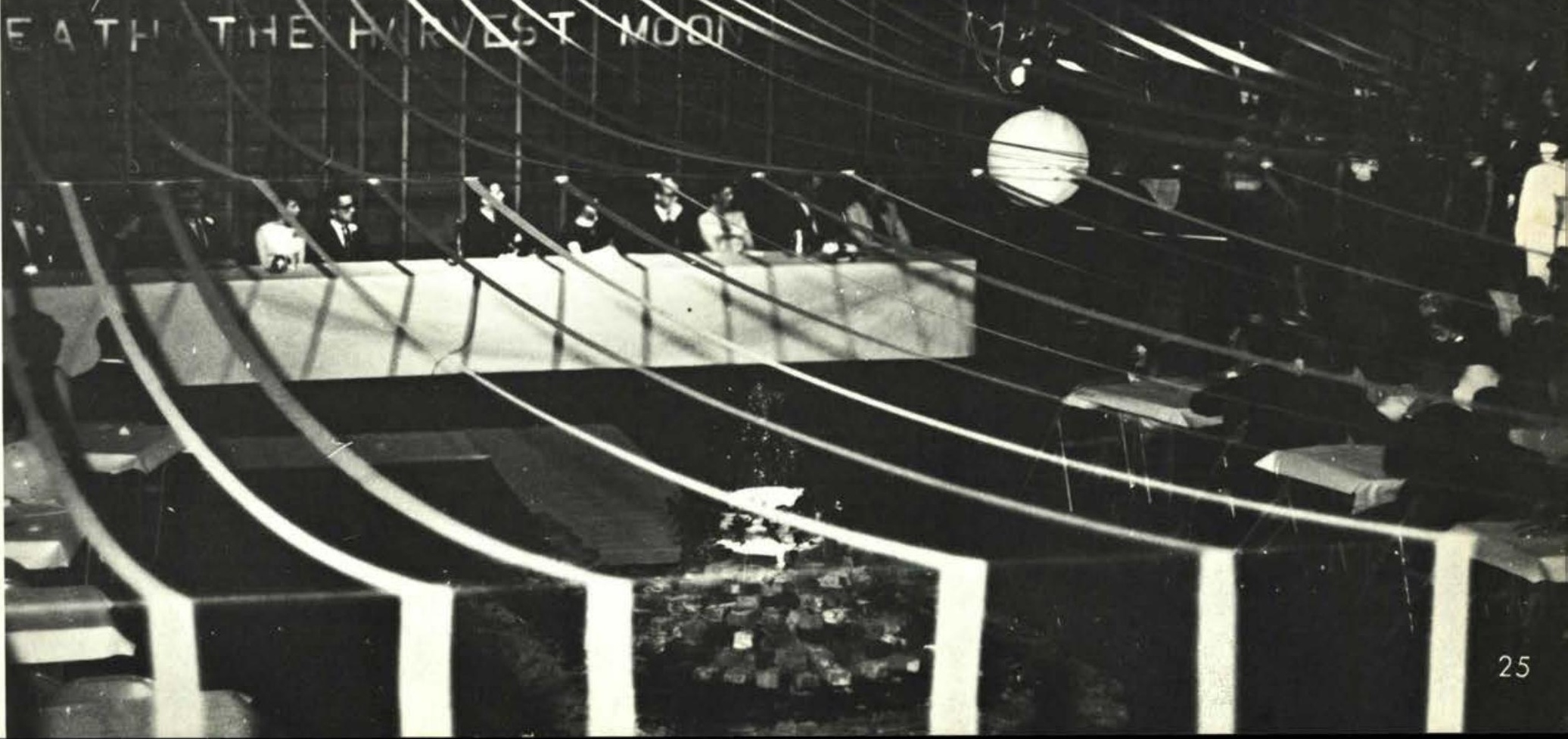


\section{ADMINISTRATION}

\section{TRUSTEES}

Dr. George S. Milner, Chairman; Rev. William Brock, Vice-Chairman; Mr. George L. Boyd, Treasurer; Rev. Thomas Younger, Secretary.

Mr. Charles Barth

Mr. Rudy Bedford

Rev. William Brown

Dr. Jack Cline

Rev. Alfred Colwell

Rev. Glenn H. Davis

Mr. John A. Draxler

Mr. Arthur Dyke

Mr. Vincent Gregg

Mr. Roy Guenin

Mr. Hugh T. Hall

Dr. James T. Jeremiah
Mr. J. Dale Murphy

Mr. George O'Bryan

Rev. Irwin Olson

Mr. William B. Patterson

Mr. James Richardson

Rev. Wilbur Rooke

Rev. Gerald V. Smelser

Rev. Robert Sumner

Rev. Earl Umbaugh

Rwv. Earl Willets

Rev. Donald Woodby

PRESIDENT - Dr. James T. Jeremiah.
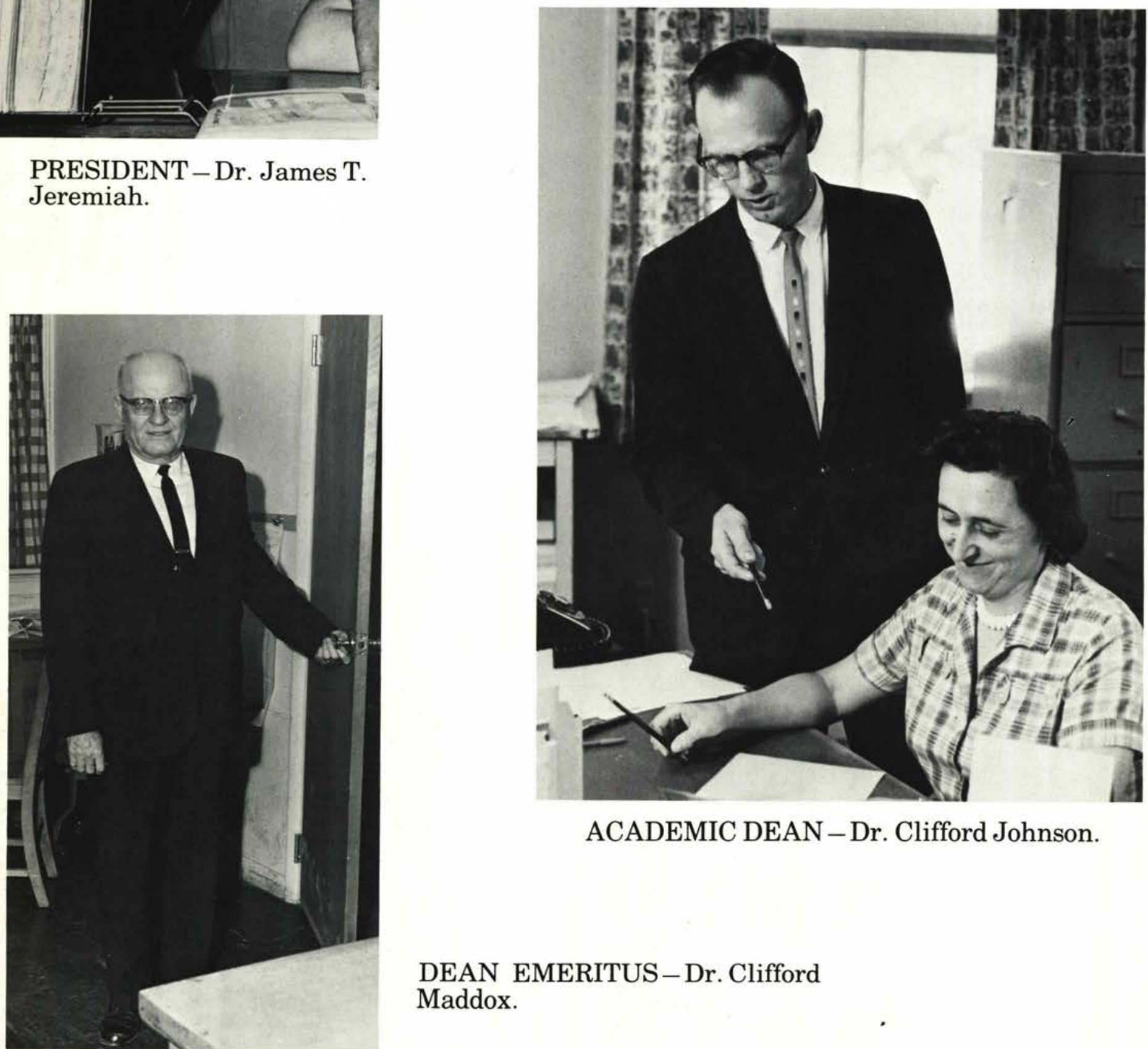

ACADEMIC DEAN - Dr. Clifford Johnson.

DEAN EMERITUS-Dr. Clifford Maddox. 


\section{Campus Cooperation}

Perhaps the most important organization on campus, the Student Council provides the link between students and the administration. In addition to negotiating with the Administration and providing certain scholarships, Student Council also sponsers many service projects such as arranging for Bloodmobile visits, caring for the vending machines on campus, and contributing to the expenses of Christian Service groups.

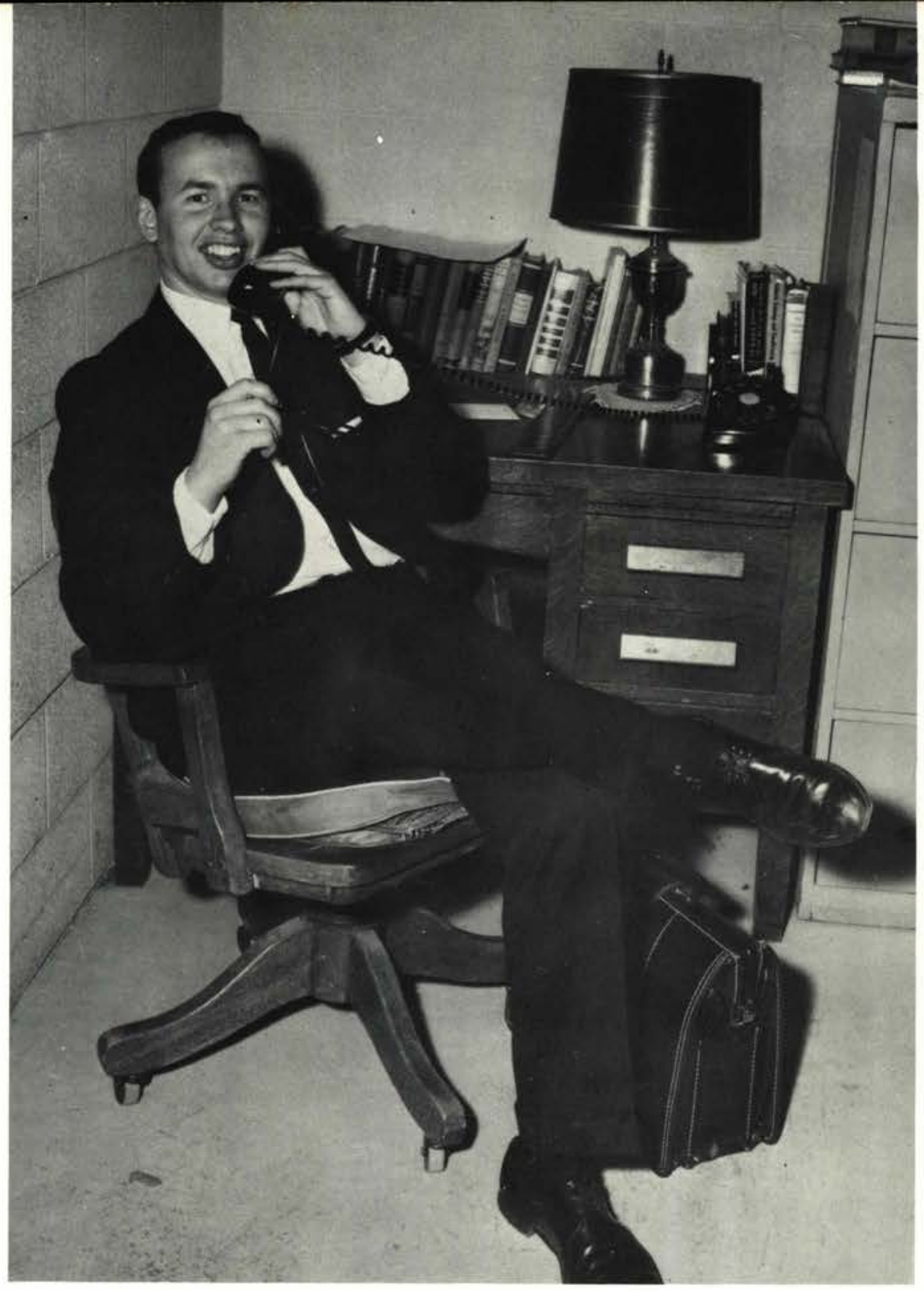

Ron McDugle-President.

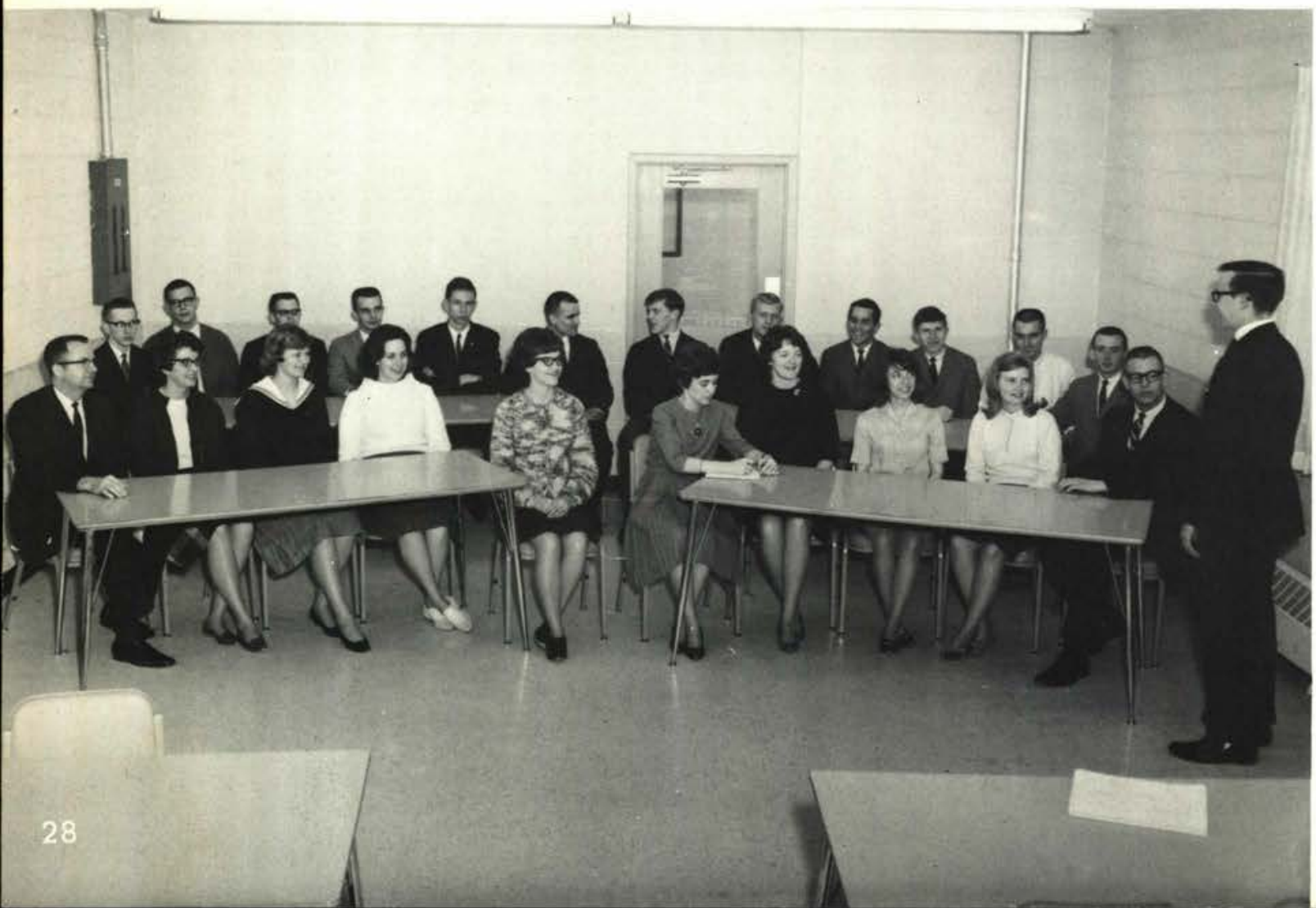

Front Row: Mr.McIntosh, Advisor; B. Beikert, C. Mikels, S. Eckert, J. Petrie, Treasurer; J. Ronk, Secretary; C. Brown, N. Stats, C. Payne, C. Wilkins, Vice-President; R. McDugle, President. BackRow: G. Griffin, T. Oakley, D. Bellew, J. Zeigler, M. Crampton, J. Walborn, J. Stow ll, V. Roloff, C. Truxton, J. Snyder, J. Branon, R. Grosh. 


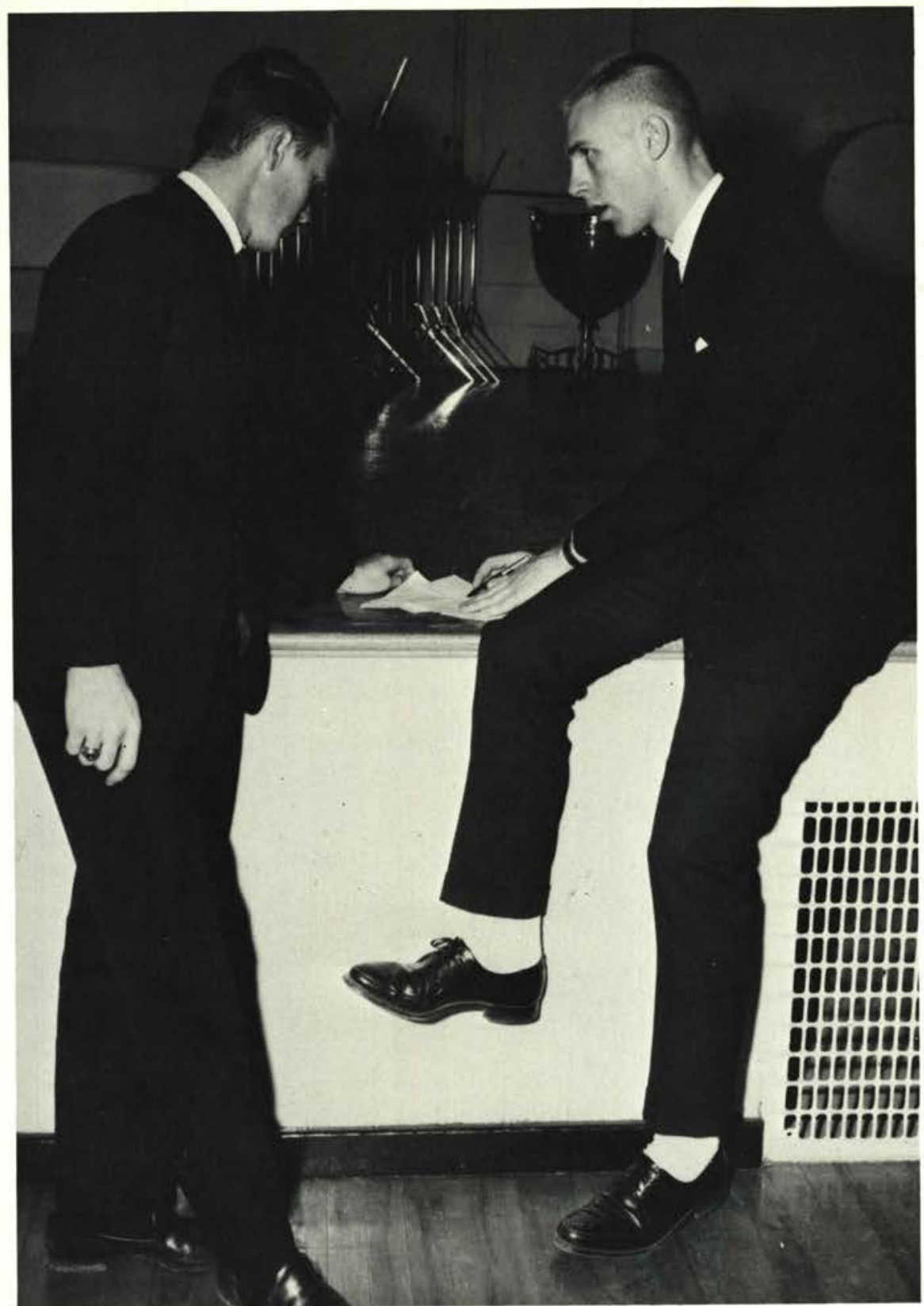

\section{Campus Improvement}

Each year the student body sets a goal for itself in order to purchase items to improve the campus. This year the goal was set for $\$ 1500$ to buy benches, bleachers, a piano, and a movie screen. Under the leadership of the co-chairmen of the project, the student body is stimulated to give through auctions, approved films shown on campus and other activities.

Duane Bales and Don Entner-Co-Chairmen

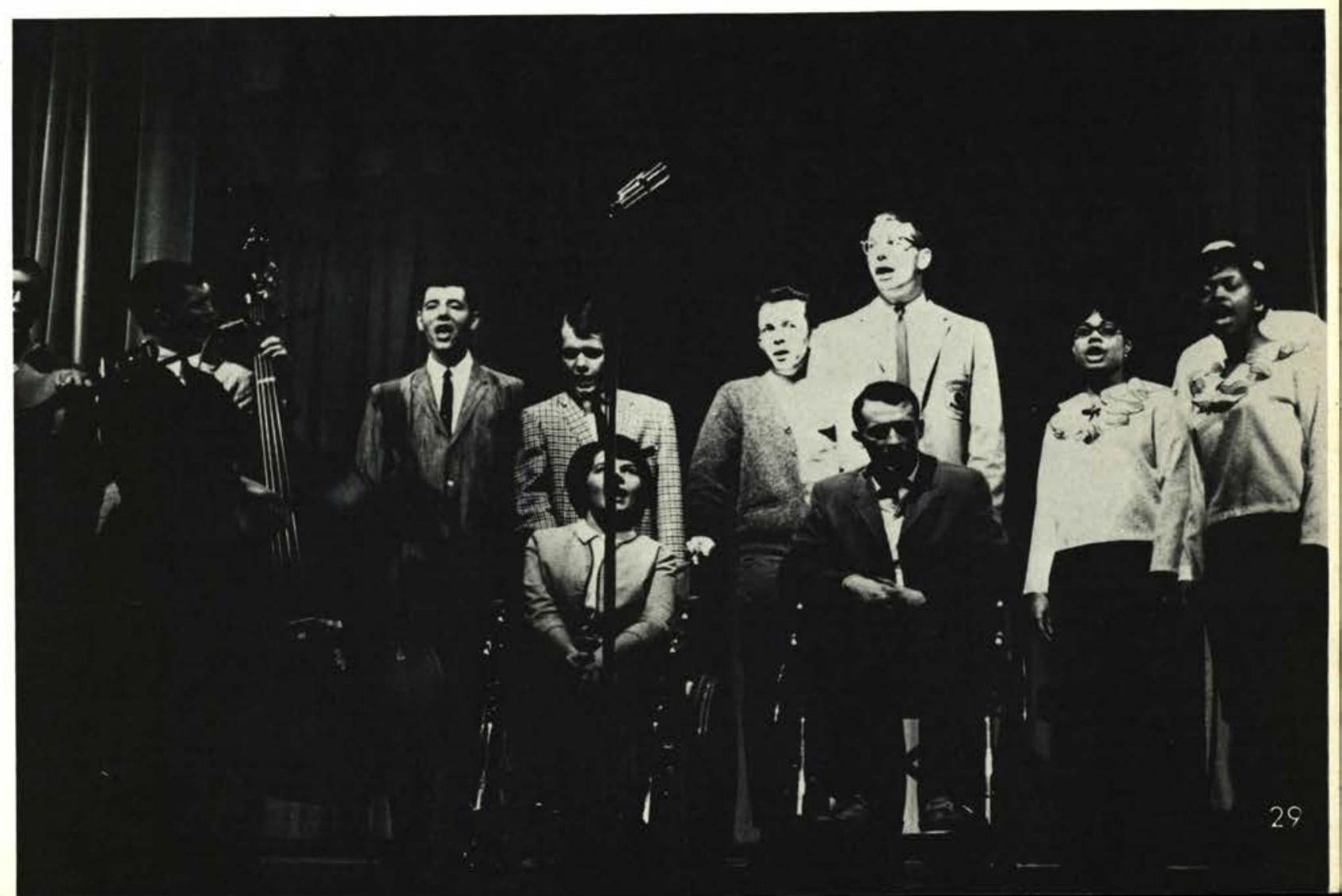




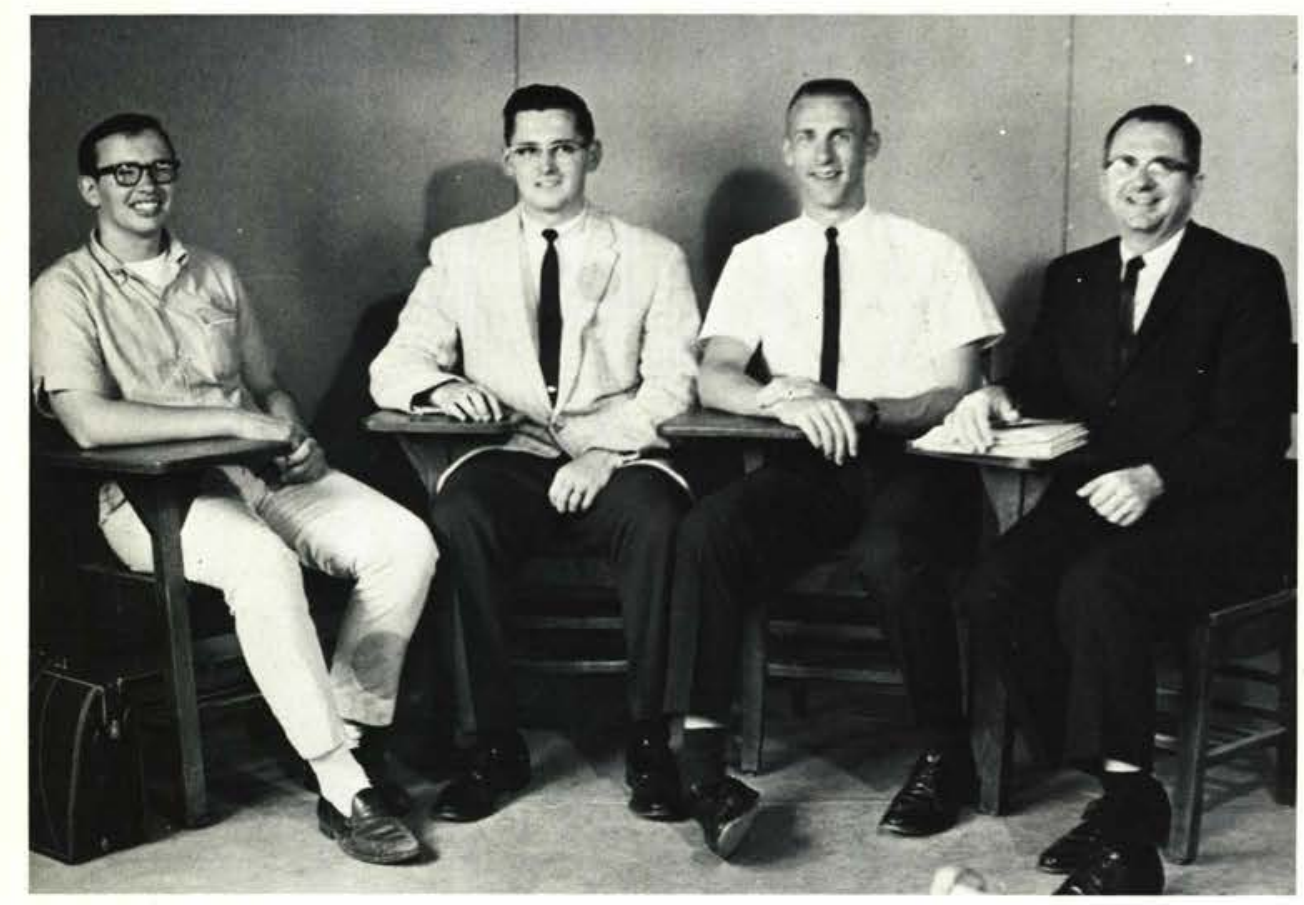

\section{Discipline Committee}

R. McDugle, Mr. Ager, D. Enter, Mr. McIntosh.

\section{Food Committee}

C. Brown, A. Sweeney, B. Beikert.
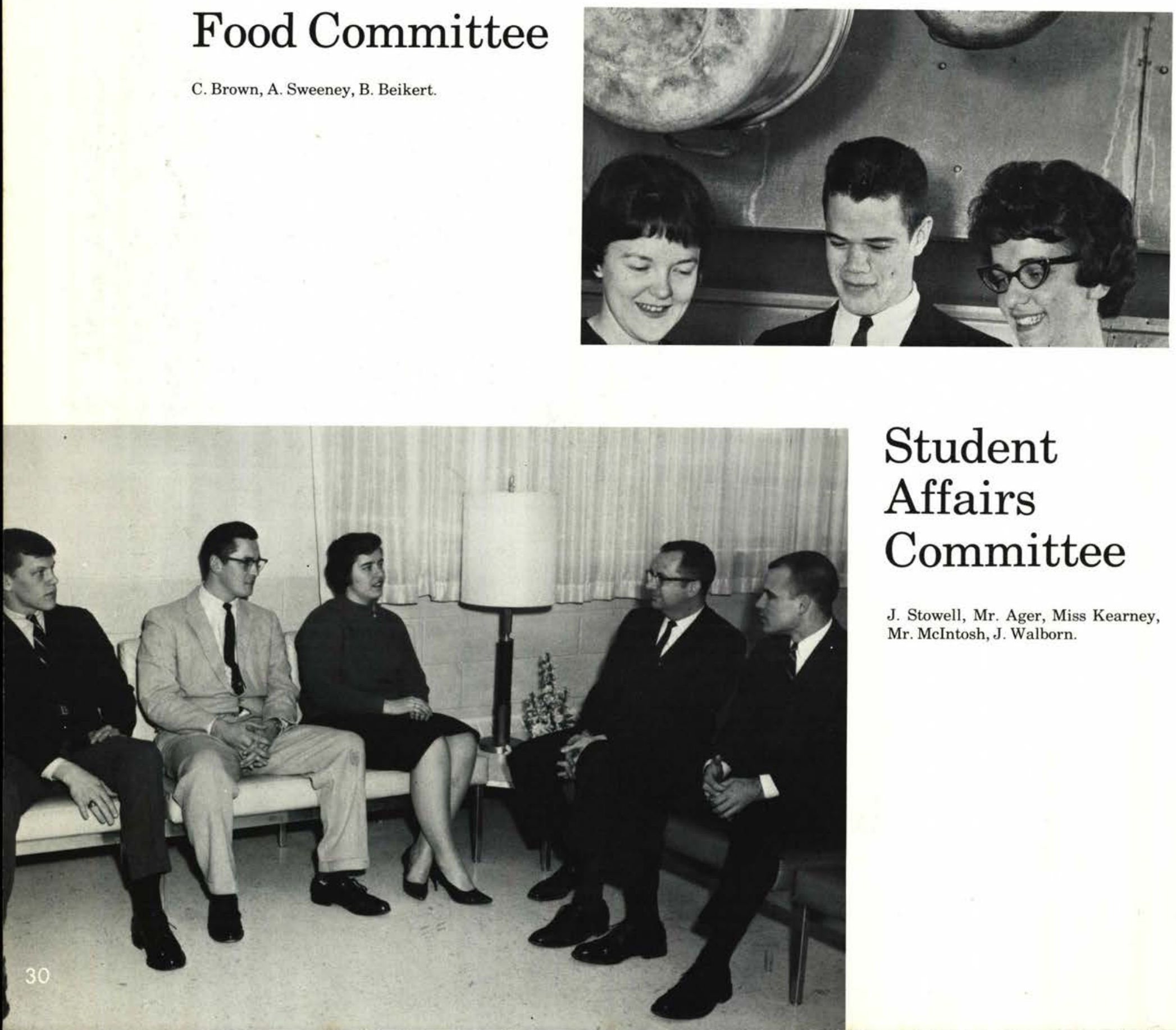

Student Affairs Committee

J. Stowell, Mr. Ager, Miss Kearney, Mr. McIntosh, J. Walborn. 


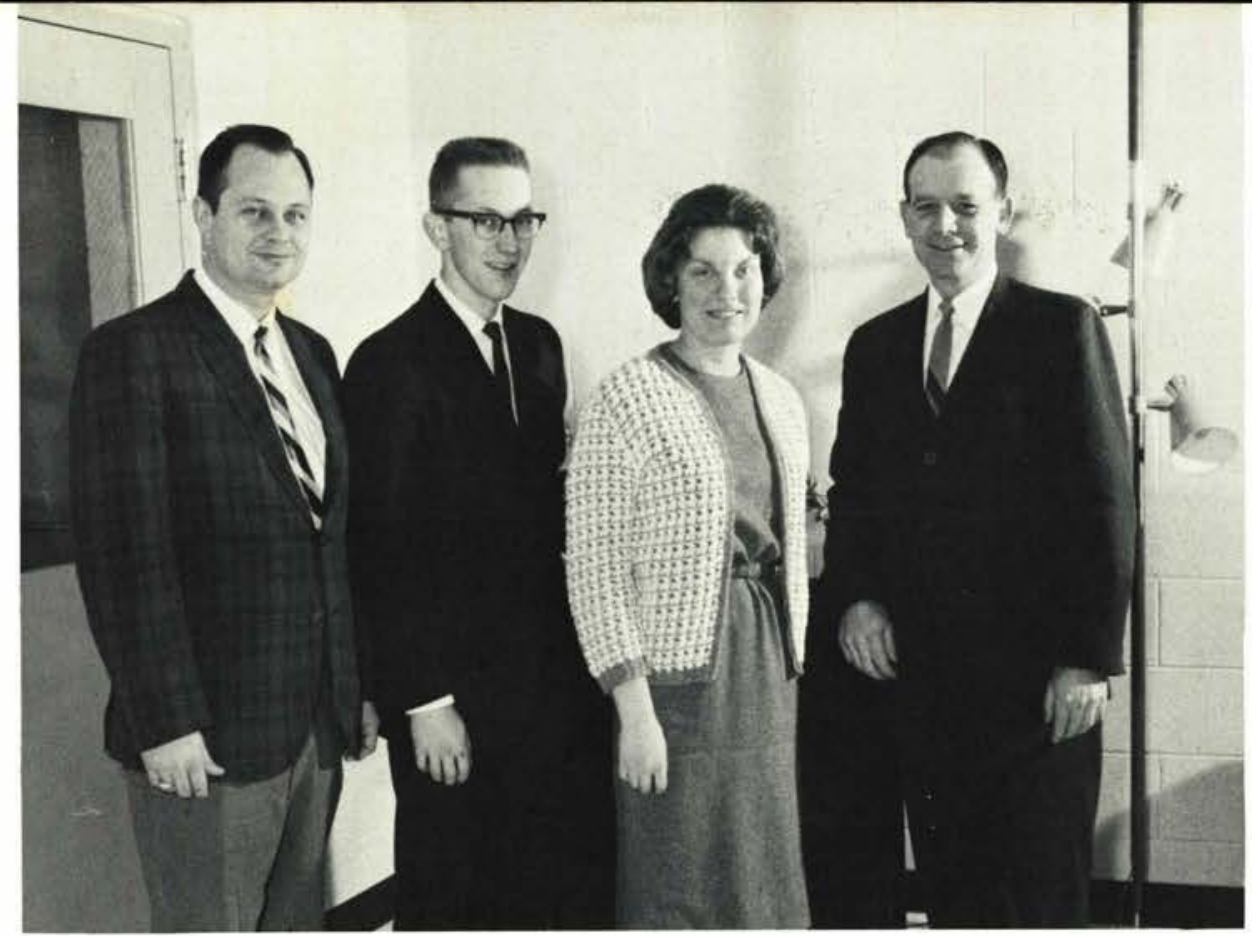

\section{Artist Series Committee}

Left to right: W. Meissner, G. Griffin, S. Byrd, W. Webber.

\section{Athletic \\ Committee}

Left to right: D. Wetzel, L. Turner, D. Callan.

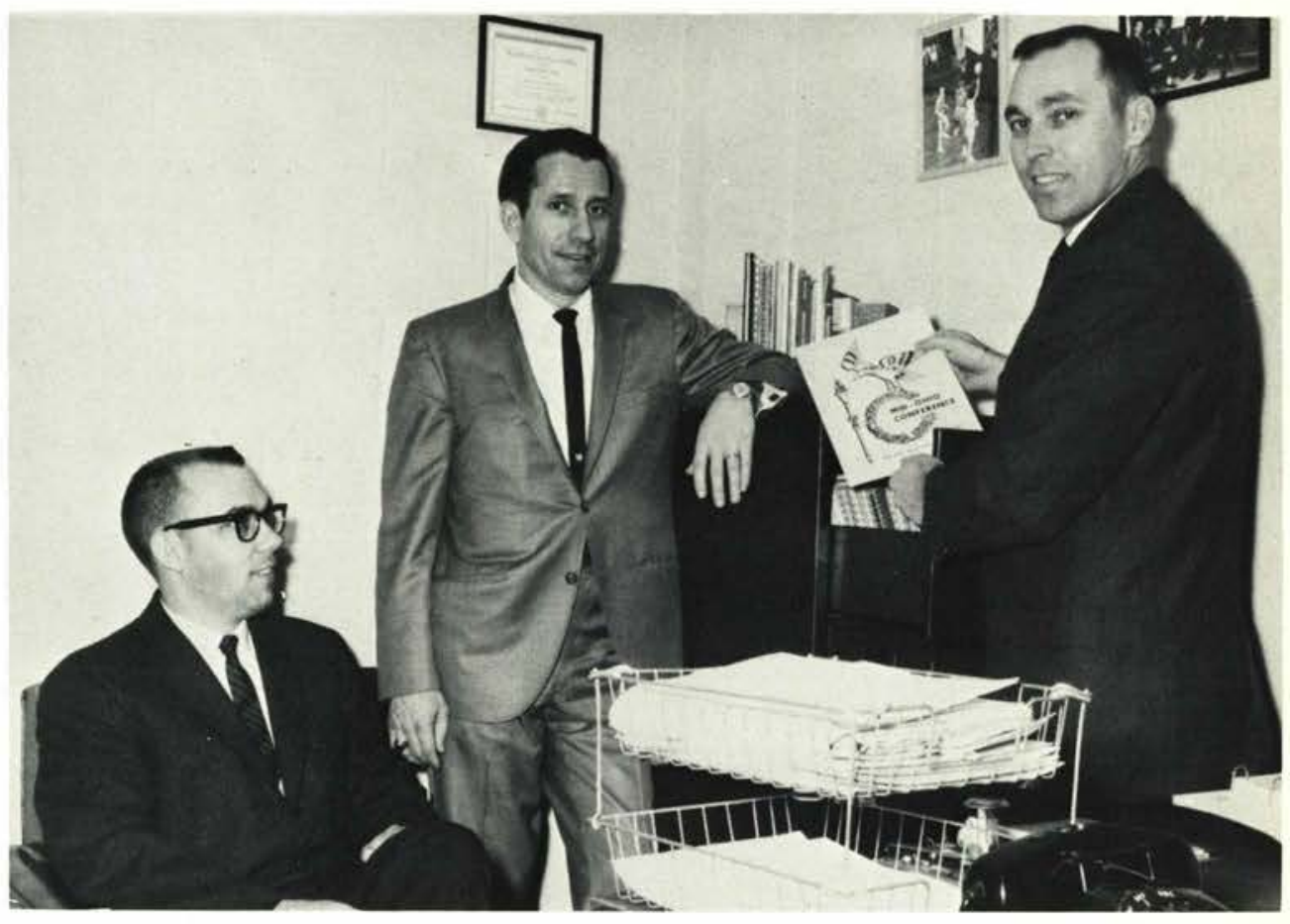

\section{Dorm Councillors}

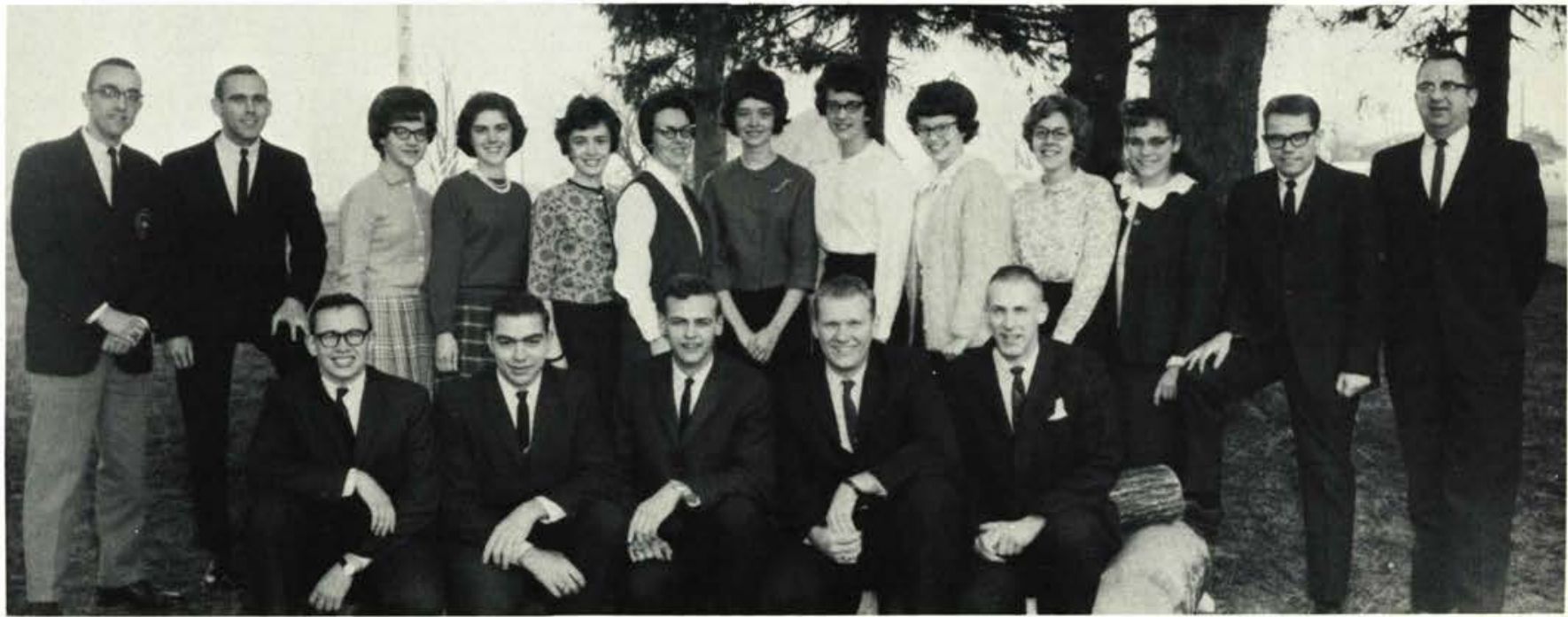

Kneeling: R. McDugle, G. Finley, G. Taylor, W. Kirtland, D. Entner. Standing: M. Stephens, D. Gordon, J. Petrie, J. VanHorn, J. Ronk, B. Murphy, B. Thomson, B. Beikert, L. Stutesman, R. O'Keefe, B. Zeigler, J. Roden, Mr. McIntosh. 

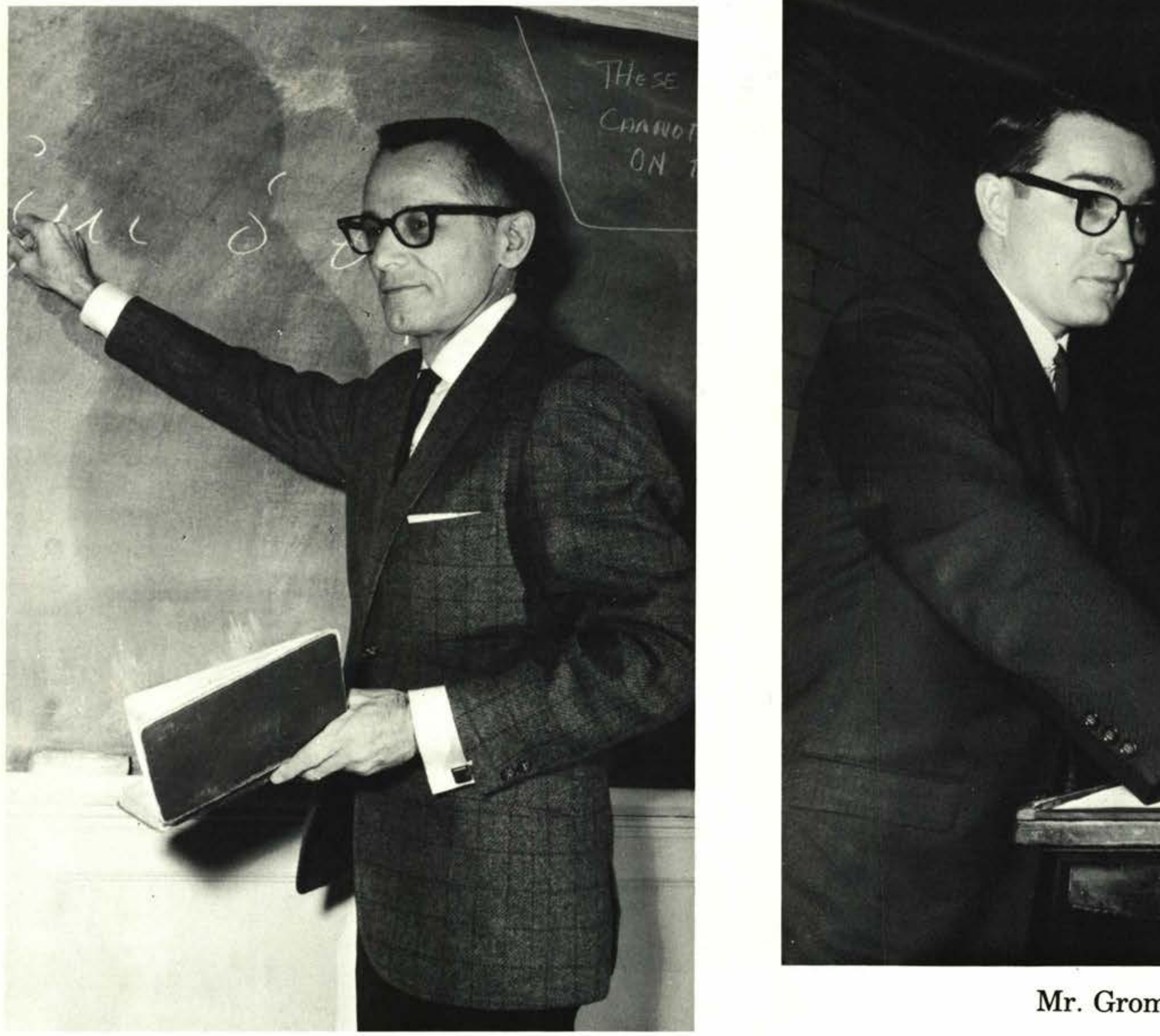

Dr. Lawlor

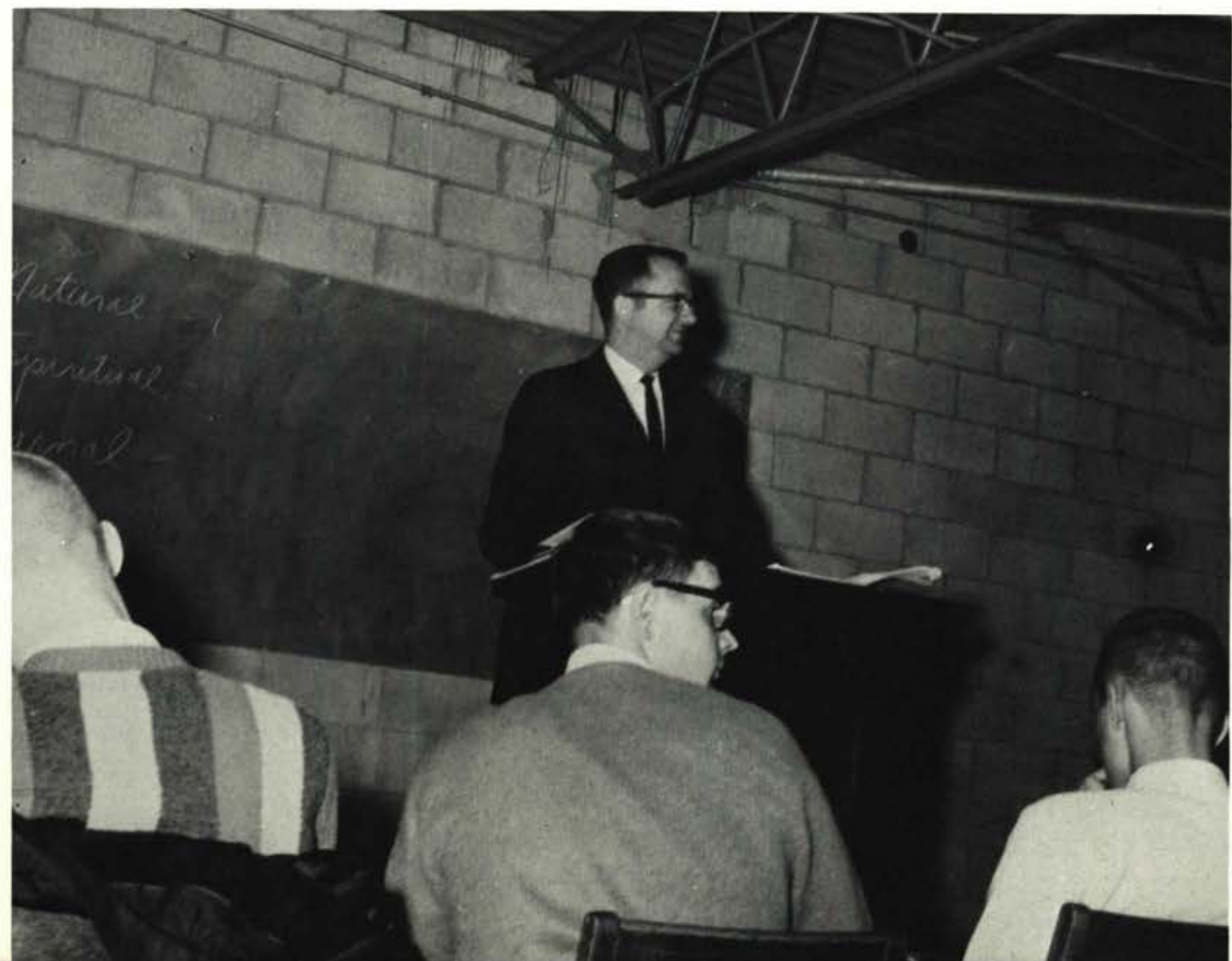

Mr. McIntosh 

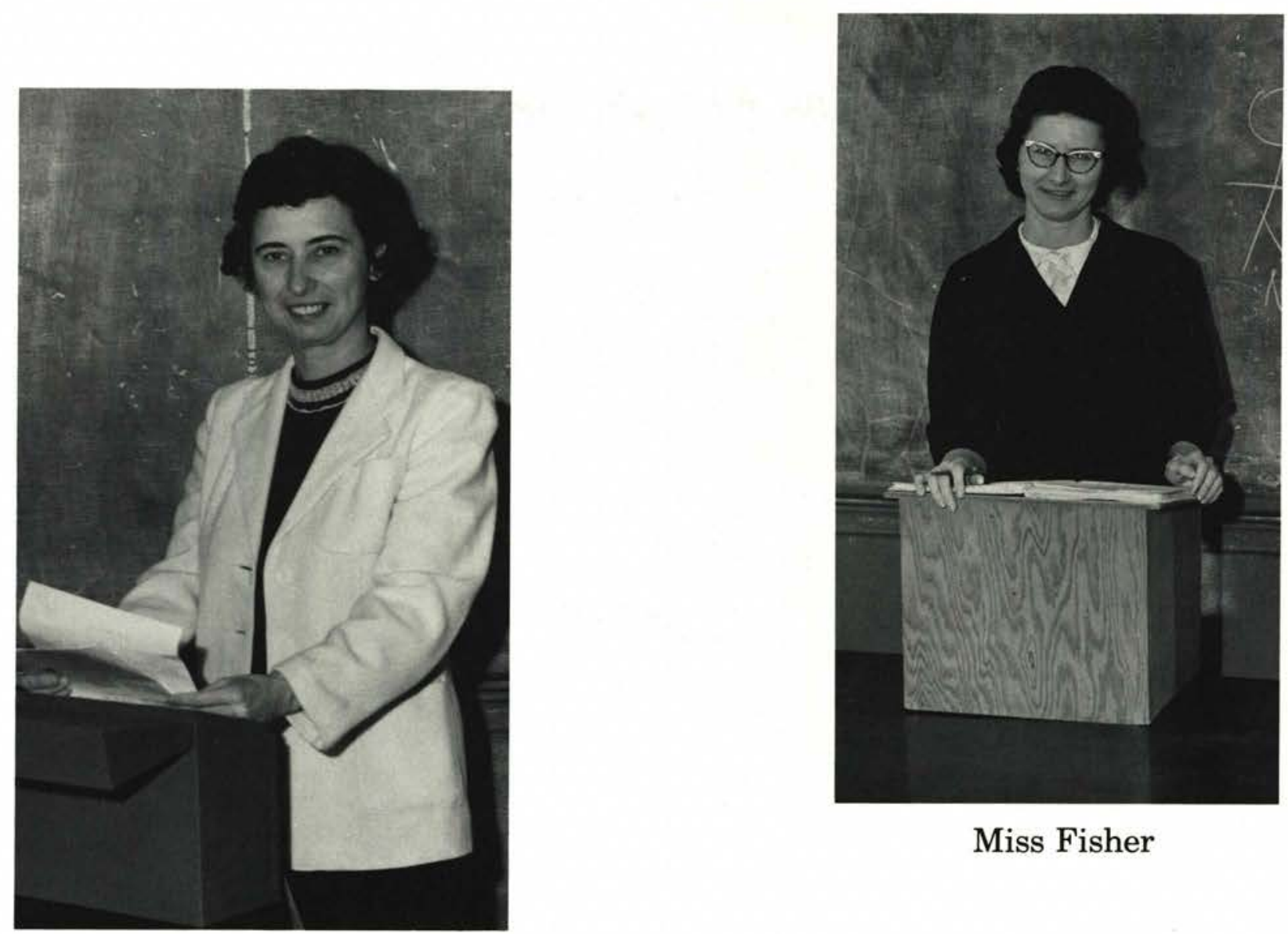

Miss Fisher

Miss Mackay

\section{Christian Education in Action}

One of the newer organizations on campus, Christian Ed. in Action, provides a place not only for learning about personal opportunities in this field but also for service to the school and area churches. In conjunction with the Music Department, the club presented the second annual Christian Education workshops program this Spring. Members also teach training classes for prospective teachers in the local churches.

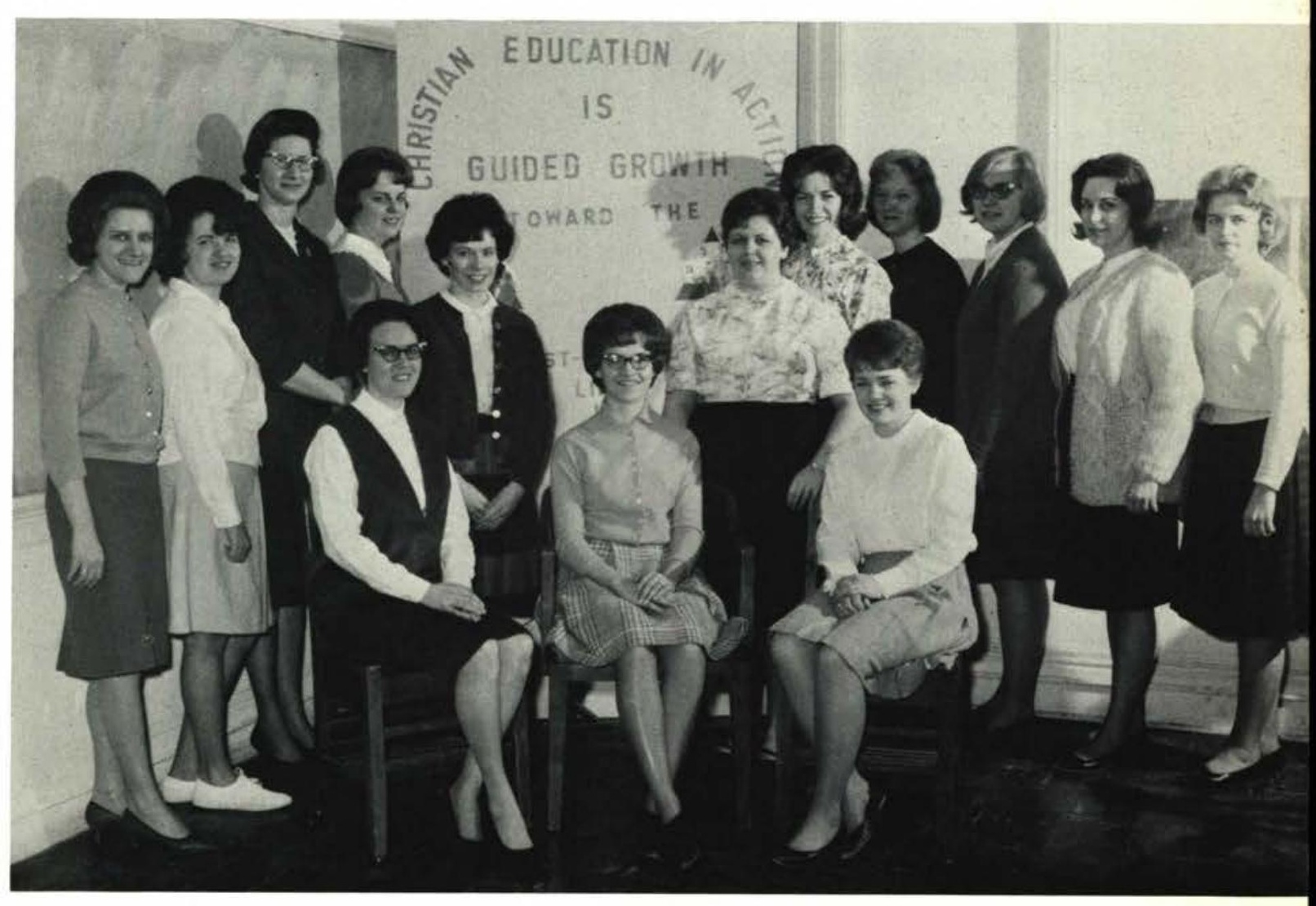

Back Row, left to right: D. Durkee, D. Simpson, Miss Fisher, D. Dunn, T. Addleman, T. Rudduck, P. Bird, B. Meyers, B. Carlson, S. Eckert, S. Dufford. Front Row: B. Murphy, T. Petrie, T. Emerson. 

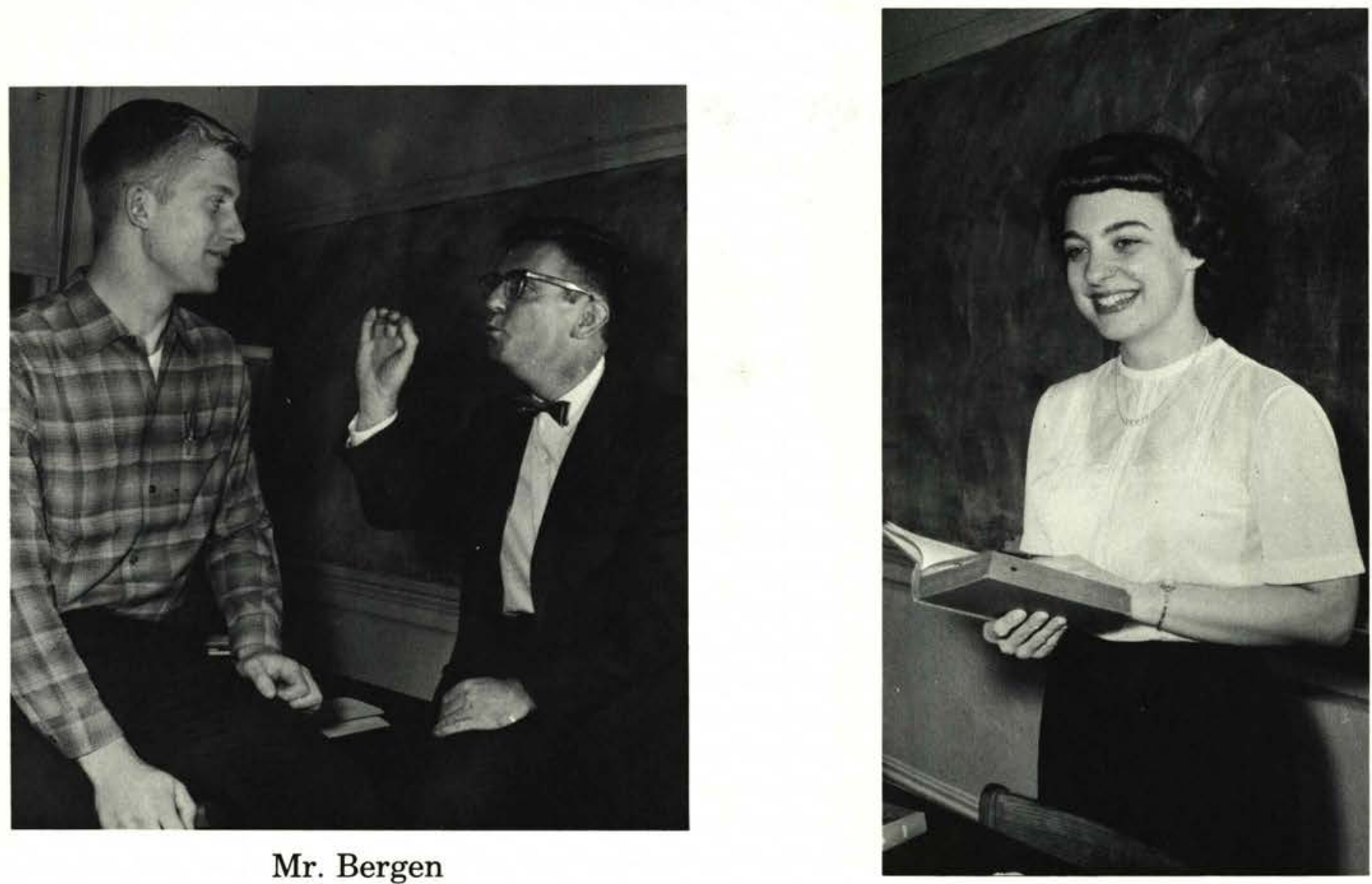

Mr. Bergen

Miss Packard

\section{Whispering Cedars}

WHISPERING CEDARS, the campus newspaper, is published regularly to serve as a means of communicating student thought and activities. Subscriptions were sold this year so that the paper now reaches many more people than it reached before. A helpful devotional column has also been included in each issue providing inspiration for many.

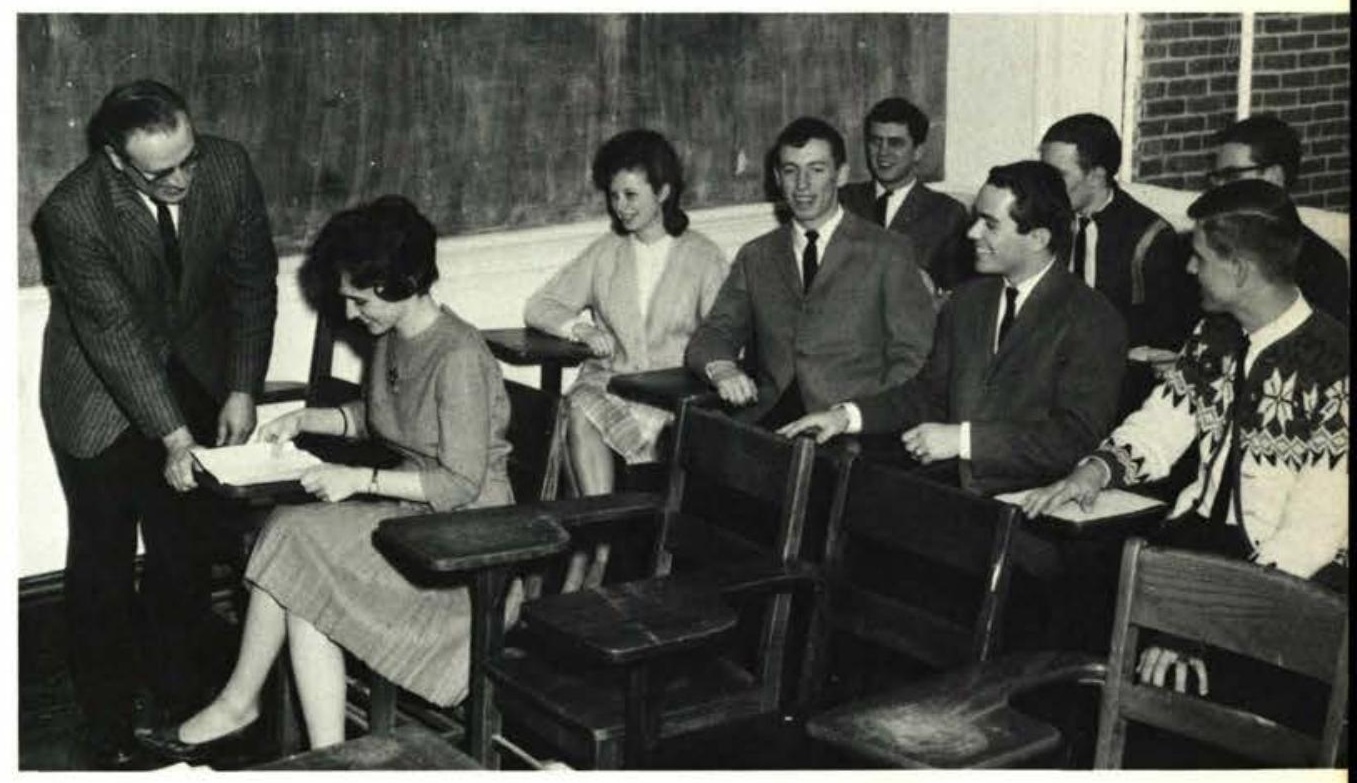

Mr. Greenwood and the Whispering Cedars staff.

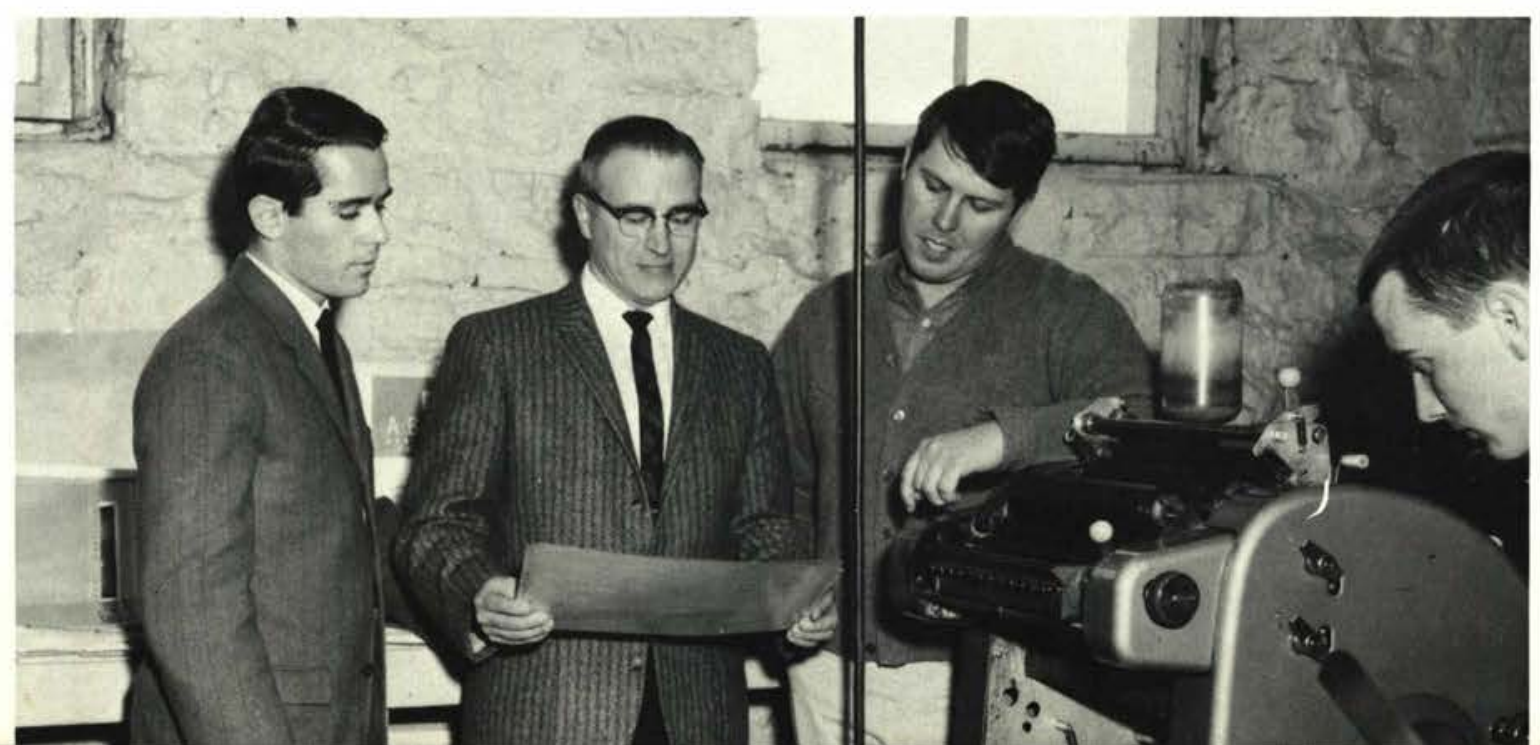

Left to right: Chuck Truxton, Mr. Greenwood, and Mike Kellogg. 


\section{Miracle}

Careful planning and hard work go to produce the Miracle. These two things are necessary to give you a book that merits the name "Miracle" and one that clearly depicts Cedarville College-its people, activities, and the testimony it has through these things.

Left to Right: P. Brower, Editor; S. Lepine, Copy Editor; Mr. Spencer, Advisor; D. Pearce, Business Manager; S. Brown, C. Wilkins, Sports Editor; M. Woodend, R. Hartikainen, K. Farrell. 


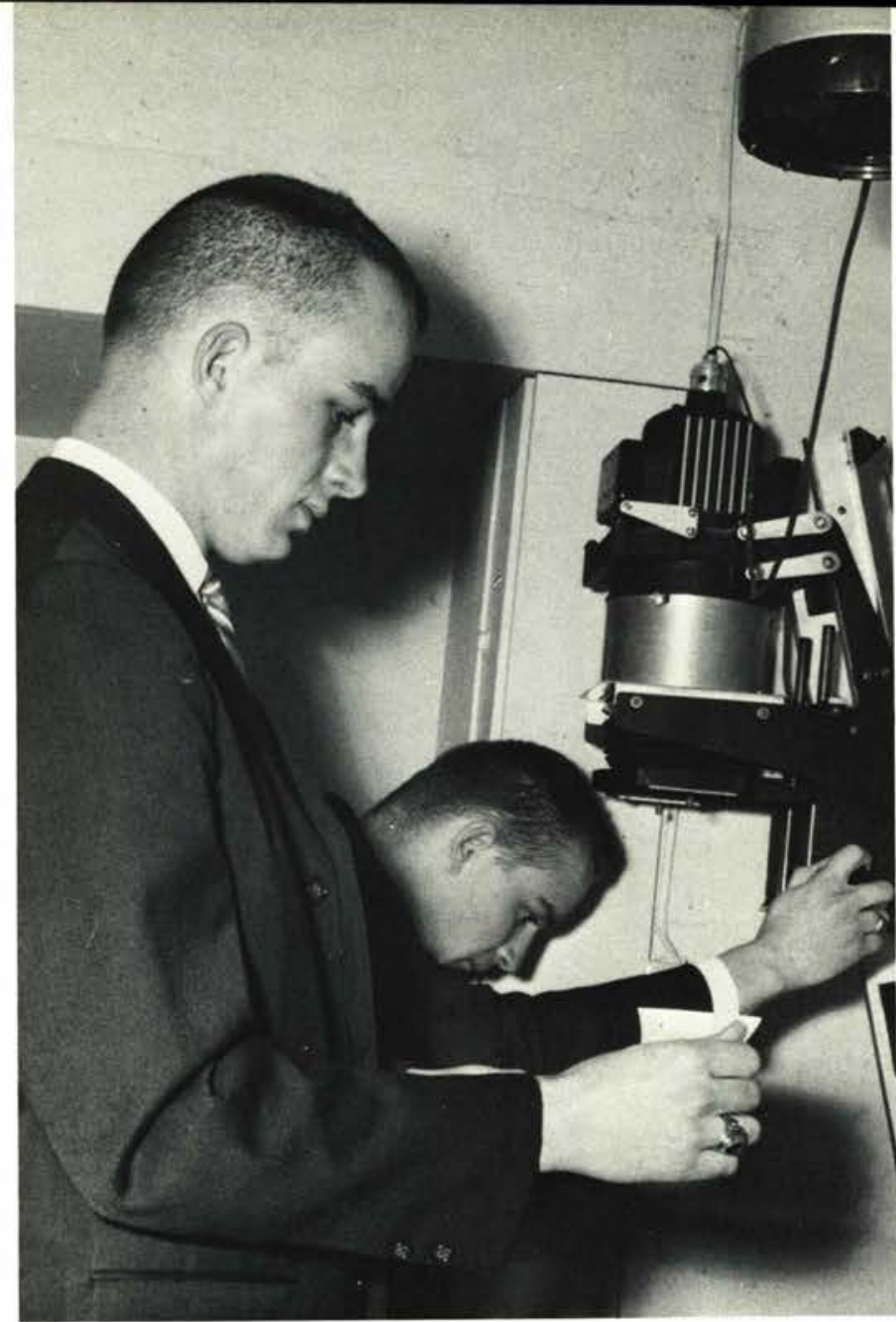

\section{Photo Club}

One of the main functions of the Photo Club is to provide pictures for various campus organizations and in particular, the yearbook. Many hours are spent in taking the pictures and processing the film. The finished product then appears in student publications and promotional literature for the school. 


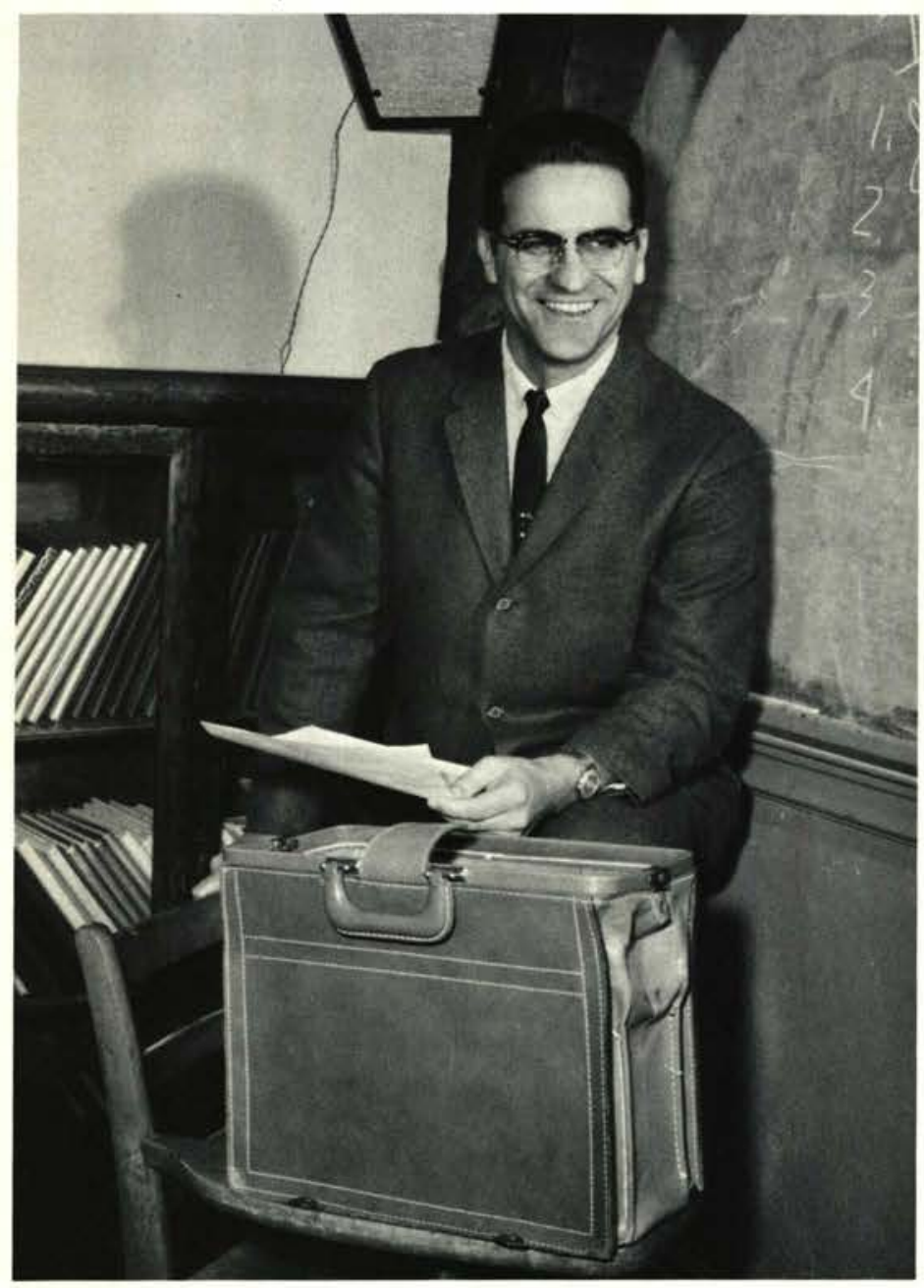

\section{WCDR-FM}

Broadcasting on 90.1 megacycles, WCDR

functions as a practical laboratory for students

in radio broadcasting. The station operates on

an educational, non-commercial basis, pro-

viding programming for the campus and com-

munity. Programming is geared to the areas of education, religion, public service, public relation, and entertainment.

Mr. John Reed

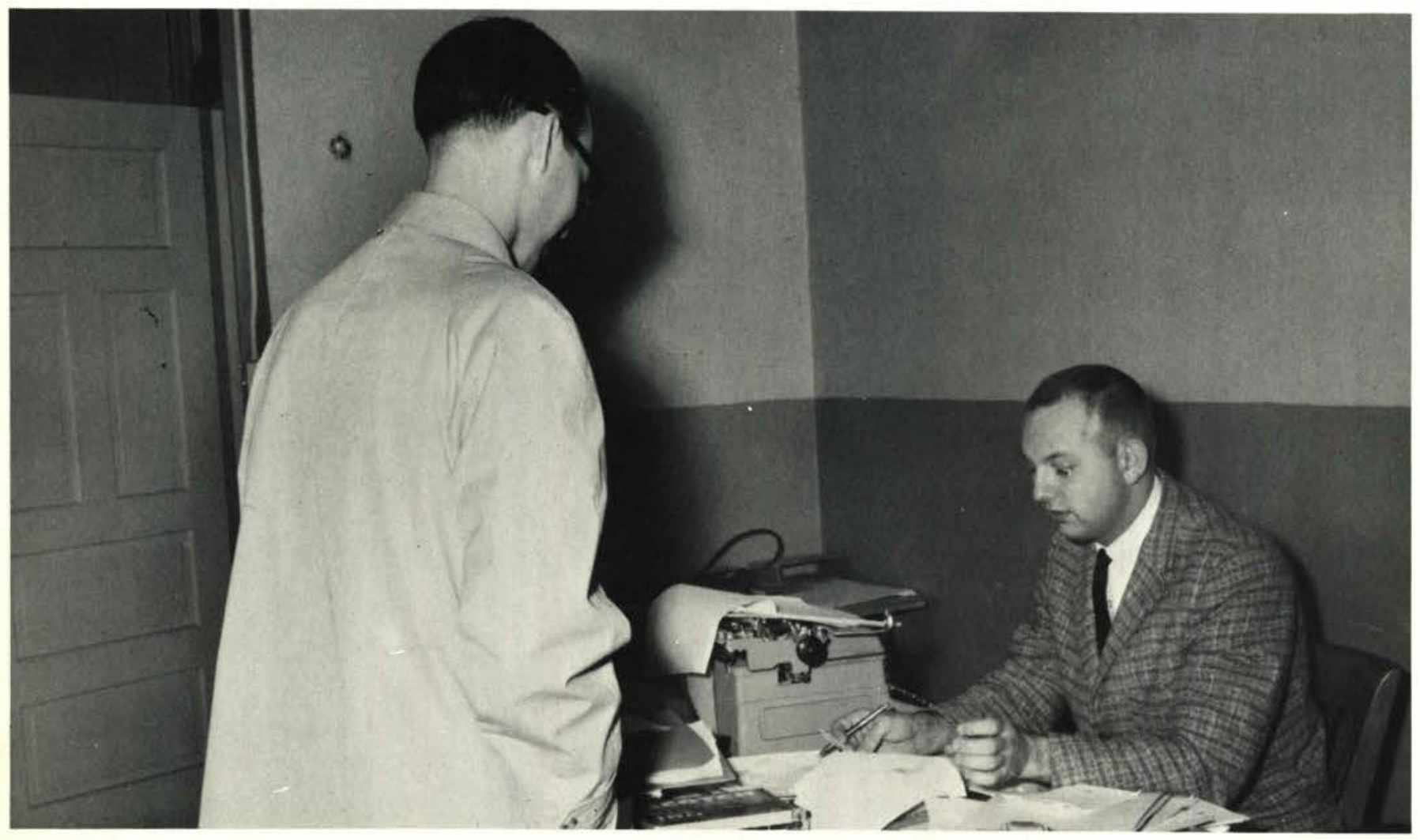

Mr. Paul Gathany 


\section{Sock 'n' Buskin}

Using traditional dramatic symbols for their club title, Sock 'n' Buskin is made up of those who have participated in campus dramatic productions. Members of this organization do most of the behind-the-scenes work on the annual plays and this year on the movie that is being filmed on campus.
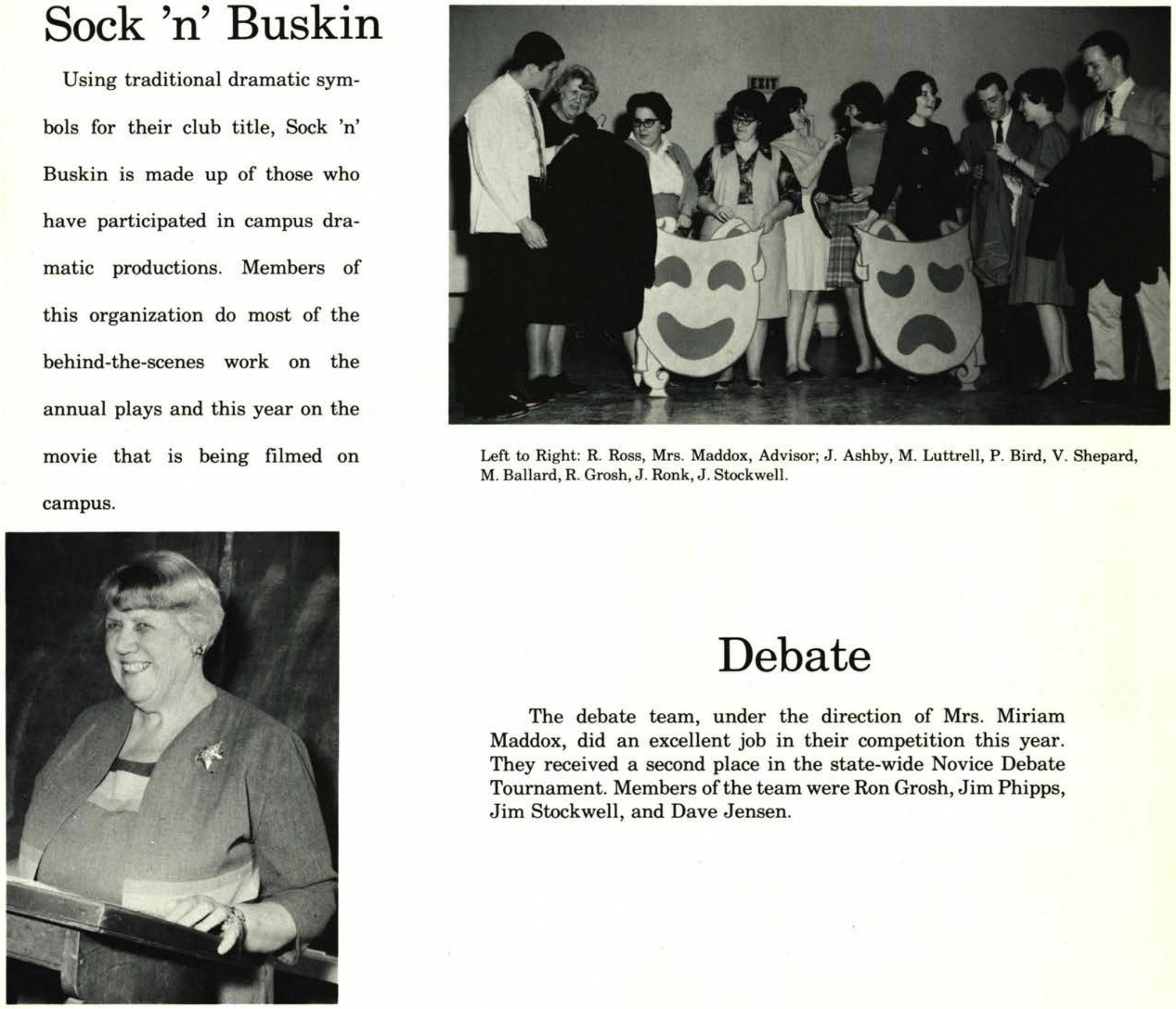

Left to Right: R. Ross, Mrs. Maddox, Advisor; J. Ashby, M. Luttrell, P. Bird, V. Shepard, M. Ballard, R. Grosh, J. Ronk, J. Stockwell.

\section{Debate}

The debate team, under the direction of Mrs. Miriam Maddox, did an excellent job in their competition this year. They received a second place in the state-wide Novice Debate Tournament. Members of the team were Ron Grosh, Jim Phipps, Jim Stockwell, and Dave Jensen.

Mrs. Maddox

NEGATIVE:

D. Jensen

J. Stockwell

AFFIRMATIVE:

R. Grosh

J. Phipps

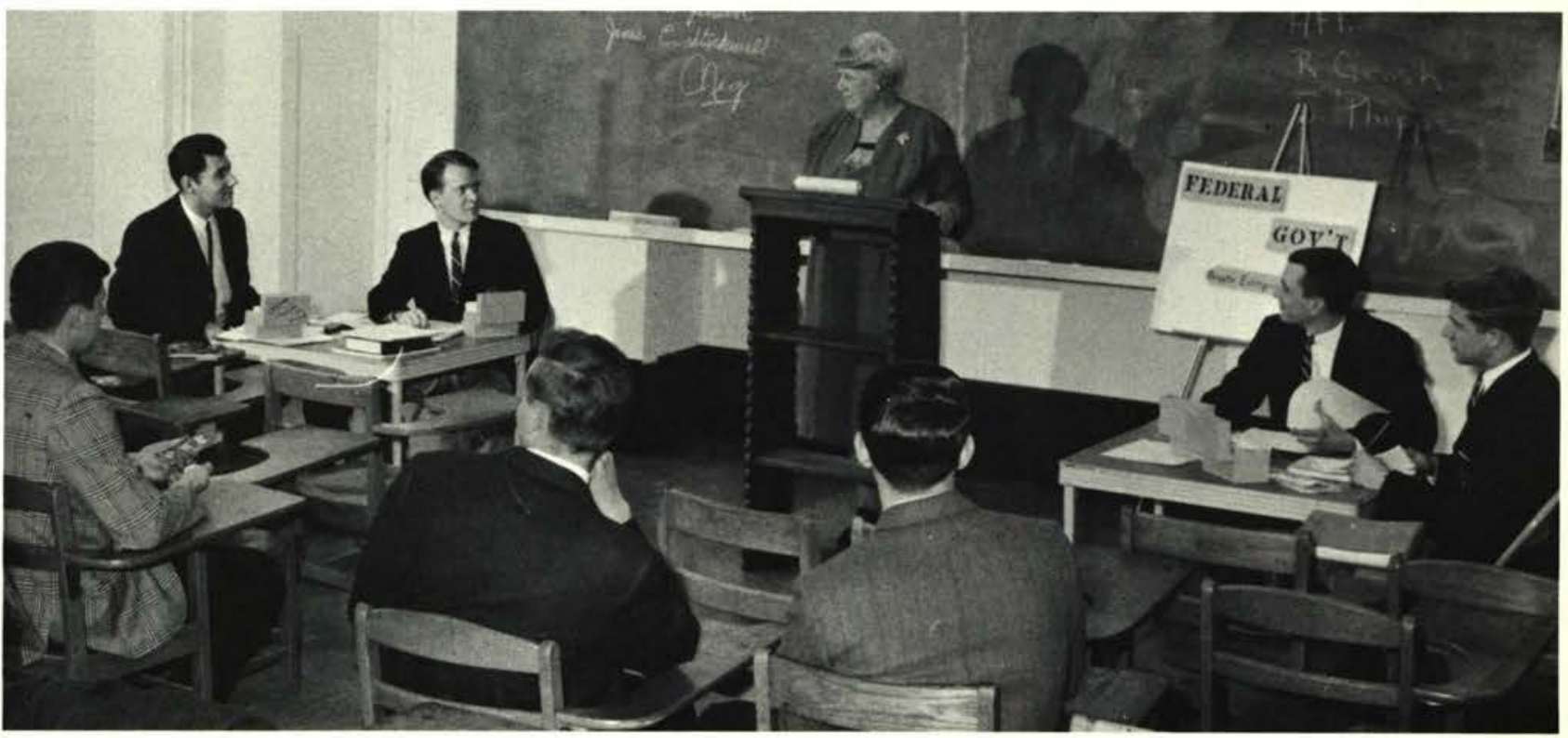




\section{SCIENCE}

"Science is vastly more stimulating to the imagination than are the classics."

- Haldane

Science is not just a static collection of facts and formulas. It is a dynamic study-one whose doors open to those who are willing to explore it. In the Science Department, math and science students are stimulated to delve beyond the formulas and equations to see why things work and behave as they do. This pursuit ultimately leads them to see God working in and through His universe; thus, strengthening their Christian faith.

\section{Mr. Elmore}

Dr. Baumann
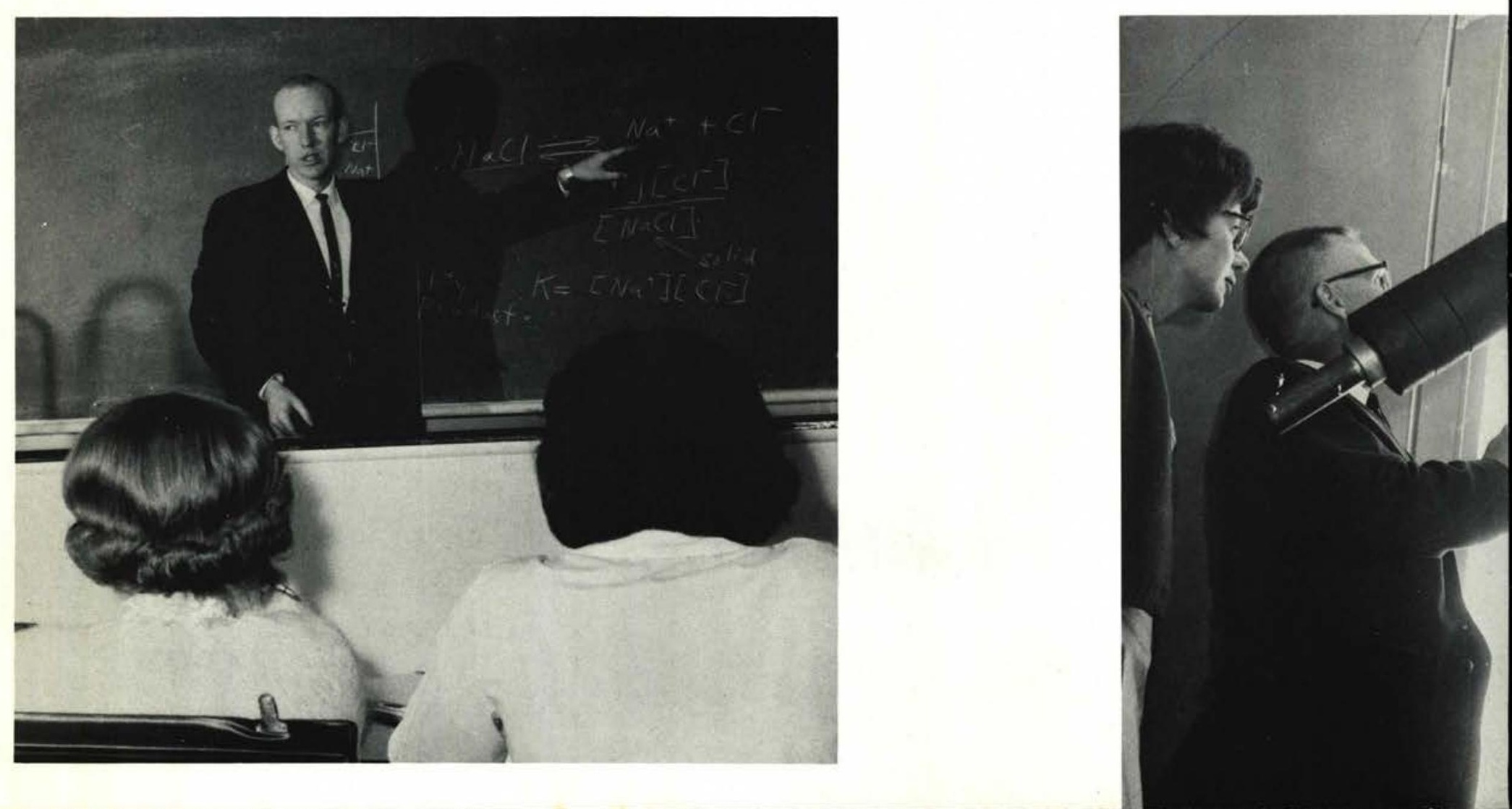


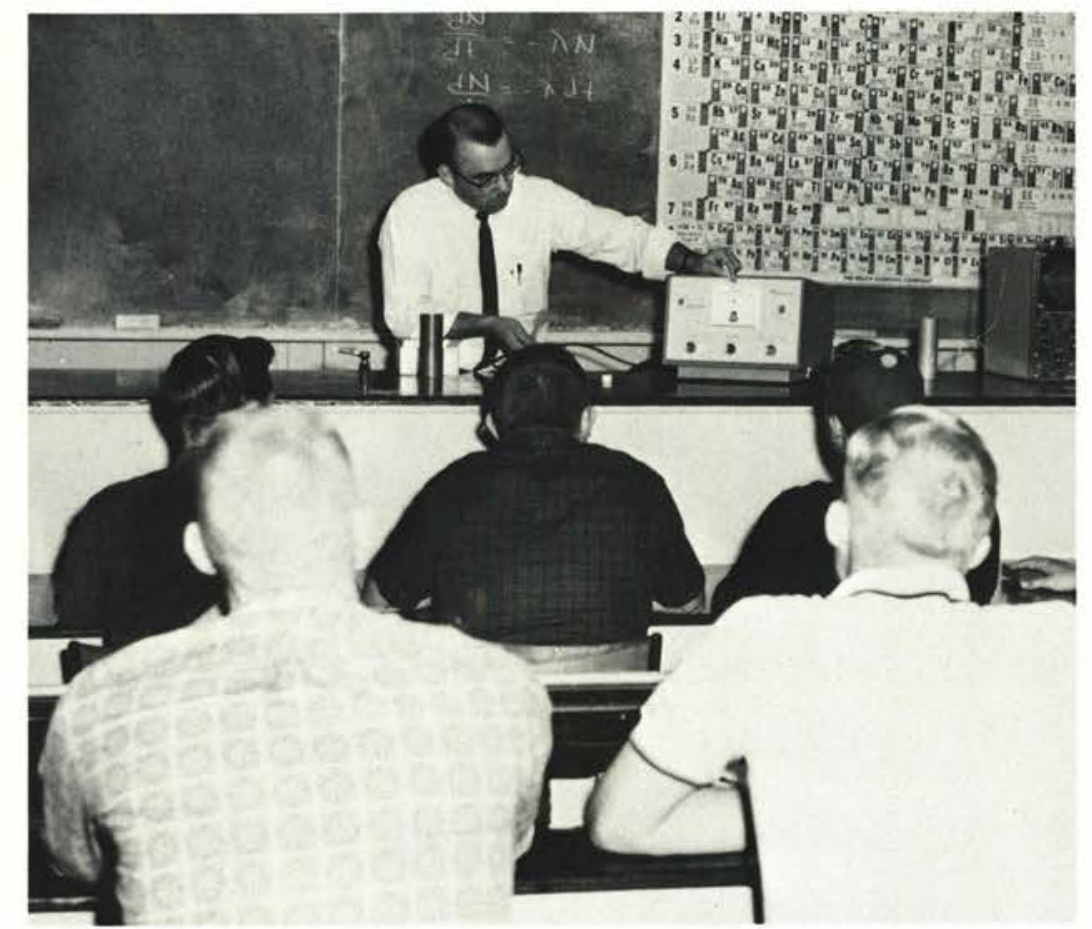

Mr. Wetzel
Left to Right: Marv Stephens, Jan Addleman, Kathie Schwebel, Mr. Elmore, Bill Kirtland.

\section{Pi Delta}

Mr. Frye

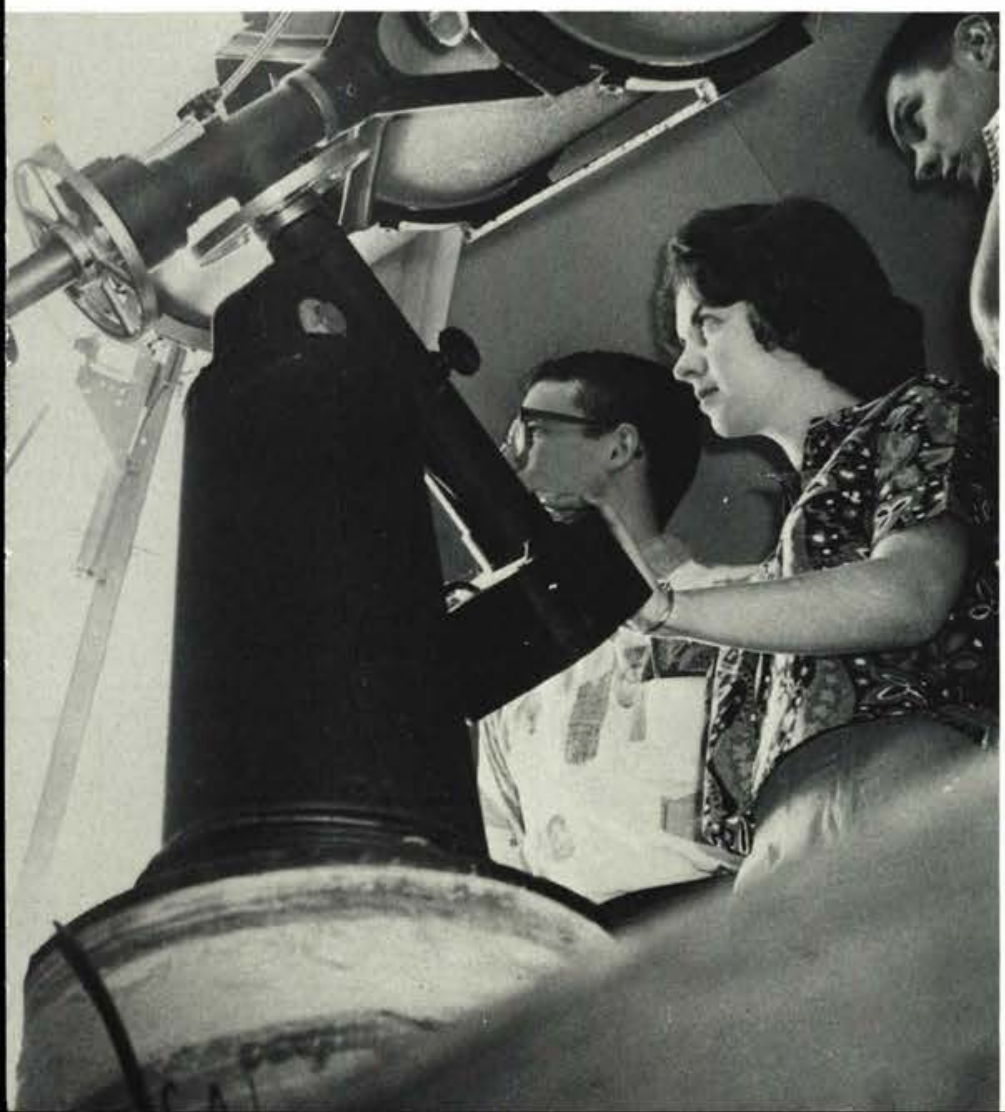

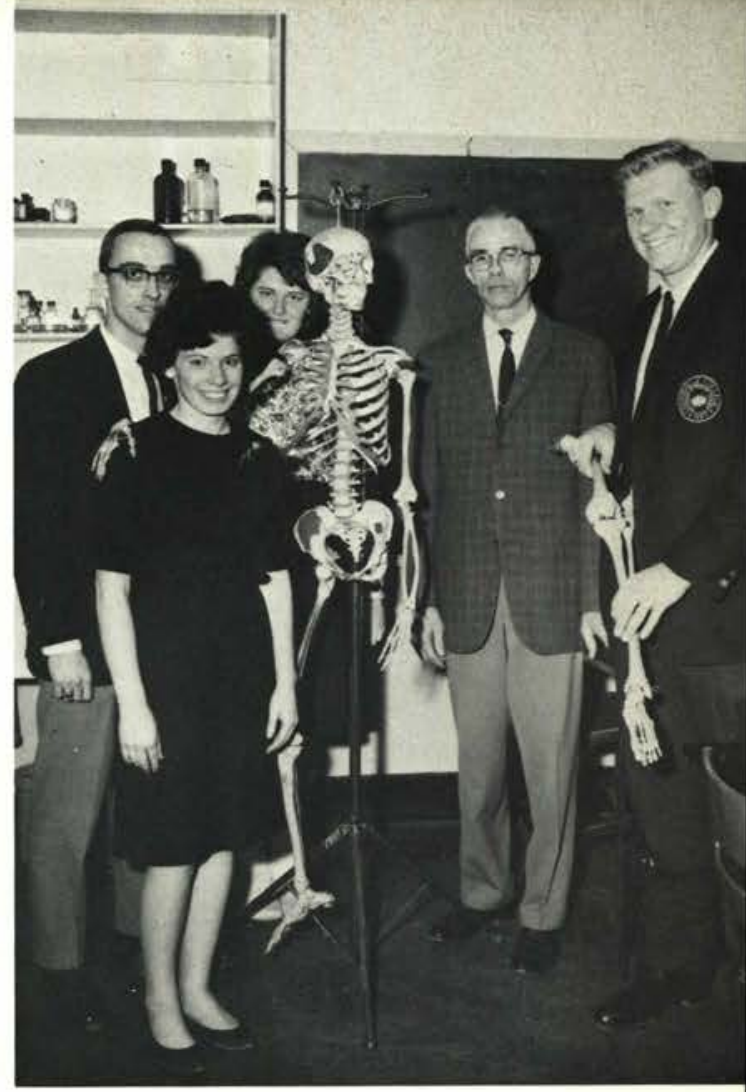

Students interested in science beyond what they learn in the classroom constitute Pi Delta. Speakers address the group on various science-related subjects and informal discussions are held. Most of the meetings are opened to the public since many timely subjects are presented.

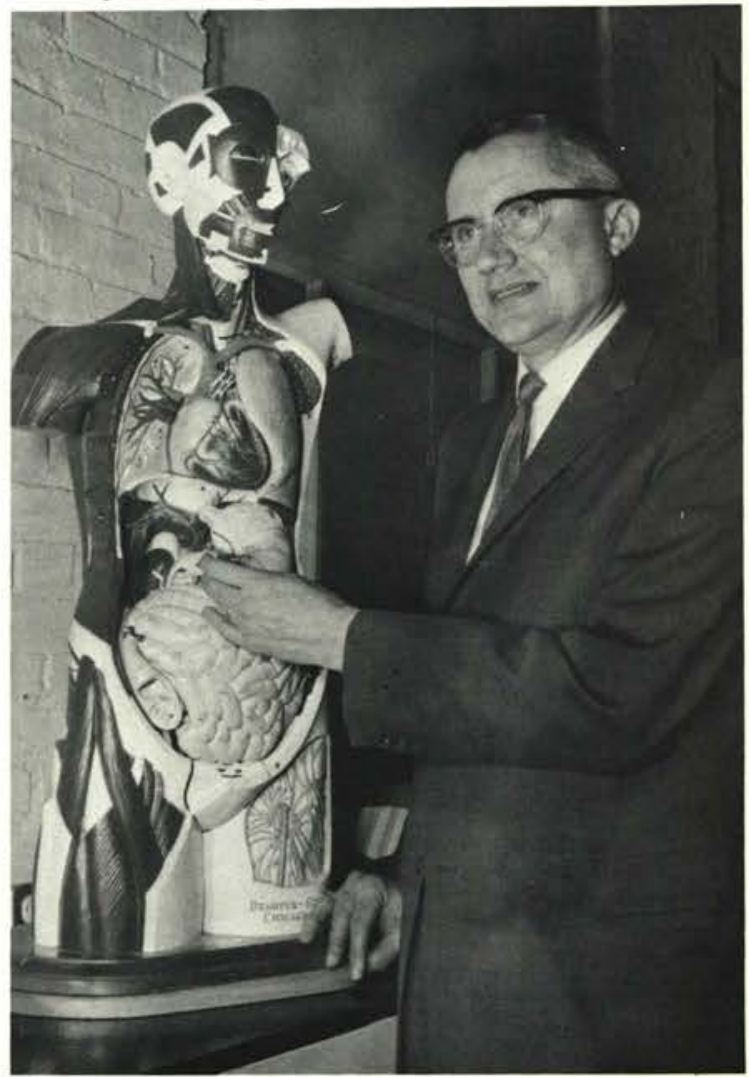

Mr. Johnson 


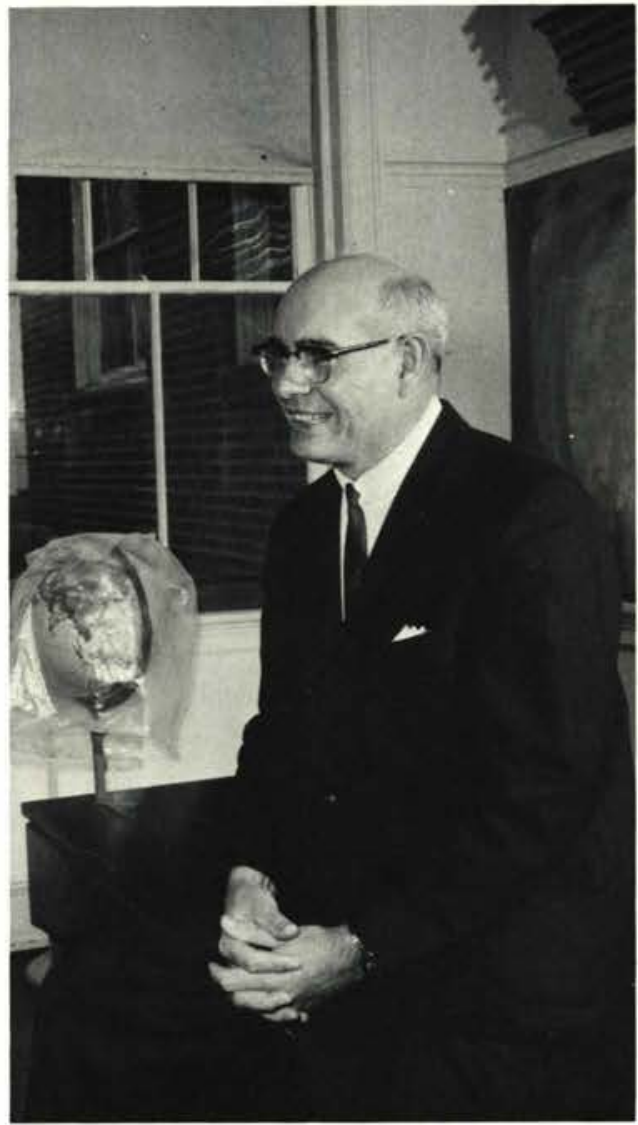

Mr. McDonald

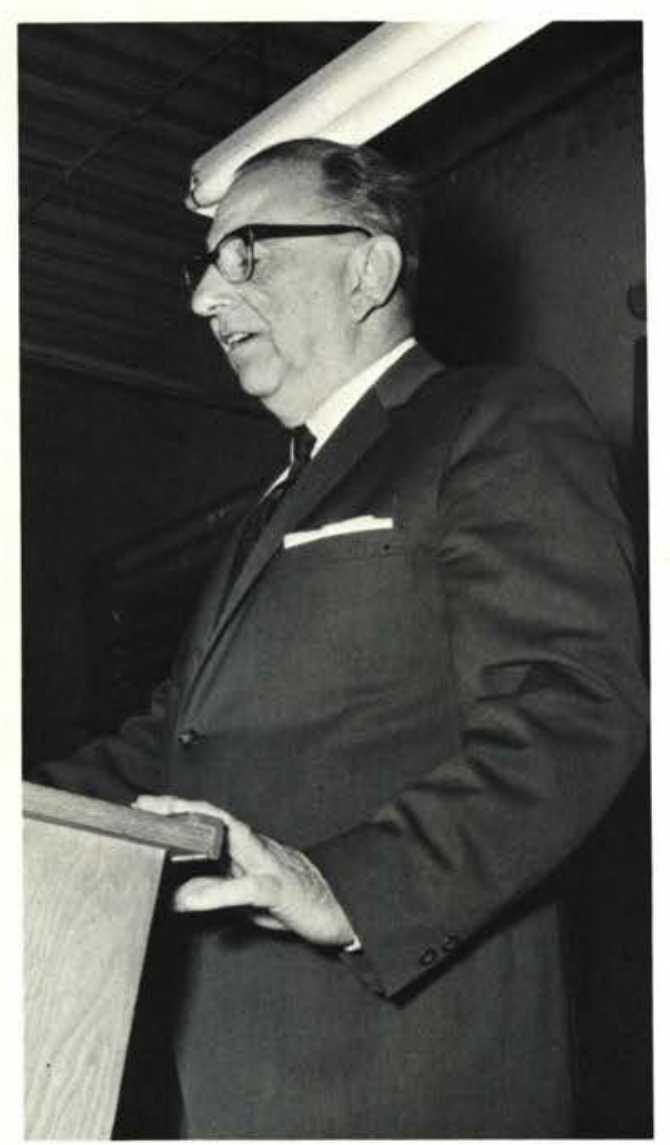

Mr. Gale

\section{SOCIAL SCIENCE}

". . A life is beautiful and ideal, or the reverse, only when we have taken into our consideration the social as well as the family relationship."

$$
\text { - Ellis }
$$

Man through the ages has had social interaction with his environment as well as with other men.

The purpose of the Social Science Department is to show the student the significance of this interaction. Courses are offered in both history and sociology since each area is important in order to understand the total picture of man's reactions and interaction. Once the overall view is achieved, courses of a more specialized nature are studied.

\section{Barry Goldwater}

\section{Conservative Club}

Left to Right: G. Fisher, R. McDugle, R. Clater, A. Sweeney, Mr. St. Clair, Advisor; I. Milliken, D. Entner, W. Pierson, J. Stockwell, T. Jager.

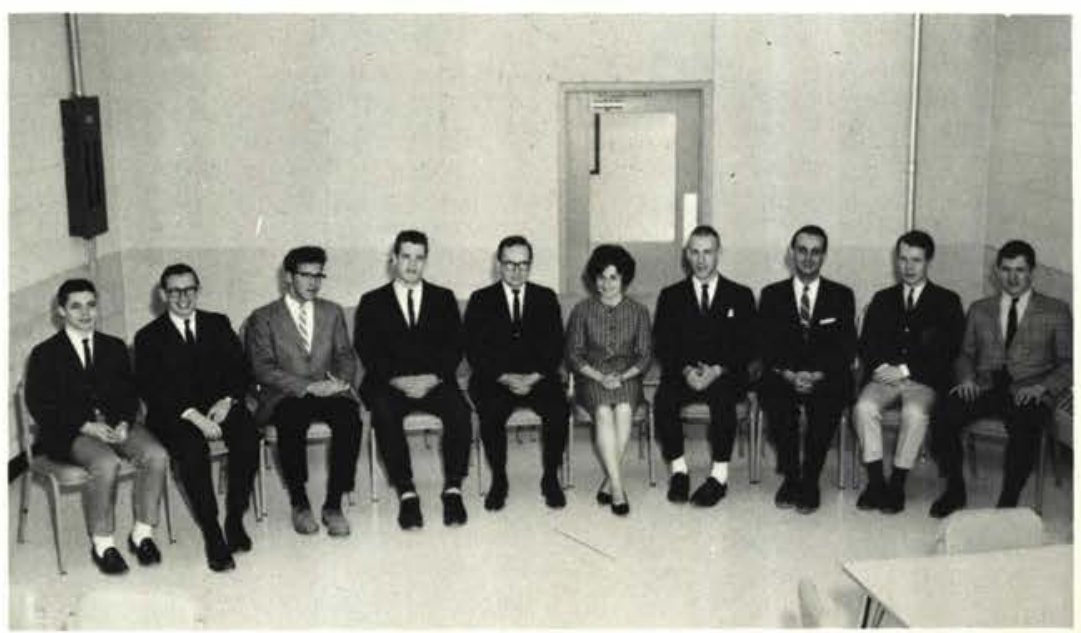




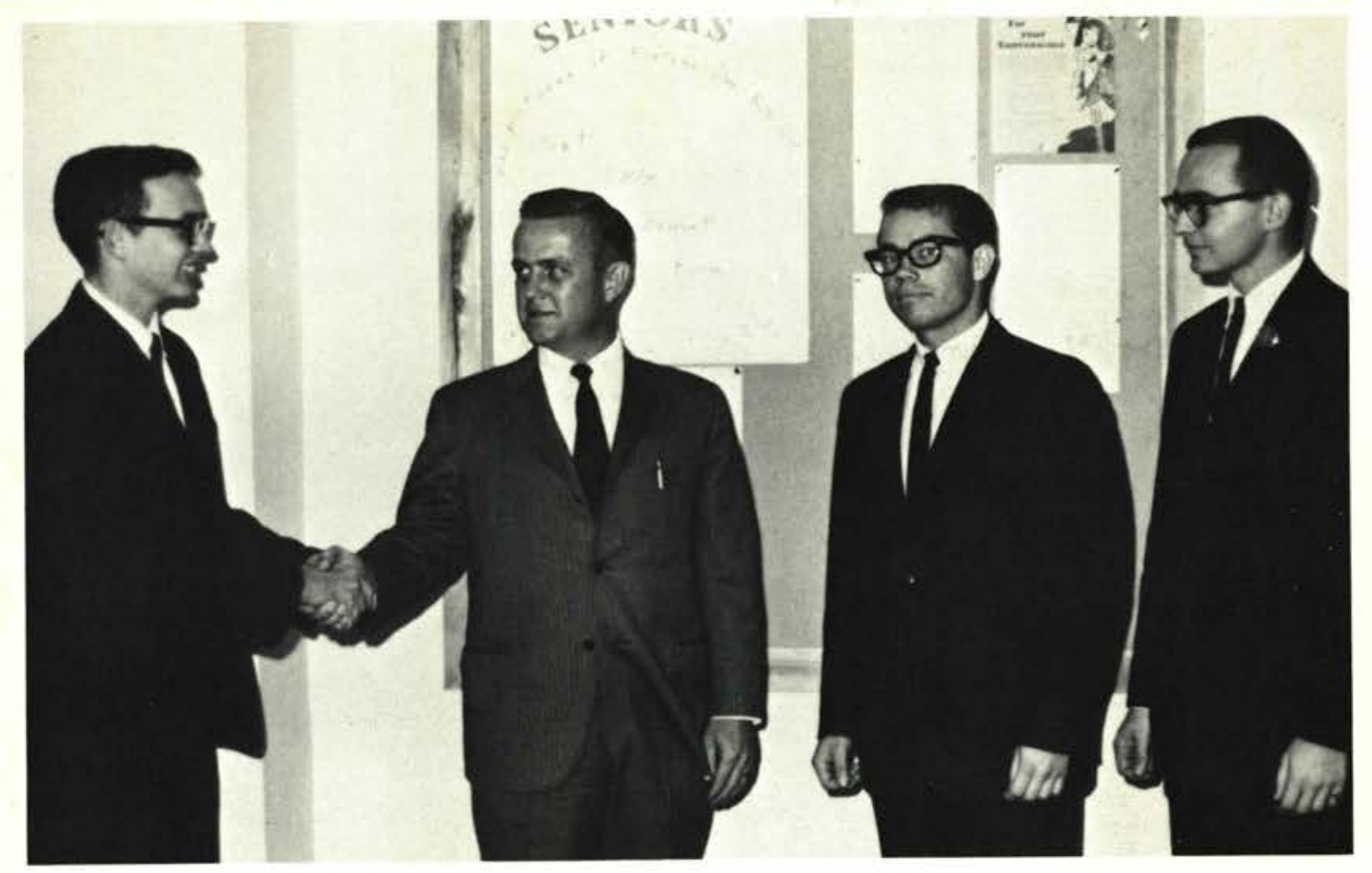

\section{Conservative Club}

An aired interest in politics is not a prerequisite for membership in the Cedarville College Conservatives; all one needs is an awareness of and an interest in what is occurring around him. The club is basically a discussion group with visiting speakers who examine and perhaps present solutions to many of the current affairs.

Also, this year the group sponsored a College Bowl on an intra-class basis.

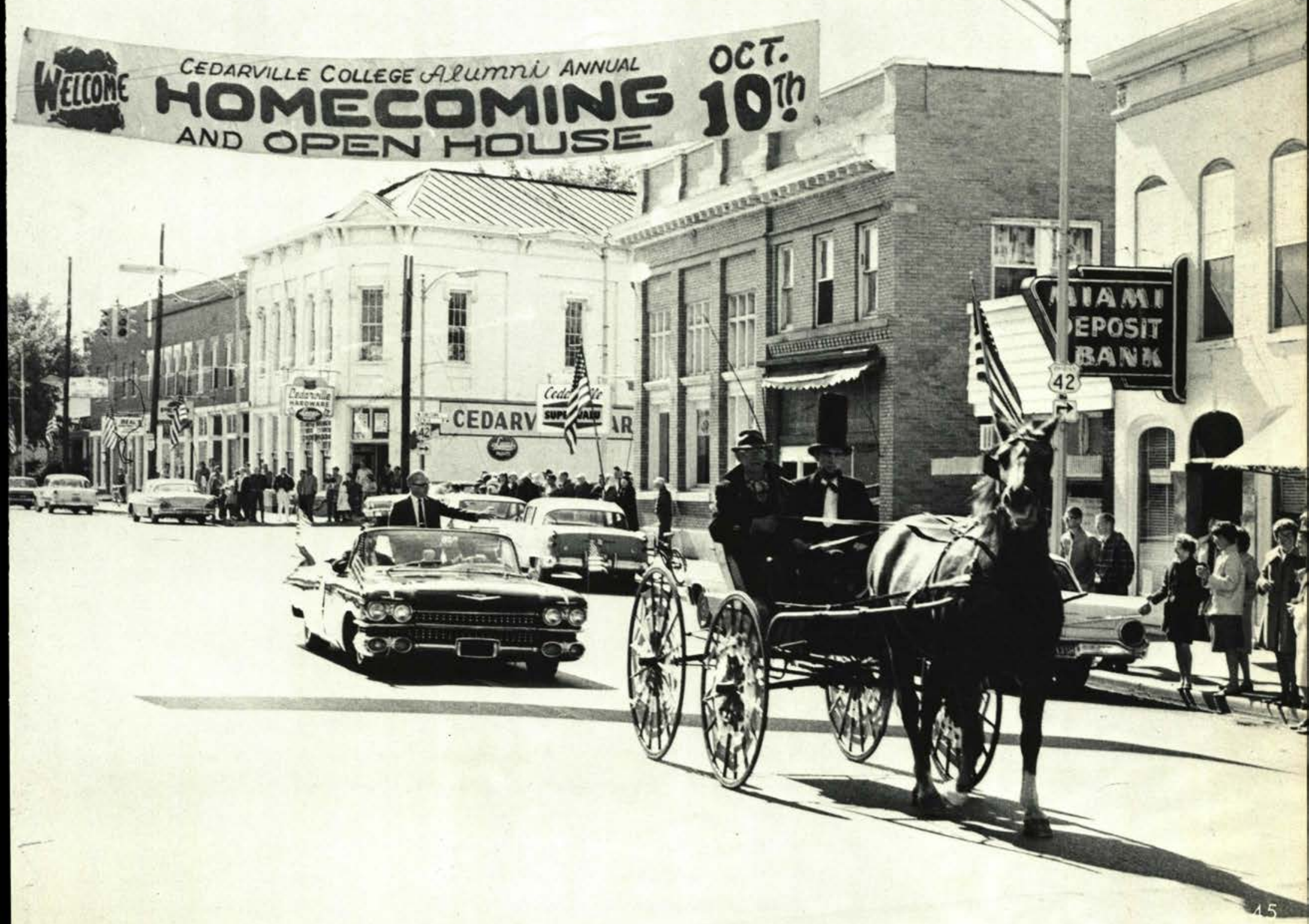




\section{BUSINESS ADMINISTRATION}

"He profits most who serves best."

-Sheldon

One step further-he serves best, who prepares best. These two ideas are literally true in the business world. Realizing this challenge, the Business Department offers excellent preparation to the student desiring a career in business. He is taught to serve in the business world and the church as a trustworthy servant of God.

Mr. Kenneth St. Clair

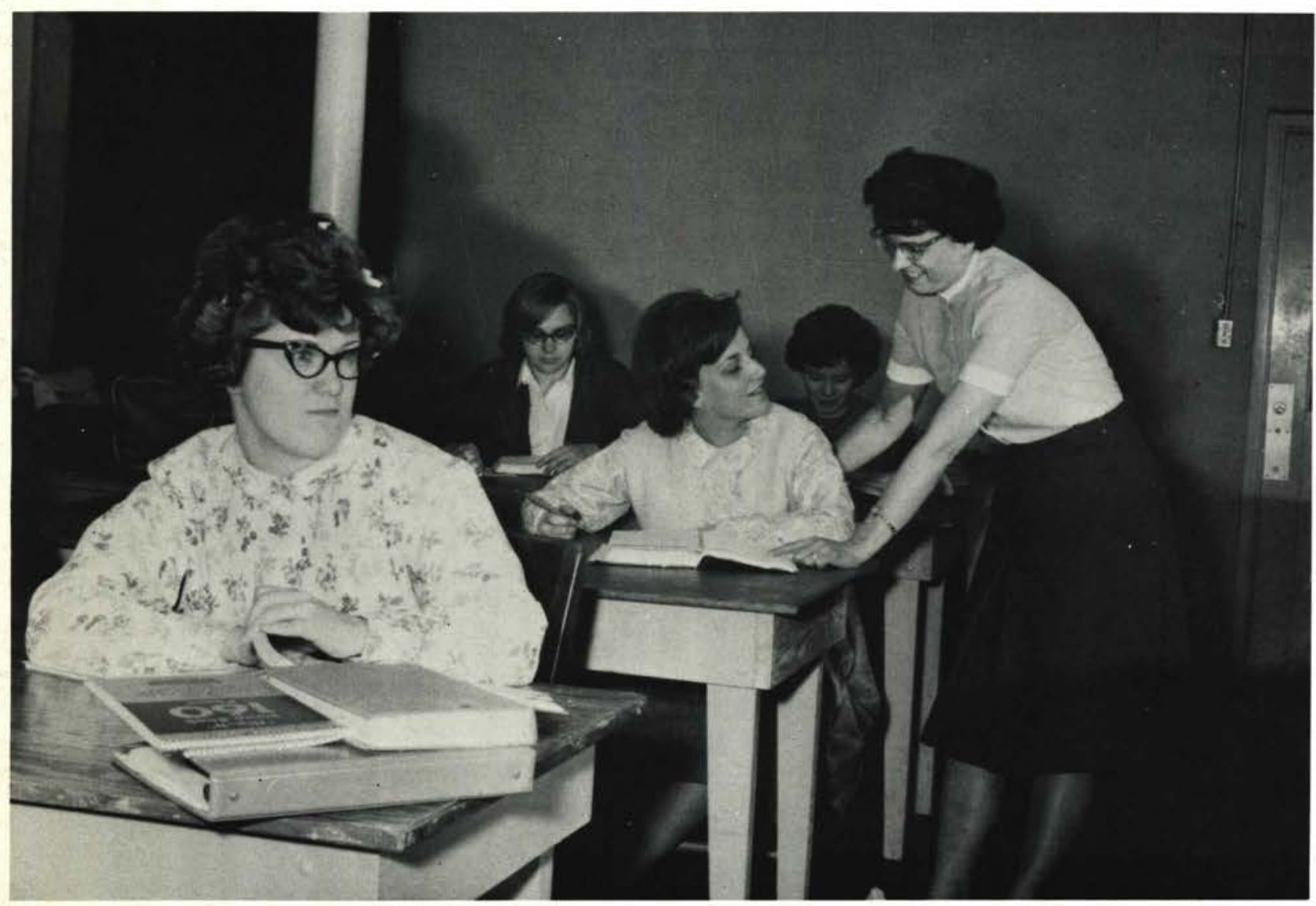

Mrs. Ardith Webber 


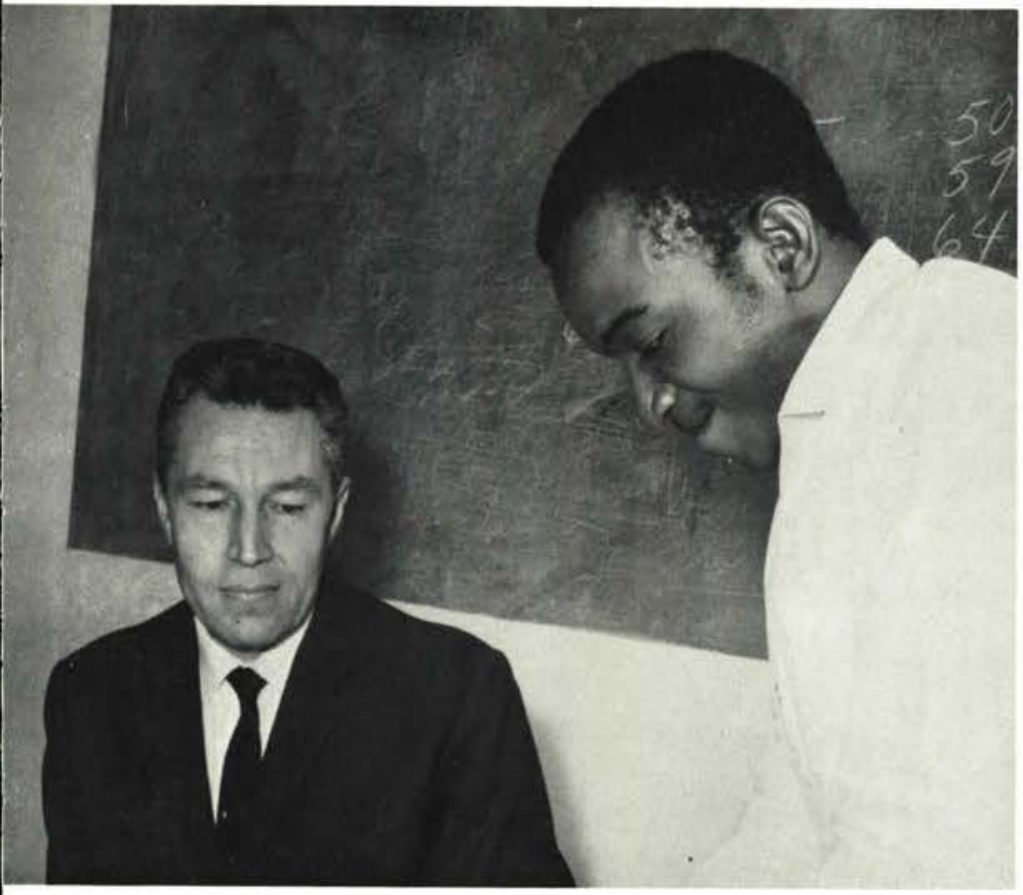

Mr. Nick Frost-ACCOUNTING

\section{Future Business (Associates)}

Future Business Associates accommodates the students of one of the fastest growing academic departments of the school. The club is organized to promote possible future employment and to provide an opportunity to view the business world in action. In order to do this, speakers from various areas of the business field address the groups and towns of plants in the vicinity are taken.

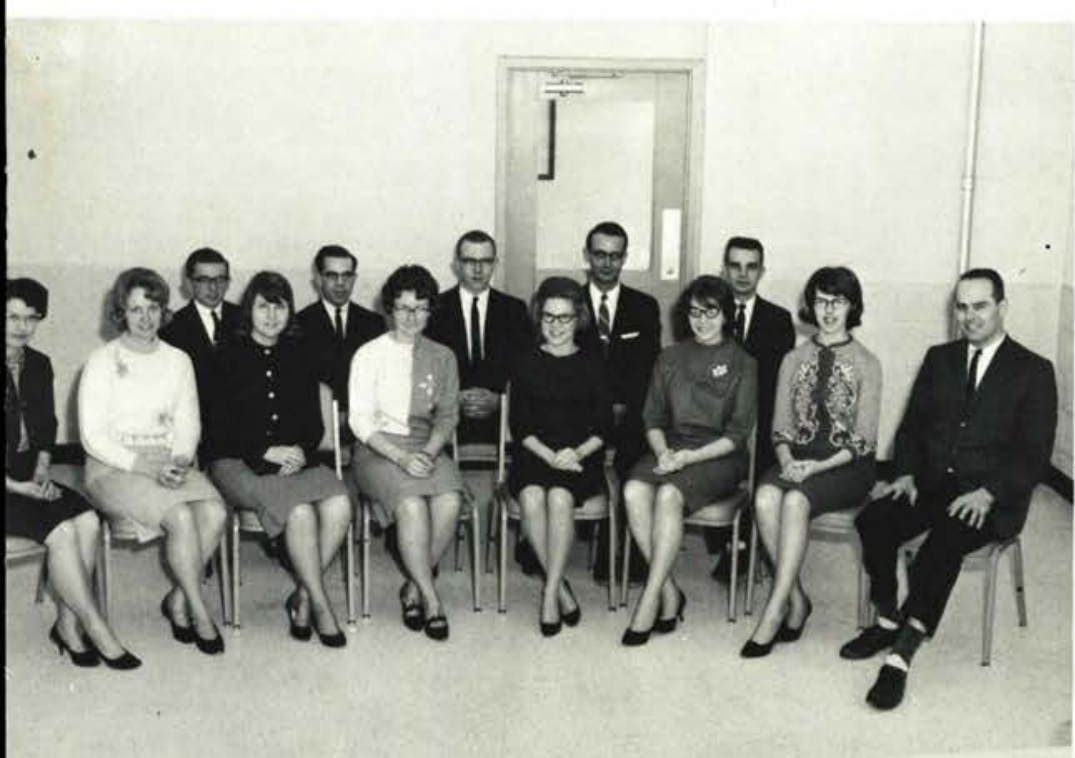

Front Row, left to right: Mrs. Webber, K. Mansfield, K. Gilden, B. Randall, L. Shaw, M. Young, L. Young, Mr. Wyse. Back Row: E. Acker, D. Wilhite, D. Stephens, W. Pierson, J. Zigler. 


\section{HEALTH AND PHYSICAL EDUCATION}

"Every man is the builder of a temple, called his body." - Thoreau

The aim of the Physical Education Department is to help build the bodies of those enrolled in its courses. For those majoring in this area, the department offers many courses designed to help the prospective physical education instructor build and train the bodies of future students. He not only learns theories of physical education but also applies them in the classroom in such courses as "First Aid," "Games and Rhythmics," and "Recreational Leadership."

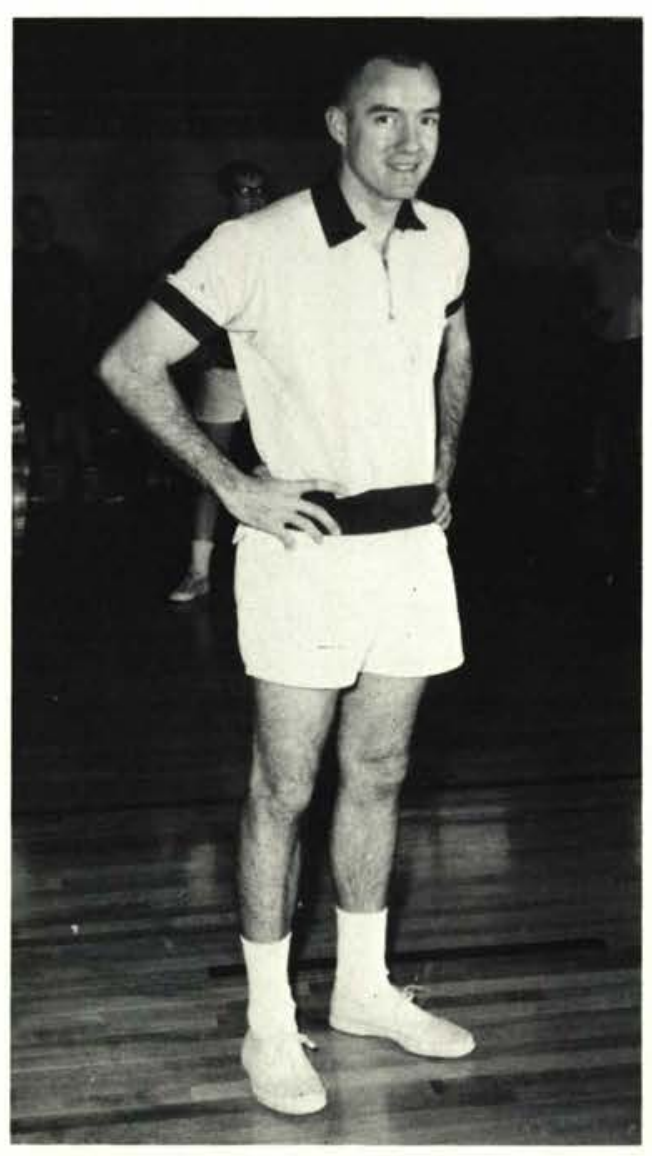

\section{Varsity C Club}

Varsity $\mathrm{C}$ is made up of those men who have ${ }^{\cdot}$ lettered in inter-collegiate sports. Perhaps the service they are remembered for most is running the concession stand at basketball games. They also perform other campus services and sponsor trips to athletic events for club members.

Mr. Moody

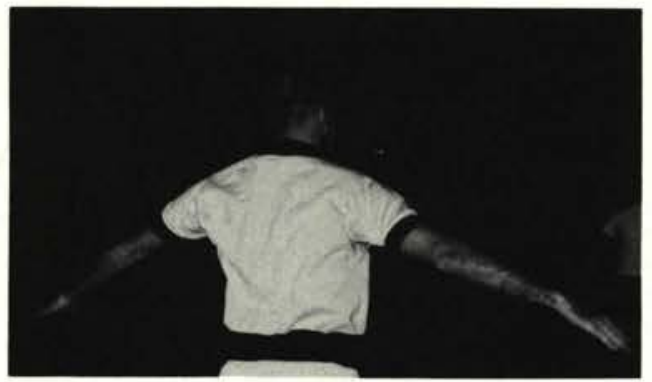




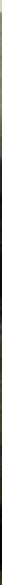

Coach Don Callan

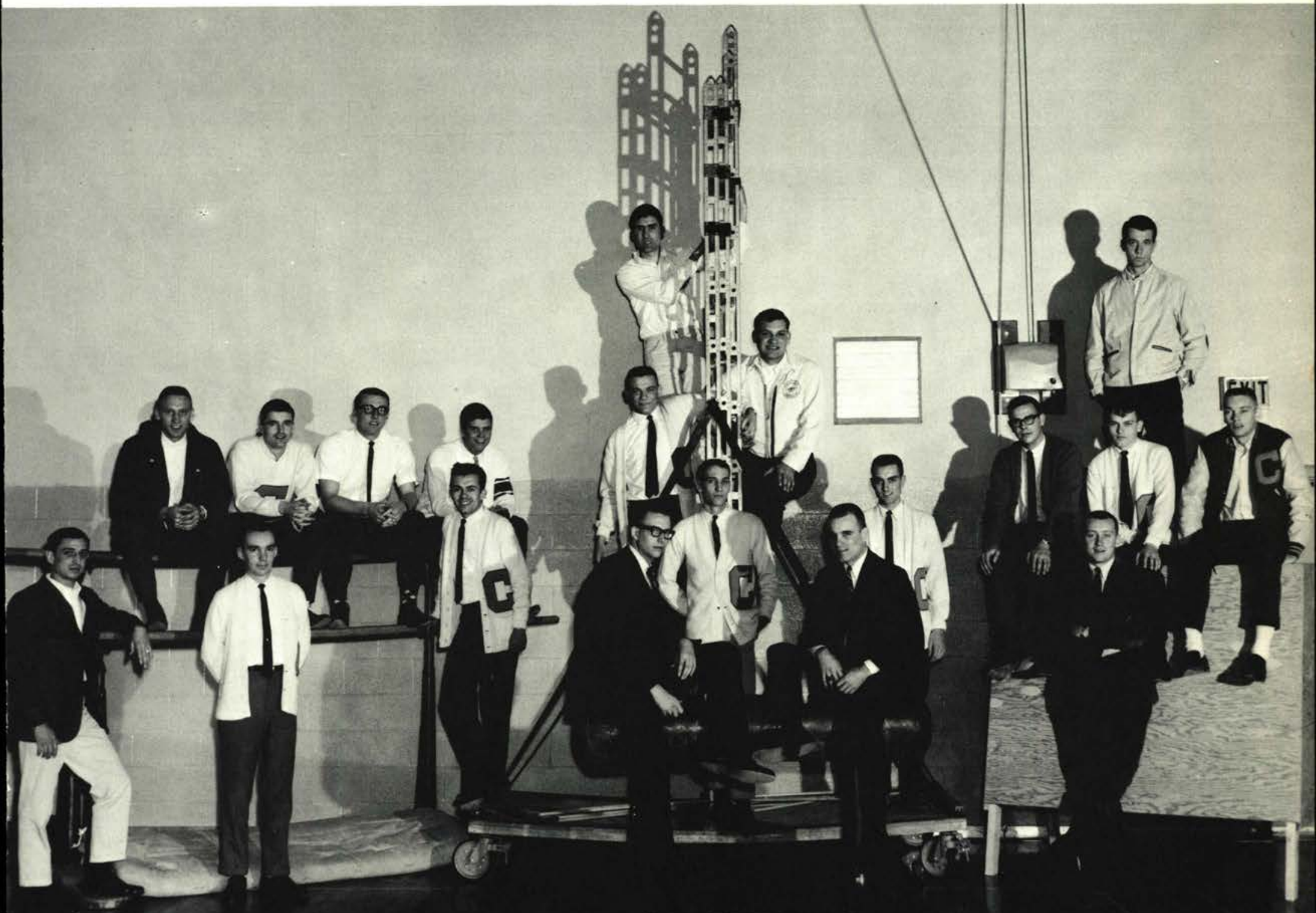

Left to Right: B. Francis, G. Culley, T. Miller, M. Combs, D. Werner, M. Pereira, R. Ross, C. Wilkins, Vice-President; C. Reed, D. McDonald, R. Davis, J. Barker, J. Walborn, President; J. Brannon, T. Oakley, A. Merwald, J. Hess, L. Richardson, R. Davis. 


\section{PSYCHOLOGY AND EDUCATION}

"A teacher affects eternity; he can never tell where his influence stops."

$$
\text { - Adams }
$$

The Psychology and Education Department has been a growing department with the granting of teacher certification in 1963. New courses are being offered each semester so that the class of 1967 will be the first class to have in its ranks certified teachers trained exclusively at Cedarville. These will be teachers who will be spiritually and academically grounded in the Word and able to teach in public schools, Christian schools, and missionary schools on the various fields.

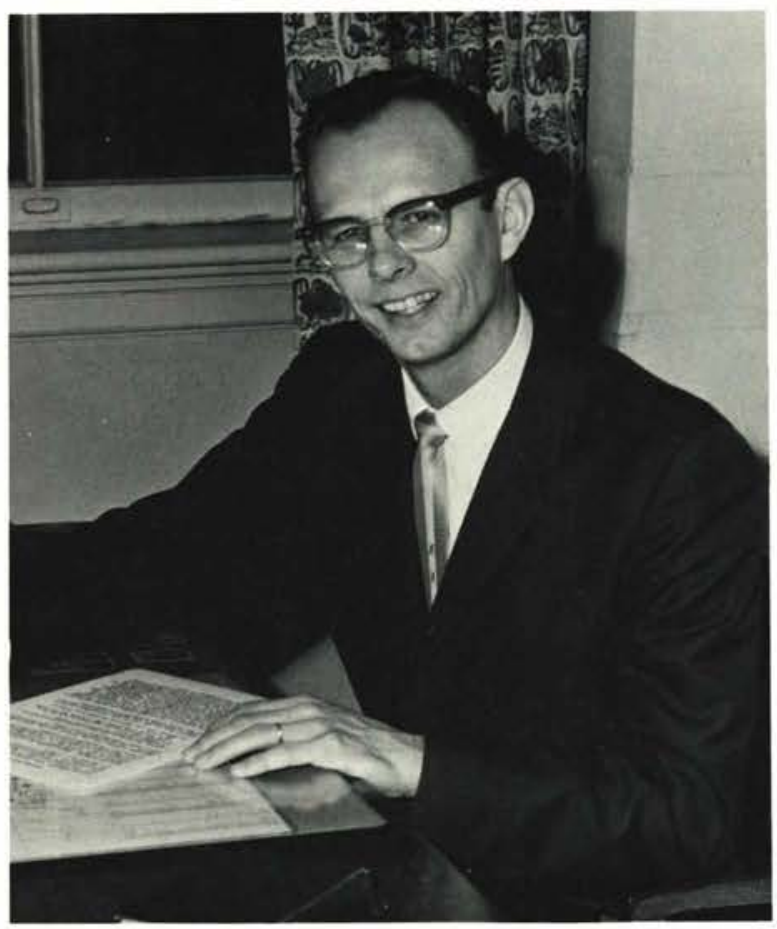

Dr. C. Johnson

Dr. C. Maddox

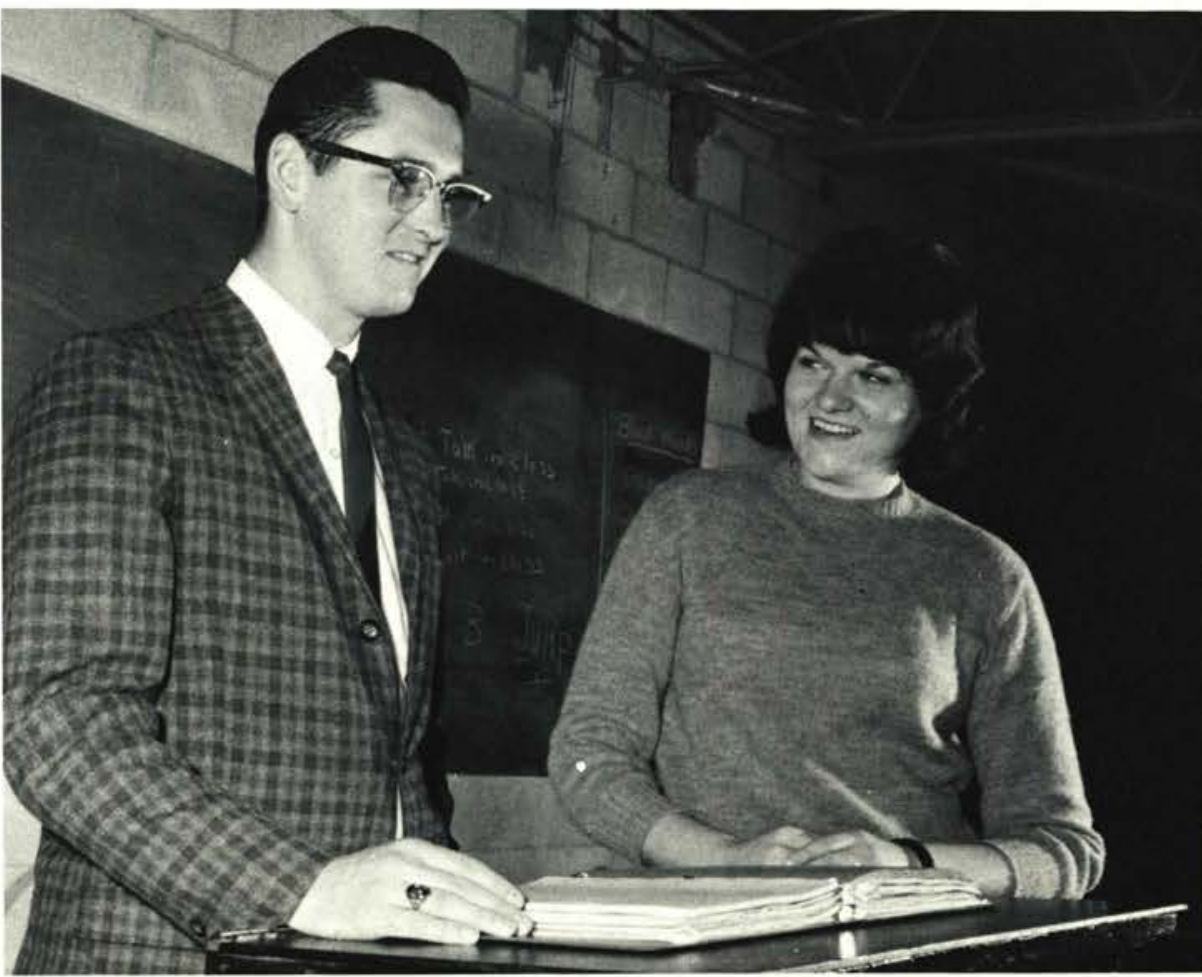

Mr. Ager 


\section{Choralaires}

Choralaires, under the direction of Miss Ruth Smith, attempts to raise the level of music appreciation through the performance of good secular music, while appropriating this opportunity to represent and glorify Christ through personal testimony. The group sings at local banquets and programs and each year is highlighted by the presentation of their annual Spring concert.

\section{Miss Ruth Smith}
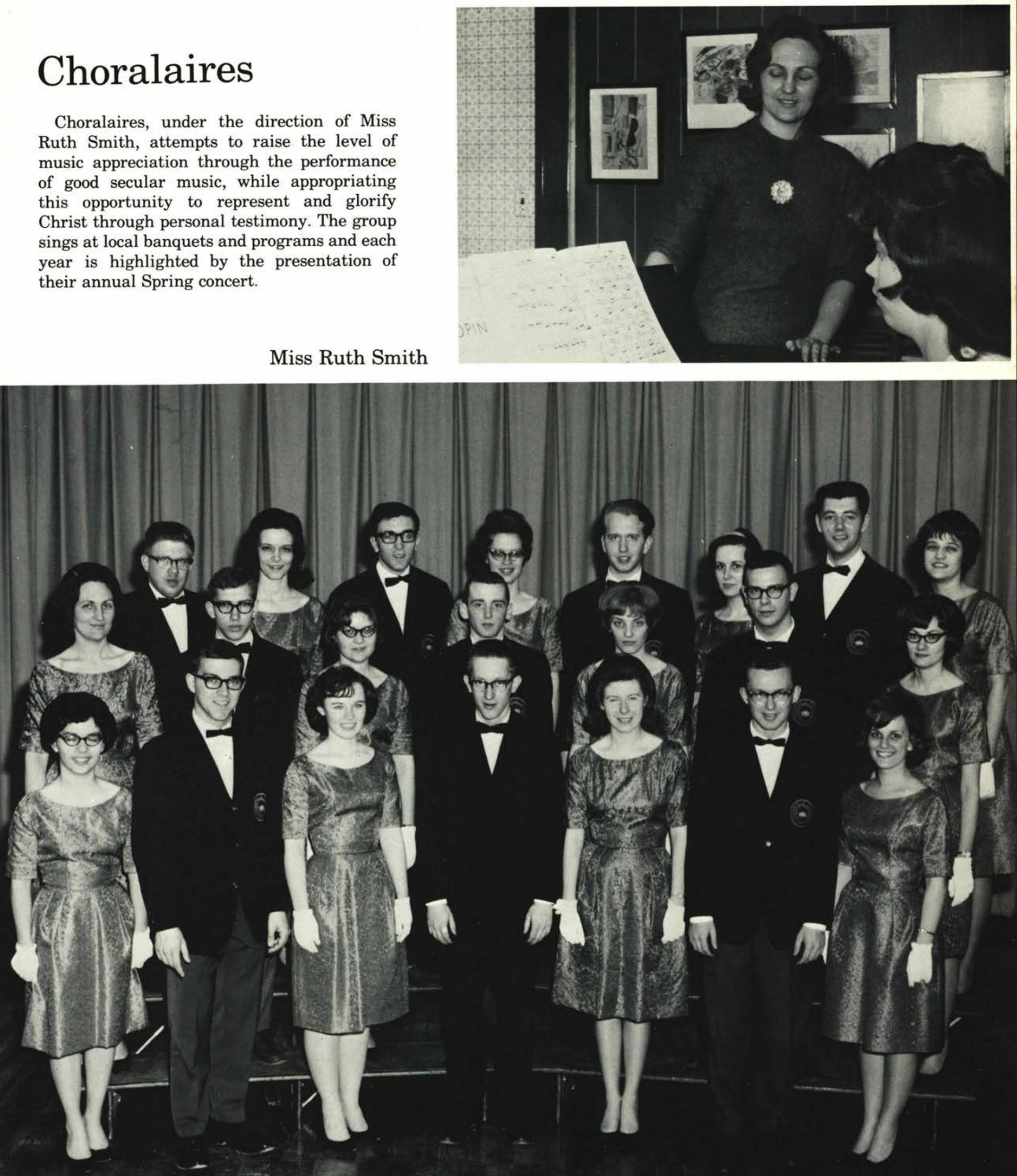

First Row: M. Davey, T. Duffie, J. Keating, G. Griffin, D. Fulkerson, P. Carlson, Vice-President; B. Grosh. Second Row: Miss Smith, J. Herwig, G. Trimble, R. Grosh, L. Hathaway, T. Oakley, G. Brown. Third Row: E. Krueger, N. Buerer, T. Miller, A. Howard, L. Welch,

S. Eckert, D. Jensen, President; V. Curcio, Secretary-Treasurer. 


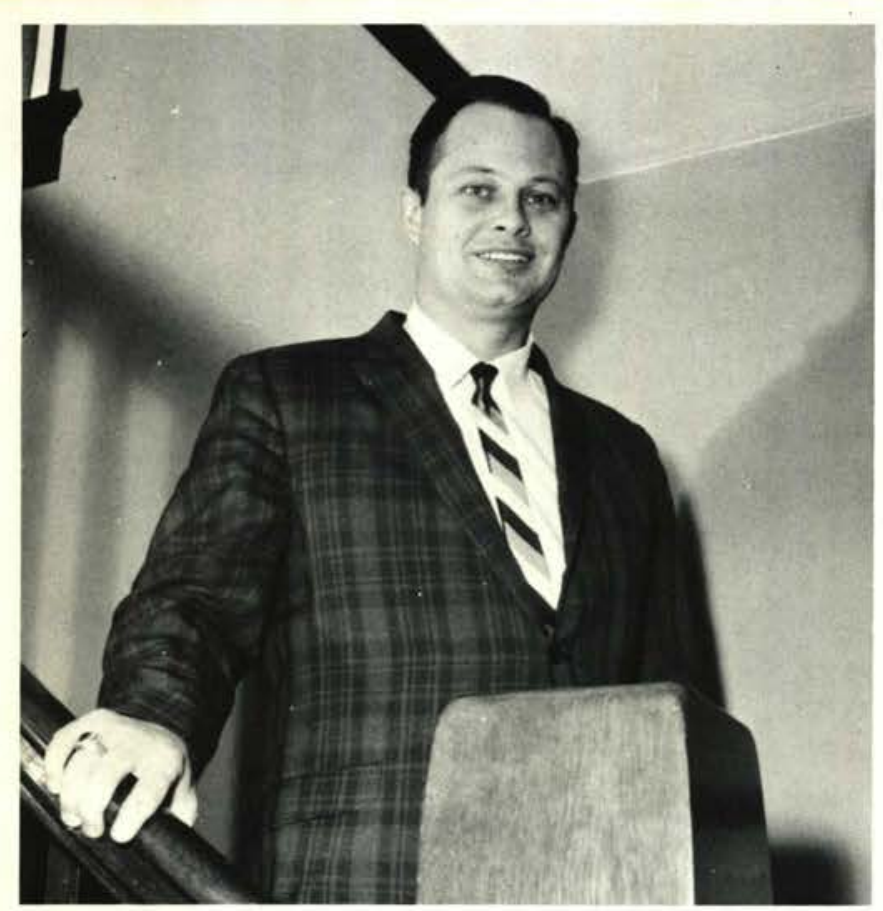

\section{College Choir}

The College choir, under the direction of $\mathrm{Mr}$.

Walter Meissner, represented the College on several weekend tours and the Spring tour. The choir performs the very necessary function of serving as a link between our College and our churches.

Mr. Meissner

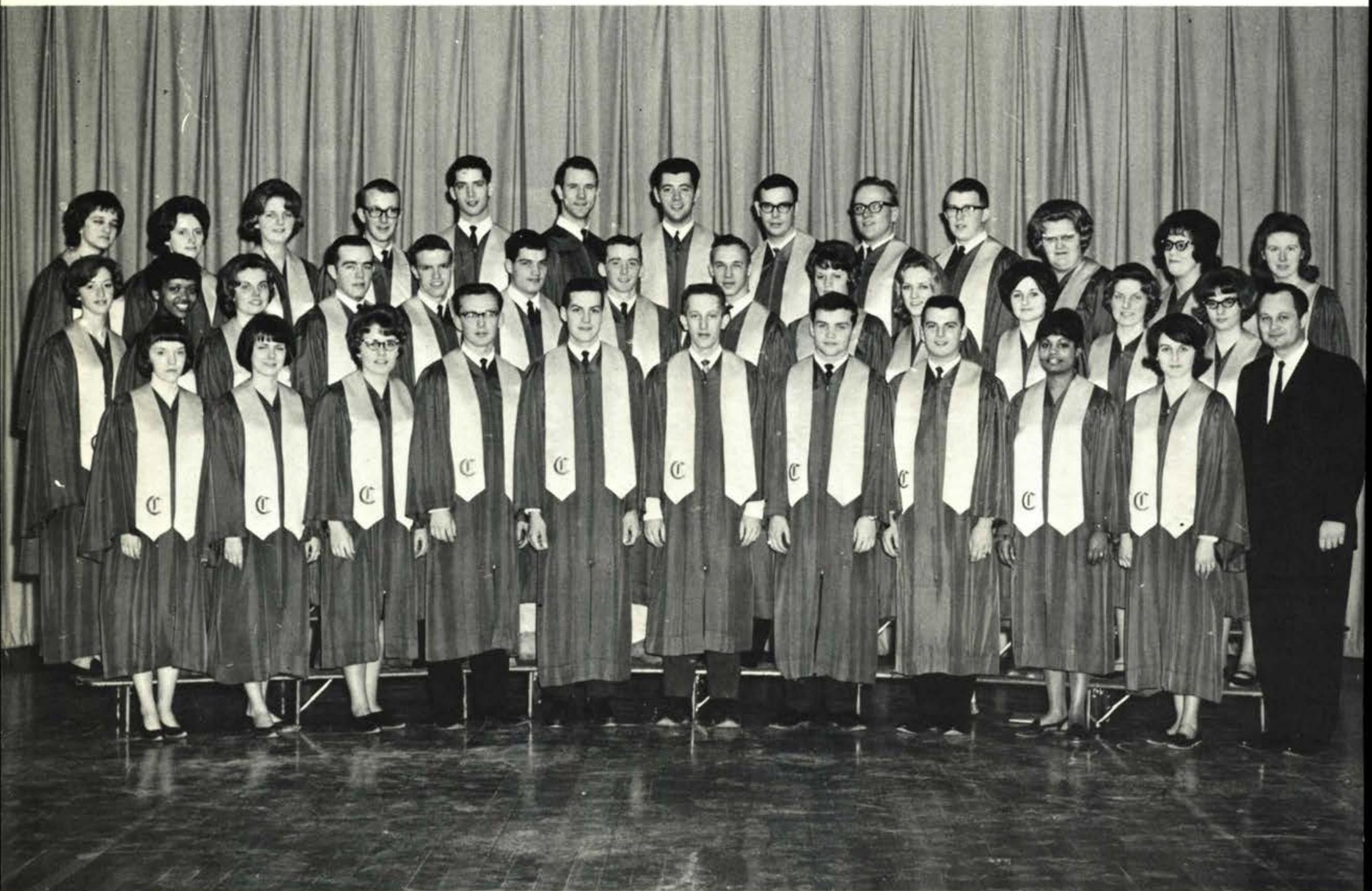

Front Row, left to right: M. Ammons, A. Cayton, M. Werner, P. Carlson, M. Searles, G. Griffin, L. Mason, A. Keim, K. Taylor, B. Millikan. Middle Row: Y. Rathfon, S. Williams, C. Wenner, T. Timmons, G. Harris, H. Loops, R. Grosh, T. Clater, K. Johnson, L. Riggs, I. Millikan, S. Bryd, M. Brown. Back Row: V. Curcio, M. Cummings, E. Johnson, D . Boyd, M. Jones, D. Nims, D. Jensen, T. Oakley, B. Griffith, G. Huber, S. Kaiser, P. Crown, D. Fulkerson, Mr. Meissner, director. 


\section{Music Groups}

Music groups have the specific ministry of representing the College throughout the year in area churches.

Many hours are spent in practicing, but the rewards are great when one sees people drawn closer to the Lord through the ministry of these groups.

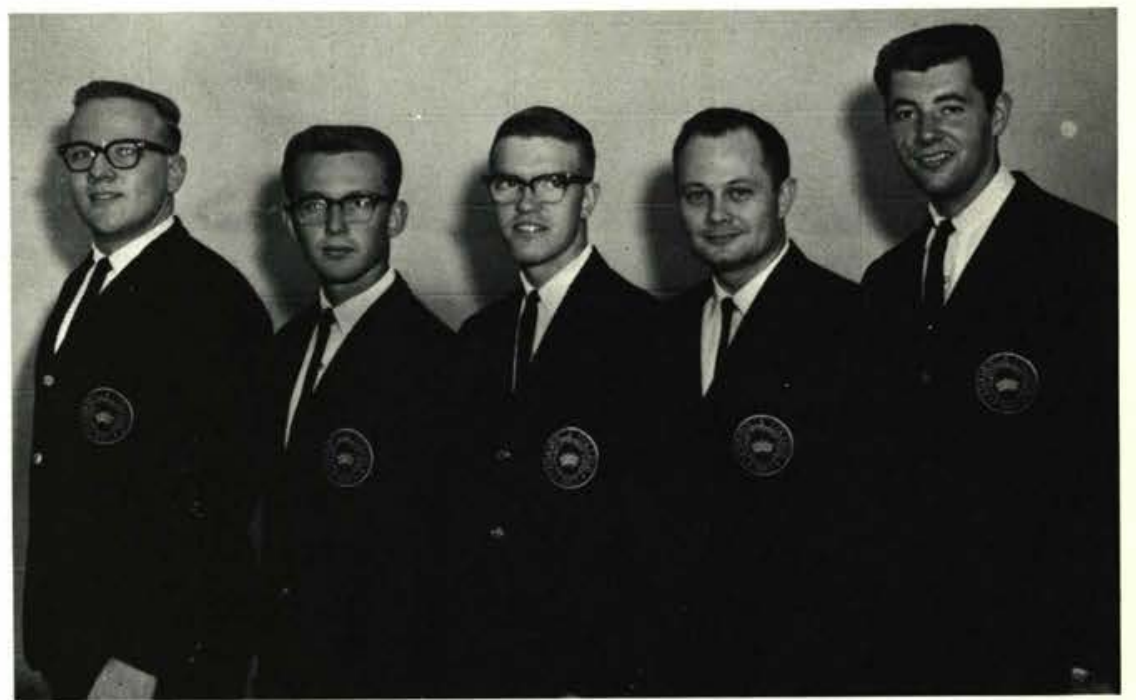

THE MESSENGERS-B. Griffith, P. Carlson, G. Harris, Mr. Meissner, D. Jensen.

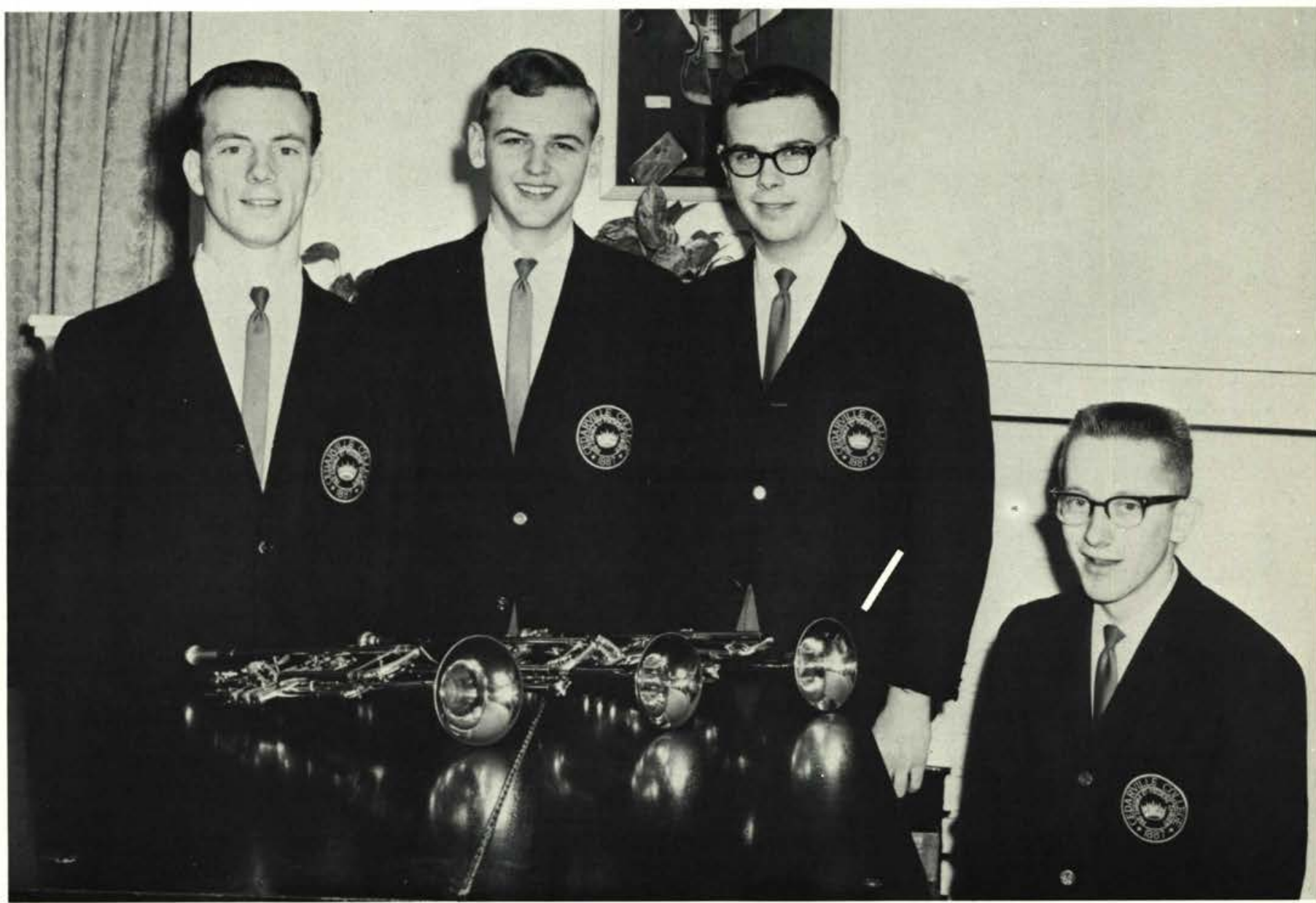

THE HERALDERS-D. Foster, G. Taylor, T. Oakley, G. Griffen. 


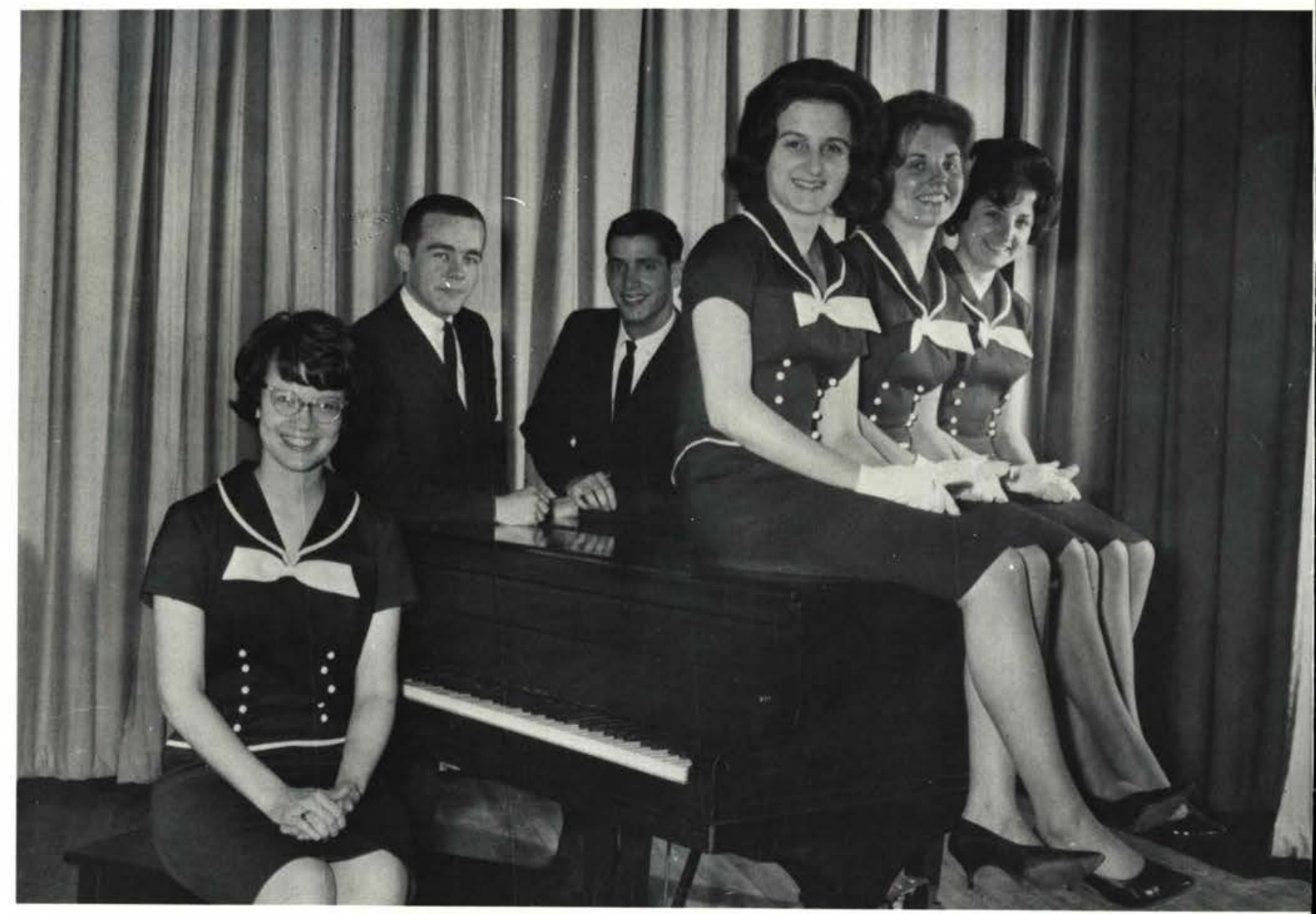

THE CONQUERORS QUINTET - L. Stutesman, T. Timmons, M. Jones, B. Millikan, C. Wenner, I. Millikan.

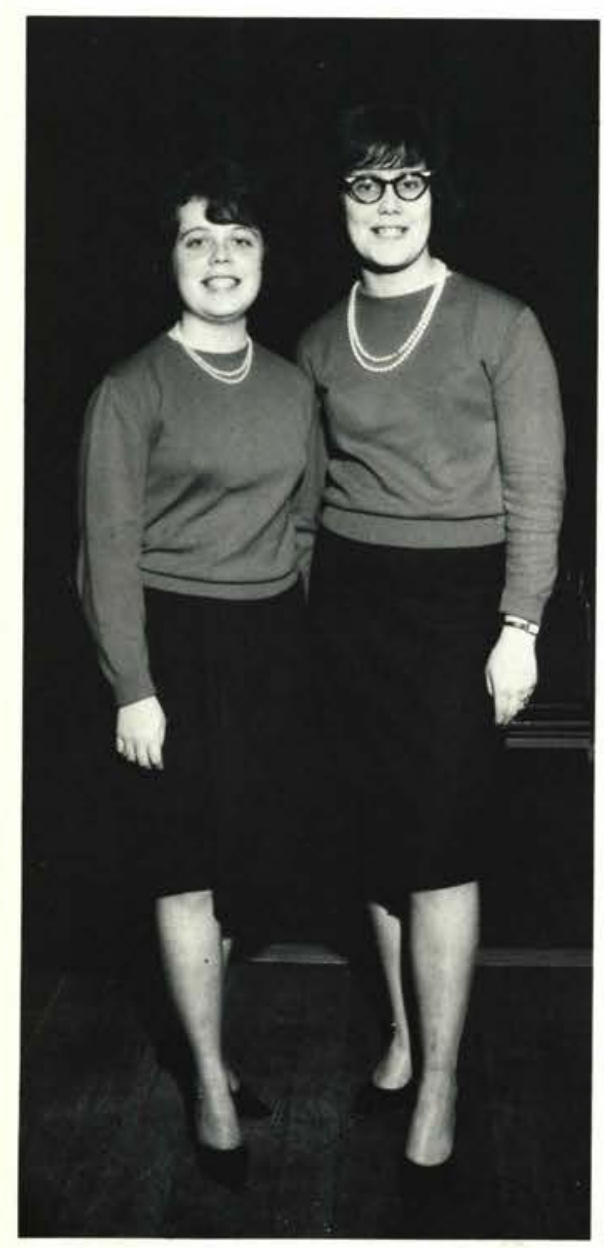

The Crown Sisters: N. Crown, P. Crown.

THE SUNLIGHTERS-G. Cooper, B. Meyers, S. Kaiser

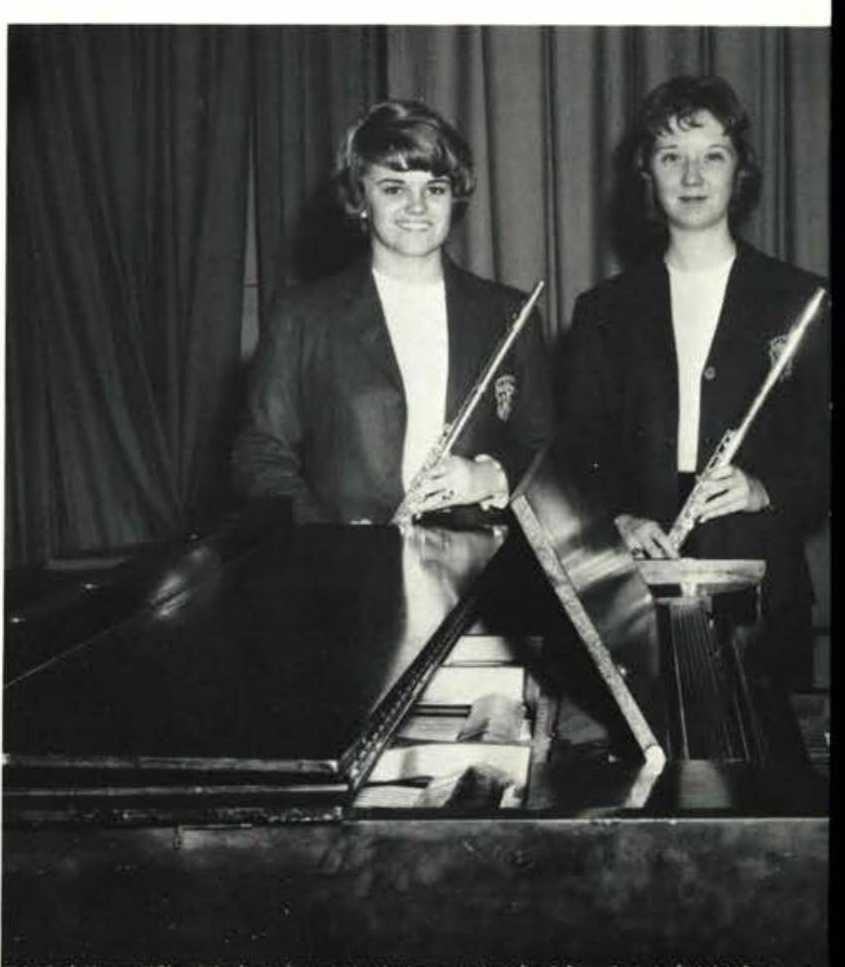




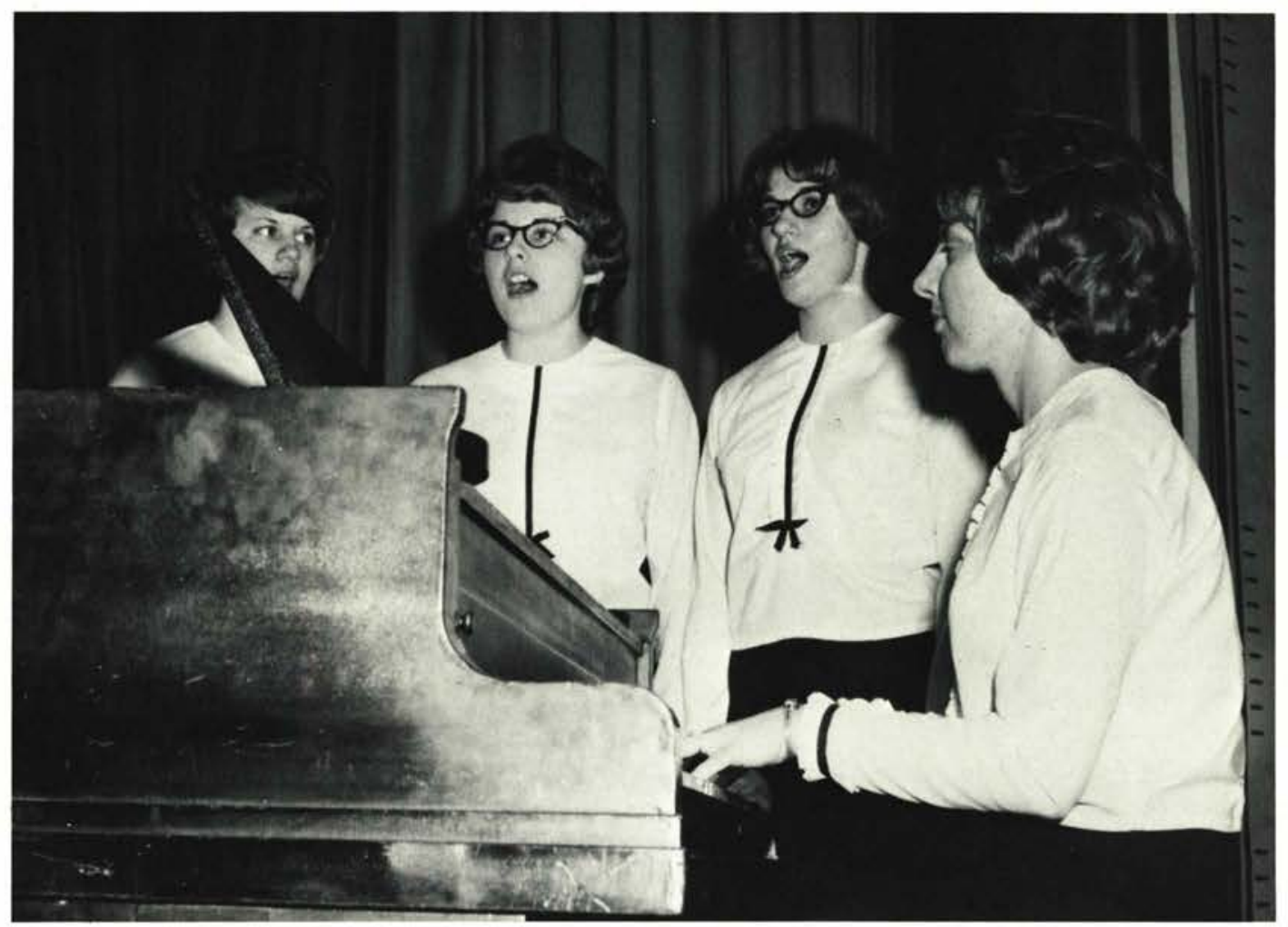

THE ROYALHEIRS-K. Johnson, M. Werner, M. Brown, S. Byrd.

THE CONQUERORS-B. Millikan, C. Wenner, I. Millikan, L. Stutesman.

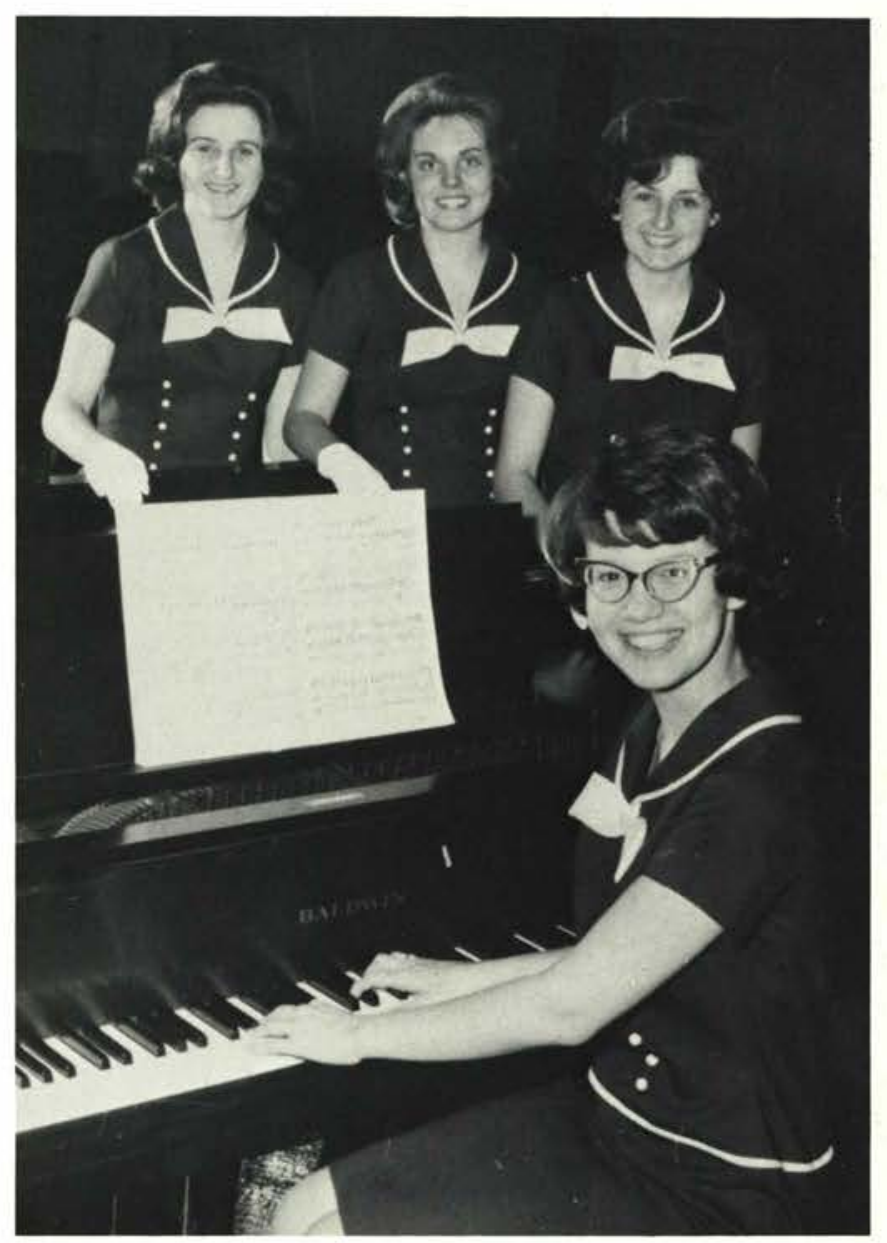




\section{Gamma Chi}

Gamma Chi is one of the two women's literary societies on campus. It seeks to promote higher spiritual, cultural, and literary interests and goals in the young women who are members. The main campus function that the organization sponsors is the annual semi-formal Valentine's banquet. In addition, the group also attends many cultured events in the area.

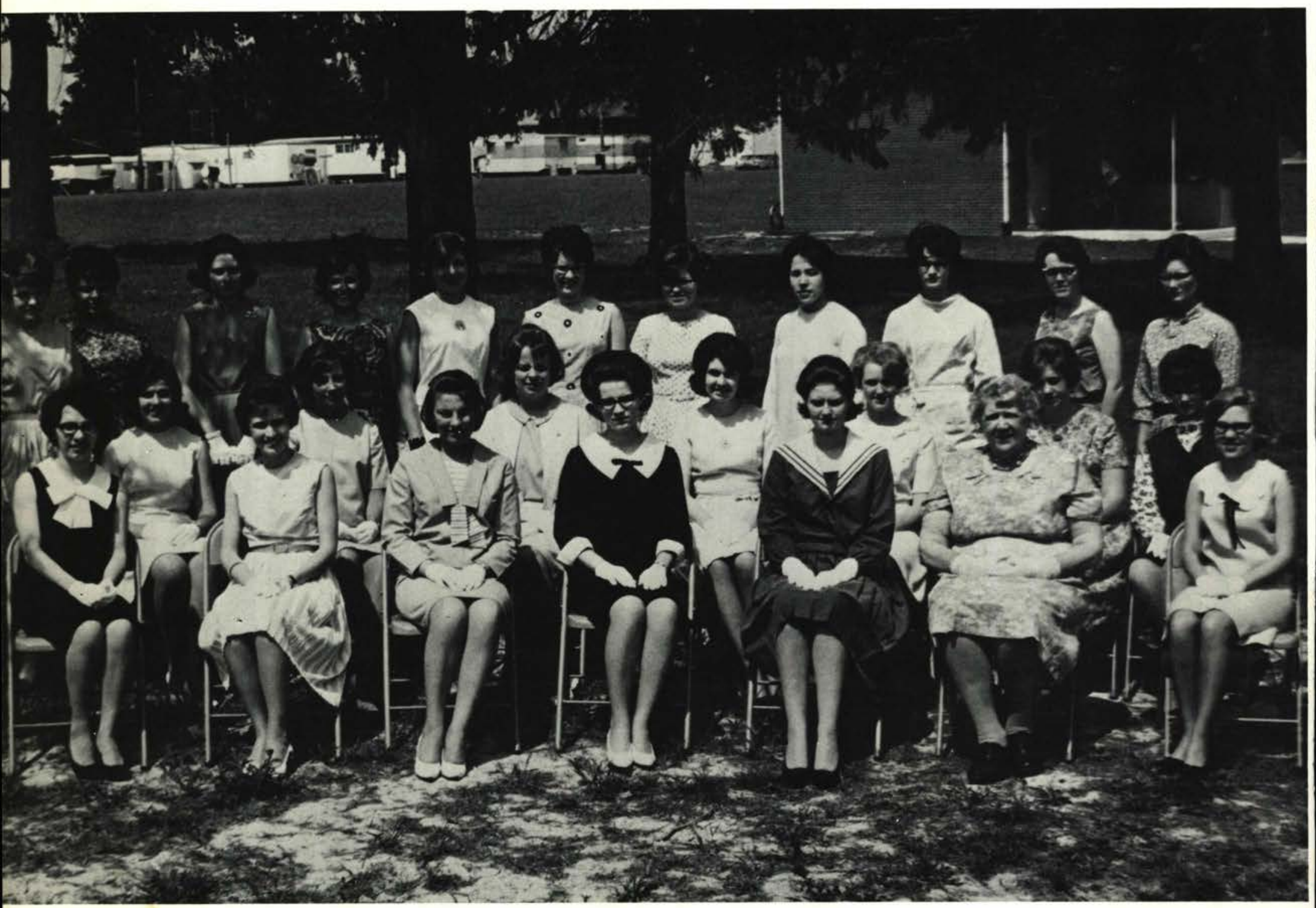

Seated, left to right: M. Davey, N. Davis, J. Ronk, C. Burcham, S. Byrd, D. Dunn, J. Petrie, M. Ballard, C. Mikels, K. Mansfield, Mrs. Maddox, B. Navorska, C. Ryan, L. Shaw. Standing: J. Emerson, J. Rudduck, L. Healy, B. Grosh, K. Patterson, S. O'Shell, K. Griffin, J. Goss, L. Wintrow, B. Murphy, B. Randall. 


\section{Alpha Chi}

Alpha Chi, the men's literary society on campus, promotes the spirit of refinement and social tastes and also develops poise, personality, and leadership at both formal and informal events. One of the major functions on campus that they sponsor is the annual Thanksgiving banquet. The club also attends other events as a group in order to accomplish the club's purposes.

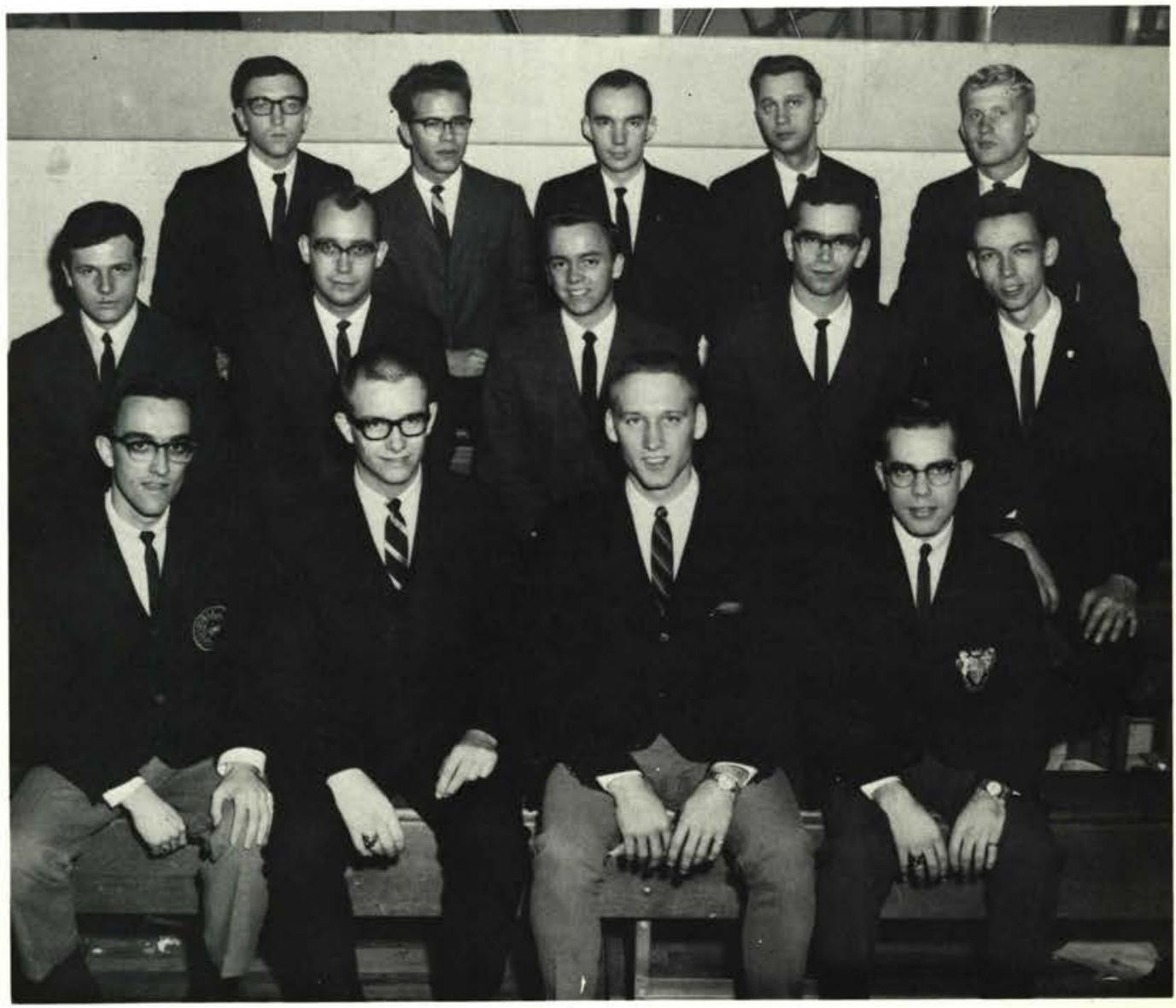

First Row, left to right: M. Stephens, President; C. Wilkins, Vice-President; G. Culley, Secretary; D. Wilhite, Treasurer. Second Row: J. Bonnema, Dr. R. Bartholomew, Adviser; R. Allerton, D. Prosser, M. Crampton. Third Row: T. Biller, R. Clater, T. Miller, R. Lamb, V. Roloff.
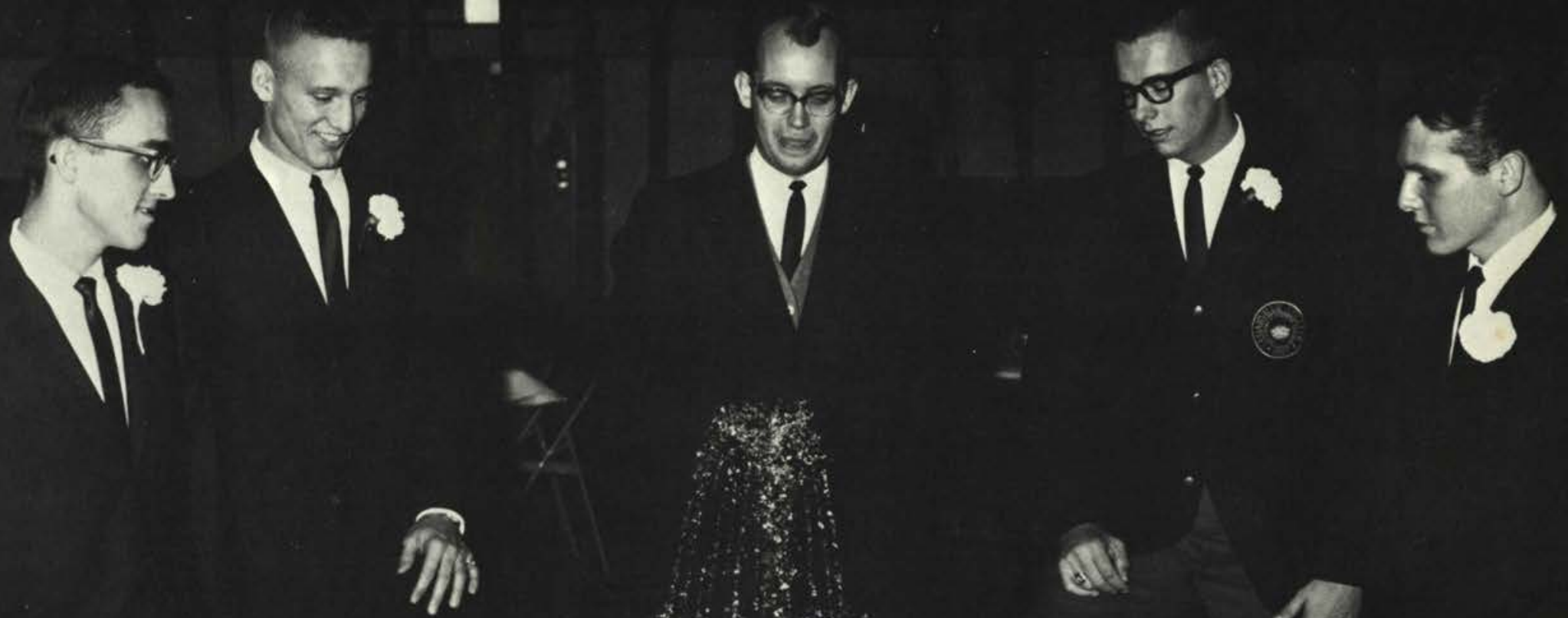


\section{Kappa Delta Chi}

Kappa Delta Chi, the newer of the two women's literary societies, is organized to stimulate an interest in gracious living and to provide an opportunity to practice this type of living in a manner pleasing to God. Members of this organization are exclusively upper classmen who meet in both formal and informal situations in order to cultivate habits and tastes becoming to Christian young women.

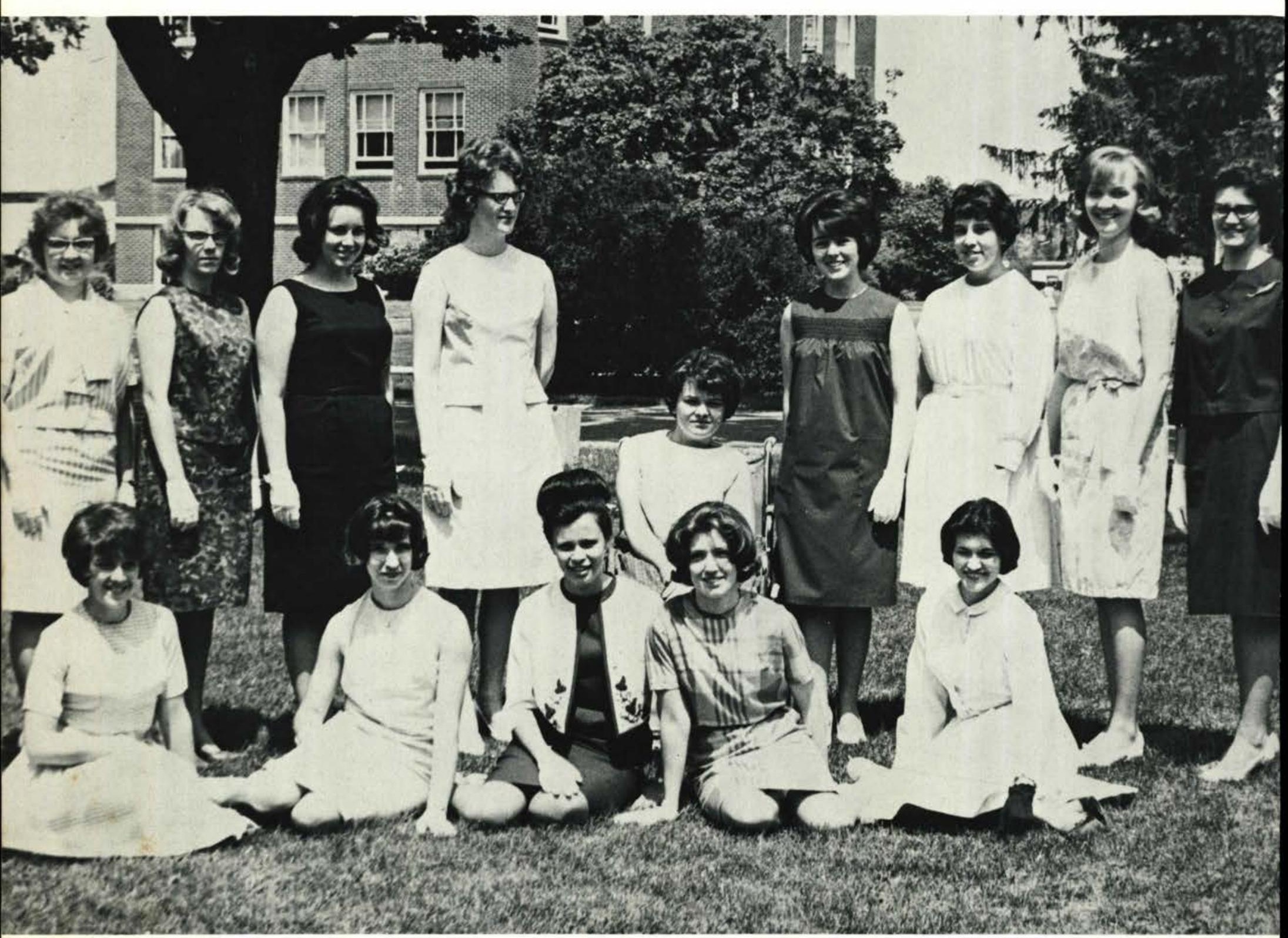

Front Row, left to right: B. Smith, S. Wing, D. Guarnere, R. Butler, S. Ackerman. Back Row: M. Brong, S. Bartheld, G. Liechty, J. Maclaren, J. Davis. 


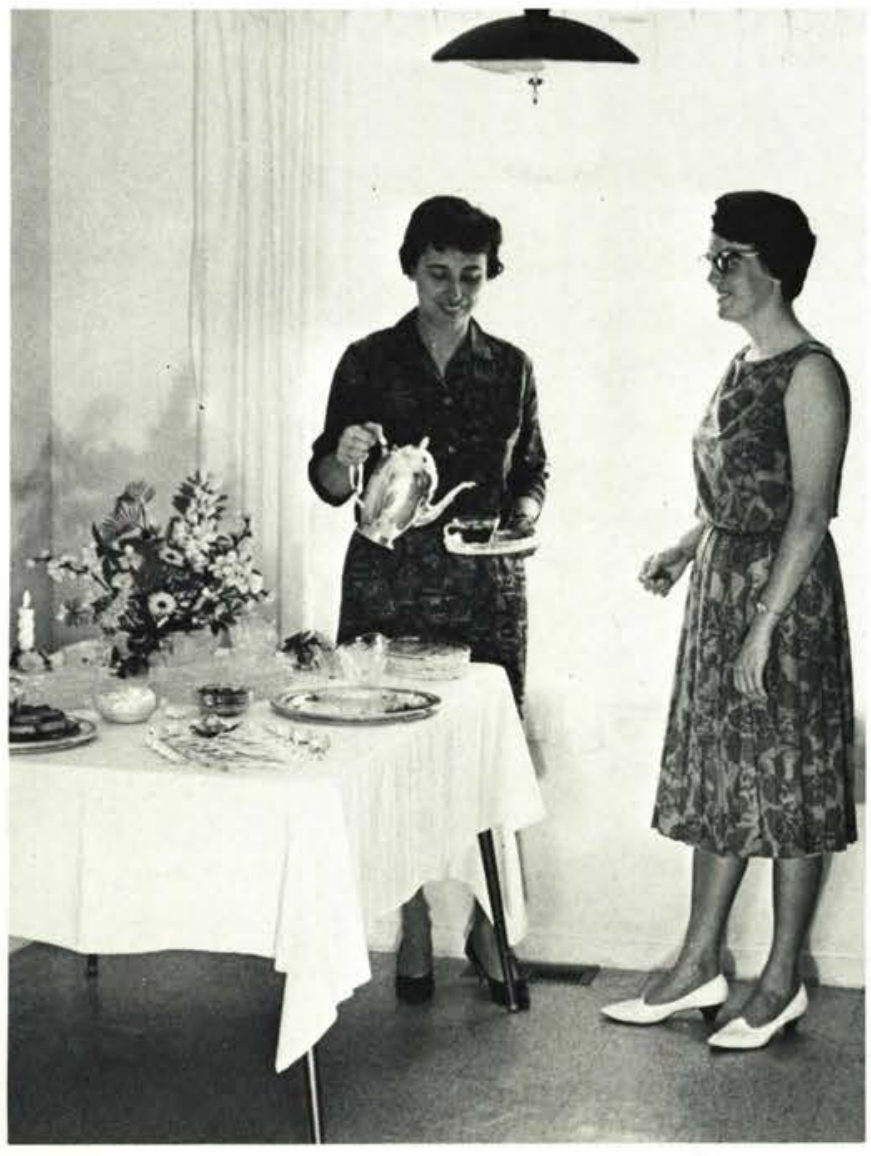

Each year Miss Joy Mackay, Dean of Women, continues and promotes the cultural interests of the women of Cedarville College by having an afternoon tea.

This social function not only provides Christian fellowship but also enables freshmen through the senior girls to practice proper and accepted social graces.

\section{An Afternoon Tea}

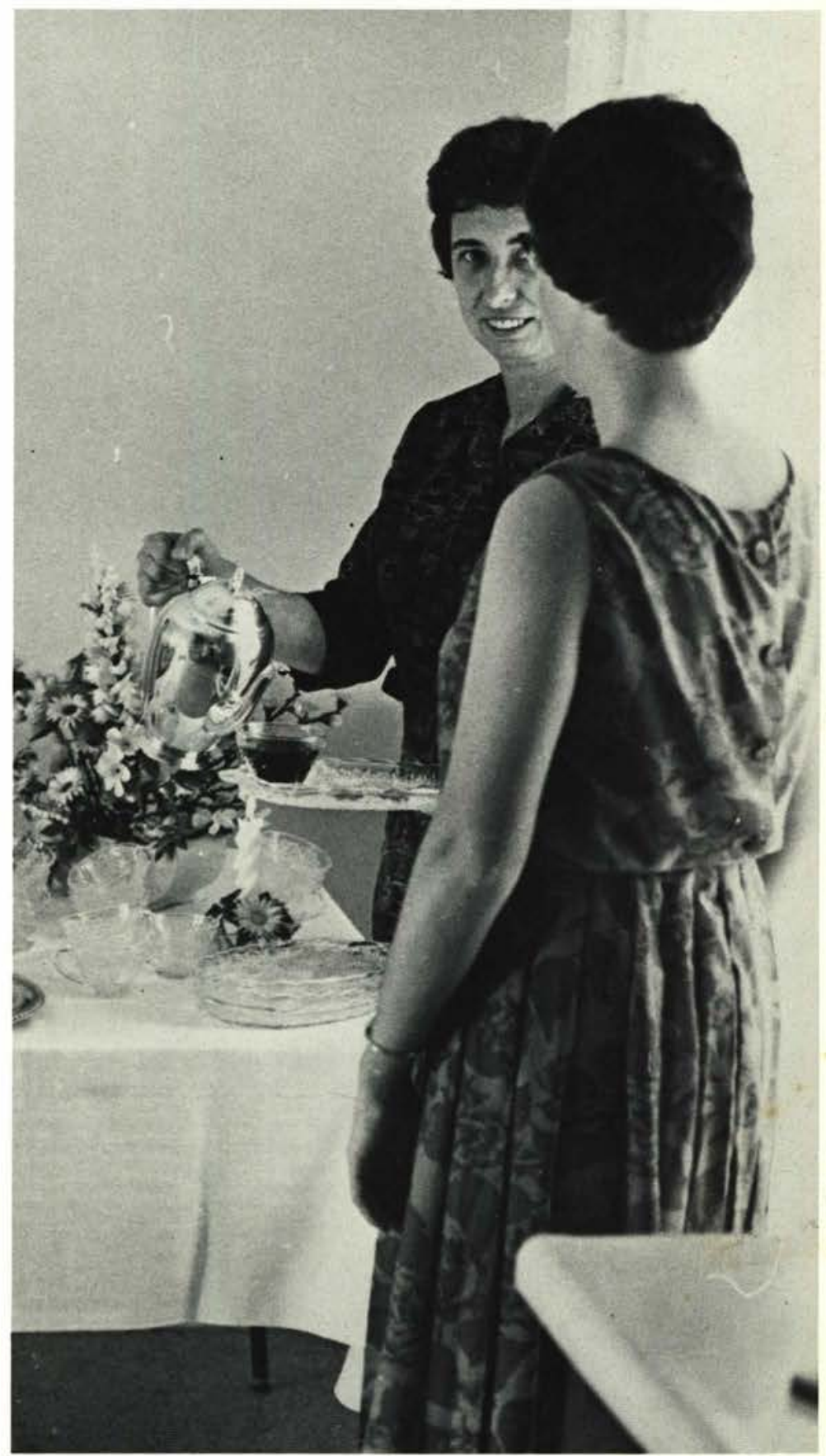




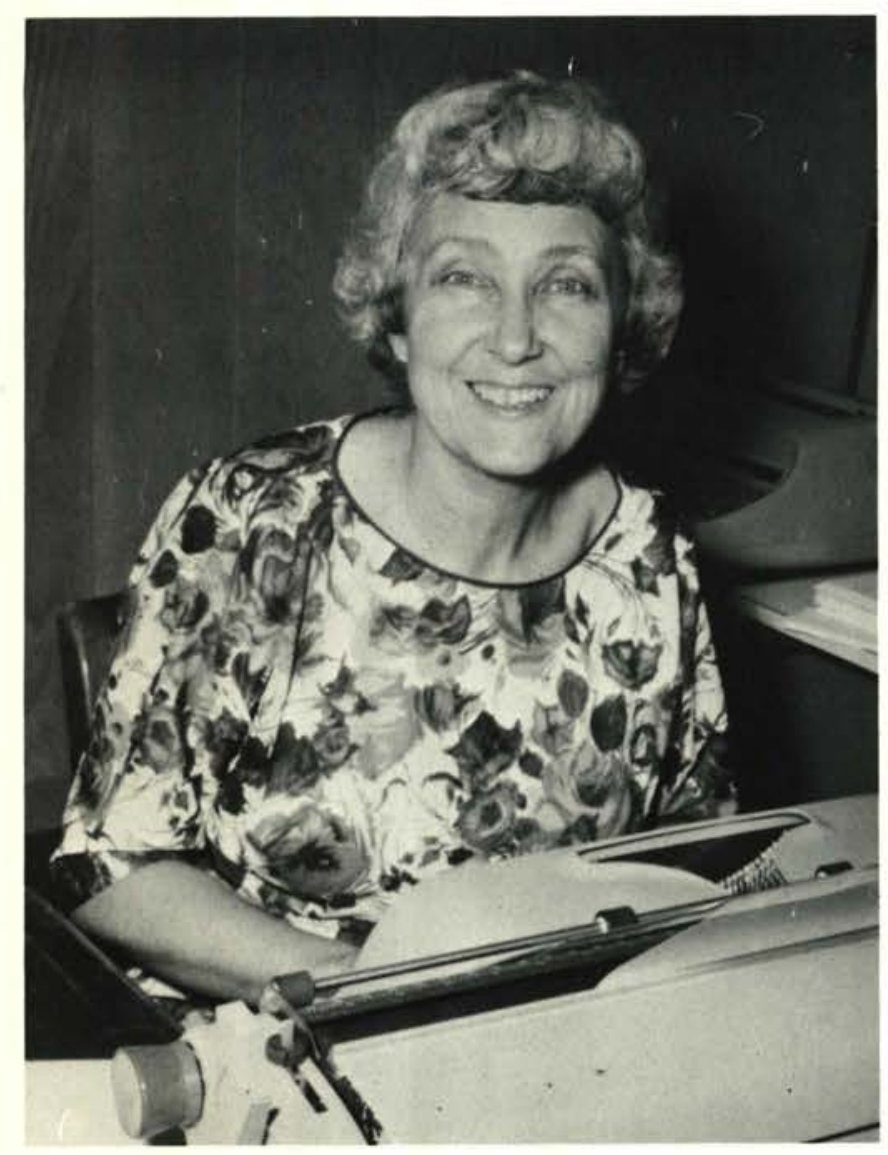

\section{Staff Secretaries}

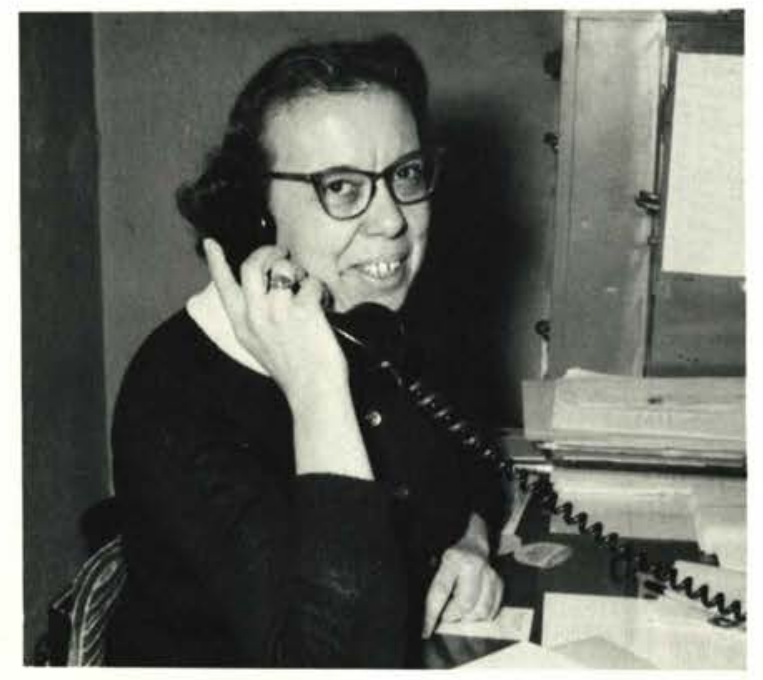

Miss Jean Hartsell, Secretary to Dean of Students.

Mrs. Brown, Secretary to President Jeremiah.

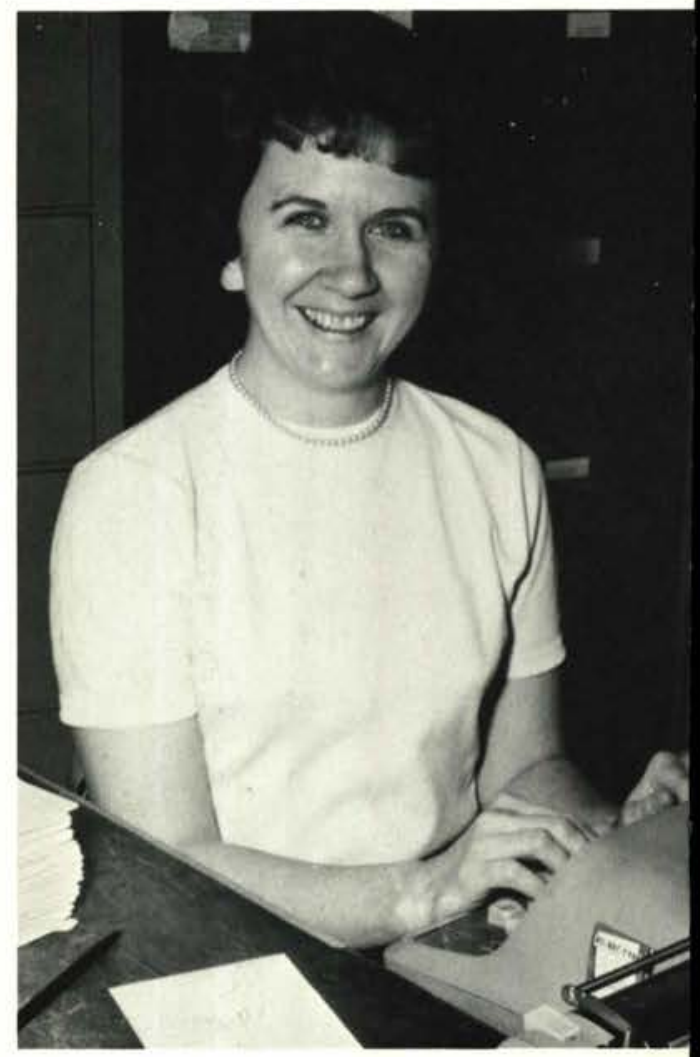

Mrs. Nancy McPheeters, Secretary to Registrar. 


\section{Business Office}

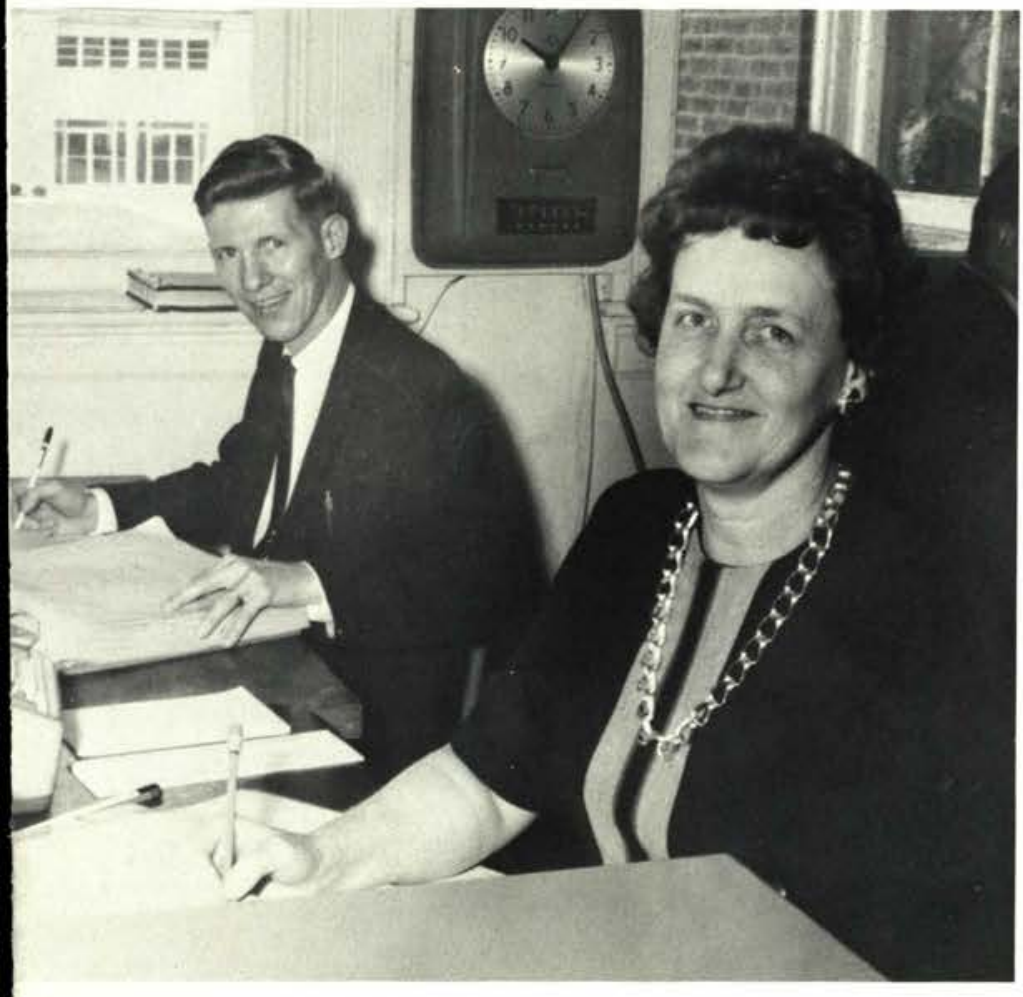

David Gidley, Bookkeeper; Dorothy Spencer, Cashier.

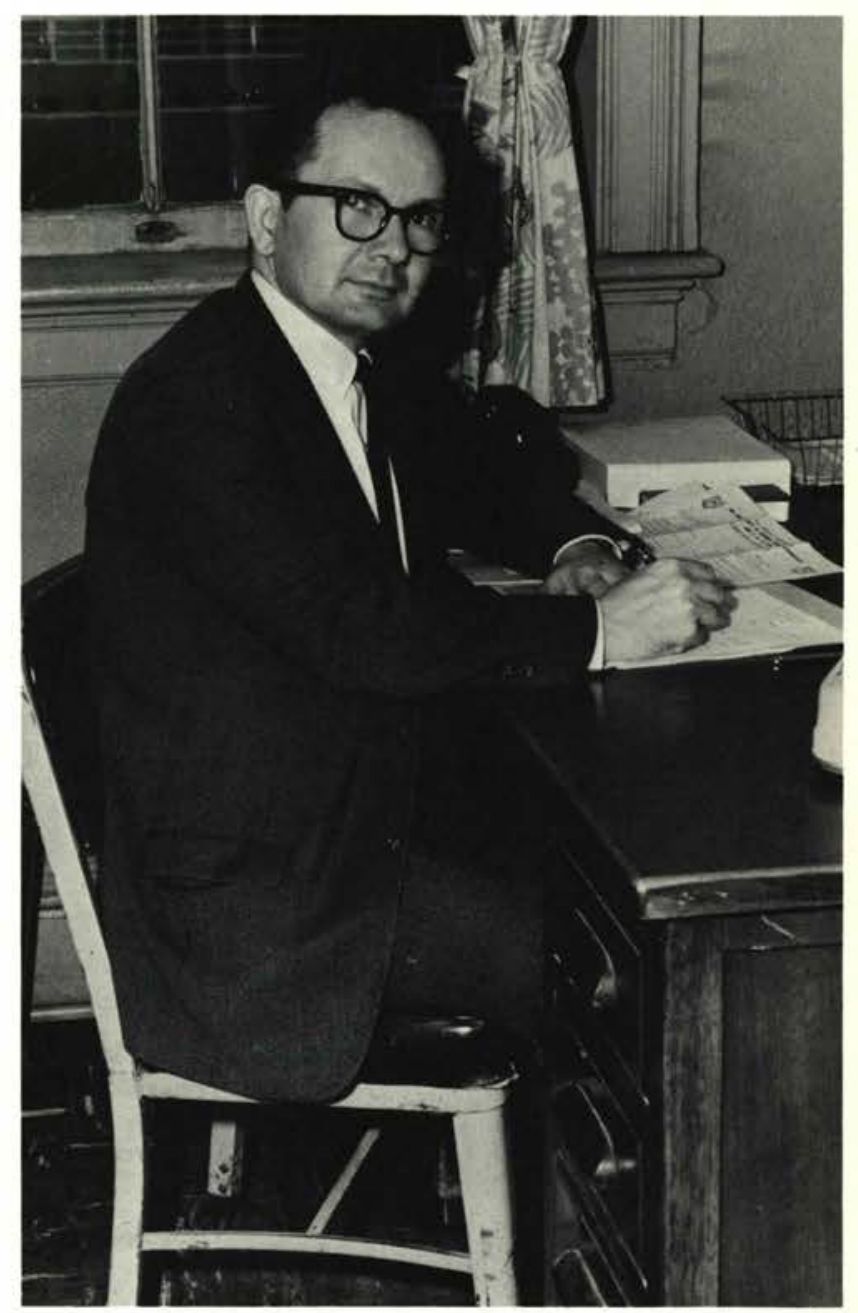

Kenneth St. Clair, C.P.A., Business Manager.

Donald Wilcoxon, Accounts Payable Clerk; Marabeth Elmore, Secretary to the Business Manager.

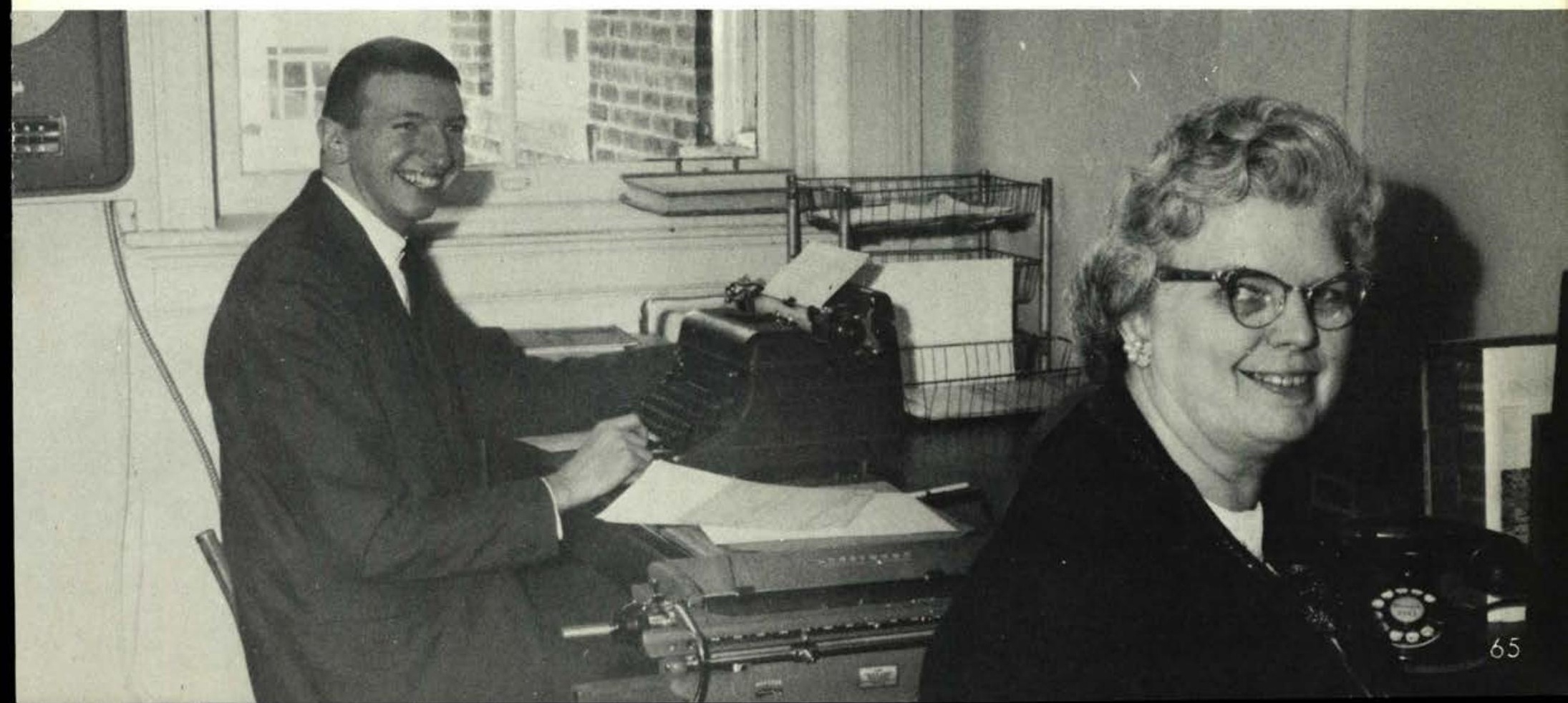




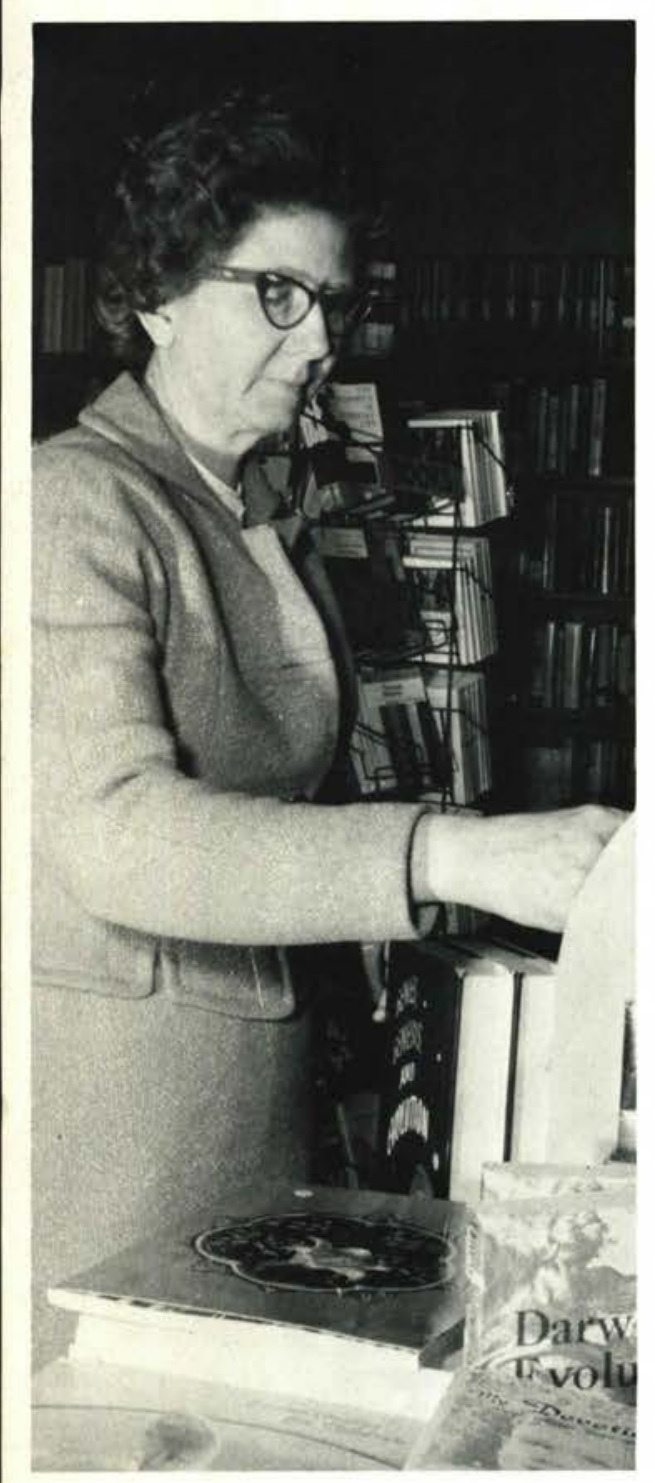

Miss B. Mick, Manager of College Book Store.

\section{College Staff}

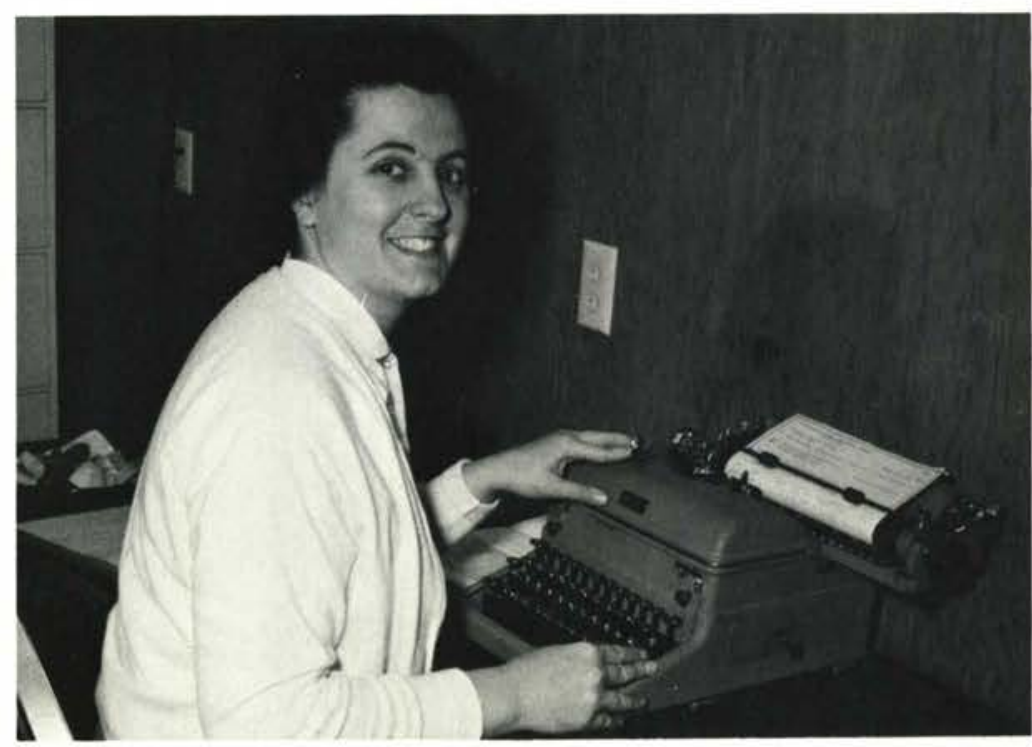

Mrs. H. Bergen

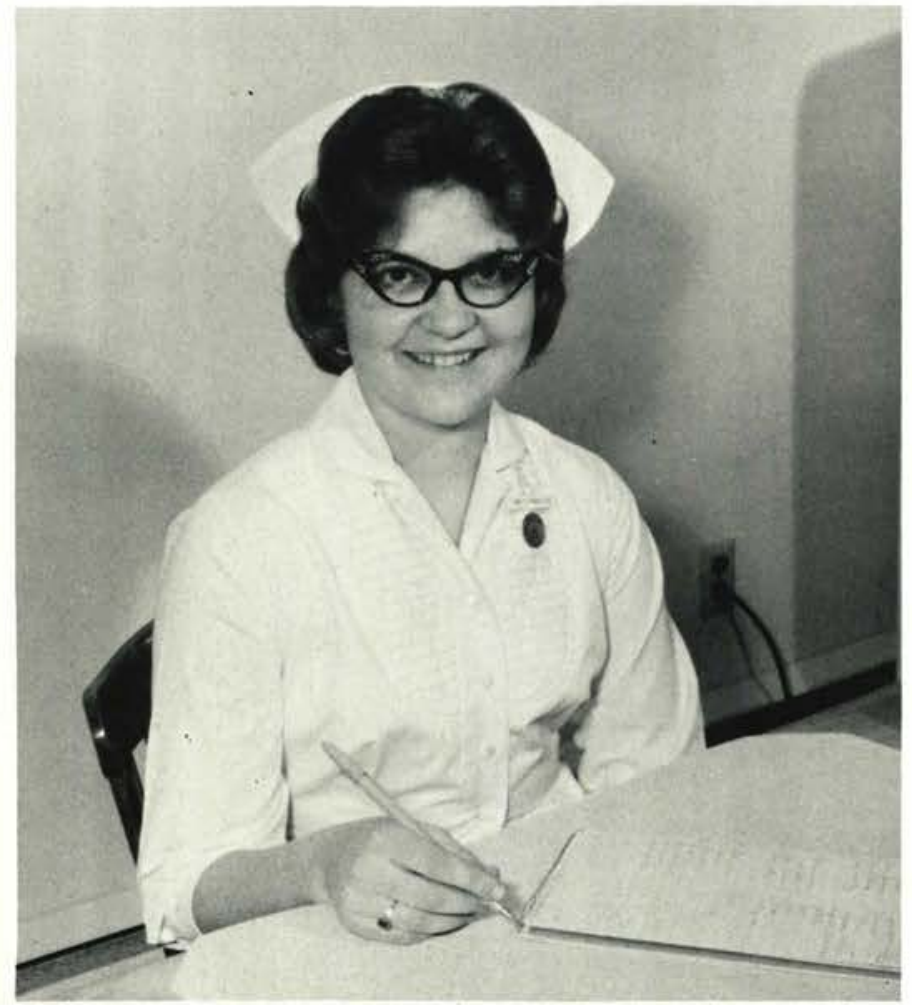

Miss G. Trimble, College Nurse.

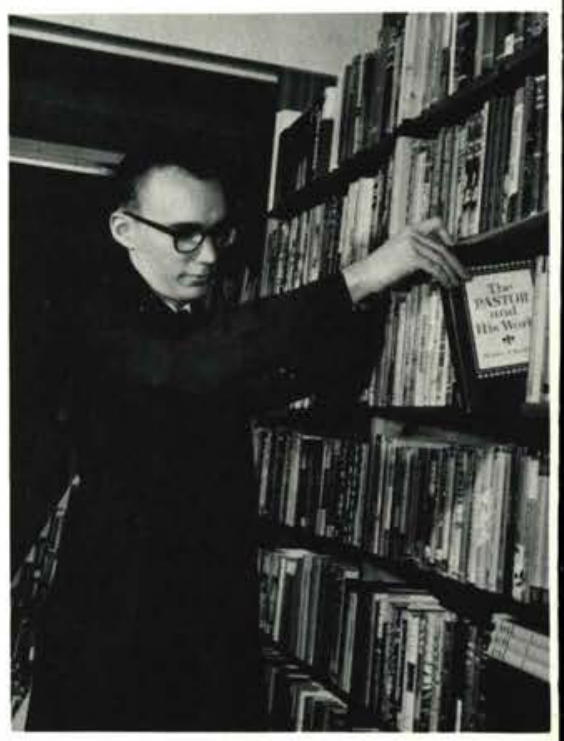




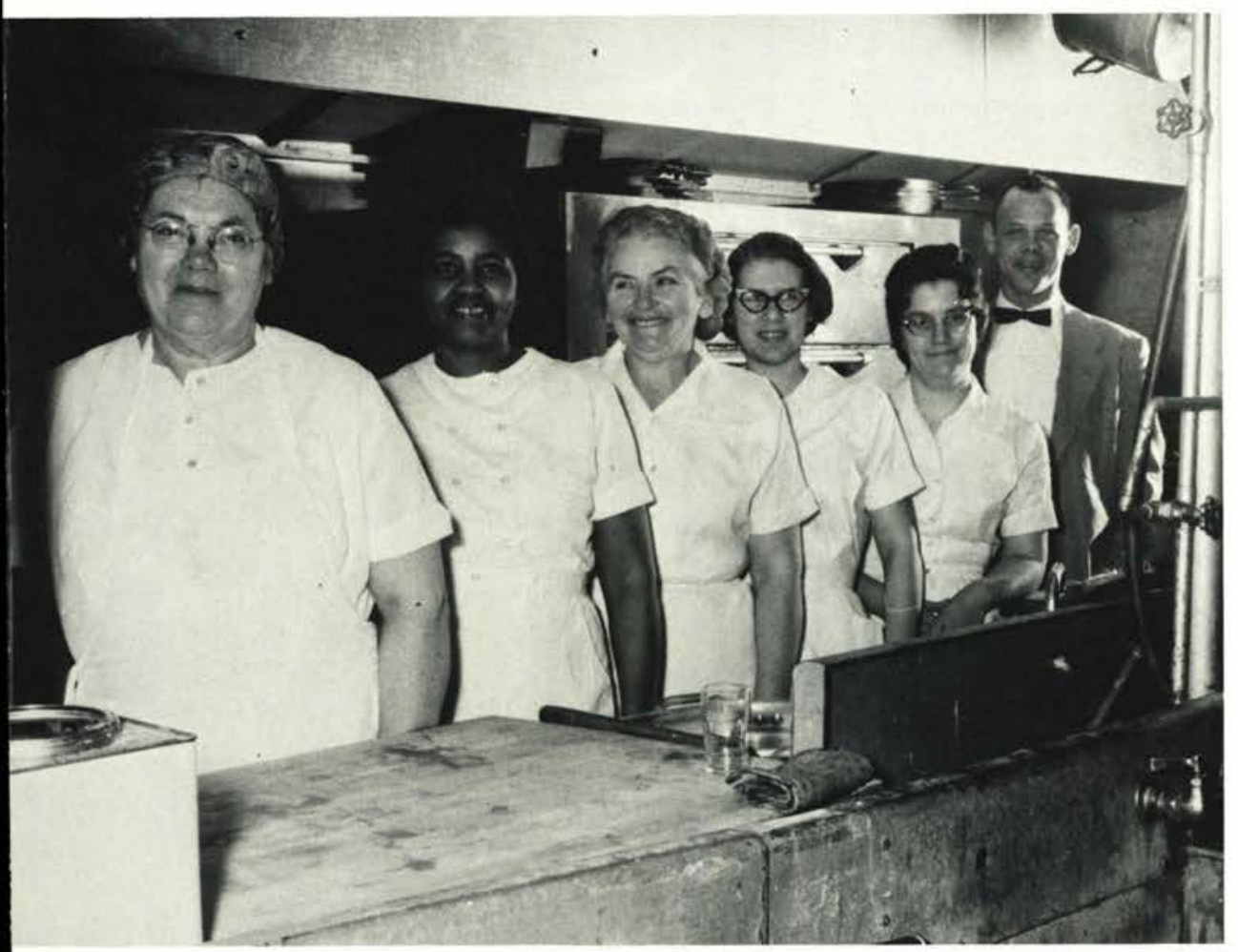

Left to Right: K. Smith, J. Wilson, M. McPherson, H. Lloyd, G. Wickell, T. Smith.

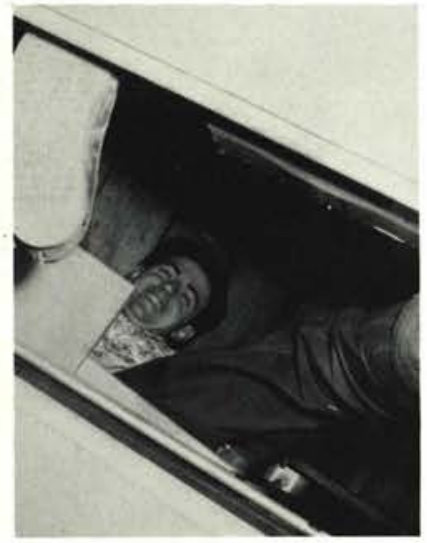

Back Row: D. Terrill, D. Stephens, G. Marshall, R. Dillon. Front Row: C. Tarter, D. Dillon, T. Folkrod.

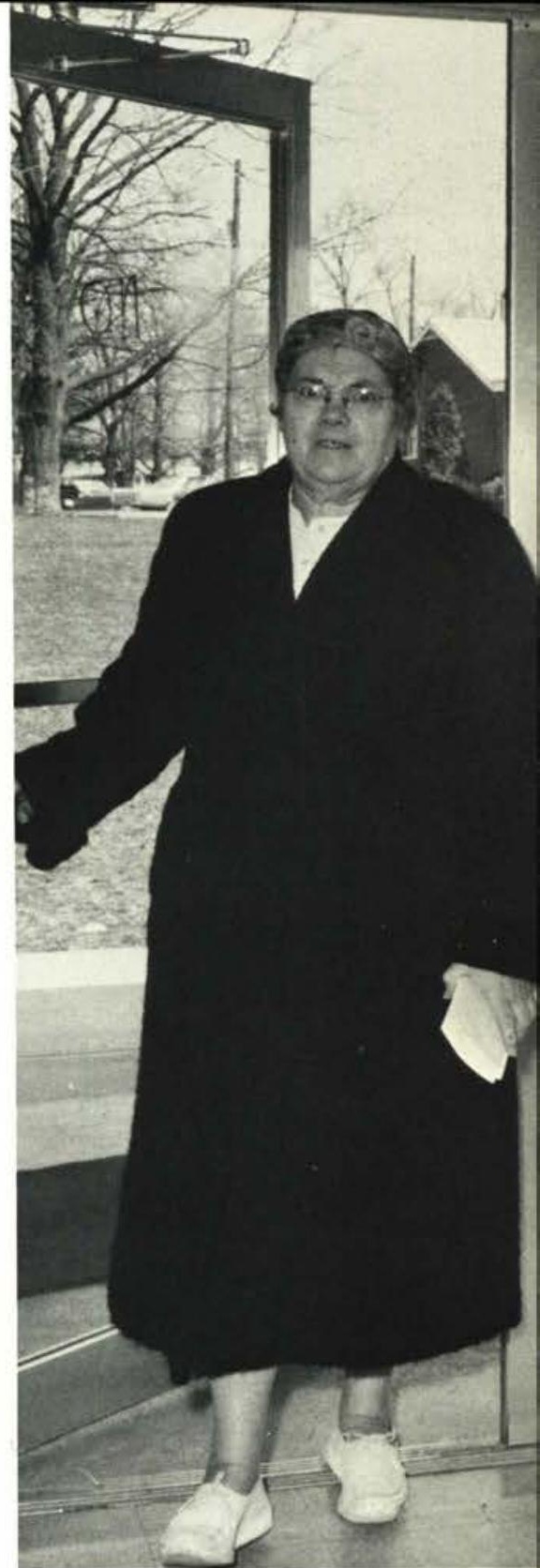

Mrs. Kate Smith.

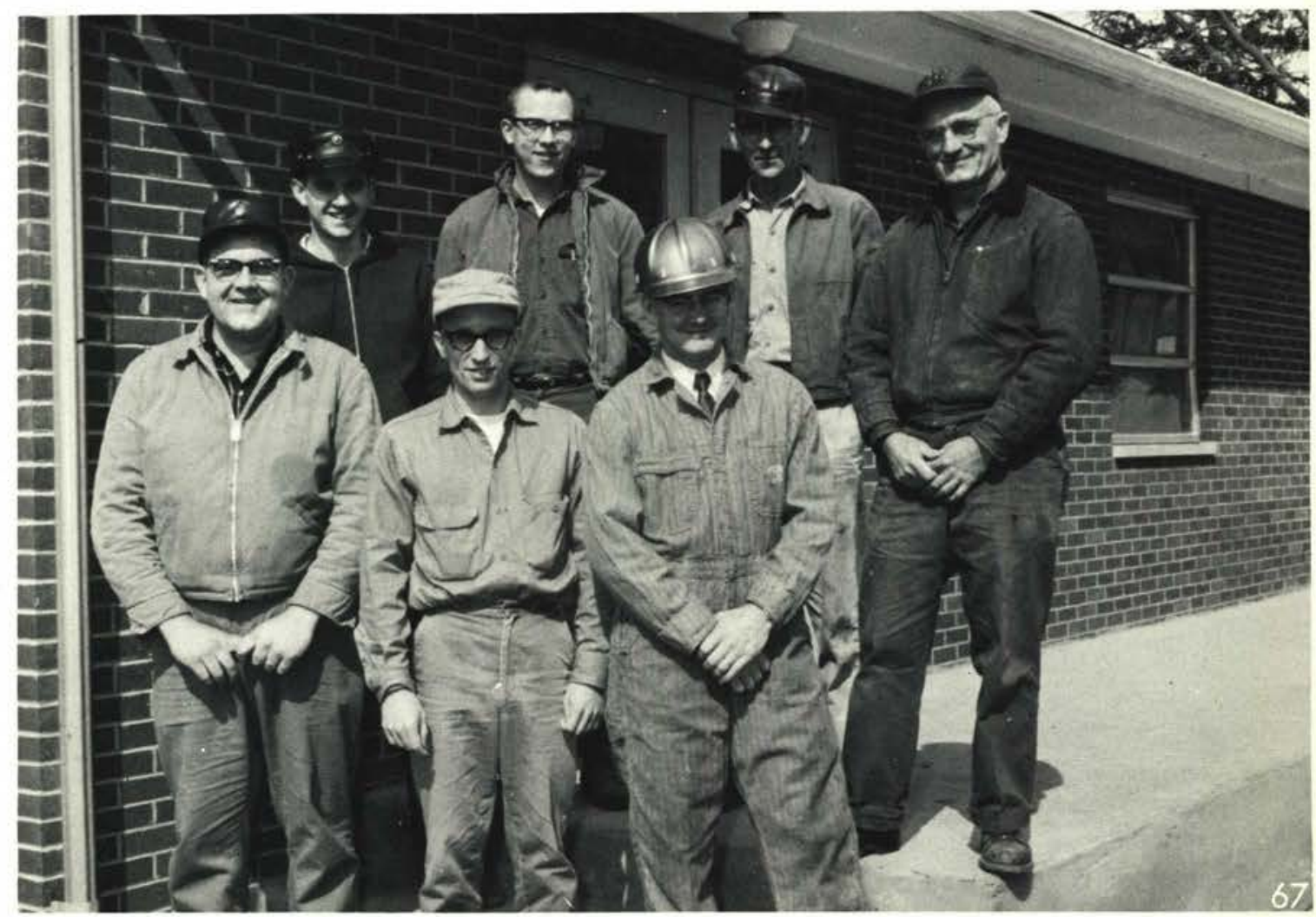




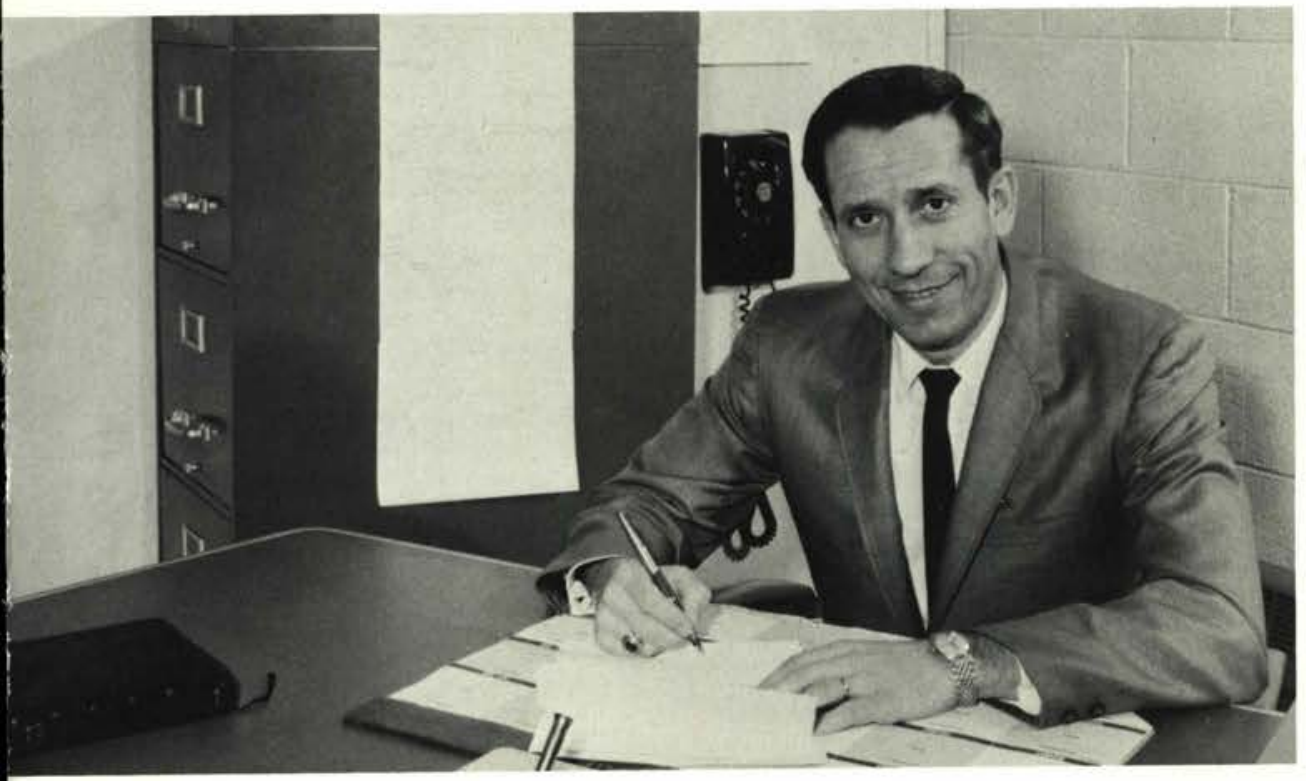

Mr. Lee Turner, Director of Public Relations.

\section{Public Relations}

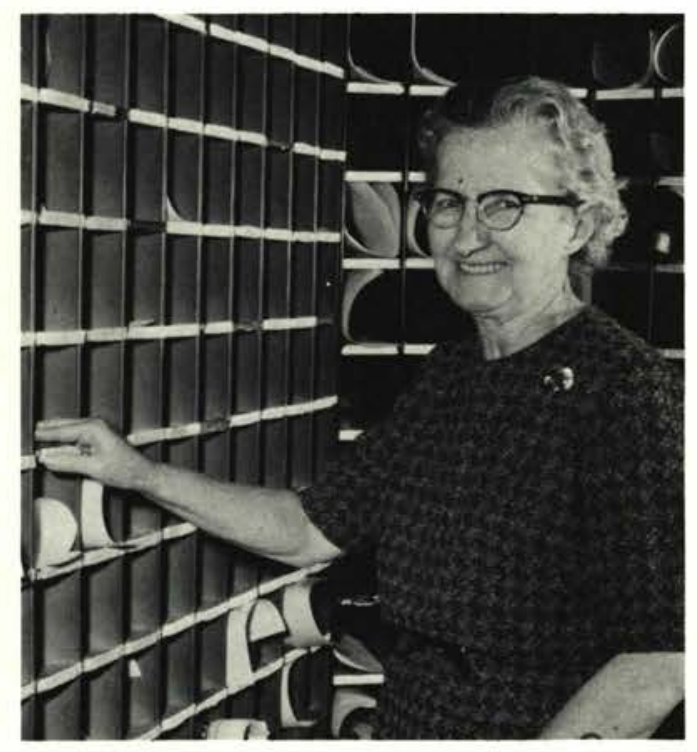

Mrs. Ruth Underwood, Publications.

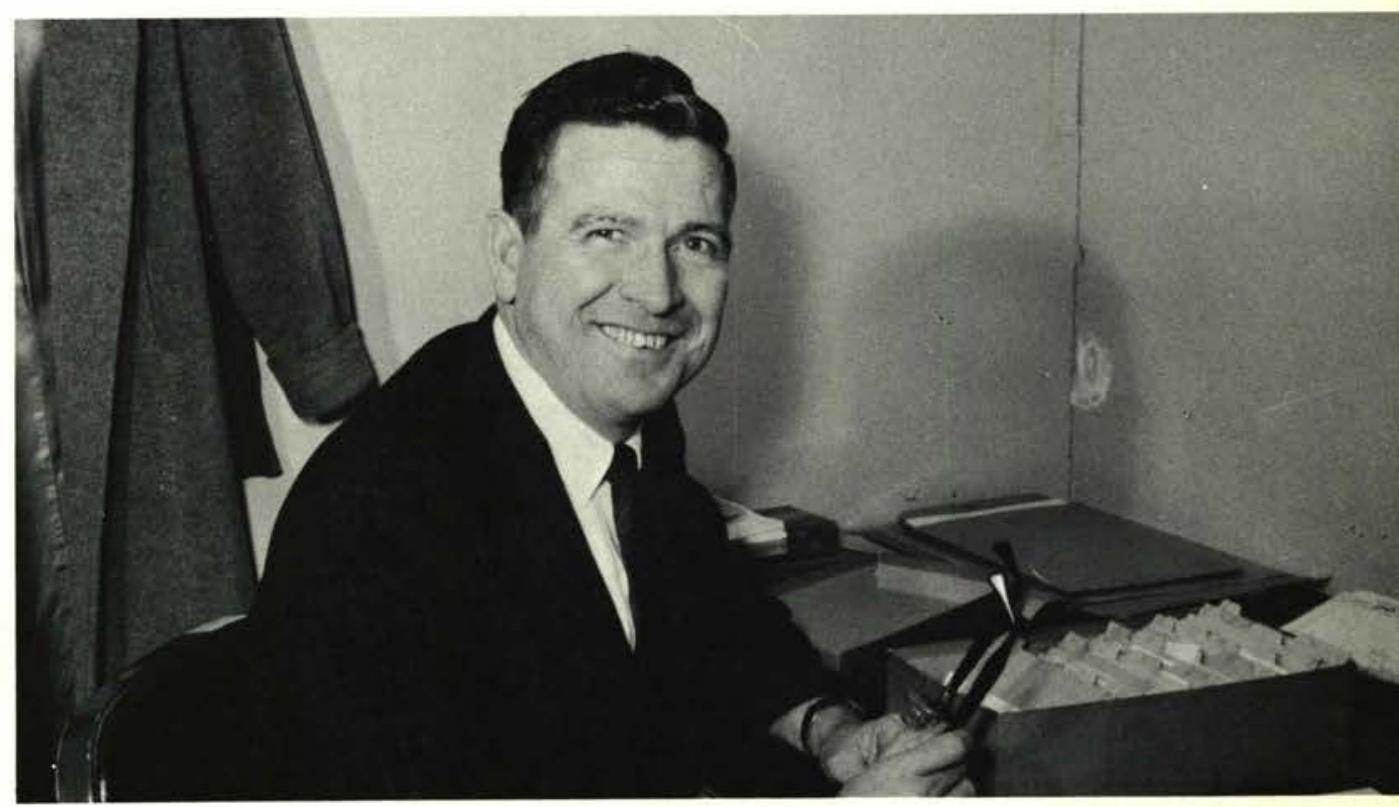

Mr. James Taylor, Publications 


\section{CHAPEL}

"Sweet Hour of Prayer" sets the mood for daily chapel services. The hour set aside for worship usually includes special music and a message from the Word of God. Speakers include pastors of local churches, representatives from Christian organizations, missionaries, laymen from the various professions, faculty and administration from the college, and sometimes students representing various campus organizations.

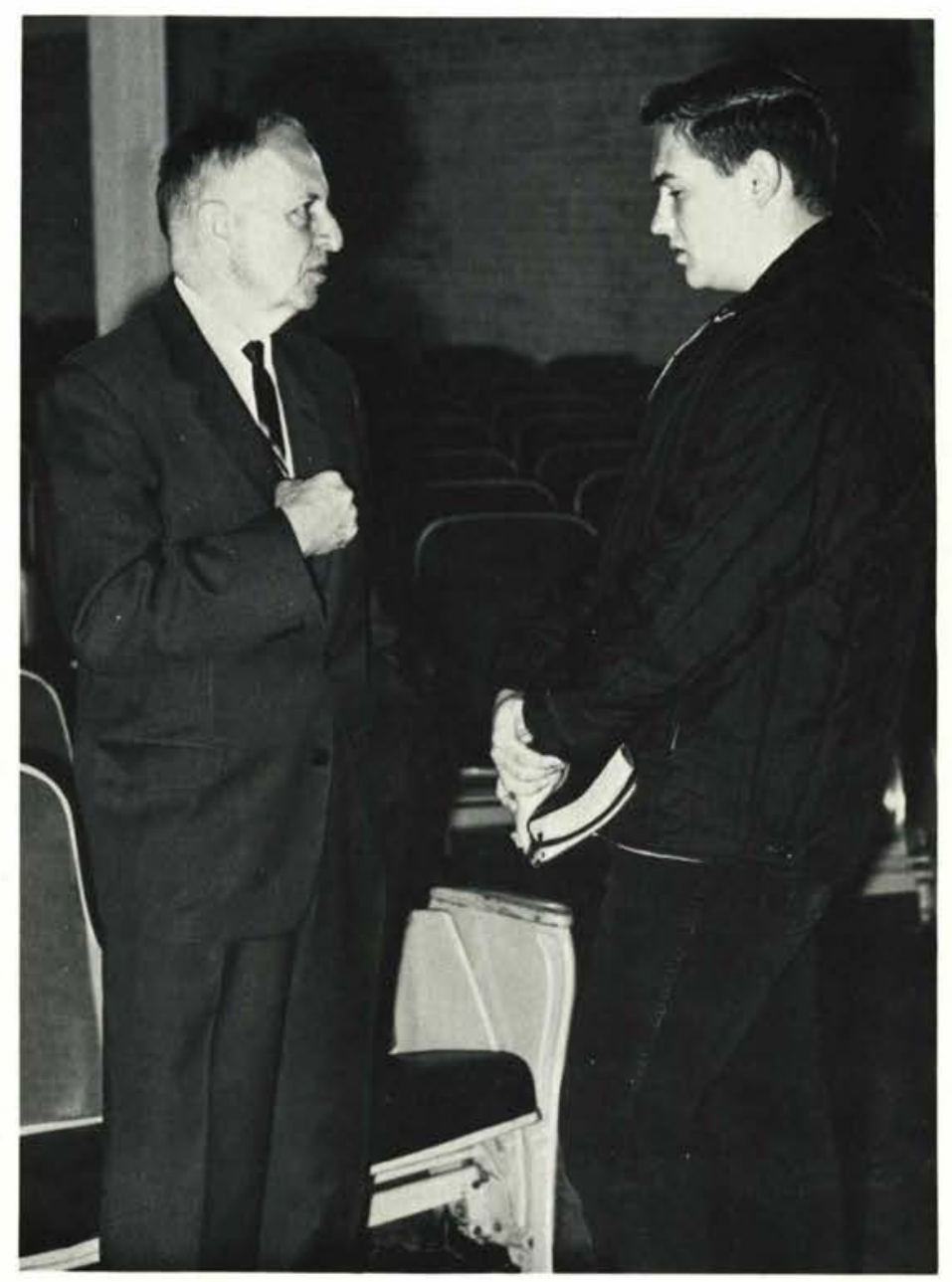

General Harrison 

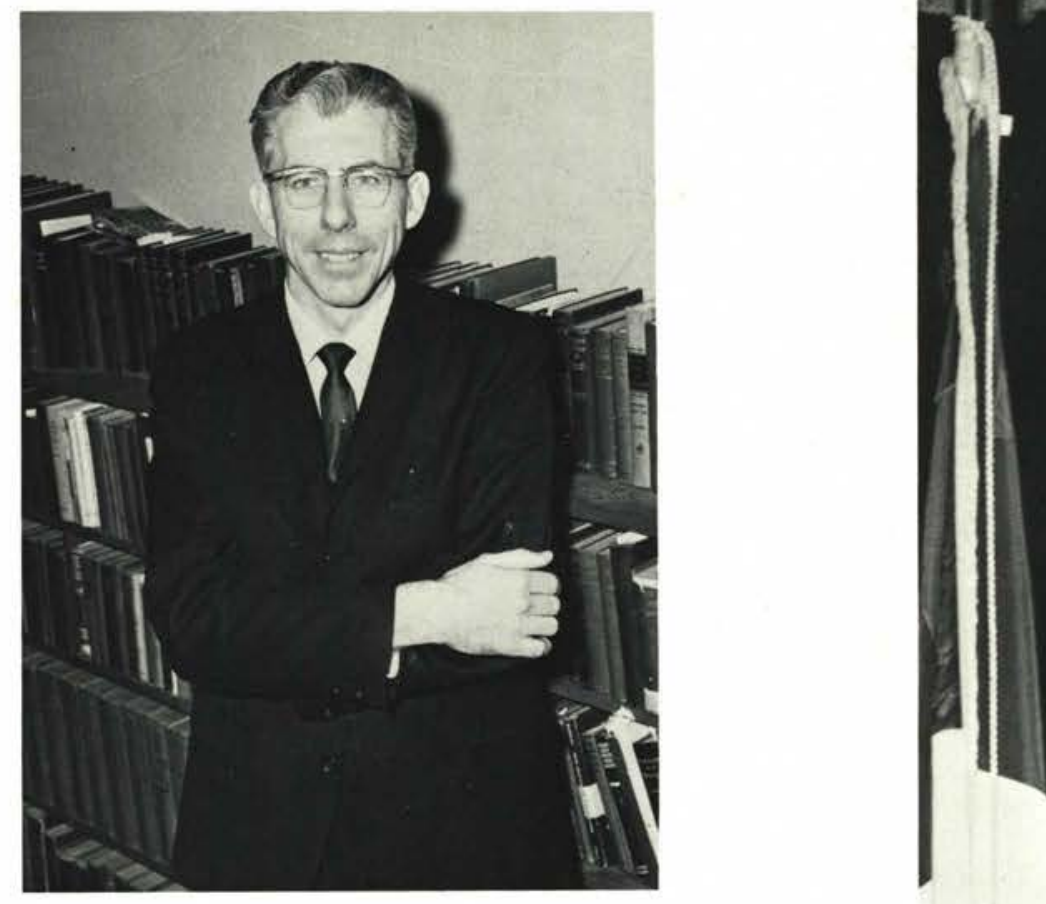

Dr. Merle Hull, editor of Regular Baptist Press

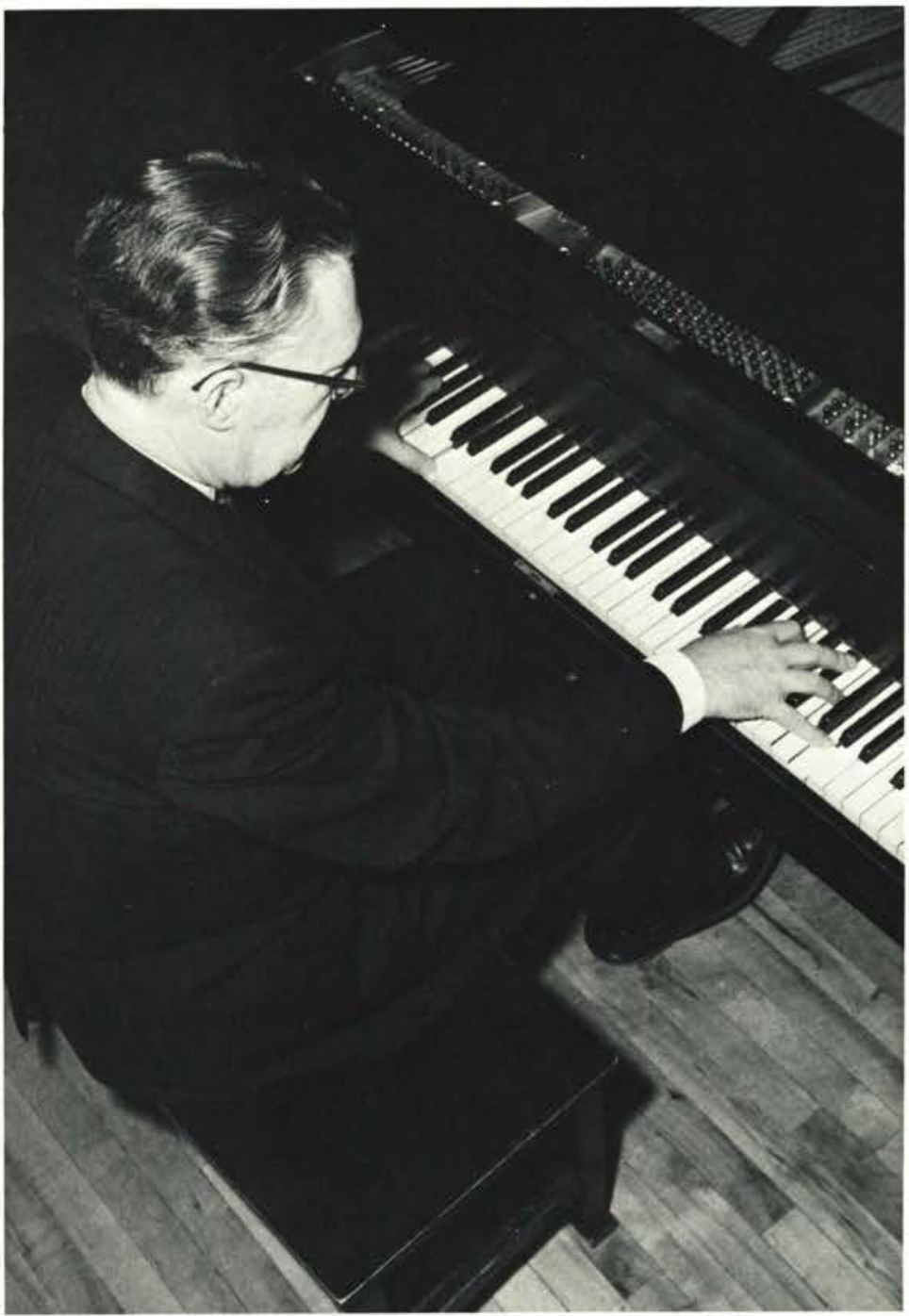

Dr. H. Dautel-First Baptist Church Harvey, Illinois 


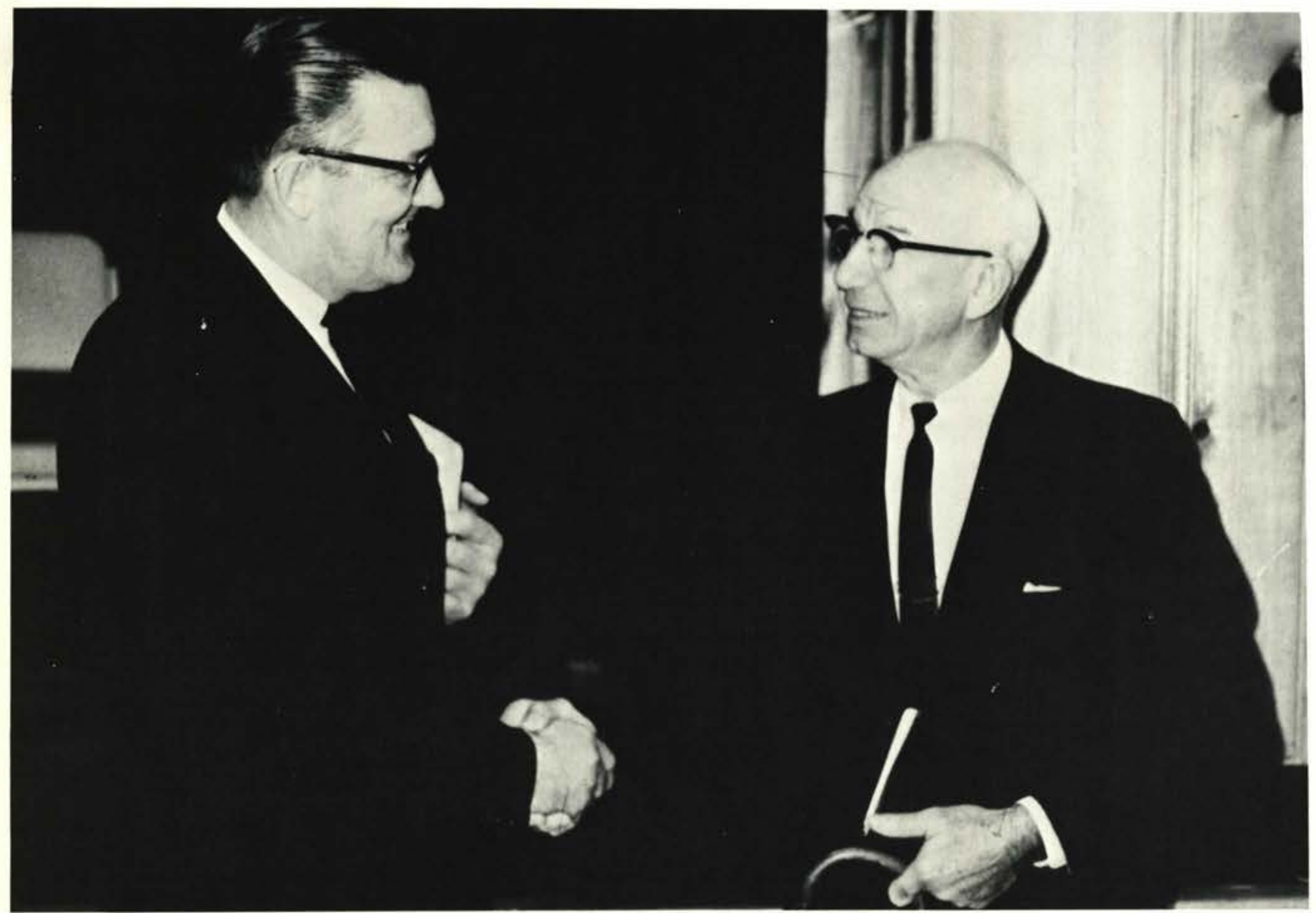

Joseph Bower-Quincy, Illinois
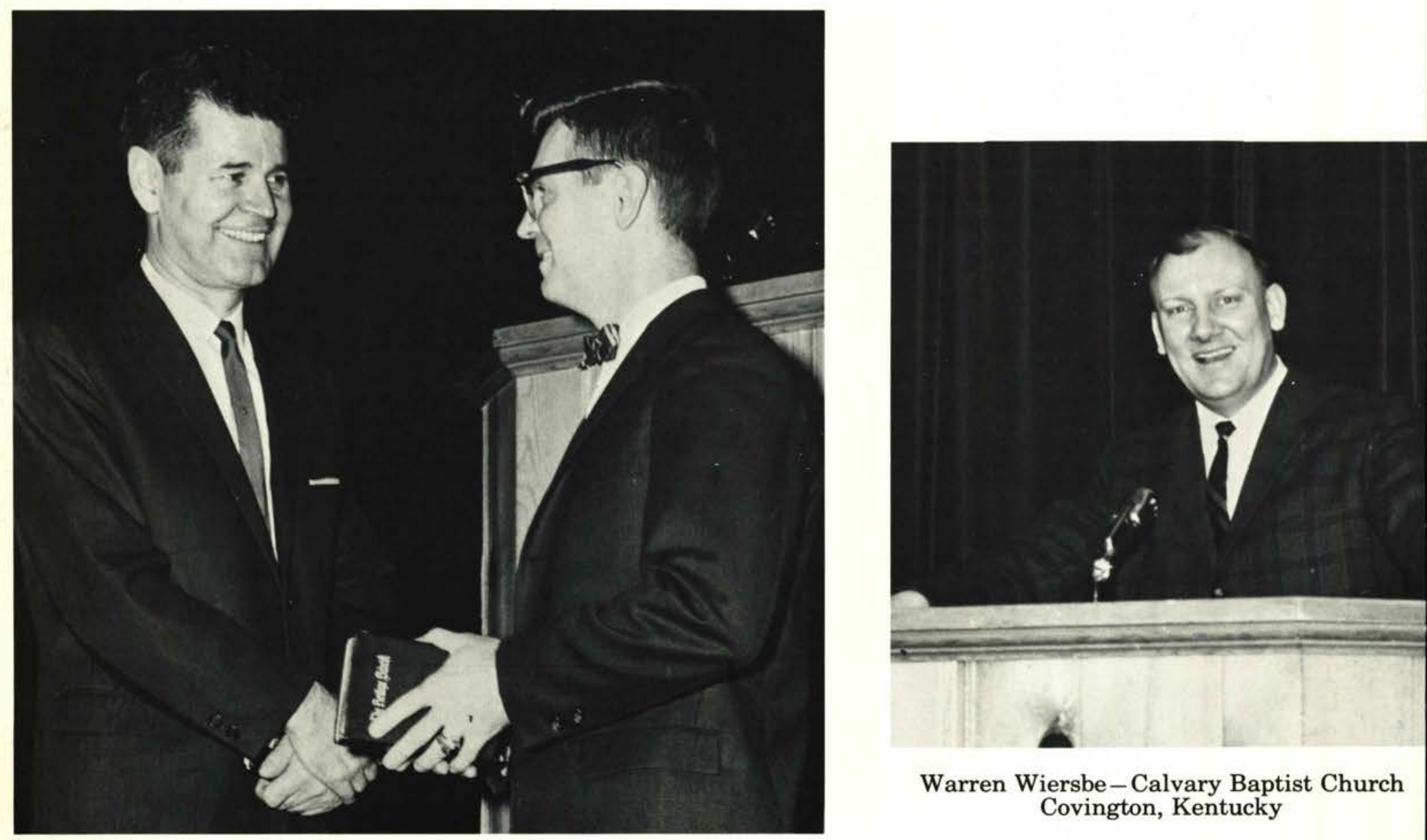

Warren Wiersbe-Calvary Baptist Church Covington, Kentucky

Dr. Hugh Horner-Sandusky, Ohio 


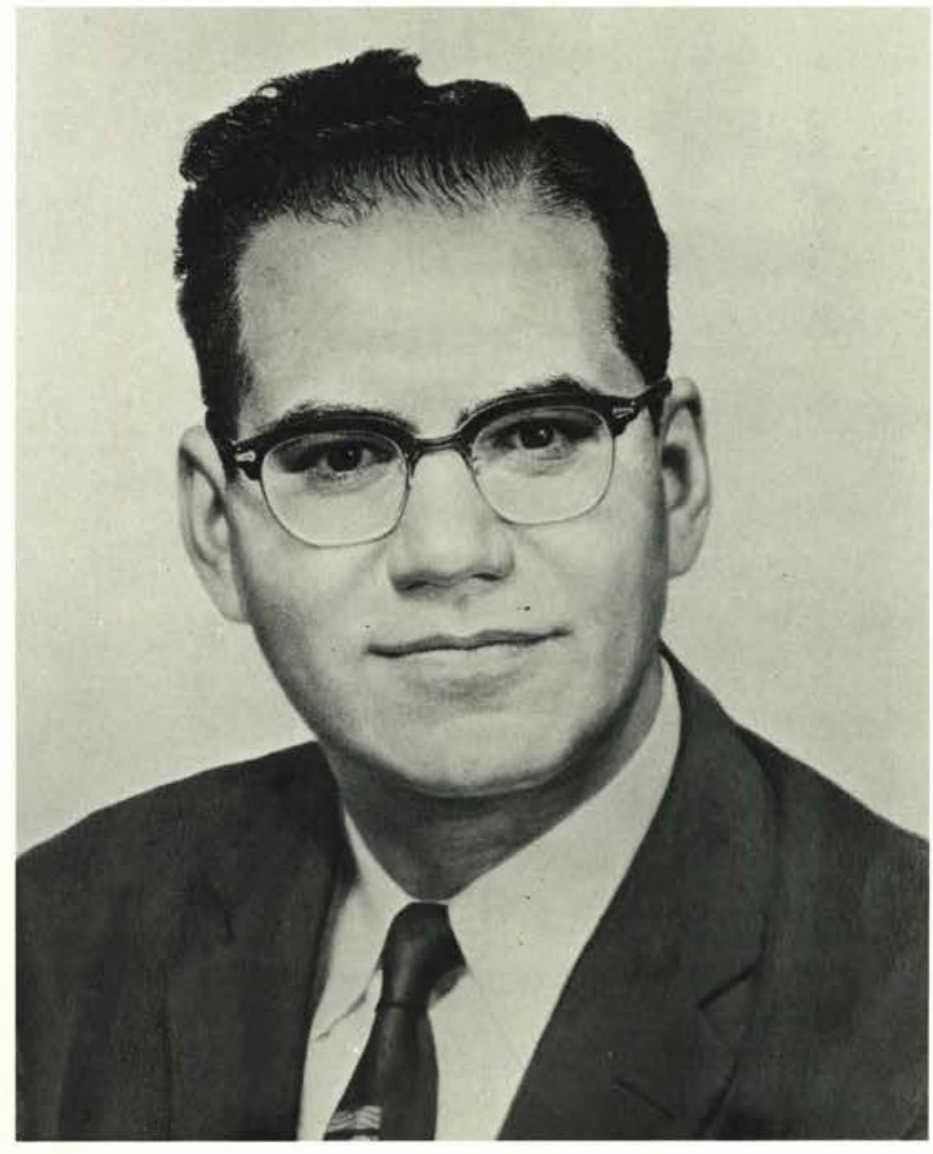

Rev. Spiros Zodhaites

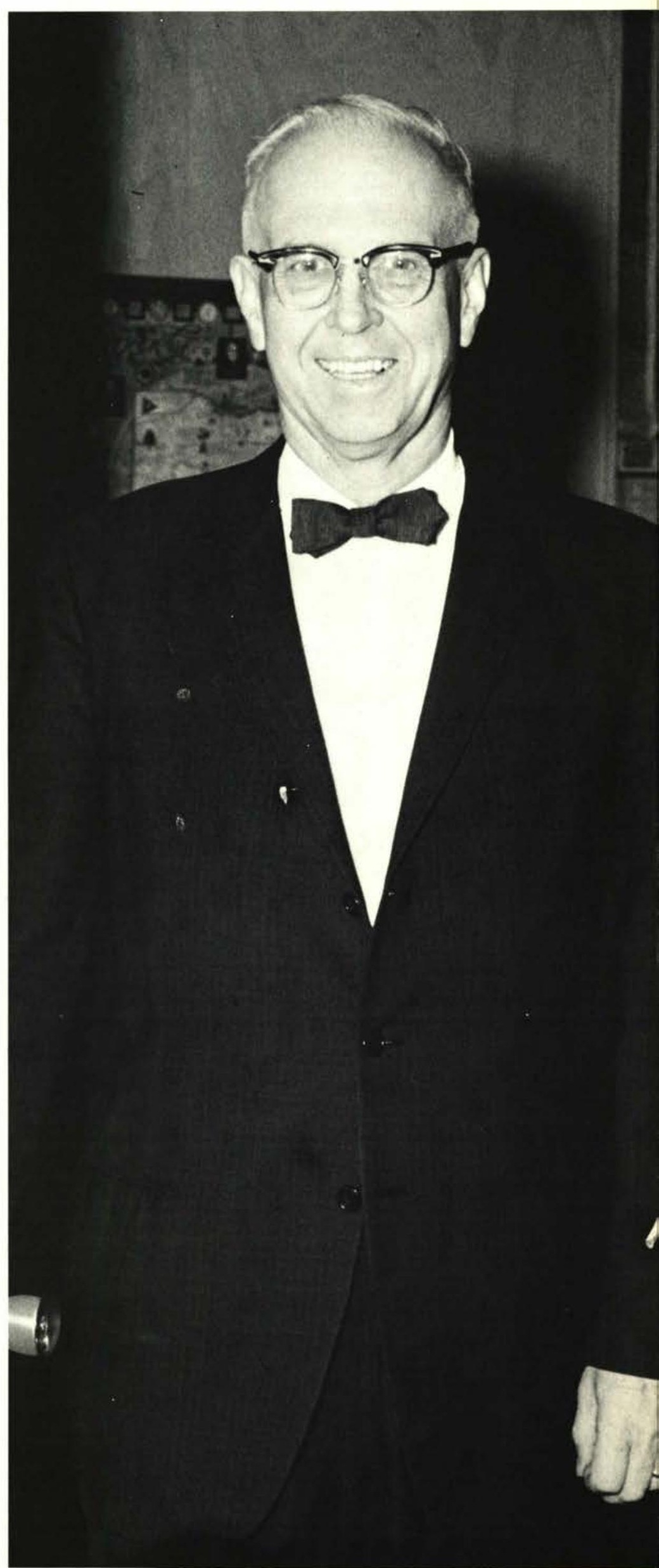

Dr. Paul Jackson 


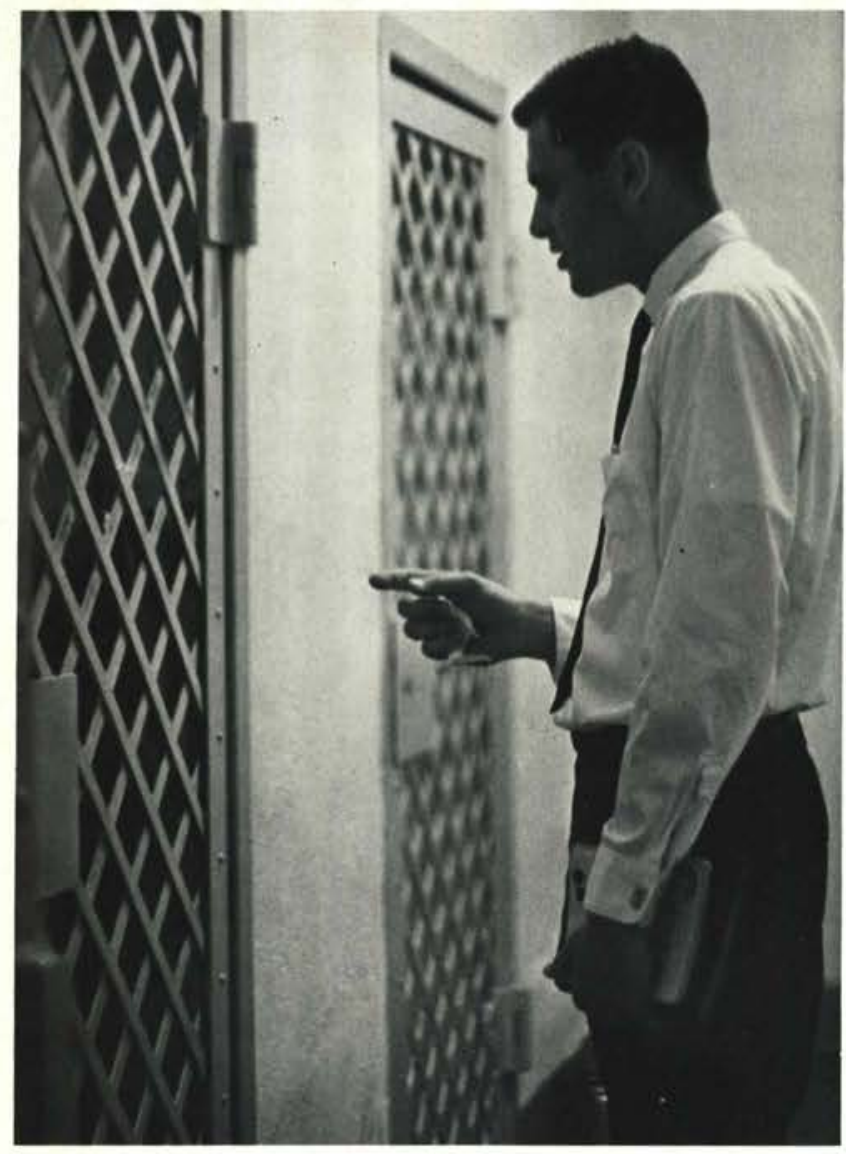

\section{Christian Service}

Each week, several college students serve the Lord in their Christian Service assignments. These are opportunities for students to apply many of the things they have studied as they talk with people: men in jails, women in the rest homes, children in Sunday School and Bible clubs. The assignments are both challenging and rewarding as many are saved through this personal contact.

SPRINGFIELD DETENTION HOME

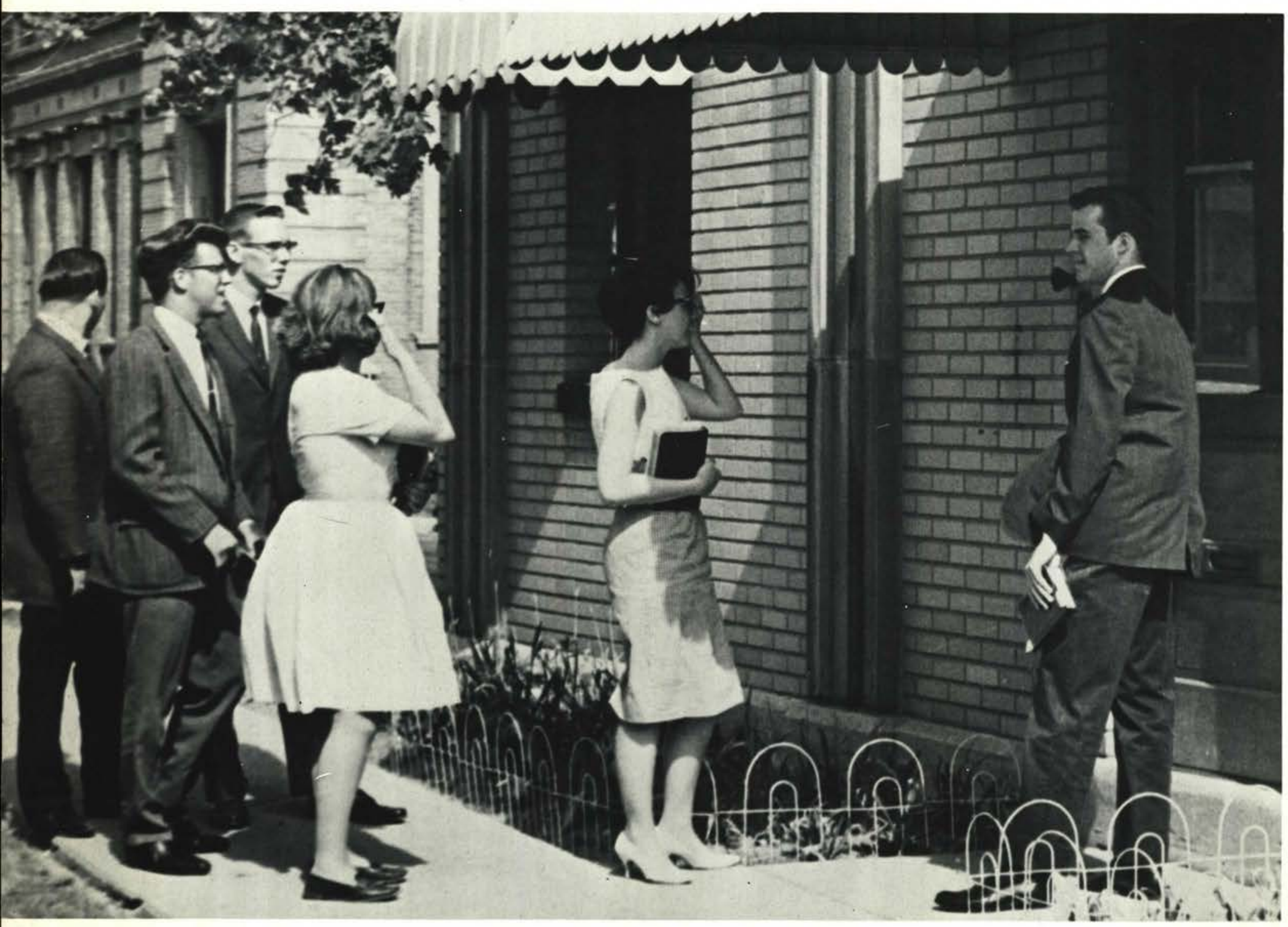




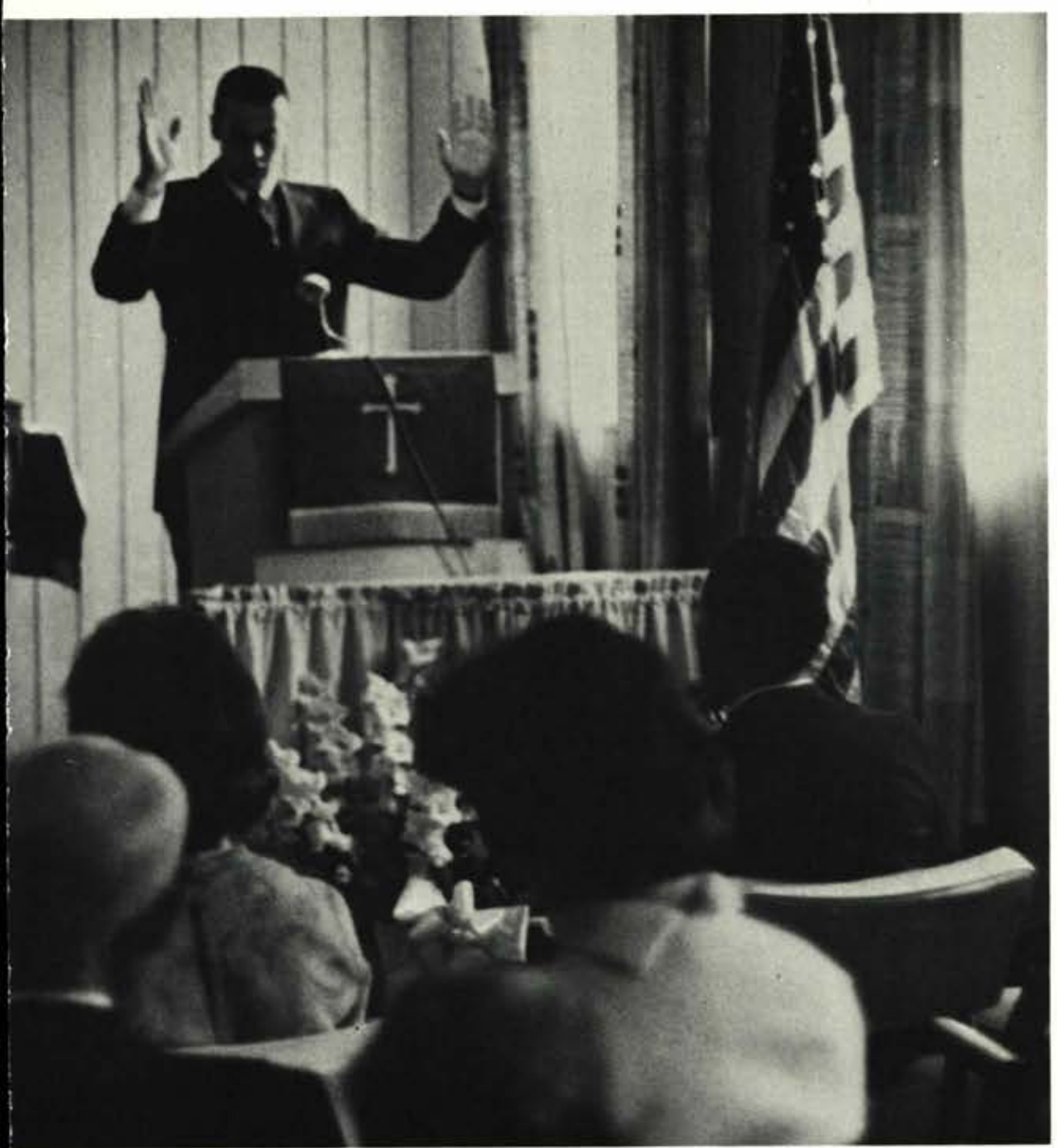

THE K OF P HOME

THE O.S.S.O. HOME

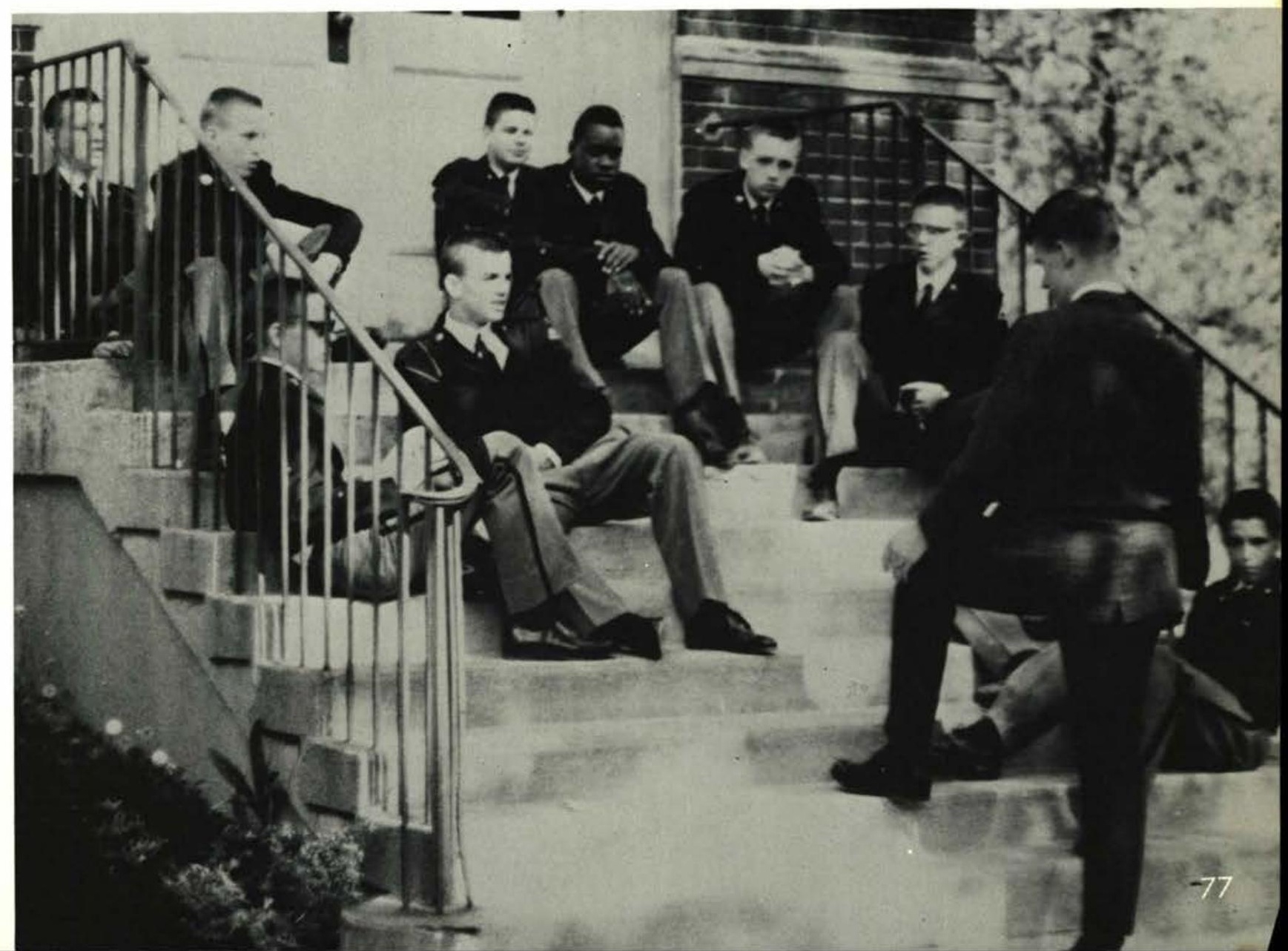


George Goodwin

Oak Forest, Illinois

David Gordon

Garrettsville, Ohio

Miriam Greiner

Wellington, Ohio

G. D. Griffin

Lincoln, Nebraska

Philip Grisso

Springfield, Ohio

James R. Halvorsen

Eagle Grove, Iowa

Arnold Hammer

Rockford, Michigan

Janet Hanlan

Ypsilanti, Michigan

Susan Harger

Titusville, Pennsylvania

Gary J. Harris

Beech Grove, Indiana

Linda Healy

Moscow, Iowa

Sherrill Hes

North Royalton, Ohio

Arlene Hoyte

Whitney Point, New York

Ted Jager

Wyoming, Michigan

Bill Kirtland

Cuyahoga Falls, Ohio

Elayne Korlewitz

Johnstown, Pennsylvania

Lloyd Lane

Coldwater, Michigan

Sue Lepine

Buffalo, New York

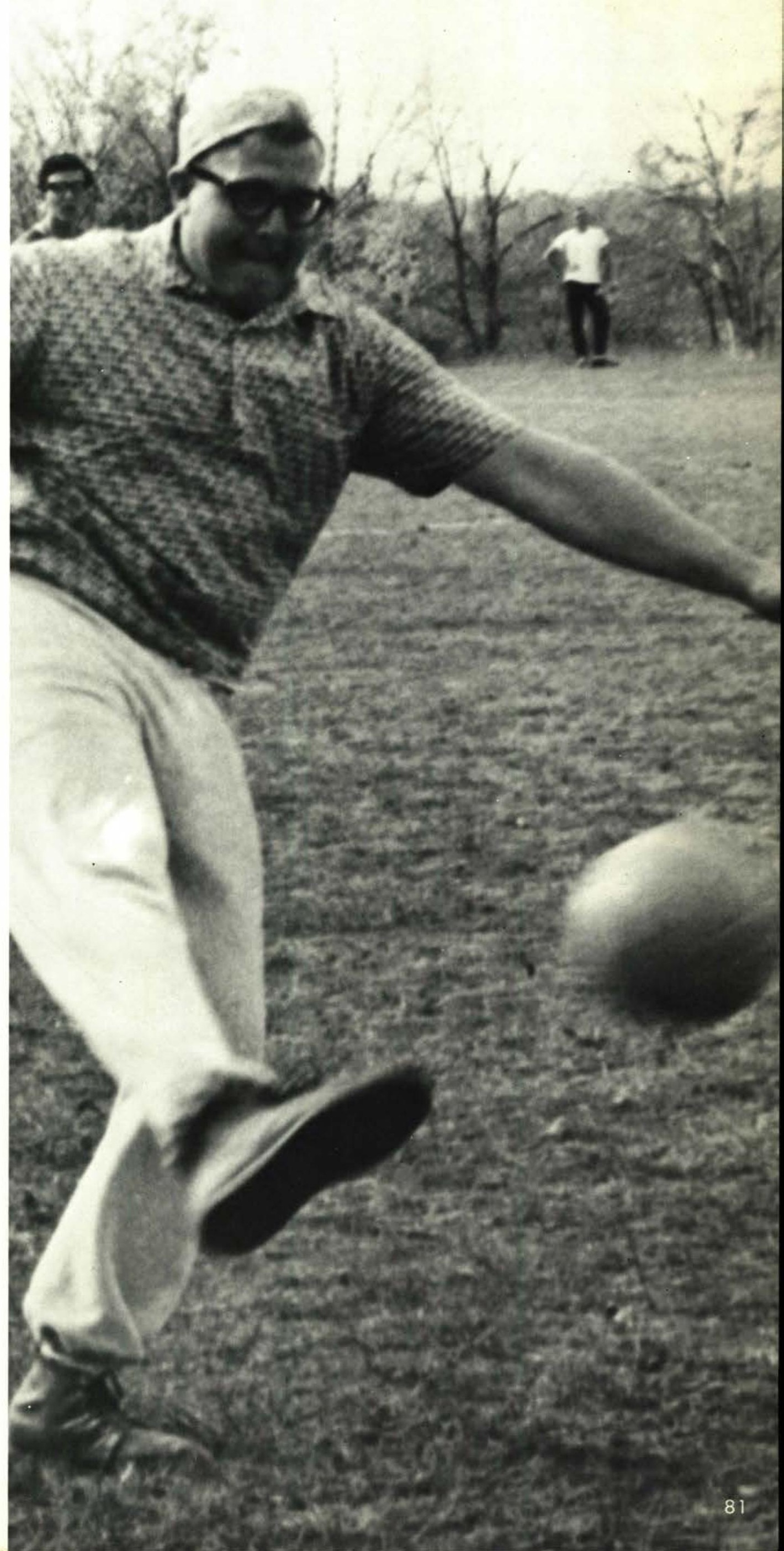


Gretchen Liechty

Hobart, Indiana

J. David Loos

Mishawaka, Indiana

Brian Mayfield

Liberty, Illinois

Buster M. McPheeters

Quincy, Illinois

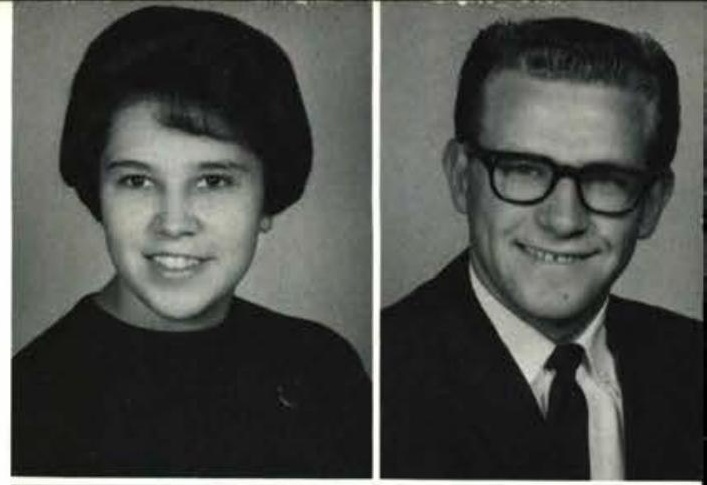

Valerie Merryman

Teaneck, New Jersey

Carol Mikels

Indianapolis, Indiana

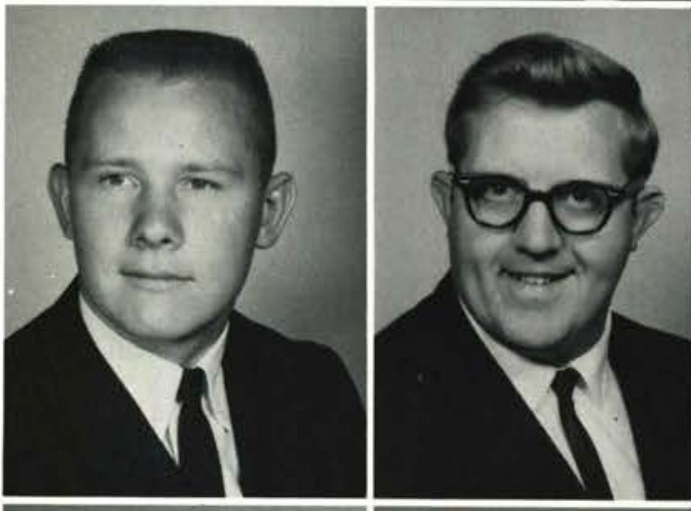

Joyce Micka

Ridgefield Park, New Jersey

Lawrence Montgomery

Columbus, Ohio

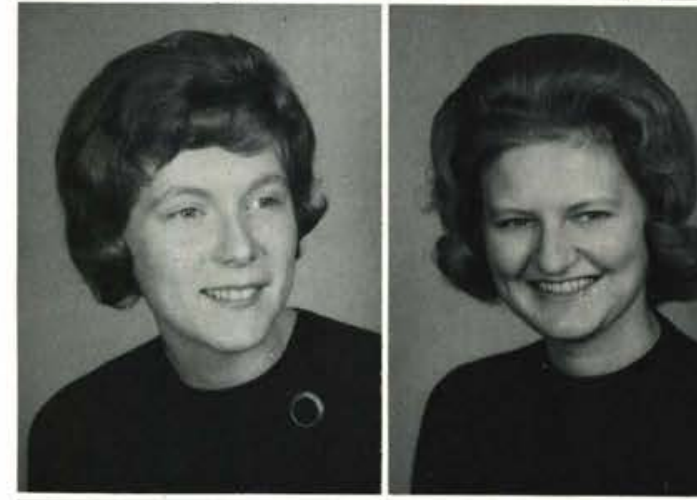

Kathy Morgan

Belle Vernon, Pennsylvania

Robert W. Newman

Hawthorne, New Jersey

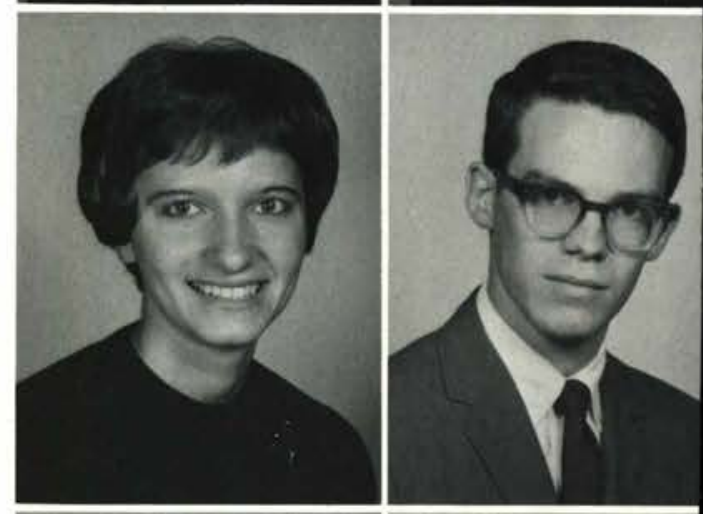

David Nims

Keene, New Hampshire

Rebecca O'Keefe

Parma, Ohio

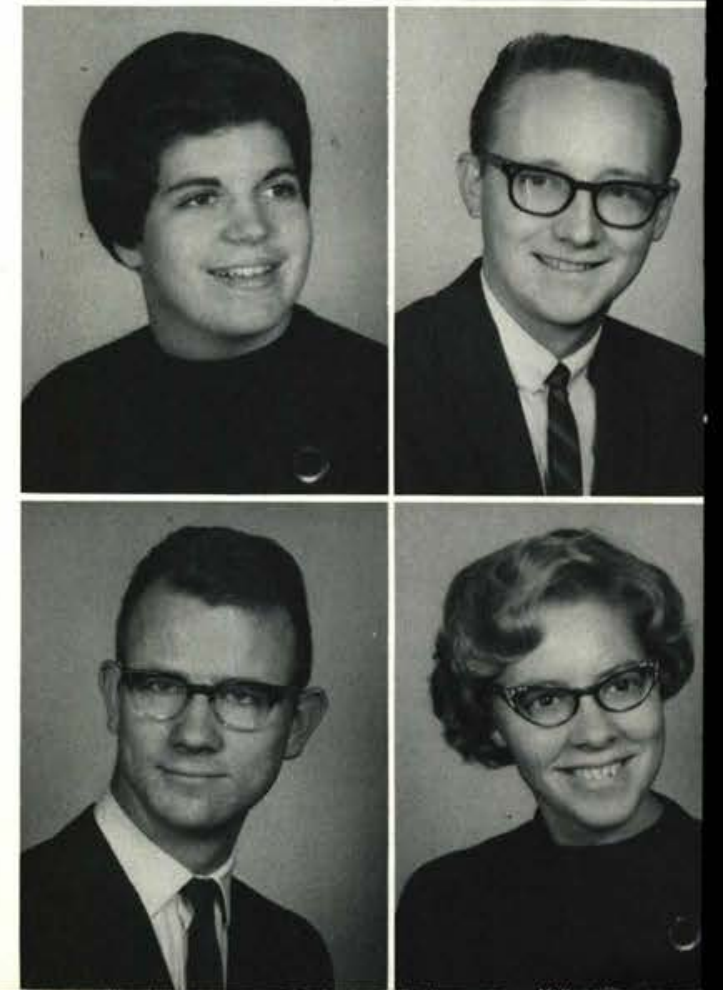


Sheryl Olson

Indianapolis, Indiana

Shirley Otto

Carroll, Iowa

Dennis Pearce

Stanwood, Washington

Manny Pereira

Kings Park, New York

William M. Pierson III

Collingswood, New Jersey

David Prosser

Warren, Ohio

Lawrence C. Richardson

Liuonia, Michigan
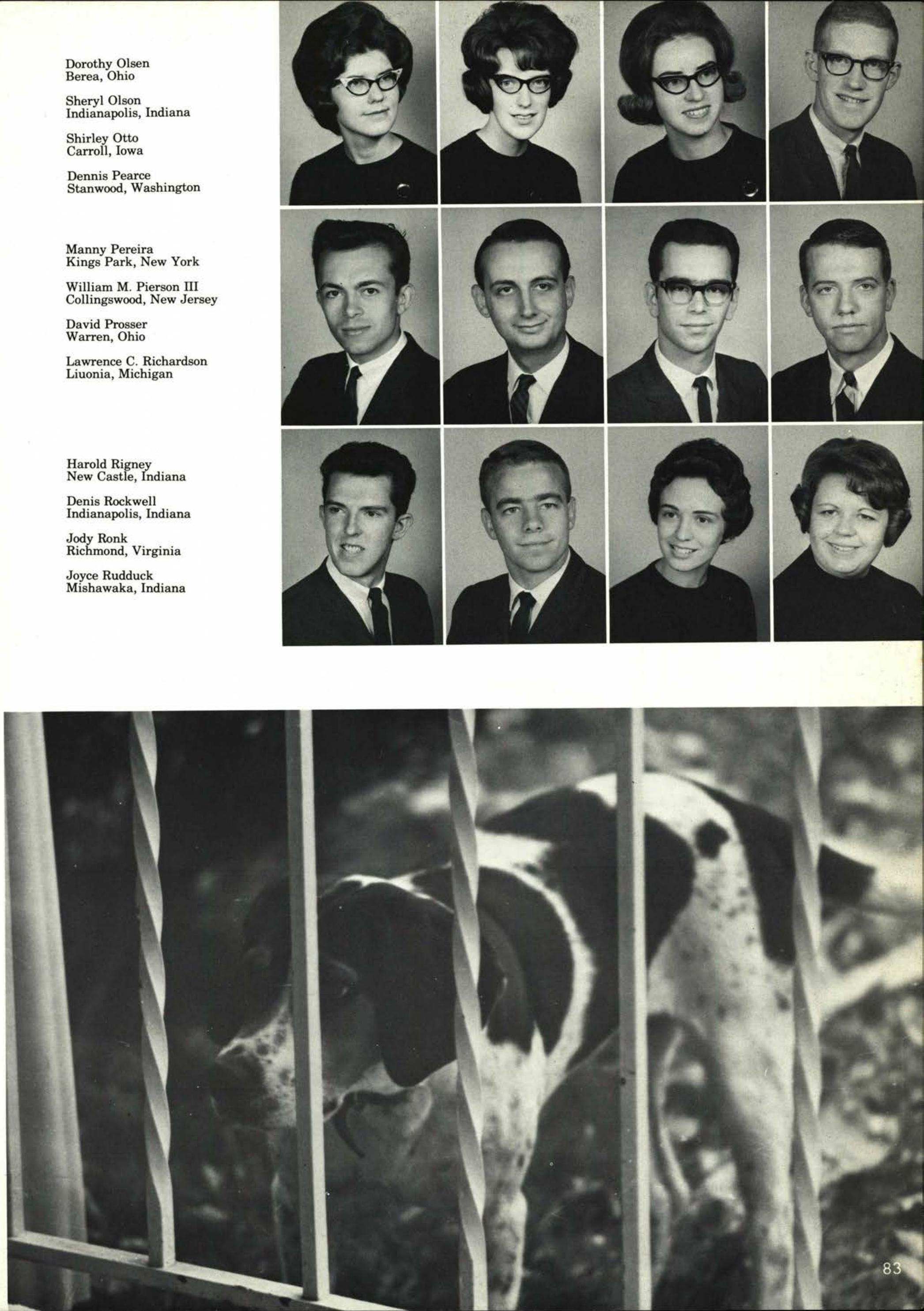


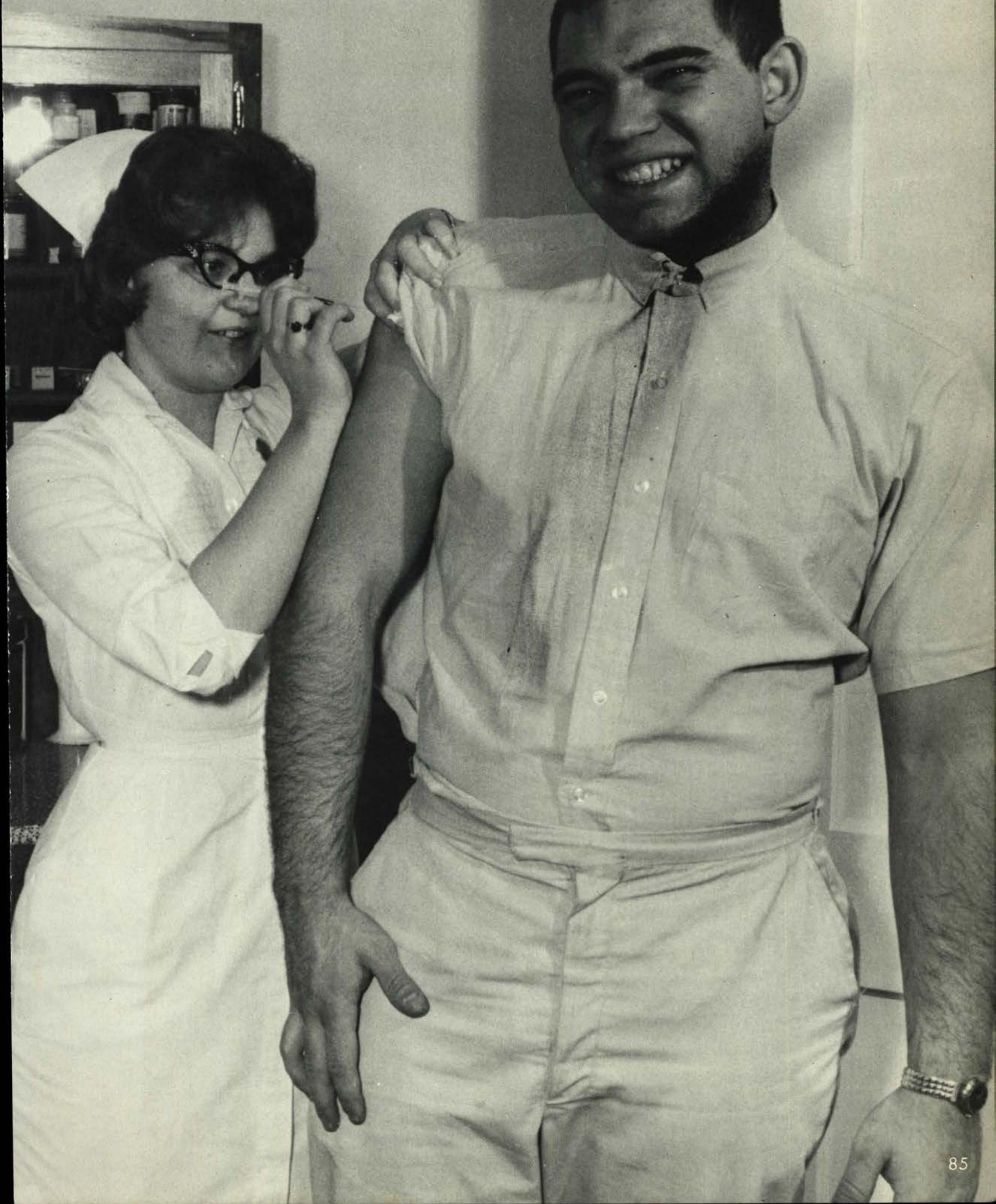




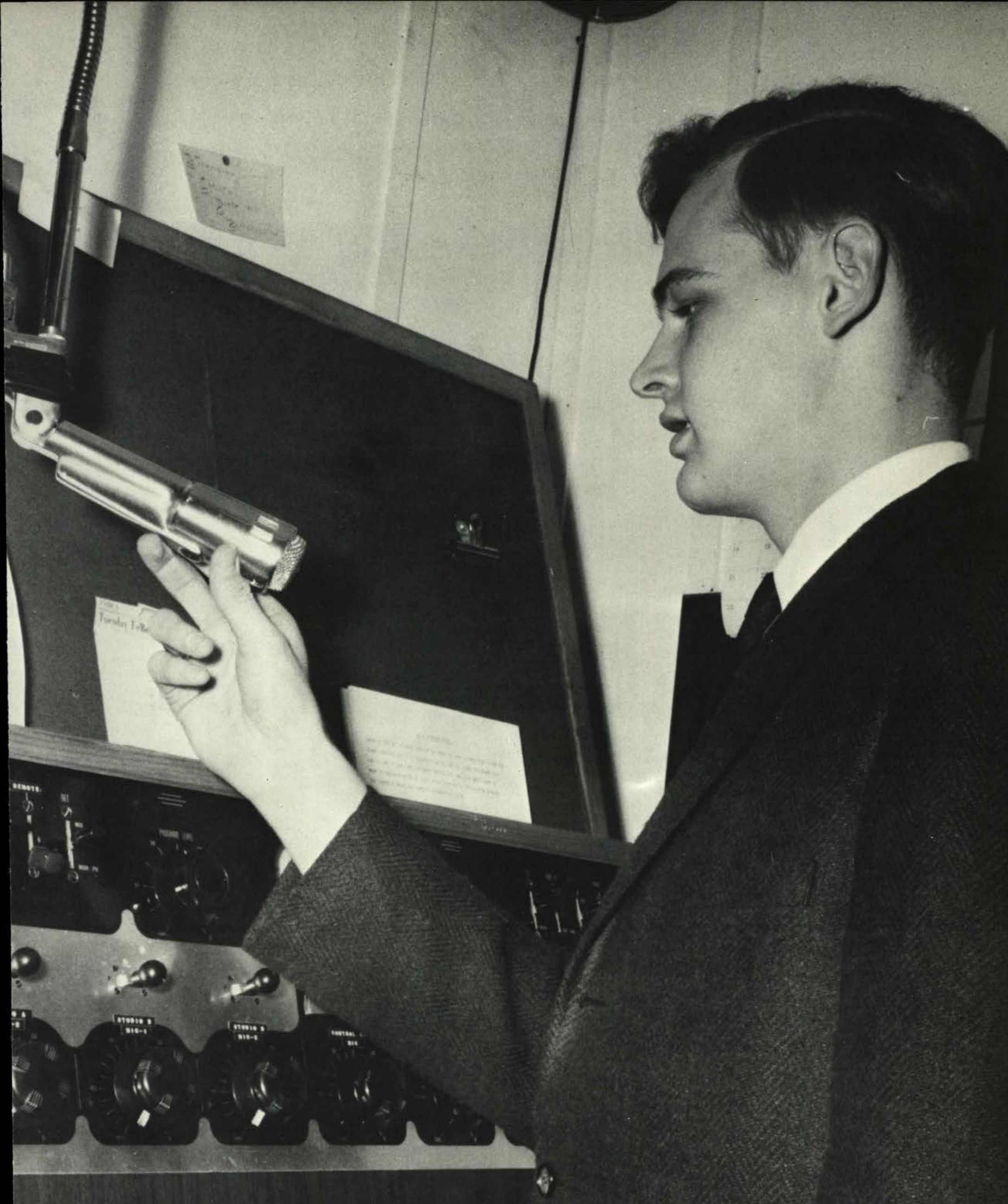

"This is WCDR-FM, 90.1 m.c. in Cedarville, Ohio." 
James E. Miller Pontiac, Michigan

James R. Miller

Valparaiso, Indiana

Linda Miller

Dayton, Ohio

Roberta Miller Lowellville, Ohio

Bonnie Millikan Bothell, Washington

Irene Millikan

Bothel, Washington

Carol Mowrey

Willowick, Ohio

Hugh Oakley

Midland, Michigan

Steve Olson

Indianapolis, Indiana

James Oswald

Millersburg, Ohio

Karen Patterson

Coshocton, Ohio

Melvin Pentecost Springfield, Ohio

Richard Pettitt

Florence, New Jersey

Jim Phelps

Binghamton, New York

Judy Phillips

Brazil, Indiana

Charles Reed

Oglesby, Illinois

Richard Robinson

Grand Rapids, Michigan

Joseph Roden

Dorset, Ohio

Jeannie Rogers

Haddon, Heights

Bonnie Rose

Crawfordsville, Indiana

Randall Ross

Jamestown, New York

Paula Sadler

Stryker, Ohio

Mary Ellen Schulte

Huntingburg, Indiana

Joan Schultz

Detroit, Michigan
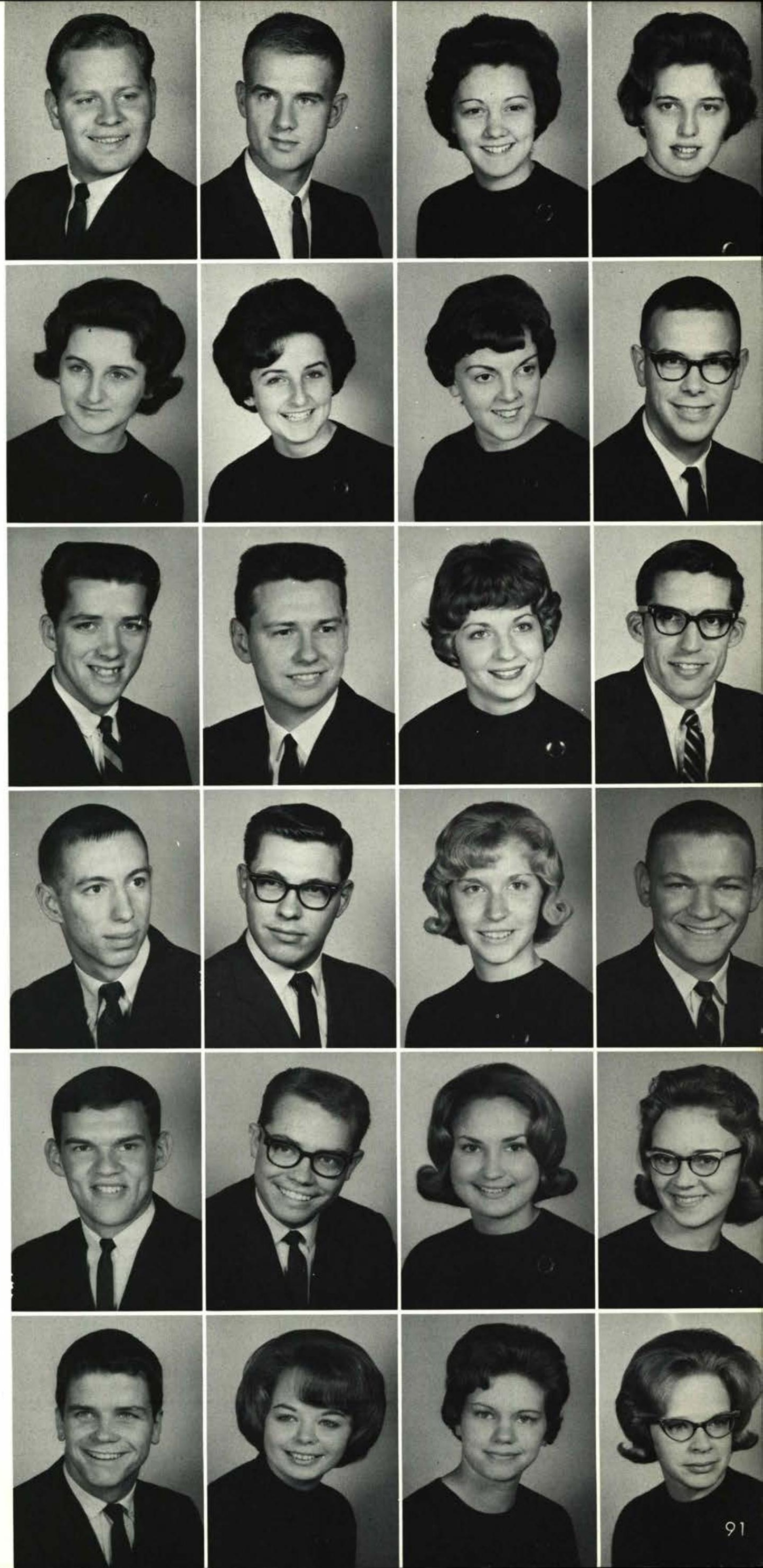


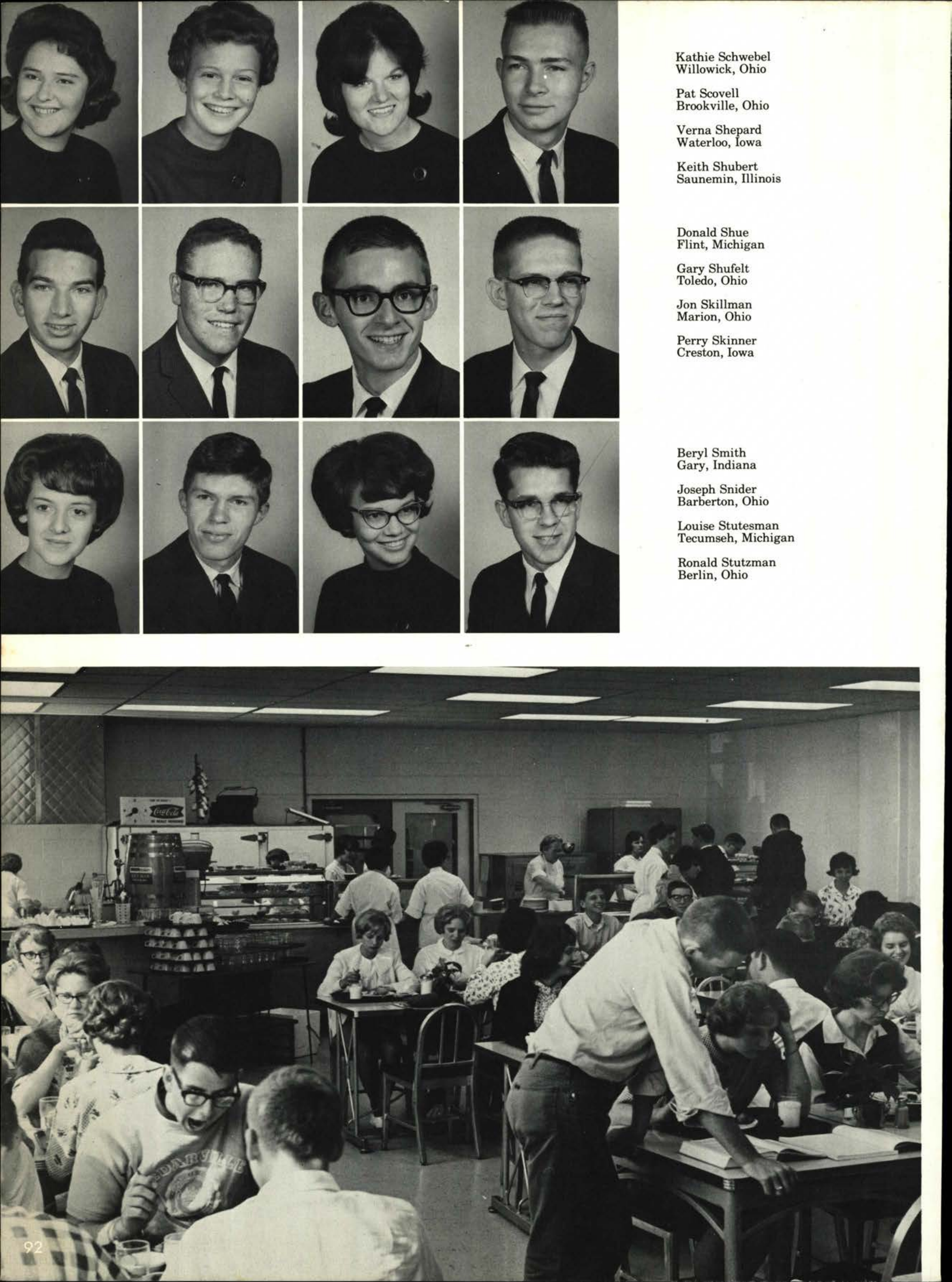




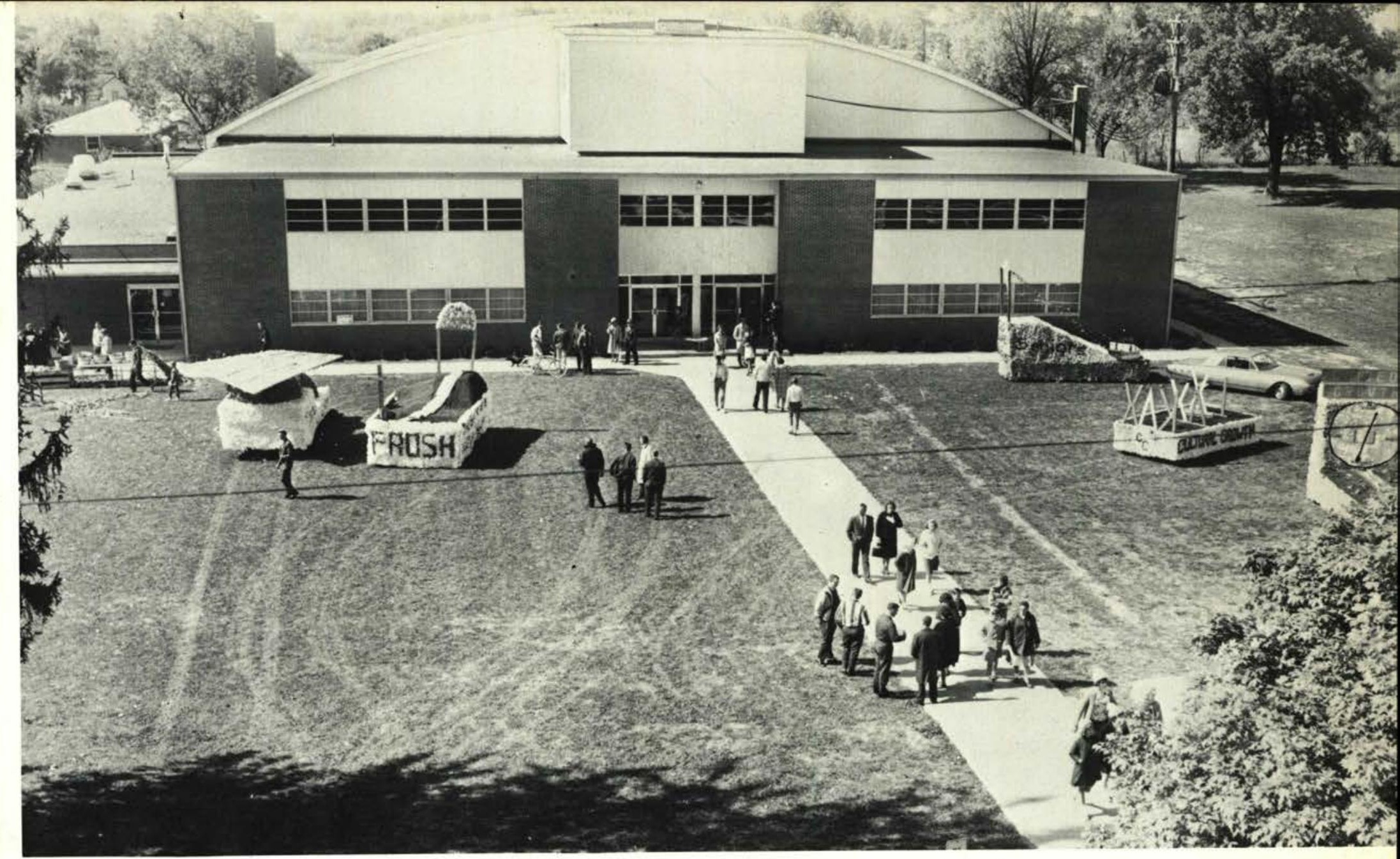

Opal Tarter

Romulus, Michigan

Howard Timmons

Blanchester, Ohio

Allen Traucht

West Liberty, Ohio

Mark Trimble

Cedarville, Ohio
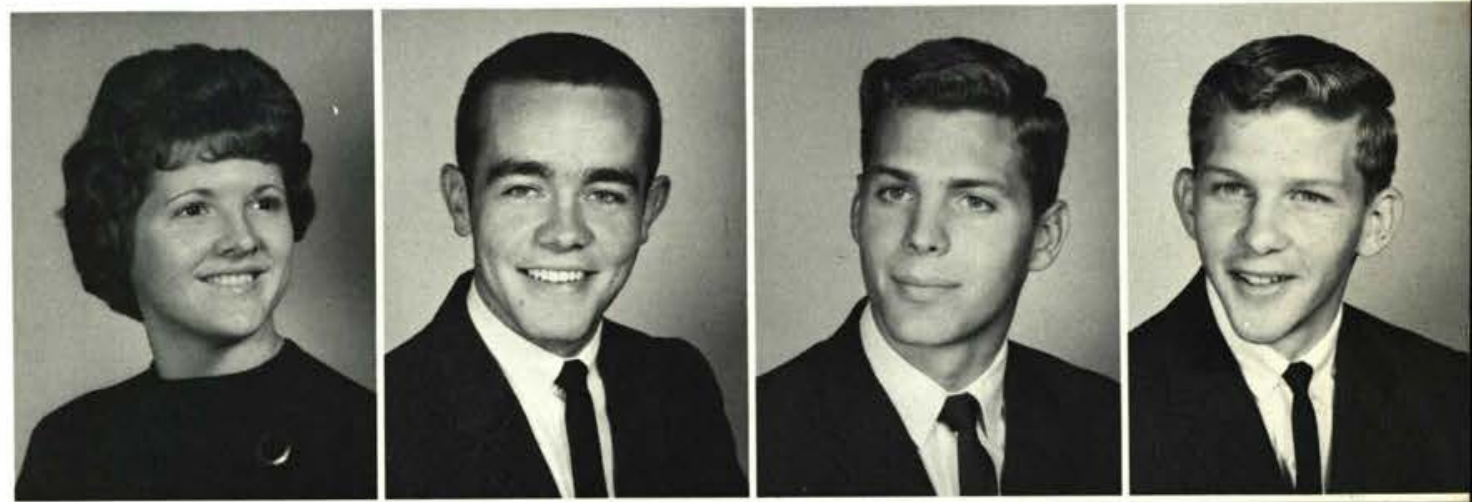

Charles Truxton

Haddonfield, New Jersey

Frank Warnken

Wheaton, Illinois

Carol Wenner

New London, Ohio

Sharon Westcott

Hanover, Pennsylvania
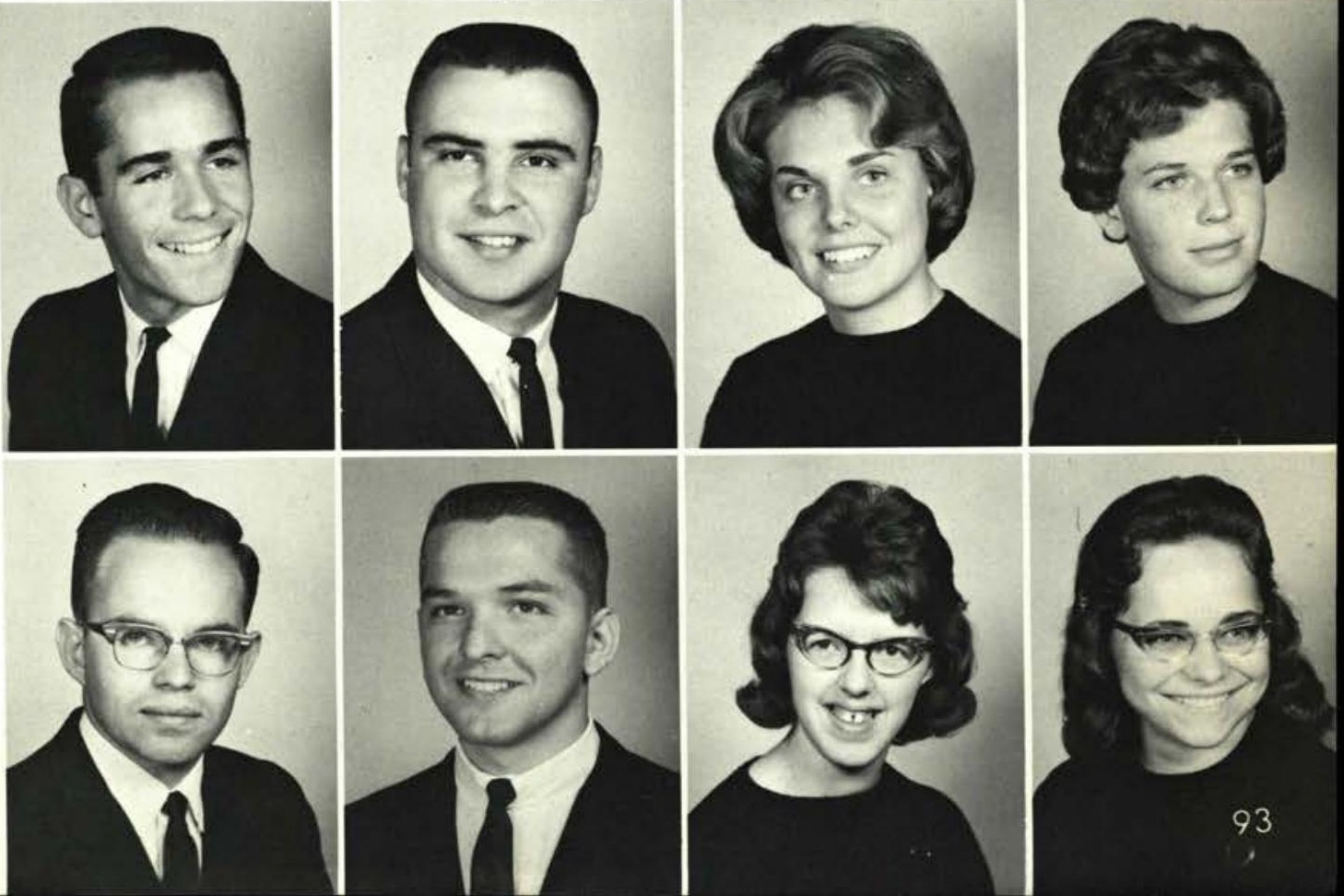

Paul Wilhite

Corwith, Iowa

Dennic Wood

Carlisle, Ohio

Linda Young

Mt. Pleasant, Michigan

Beth Zeigler

Wellington, Ohio
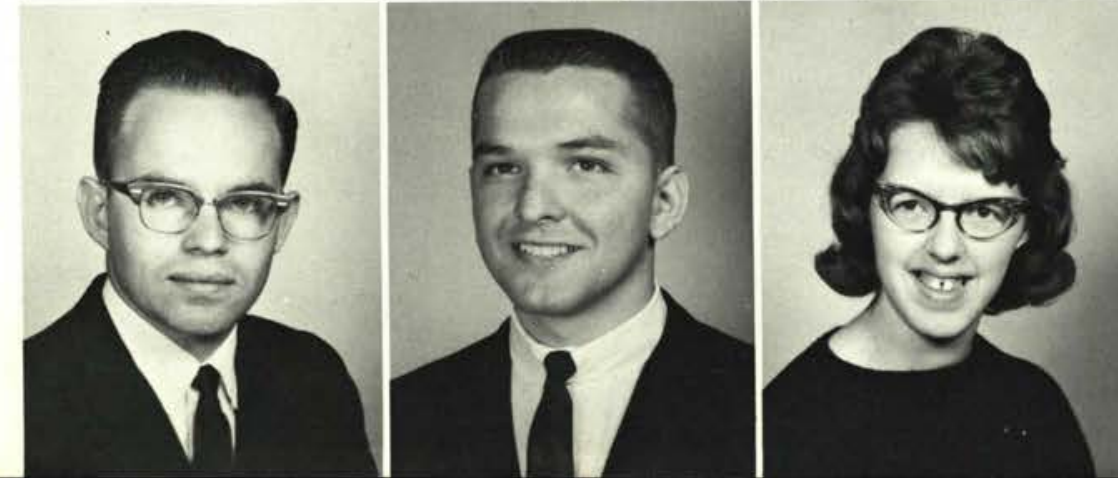
Lynn Brock

Columbus, Ohio

Jon Brown

Cedarville, Ohio

Martha Brown

Holly, Michigan

Carol Burcham Mattoon, Illinois

Tom Burcham Mattoon, Illinois

Kris Burroughs Highland, Indiana

Audrey Cady

Troupsburg, New York

Beverly Carlson Decatur, Georgia
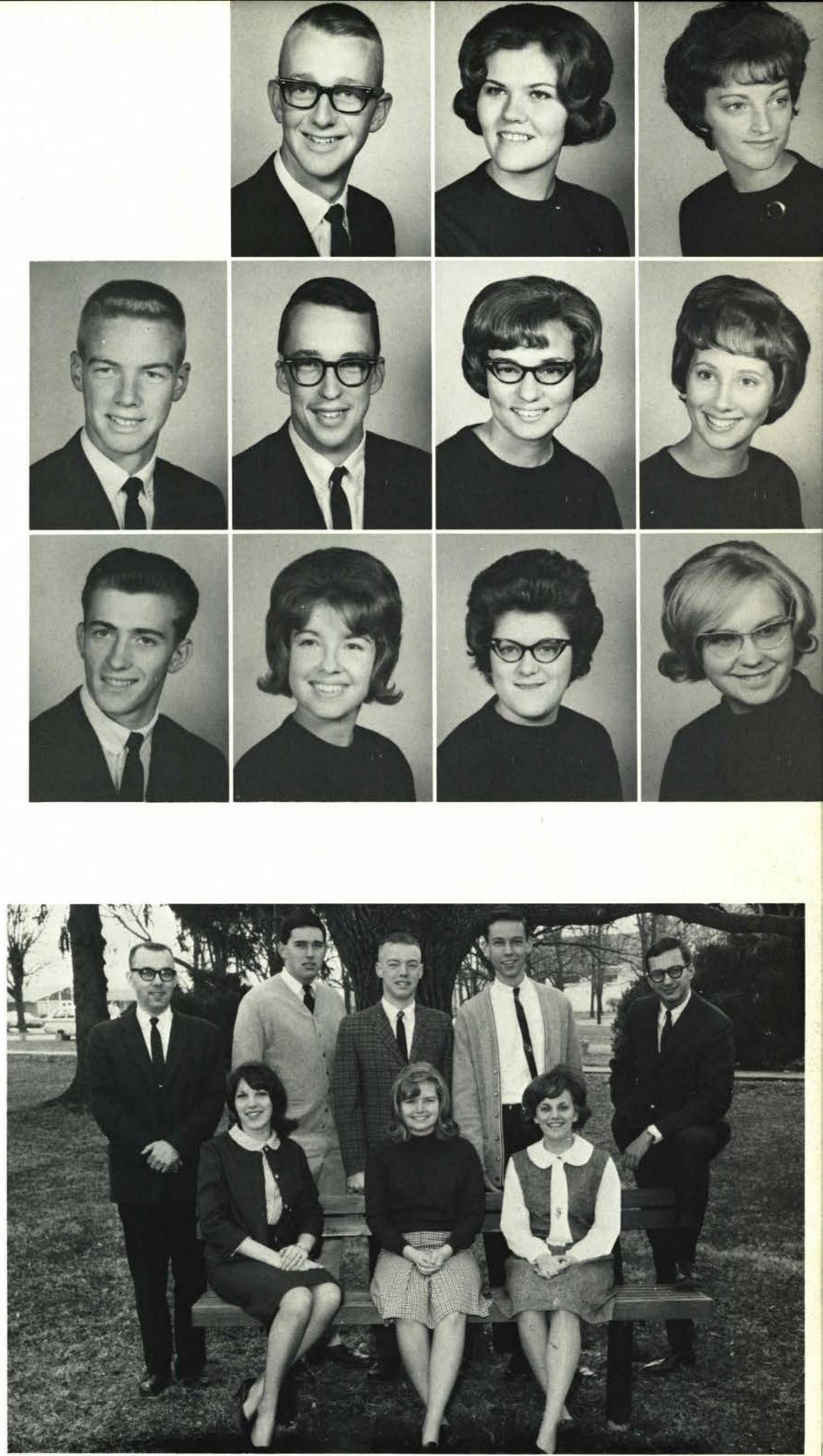

Seated: N. Mercer, Secretary; C. Payne, Student Council Rep.; B. Grosh, Treasurer. Standing: Mr Wetzel, Advisor; M. Woodend, Vice-President; L. Brock, President; M. Crampton, Student Council Rep.; D. Jones, Chaplain. 



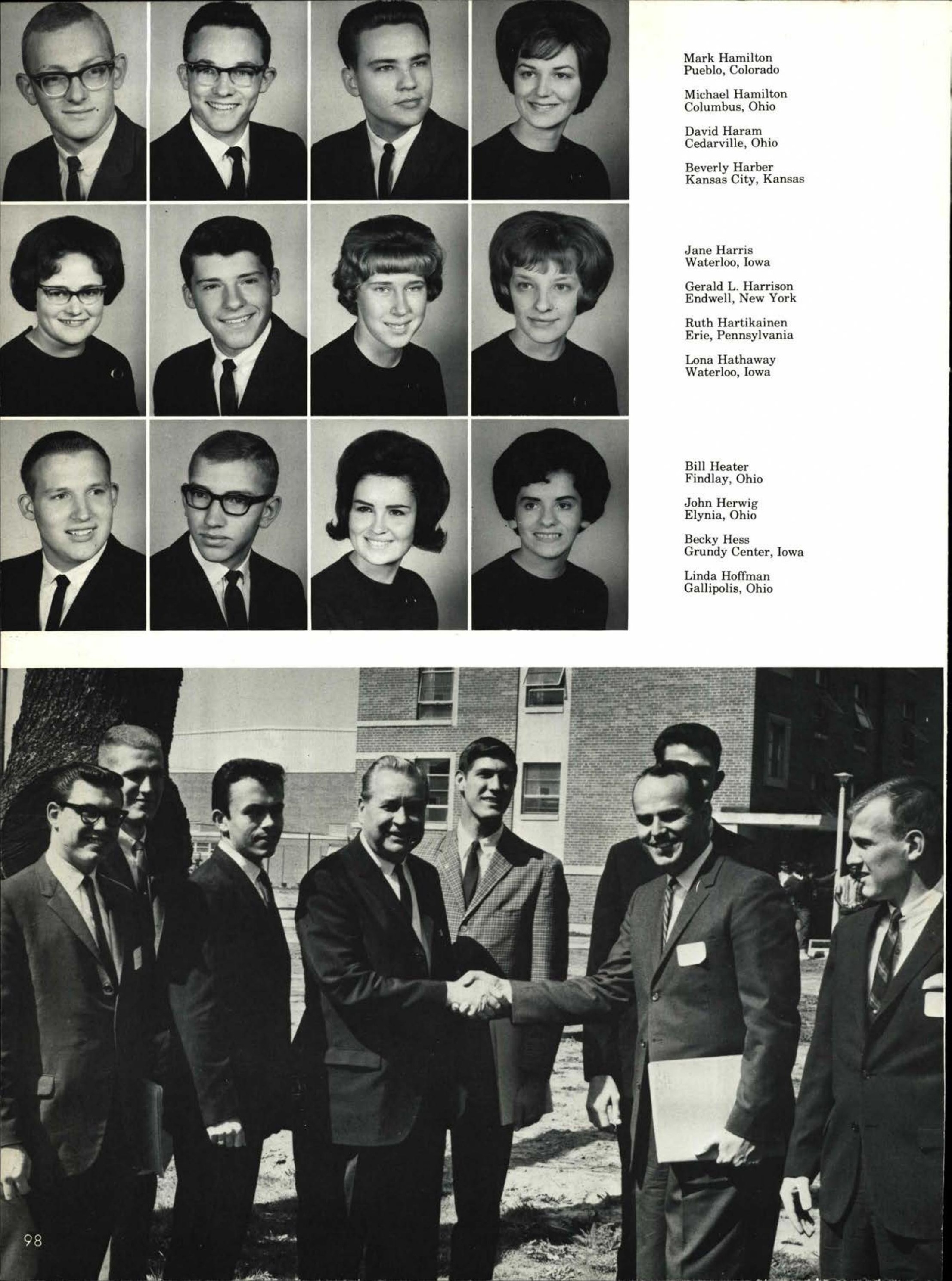


Joseph Hollaway

Columbus, Ohio

Beverly Homanick

Fairborn, Ohio

Anne Howard

Traer, Iowa

Eleanor Howard

Bucyros, Ohio

Shirley Hudson

Marion, Ohio

Glenn Huebner

Union Grove, Wisconsin

Charles Hull

Cortland, Ohio

James Hume

Dayton, Ohio

Charles Hunter

Jesup, Iowa

Sharon Hunter

Decatur, Illinois

Judy Jager

Wyoming, Michigan

Sharon Jamison

Flint Michigan

Frank Jenista

Bloomfield, New Jersey

Elaine Johnson

West Palm Beach, Florida

Kathy Johnson

Seattle, Washington

Joyce Johnson

Cleveland, Ohio

Judy Johnston

Galion, Ohio

David Jones

Arlington, Virginia

Sheryl Kaiser

Plymouth, Indiana

Marian Kapp

Erie, Pennsylvania

Ruth Kauffold

Pana, Illinois

Janice Keating

Wausaukee, Wisconsin

Alan Keim

Holsobble, Pennsylvania

Barbara Kensil

Decatur, Illinois
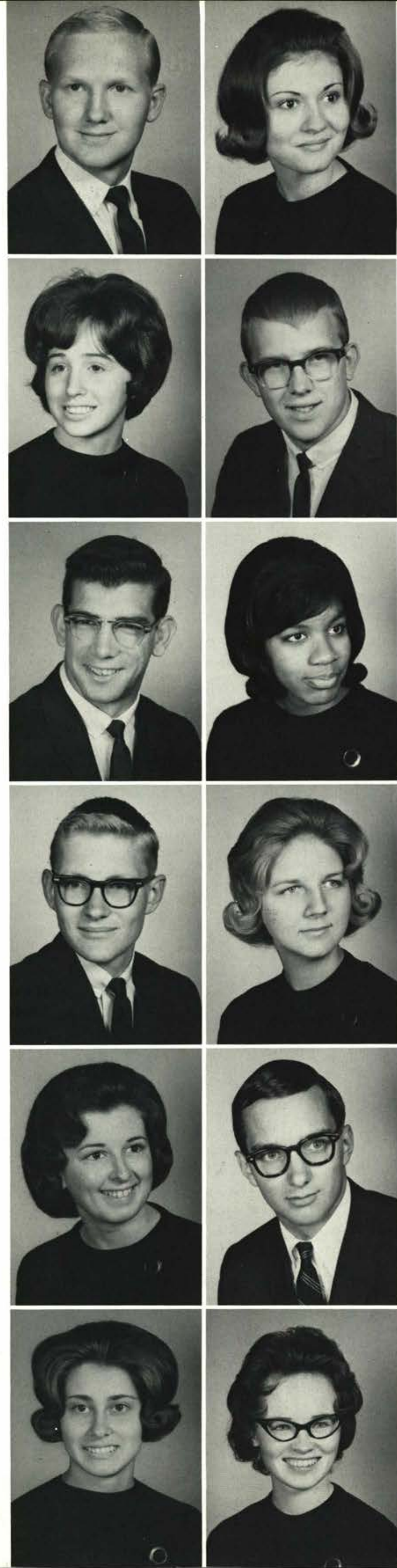
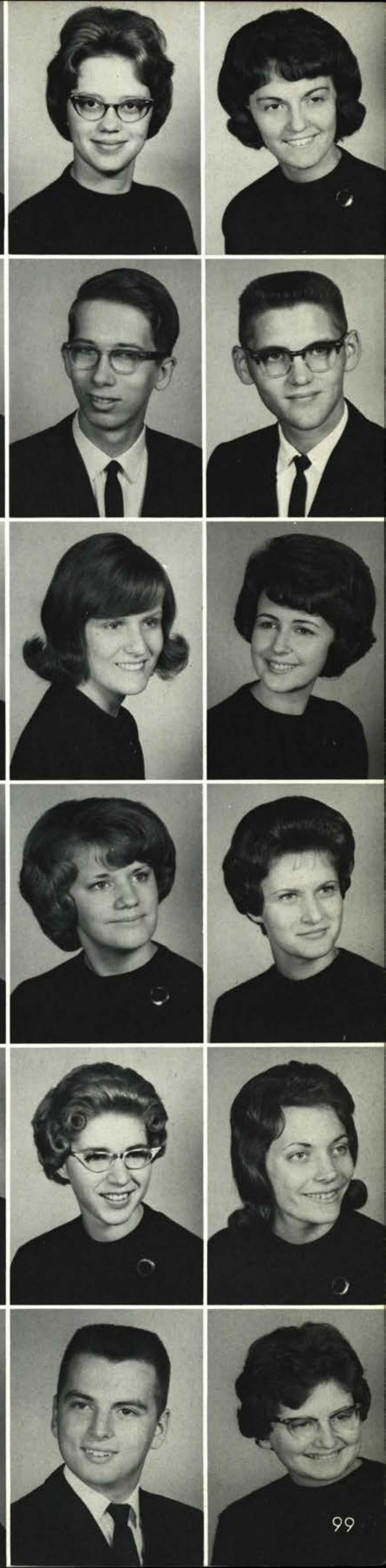



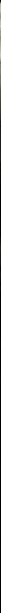

$\begin{array}{ll}\text { Karen Taylor } & \text { Gail Tompkins } \\ \text { Dayton, Ohio } & \text { Sherman, New York }\end{array}$ Columbus, Ohio

Jennifer S

Pontiac, Illinois

Lancaster, New York

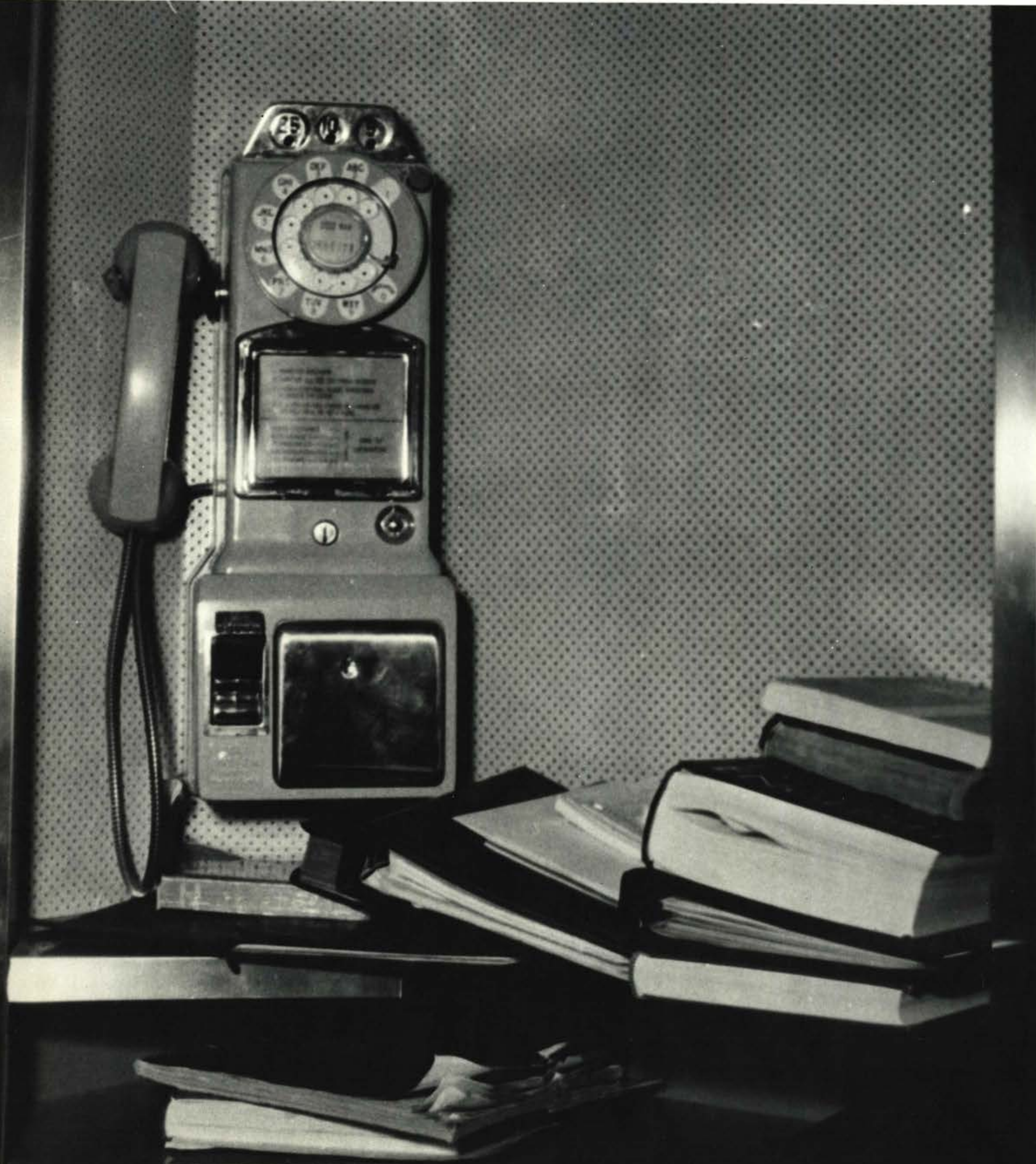




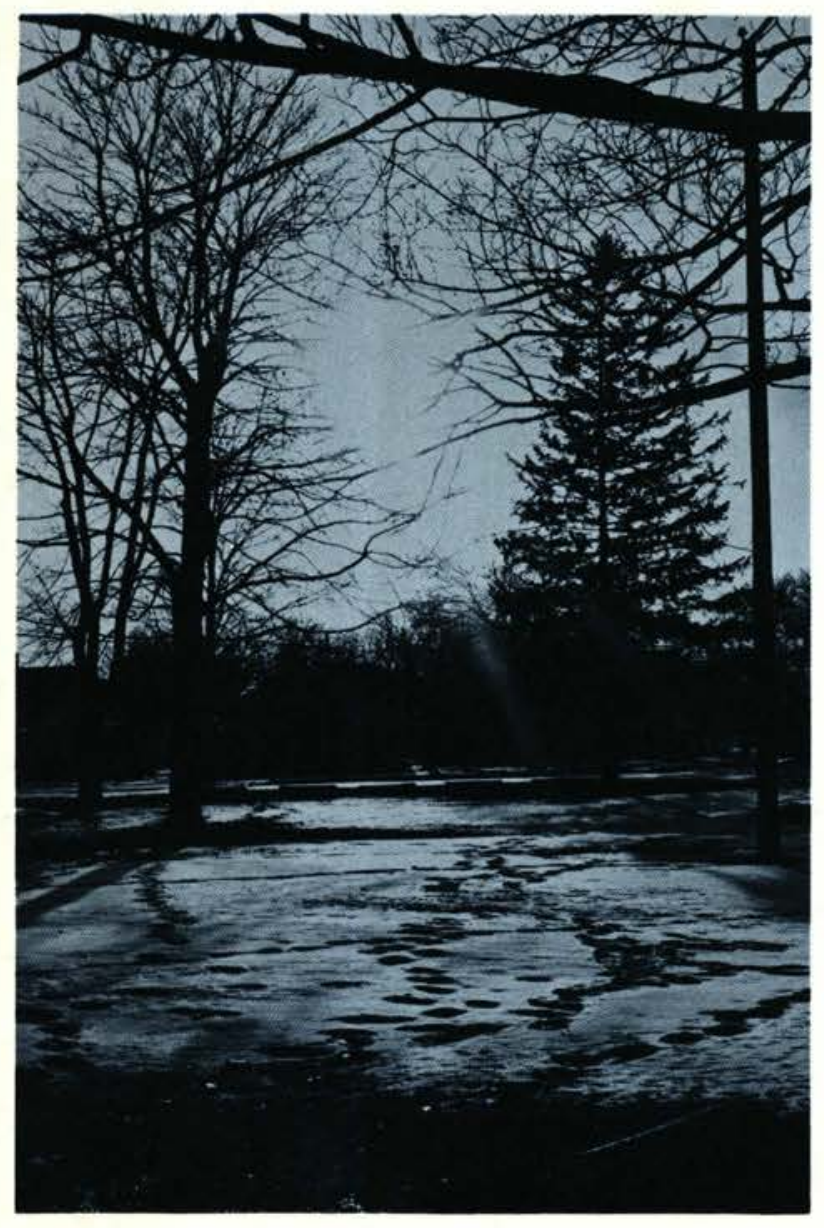

\section{WINTER}

Winter is many things . . . cold mornings ... twinkling snow ... icy walks ... steamed windows ... crunching snow ... mittens . . . ice skating . . . brisk walks . . crackling fire places ... Christmas ... hot chocolate . . sleigh rides . .. and God's handiwork in snow. 

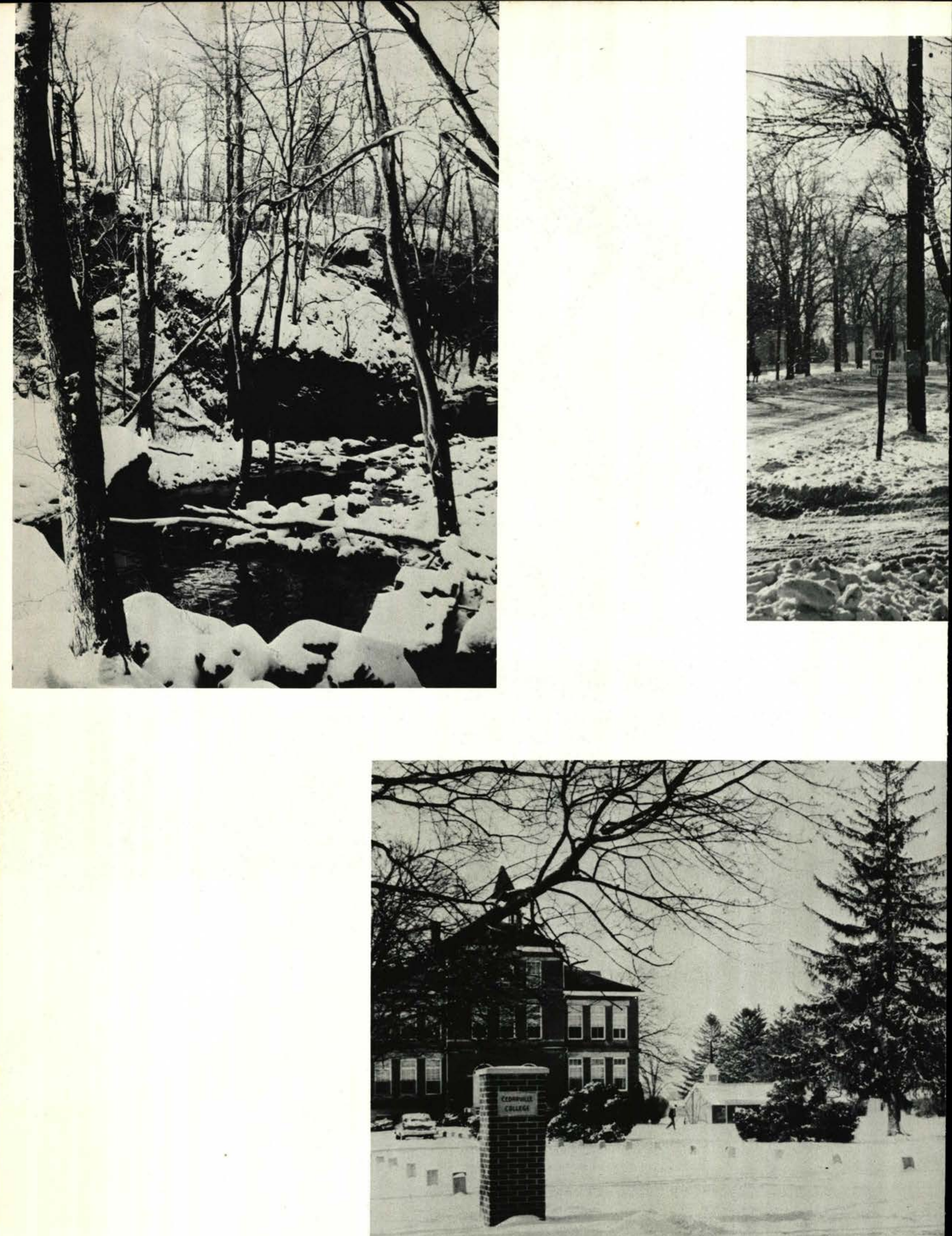

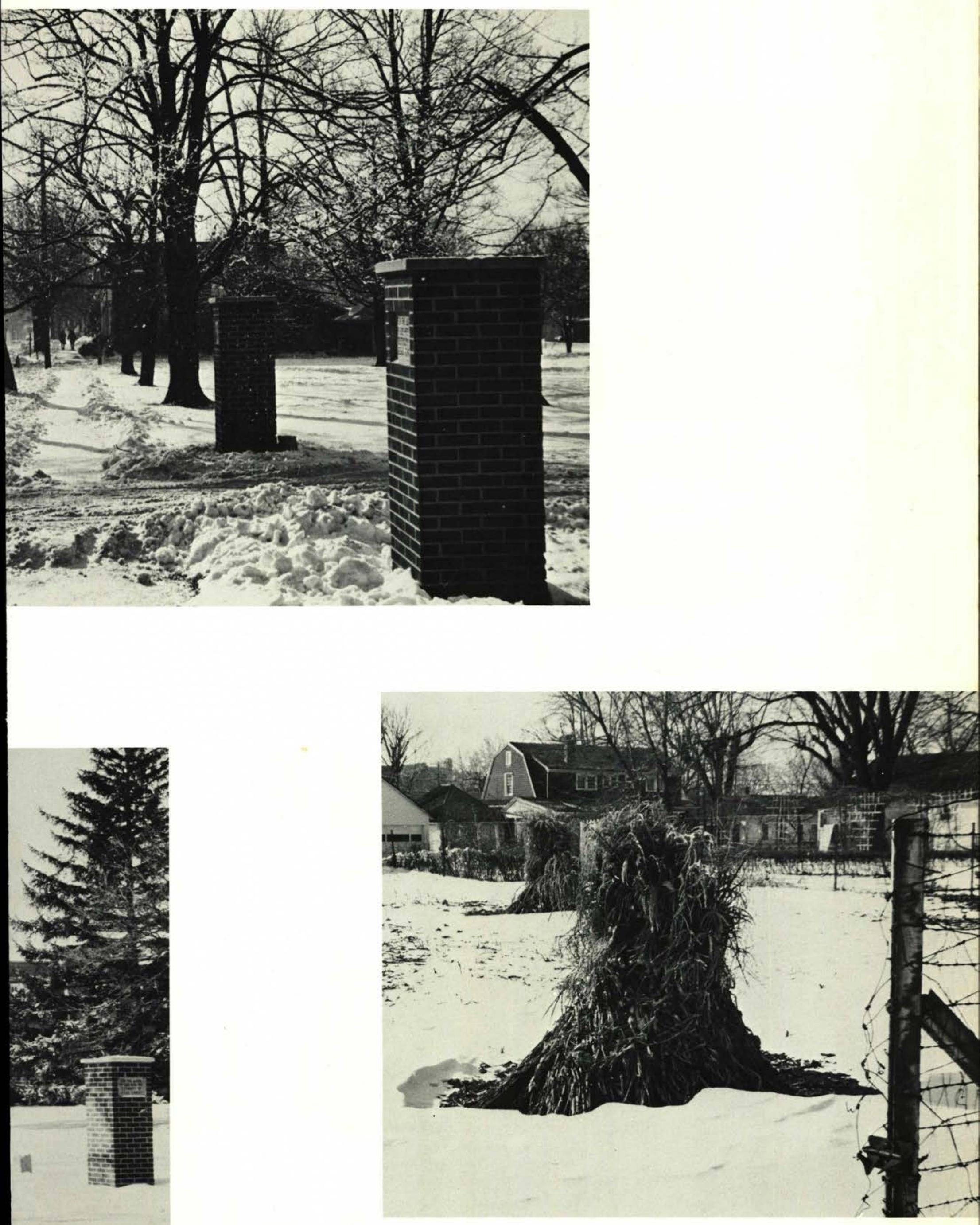


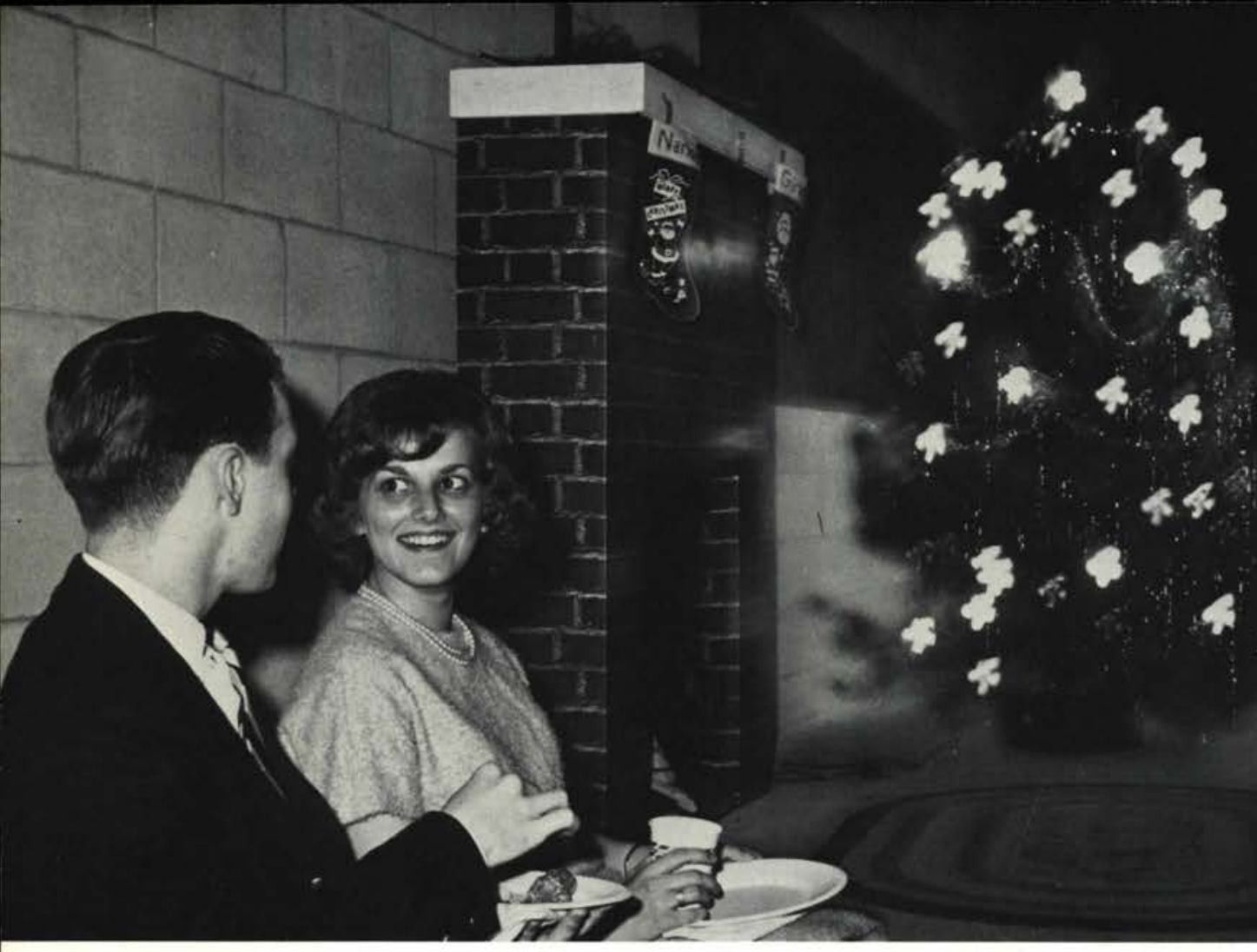

\section{ALL-SCHOOL CHRISTMAS PARTY}
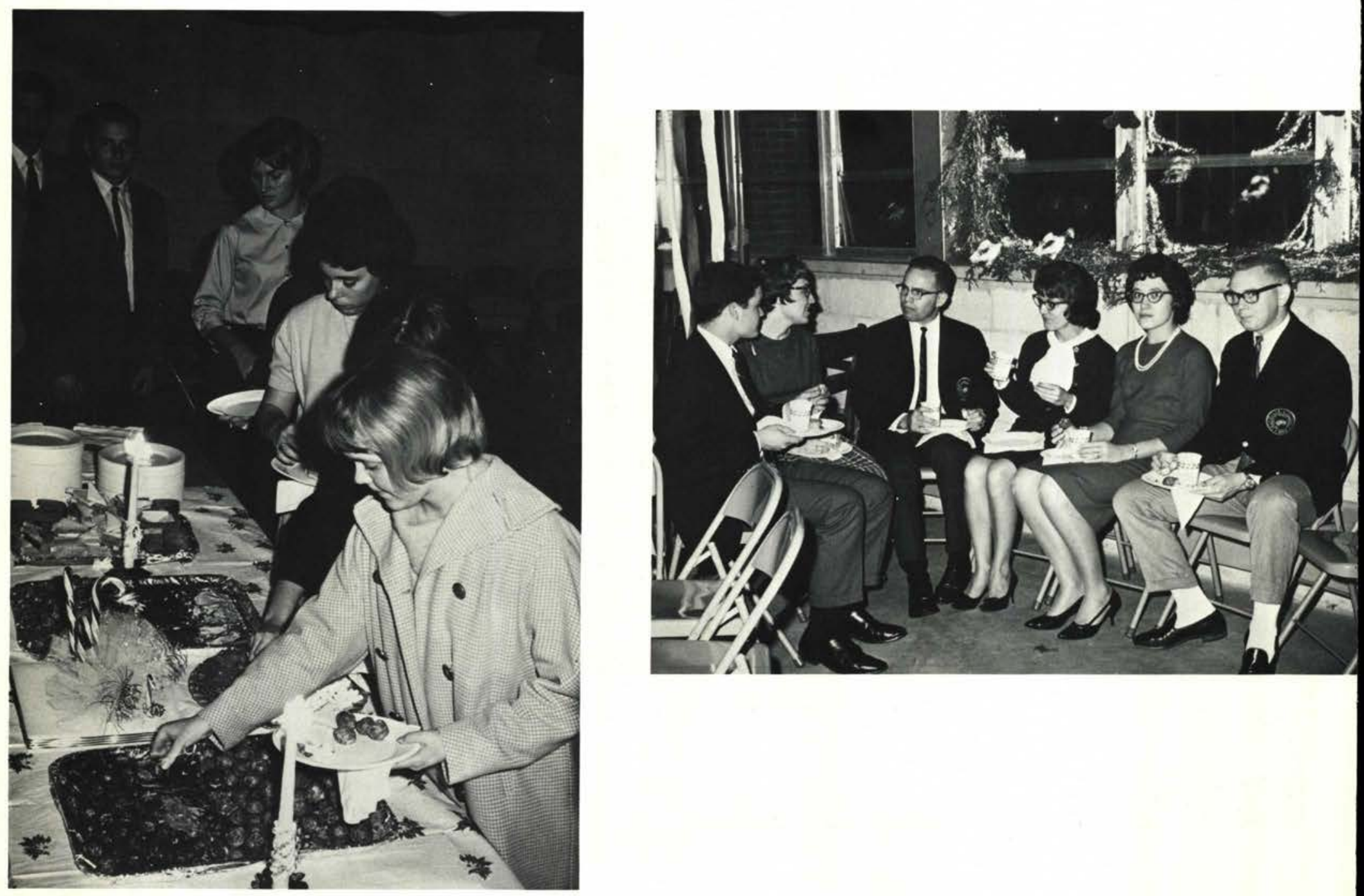
Music

Department

Presents

The

MESSIAH

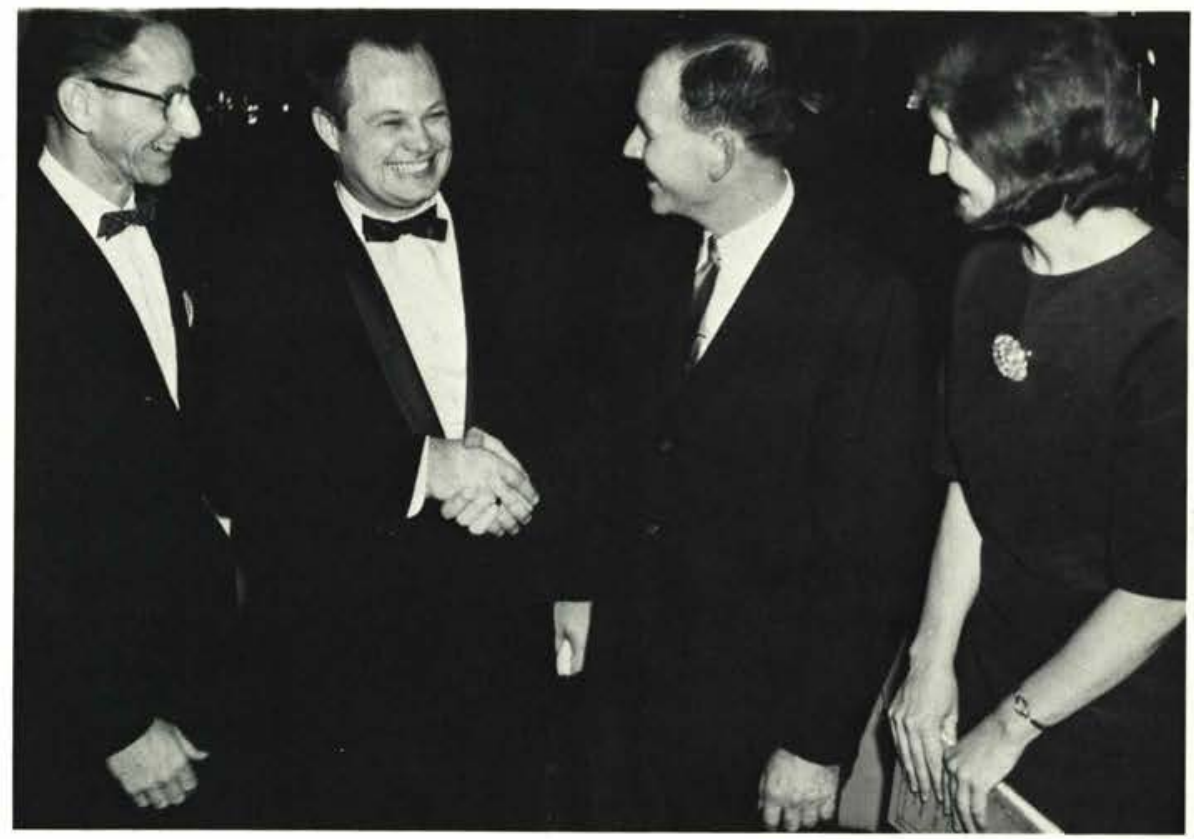

W. Thornton, W. Meissner, W. Webber, Ruth Smith.

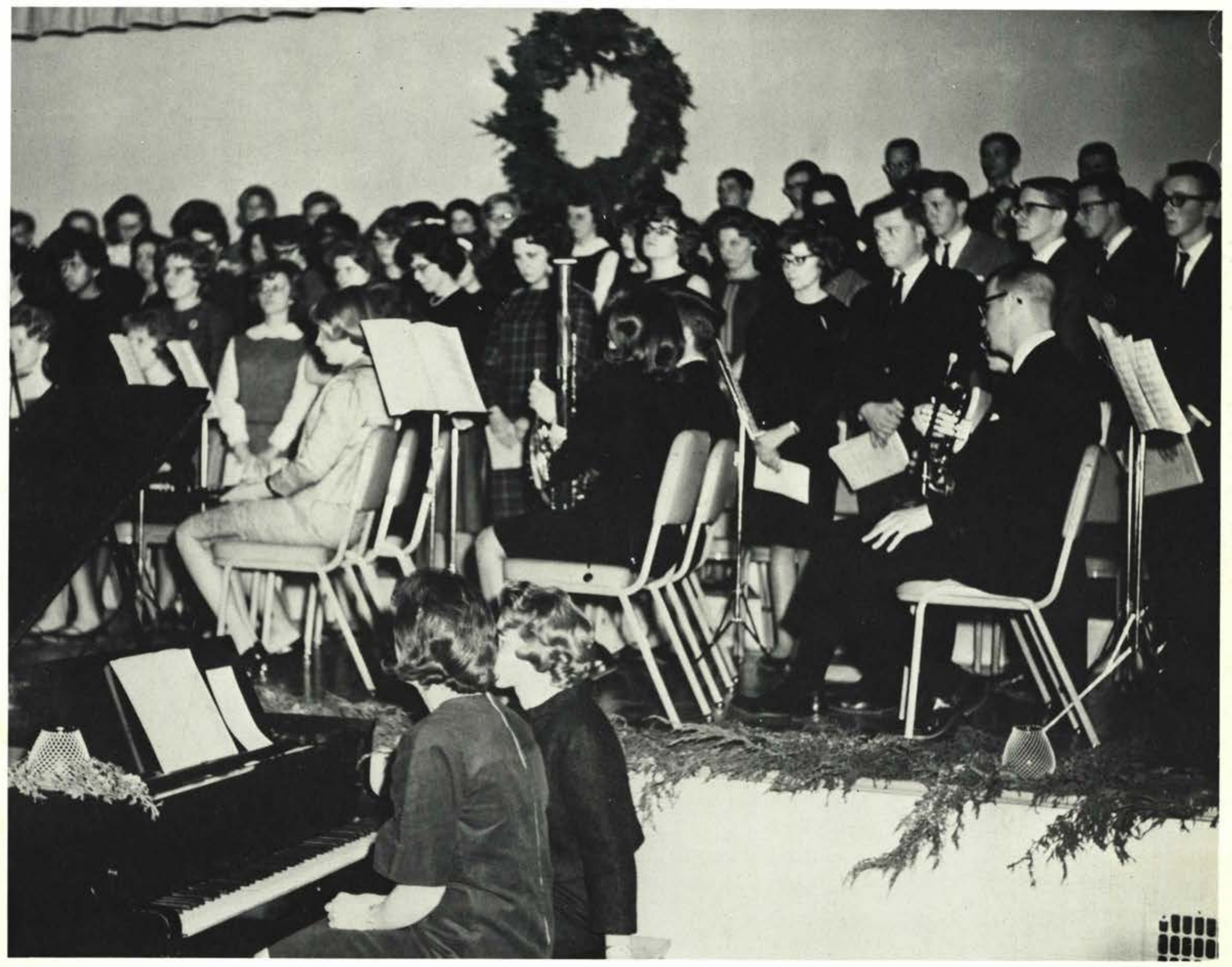




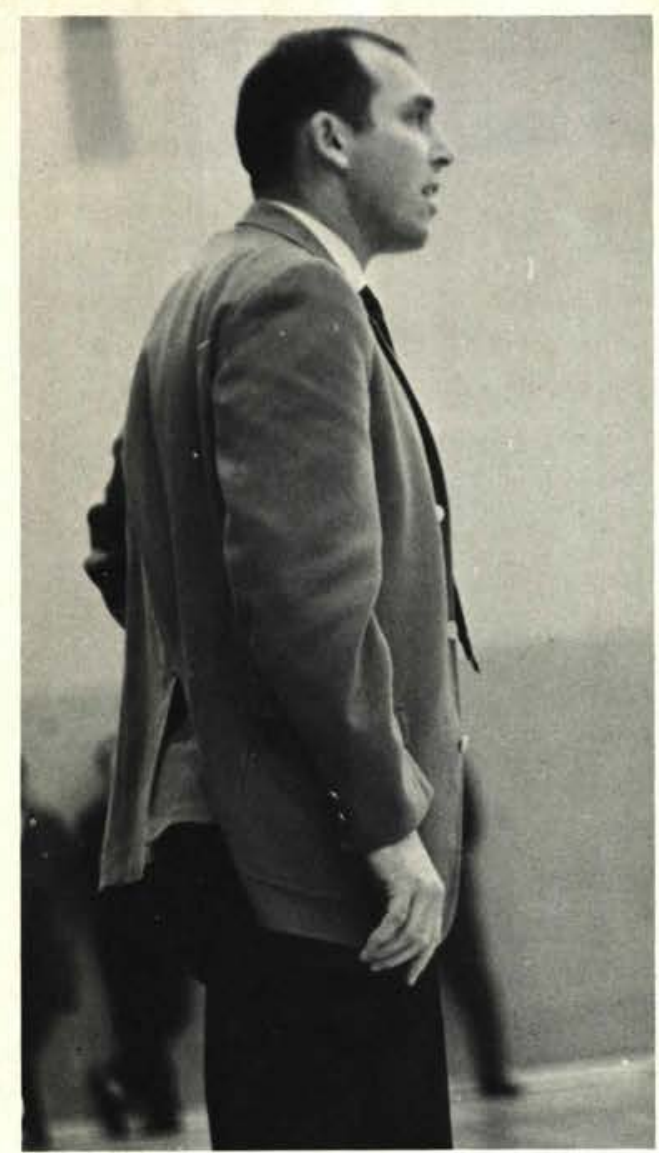

\section{LEAGUE CHAMPS}

Basketball Season 1964-65 proved to be one which will long be remembered. It saw the Jackets compile a fine 23-4 record, capture the M.O.L. Trophy, going undefeated in the league, and for the second year in a row, advance to the finals of the N.A.I.A. District \# 22 Playoffs.

The Jackets dominated the league in scoring, defense, rebounding, and shooting percentages. Also, three Jackets were named to the All-League First Five.

Although Coach Callan is losing the likes of Mark Combs, Gary Walthall and Dozier Carter, the future bright with such freshmen as Larry Waite, John Watson, Gayle Bradds, and Al Knott.

Al Knott, in particular, finished the season averaging 19 points per game, second to "Mr. Basketball"Dozier Carter at 19.5 points per game.

Coach Callan

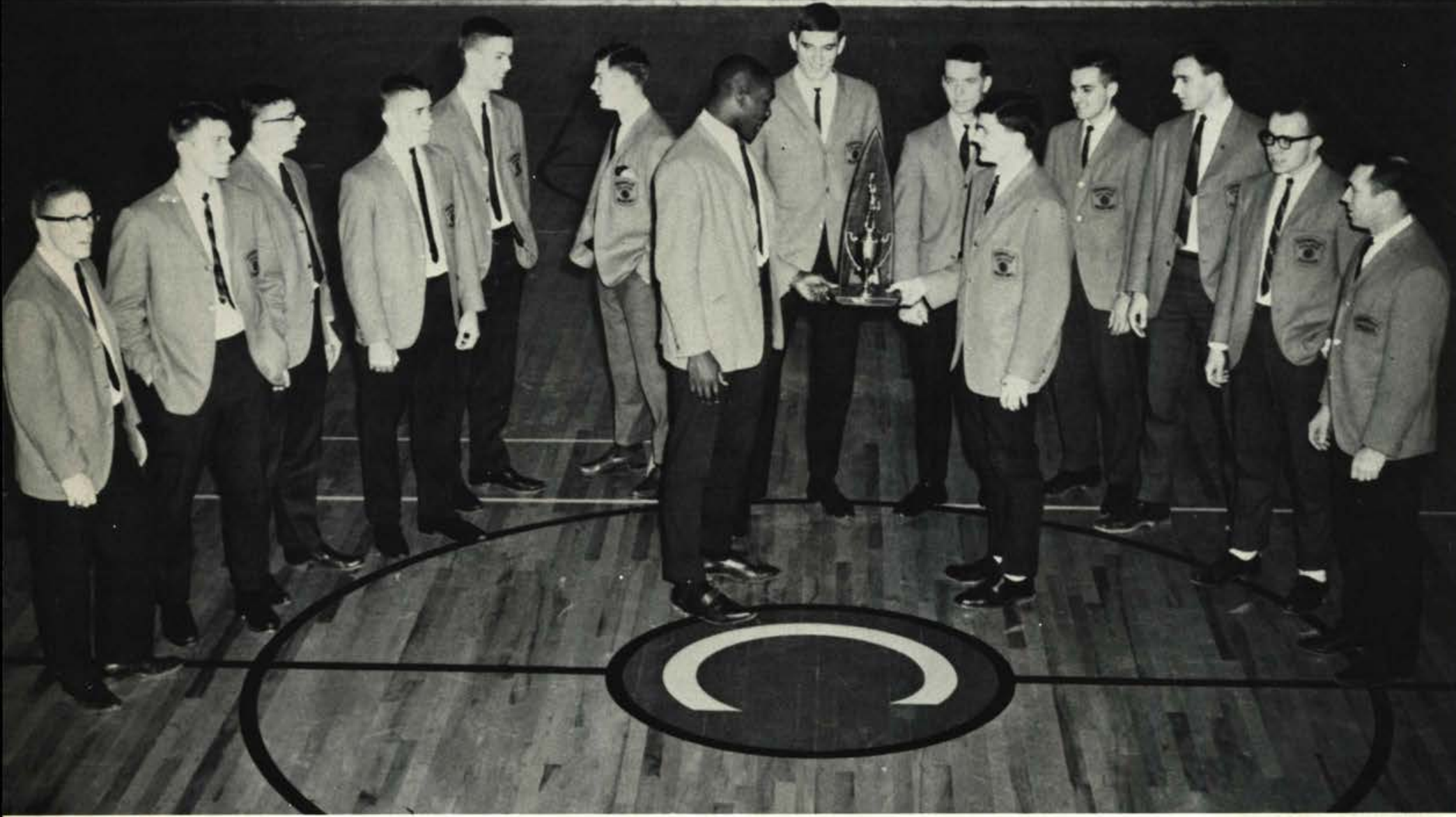

Left to right: Gary Shufelt, Gary Drill, Vern Hooper, Don Cooper, Gayle Bradds, John Watson, Gary Walthall, Larry Richardson, Jack Brannon, Mgr., Al Knott, Dave Thorne, Mgr., Coach Donald Callan. Foreground: Co-Captains Dozier Carter and Mark Combs with M.O.L. Trophy. 



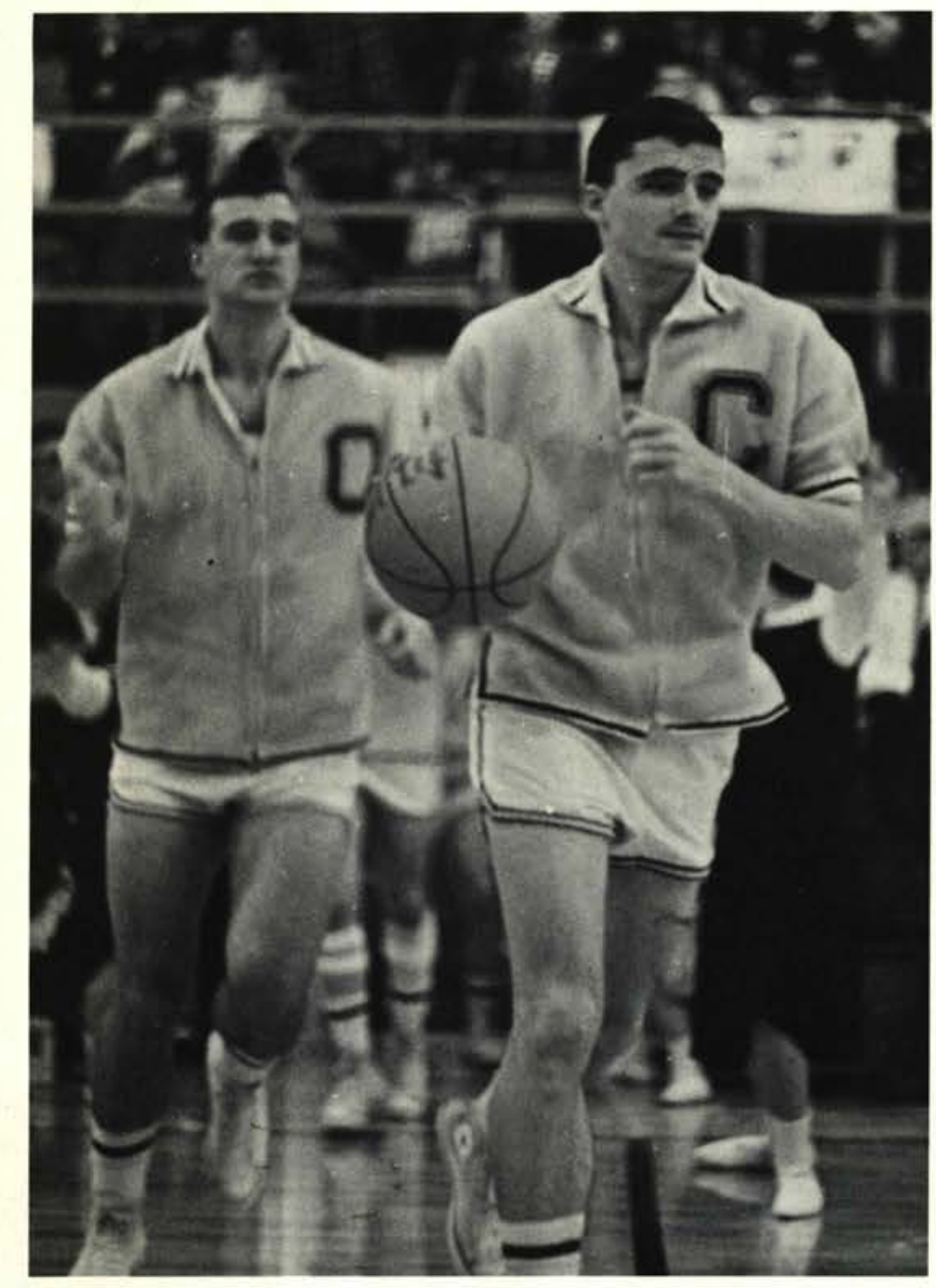

Captain Mark Combs
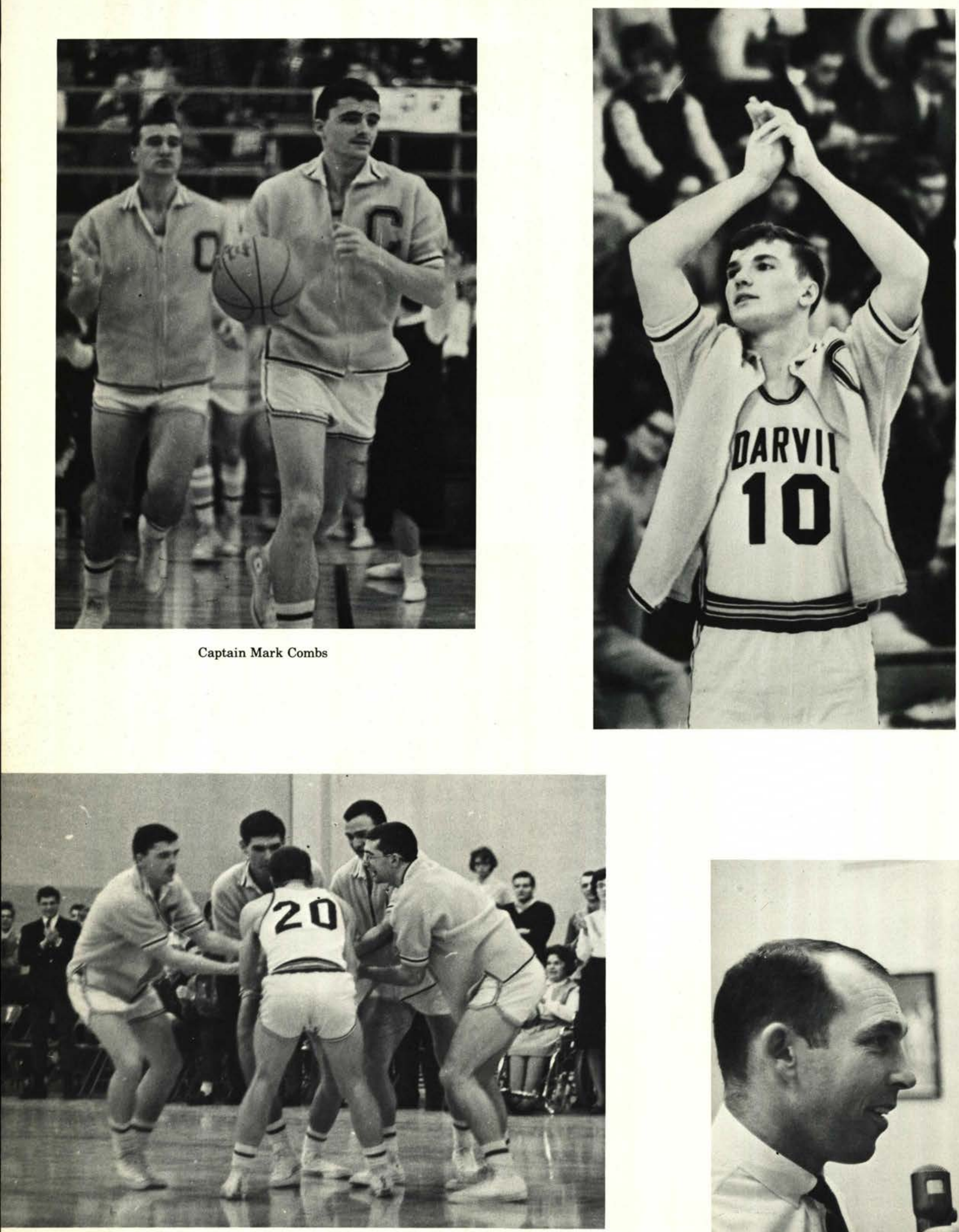

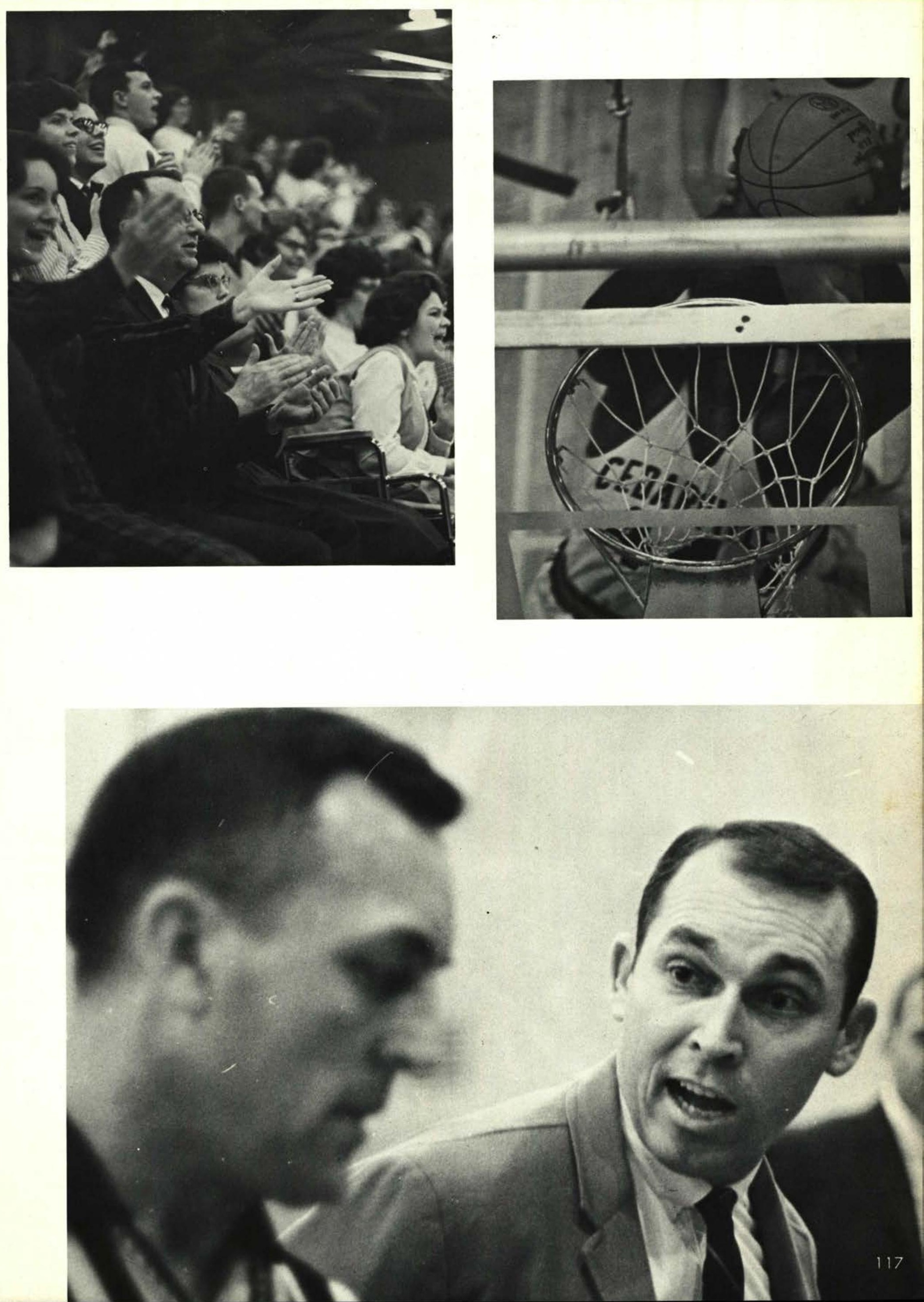


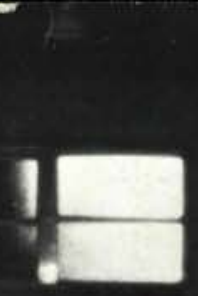

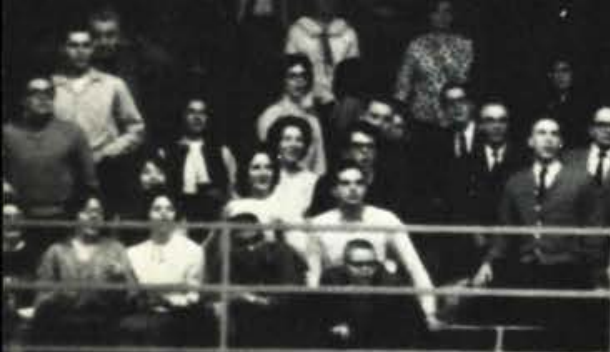
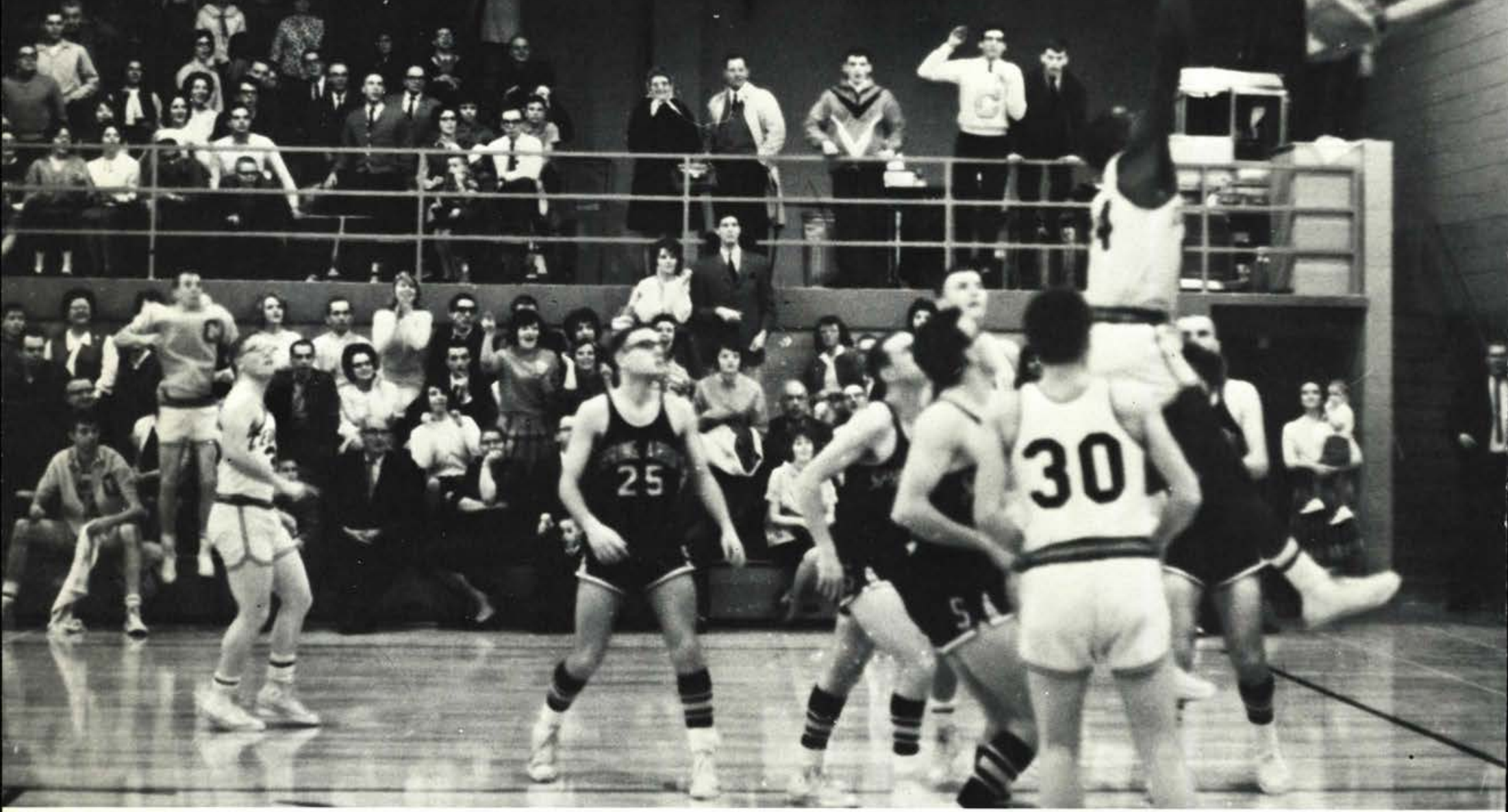

Dozier Carter caps record breaking performance with final basket.

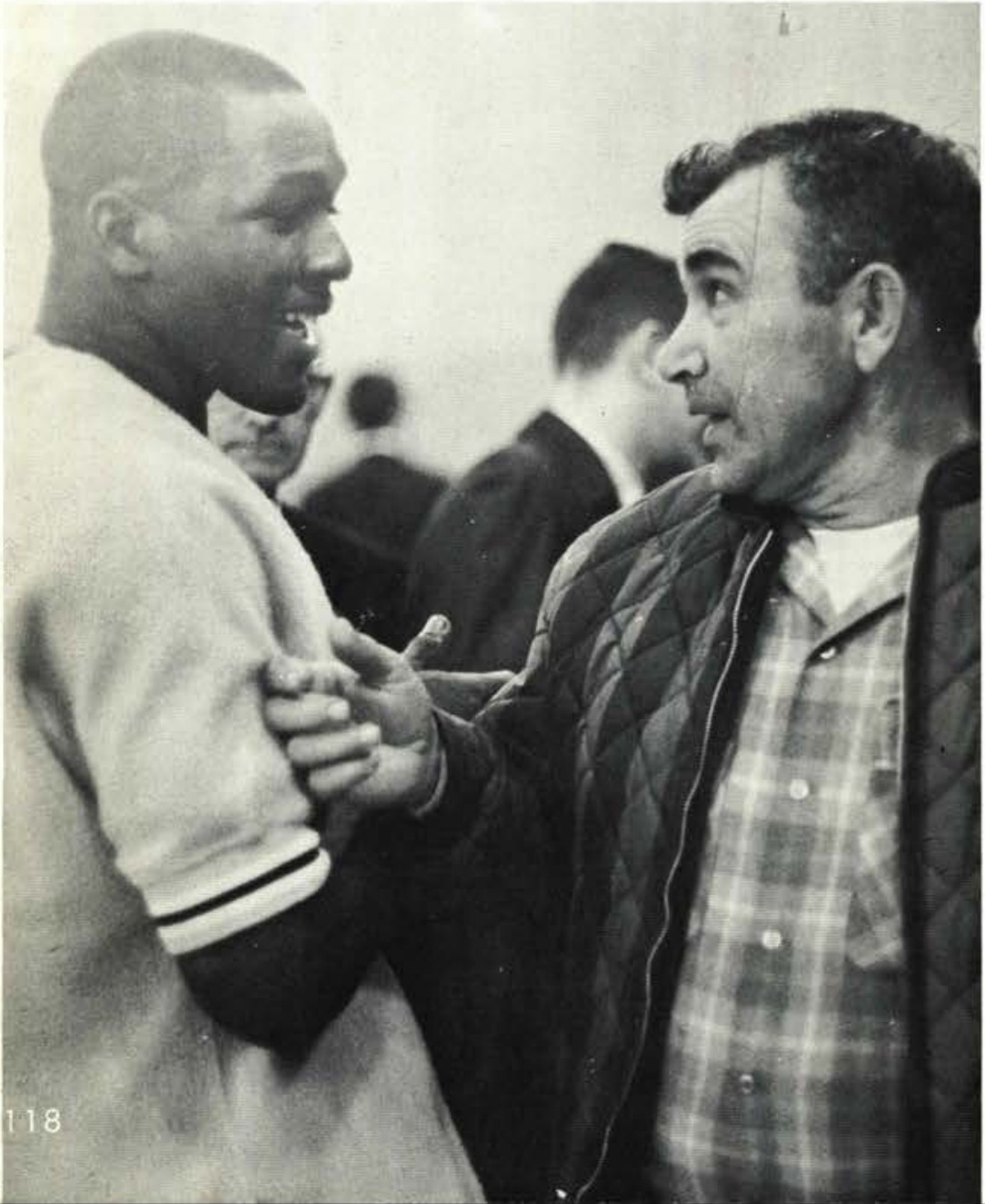

\section{CARTER BREAKS RECORDS}

Dozier Carter will undoubtedly go down in the annals of Cedarville as one of the greatest players ever to grace its basketball court.

During his four years at Cedarville he amassed a total of 1774 points and over 1500 rebounds. The following awards were accorded him: All-M.O.L. all four years, AllN.A.I.A. District \# 22 First team, his junior and senior years, captain of the Jackets his last two years, and M.V.P. his junior and senior years.

His accomplishments include setting the single season scoring mark at 559 his junior year, and shooting over 50 per cent from the field his last two yèars. Perhaps his most memorable feat occurred January 15, 1965, setting not only a single game record, but also a field house record of 49 points against Spring Arbor College.

Dozier Carter, basketball player supreme, sportsman, and one of the finest Christian athletes to pass through the halls of Cedarville will be missed by all and forgotten by none. 


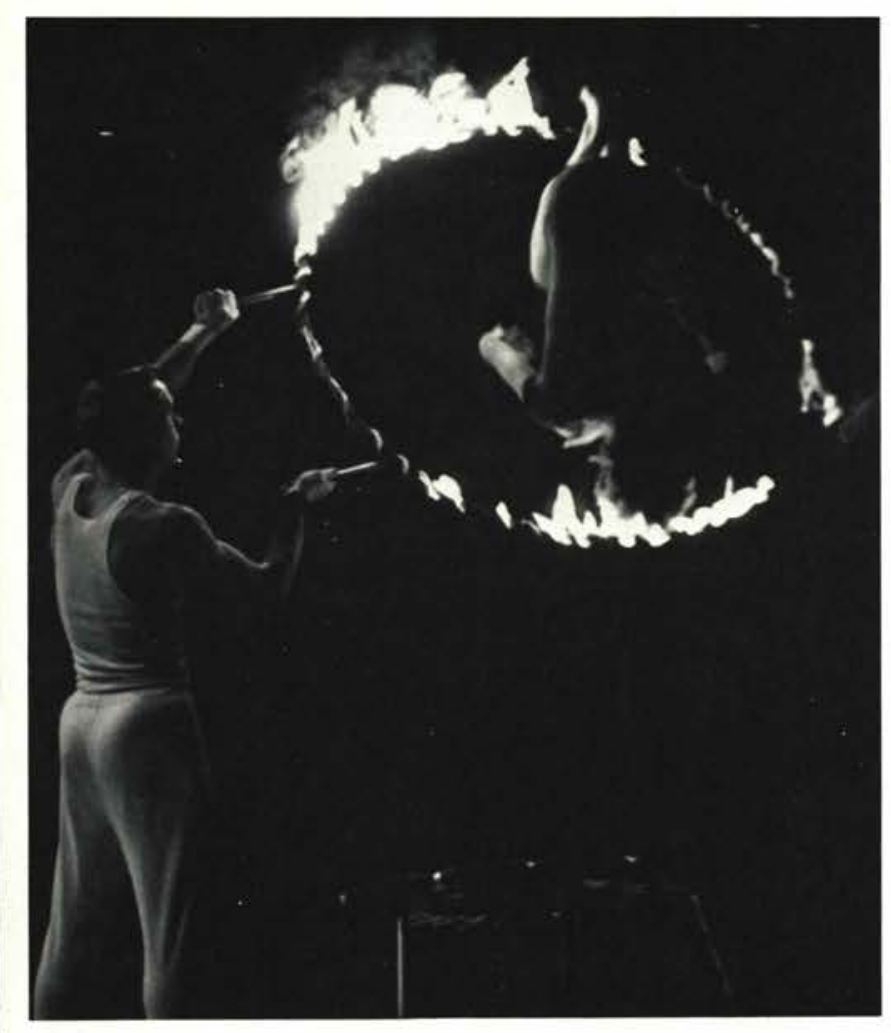

Wyoming, Michigan Junior Olympians
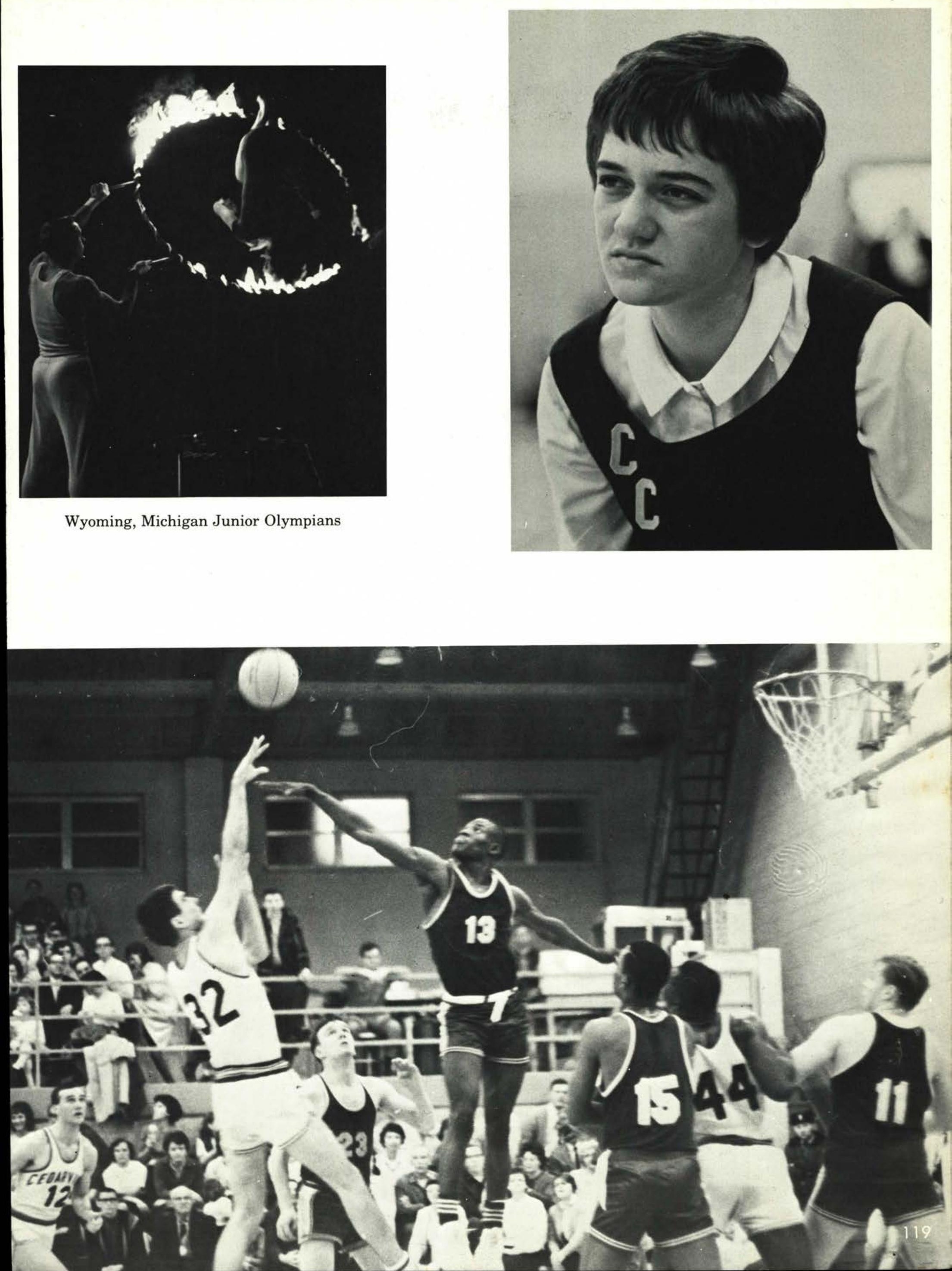
tolno non
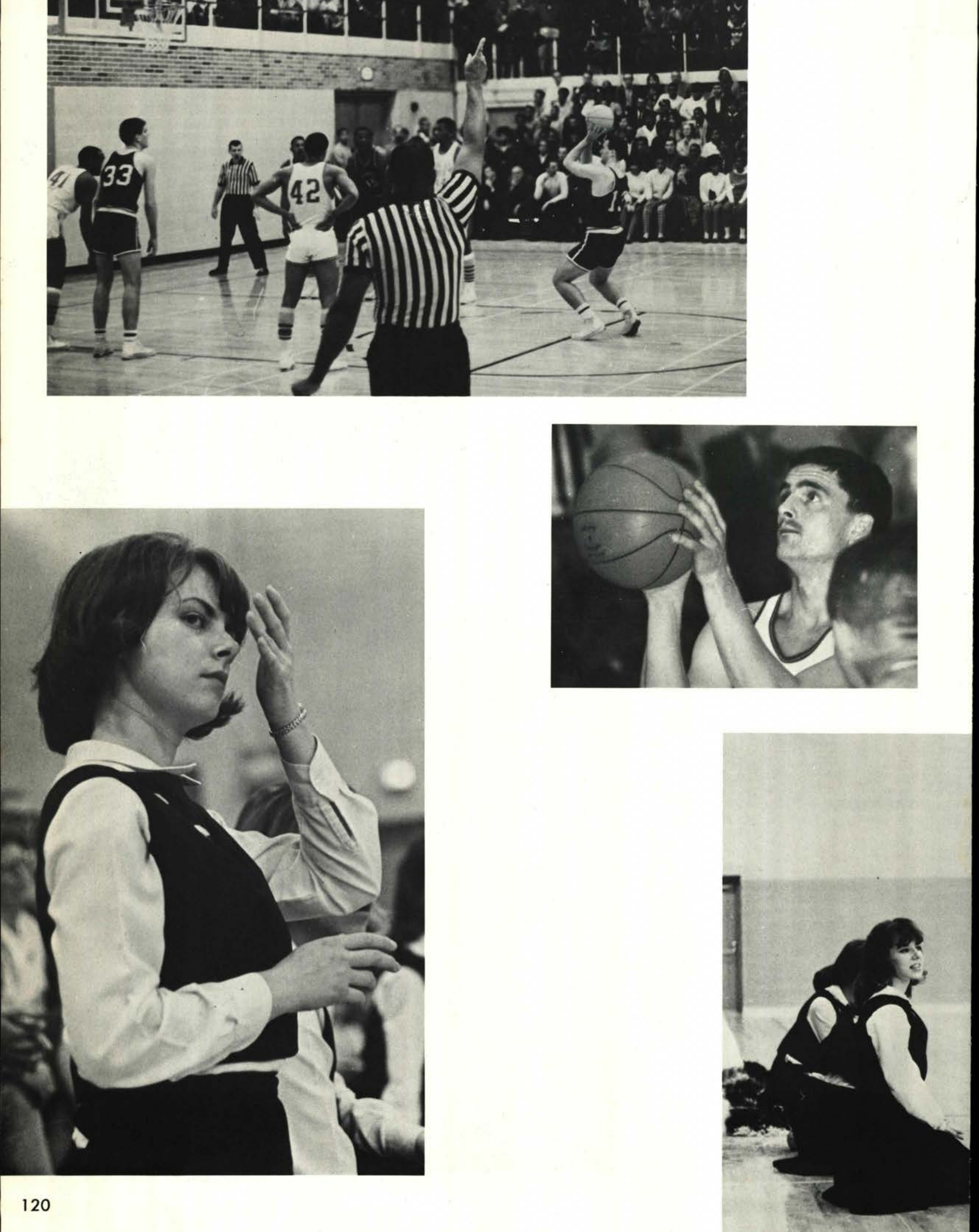


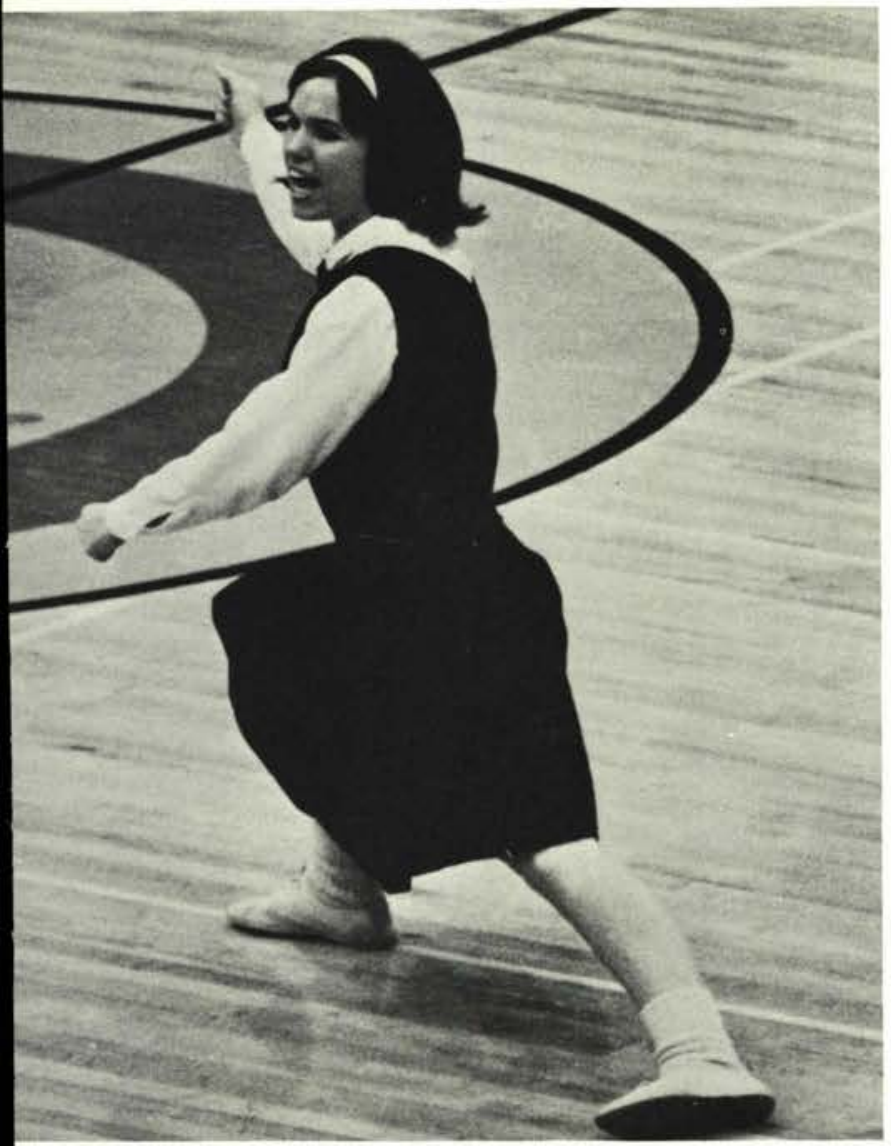

Martie Bennett, captain of cheerleaders
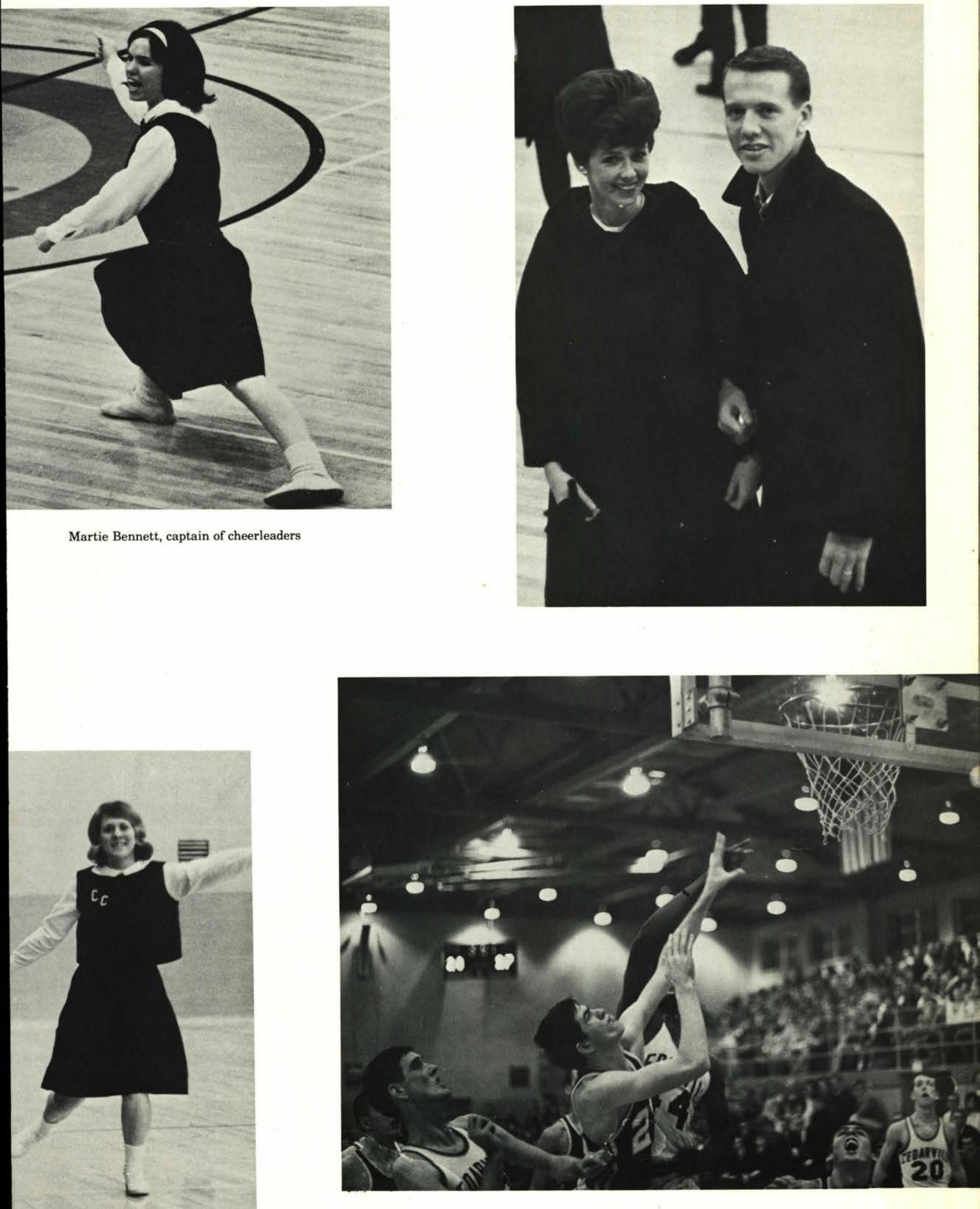


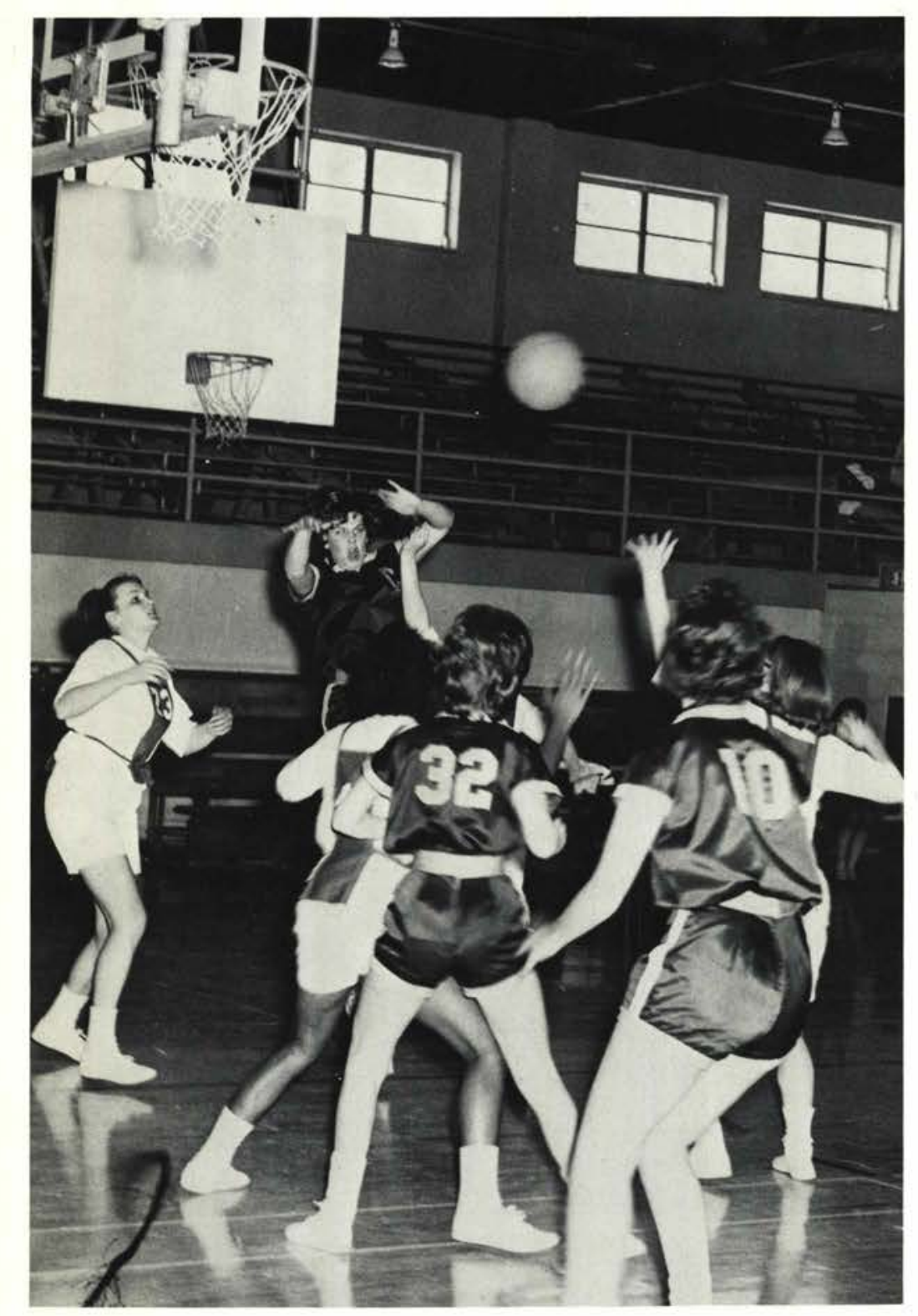

Girl's Basketball

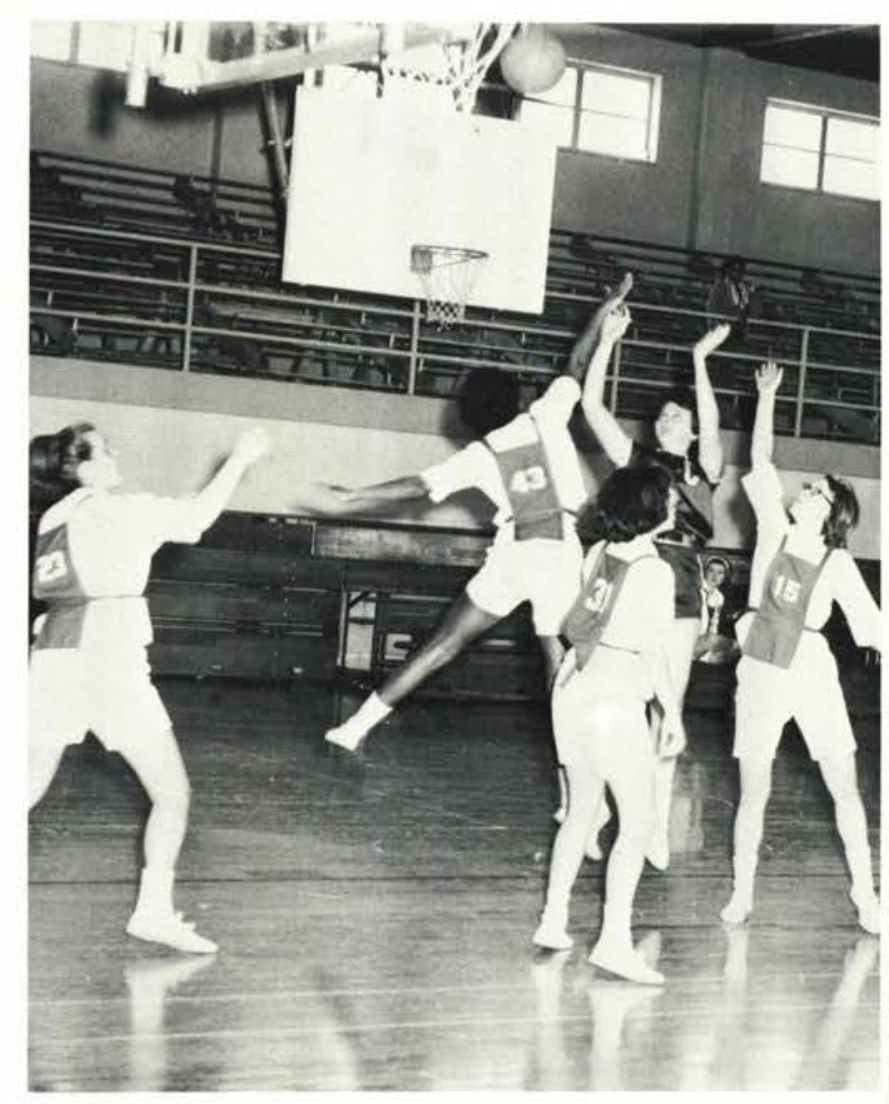

Captain, Mary Alice Jeremiah

Wescott to Jeremiah . . .

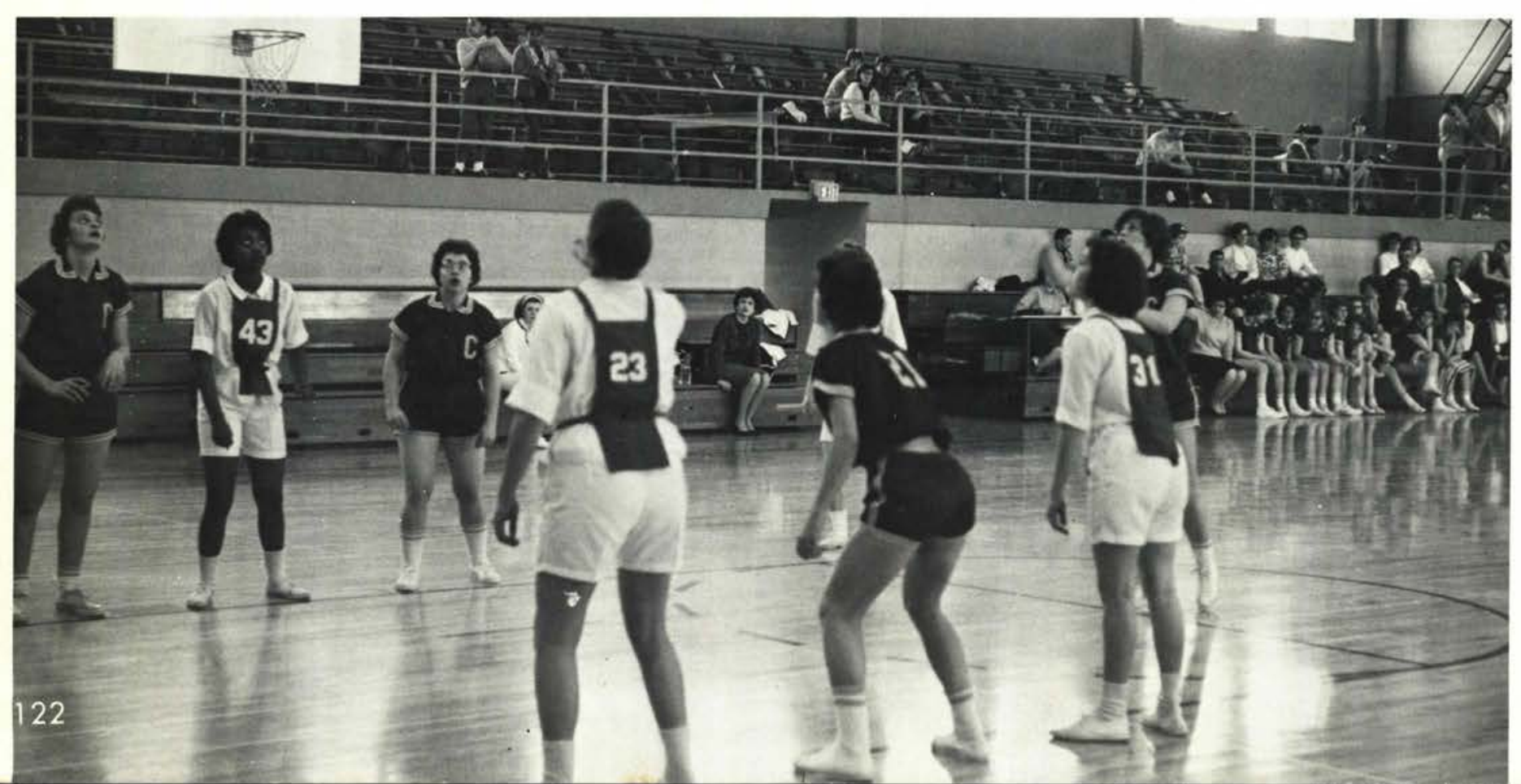




\section{Girl's Basketball}

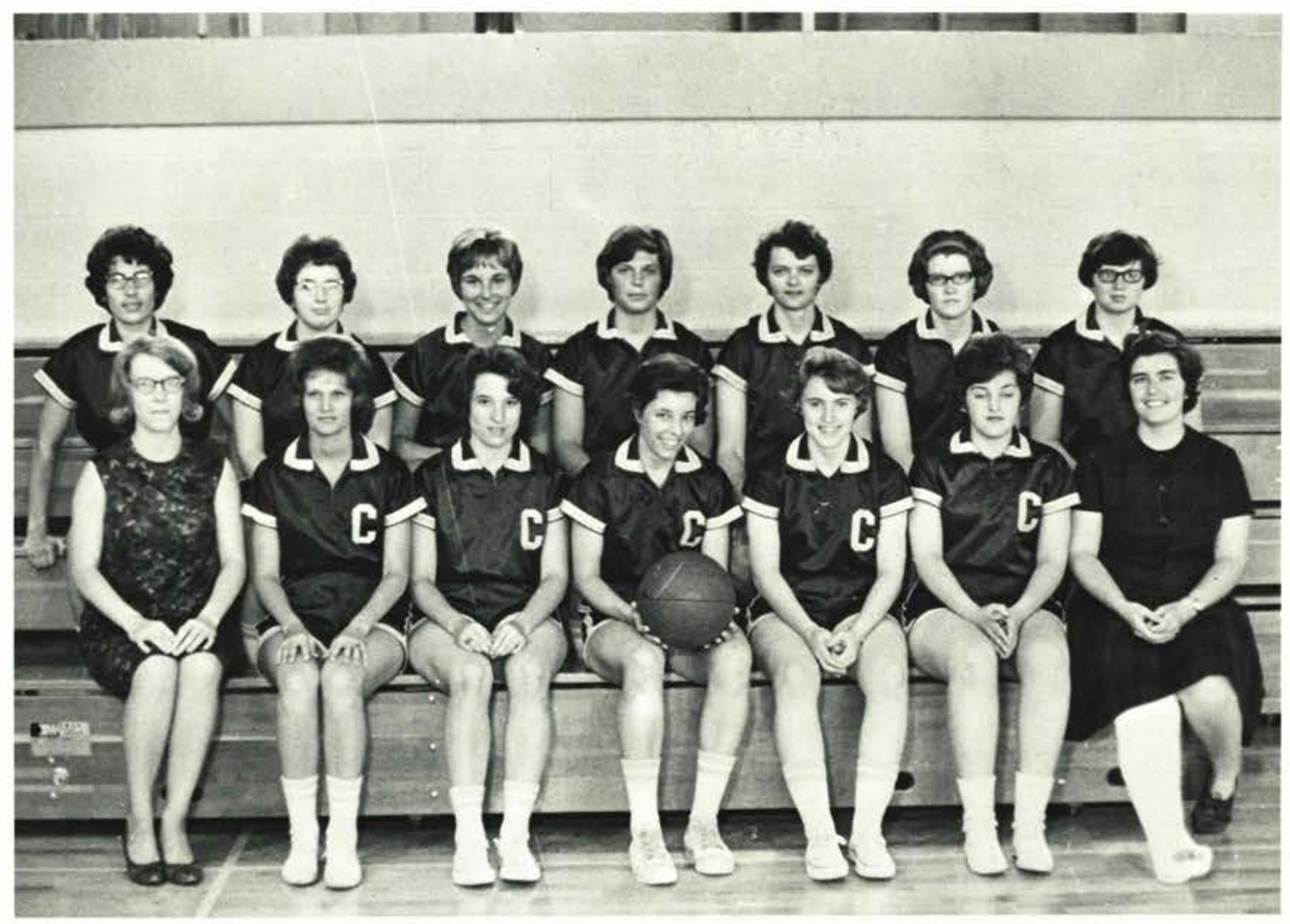

Front Row, left to right: C. Ward, J. Johnson, E. Shugg, G. Bonnema, A. Damon, G. Tompkins, Miss Kearney. Back Row: S. Johns, R. Grant, T. Wing, S. Westcott, M. Jeremiah, B. Ross, B. Kensil.

\section{Girl's Volleyball}

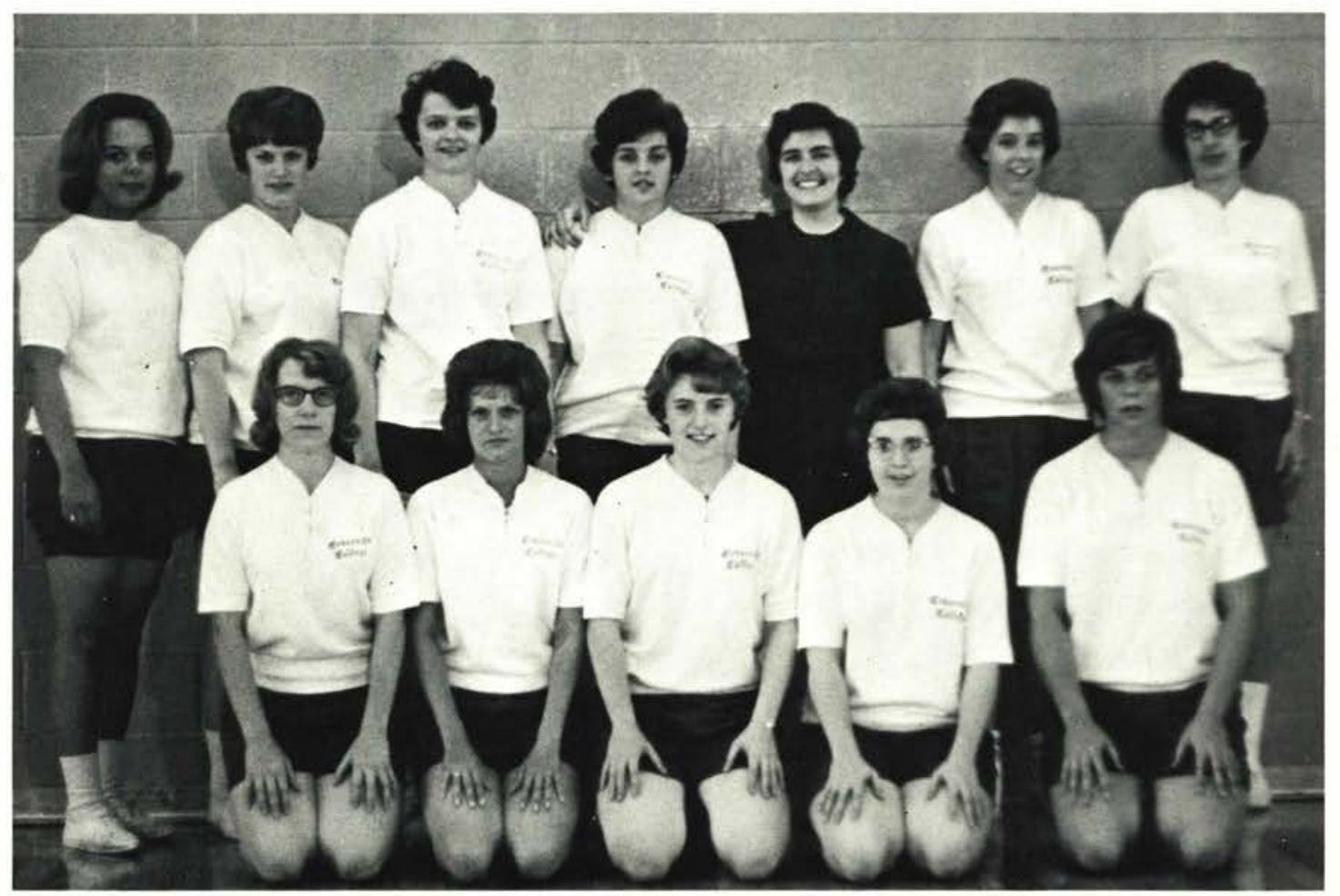

Kneeling, left to right: C. Ward, J. Johnson, A. Damon, R. Grant, S. Westcott. Standing: P. Sadler, K. Johnson, M. Jeremiah, G. Tompkins, Miss Kearney, G. Bonnema, S. Johns. 


\section{Gamma Chi Presents ...}
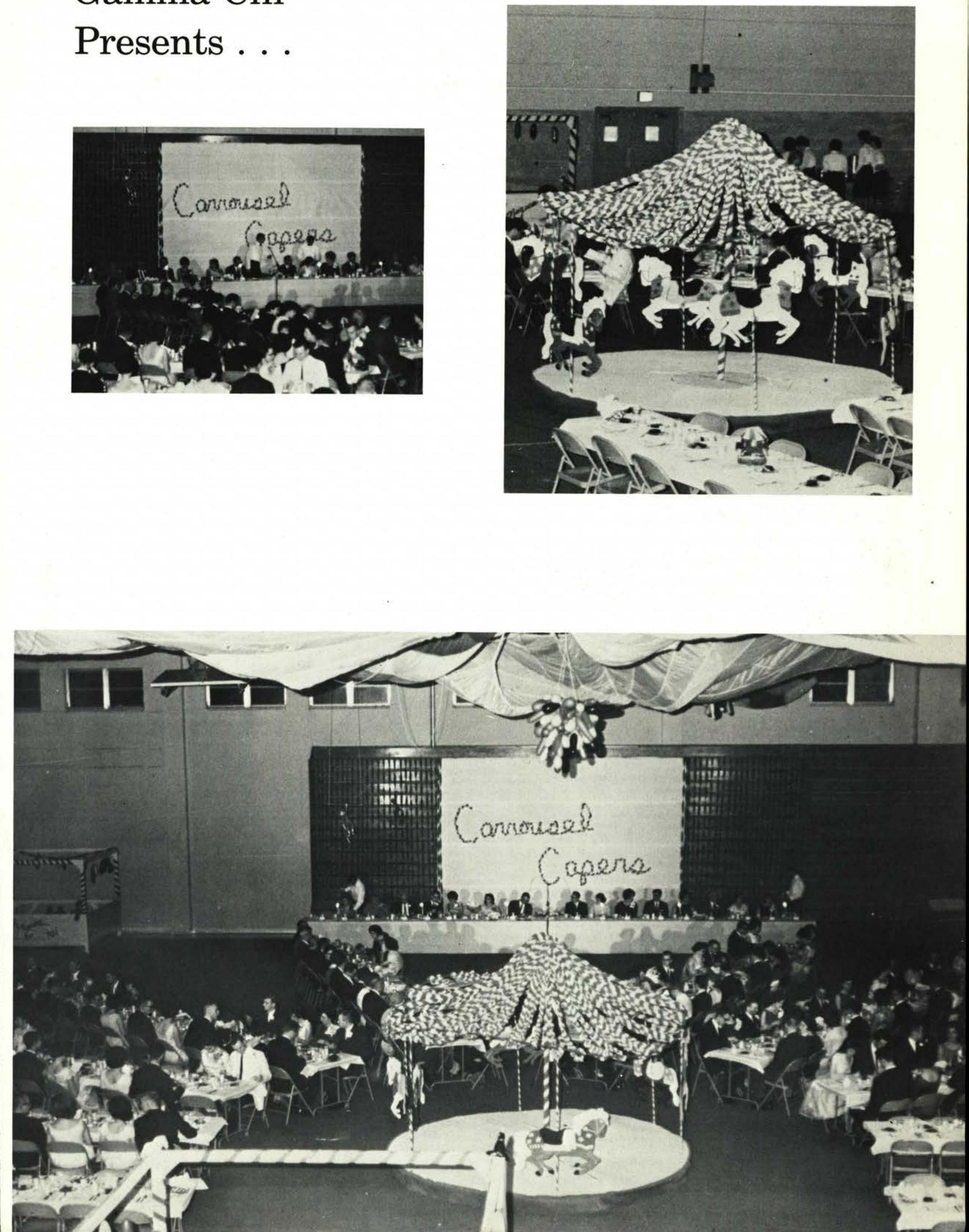


\section{First Annual Operetta}

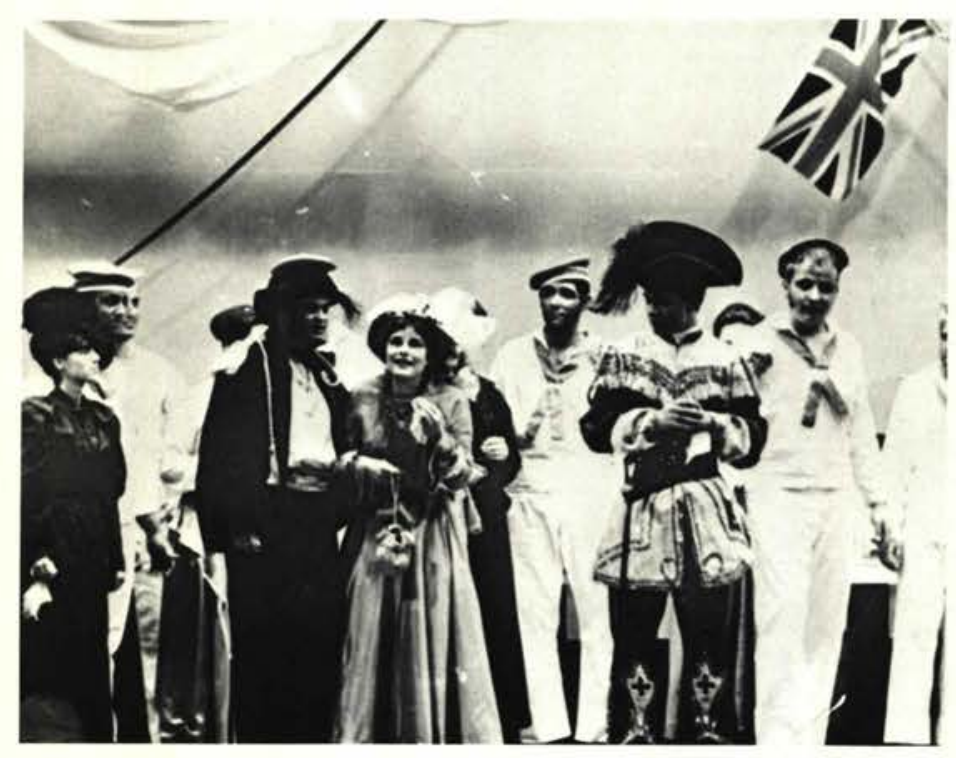

The Music Department marked another

first by presenting the first annual operetta $-\mathbf{a}$

Gilbert and Sullivan favorite, "H.M.S. Pina-

fore." It was performed on March 5 under the

direction of Miss Ruth Smith and Mr. Walter

Meissner.
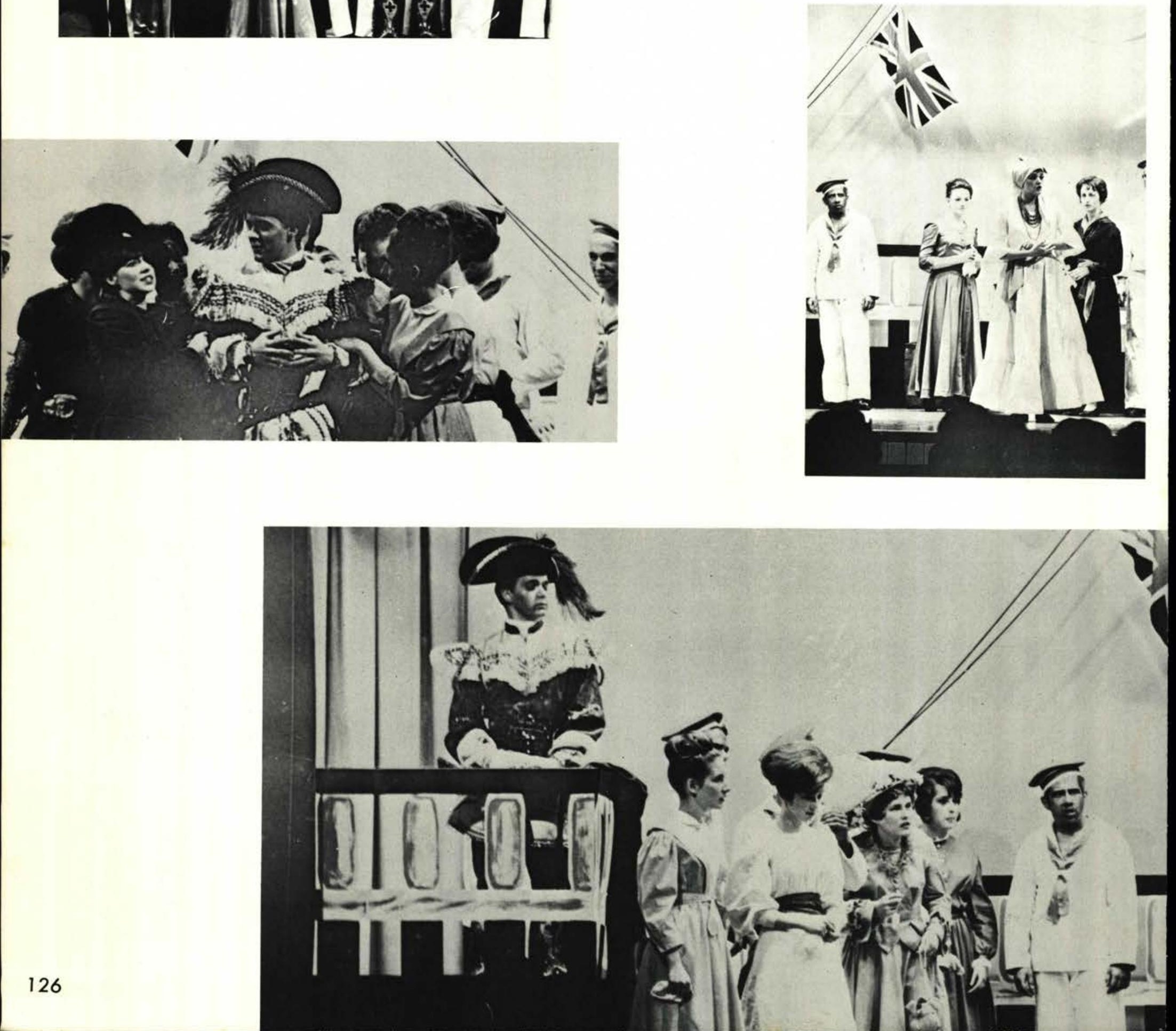


\section{Artist Series}

Artist series were fewer in number this year but their quality was by no means reduced from previous years. Presentations this year were by Miss Joyce Jones from Corpus Christi, Texas who performed brilliantly at the organ and a duo-piano concert by Miss Ruth Smith and Mr. Joe Zimmerman.

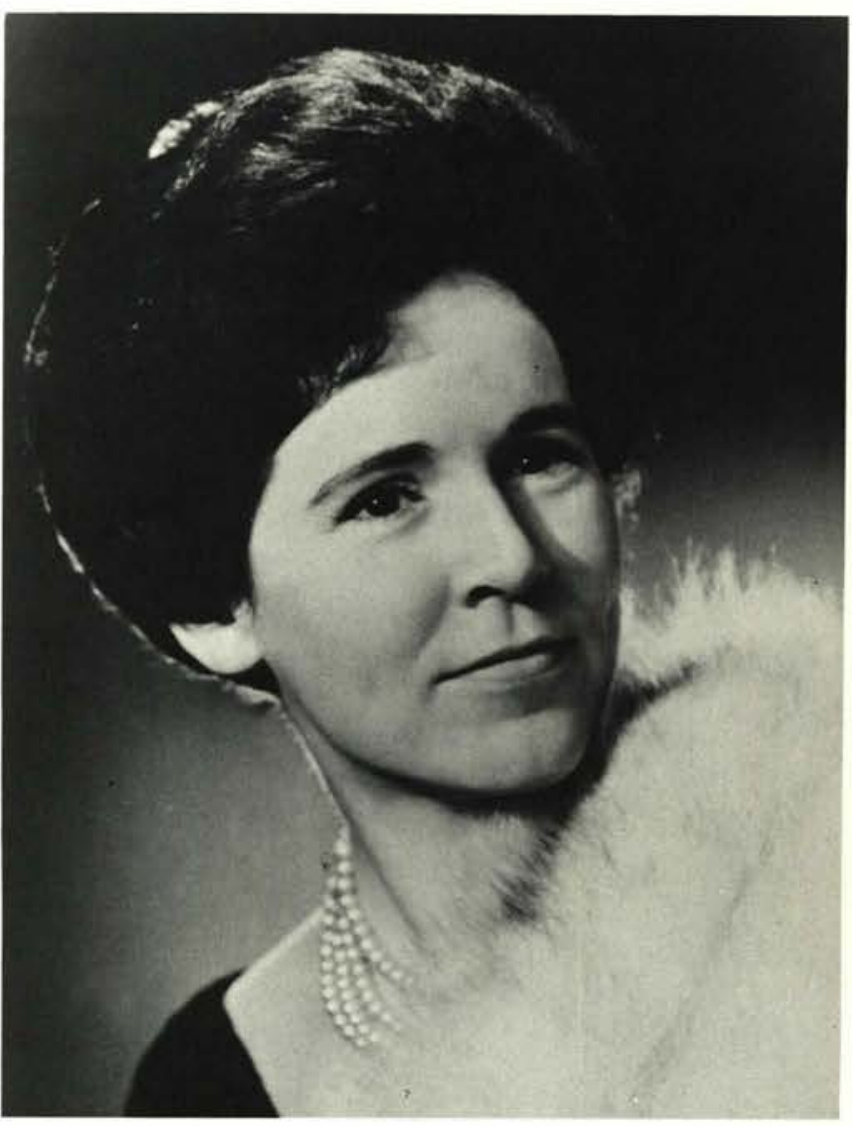

Miss Joyce Jones

Miss Ruth Smith and Mr. Joe Zimmerman.

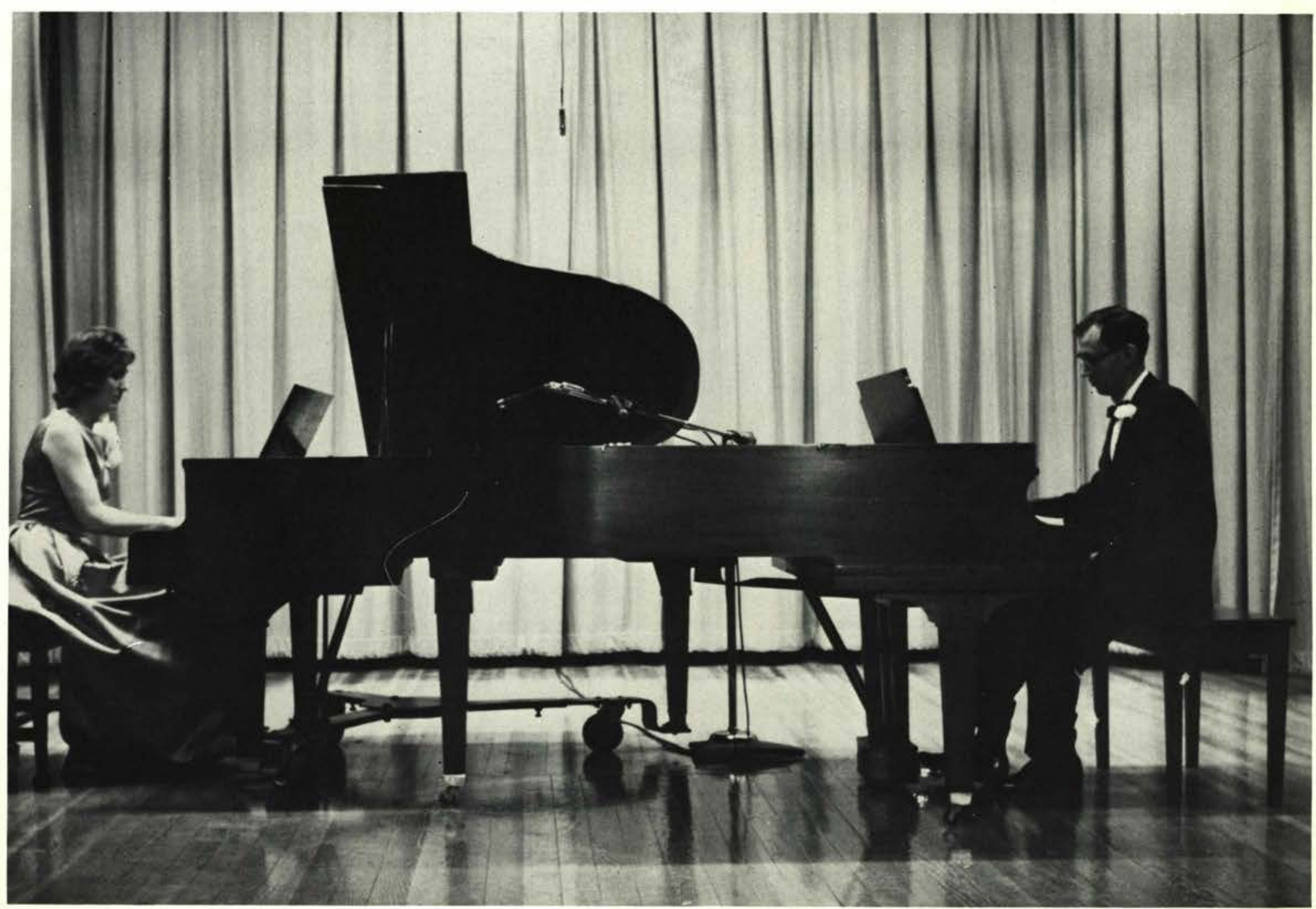




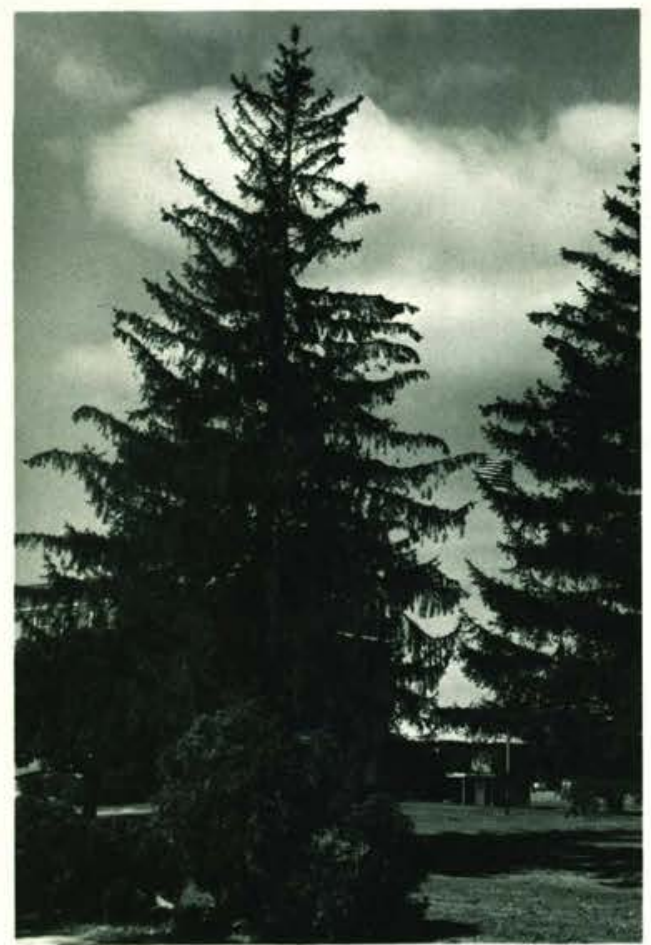

\section{SPRING}

Spring is the wonderful renewal of many things ... grass turns green again ... campus benches and walks are used again for leisurely evenings together . . . the turtle tree reassumes its shape ... after-supper softball games are organized ... Mr. St. Clair's bicycle business booms .... and convertible tops come down.
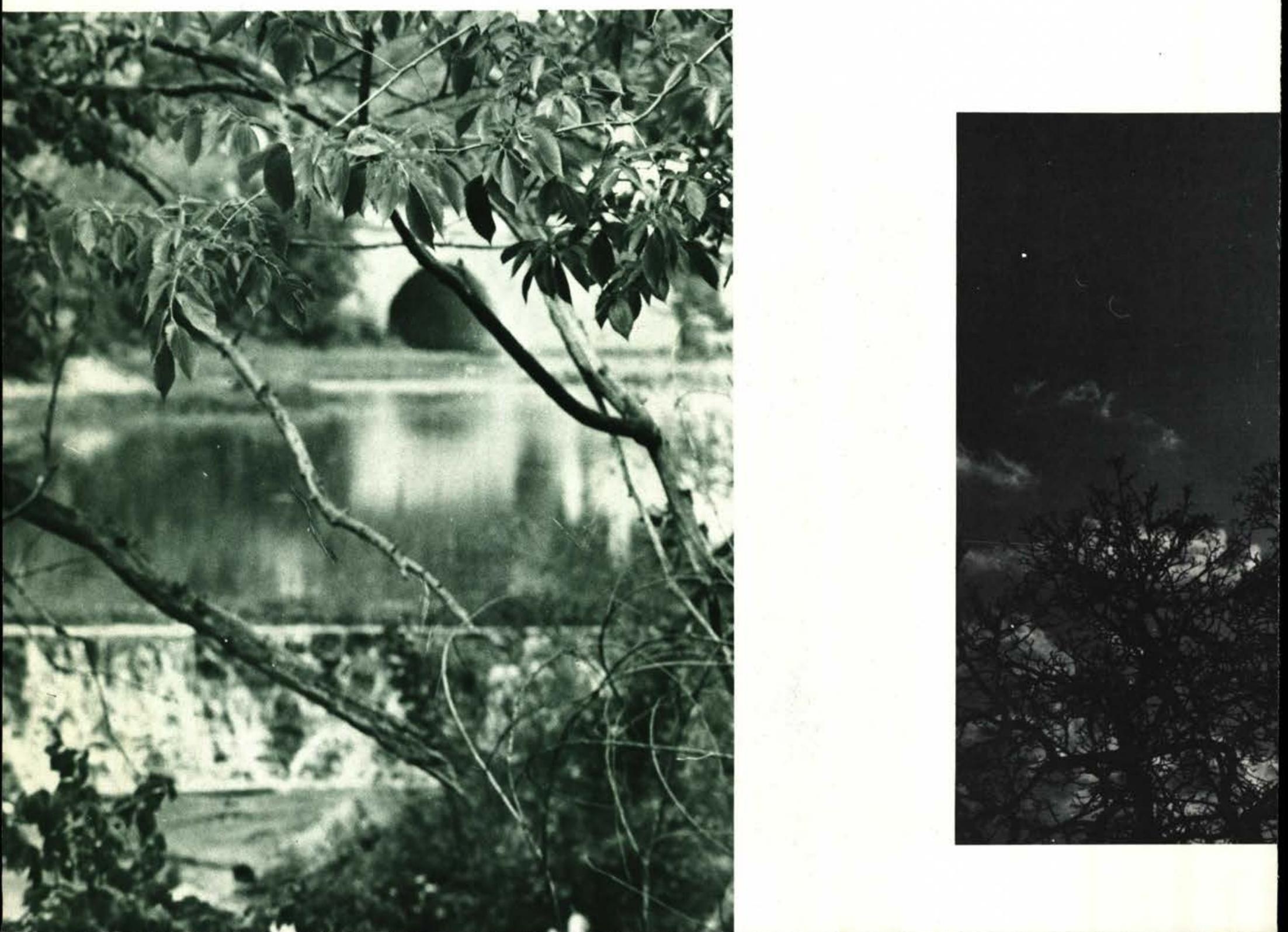
If

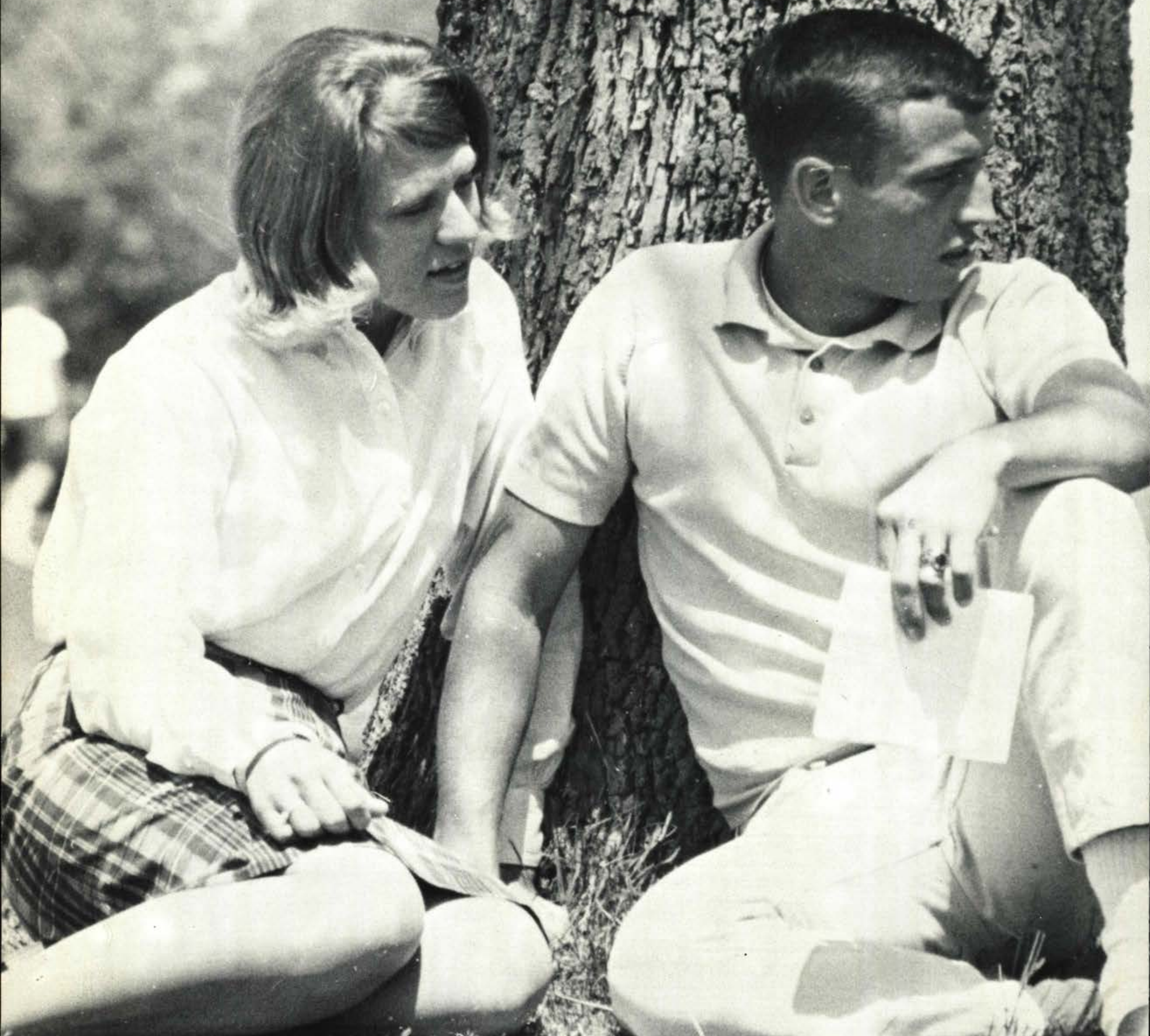

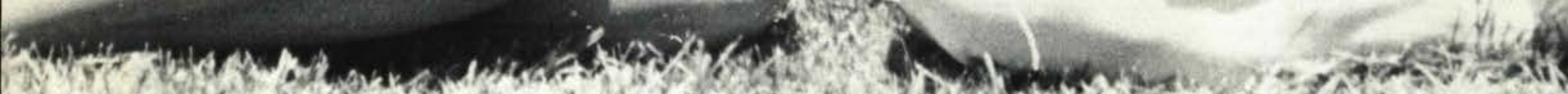

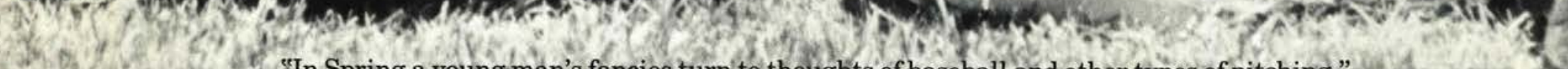
(2) "In Spring a young man's fancies turn to thoughts of baseball and other types of pitching." whe

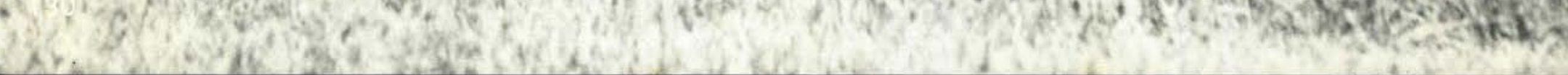




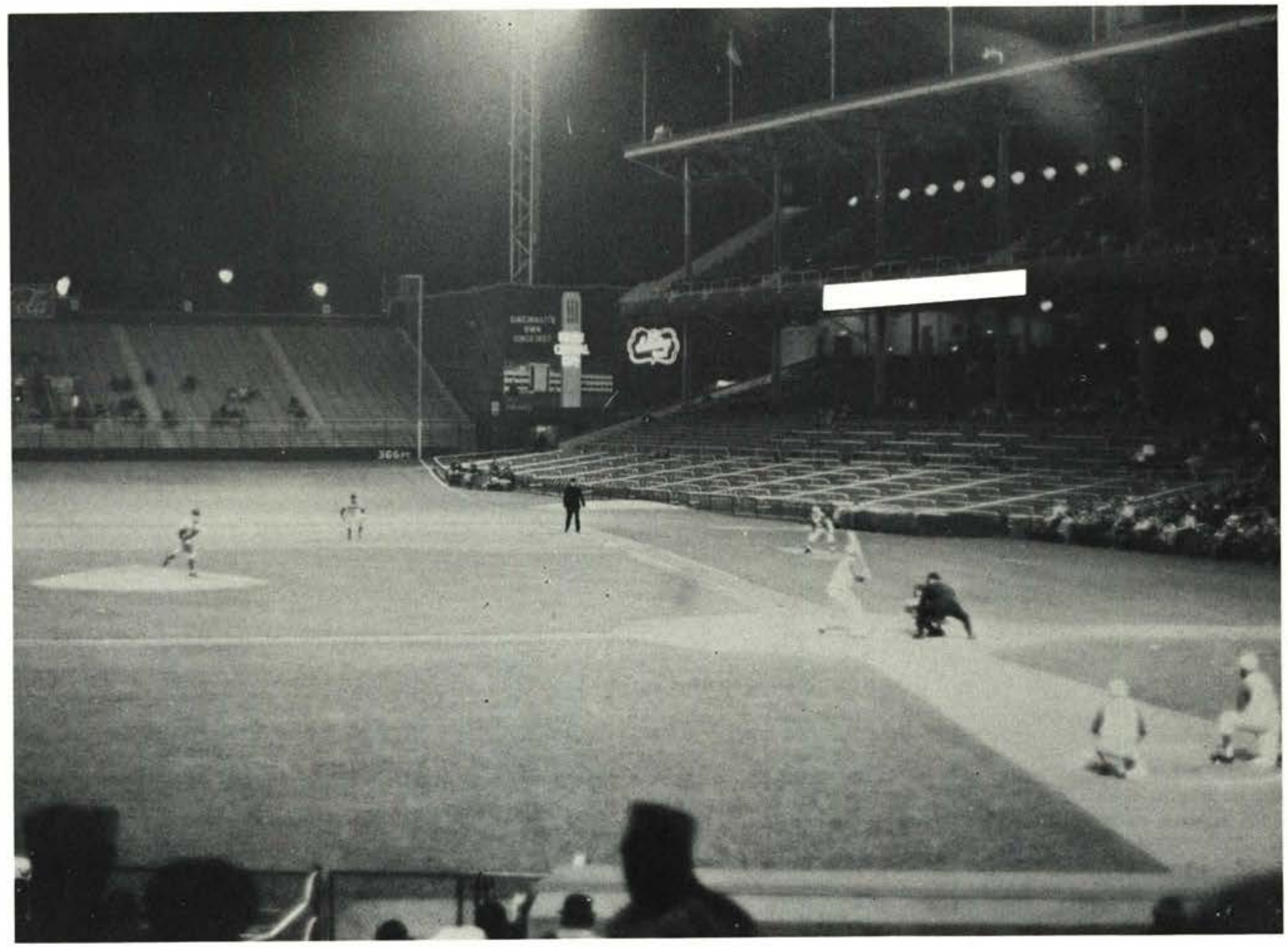

Spring is trips to night baseball games at Cincinnati.

Spring is mixing sunbathing with studies.
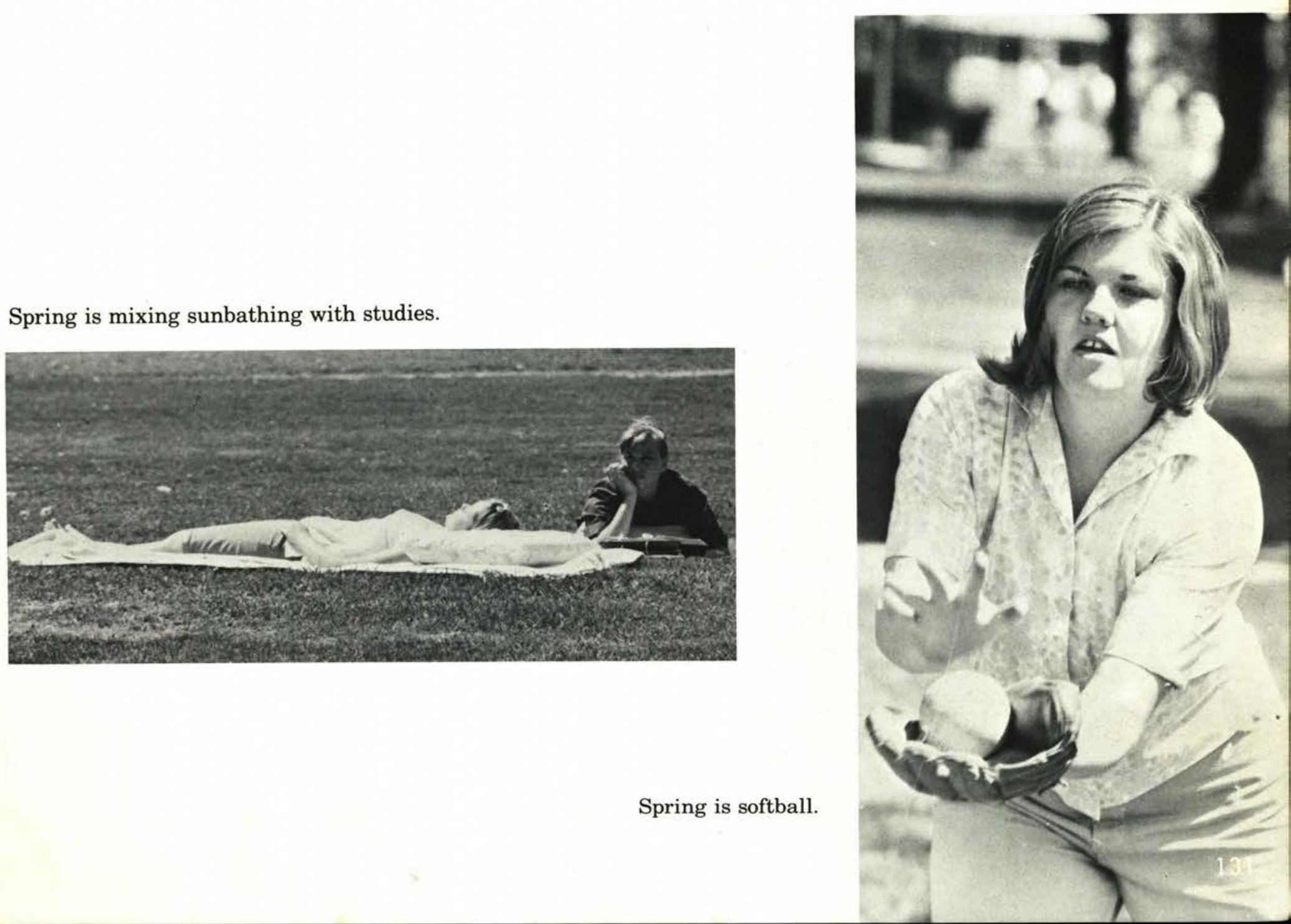

Spring is softball. 

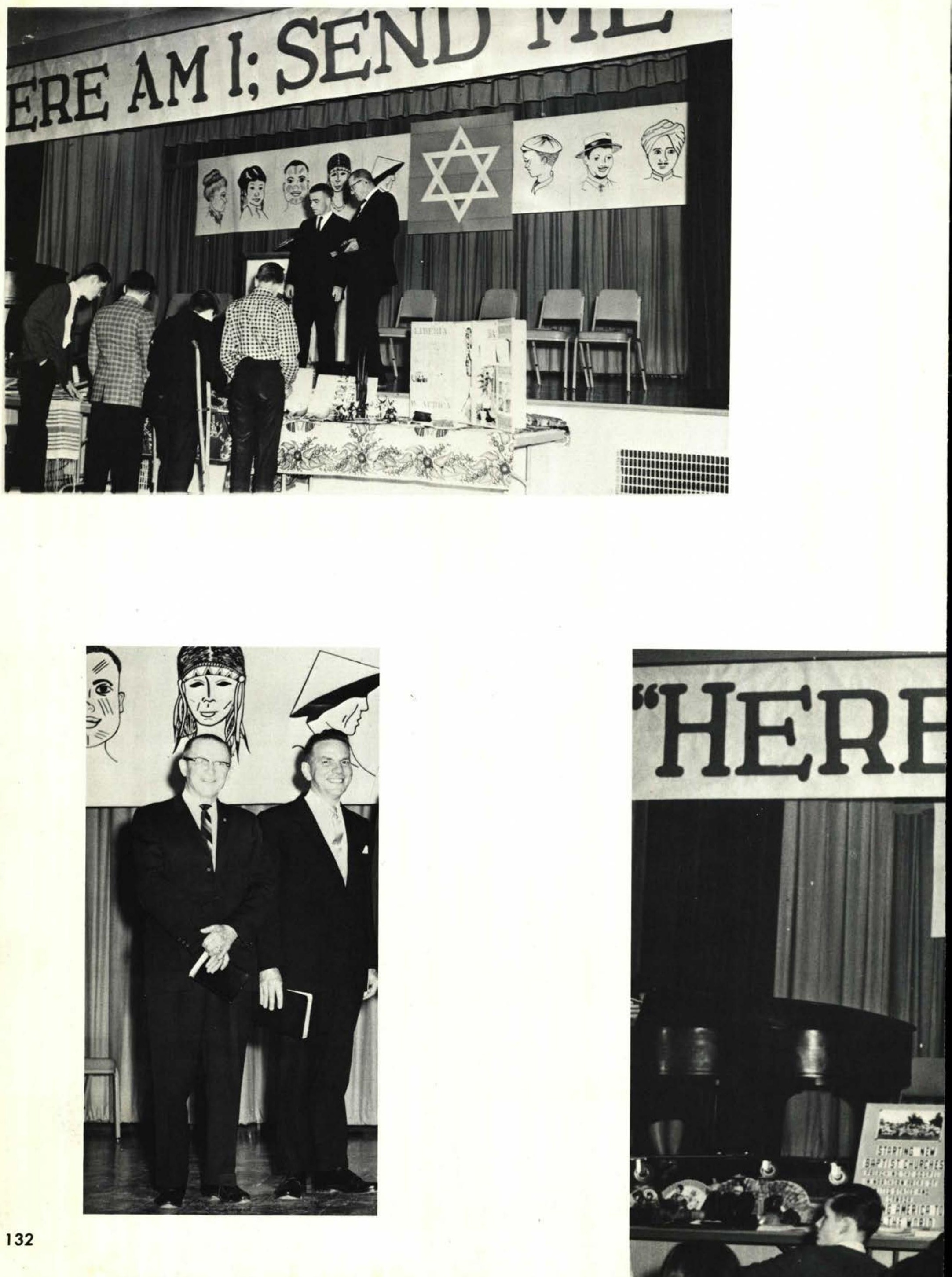


\section{SPRING MISSIONARY CONFERENCE}

March 31 to April 4 marked the sixth annual Missionary Conference sponsored by the Fellowship for World Missions. Missionaries, representing five different fields, challenged the student body during the morning chapel periods and the evening services. The Conference was held in conjunction with area churches so that they too could benefit from these excellent speakers.
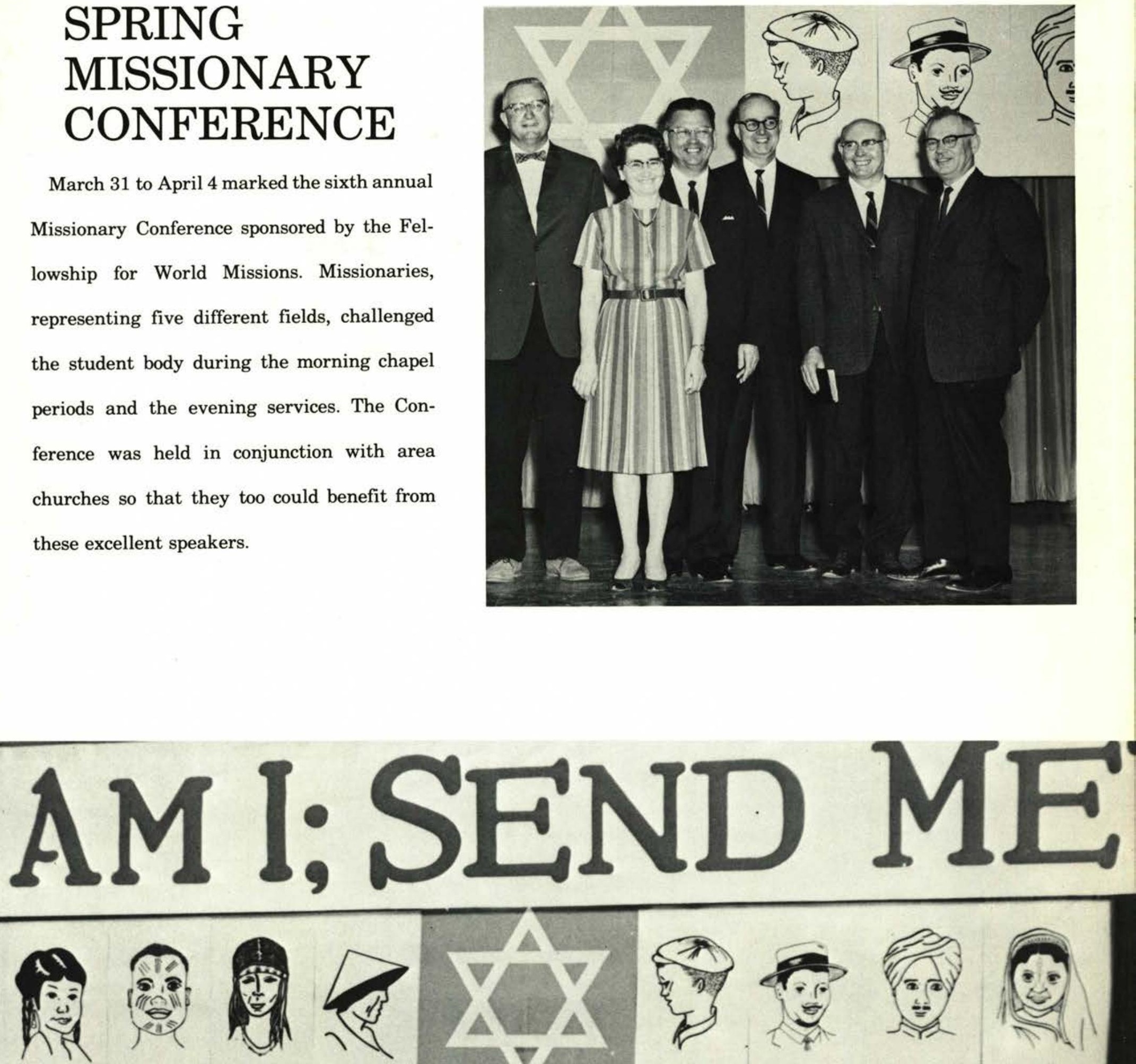

\section{.}

r
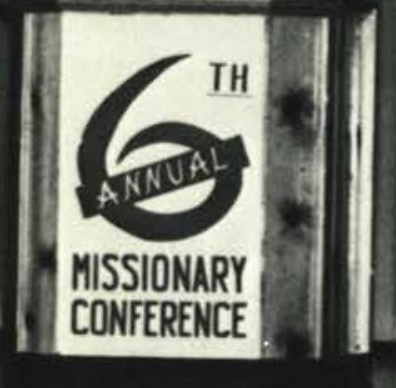

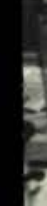

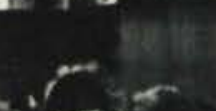

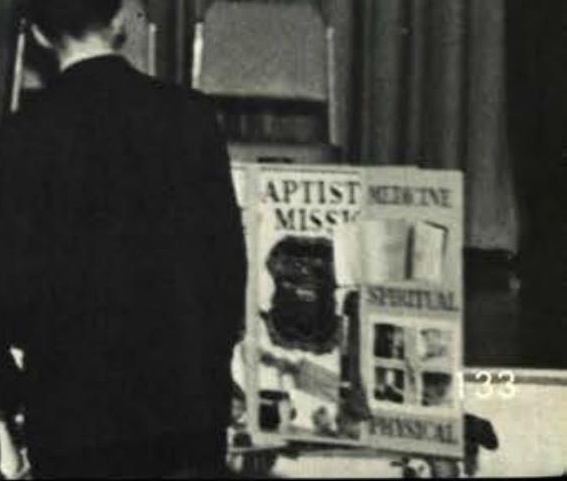




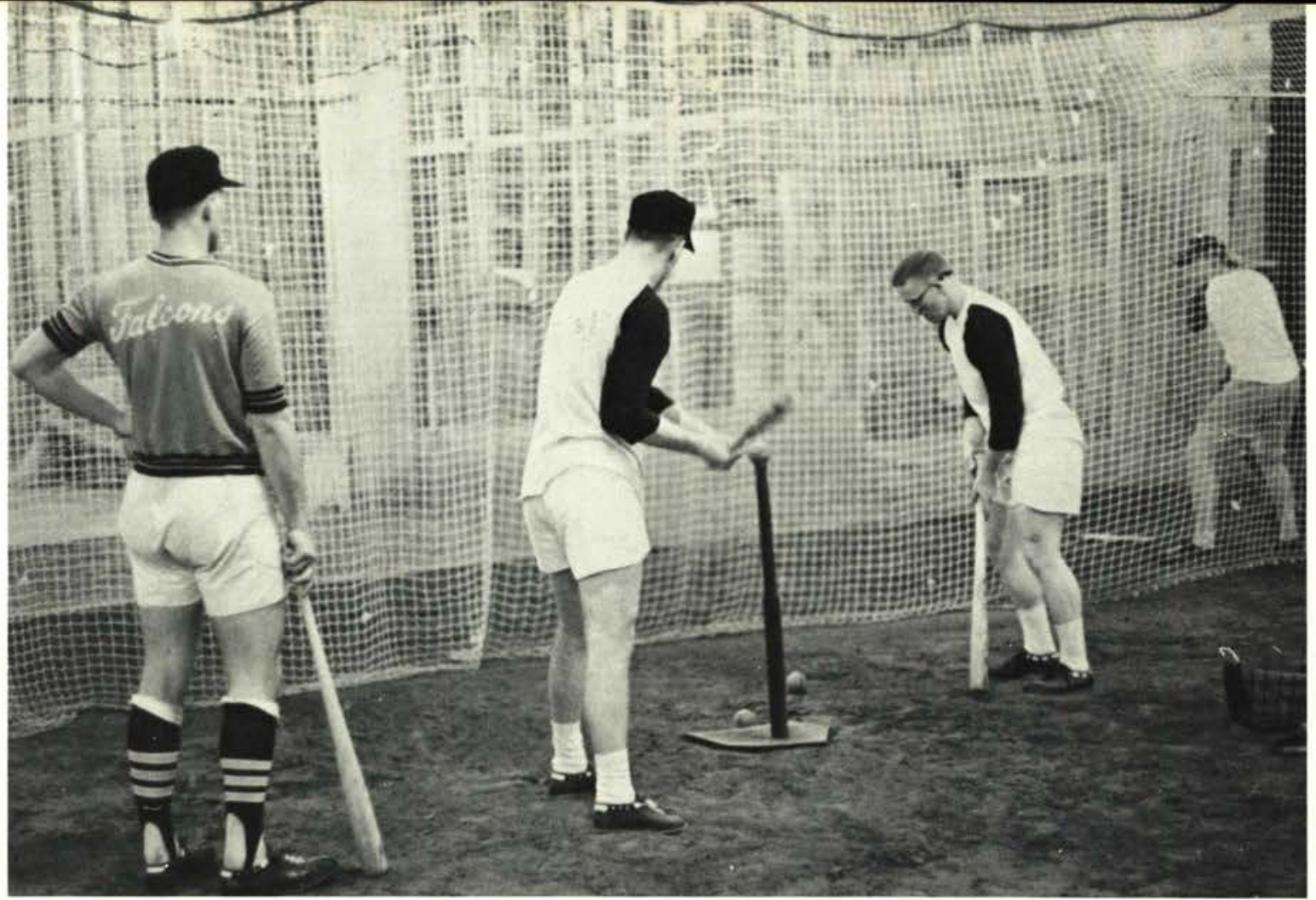

Indoor practice.
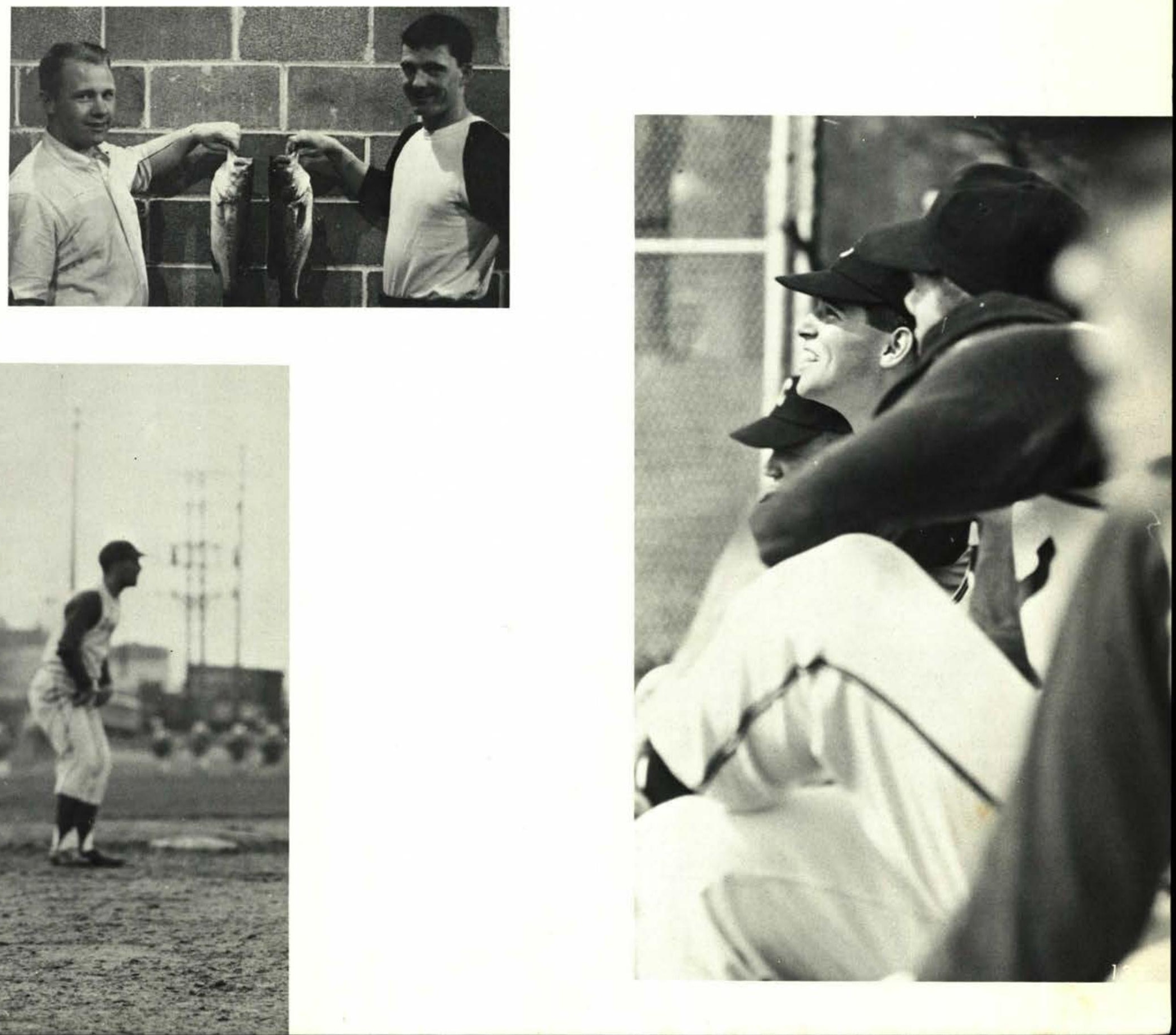

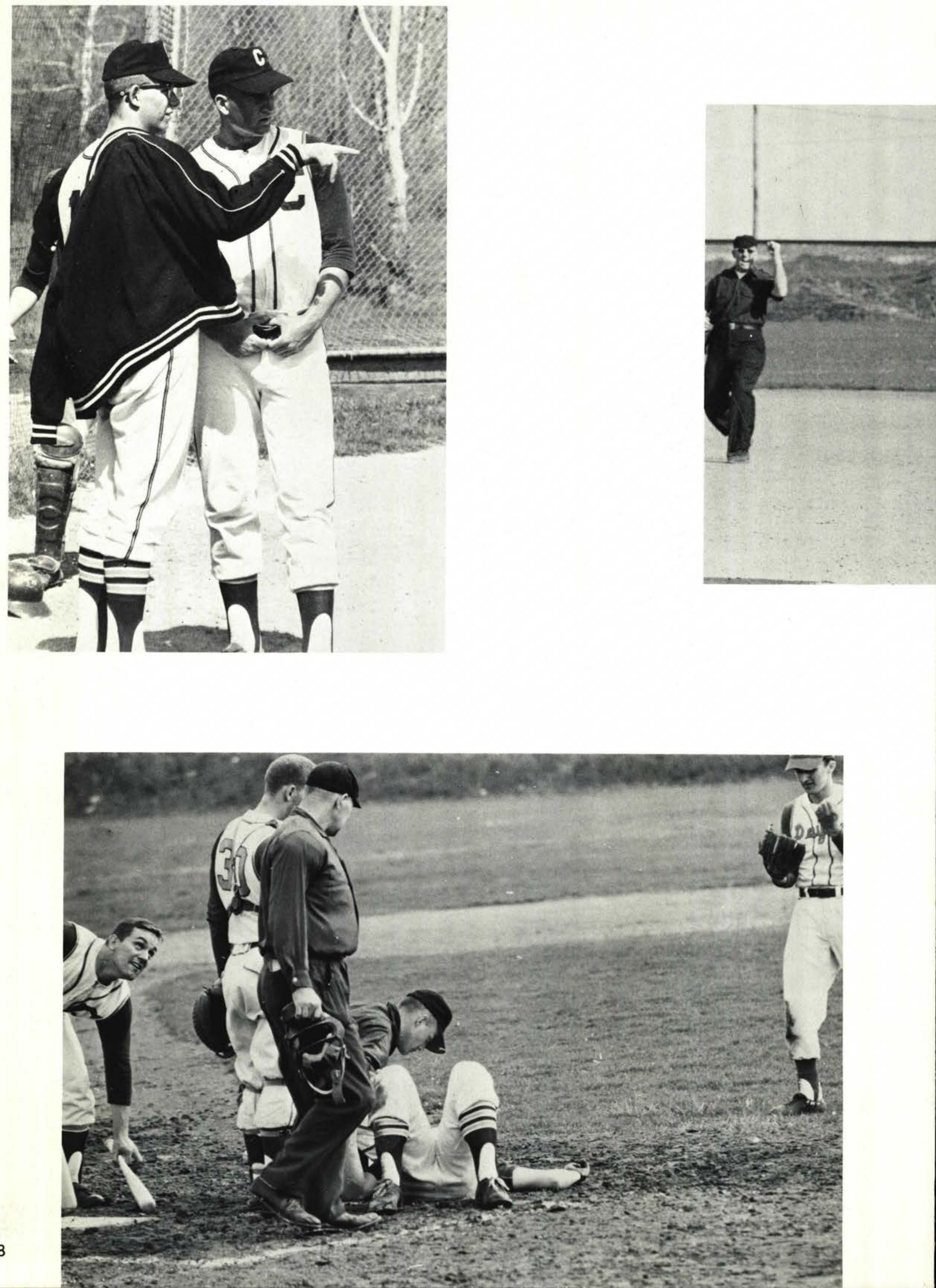

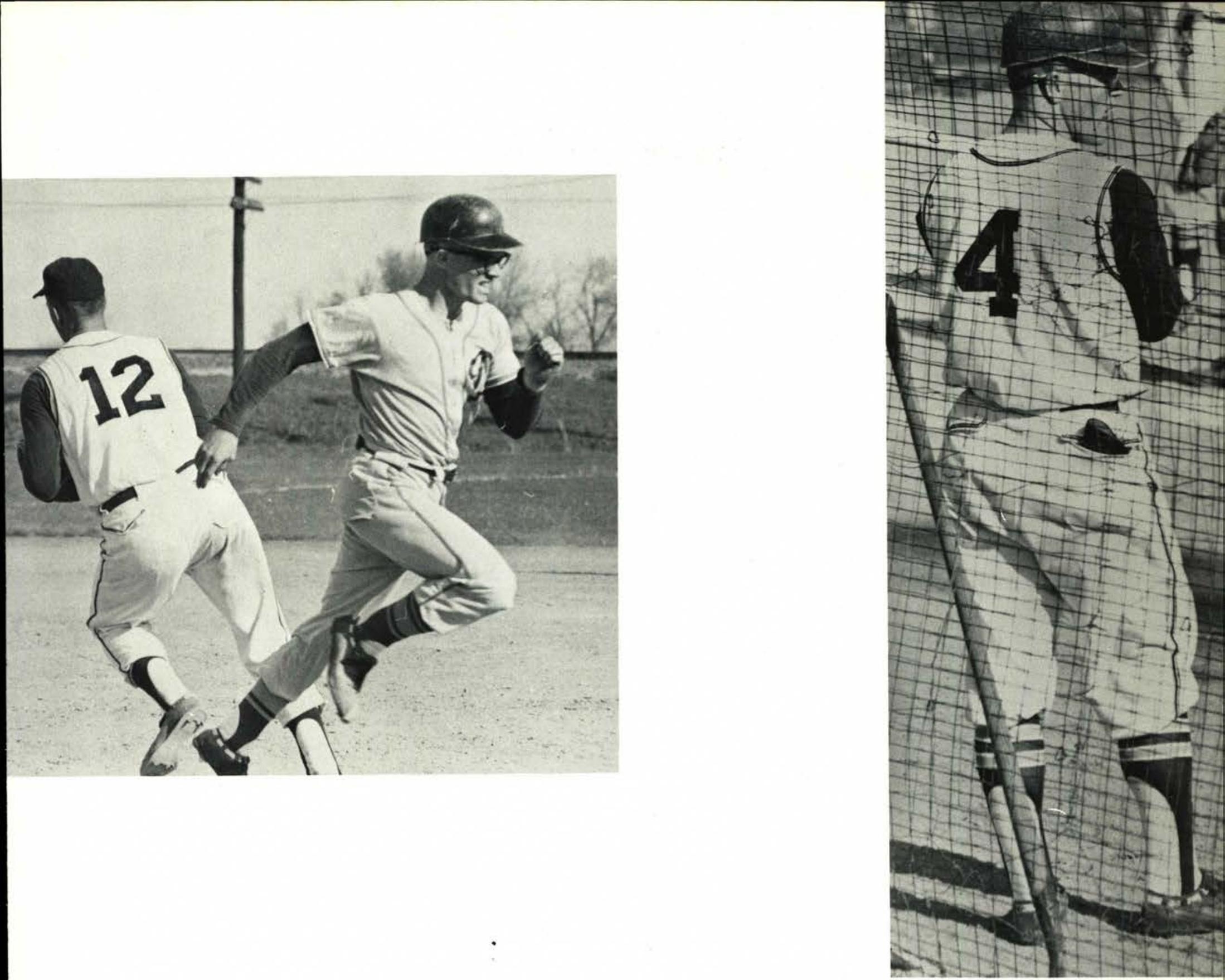

\section{6-2021 Ph.766480ited Ph. 7662571}

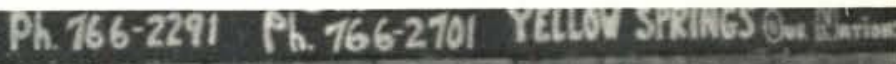

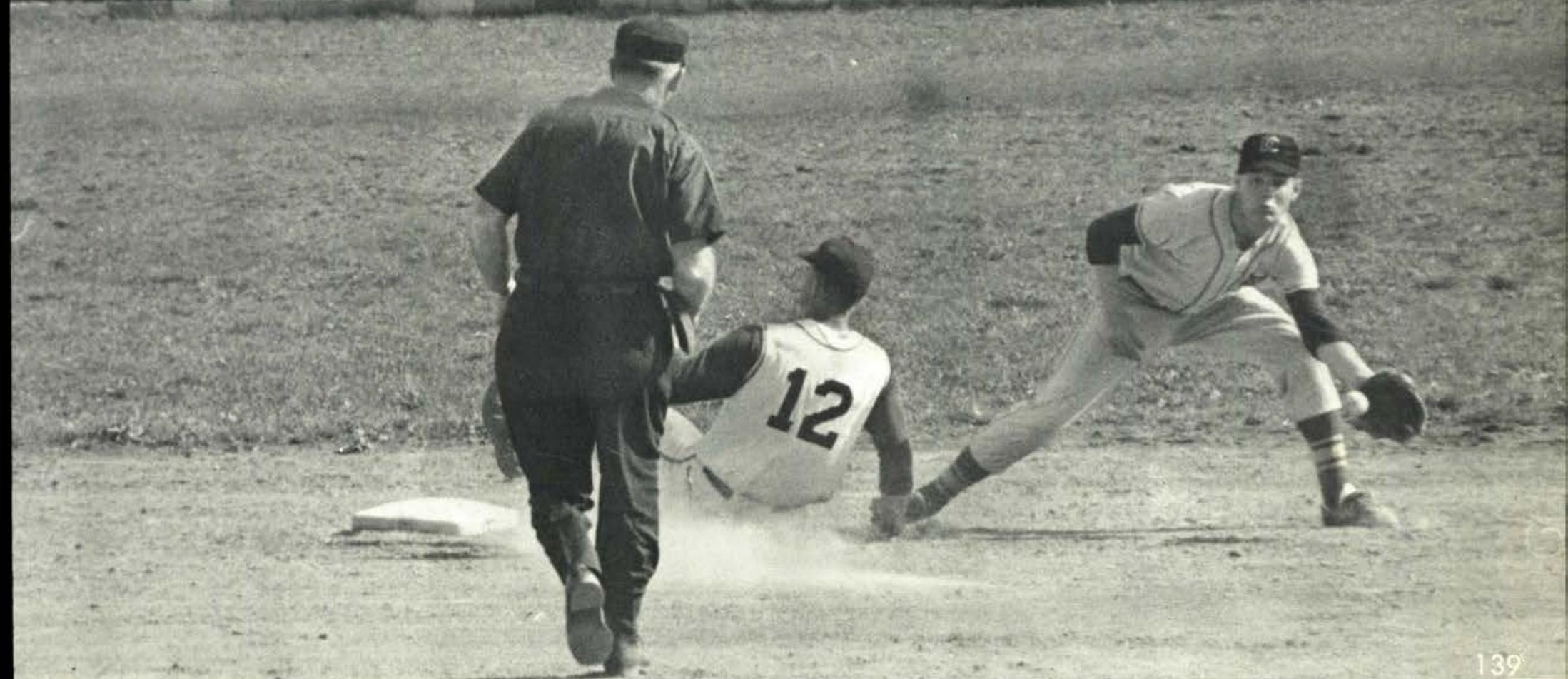



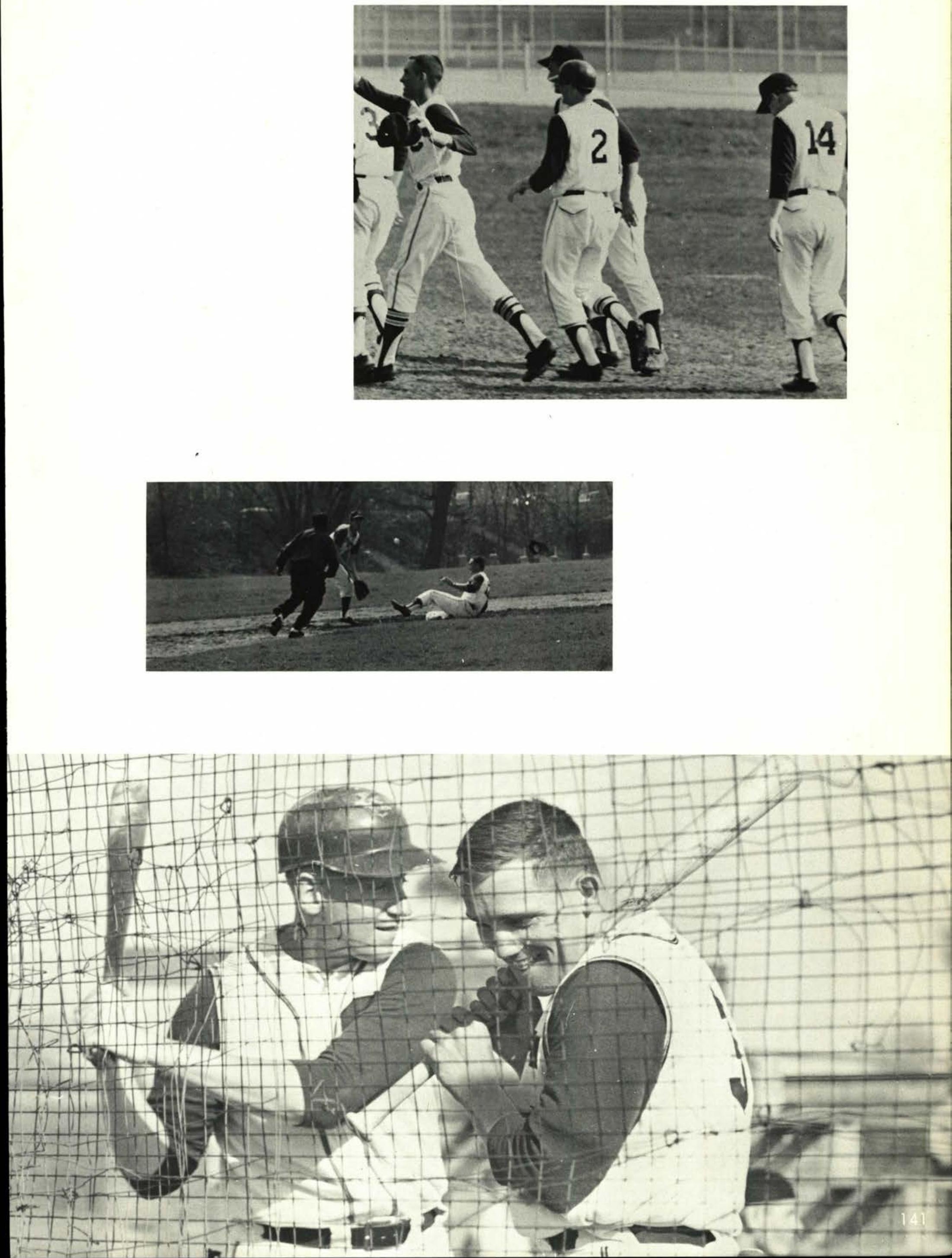
Due to lack of depth, the team could not compile enough points to have good showings in their meets, although they continually took many 2nd places. With returning lettermen such as distance men, Rich Davis, Tom Eliot and $\mathrm{Al}$ Traucht, the future for the Cedarville track team appears brighter.
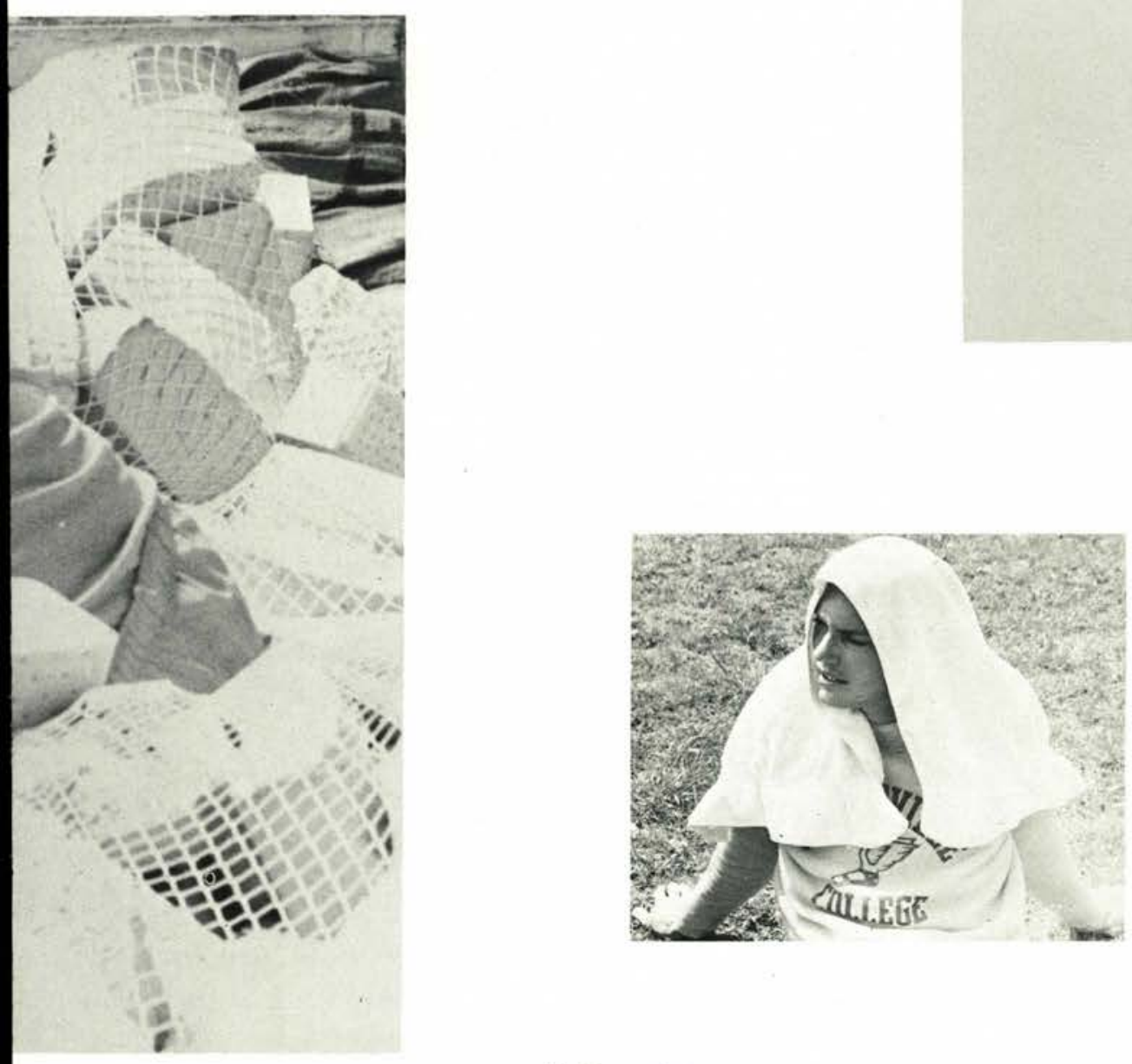

Al Traucht

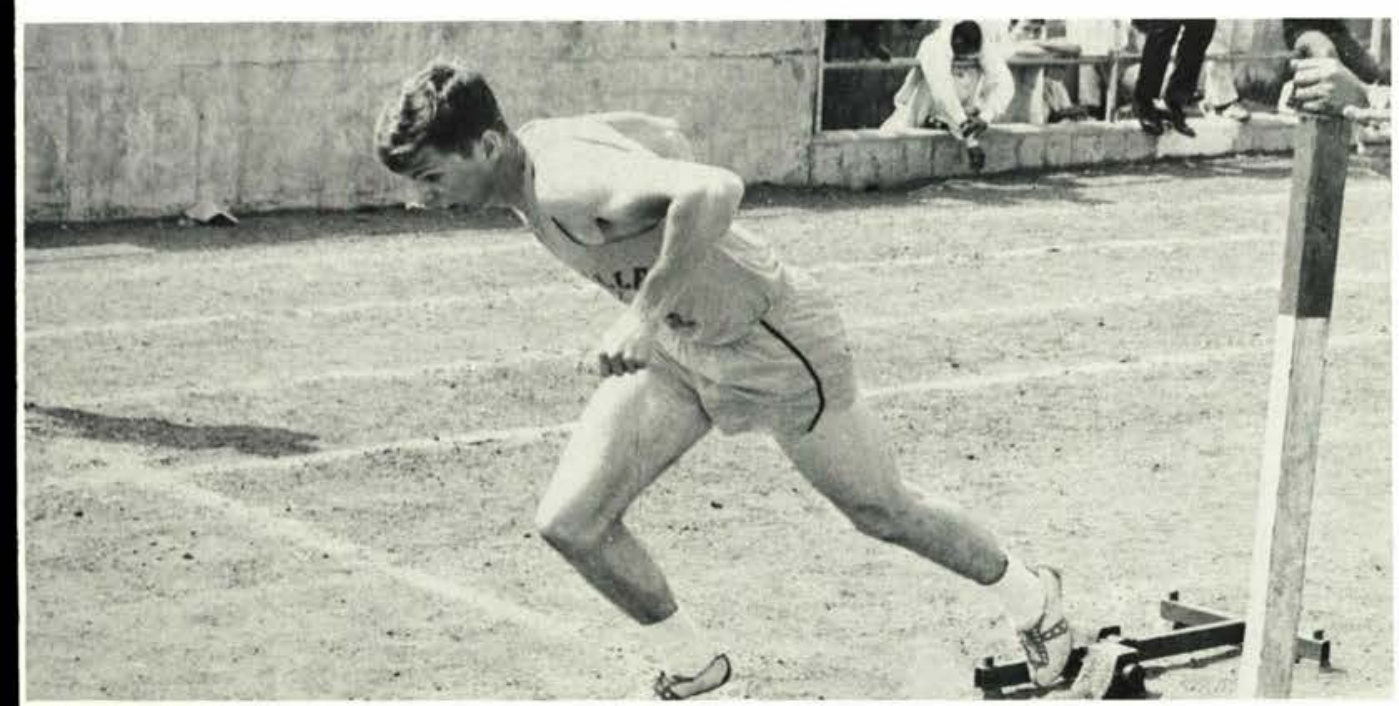

Bill Francis

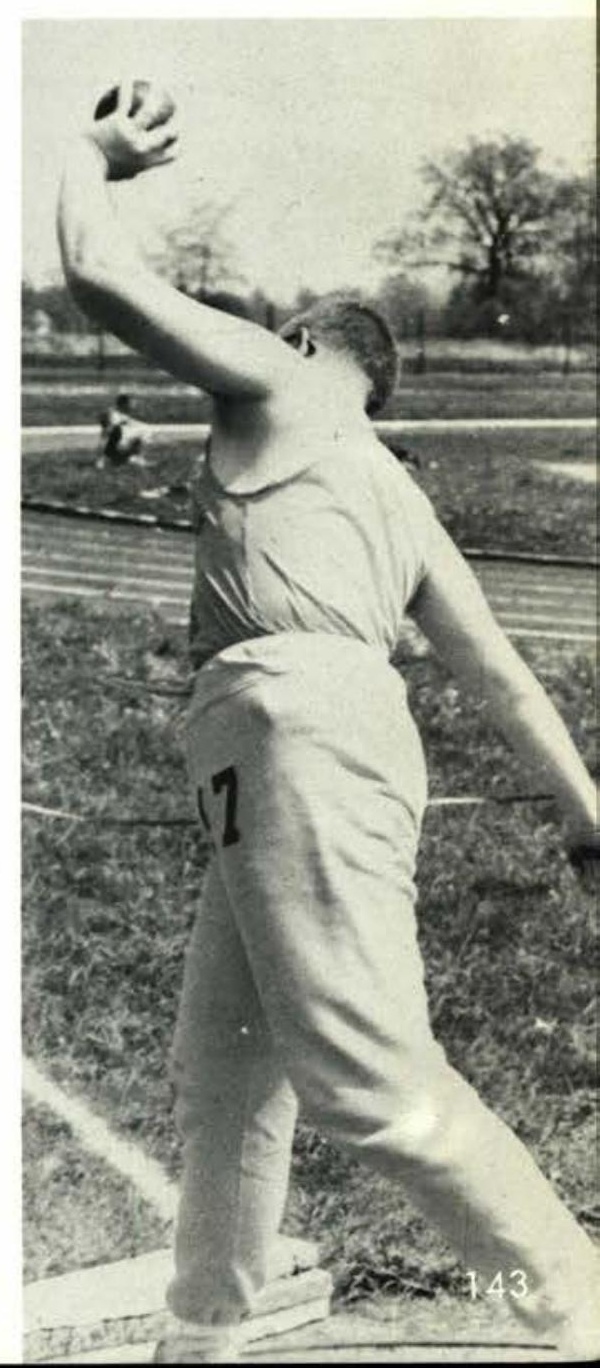




\section{Tennis}

The tennis team, 1-5 on the year, seemed to have

had a disappointing season. Although this year was a

rebuilding year, the team faired quite well, In the

league meet in May, the Jackets finished 4th in the

standings.

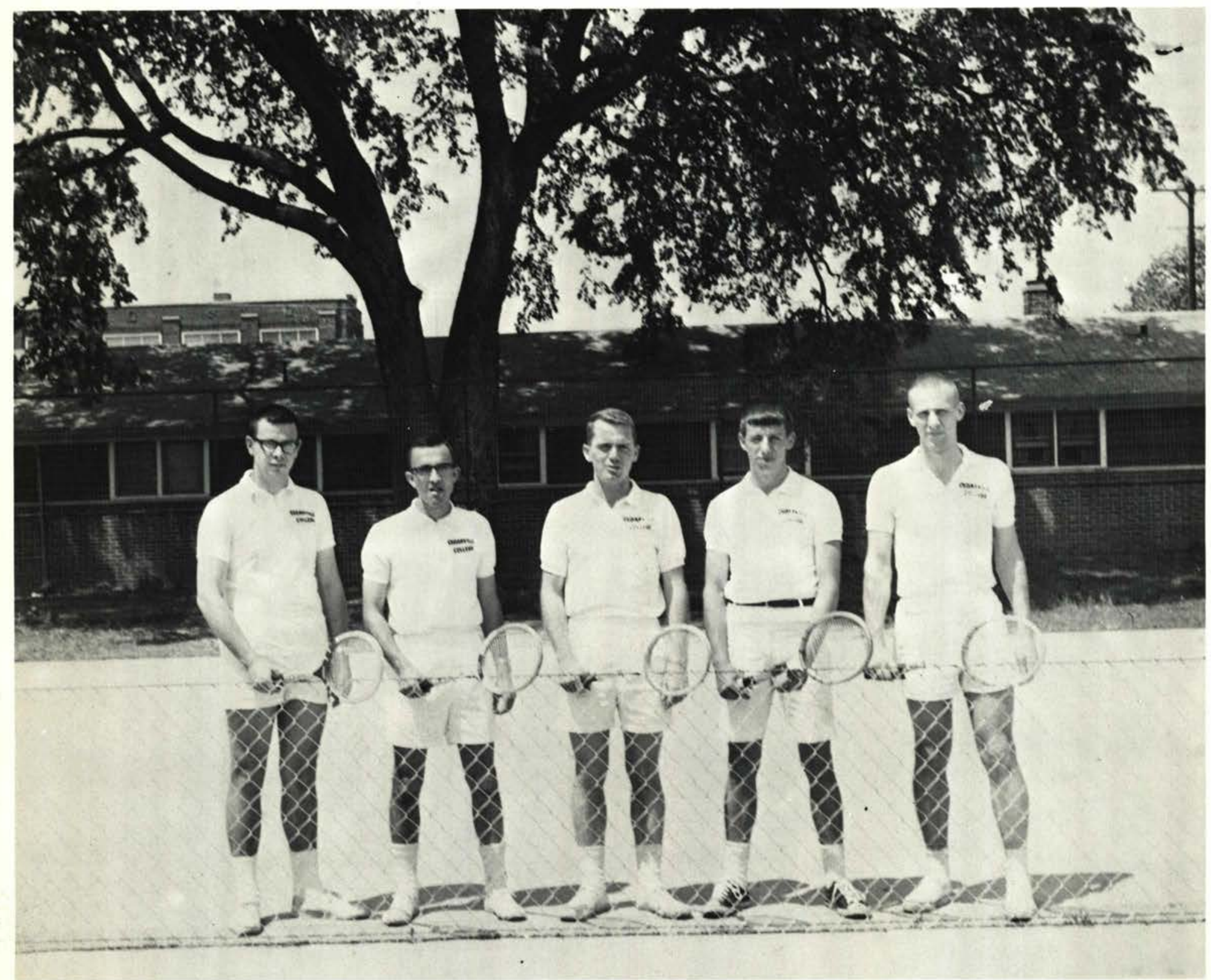

Left to Right: T. Oakley, M. Stephens, D. Bales, D. Pettit, D. Entner. 


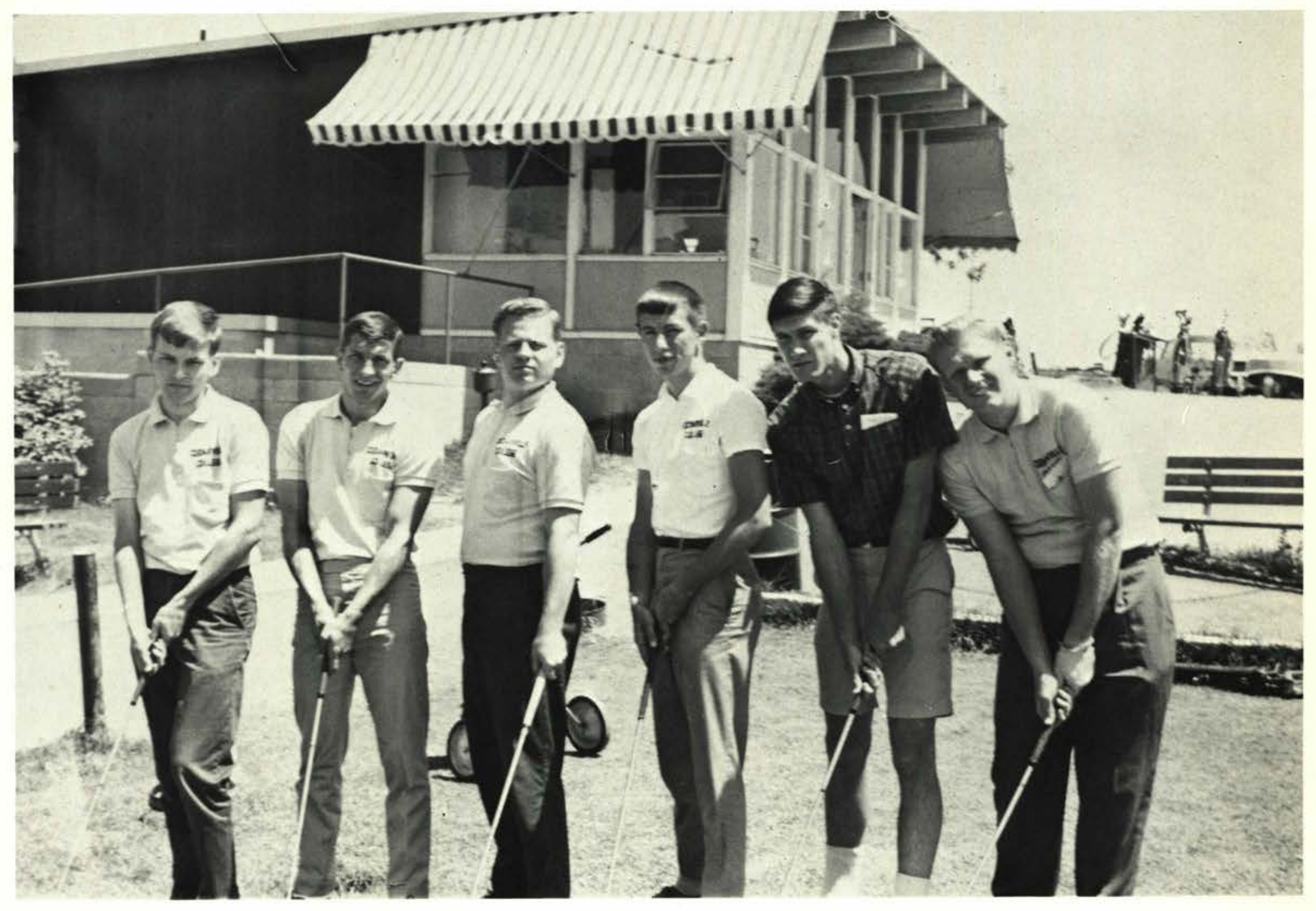

Left to Right: J. Hess, J. Engelmann, J. Miller, D. Pettit, J. Stadt, B. Kirtland.

\section{Golf}

The golf team this year did considerably better than in previous years, although victories were few.

With practically all returning lettermen, the scores should go down and the victories up this coming year.

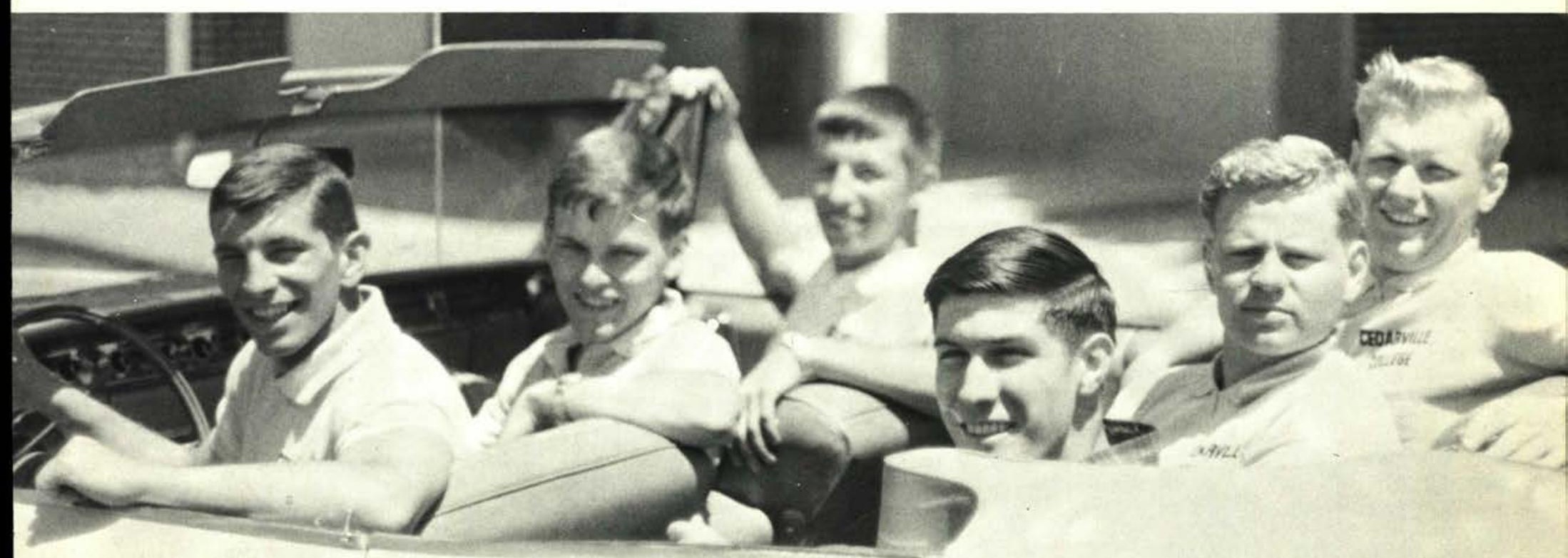




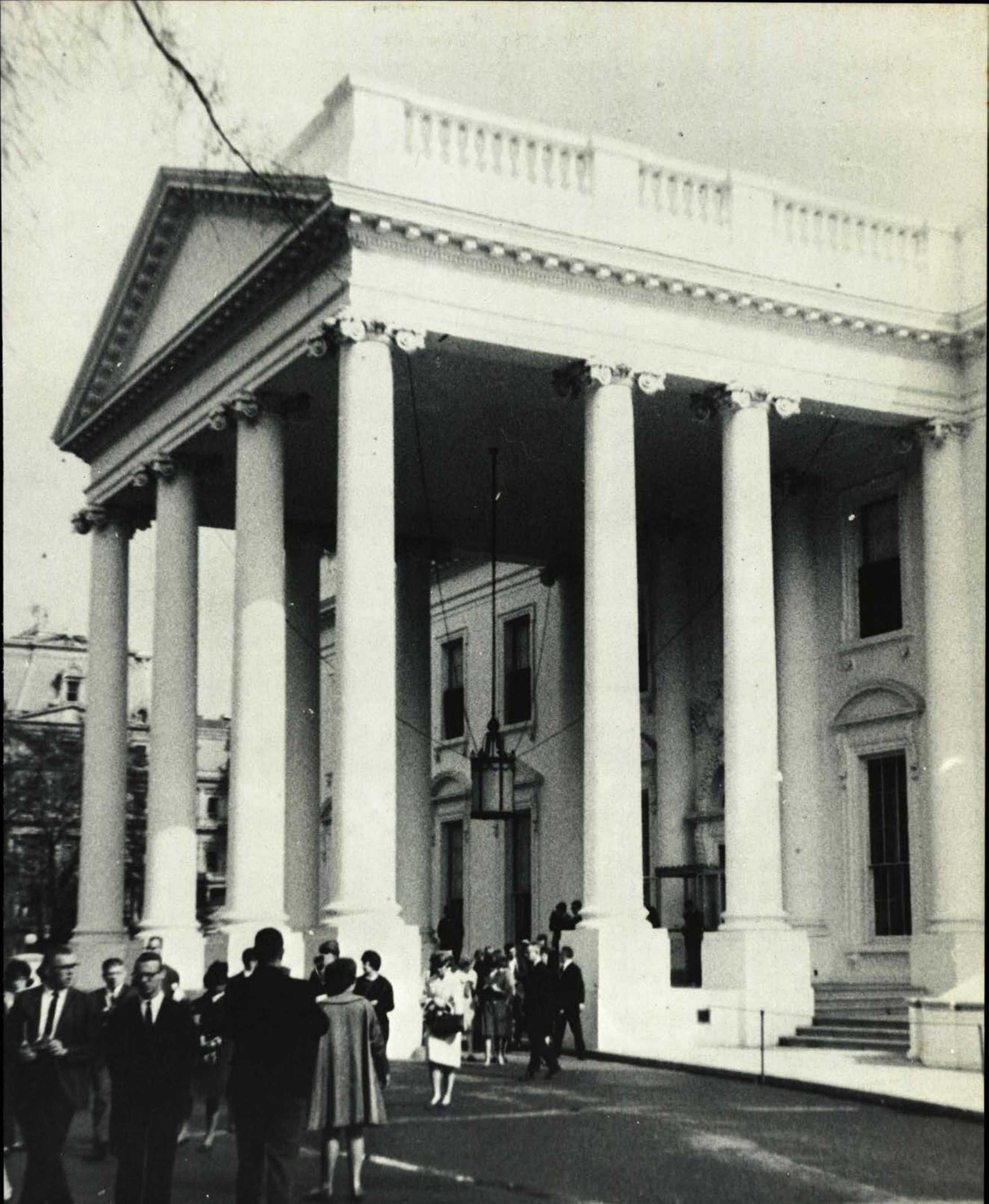

Wheyente 

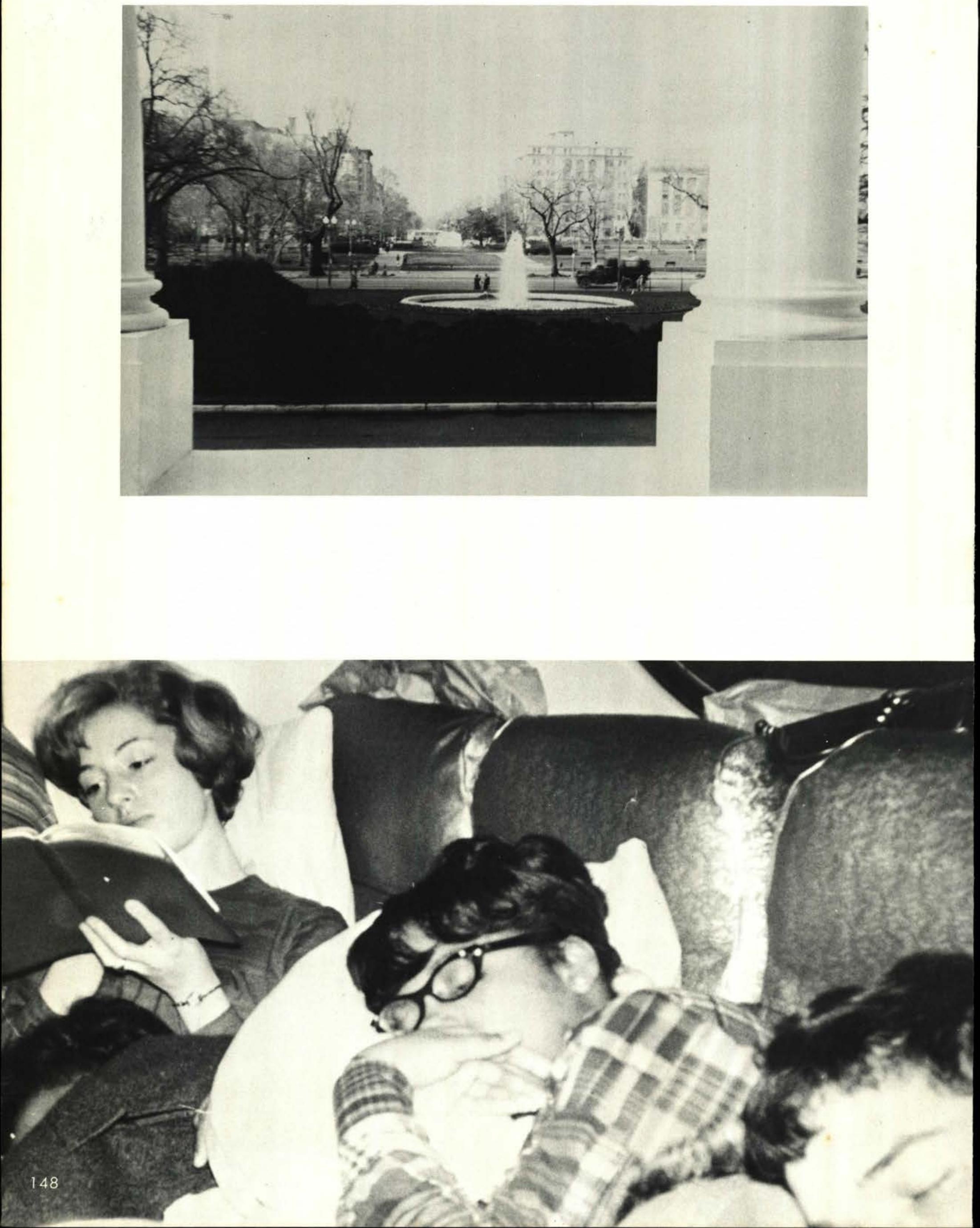


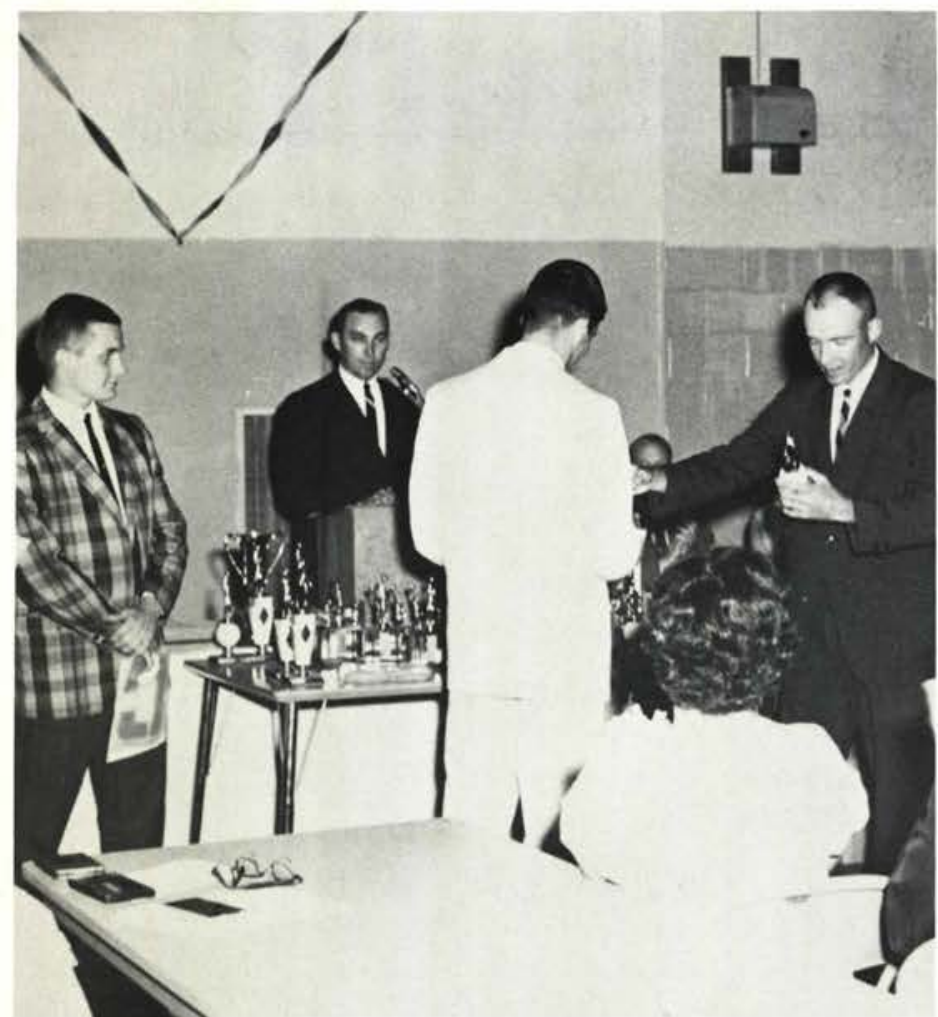

Randy Ross, Soccer M.V.P.

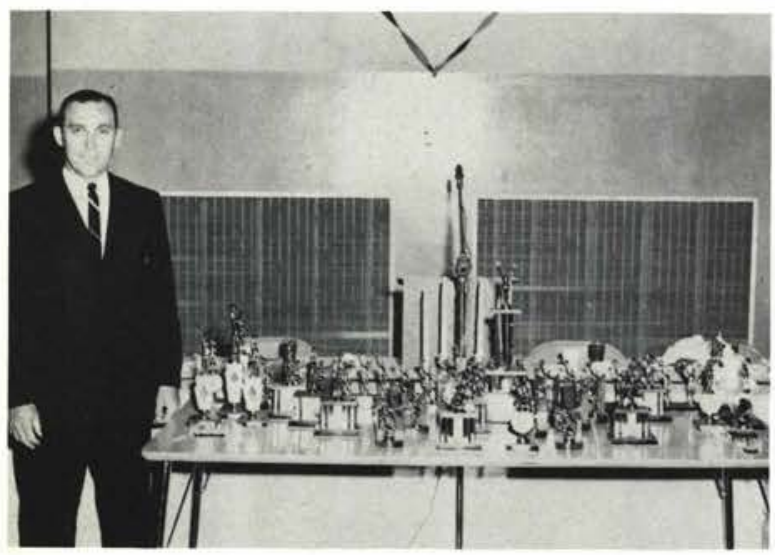

Mr. Callan, Athletic Director.

\section{Athletic Banquet}

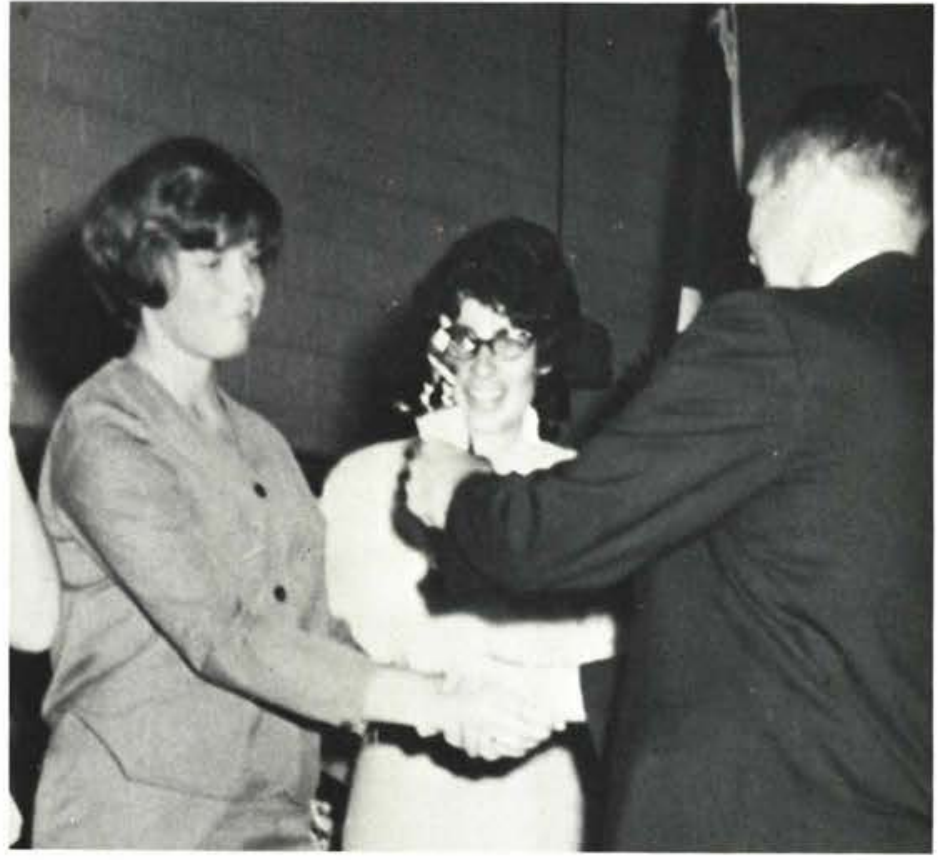

Sharon Wescott, Volleyball M.V.P.

The Mid-Ohio League basketball champions.

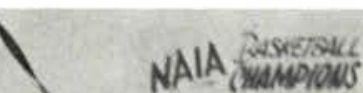

1904

(

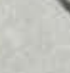

1
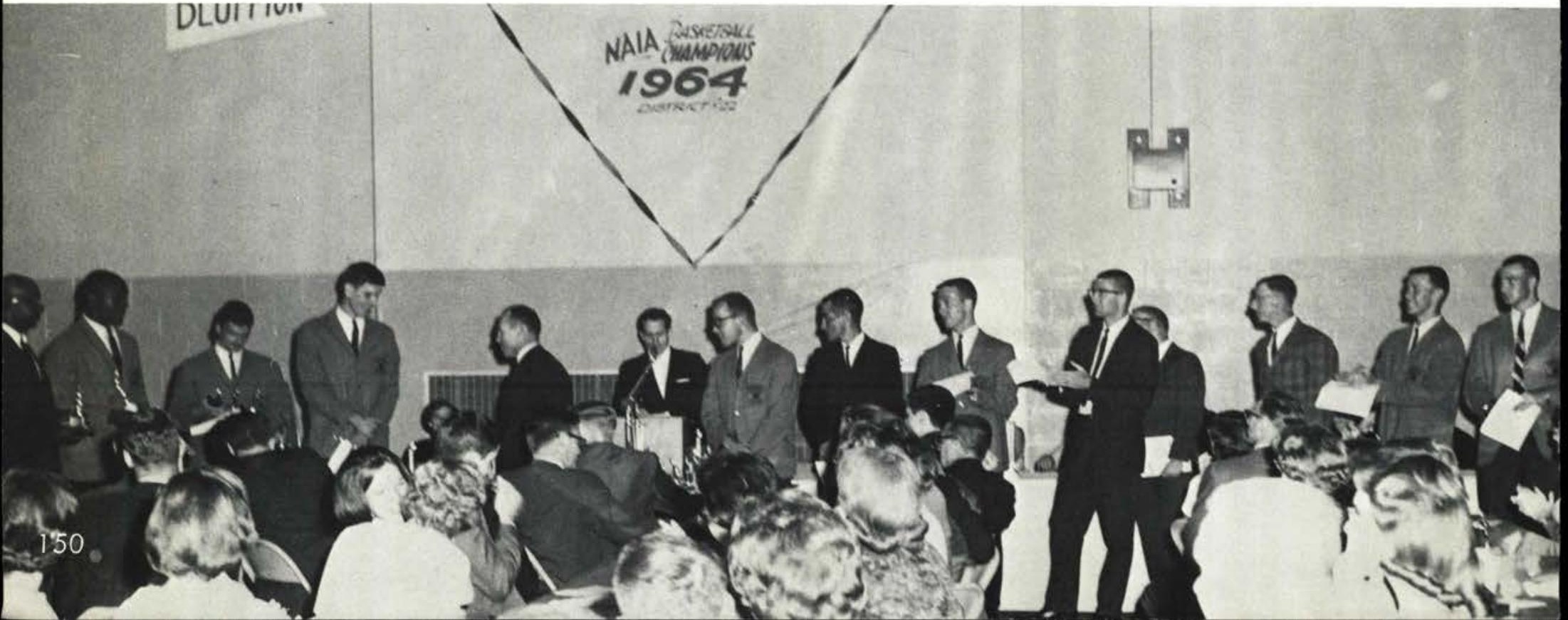


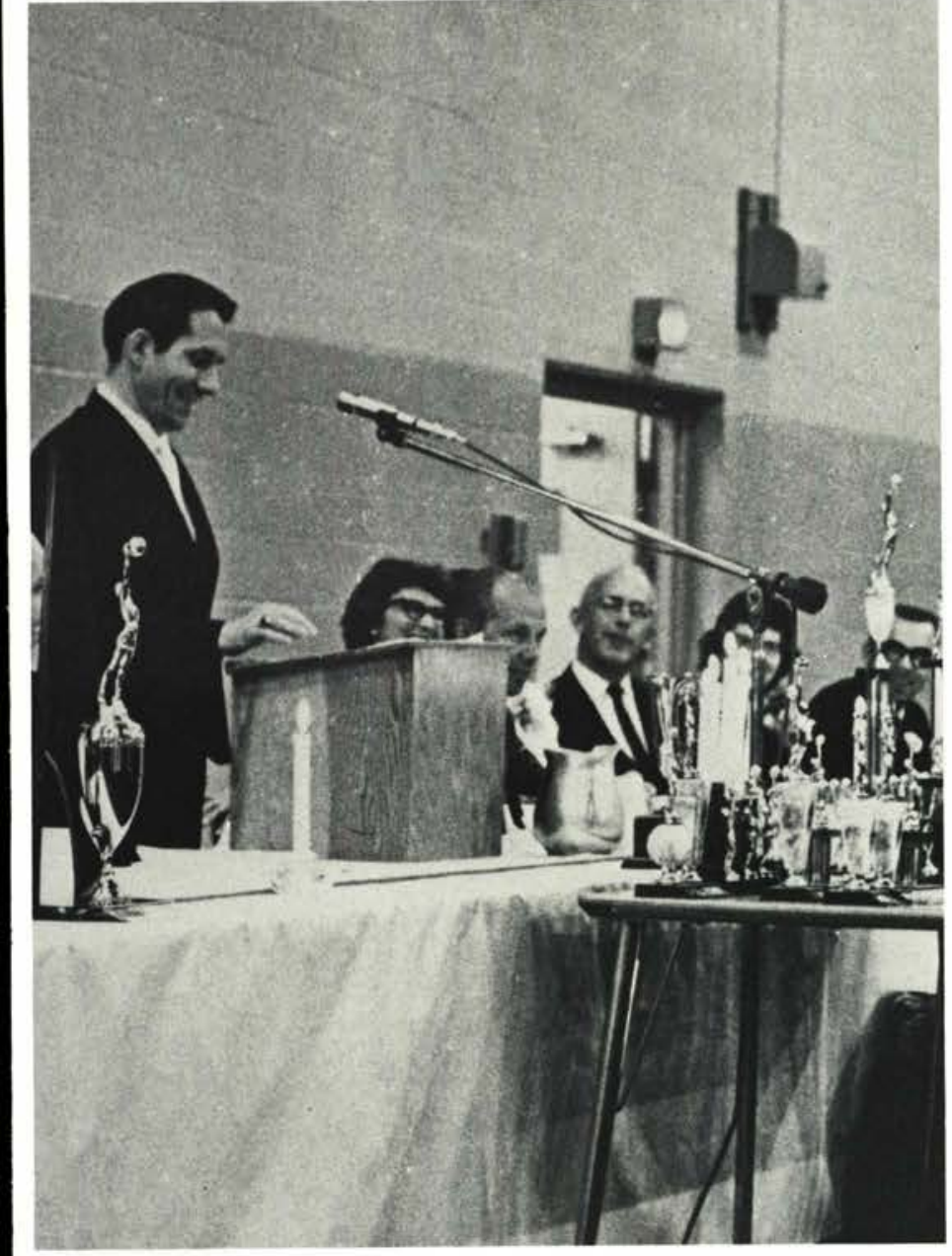

Mr. Turner, Publicity Director.

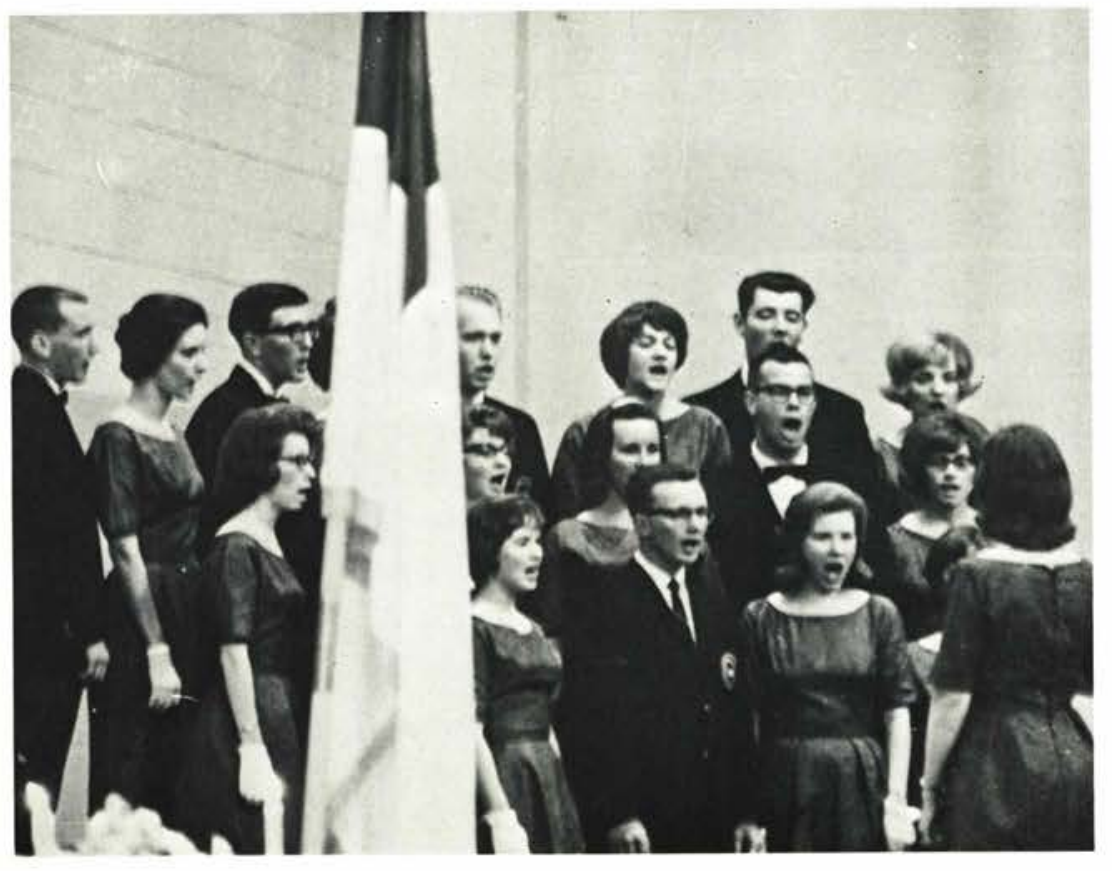

The Choralaires.

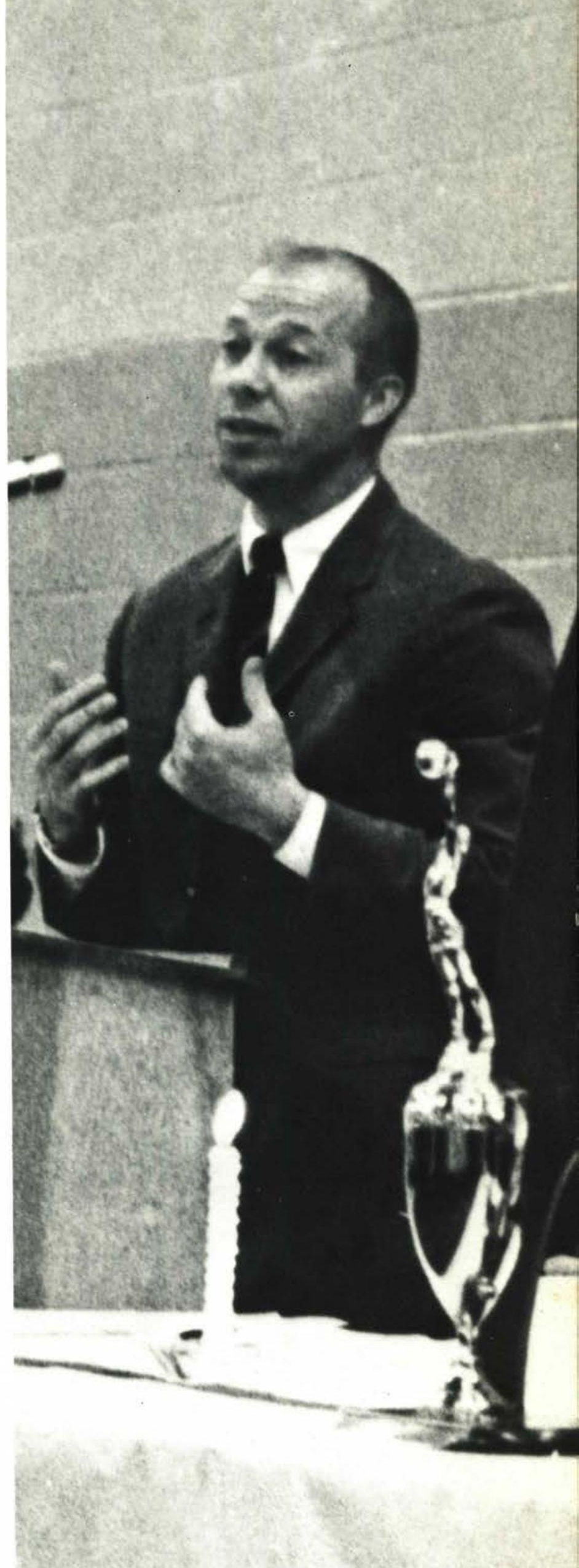

Bill Swain, Athletic Banquet speaker. 


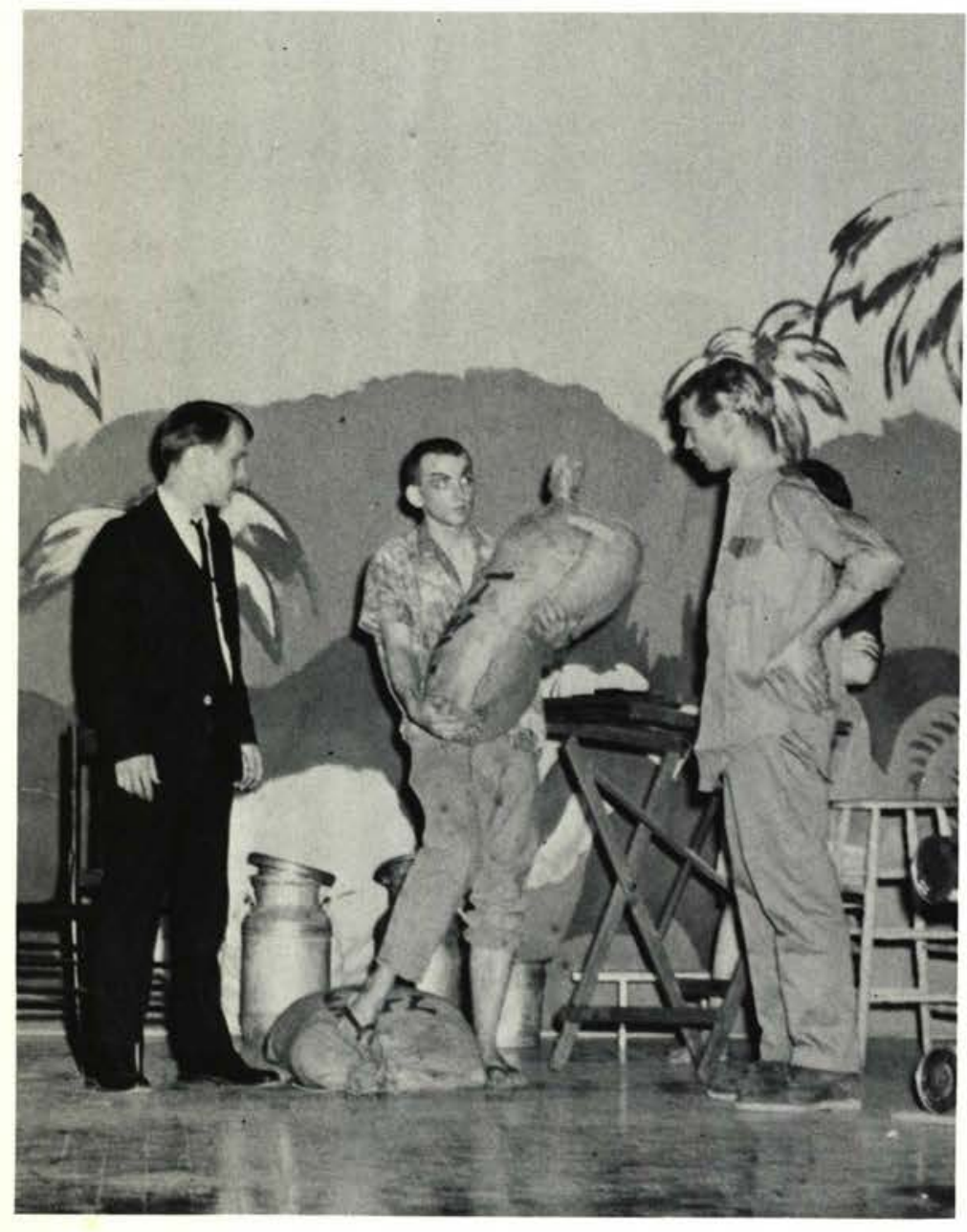

\section{THE UGLY AMERICAN}

On May 7 and 8, the Dramatics Department presented "The Ugly American" by Bernard Lubar, an adaptation of the novel by Lederer and Burdick. The play was under the direction of Mrs. Miriam Maddox.
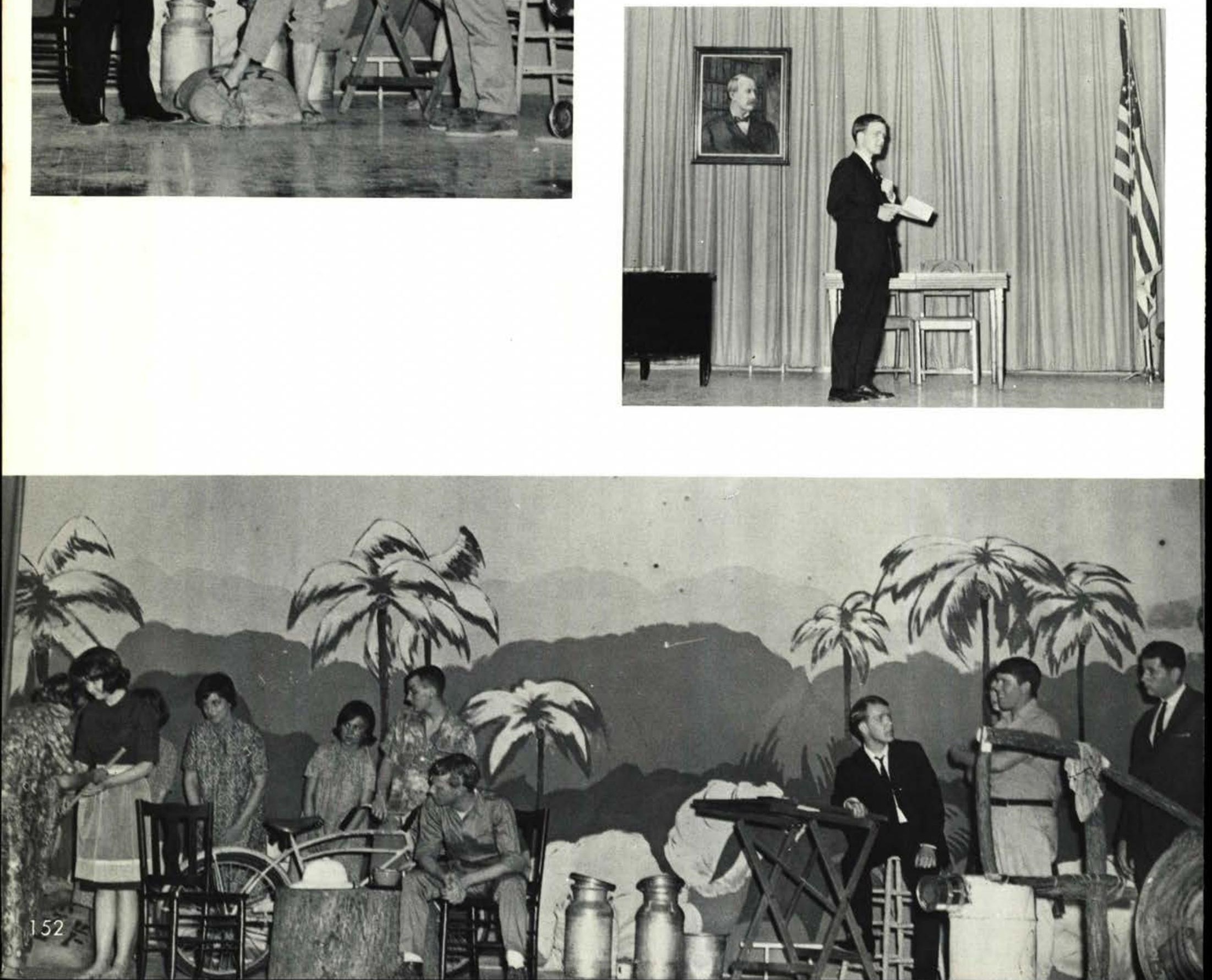


\section{Honors Day}

Honor students were recognized this year at a special chapel service on May 8th. Certificates were awarded at this time for academic achievements throughout the year. A luncheon was also held on Saturday afternoon to honor these students.

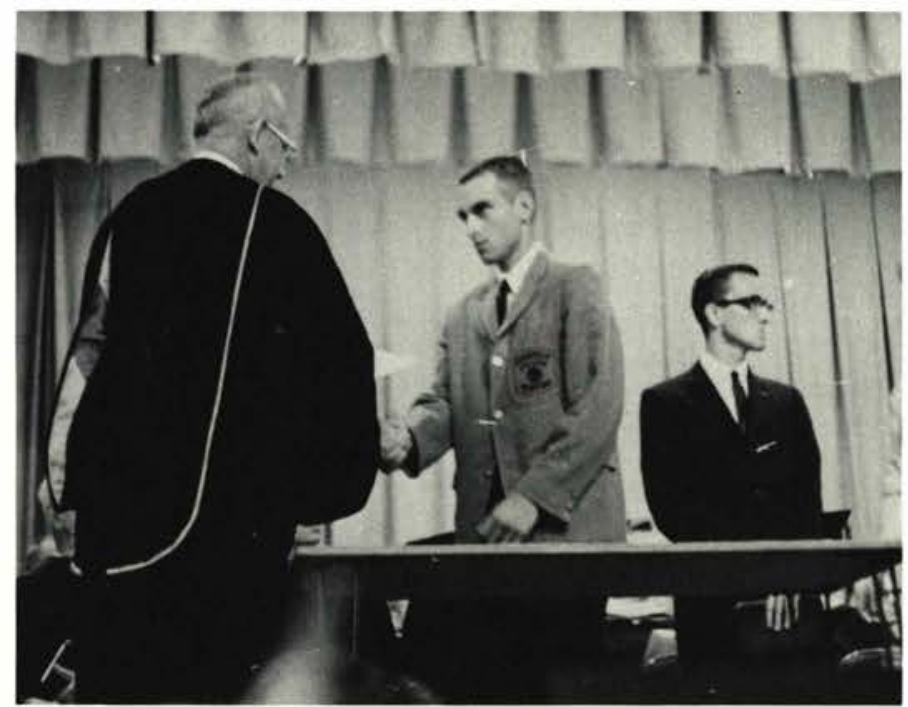

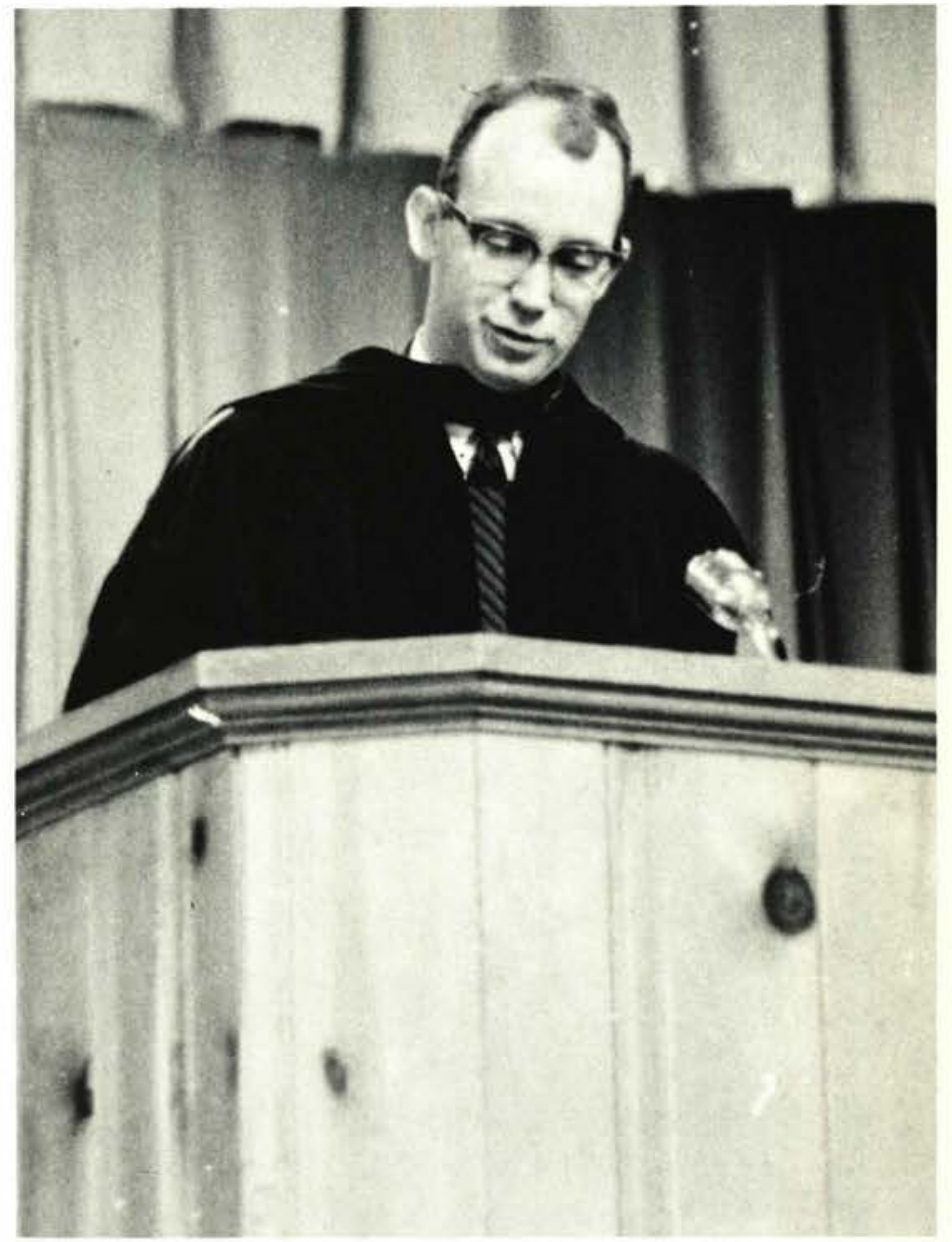

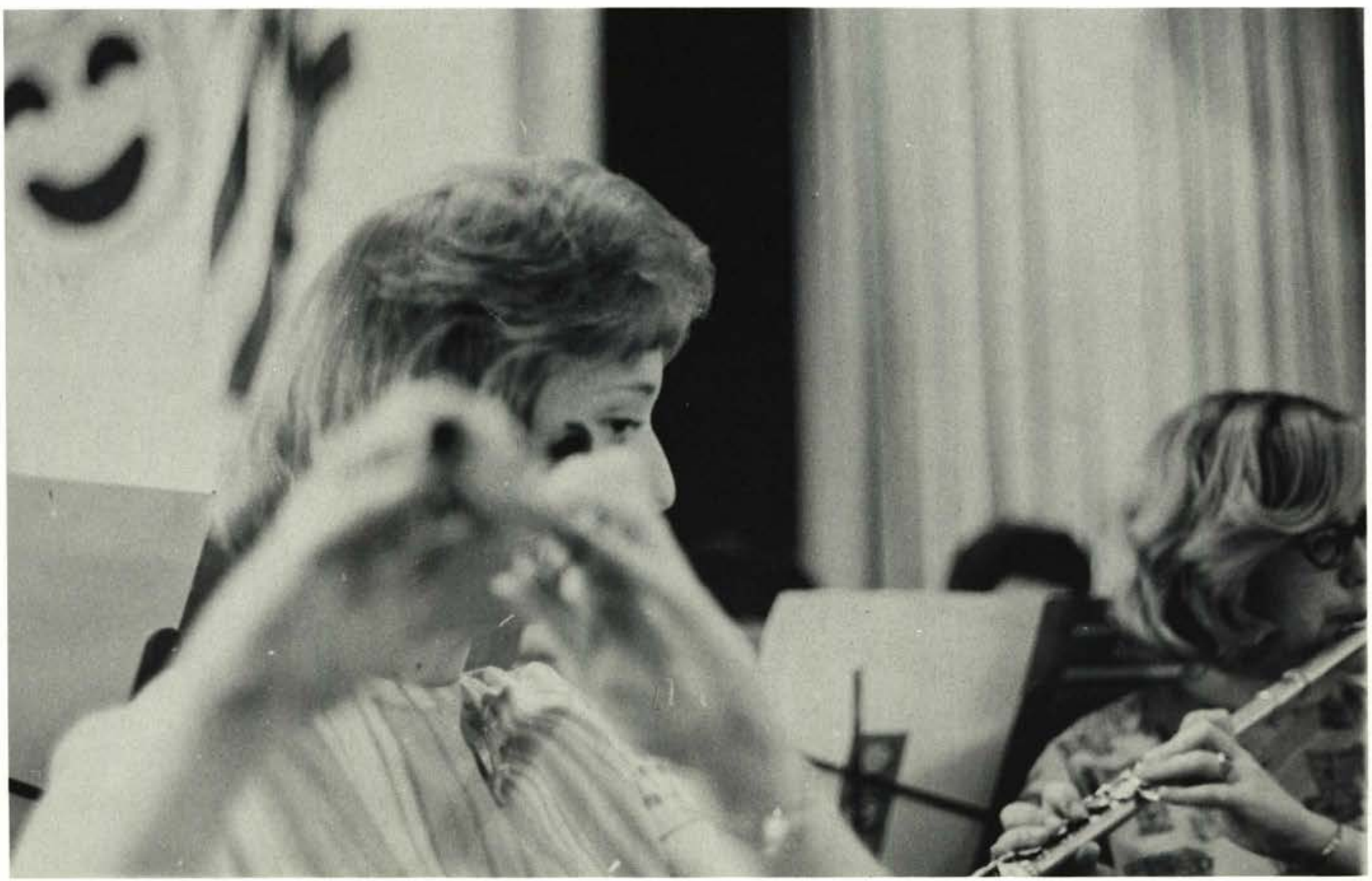



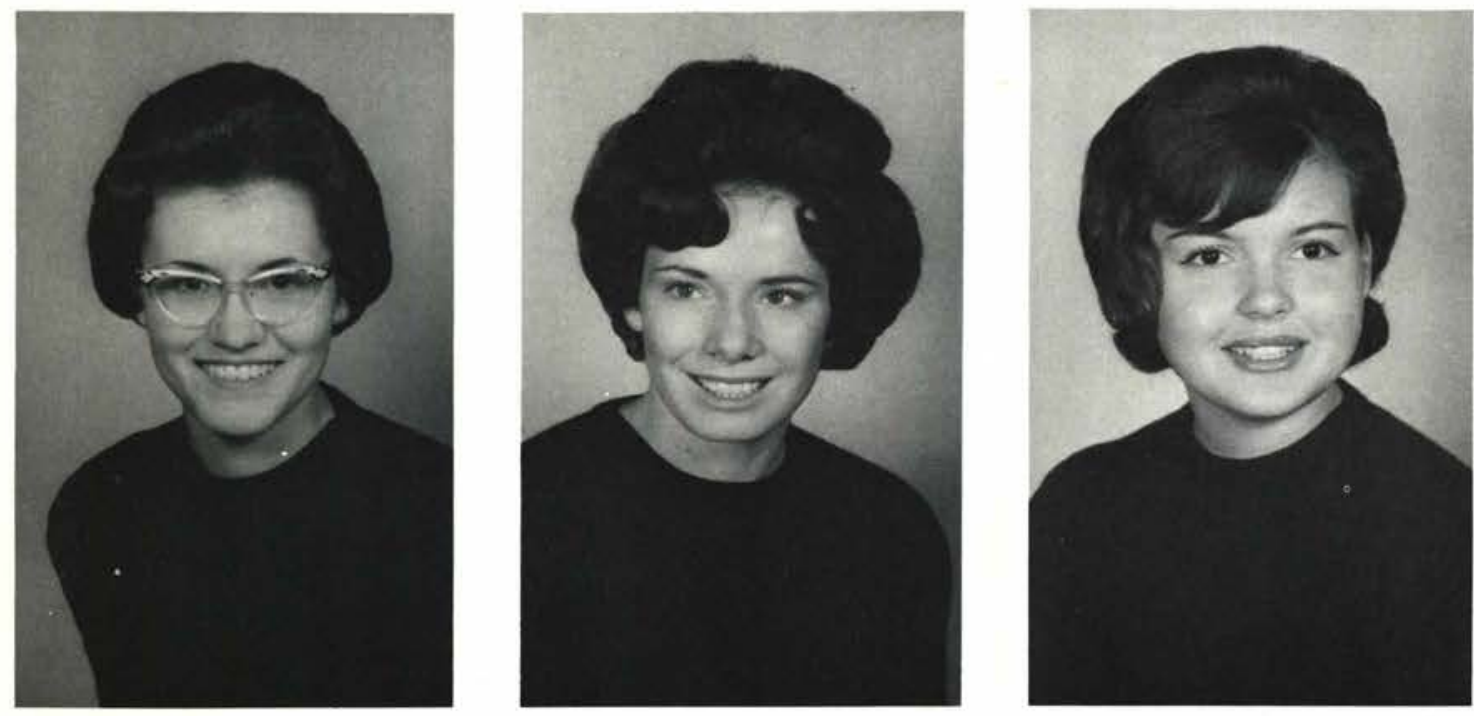

Sharon Ann Ackerman

Frankfort, Michigan

Major: Sociology-Degree B.A.

Janice Ann Addleman

Hillsdale, Michigan

Major: Christian Education-Degree B.A.

Kathleen Mae Alberter

Johnstown, Pennsylvania

Major: English-Degree B.A.

Martha Joy Ammons

Gardena, California

Major: English-Degree B.A.

Duane Russell Bales

Pana, Illinois

Major: Christian Education-Degree B.A.
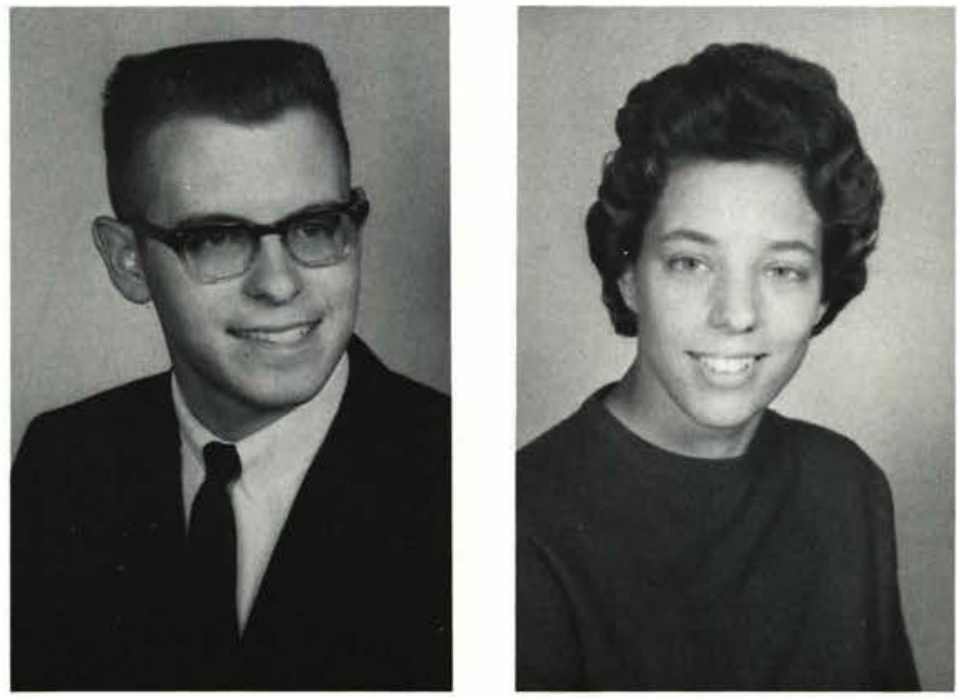

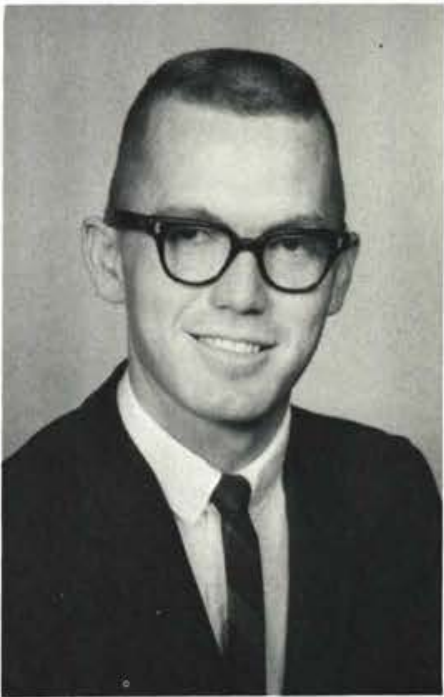

Suzanne Martha Bartheld

Valley Stream, New York

Major: Elementary Education-Degree B.A.

Dennis Alan Bellew

Cincinnati, Ohio

Major: Bible-Degree B.A.

Grace Gertrude Bonnema

Perry, Ohio

Major: Nursing-Degree B.S. 
Mary Jane Brong

Clear Lake, Iowa

Major: Sociology-Degree B.S

Paul S. Brower

Rockford, Michigan

Major: English-Degree B.A.

Clara Nancy Brown

Marathon, New York

Major: Nursing-Degree B.S.
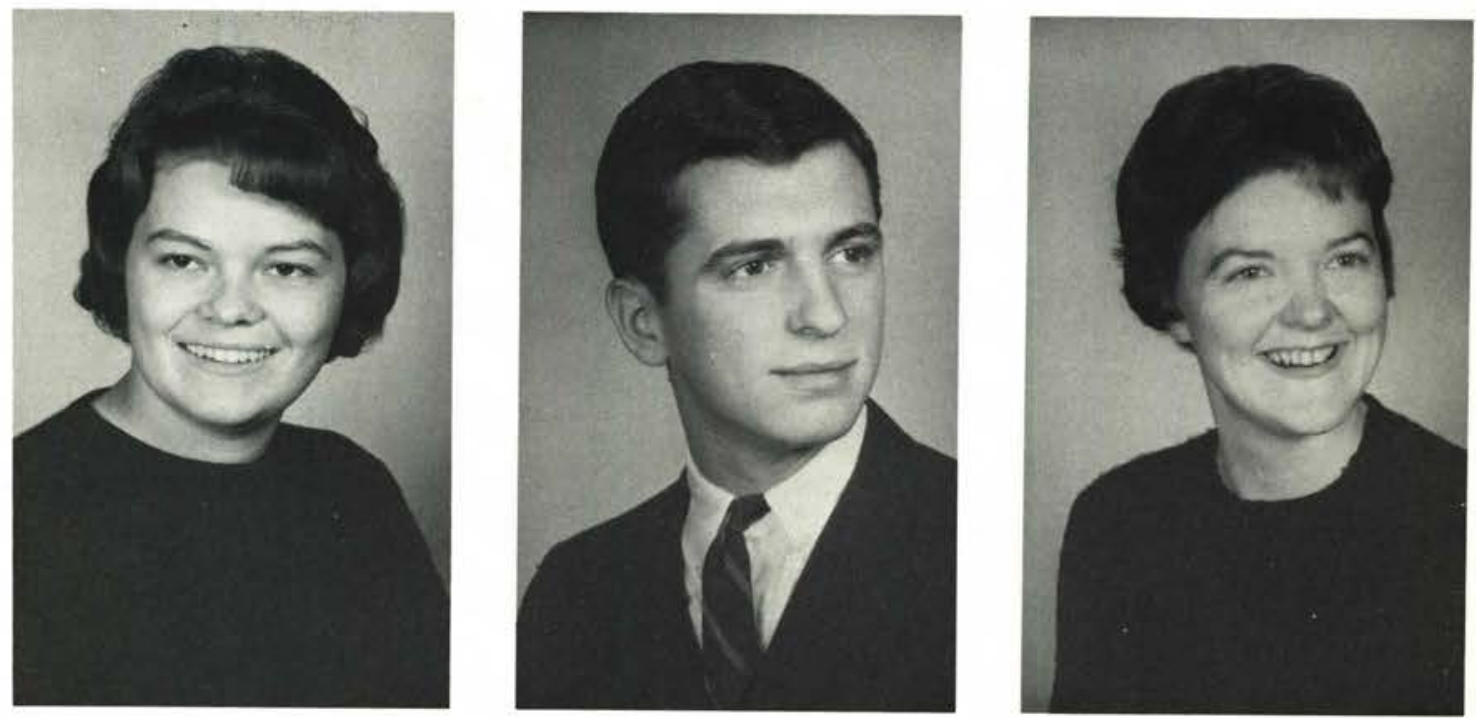

Ronald Gerald Butler Springfield, Ohio Major: Bible-Degree B.A.

Shirley Ann Byrd

Hammond, Indiana

Major: Music-Degree B.A.

Paul Gerald Carlson

Gary, Indiana

Major: Speech-Degree B.A.
Nancy Grace Buerer

Cedarville, Ohio

Major: Biology-Degree B.A.

Rayna Kay Butler

Corning, New York

Major: Elementary Education-Degree B.A.
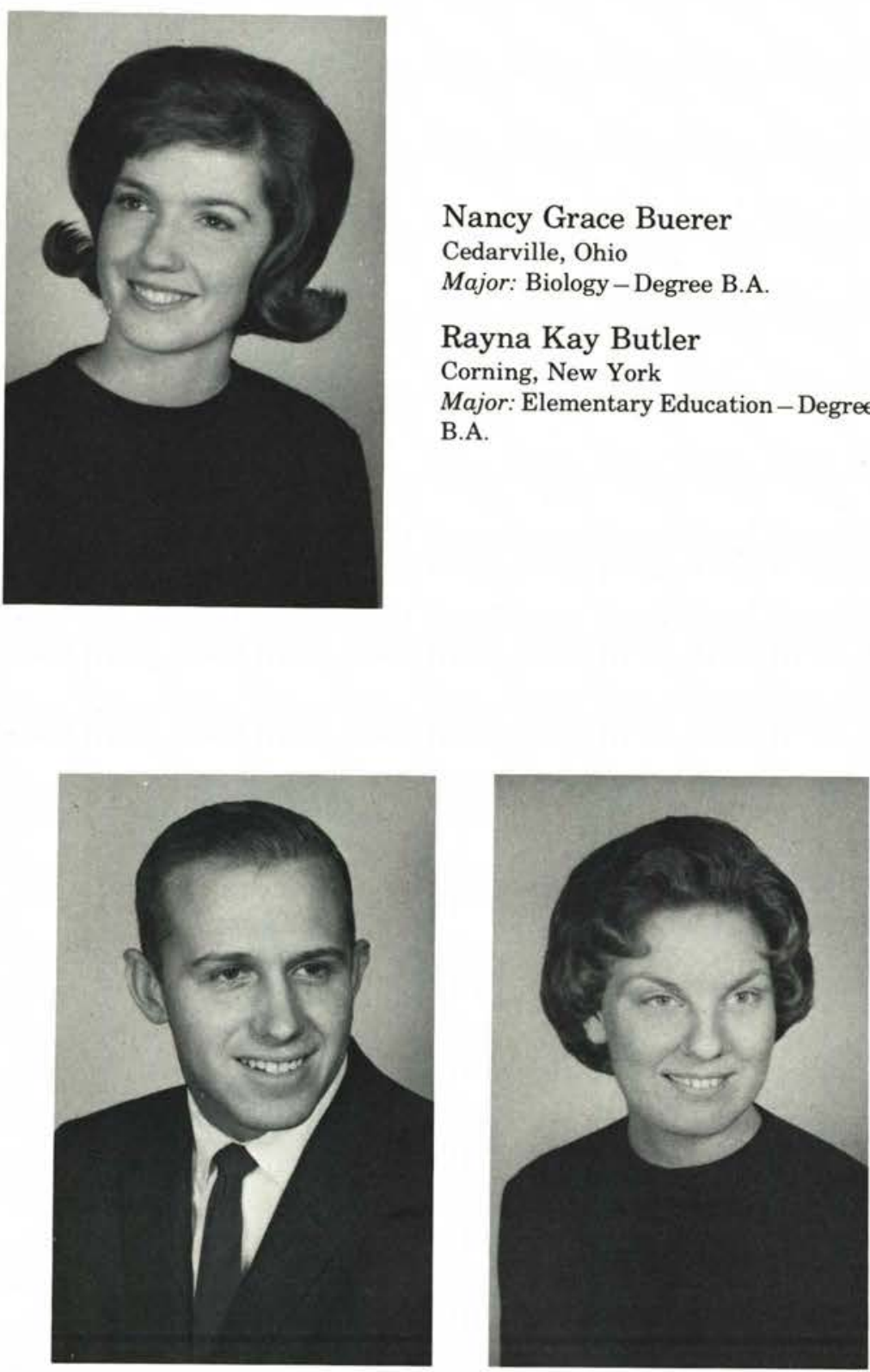

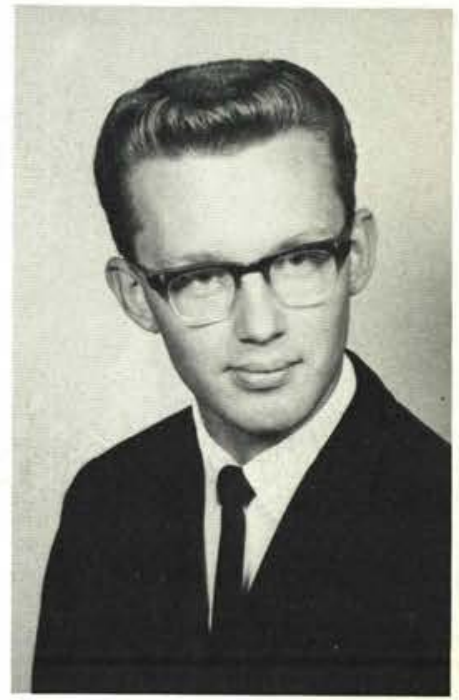



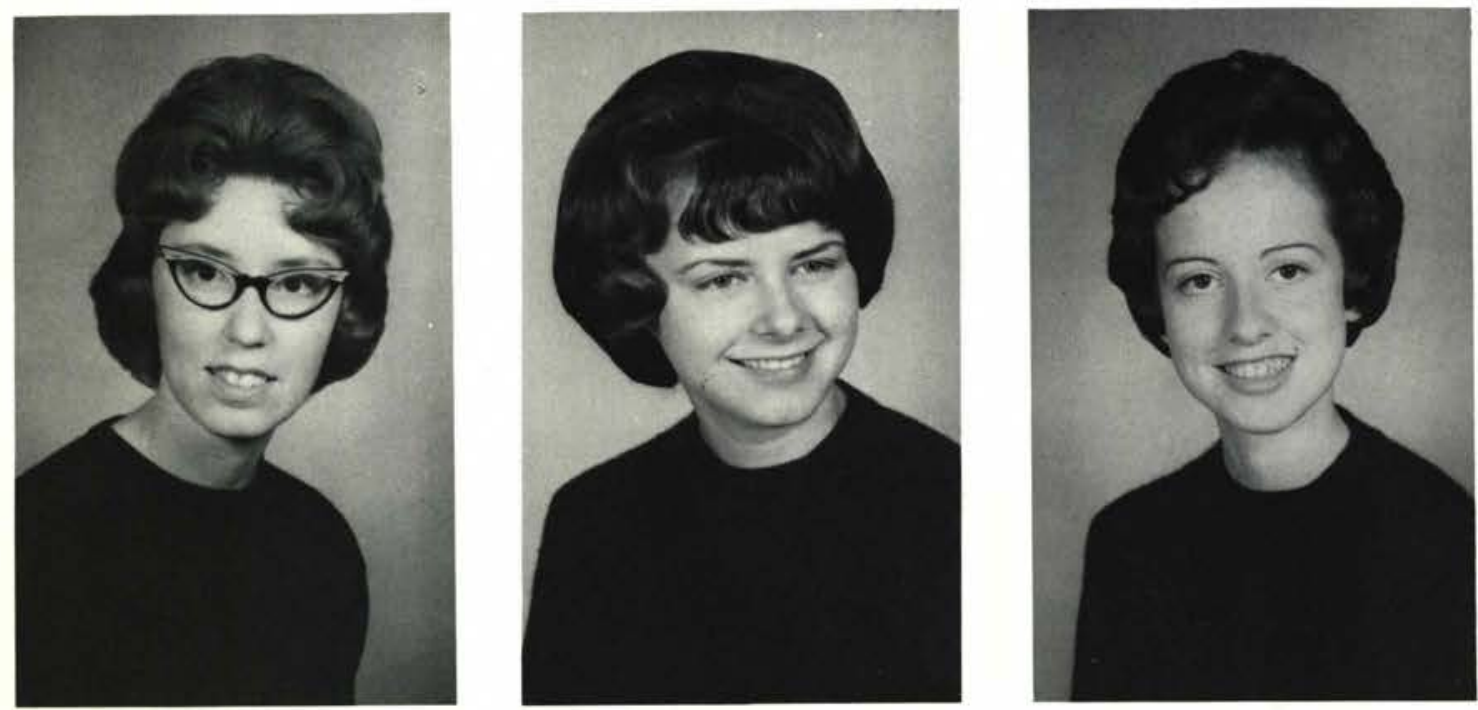

Joan Darlene Carter

Sinclairville, New York

Major: English-Degree B.A.

Ardith R. Cayton

Milwaukee, Wisconsin

Major: English-Degree B.A.

\section{Lynn Cayton}

Shorewood, Wisconsin

Major: Elementary Education-Degree

B.A.

Marcus Combs

Dayton, Ohio

Major: Social Studies-Degree B.S.

Carolyn Sue Coppock

Xenia, Ohio

Major: English-Degree B.A
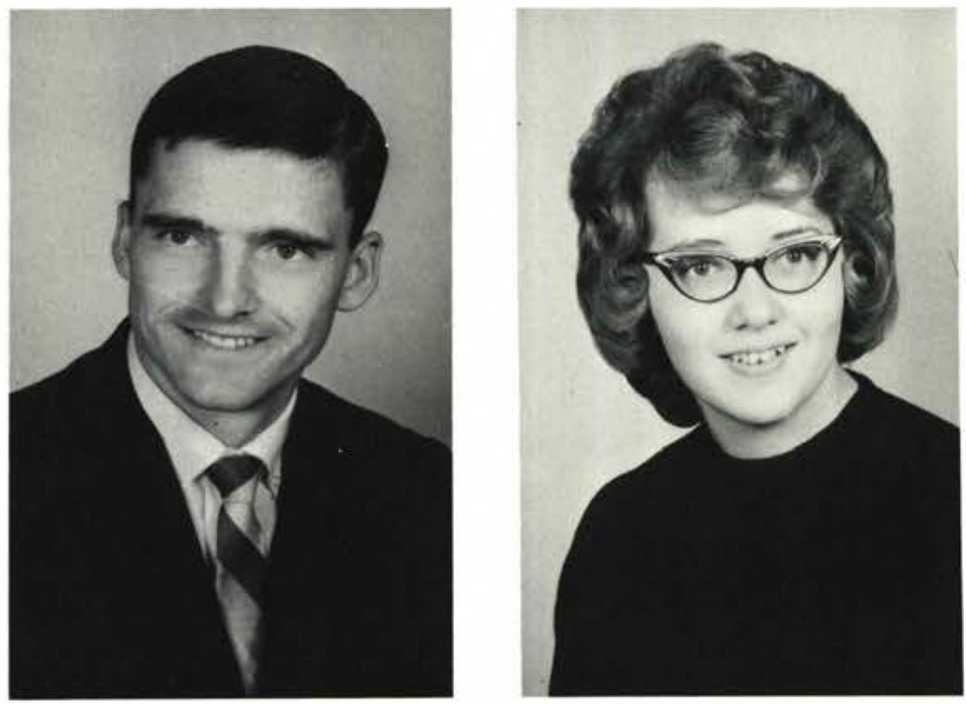

Mary Martha Cunningham

Bradenton, Florida

Major: Elementary Education-Degree

B.A.

Victoria Lee Curcio

Portsmouth, Ohio

Major: Music-Degree B.A.

Merry Jessica Davey

Bothell, Washington

Major: English-Degree B.A. 
Janice Carol Davis

Mentone, Indiana

Major: Elementary Education-Degree B.A.

Ray A. Davis

Sherman, New York

Major: Physical Education - Degree B.S.

Howard Lawrence Day

Portsmouth, Ohio

Major: History - Degree B.S
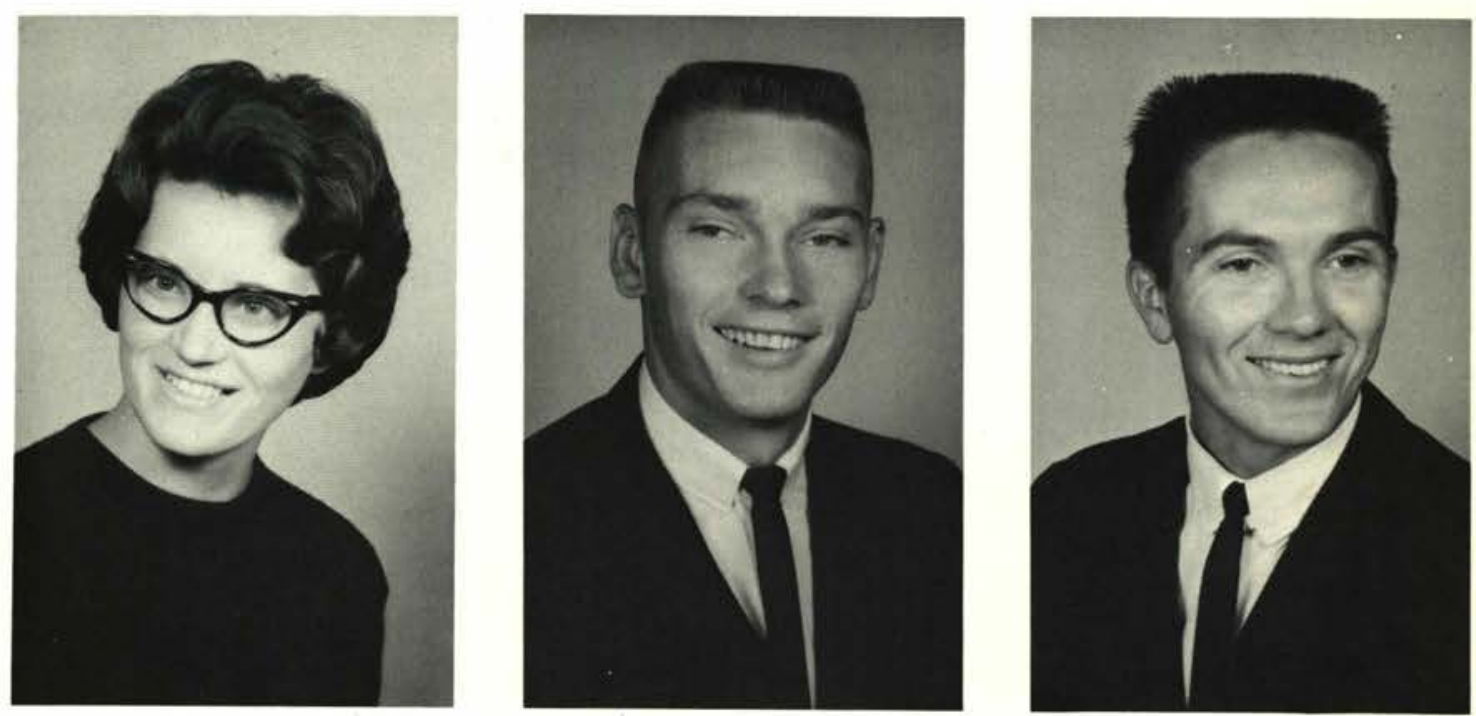

John William Donaldson

Rochelle, Illinois

Major: Social Studies-Degree B.S.

Jerry M. Eaton

Rochester, Indiana

Major: Elementary Education-Degree

B.A.

\section{Susanne Louise Eckert} Berea, Ohio

Major: Christian Education-Degree B.A.

\section{David Edward Elmore}

Cedarville, Ohio

Major: Bible-Degree B.A.

\section{Donald Entner}

\section{Dunkirk, Indiana}

Major: Physical Science-Degree B.S
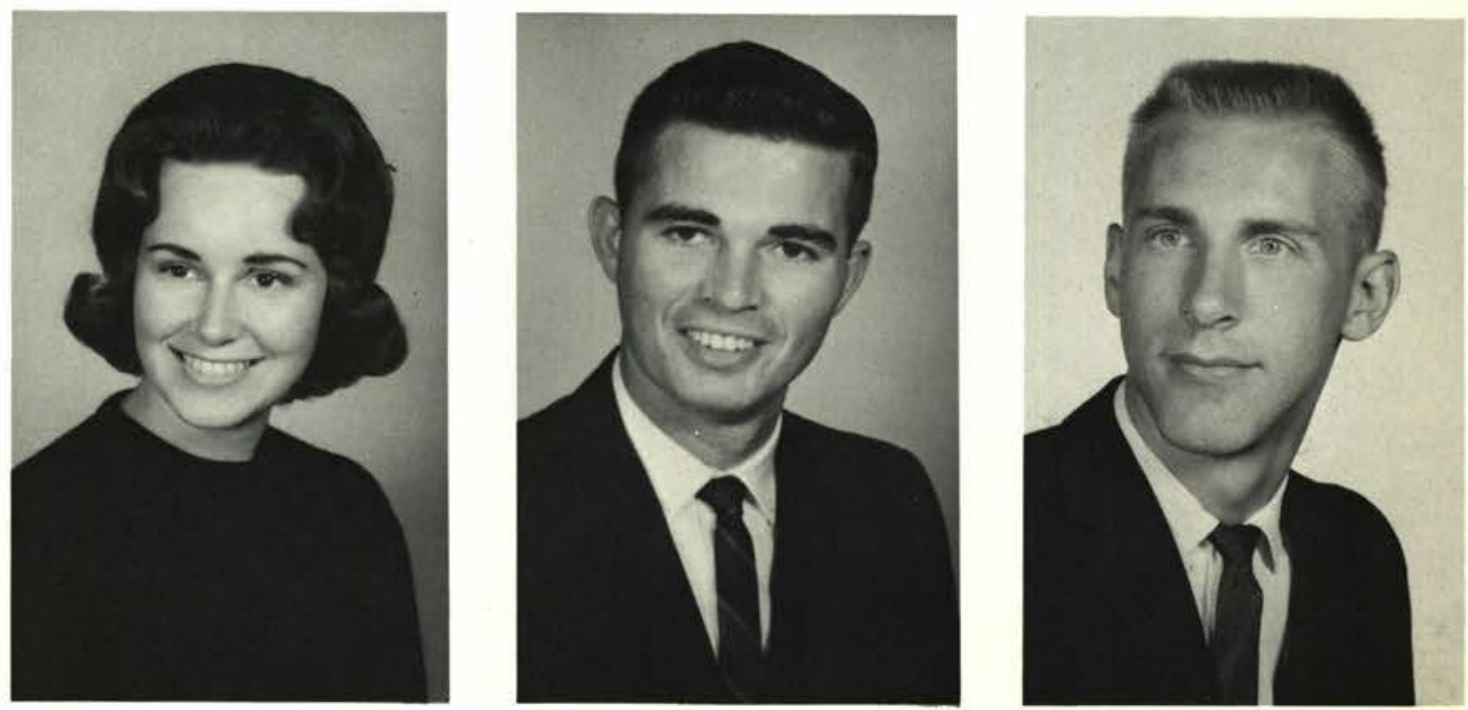

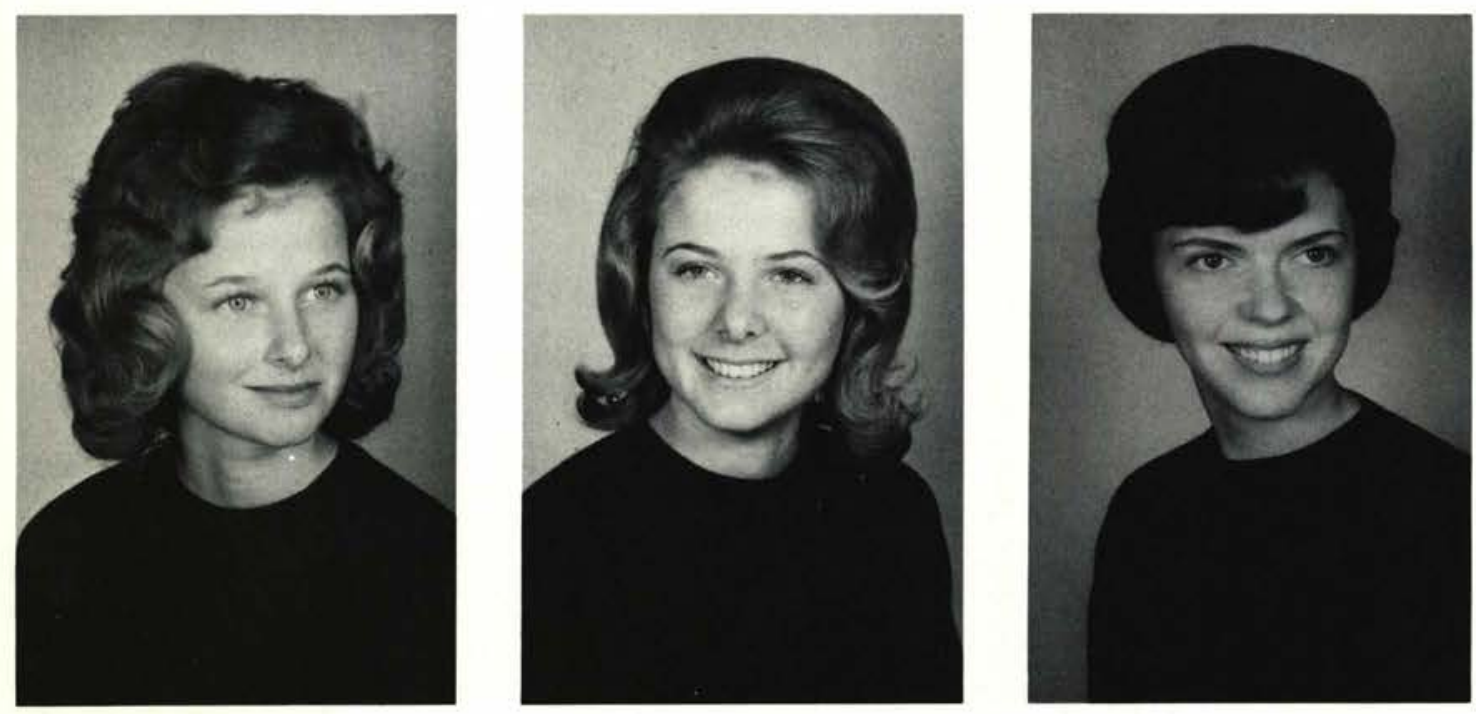

Dawne Elaine Evans

New Carlisle, Ohio

Major: Secretarial Science-Degree B.S.

Diane Elise Evans

New Carlisle, Ohio

Major: English-Degree B.A.

Suzanne Kay Fennell

Sturgis, Michigan

Major: Elementary Education-Degree B.A.

Gordon Lloyde Finley

Faribault, Minnesota

Major: Bible-Degree B.A.

Mary Ellen Floyd

Galion, Ohio

Major: Secretarial Science-Degree B.S.
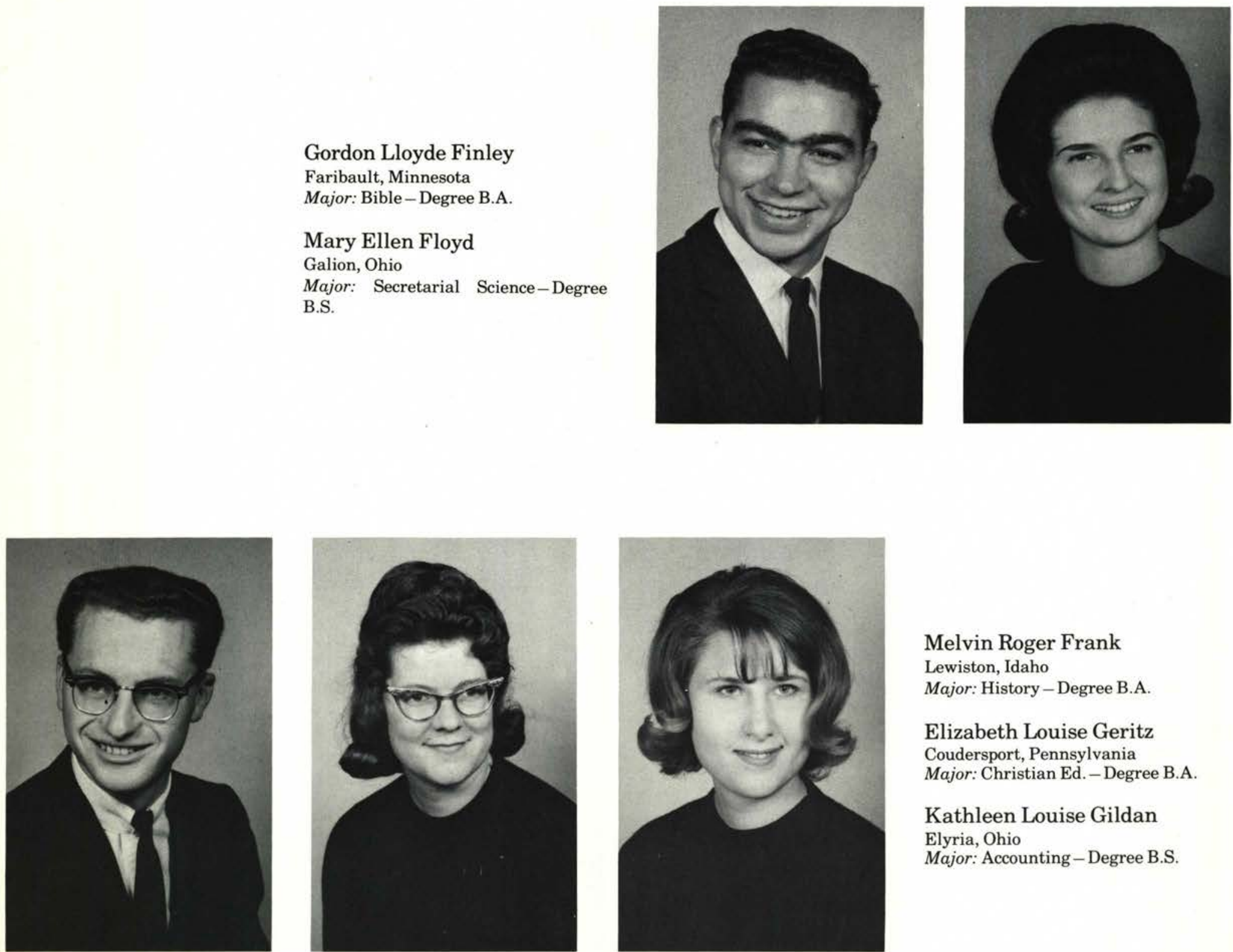

Melvin Roger Frank

Lewiston, Idaho

Major: History-Degree B.A.

Elizabeth Louise Geritz

Coudersport, Pennsylvania

Major: Christian Ed. - Degree B.A.

Kathleen Louise Gildan

Elyria, Ohio

Major: Accounting-Degree B.S. 
Joanne Louise Goss

Bay City, Michigan

Major: English-Degree B.A.

Boyd Kenneth Griffith

Creston, Iowa

Major: Music-Degree B.A.

\section{Faye Haas}

Randolph, New York

Major: Music-Degree B.S.
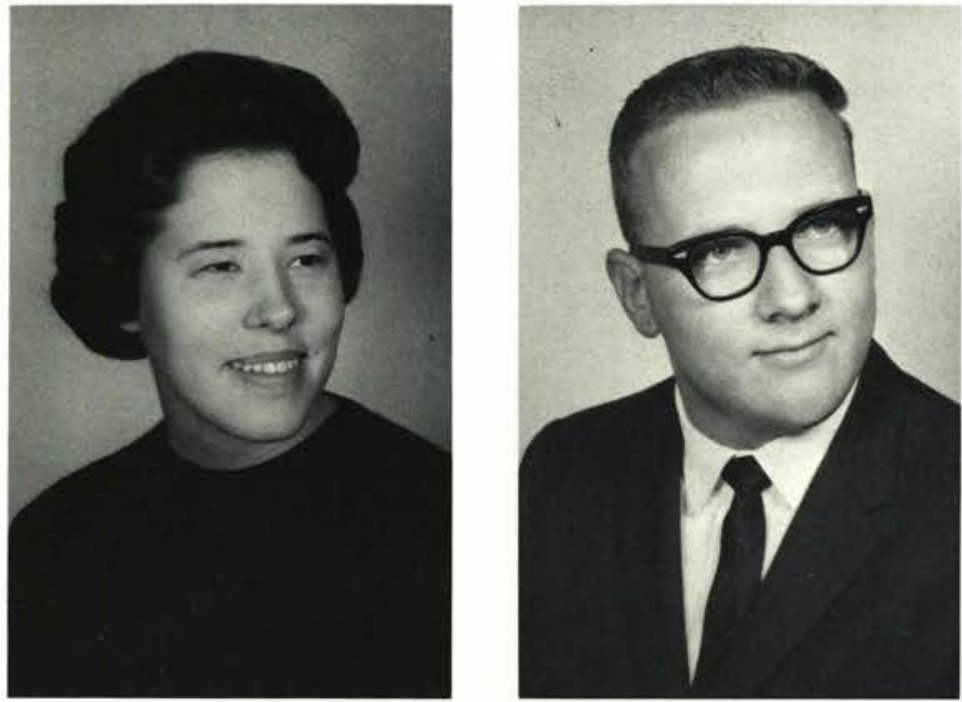

Joanne Helen Hamilton

Alton, Illinois

Major: Elementary Education-Degree B.A.

\section{Judith Ann Hartzell}

Butler, Pennsylvania

Major: Elementary Education-De-

gree B.A.

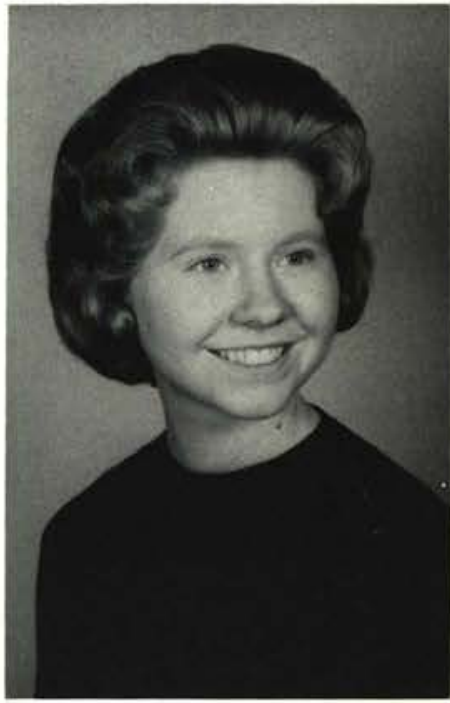

\section{Faith Hocking}

Hazel Park, Michigan

Major: Social Science-Degree B.A.

Raymond Eugene Hoffeditz Linton, Indiana

Major: Bible-Degree B.A.

David Richard Jensen

Wellington, Ohio

Major: Speech-Degree B.A

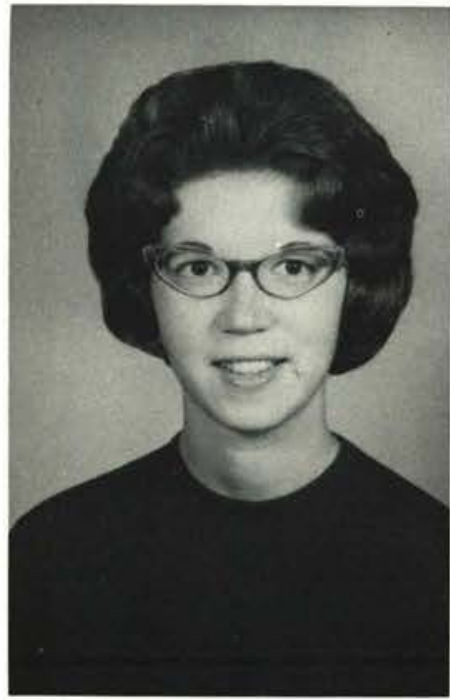

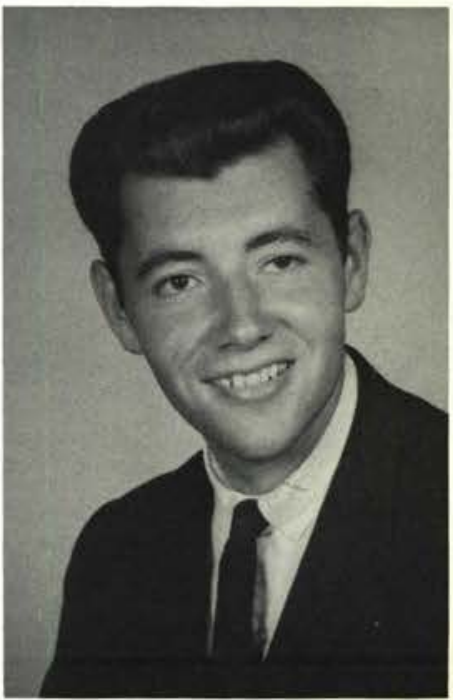



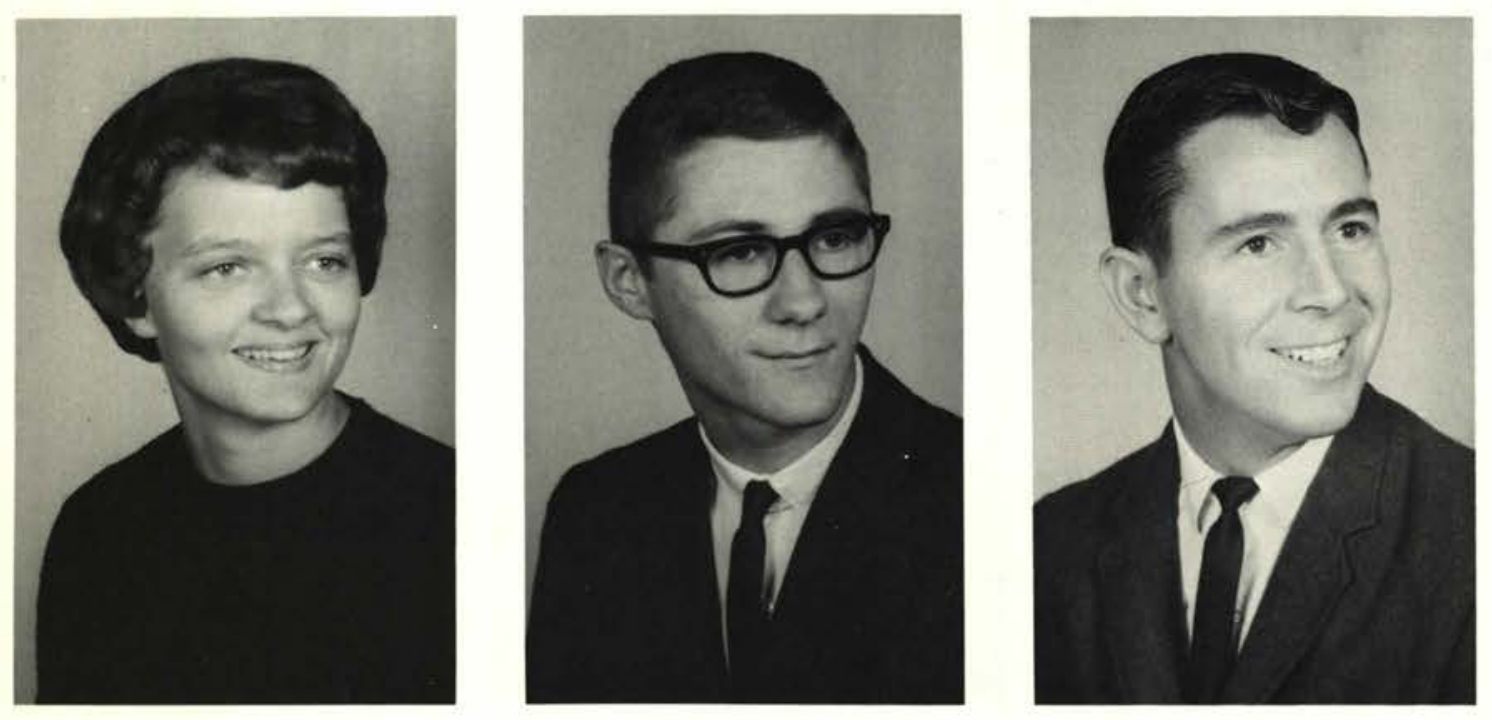

Maryalyce Jeremiah

Cedarville, Ohio

Major: Physical Education-Degree B.A.

Paul Edwin Jodry

Stryker, Ohio

Major: Elementary Education-Degree B.A.

James L. Joiner

Cedarville, Ohio

Major: English-Degree B.A.

Susan June Johns

East Randolph, New York

Major: Physical Education-Degree B.A.

Ruth Ann Johnson

Portsmouth, Ohio

Major: Elementary Education-Degree B.A.
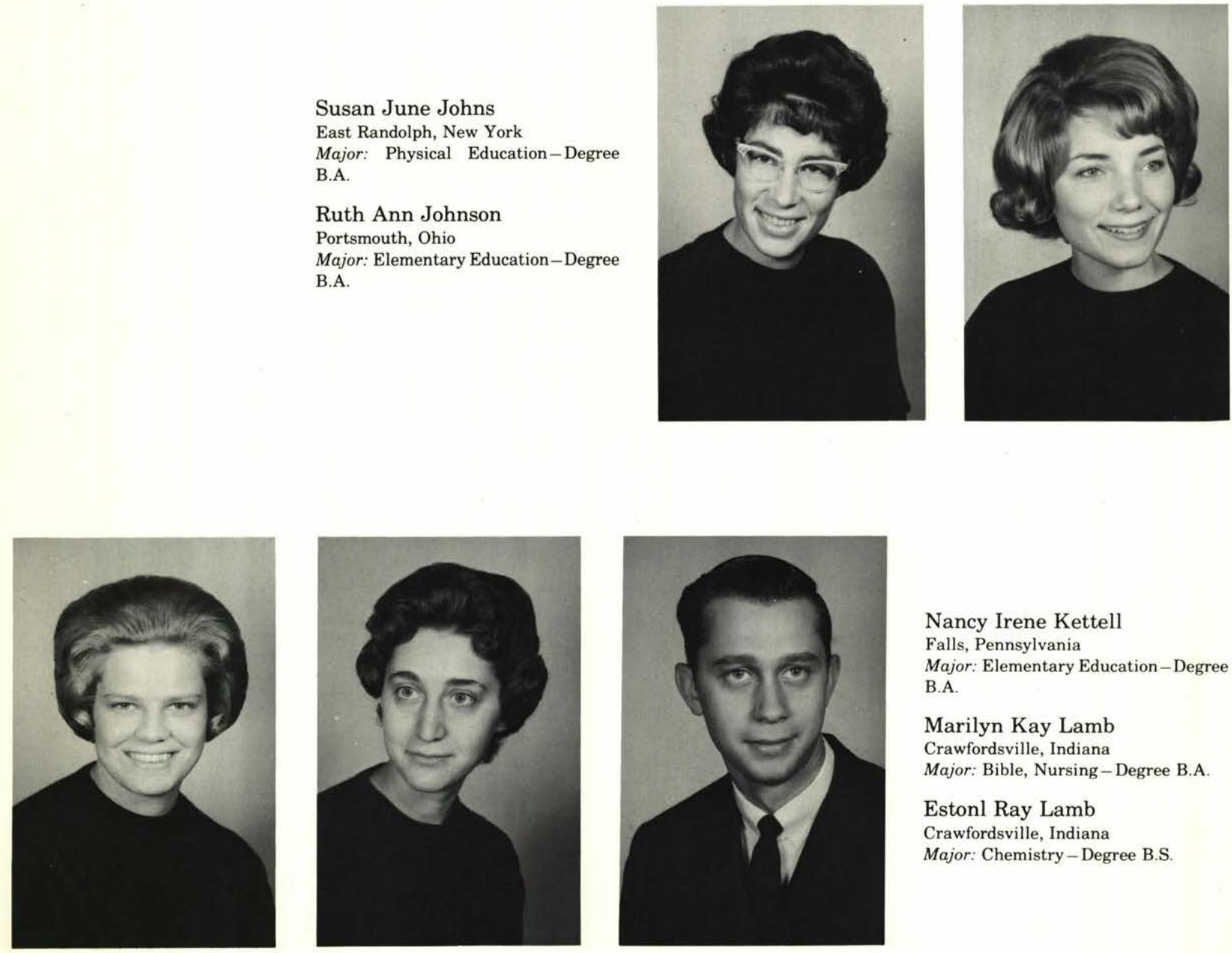

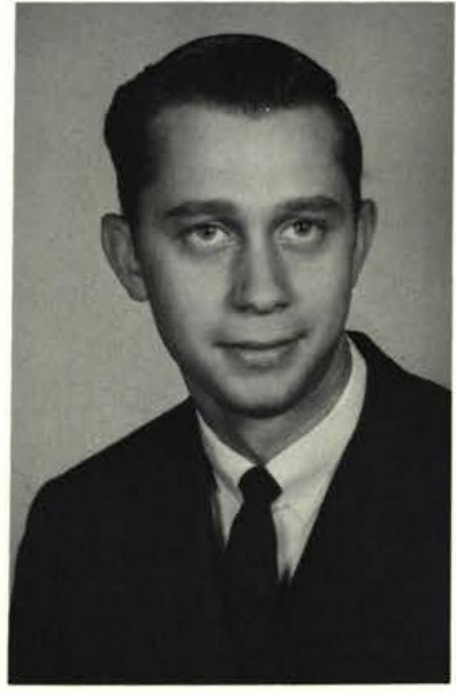

Nancy Irene Kettell

Falls, Pennsylvania

Major: Elementary Education-Degree B.A.

Marilyn Kay Lamb Crawfordsville, Indiana Major: Bible, Nursing-Degree B.A.

Estonl Ray Lamb

Crawfordsville, Indiana

Major: Chemistry-Degree B.S. 
Susan Doris Lane

Cedarville, Ohio

Major: English-Degree B.A.

Ronald Lewis

Edmore, Michigan

Ronald Eugene McDugle Pontiac, Illinois

Major: English-Degree B.A.
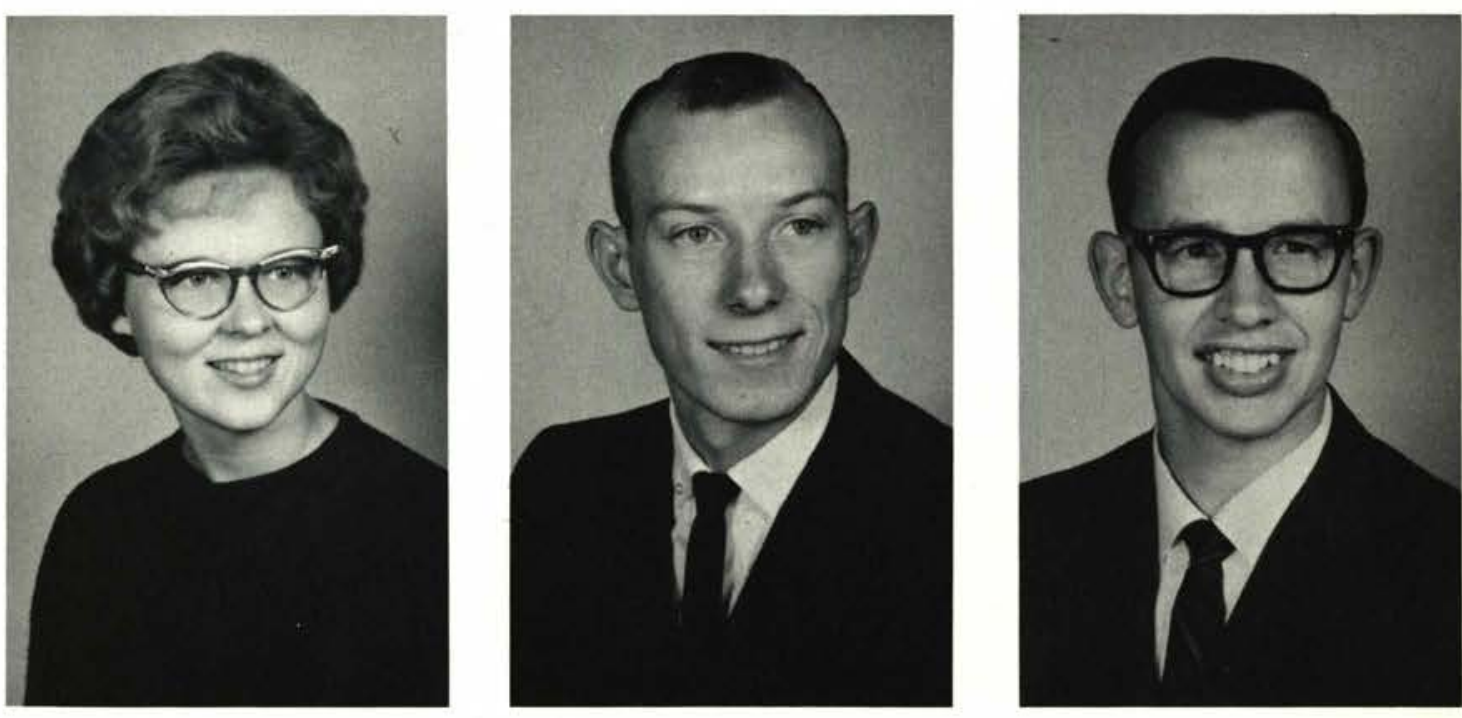

Donald J. McGinnis

Sciotorville, Ohio

Major: Accounting-Degree B.S.

Jean I. Maclaren

Harpster, Ohio

Major: Elementary Education-Degree

B.A
Alfred Arthur Merwald

Portage, Indiana

Major: Speech - Degree B.A.

Terry Sherman Miller

Bay City, Michigan

Major: Physical Science-Degree B.S.

Luann Gale Mundhenk

Pittsburg, Ohio

Major: Elementary Education-

Degree B.A.
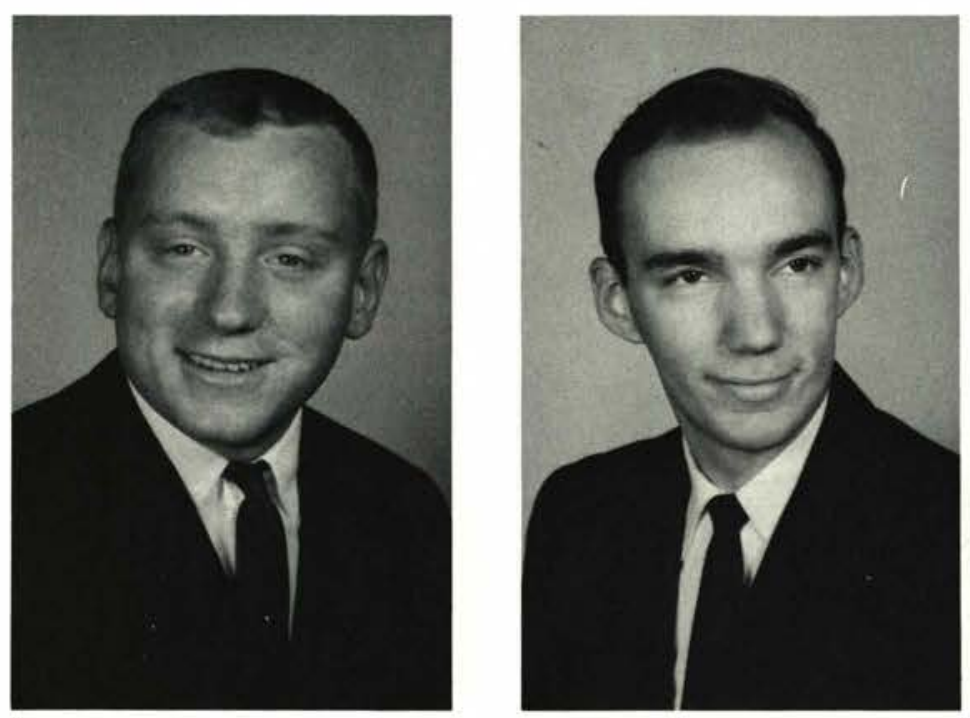

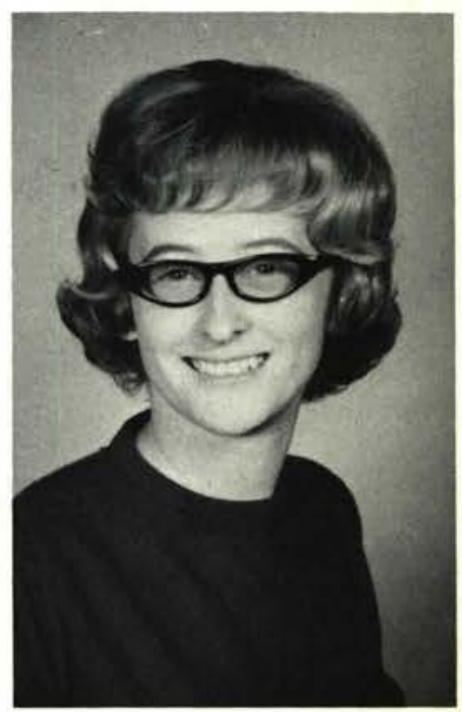



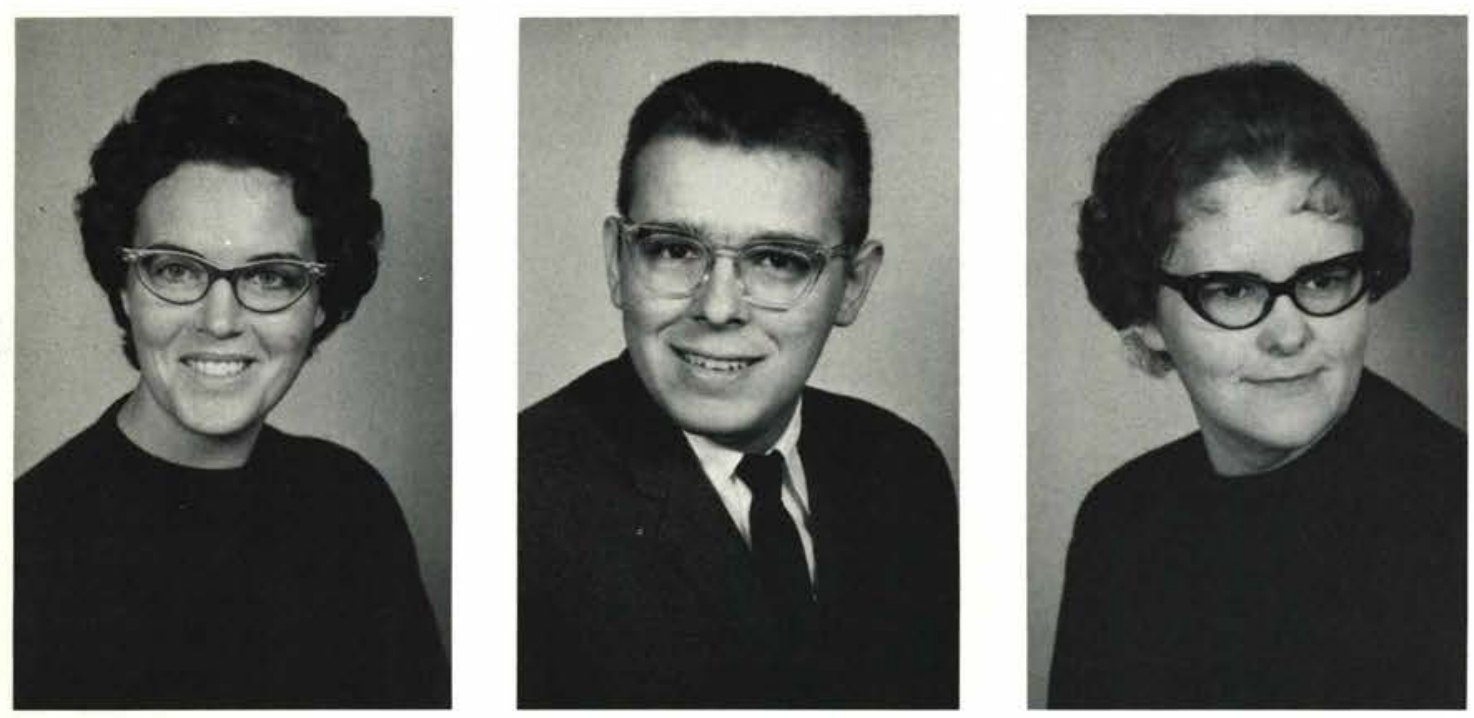

Bessie Viola Murphy

Crane, Indiana

Major: Christian Education-Degree B.A.

Leonard Lee Myers

Marathon, New York

Major: Education-Degree B.S.

Arlene Lee Nightingale

Cleveland, Ohio

Major: Elementary Education-Deggree B.A.

Ed Allen Norris

Rochester, Indiana

Major: Business-Degree B.S.

Phillip Irving Olsen

Lapeer, Michigan

Major: Bible-Degree B.A.
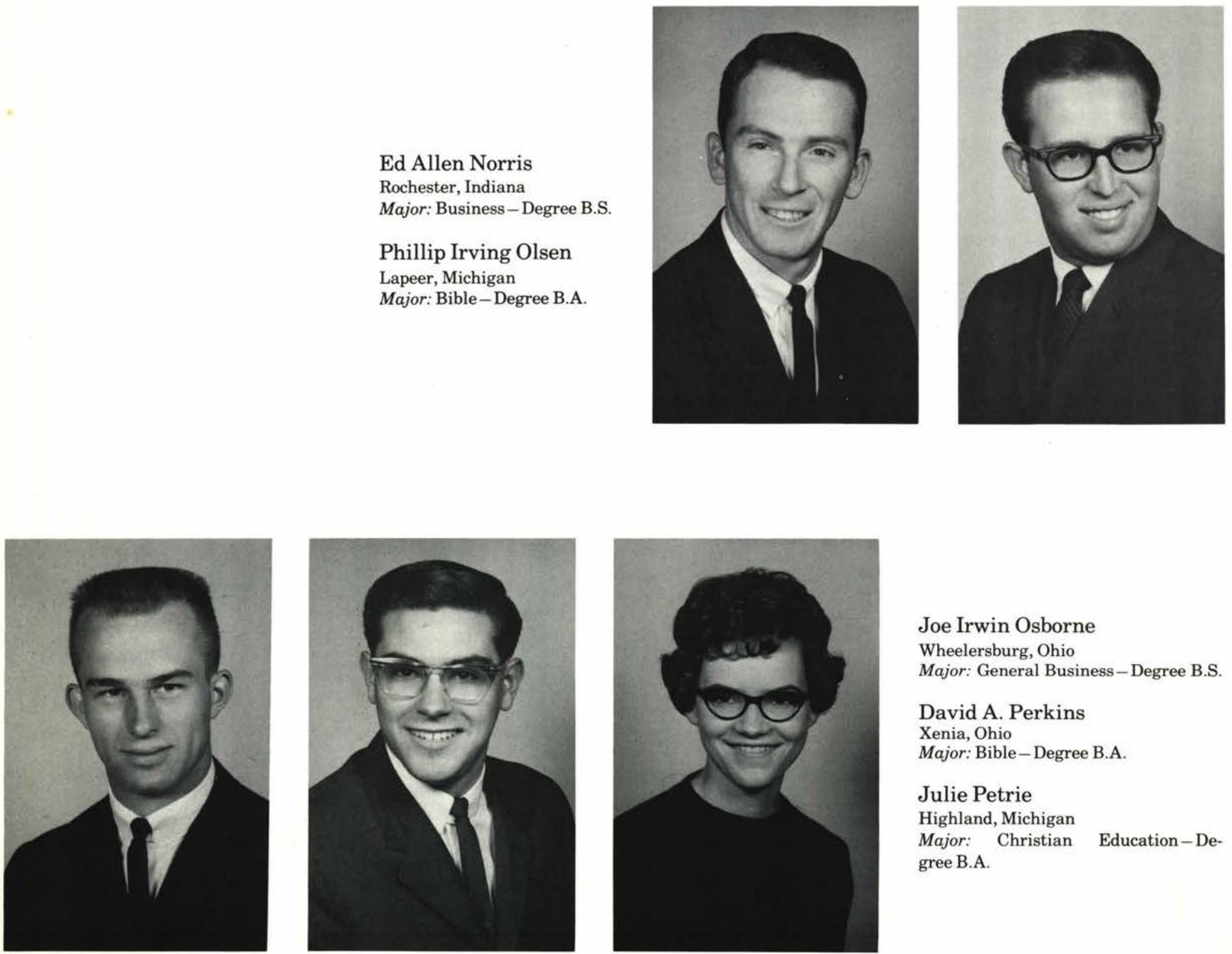

Joe Irwin Osborne

Wheelersburg, Ohio

Major: General Business-Degree B.S

David A. Perkins

Xenia, Ohio

Major: Bible-Degree B.A

Julie Petrie

Highland, Michigan

Major: Christian Education-Degree B.A. 
Barbara Lee Randall

Essexville, Michigan

Major: Accounting-Degree B.S.

\section{G. Eugene Ream}

Brookfield, Wisconsin

Major: Physical Science-Degree B.S.

\section{J. Paul Reno}

Cedarville, Ohio

Major: Physical Education-Degree B.S.
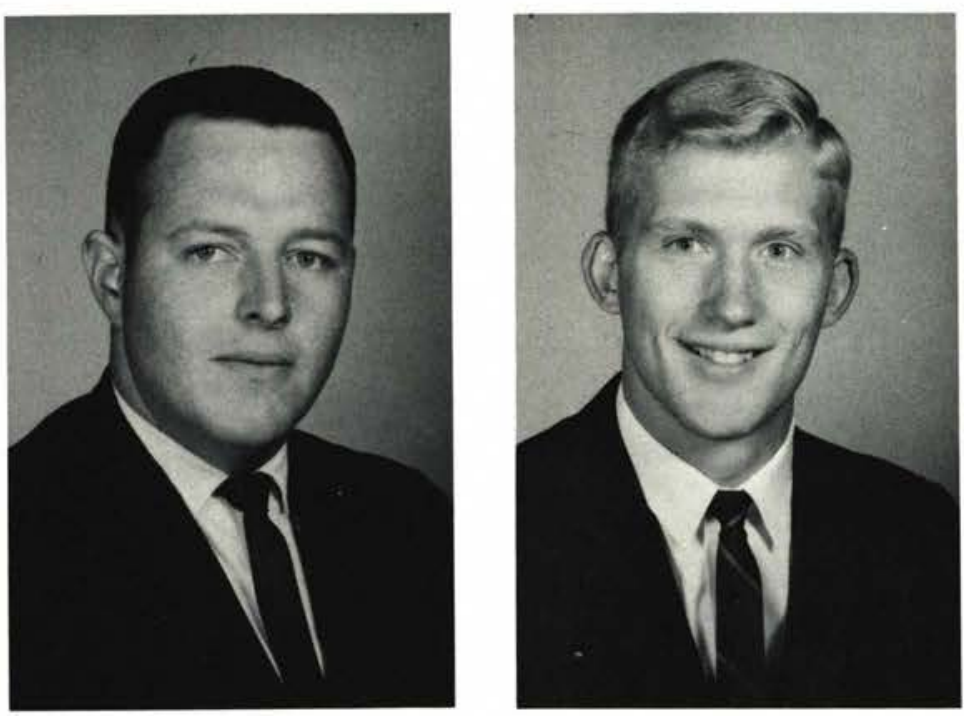

Richard Lee Rich

Louis, Michigan

Major: Bible-Degree B.A.

\section{Marston Val Roloff}

Charles City, Iowa

Major: Chemistry-Degree B.A.
Mary Lisa Rulison

Amsterdam, New York

Major: Elementary Education-Degree B.S.

\section{Fay Joan Sesslar}

Jamestown, Ohio

Major: Physical Education-Degree B.A.

\section{Linda M. Skeie}

Ames, Iowa

Major: Elementary Education-Degree B.S.
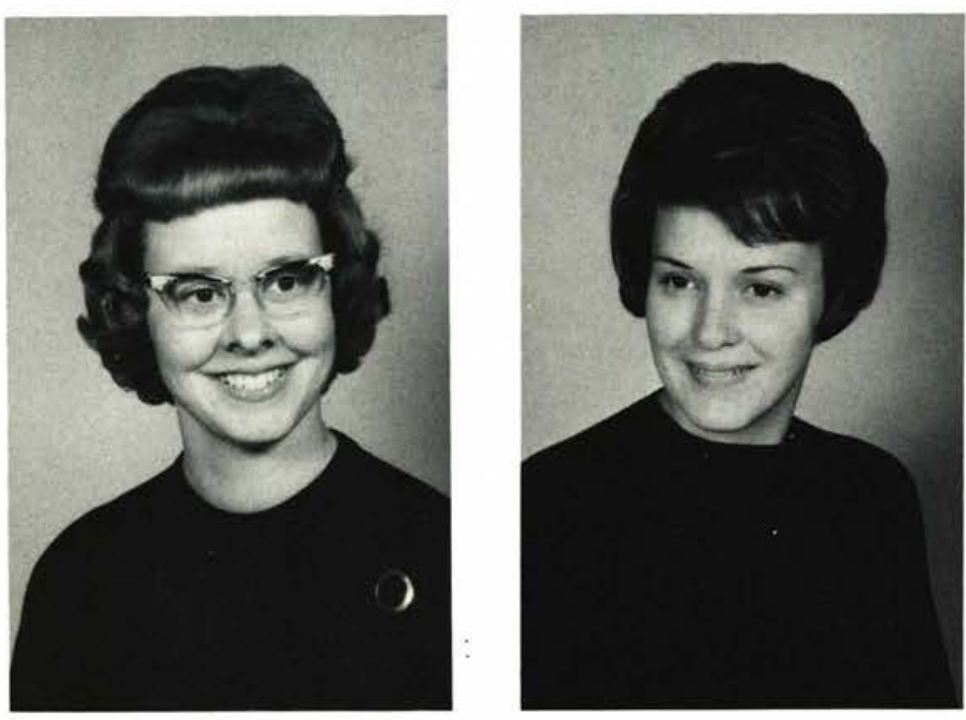

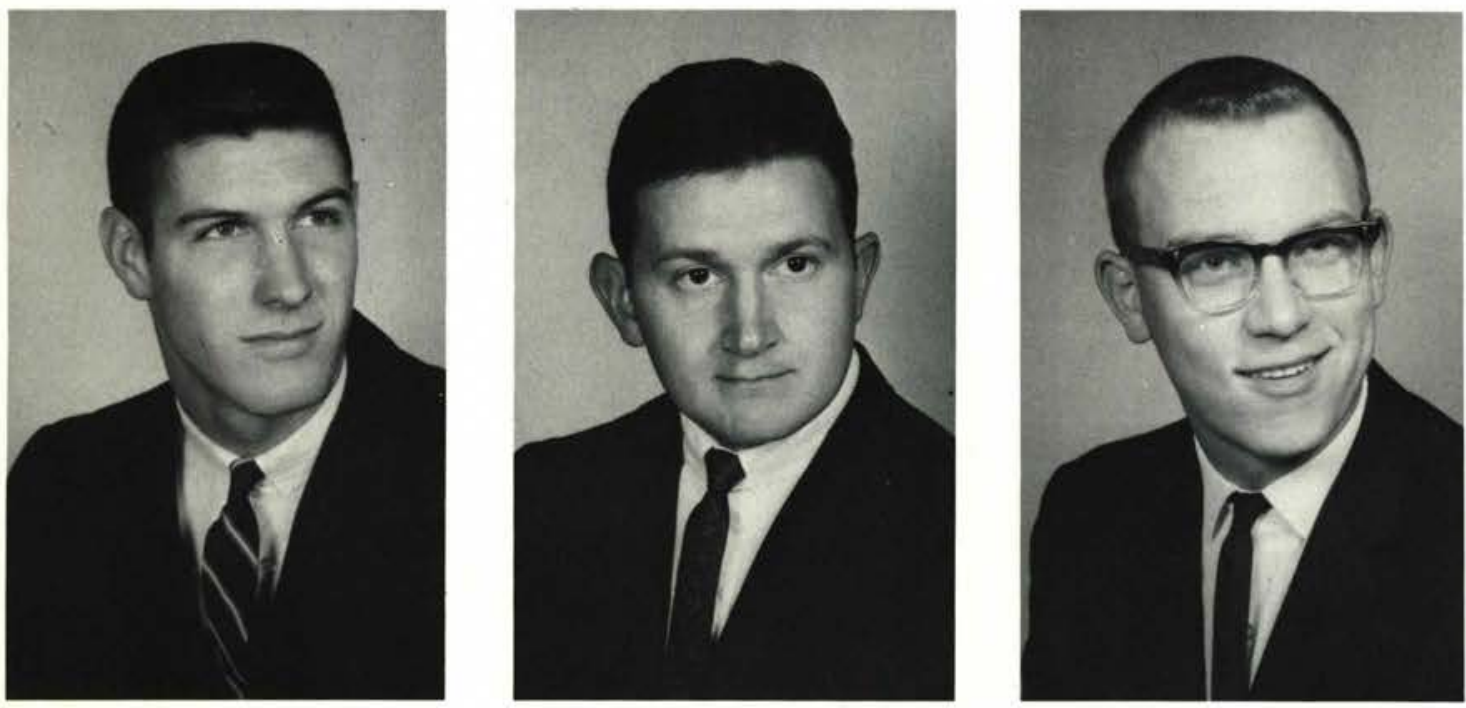

Gerald Lynn Stadt

Deerfield, Illinois

Major: History-Degree B.A.

Gerald E. Stafford

Baltimore, Maryland

Major: Social Science-Degree B.A.

\section{Dan Stevens}

McMillan, Michigan

Major: Accounting-Degree B.S.

Marvin Wayne Stephens

Grand Rapids, Michigan

Major: Chemistry-Degree B.S.

David Earl Taylor

Blanchester, Ohio

Major: PreSeminary-Degree B.A.
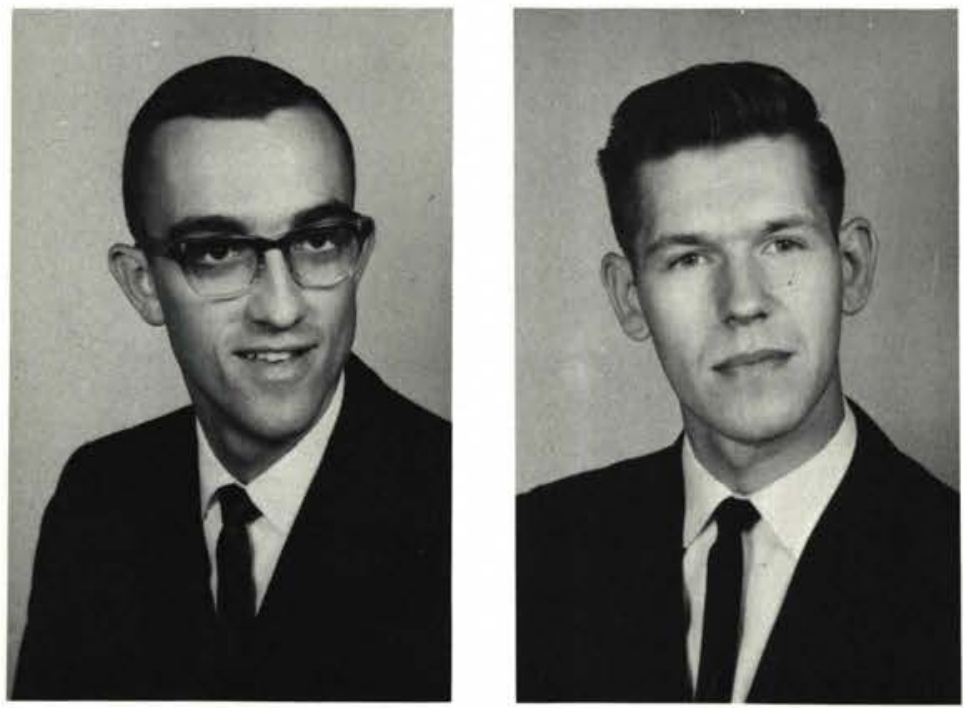
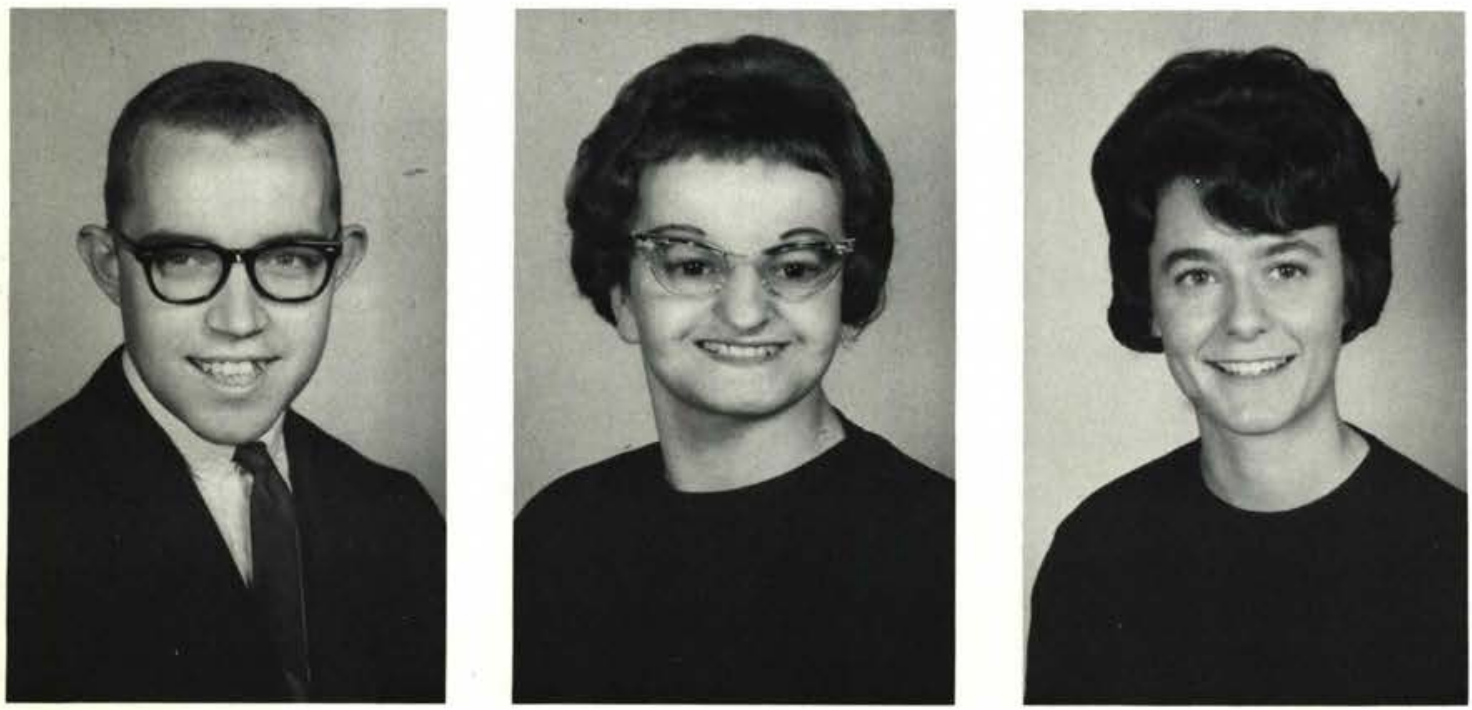

Arthur David Thorne

Medford, New Jersey

Major: History-Degree B.S.

Barbara Alice Thorne

Medford, New Jersey

Major: Elementary Education-Degree B.A.

Janet Gayle Tremba

Youngstown, Ohio

Major: Elementary Education-Degree B.A 
Patricia Versuluis

Marne, Michigan

Cornelia Ann Ward

Collins, Ohio

Major: Physical Education-Degree B.S.

Jon Walborn

Euclid, Ohio

Major: Science-Degree B.S.
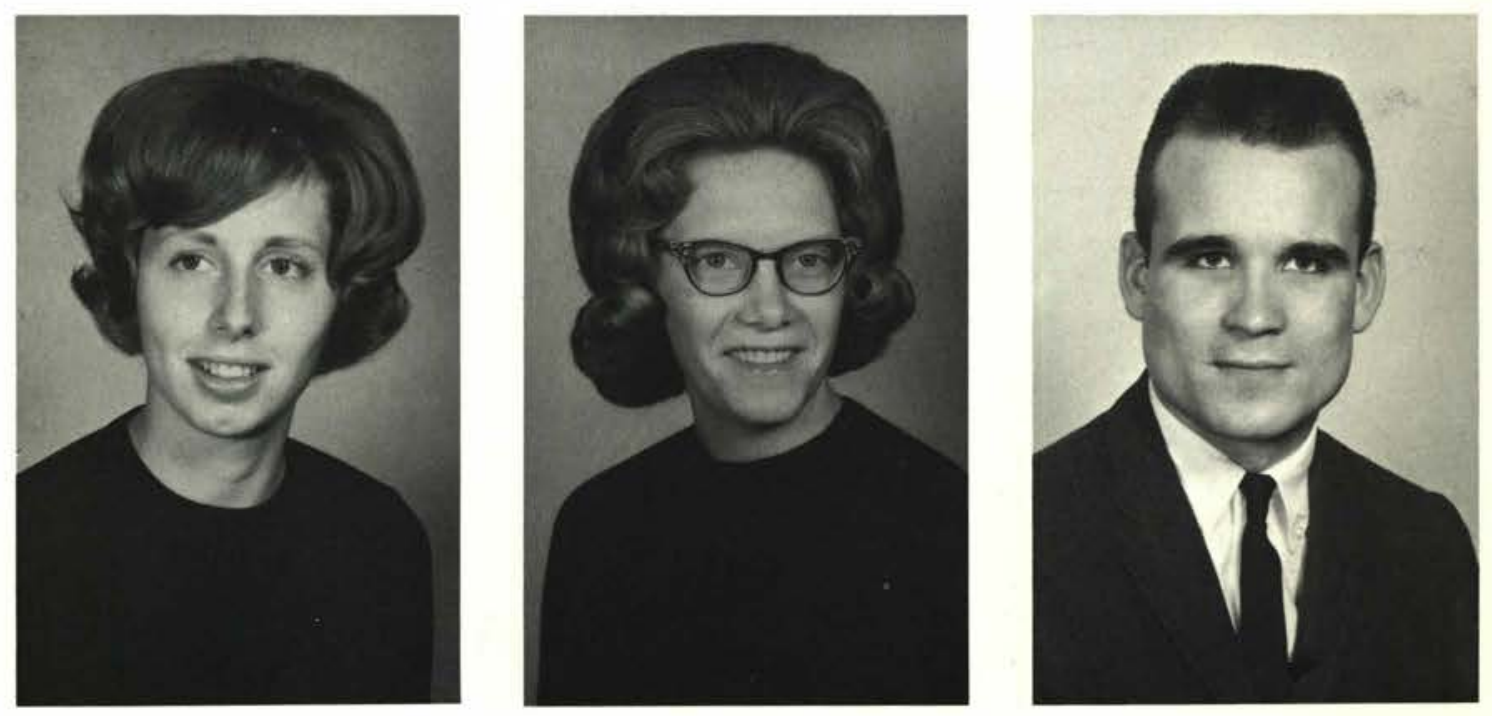

Eugene Wallis

Thurston, Ohio

Major: Science-Degree B.S.

Roosevelt Walker

Cleveland, Ohio

Major: Social Science-Degree B.S.

\section{David Arthur Werner}

Columbia Falls, Montana

Major: Christian Education-Degree B.S.

\section{Donald Wilcoxon}

Cedarville, Ohio

Major: Secretarial Science-Degree B.S.

David Leroy Wilhite

Corwith, Iowa

Major: Accounting-Degree B.S.
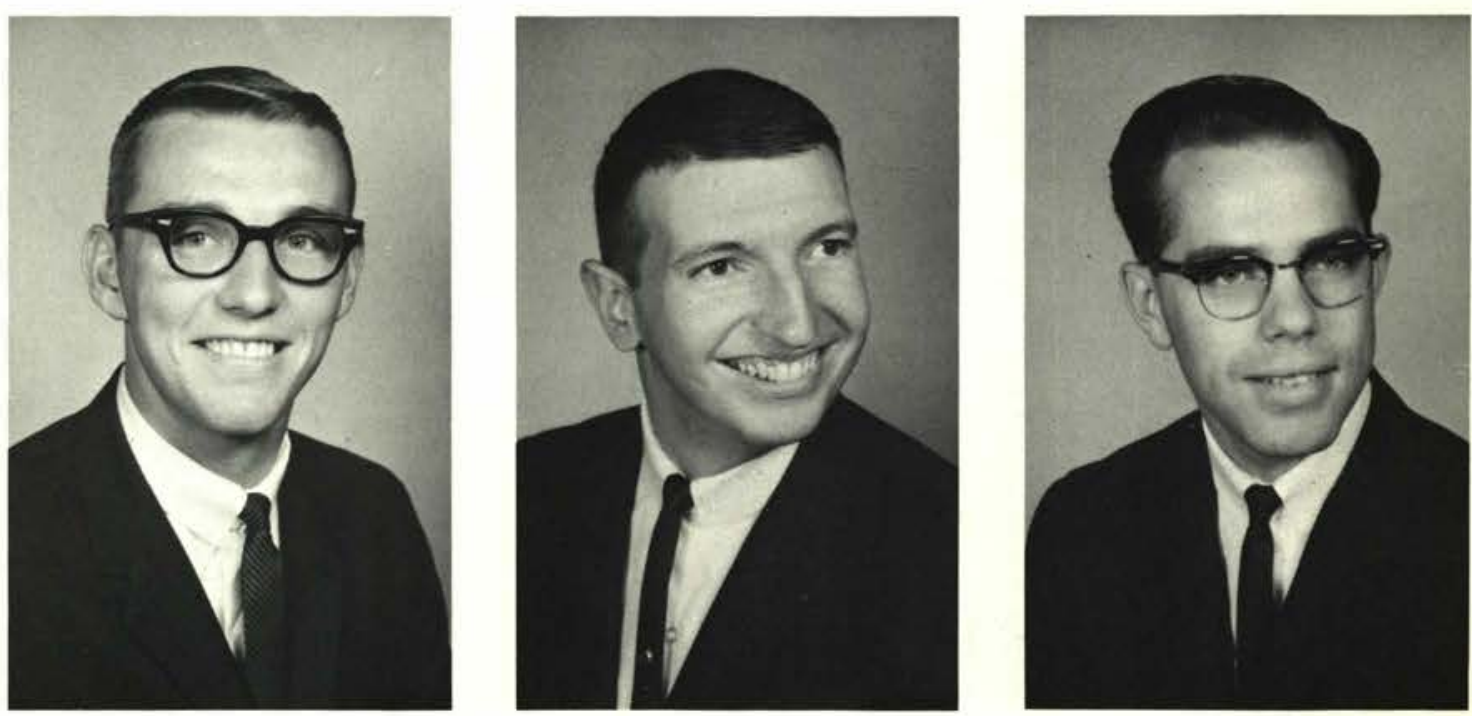

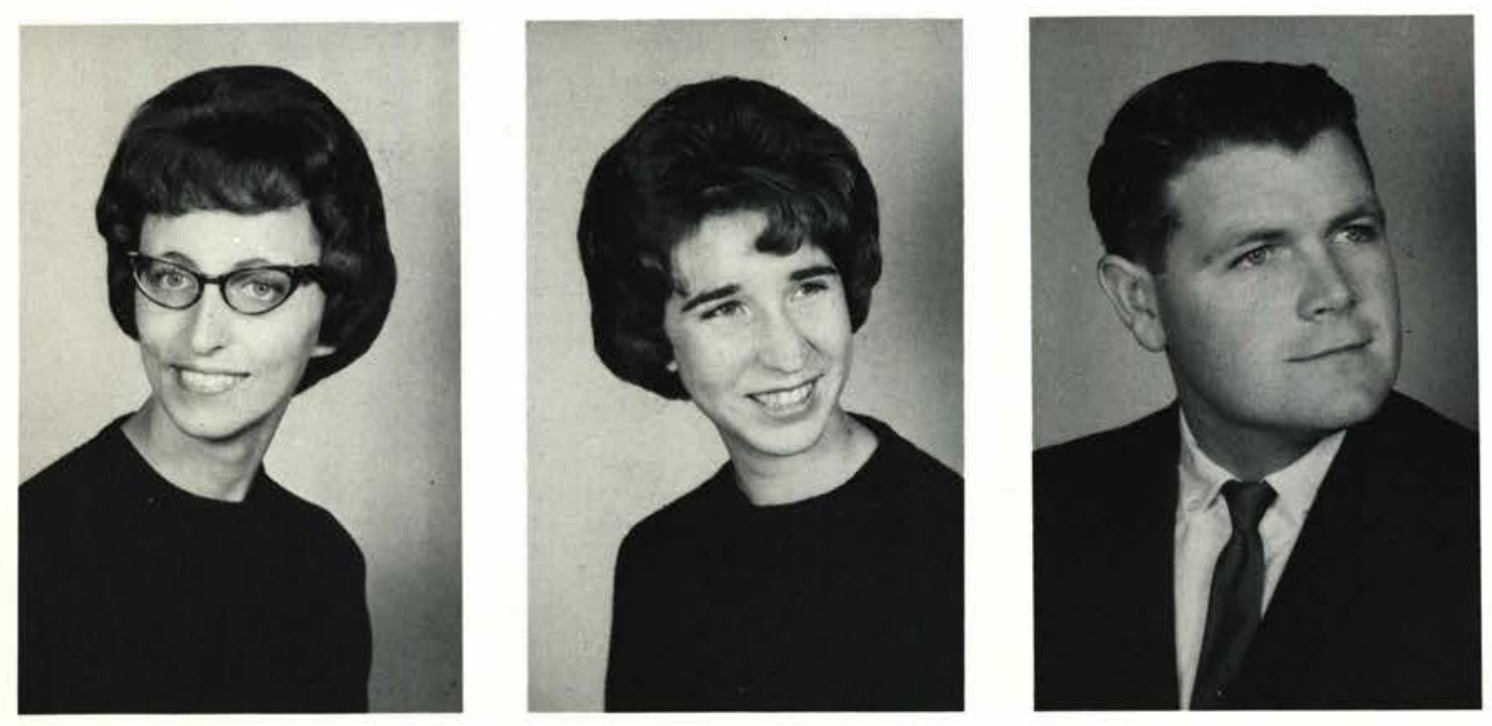

Helen Rae Wing

Wyoming, Michigan

Major: Elementary Education - Degree B.A.

Sylvia Ingrid Wing

Winfield, New York

Major: Physical Education-Degree B.A.

\section{David Woodman}

Bay City, Michigan

Major: Bible-Degree B.A.

Richard Zandstra

Grand Rapids, Michigan

Major: Biology - Degree B.S.

Paul Titus Zemek

Binghampton, New York

Major: Bible-Degree B.A.

Mervin Lee Ziegler

Wellington, Ohio

Major: Speech-Degree B.A.
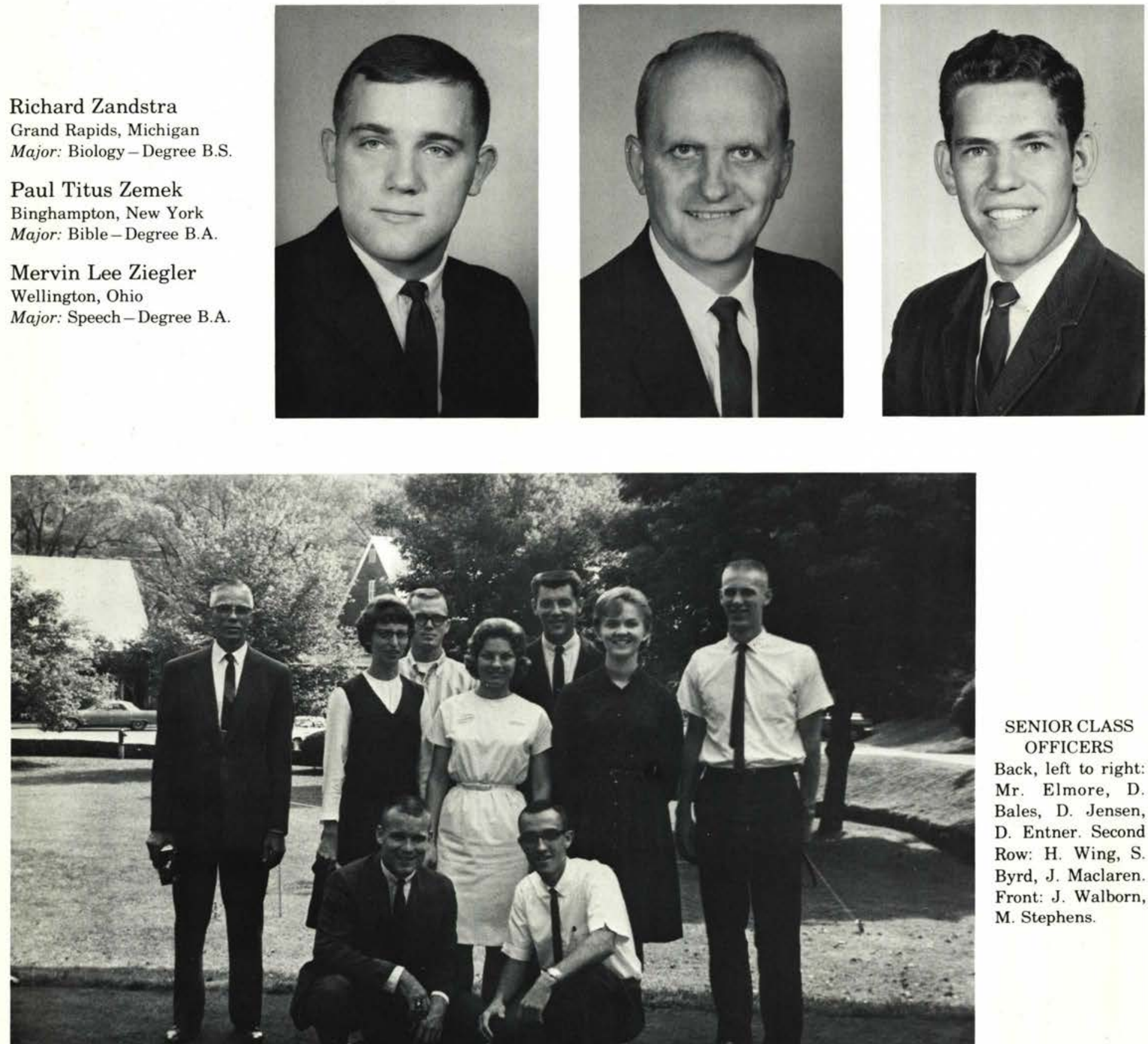

SENIOR CLASS OFFICERS

Back, left to right: Mr. Elmore, D Bales, D. Jensen, D. Entner. Second Row: H. Wing, S. Byrd, J. Maclaren. Front: J. Walborn, M. Stephens 
The Many Faces of The Senior Class
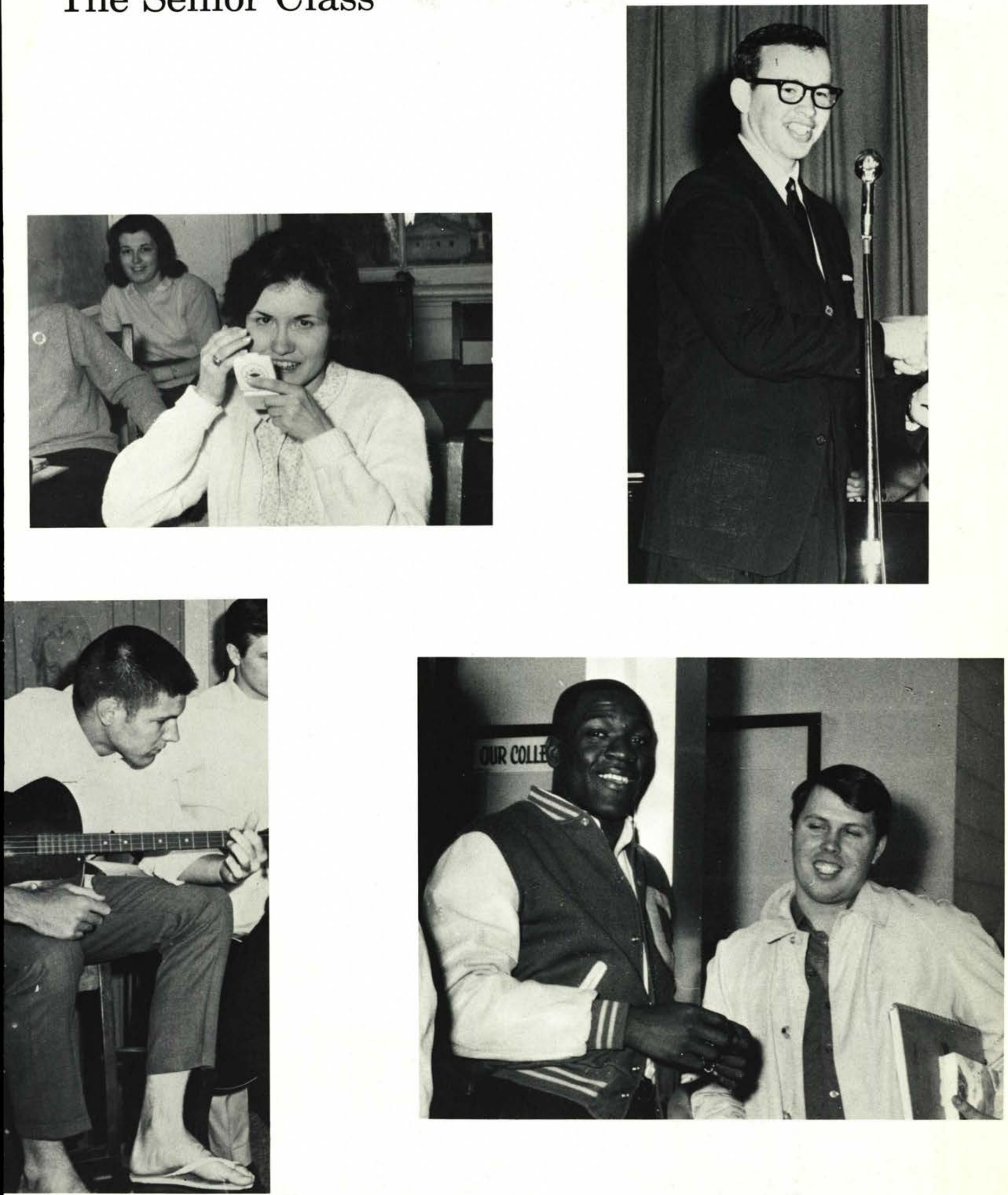


\section{SIXTY-NINTH ANNUAL \\ BACCALAUREATE}

CLASS OF 1965

\section{CEDARVILLE COLLEGE}

CEDARVILLE, OHIO

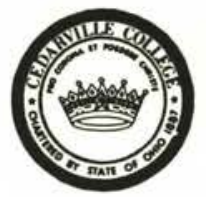

"For the Word of God and the Testimony of Jesus Christ" Revelation 1:9

\section{THE GYMNASIUM}

Friday

May 28

7:30 P.M.

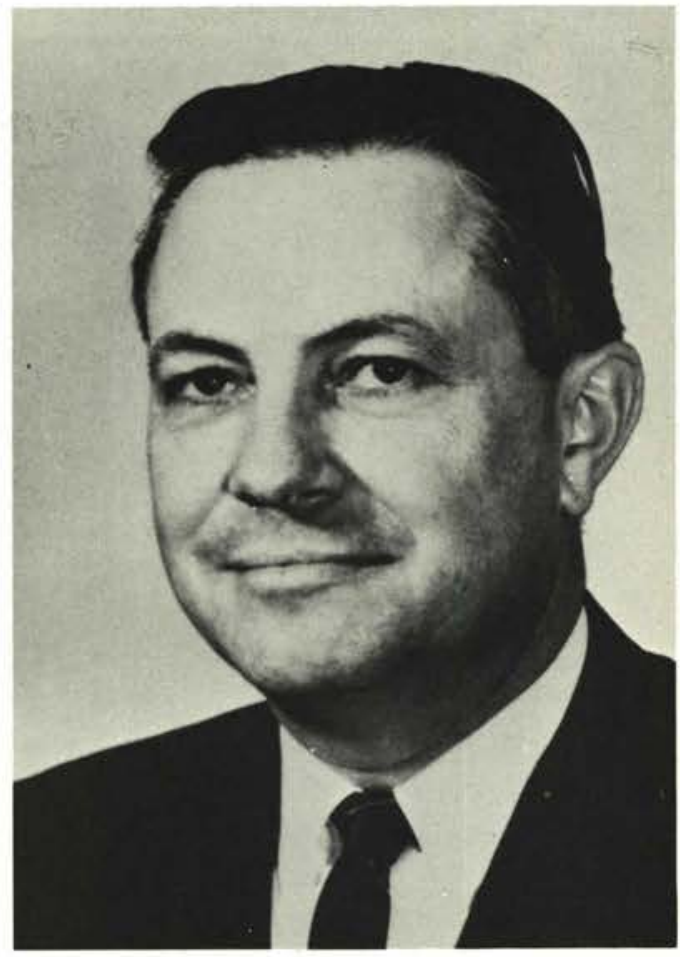

Rev. Donald Brong

Baccalaureate services, the prelude to Commencement, were held on Friday evening, May 28. The Seniors and their guests heard an inspiring message by Rev. Donald Brong, the state missionary from Iowa.

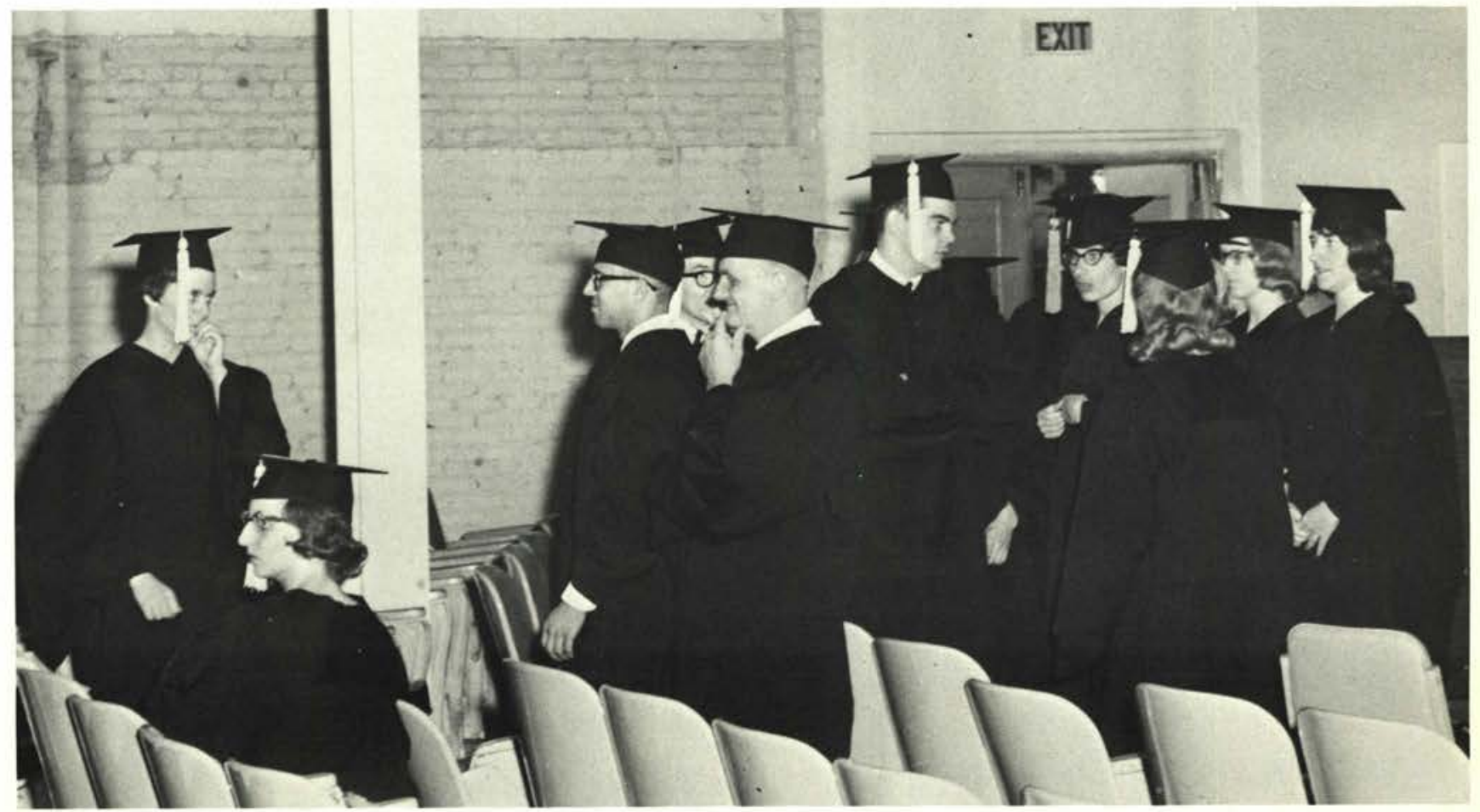




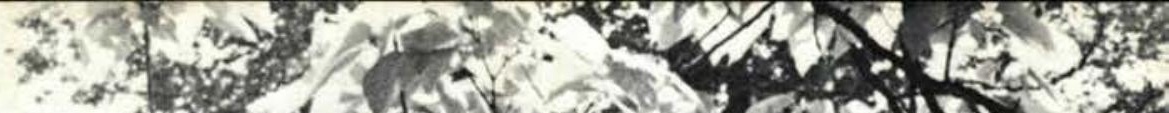

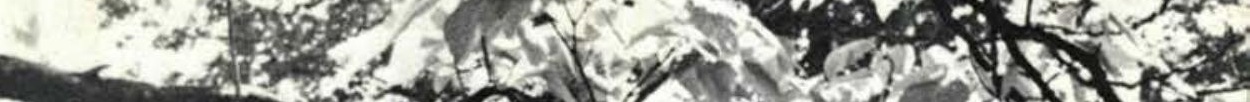

2. 350

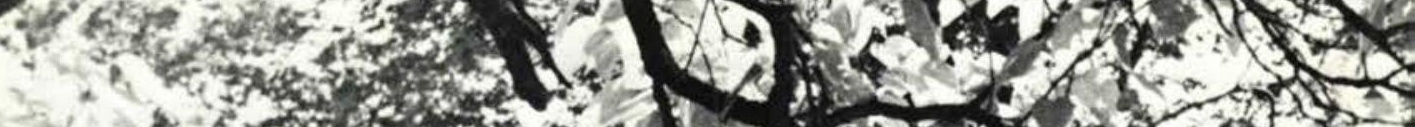

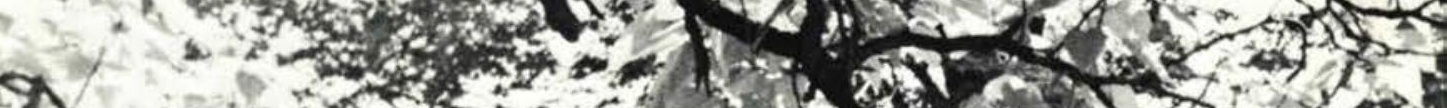

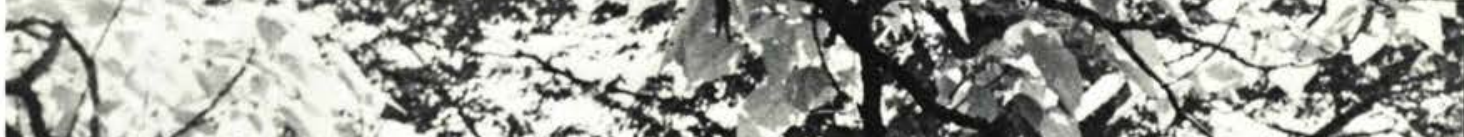

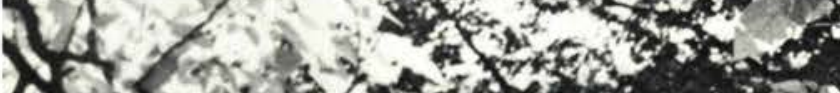
(1) 10.20 C $1,+,<$,

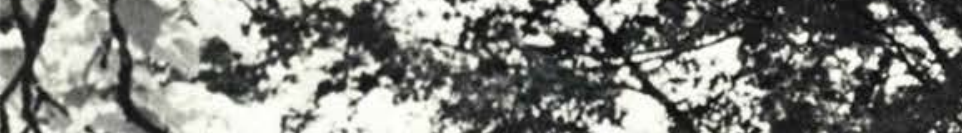

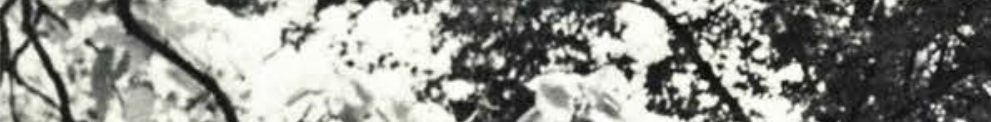
$120 \mathrm{~m}, 5 \%$ ard

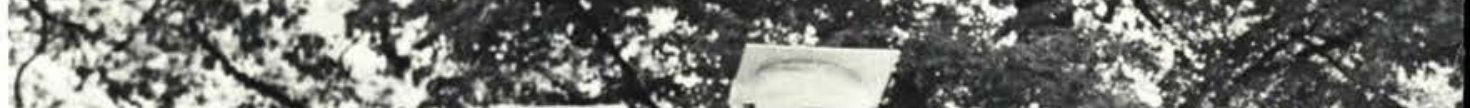

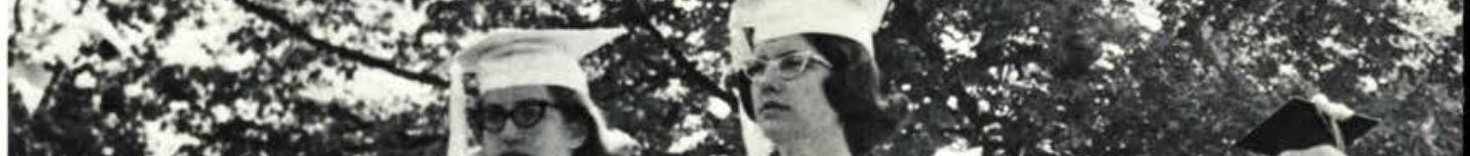
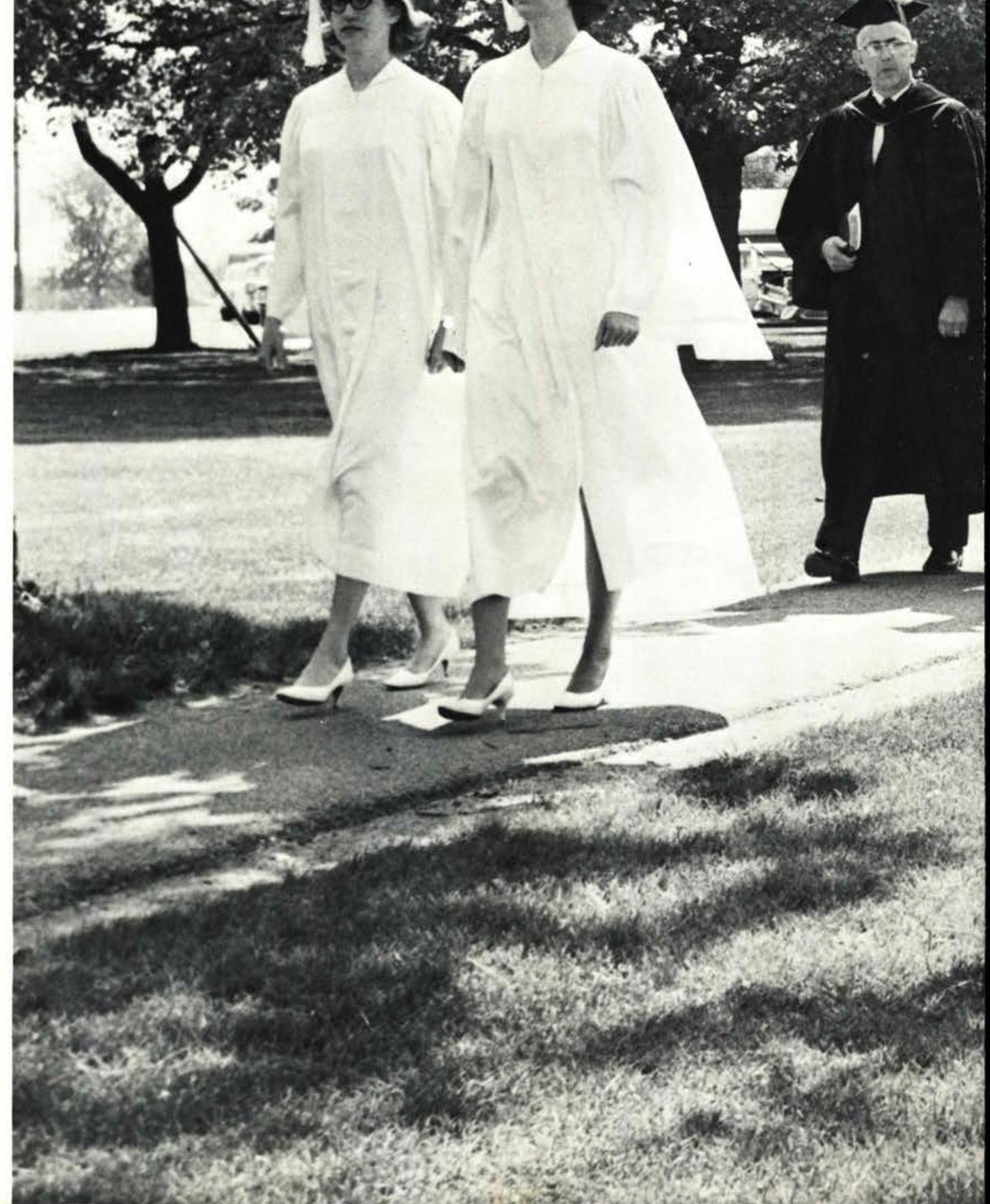


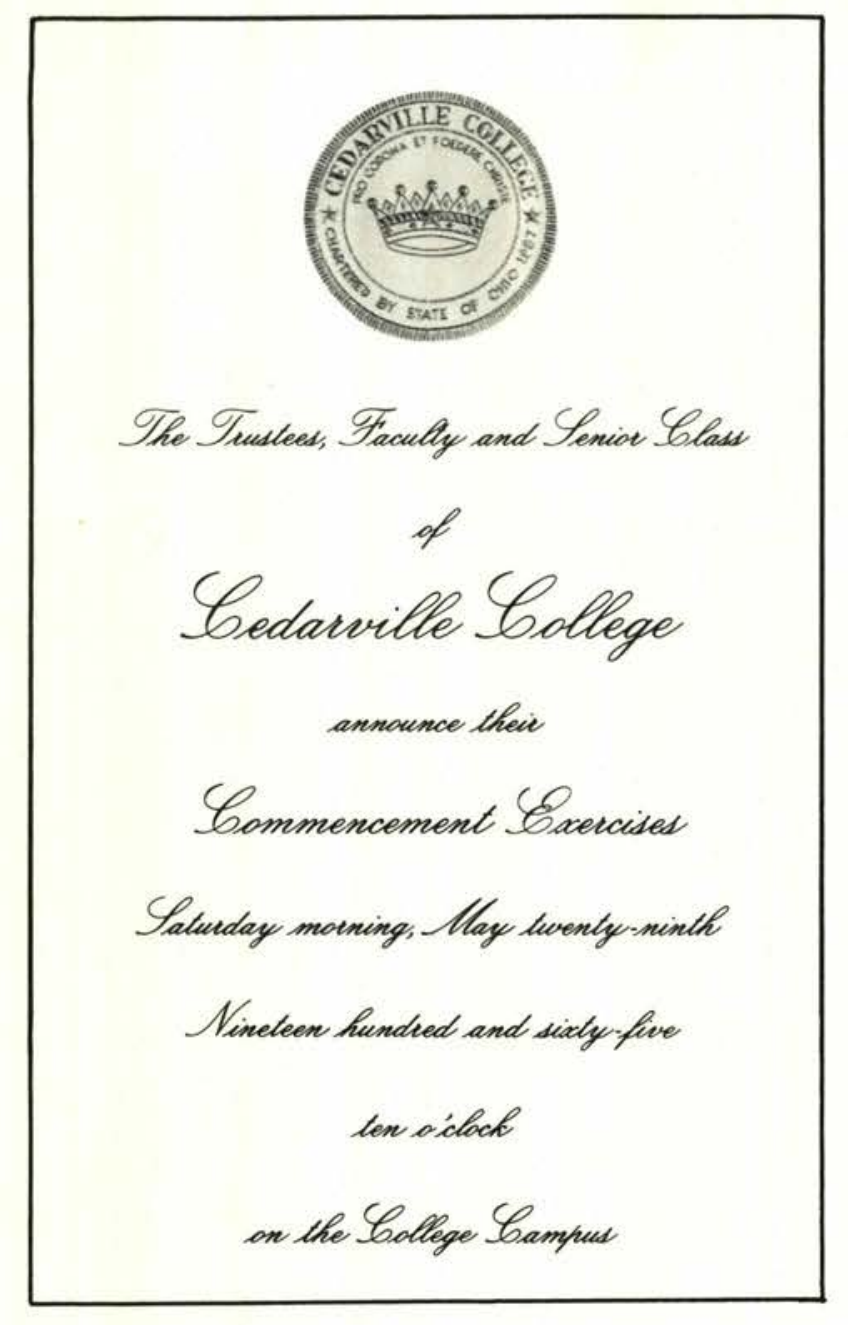

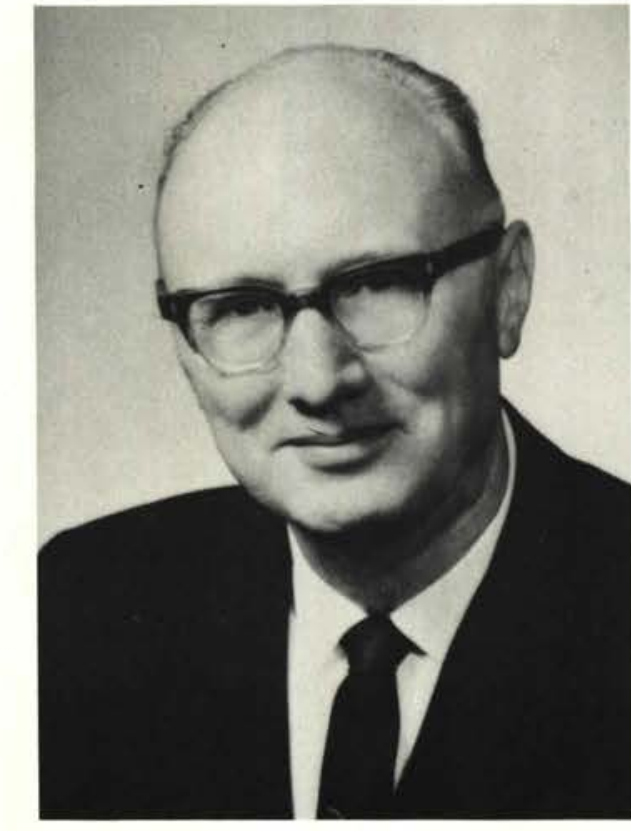

Dr. Walvoord

An impressive Commencement exercise was held on May 29 in the Student Center. The procession was led by four Junior girls in white robes. After a number by the Senior Trio and the Messengers Quartet, Dr. John F. Walvoord, President of Dallas Theological Seminary, Dallas, Texas, presented a challenge to the graduates and the audience.

Miss Ruth Hege was the candidate for the Doctor of Humanities Degree this year.

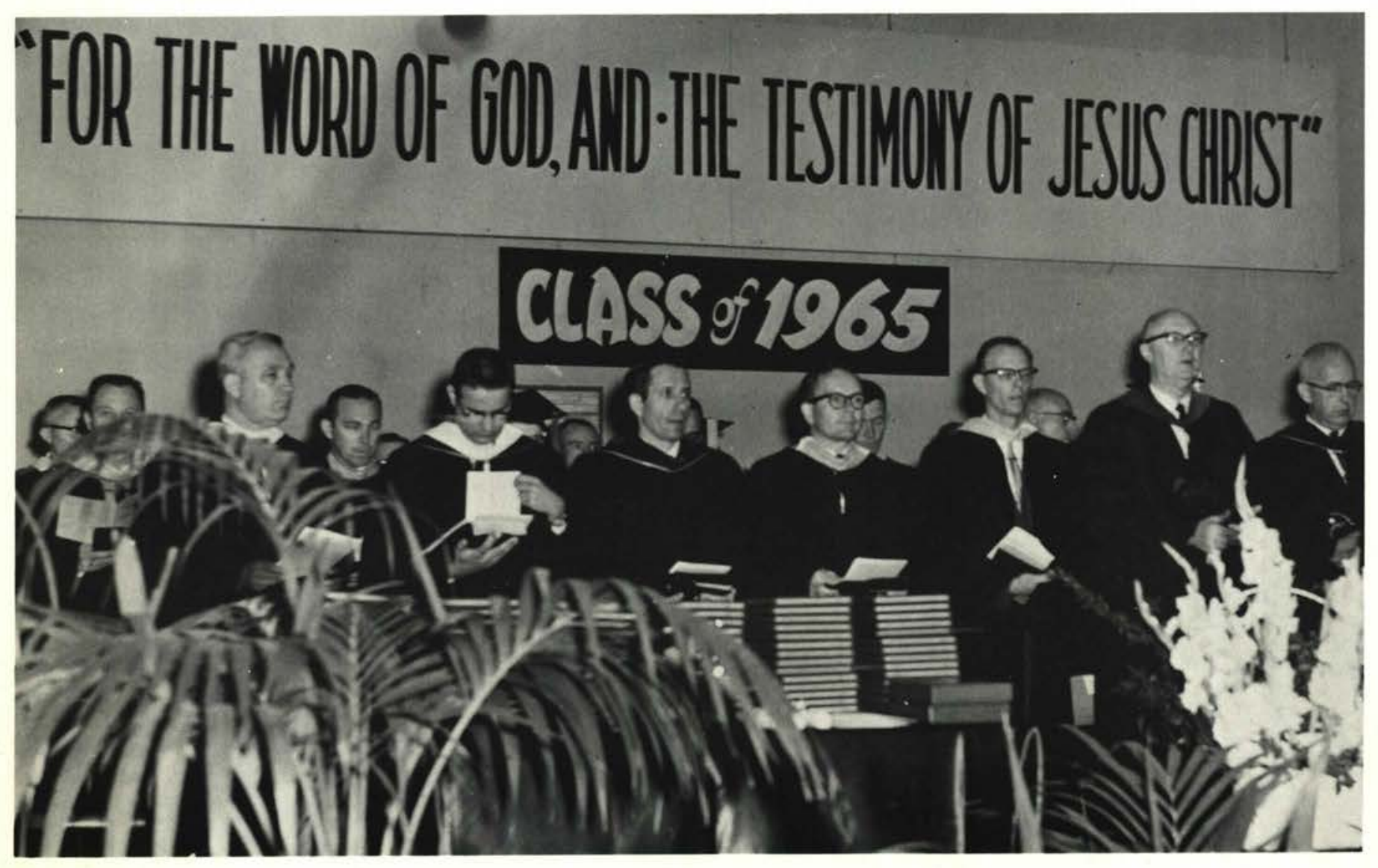




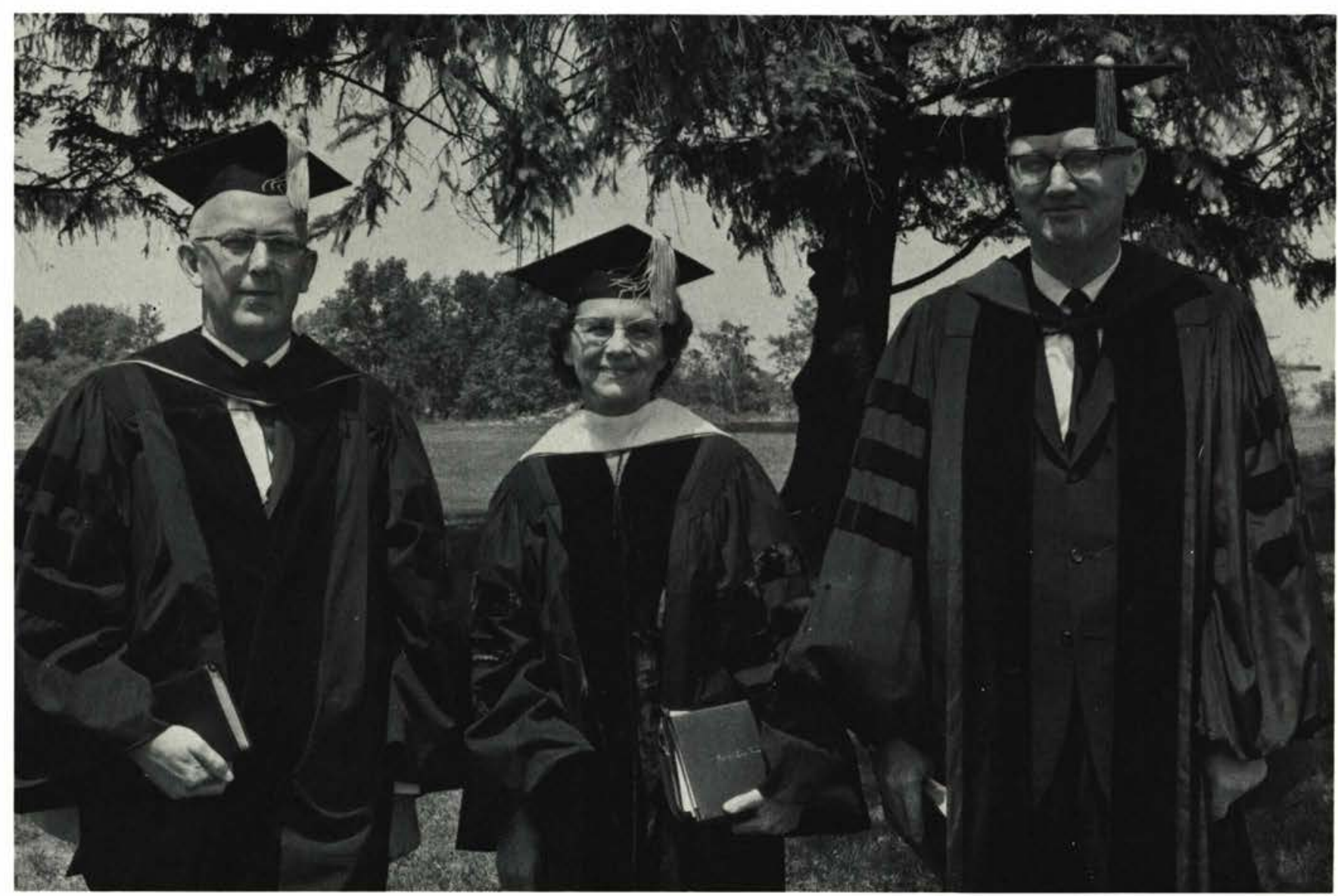

Dr. James T. Jeremiah, Dr. Ruth Hege, and Dr. John F. Walvoord.

\section{CL.15S of 15.55}

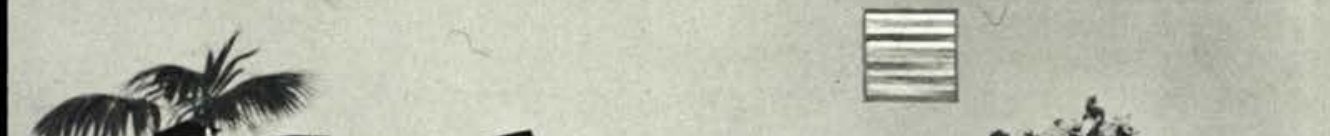

(3)

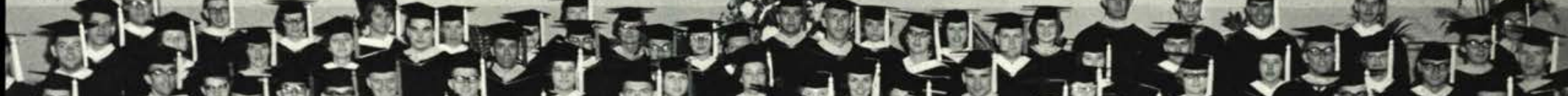

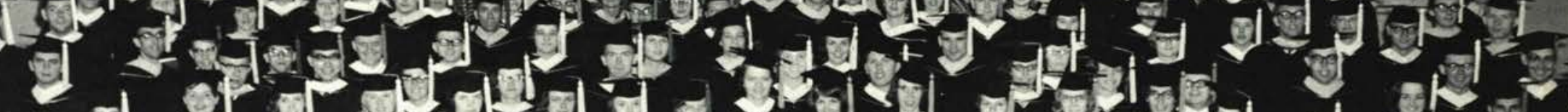

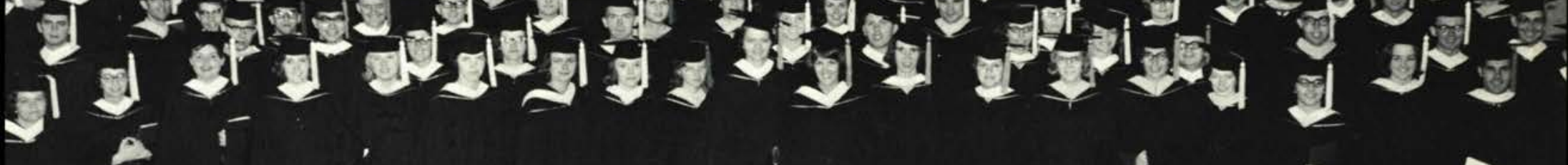

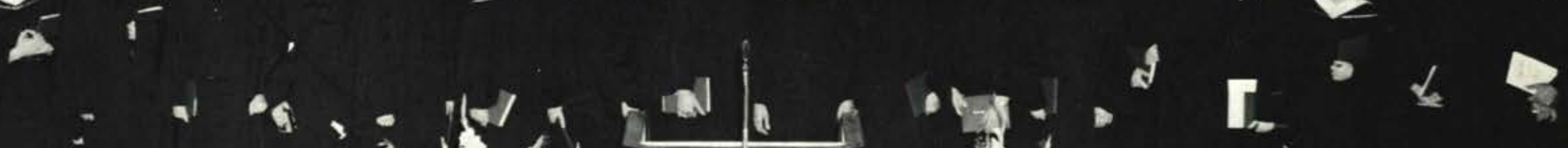




\section{FIRST \\ BAPTIST CHURCH}

602 Illinois Avenue

McDonald, Ohio

9:30 Sunday School

10:30 Morning Worship

6:30 Family Study Hour

7:30 Evening Service

Midweek Service, Wednesday 7:30

J. E. Beckley, Jr., Pastor

\section{SILVIS HEIGHTS}

\section{BAPTIST CHURCH}

Middle Road and Tenth Avenue

Silvis Heights, Silvis, Illinois

Gordon H. Perry, Pastor

Sunday

9:30 Sunday School

10:30 Morning Worship

6:00 Baptist Youth Fellowships

7:00 Evening Gospel Service

Tuesday

6:00 Junior Choir Practice

Wednesday

7:00 Midweek Bible Study \& Prayer

8:00 Adult Choir Practice

"Holding forth the word of life;

that I may rejoice in the day of Christ."

Phil, 2:16
FIRST BAPTIST CHURCH

123 W. Liberty Street

Medina, Ohio

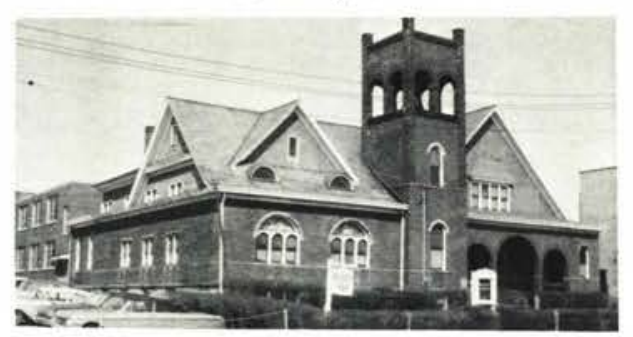

WELCOME

"In the heart of Medina with Medina on its heart"

\section{GRANDVIEW PARK} BAPTIST CHURCH

1701 East 33rd Street

Des Moines 17, Iowa

David Nettleton, Pastor

Steve Boalt,

Minister of Youth and Music

You are Always Welcome at the

Grandview Park Baptist Church

Congratulations to the Graduating

Class of 1965 from all of us at

Grandview

\section{SOUTH \\ BAPTIST CHURCH}

\author{
3212 South Saginaw Street \\ Flint 3, Michigan
}

Kenneth K. Elgena, Pastor

"An Industrious Church in an Industrial Center with your Soul as its central business." 
Abbott, Marion

Abshear, Keith Bryson

1908 Kylemore Drive, Xenia, Ohio .302 E. McCreight, Springfield, Ohio Abston, Howard R.R. No. 5, Crawfordsville, Indiana Acker, Elton Dale 75 Jamestown Street, Randolph, N.Y. 3001 Derr Rd., Springfield, Ohio R.D. No. 1, Frankfort, Mich. 867 Valdes Ave., Akron, Ohio R.R. No. 1, Hillsdale, Mich. 47 Lagos St., Lagos, Nigeria 208 Orchard Way, Johnstown, Pa. 208 Orchard Way, Johnstown, Pa. 503 W. Funderburg Rd., Fairborn, Ohio Tiffin Rd. R.R. No. 2, Findlay, Ohio ................ Prospect, $\mathrm{Pa}$ 13504 Ardath Ave., Gardena, Calif. 321 Sheffield Rd., Cherry Hill, N.J. R.R. No. 1, Taylors Falls, Minn. 1216 Mackinaw Ave., Cheboygan, Mich. 180 E. Main St., Holland, N.Y. 1107 Park Ave., Chariton, Iowa 296 Moore Rd., Avon Lake, Ohio 1045 Main St., Charleston, W. Va. 1110 Jerry Ave., Schenectady, N.Y. 409 E. 6th St., Pana, III. 48 South Biggs Ave., Belleville, Mich. 403 E. Hunt St., Adrian, Mich. 2438 182nd St., Highland, Ind. 408 W. North St., Arcanum, Ohio Box 237, Kingman, Ind. 807 Harding Rd., Elkhart, Ind. 5 Bittersweet Lane, Valley Stream, N.Y. 249 Mansion Ave., Audubon, N.J. . 201 Palmer Dr., Cedarville, Ohio 1249 Canton St. Elkhart, Ind. R.R. No. 4, Saxonburg Rd., Butler, Pa. R.F.D. No. 3, Jerseyville, III. 175 Greenlea Drive, Coraopolis, $\mathrm{Pa}$. 1398 Columbus St., Cincinnati, Ohio R.R. No. 2 N. Mitchell St., Boise, Idaho 1152 Oxford Rd., Cleveland Heights, Ohio Box 146, Anchor, Alaska 33 Arrowhead Trail, Fairborn, Ohio 232 W. Bradford St., Stanton, Mich. 4214 Leonard St., N.W., Grand Rapids, Mich. . R.R. No. 3, Xenia, Ohio R. 3, Beloit, Ohio Cedarville, Ohio 5810 W. 5 th Ave., Gary, Ind. R.D. No. 1, Homerville, Ohio 834 Tralger Dr., Toledo, Ohio 960 Judd Rd., Saline, Mich. 6776 Rose Crest, Cincinnati, Ohio R.R. No. 1, Algona, Iowa 623 Fairfield Ave., Fairborn, Ohio 1920 Loow Rd., Sagiwaw, Mich.

500 W. Madison St., New Carlisle, Ohio 7801 W. 27th St., Palos Park, Ill. 3659 S. Ridge Rd., Perry, Ohio 3659 S. Ridge Rd., Perry, Ohio Vrooman Rd, Painesville, Ohio 83 Cedar St., Garfield, N.J. Washington St., New Bethlehem, Pa. R.D. No. 4, Grove City, Pa. R.R. No. 1, Argenta, IIl. 4 A. Church St., Jamestown, Ohio Colchester, III. 1139 Normandy Dr., Portsmouth, Ohio 750 Milan Ave., Amhurst, Ohio 259 Jackson Pike, Gallipolis, Ohio 289 Bennett Rd., Cheektowaga 25, N.Y. 1636 Ongaro Dr. W., Columbus, Ohio North Shore Dr. Box 47, Clear Lake, Iowa 1756 Breese Rd., Lima, Ohio 227 Courtland St., Rockford, Mich. 56 Cortland St., Marathon, N.Y. 307 North St., Holly, Mich.
Brown, Jonathan

671 Walnut St., Cedarville, Ohio Brown, Martha Louise Brown, Stephen Paul 307 North St., Holly, Mich. Buckle, Marian Jane Buerer, Nancy Grace Burcham, Carolyn Fern Burcham, Thomas, A. Burroughs, Kristine Bush, Dorinda Rae Butler, Rayna K. Byrd, Shirley Ann Cady, Audrey Jean Cagwin, Raymond H.

Cain, Daniel Fred Carlson, Beverly Jean Carlson, Paul Carr, Carol Lucille Carroll, William Martin Carson, Lee Harold

Carter, Dozier Carter, Joan Darlene

Cayton, Ardith R. Cayton Lynne Elizabeth Chumbler, David Willard Claflin, Jane Elizabeth

Clater, Robert D.

Clater, Thedore Edward

Clement, Michael Ardin Colley, Sandra Lynn Combs, Marcus . Compton, Gary Lee Cooper, Donald William Cooper, Gwytha Dawn Coppock, Carolyn S. Corn, Larry Allan Corns, Marion Rose Cosgray, Robert Eugene

Cox, Martha Louise Crampton, Michael William Crampton, Roger Gary Crouch, Frederick Anthony Crown, Nancy Carol Crown, Pat, Karen Culberson, Lyle Edwin 08 N. Mantorville Ave., Kasson, Minn. 400 Irwin St., Lockhaven, $\mathrm{Pa}$. Main St., Cedarville, Ohio R.R. No. 2, Mattoon, III. R.R. No. 2, Mattoon, III. 2615 Wicker St., Highland, Ind. 749 Northampton Rd., Cuyahoga Falls, Ohio 195 Cutler Ave., Corning N.Y. 6732 Parrish Ave., Hammond, Ind. Box 45, Troupsburg, N.Y. 9961 Jennings Rd., Eden, N.Y. 515 E. 45th St., Lorain, Ohio 2570 Pangborn, Circle, Decatur, Geo. 336 Porter St., Gary, Ind. R.F.D. No. 1, Gerry, N.Y. 11 Campbell Ave., Buffalo, N.Y. 333 W. Cortland, Jackson, Mich. 1126 15th St., Portsmouth, Ohio 9 Park St., Sinclairville, Ohio 2740 N. 86 th St. Milwaukee, Wis. 4203 N. Ardmore Ave., Milwaukee, Wis. 5361 Marcella Rd., Gary, Ind. 937 Blaine Ave., Racine, Wis. 110 N. Columbia St., Warsaw, Ind. 110 N. Columbia St., Warsaw, Ind. 2968 Tiffin Ave., Des Moines, Iowa .. Burma Rd., Southbury, Conn. 1644 Bauer Ave, Dayton, Ohio 153 10th St., Silvis, III. R.R. No. 2, Belle Center, Ohio 1058 Lester St., Ypsilanti, Mich. R. No. 1, Xenia, Ohio 402 South Grant, Brownsburg, Ind. 750 Kipling St., Akron, Ohio R.R. No. 1, Jamestown, Ohio 2317 De Camp Ave., Elkhart, Ind. R.R. No. 4, Columbia City, Ind. 8202 South Riverside Dr., Marine City, Mich. 61 Boulavard, Newtown, Conn. 314 1st St., Findlay, Ohio 314 1st St., Findlay, Ohio R.R. No. 4, Pana, IIl. Culley, Ronald Eugene ............................. 2029 Helen Dr., Kalamazoo, Mich. Cummings, Marsha Louise Cunningham, Mary M. 521 Ringgold St., Indianapolis, Ind. M. ...................... 100 Carrollton Ave. E., Bradenton, Florida Curcio, Vicki Lee ........................... 1017 Bronson St., Portsmouth, Ohio Curless, Caryl Anne .............................. 1136 North Detroit, Xenia, Ohio Curtis, David Allyn Curtis, Mary Jane 1107 E. 169th St., Cleveland, Ohio .......... Cedarville, Ohio

Damon, Ann Marie Daubert, Russell Eugene Davey, Merry Jessica Davis, Andrew Lewis Davis, Janice Carol Davis, Nancy Lynne Davis, Ray A. Davis, Richard T Dawes, Dennis F Day, Howard L. Day, Juanita June Defibaugh, James DePuy, Dianna Lee Dillon, Donald Dean Dillon, Leslie S. Donaldson, John W. Donovan, Richard L Drill, Gary Lavern DuBois, Janice E. Dudley, Eunice V. Duff, Thomas Michael Duffie, Timothy Alded Dufford, Shirley Belle Duggan, Virginia Arlene
R.D. No. 1, Bemus Point, N.Y. 2303 Oakenwald Dr., Michigan City, Ind. 216 224th S.W., Bothell, Washington 2200 Lilly Dr., Charleston, W.V. Box 224, Mentone, Ind. 601 Rhode Island St., Gary, Ind. R.D. No. 2 Potter Rd, Sherman, N.Y. 119 Lindbergh Ave., Johnstown, Pa. Hamburg Rd., Greenville, $\mathrm{Pa}$. Cedarville, Ohio 2908 Nepperhan Rd., Louisville, Ky. R.R. No. 2 Box 488 , Richmond, Ind. Upper Ferndale Rd., Liberty, N.Y. 499 Walnut St., Cedarville, Ohio 14th St., Burlington, N.J. Box 238, Rochelle, III. 19 Ohio St., Fairborn, Ohio 419 Residence St., Winchester, Ind. 1215 County Rd., Milan, Mich. 3rd St., Roulette, $\mathrm{Pa}$.

719 Staunton Rd., Troy, Ohio 30 W. South St., West Alexandria, Ohio Route 1, Shell Rock, lowa 6965 W. 29th St., Denver, Colo. 


\section{BAPTIST CHILDREN'S HOME-}

BOARD OF DIRECTORS

Rev. Don Worch, Gary, Ind.

Rev. Geo. Badger, LaPorte, Ind.

Dr. Tom Walker, Brownsburg, Ind.

Rev. Will Davis, Gary, Ind.

Rev. Leo Gathany, Highland, Ind.

Mr. Martin Rust, Ft. Wayne, Ind.

Mr. Frank Keifer, Bremen, Ind.

Staff

Rev. \& Mrs. Ernest L. Francis,

Founder \& Supt.

Miss Alice Stroup, Secretary

Mrs. Mary Klinger, Cook

Miss Esther May Handy

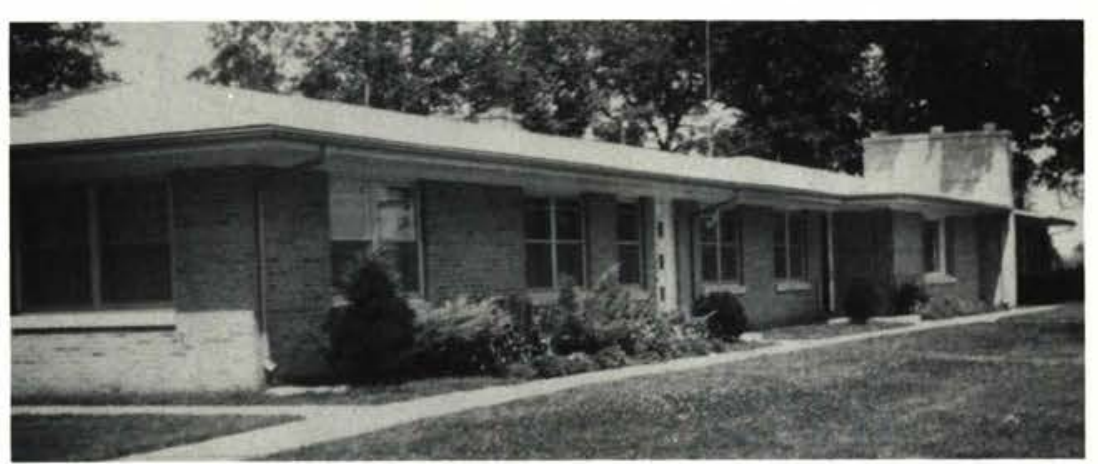

A G.A.R.B. Home for boys and girls who need a Home

\section{CEDARVILLE COLLEGE BOOKSTORE}

Student Supply Center

Bernice Mick, Manager

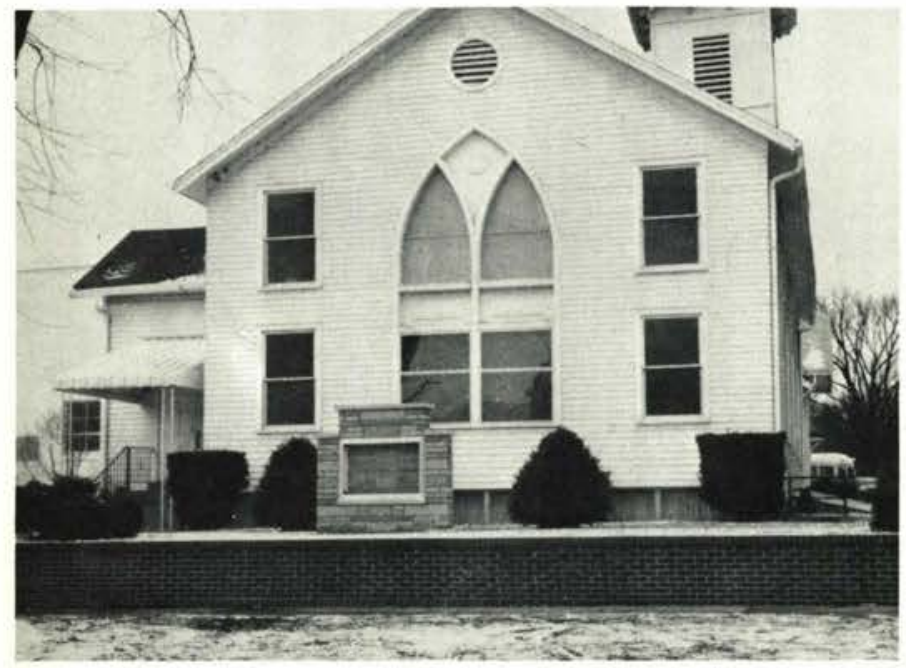

\section{WHEELERSBURG BAPTIST CHURCH}

GALLIA AT SOUTH STREET

WHEELERSBURG, OHIO

THE FRIENDLY CHURCH WITH AN

ETERNAL MESSAGE

DONALD GROLLIMUND, PASTOR 
Dunn, Ruth Damaris

Dunstan, Donald

Durkee, Darlene Ann

Earl, Pamela

Earles, Nancy Carol

Eaton, Jerry M.

Eckert, Louise Susanne

Edmiston, David A.

Edminston, Naomi Ann

Edwards, Dorinne M.

Edwards, George John

Ehnis, Mary E

Elkin, June Ellen

Elliott, Tom

Elmore, David Edward

Emerson, Janice Marie

Engelmann, James Douglas

England, Loren R.

Engle, Sharon Faith

Entner, Donald

Evans, Dawne Elaine

Evans, Diane Elise

Farrell, Mary Kathleen

Felton, Mary Louise

Fennell, Suzanne Kay

Field, Jennifer Lynn

Finley, Gordon Lloyde

Fisher, Gerald A.

Fissel, Nancy Jane

Fleming, Barbara Jean

Floyd, Mary Ellen

Flury, Gerald G.

Ford, George Lee

Foster, Howard Dale

Foulkrod, Lois Beverly

Foulkrod, Thomas E.

Fourman, Nancy Carol

Fox, Loris Walter

Francis, William

Frank, Melvin R.

Frink, Mary Lynn

Frost, Nick ...

Fulkerson, Dorothy Jane

Gann, Wilma Joyce .

Gapinski, Donna Grace

Gardner, Carol Jean

Garlow, David Ellsworth

Gathany, James W.

Gavitt, Eugene Leroy

Geer, Wynona Lee

Geritz, Elizabeth Louise

Gildan, Kathleen Louise

Goethals, James Richard

Good, Patricia C.

Goodwin, George R.

Goodwin, James Warren

Gordon, Edward David

Goss, Joanne Louise

Grant, Ruth Eilene

Greiner, Miriam L.

Griffin, Gordon Donald

Griffin, Sylvia Kay

Griffin, Boyd K.

Griffiths, Jean Penny

Grisso, Philip Danie

Grosh, Barbara Lee

Grosh, Ronald Milton

Guarnere, Donna Grace

Gunlock, James Richar

Hall, Deloris Jean

Halvorsen, James R.

Hamilton, Joanne Helen

Hamilton, Michael Jay

Hammer, Arnold Dayton

Hanson, Carol J.

Haram, David L.
154 E. 214th St., Euclid 23, Ohio R.F.D. No. 2, Randolph, N.Y 9555 Eastern Ave. S.E., Grand Rapids, Mich 10 Garrison Ave., Hasbrouck Heights, N.J. 3332 Hiwood Ave., Stow, Ohio

R. R. No. 2, Rochester, Ind 280 Wayne Dr., Berea, Ohio

322 Shade Dr., Fairborn, Ohio .R.R. No. 1, Wakarusa, Ind Sharon Springs, Schohanie, N.Y 1903 Manchester Rd., Akron 14, Ohio 153 Sherman St., Brooklyn, N.Y

64 Lyndale Dr., Painsville, Ohio 6541 W. Marlette, Marlette, Mich Cedarville, Ohio

12130 Dearmyer Rd., Brooklyn, Mich. 2159 N. Belsay Rd., Flint, Mich. 326 S. Sullivan St., Gary, Ind 406 W. George St., Arcanum, Ohio 301 S. Franklin, Dunkirk, Indiana R.R. No. 1, New Carlisle, Ohio R.R. No. 1, New Carlisle, Ohio 237 W. F St., Phillipsburg, Kansas 1966 Arlene Ave., Dayton, Ohio 507 w. West St., Surgis, Mich. 1708 Park Blvd., CherryHill, N.J. 329 Brand Ave. S.E., Fairbault, Minn. 324 W. Norht St., Lebanon, Ind. Box 116, West Liberty, Ohio 410 N. Maple St., Lancaster, Ohio 409 West Railroad St., Galion, Ohio 18018 Flamingo St., Cleveland, Ohio R.D. No. 1, Turtlepoint, $\mathrm{Pa}$. 2526 Oakwood Dr., Ft. Wayne, Ind. 83 Elm St., Cedarville, Ohio 83 Elm St., Cedarville, Ohio Box 1612, Stow, Ohio R.R. No. 3, Naples, N.Y. R.R. No. 1, Koutz, Ind. 904 3rd St., Lewiston, Idaho 6423 Arizona Ave., Hammond, Ind. 25 Milburn St., Cedarville, Ohio R.R. No. 2, Belle Center, Ohio 2405 West High St., Jackson, Mich. 1627 Sixth St., Peru, IIl. 3507 Welch Dr., Indianapolis, Ind. Bazaricherra P.O., Assam, India 8721 Cottage Grove Ave., Highland, Mich. 1020 Center St. W., Warren, Ohio 2127 Coventry Rd., Cleveland Heights, Ohio R.D. No. , Coudersport, Pa. 318 Eastern Hts. Blvd., Elyria, Ohio 51894 Elm Rd., Granger, Ind. 318 Lamont Dr., Eggertsville, N.Y. 5142 W. 151st St., Oak Forest, Ill. 5142 W. 151st St., Oak Forest, II.

R. No. 2 Box 248XA, Garrettsville, Ohio 806 King St., Bay City, Mich. 2411 Third St., East Moline, IIl. R.D. No. 3, Wellington, Ohio 2741 South 39th St., Lincoln 6, Neb. 3027 Mounds Rd., Anderson, Ind. 502 S. Walnut St., Creston, Iowa R.R. No. 1 Box 3A, Union Grove, Wisc. 1270 Perry St., Springfield, Ohio 14921 Bennett Rd., North Royalton, Ohio 14921 Bennett Rd., North Royalton, Ohio 278 Veterans Highway, Commack, Long Island, N.Y. 426 Bridle Lane, Wheaton, $\mathrm{ml}$. 3877 Pat St., Ypsilanti, Mich. 211 King Ave., Eagle Grove, Iowa 3123 Brown St., Alton, Ill. 1073 Urana Ave., Columbus, Ohio 9313 Peterson St., Rockford, Mich. R.R. No. 1 Box 25, Union Grove, Wisc 116 W. Xenia Ave, Cedarville, Ohio
Harger, Susan E.

R.D. No. 1, Titusville, $\mathrm{Pa}$

Harris, Gary James 247 S. 10th St., Beech Grove, Ind

Harris, Jane Ann 325 Ivanhoe Rd., Waterloo, Iowa

Hartikainen, Ruth

Hartzell, Judith Ann

3437 Peach St., Erie, Penn.

Hartzell, Martin Eugene

Hathaway, Lona Frances

Hay, James Robert

Headlee, Catherine Doris

Healy, Linda Elaine

Heater, William R.

Hendrickson, Rose Emily

Herwig, John Ralph

Hes, Nancy Jeanne

Hes, Sherrill Louise

Hess, John Harvey

Hess, Rebecca Rae

Hiller, Doris May

Hocking, Faith Rebecca

Hoffeditz, Raymond Eugen

Hoffman, Linda Lou

Hollaway, Joseph Eric

Holmes, Dale Alton

Homanick, Beverly Ann

Hooper, Vernon Jones

Howard, Anne :.

Howard, Eleanor

Hoyte, Arlene Elizabeth

Huebner, Glen Melvin

Hull, Charles Olin

Pittsburg Rd., Butler, $\mathrm{Pa}$

646 Pittsburg Rd., Butler, $\mathrm{Pa}$.

824 W. 3rd St., Waterloo, lowa

Box 127, Addis Ababa, Ethiopia 700 Sherwood Dr., Crawfordsville, Ind.

R.R. No. 1, Moscow, lowa

341 Davis St., Findlay, Ohio

R.R. No. 3 Box 122, Cedarlake, Ind. 311 Gulf Rd., Elyria, Ohio 6616 Royalton Rd., North Royalton, Ohio 6616 Royalton Rd., North Royalton, Ohio 19844 Southland Ave., South Bend, Ind. R.R. No. 1, Grundy Center, Iowa 14639 Lakeshore Blvd., Cleveland, Ohio 30 East Evelyn St., Hazel Park, Mich R.R. No. 3, Linton, Ind. 136 State St., Gallipolis, Ohio 320 Deerfield Rd., Columbus, Ohio R.R. No. 1, Litchfield, Ohio 01 East Main St., Fairborn, Ohio R.R. No. 2, Sabina, Ohio Tracer, Iowa

Hume, James Douglas

Hunter, Charles Eugene

Hunter, Sharon Ann

Hussey, Ruth Elizabeth

Jackson, William Paul

Jager, Ted

Jamison, Sharon Lee

Jenista, Frank L.

Jensen, David Richard

Jeremiah, Maryalyce

Jodry, Paul E.

Johns, Susan June

Johnson, Elaine Marie

Johnson, Eunice Gertrude

Johnson, Gary Lee

Johnson, Joyce May

Johnson, Kathy Joan

Johnston, Judith Kay

Jones, David Alan

Jones, Mary Elizabeth

Jones, Merlyn Earl

Kaiser, Sheryl Mae

Kapp, Marian Elizabeth

Kauffold, Ruth Elizabeth

Keating, Janice Ann

Keim, Alan L.

Kellogg, Michael S.

Kensil, Barbara K.

Kettell, Nancy Irene

Kever, Janet Elaine

Kidd, Robert $T$.

King, John William

Kirtland, Wilbur Howard

Kline, John Wilson

Kniffen, Mark W

Knott, Allen Dale

Krueger, Edwin R

Kuhns, Robert Lee

Kunkle, Kenneth Richard

Kytle, Laurene Marye

Lamb, Marilyn Kay

Lamb, Estol Ray

Lane, Lloyd Leon

Diebler Rd., Bucyrus, Ohio Box 424, Whitney Point, N.Y.

3634 N. 24th Place, Milwaukee, Wisc

R.D. No. 4 Box 88 , Cortland, Ohio 3209 Delaney St., Dayton, Ohio Jesup, Iowa

1293 West Forest St., Decatur, III 615 Washington Ave., Niles, Ohio 1505 Canadian Ave., Akron 6, Ohio 2733 Newstead S.W., Wyoming, Mich. G-3540 W. Concord St., Flint, Mich. .1388 Broad St., Bloomfield, N.J.

R.D. No. 2 Smith Rd., Wellington, Ohio Elm St., Cedarville, Ohio West Lynn, Stryker, Ohio 1 Hall St., East Randolph, N.Y 84 Beech Dr., West Palm Beach, Florida. S. Main St., Cedarville, Ohio 409 11th St., Silvis, IIl.

1772 Fay Ave., East Cleveland, Ohio 718 16th St., Seattle 2, Wash 483 Grand St., Galion, Ohio

1138 S. Thomas St., Arlinton, Va

Lane, Susan .........

Lauener, Dale Edward

Lautenschlager, Daphne, J.

R. No. 2 Box 190, Plymouth, Ind. 1553 Winslow Dr., Erie, Pa

R.R. No. 3 Lake Pana, Pana, III.

R.F.D. No. 2, Wausaukee, Wisc

R.D. No. 1 Box 24, Holsobble, Pa 17635 W. Chicago St., Detroit, Mich. 440 S. Westlawn Ave., Decatur, III R.D. No. 1 Star Route, Falls, Pa 9435 N. Bedford Rd., Macedonia, Ohio 1211 E. 93rd St., Brooklyn, N.Y R. R. No. 1, South Vienna, Ohio 481 Northland St., Cuyahoga Falls, Ohio Box 75, South Solon, Ohio 3597 Geddes Rd., Ann Arbor, Mich 


\section{FIRST BAPTIST}

\section{CHURCH}

Main at Lawrence

Mishawaka, Indiana

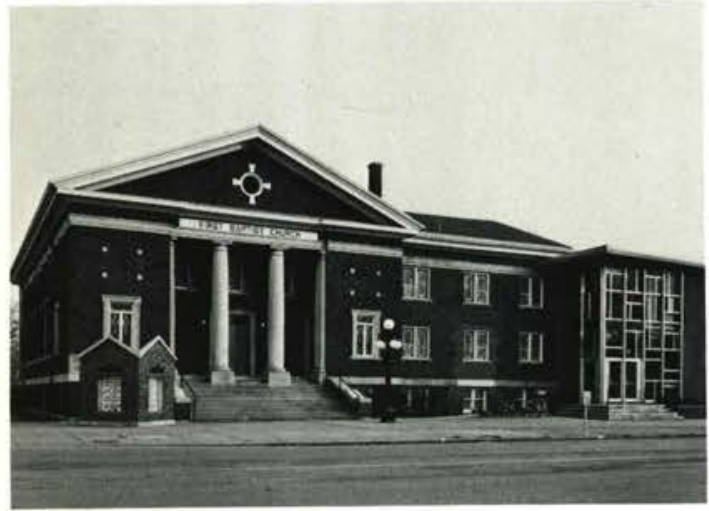

"The Bible as it is for men as they are."
SUNDAY SCHOOL MORNING WORSHIP EVENING WORSHIP WED. VICTORY HOUR THURS. VISITATION
9:25 a.m.

10:30 a.m.

7:30 p.m.

7:30 p.m.

6:30 p.m.
Five youth groups; eight graded choirs; radio and telephone ministries.

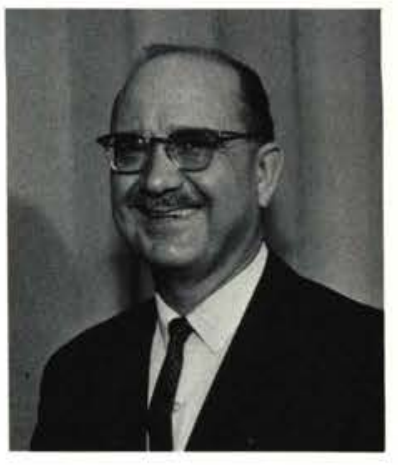

Rev. Roy G. Hamman Pastor

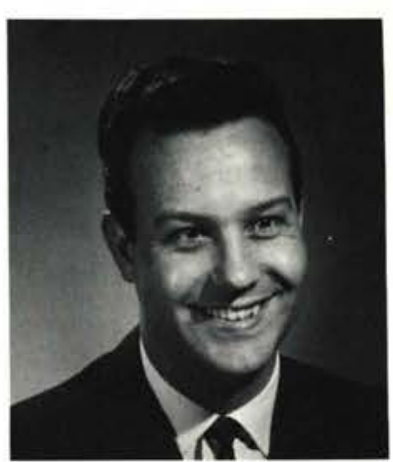

Rev. Robert C. Messener Education, Music and Youth

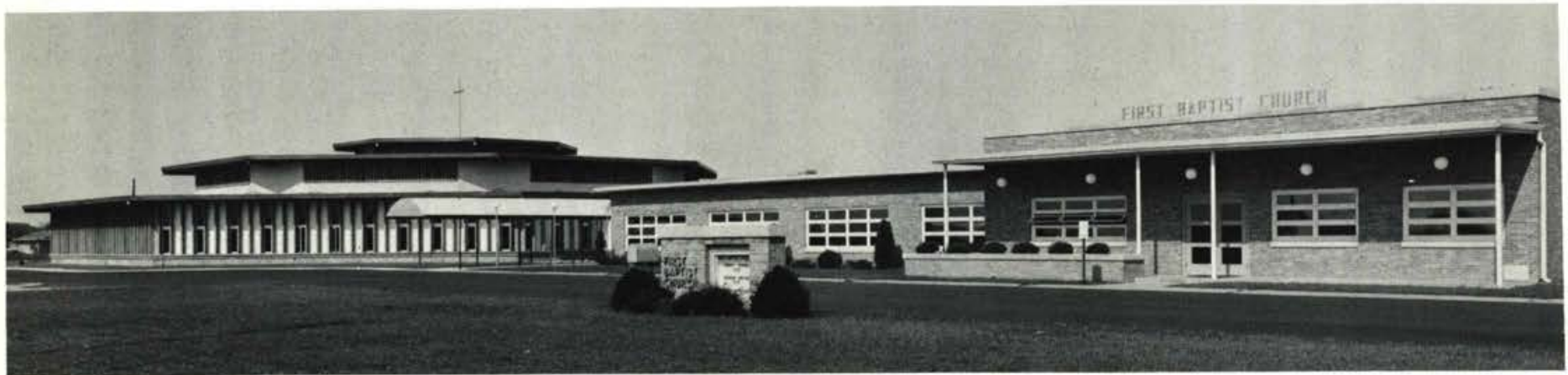

\section{FIRST BAPTIST CHURCH}

Sunday

9:00 a.m. Sunday School

10:15 a.m. Morning Worship

5:00 p.m. Youth Activities, including Choirs

7:00 p.m. Evening Gospel Service

Wednesday 7:00 p.m. Bible Study and Prayer completely departmentalized

8:00 p.m. Adult Choir Practice

\section{Personnel}

Dr. Hugh T. Hall, Pastor

Mr. Donald E. Sandberg, Director of Christian Education

Miss Ruth E. Curran, Secretary and Assistant in Christian Education 
Lawhead, Cheryl Lee

Leach, Donald Paul

Leach, Ronald Kent

Lennox, Jessie M.

Lepine, Suzanne Ruth

Lescure, Lynn Louise

Lewis, Ronald Lester

Lewis, Susan Elaine

Liechty, Gretchen Ruth

Lightly, Douglas Arnold

Linder, Julienne

Lloyd, Kathleen May

Lockhart, Mary Anne

Loops, Herbert Sherwood

Loos, John David

Louwerse, Marsha Marie

Luttrell, Mary Alice

Maclarn, Jean

Maidment, Lee Robert

Mansfield, Kathy

Marsh, Candace Gail

Marshall, Steven Leon

Martens, Francene May

Marvin, Lois Elaine

Mason, Larry Lee

Mayfield, Brian Kent

Mayo, Shelby

Mays, Brian Daved

Mc Caskey, William A.

Mc Donald, David Alan

Mc Dugle, Ronald Eugene

Mc Innes, Roberta Jean

Mc Intosh, Barbara Ellen

Mc Kenney, Roger H.

Mc Pheeters, Buster Mayhew

Mc Vey, William Lee

Mercer, Nancy Ruth

Merryman, Valerie Lee

Merwald, Al Arthur

Meyers, Betty Mae

Micka, Edward John

Micka, Joyce Elaine

Mikels, Carol Joyce

Miller, Douglas Wade

Miller, James E.

Miller, James Richard

Miller, Linda Jean

Miller, Roberta Raye

Miller, Terry Sherman

Millikan, Bonnie

Millikan, Irene Alice

Mines, Herman Clifford

Mitchell, Charles

Mitchell, Lola Louise

Mogle, Claire Ann .

Monroe, Sharon Ann

Montgomery, Lawrence A.

Moore, Joyce Wood

Morgan, Donna Rae

Morgan, Kathleen Ann

Mosely, Beverly Kay

Mowery, Carol Ann

Mundhenk, Luann Gale

Murphy, Bess V. .

Myers, James Arthur

Myers, Leonard Lee

Navorska, Becky Ann

Newman, Robert Wesley

Nichols, Ken Warren

Nims, David Arthur

Norris, Edward A.

Norris, Maynard Ray

Oakley, Hugh Theodore

Ogrodowske, Joseph George

O'Keefe, Rebecca Sweet

Oliver, Donna Louise
R.R. No. 2, Crull Rd., Minford, Ohio 126 Ketay Dr. South, East Northport, N. Y R.R. No. 1, Jamestown, Ohio 5914 Middlebrook Blvd., Cleveland, Ohio 106 Westbrook Dr., Cheektowaga, N. Y. 1545 Chestnut Ave., Haddon Heights, N. J. Box 301, Edmore, Mich. R.R. No. 1, Bainbridge, Ohio 1359 S. Lake Park, Hobart, Ind

R.R. No. 3, Box 184, Austin, Minn R.R. No. 2, Jesup, lowa 3403 13th St., Racine, Wisc 1226 Mayo St., Sciotoville, Ohio Ventura, Iowa

307 E. Grove St., Mishawaka, Ind 1221 Riverside, Decatur, III 1838 Highview Ave., Akron 19, Ohio Box 38 Wyandot Ave., Harpster, Ohio 5208 Bennett Rd., Toledo, Ohio 5191 Broadway St., Lorain, Ohio R.D. No. 4 Hereford Rd., Corry, Pa. 118 W. Grant St., Bremen, Ind. 1818 Fairwinds Dr., Cedar Falls, Iowa . R.R. No. 3, Grant, Mich. R.R. No. 1, Carlisle, Ohio R.R. No. 2, Liberty, Ohio 1025 Sheffield Dr., Mason, Ohio 249 East 317th St., Willowick, Ohio 233 S. Davis Ave., Audubon, N.J. 3365 Lake George, Lake Orion, Mich. . 519 Barber St., Pontiac, III. R.R. No. 3, Columbia City, Ind. 215 Palmer Dr., Cedarville, Ohio 15355 Evergreen St., East Detroit, Mich. 624 Kentucky St., Quincy, III. 2733 N. 52nd St., Kansas City, Kansas 3701 N.E. 16 th Ave., Pompano Beach, Flor. 1137 Queen Ann Rd., Teaneck, N.J. 2555 Willowdale Rd., Portage, Ind. 4336 7th St., East Moline, III.

123 Paulison Ave., Ridgefield Park, N.J. 123 Paulison Ave., Ridgefield Park, N.J. $6102 \mathrm{~N}$. Dearborn St., Indianapolis, Ind. 336 Columbus Park Rd., Cedarville, Ohio 2456 Richwood St., Pontiac, Mich. R.R. No. 2, Box 32, Valparaiso, Ind. 3282 Revlon Place, Dayton, Ohio 6570 McGuffey Rd. Ext., Lowellville, Ohio 420 Fraser St., Bay City, Mich. 226059 th St., Bothell, Wash. 22605 9th St., Bothell, Wash.

1601 E. 94th St., Cleveland, Ohio 40 Highland St., Sharon, Mass. 2113 North Phillippi St., Boise, Idaho 302 N. Detroit St., Xenia, Ohio 411 Sturgis Ave., Sturgis, Mich. .350 Tibet Rd., Columbus, Ohio 77 Sunset Ave., Mattituck, N. Y. 207 S. Joslin St., Charles City, Iowa Box 199 R.D. No. 4, Belle Vernon, Pa. 2530 Grace St., Melvindale, Mich. 638 Pendley Rd., Willowick, Ohio Box 136, Pitsburg, Ohio 148 Larrimer St., Crane, Ind 671 High St., Van Wert, Ohio . 6 Warren St., Marathon, Ohio 6985 Lewis Rd., Olnsted Falls, Ohio 1080 Laffyette Ave., Hawthorne, N.J. 237 Euclid Ave., Byerville, Ohio Nelson Star Route, Keene, N. H. R.R. No. 3, Box 121, Rochester, Ind. R. R. No. 3, Box 121, Rochester, Ind. 416 Cronkright St., Midland, Mich. 9 Franklin St., South River, N. J. 1203 Brookview Blvd., Parma 34, Ohio .. 2580 Kunz Rd., Columbus, Ohio
Olsen, Dorothy Anne Olson, Sheryl Joan

Olson, Steven Bradley

Osborne, Joseph Irwin

O'Shell, Suzanne Elizabeth

Oswald, James Oliver

Otto, Shirley Marie

Patterson, Karen Eileen

Payne, Charlotte A.

Pearce, Dennis John

Pereira, Manny

Perkins, David Arthur

Perrin, Bonita Lou

Pestel, Gary Michael

Petrie, Juliann

Pettitt, Richard Norman

Phelps, James R.

Phillips, Judy Evyonne

Phipps, James Ronald

Phyillaier, John R.

Pierson, William Morris

Plough, Dianna Kay

Prather, Eugene Kenneth

Preston, Donna Lee

Price, Dan Lee

Price, Nedra Ann

Pritchett, Dale Robert

Prosser, David Harvey

Randall, Barbara Lee

Rathfon, Yvonne Sue

Ream, G. Eugene

Reed, Charles Robert

Reed, David Charles

Renfrow, Linda Lou

Reno, John Paul

Rich, Richard Lee

Richardson, Gerald Herbert

Richardson, Lawrence Clark

Riggs, Linda Sue

Rigney, Harold Dean

Robinson, Richard Wayne

Rockwell, Denis Ross

Roden, Joseph George

Roderick, Richard Arden

Rogers, Jean Ann

Rohm, Robert Karl

Roloff, Marston Val

Ronk, Phyllis Joanna

Rose, Bonnie Carol

Rose, Russell Lee

Ross, Randall Alan

Ross, Rebecca Ann

Rotondi, Tony V.

Rudduck, Joyce Lynn

Ruder, Esther L

Rulison, Mary Lisa

Ryan, Colleen Mae

Sadler, Paula Mae

Sammons, John $\mathrm{H}$.

Satterblom, Sheryl Rae

Schaeffer, James William

Schimkus, Richard C

Schlechty, Sandra Jan

Schonscheck, Marlene Jean

Schulte, Mary Ellen

Schultz, Joan Elizabeth

Schwanbeck, Carl Arnold

Schwebel, Kathie Ruth

Scott, Carol Sue

Scovell, Patty Ann

Searles, Marshall William

Seeley, Jeannette Kathleen

Sesslar, Fay Joan

Sharpless, Larry Dean

Shaw, Lavonne K.

Shearer, R. Byron

263 Oakdale Rd., Berea, Ohio 9461 Lincoln Blvd., Indianapolis, Ind 9641 Lincoln Blvd., Indianapolis, Ind

R.R. No. 2, Box 214, Wheelersburg, Ohio 8970 Lindberg Blvd., Olmsted Falls 38, Ohio R.R. No. 5, Millerburg, Ohi Carroll, lowa

R.R. No. 3, Coshocton, Ohio 4214 South Logan St., Englewood, Colorado R.R. No. 2 Box 417, Stanwood, Wash 50 East Northport Rd., Kings Park, N. Y 499 Center St., Xenia, Ohio .4191 West 23rd St., Cleveland, Ohio 516 Sabin Ave. E., Ladysmith, Wisc 594 Allen Dr. R.R. No. 2, Highland, Mich 510 W. Front St., Florence, N.J. . 30 Jutland Rd., Binghamton, N.Y 2805 S. $11 \frac{112}{2}$ St., Terre Haute, Ind. 598 Walnut Ave, Red Bluff, Calif 206 High St., Pleasent, Ohio

14 Ardmore Terrace, Collongswood, N.J R.R. No. 1, Wabash, Ind .. 909 Hartz Dr., Lebanon, Ohio 3705 135th St., Belleville, Wash 5339 Lindburg Blvd., Dayton, Ohio 384 Olympic Ave., Buffalo, N.Y 249 W. Summit Ave., Haddonfield, N.J. 1497 North Rd. S.E., Warren, Ohio 615 Scheurmann St., Essexville, Mich R.R. No. 2, Rochester, Ind 512 14th St., Hampton, III 322 Fitzgerald St., Oglesby, III 89 Eastwood Dr., Norwalk, Ohio R.R. No. 3, Indianola, Iowa 280 Bridge St., Cedarville, Ohio R.R. No. 3, St. Louis, Mich R.R. No. 2 Box 263 Zoor Rd., Morrow, Oh 29015 Five Mile, Livonia, Mich 1410 Meadowlawn Dr., Northfield, Ohio 2504 East Spring St., New Castle, Ind 2493 Northville Dr., N.E., Grand Rapids, Mich. 2637 E. Walker Ave., Indianapolis, Ind R.D. No. 1, Dorset, Ohio R.R. No. 2, Warsaw, Ind

7 First Ave., Haddon Heights, N.J. 243 Maple Ave., Audobon, N.J. 602 Fourth St., Charles City, lowa 2415 Aberdeen Rd., Richmond, Va. R.R. No. 4, Crawfordsville, Ind R.R. No. 4, Crawfordsville, Ind 42 Linwood Ave., Jamestown, N.Y 42 Linwood Ave., Jamestown, N.Y 323 Ogden Ave., Teaneck, N.J. R.R. No. 2, Cedar Falls, Iowa 42 College St., Cedarville, Ohio R.D. No. 2, Amsterdam, N.Y 1715 Bigelow St., Toledo, Ohio 402 West Lynn St., Stryker, Ohio Kermit, W. V

3544 43rd Place, Highland, Ind 2840 Lantz Rd., Xenia, Ohio 216 N. Central Dr., Dayton 19, Ohio 17020 Lakewood Hts. Blvd., Lakewood, Ohio 16188 Thomas St., Allen Park, Mich 


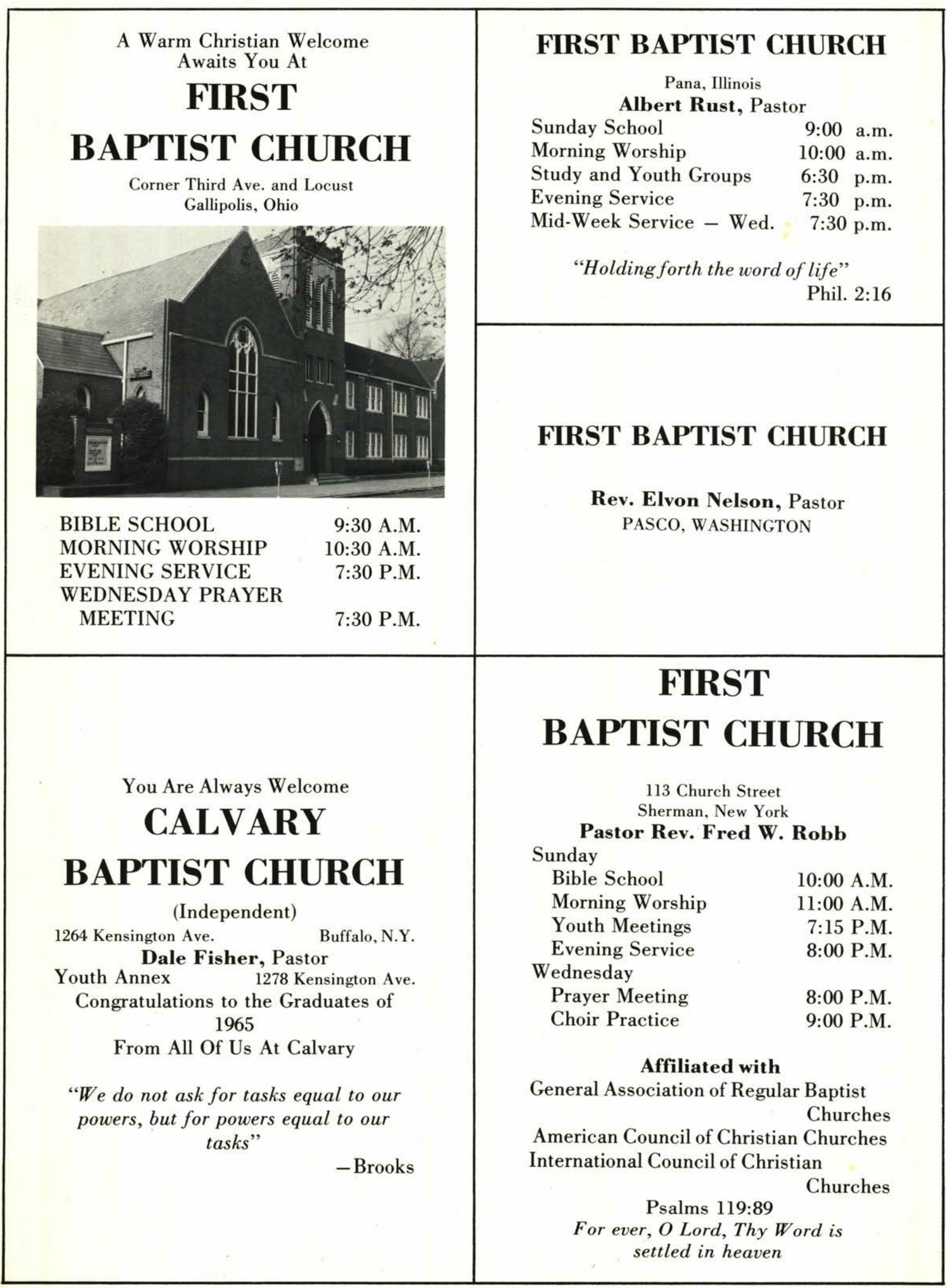


Shelton, James 312 E. Clinton St., Howell, Mich Shepard, Verna Del 211 2nd St. S.W., Waverly, lowa Shriver, Allen Madison Ursa, III. Shubert, Keith Allen Shue, Donald 0 . Shufelt, Gary Lee Shug, Elma Kay R.R., Saunemin, Il 5834 Elden Dr., Sylvania, Ohio Simpson, Donna Sue ........................ 2215 Churchman St., Indianapolis, Ind Skillman, Jon Charles ......................... 237 Homer St., Marion, Ohio Skinner, Perry L. ............................. $500 \mathrm{S.} \mathrm{Mulberry} \mathrm{St.,} \mathrm{Creston,} \mathrm{Iowa}$ Smith, Beryl R. ............................... 4128 Washington St., Gary, Ind Smith, Douglas Courtney ............................... 700 Ridge Ave., Bucyrus, Ohio Smith, Eddy Scott Washington St., Randolph, N.Y. Smith, Nancy Lee .............................. 411 Main St., Cedarville, Ohio Smoker, Jane Emily Snider, Joseph Bernard Spear, Judy Ann 2915 Peoria St., Elkhart, Ind 2071 McCoy Rd., Barberton, Ohio 7998 Deckerville Rd., Decker, Mich Spears, Susan Elizabeth ................... 26207 Harmon St., St. Clair Shores, Mich. Spinks, Linda Lee ..................................... R.D. No. 1, Sherman, N.Y Spong, Kathleen Ruth .......................... 7185 Butternut Lane, Mentor, Ohio

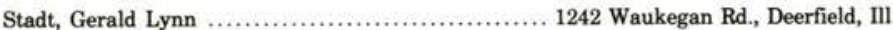
Stafford, Gerald Edward ....................... 109 Camrose Ave., Baltimore, Maryland Stanberry, Maxine .............................. 4110 Harvard Ave., Peoria, III. Stanberry, Pauline Fay ......................... 4110 Harvard Ave., Peoria, Ill

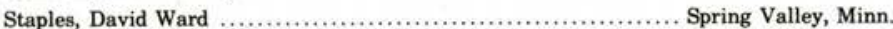
Stats, Nicki Rae ..................................... Box 300, Cambridge, Ohio

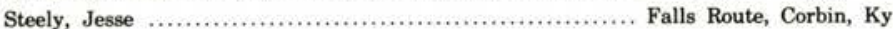
Stephens, Don Elbert R. No. 1, McMillan, Mich. Stephens, Marvin Wayne ................... 1254 Lewison N.E., Grand Rapids, Mich Stillwell, Craig Richard ............................. 94 Roosevelt Blvd., Oakland, N.J. Stockwell, John C. ................................ 117 Elm St., Cedarville, Ohio Stockwell, James Earl ................................ Box 344, Cedarville, Ohio Stone, Cheryl G. .............................. 18205 Fern Lane, Walton Hills, Ohio Stout, Roger ........................................... Springfield, Ohio Stowell, Joseph Mishael .......................... 471 Summit Ave., Hackensack, N.J. Stutesman, Louise Elaine .........................113 S. Maumee St., Tecumseh, Mich. Stutzman, Ronald Lee ................................... Box 155, Berlin, Ohio Swank, Linda Lou .............................. 112 Shawnee Dr., Greenville, Ohio Sweeney, Adrian David ............................ Folkstone Way, Cherry Hill, N.J. Tallman, Sharon Kay ....................... 405 SW. 8th St., Hallandale, Fla Tartar, Opal Jean ............................ 18276 Inkster St., Romulus, Mich. Taylor, David Earl .......................... 505 E. Main St., Blanchester, Ohio Taylor, Delmer Omrie ................................. 38 Knox St., Ecorse, Mich. Taylor, Gordon Allen .................................... Steamboat Rock, Iowa Taylor, Karen Claire ...................... 4560 Dayton-Liberty Rd., Dayton, Ohio Terrill, Douglas T. ........................................ Fabius, N. Y. Thompson, Brenda Lee ........................ 830 S. Terrace Ave., Columbus 4, Ohio Thompson, Connie L. ............................ 3363 Greensburg Rd., N. Canton, Ohio Thompson, Jennifer Sue .............................. 4130 Hudson Dr., Stow, Ohio Thompson, Pat Mae ................................. 1101 N. Hazel St., Pontiac, Ill. Thompson, Beth Elaine ........................ 18610 N.W. 8th Rd., Miami, Flor Thorne, Arthur David .............................. Stokes Rd., Medford, Ohio Thorne, Barbara A. ....................................... Stokes Rd., Medford, Ohio Timmons, Howard Edwin .......................... 638 w. Main St., Blanchester, Ohio

Titus, Anna ................................... City Mounted Rd., Crawfordsville, Ind. Tompkins, Gail L. Traucht, Al Wayne Tremba, Janet Gayle Trimble, Glen Mark Trimble, Grace Ann R.R. No. 2, Sherman, N.Y.

R.R. No. 2, West Liberty, Ohio 568 Sherwood Ave., Youngstown, Ohio Caixa 372, Manaus, Amazonas, Brazil Caixa 372, Manaus, Amazonas, Brazil
Truxton, Charles G.

Underwood, Norman Lee

Vance, Sharon Elaine

Vanderground, David John

Van Horn, Janice Gale

Versluis, Patricia Madge

Vincent, Beverly Reynolds

Von Hagel, Charles Paul

Votaw, Robert Howard

Wahl, Dona Louise

Walborn, Jon B.

Walker, Leland

Waite, Larry

Walker, Roosevelt

Wall, David Roy

Wallis, Eugene

Walthall, Gary

Ward, Cornelia A.

Ward, Diana Louise

Wareham, Jane $\mathrm{H}$.

Warnken, Frank A

Watson, John H

Webster, Vicki Joy

Welch, Loren Melvin

Werner, Carol Jean

Werner, David Arthur

Werner, Marlene Kay

Westcott, Sharon Lee

Wilhite, David T.

Wilhite, Paul Jay

Wilkins, Charles L.

Williams, Gay Lynn

White, Janice Joan

Whiting, Dean Allen

Whitmire, James William

Wickell, E. Geraldine

Wilcoxson, Donald Thaine

Williams, Lawrence $\mathrm{H}$.

Williams, Sarah Esther

Willsie, Margaret Jane

Wilson, Michael

Wing, Helen Rae

Wing, Sylvia Ingrid

Wing, Twila Grace .

Wintrow, Linda Carol

Wood, Dennis Fredrick

Wood, Joyce A

Woodend, Michael Joseph

Woodman, David A.

Wymer, Ralph Ronald

Wyse, Vera Geraldine

Young, Maxine

Young, Linda Lou

Young, M. Louise

Zanstra, Richard

Zeigler, James D.

Zemek, Paul Titus

Zeigler, Beth Louise

Ziegler, Mervin Lee

Zills, Helen Ruth

Zimmerman, Barry Kent
511 Graisbury Ave., Haddonfield, N.J. 36 Jones St., Dayton, Ohio

220 Pole Lane Rd., Marion, Ohio 4115 Warrensville Rd., Cleveland, Ohio R.R. No. 3, Plainfield, III 097 Jahnson N.W., Grand Rapids, Mich 139 Brunck Rd., Lancaster, N.Y 1339 Harshaw Rd., Chester, Pa R.R. No. 1 Box 252, Decatur, III

143 Main Ave., Warwick, R.I 13716 Superior Rd., East Cleveland, Ohio R.D. No. 2, Carlisle, Iowa 5248 32nd Ave., Hudsonville, Mich. 61 Church St, Cedarville, Ohio 360 N. 34th St., Decatur, III Box 78 Thurston, Ohio 207 Dayton Ave., Xenia, Ohio .. R.D. No. 1, Collins, Ohio

15716 Oakhill Rd., East Cleveland, Ohio 173 West Main St., Sherman, N.Y. 70 Herb Stickel, Wakarusa, Ind. 433 E. Mishawaka Ave., Mishawaka, Ind. 13 Lincoln Dr., Cambridge City, Ind. 14 Plum Ave., Shelby, Ohio Box 108 R.D. No. 1, New London, Ohio 819 9th St., Columbia Falls, Mont. 819 9th St., Columbia Falls, Mont. R.R. No. 3, Hanover, Pa R.R. No. 2, Corwith, Iowa R.R. No. 2, Corwith, Iowa 3310 Westline Dr., Columbus, Ind 382 Hendon Circle, Cuyahoga Falls, Ohio 4313 Oriole Dr., Jackson, Mich. 3278 E. Clineon, Des Moines, Iowa 421 North Duffy Rd., Butler, Pa South 3rd, Canton, Miss R.R. No. 3, Villisca, Iowa 518 S. West St., Greenfield, Ind. 694 E. 124th St., Cleveland, Ohio ... Freeland, Mich 402 East St., Rochester, Mich 2038 La Crosse St., Wyoming, Mich. R.D. No. 1, West Winfield, N.Y R.D. No. 1, West Winfield, N.Y. R.F.D., Carlisle, Iowa 1107 th St., Clarkston, Wash 10 Mesa Way, Alexandria, Va. 215 32nd St., Bay City, Mich . 5602 Palm River Rd., Tampa, Fla Cedarville, Ohio 1024 S. College Ave., Mt. Pleasant, Mich 1024 S. College Ave., Mt. Pleasant, Mich 900 East Main St., Albion, Ind 2213 Stafford S.W., Grand Rapids, Mich. 708 S. Whitman Dr., Turnersville, N.J. . 5 Park St., Binghamton, N.Y. R.D. No. 2, Wellington, Ohio R.D. No. 2, Wellington, Ohio Box 124, Silver Lake, Ind.
Jeremiah, James T., D.D.

Ager, Merlin, M.S.

Bartholomew, Raymond E., Ph.D

Baumann, Donald, P., Ph.D.

Bergen, Harmon, B.A

Callan, Donald, M.S.

Chaffe, Alberta L., B.A

Dowell, Max Boyd, M.S.

Elmore, Austin D., M.A.T

Fisher, Jean, M.A.

\section{Faculty}

Frye, Bert L., M.A

Gale, Ralph B., M.A.

Greenwood, Edward L., B.A.

Gromacki, Robert, Th. M

Johnson, Clifford W., D.Ed.

Johnson, Howard R., B.A.

Kearney, June F., B.S.

Lawlor, George L., Th.D.

Mackay, Joy, M.A.

Maddox, Clifford R., Ph.D.
Maddox, Miriam B., B.A.

McDonald, Cleveland M., M.Litt.

McIntosh, Richard T., Th.M.

Meissner, Walter, M.A.

Moody, Lane W., M.A.

Packard, Carla, M.A.

Reed, John William, M.A.

Russell, J. Emerson, M.A

Smith, Ruth E., M.Mus
Spencer, Edward E., B.A.

St. Clair, Kenneth, M.S., C.P.A.

Thomson, Dale Stirling, M.S.

Thornton, William D., M.A

Webber, Ardeth, B.A.

Webber, Warren L., M.M.E.

Wetzel, Daniel E., M.S

Williams, Arthur Franklin, D.D.

Wyse, Rodney E., M.B.A. 


\section{CALVARY BAPTIST CHURCH}

COVINGTON, KENTUCKY

Warren W. Wiersbe, Pastor

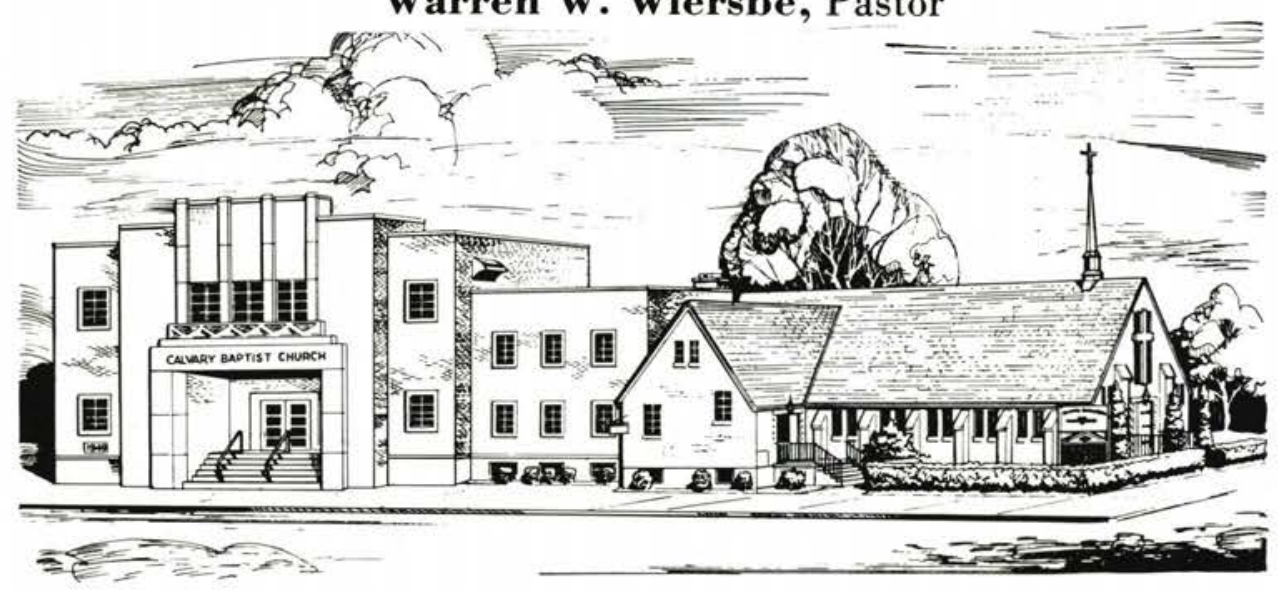

^ TEACHING THE WHOLE BIBLE * MINISTERING TO THE WHOLE FAMILY

Calvary Baptist Church is the home of the "Whole Bible Study Course" developed by Dr. D. B. Eastep, who pastored the church for thirty-five years. The course takes the student through the Bible, book by book and chapter by chapter in seven years.

Calvary Book Room, the literature ministry of the church, publishes and distributes true-to-the-Bible literature across the world.

Write for free information and book list.

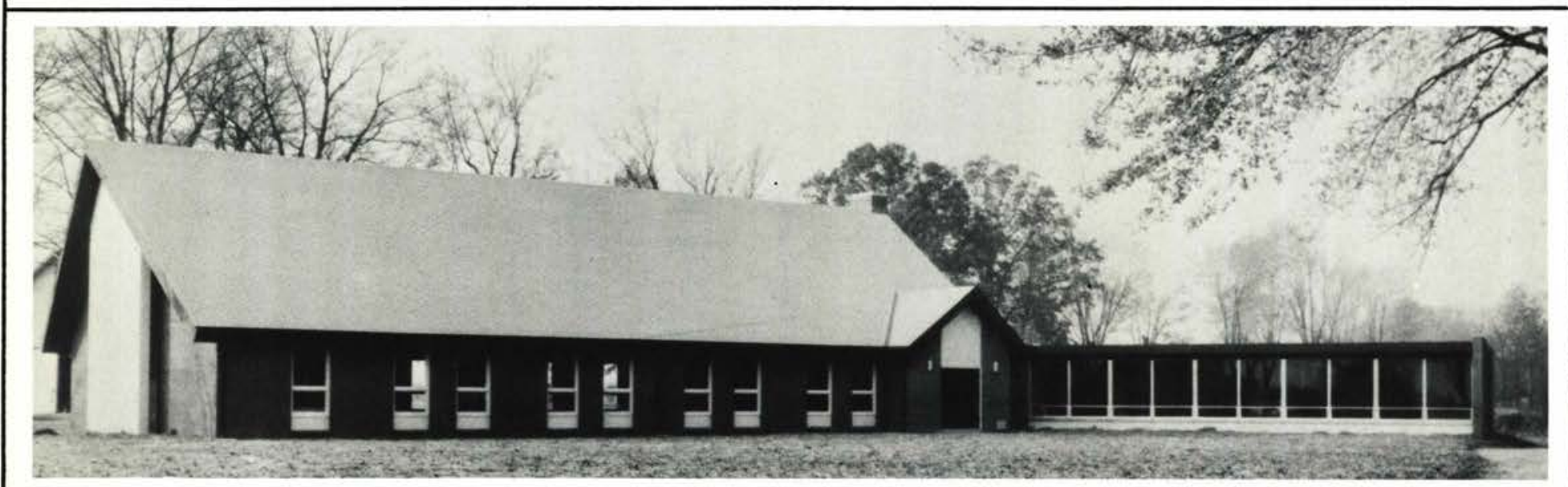

\section{CALVARY BAPTIST CHURCH}

NORWALK, OHIO

Best Wishes to Class of ' 65

And to Our First Graduate

CORNELIA ANN WARD

1 Corinthians 10:31 


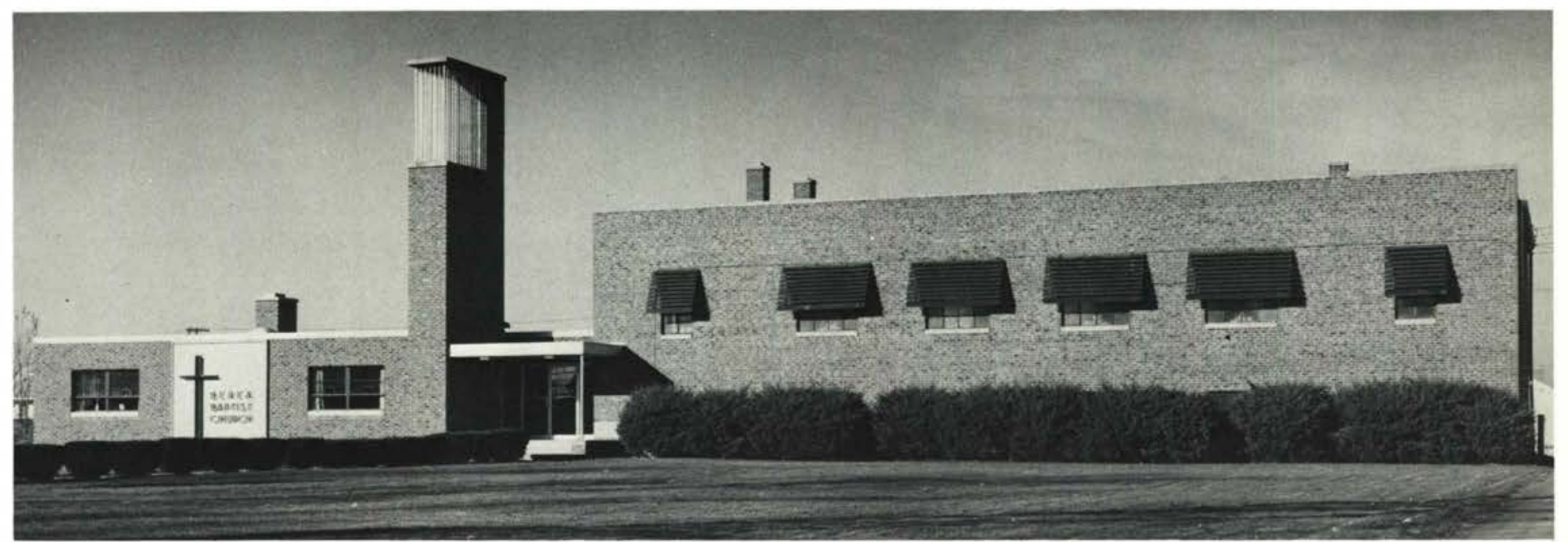

\section{BEREA BAPTIST CHURCH}

250 West St., Berea, Ohio

You Are Cordially Invited to Attend Our:

$\begin{aligned} \text { NURSERY } & \text { Bible School at } & \text { 9:30 A.M. } & \text { AMPLE } \\ \text { FACILITIES } & \text { Morning Worship at } & \text { 10:30 A.M. } & \text { PARKING } \\ & \text { Youth Hour at } & 6: 30 \text { P.M. } & \\ & \text { Evening Worship at } & 7: 30 \text { P.M. } & \\ & \text { and Wednesday Prayer Meeting at } & 7: 30 \text { P.M. } & \end{aligned}$

In Fellowship with the General Association of Regular Baptist Churches

\section{TEMPLE BAPTIST CHURCH}

Portsmouth, Ohio

Otis R. Holmes, D.D., Pastor

Rev. Billy Carl Rice, Minister of Visitation

SUNDAY SERVICES

9:15 A.M. Bible School

10:30 A.M. Morning Worship

Broadcast over WNXT, Portsmouth (1260 on your dial)

5:30 P.M. Broadcast over WPKO, Waverly, Ohio ( 1380 on your dial)

6:30 P.M. Youth Groups

7:30 P.M. Evening Services

9:30 P.M. Delayed broadcast of evening service over WNXT, Portsmouth "TIMELY TRUTHS FOR TRYING TIMES" 


\section{CONGRATULATIONS}

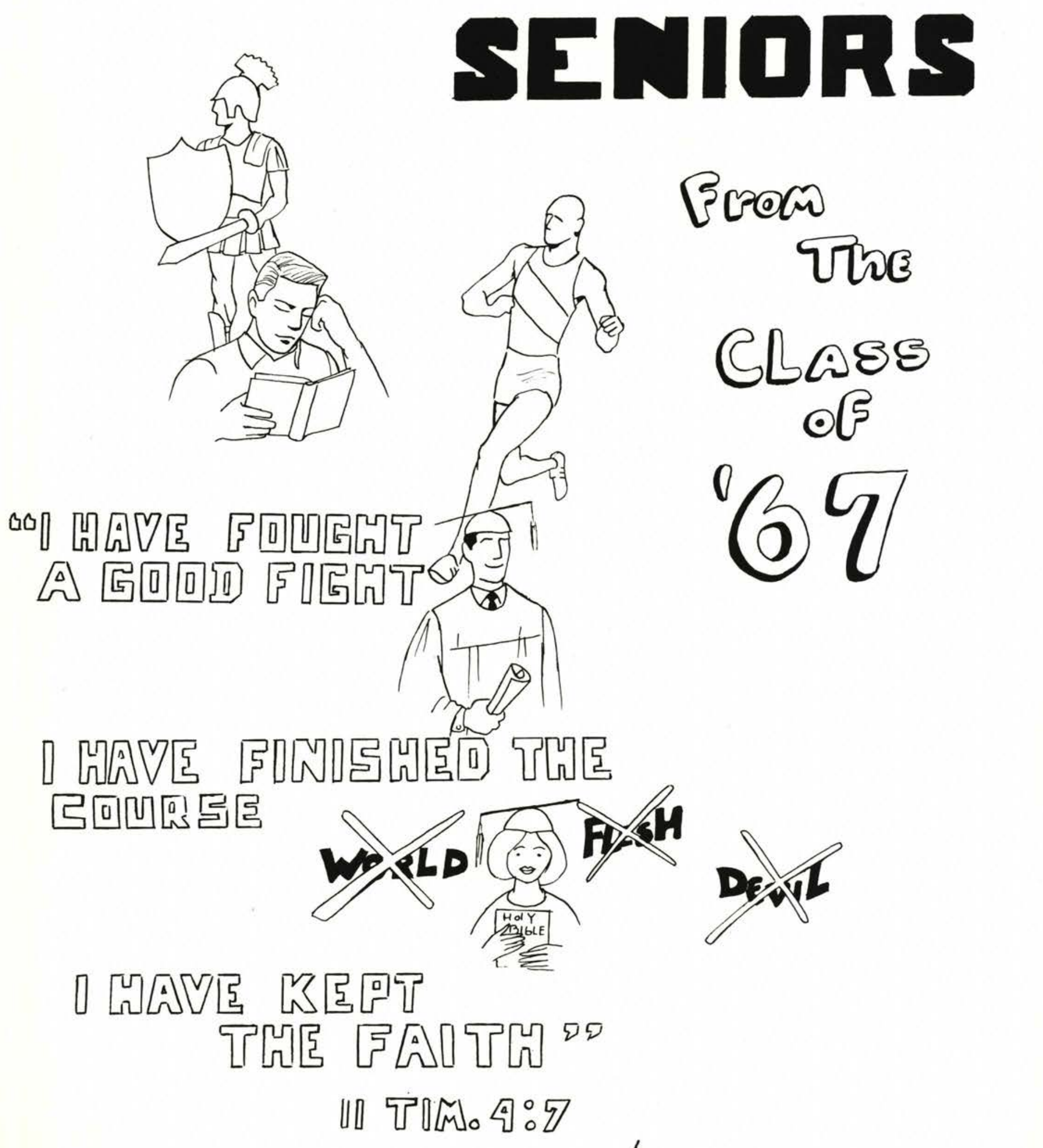




\section{CLINTONVILLE BAPTIST CHURCH}

35 Oakland Park Avenue

Columbus 14, Ohio

\section{Founded on THE WORD of GOD \\ Grounded in THE WORD of GOD \\ Bounded in THE WORD of GOD}

“CLINTONVILLE BAPTIST CHURCH CONGRATULATES CEDARVILLE GRADUATES AND COMMENDS CEDARVILLE COLLEGE TO ONE AND ALL."

Glenn H. Davis, Pastor

\section{PENFIELD}

\section{JUNCTION}

\section{BAPTIST CHURCH}

5046 Broadway

Lorain, Ohio

Norman E. Hoag, Pastor

9:30 AM - . . . Sunday School

10:30 AM - . . . Morning Worship

Beginner Church

Primary Church

6:00 PM - . . . Youth Groups

7:00 PM - . . . Evening Service

7:00 PM -... Prayer Meeting

Wednesday

OUR STUDENTS ATTENDING

CEDARVILLE

Margaret Katherine Mansfield

Daniel Cain

\section{FIRST BAPTIST CHURCH}

Galion, Ohio

Rev. Wilfred Booth

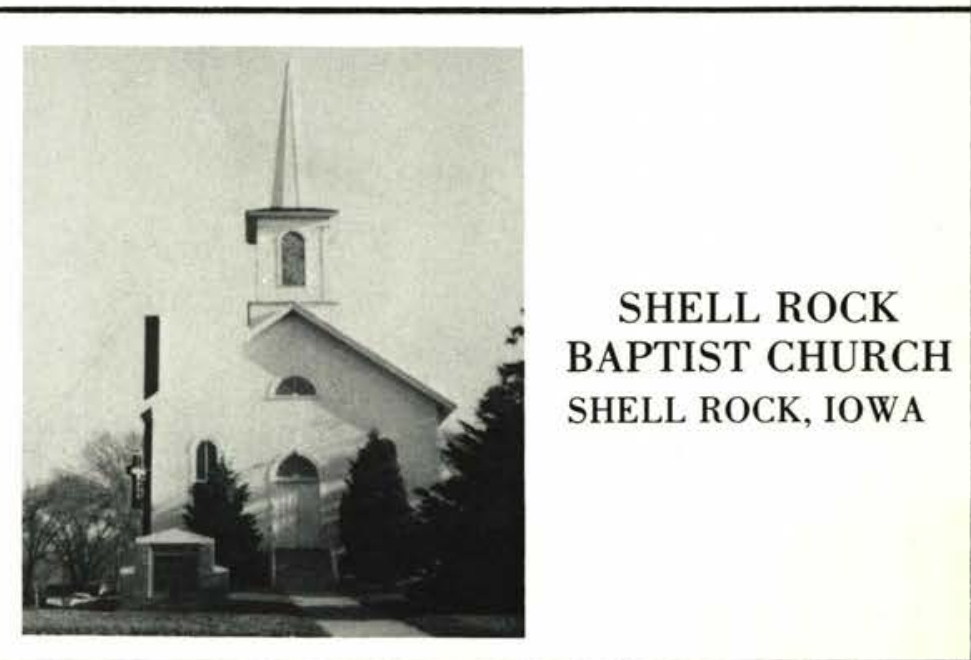




\title{
HADDON HEIGHTS BAPTIST CHURCH
}

\author{
THIRD AND STATION AVENUES \\ HADDON HEIGHTS, NEW JERSEY \\ "Holding forth the Word of Life"
}

REV. K. W. MASTELLER, Pastor

F. D. HANZLER, Minister of Christian Education

Sunday

- Weekly Schedule of Events -

$$
\begin{aligned}
& \text { 9:45 A.M. - Bible School } \\
& \text { 11:00 A.M. - Morning Worship and Children's Churches } \\
& \text { 6:30 P.M. - Six Thriving Youth Groups } \\
& \text { 7:30 P.M. - Gospel Service }
\end{aligned}
$$

Wednesday 7:45 P.M. - Prayer Meetings (Youth and Adults)

Friday $\quad$ 7:30 P.M. - Youth Activity Night

Saturday ALL DAY RECREATION PROGRAM IN RECREATION ROOM

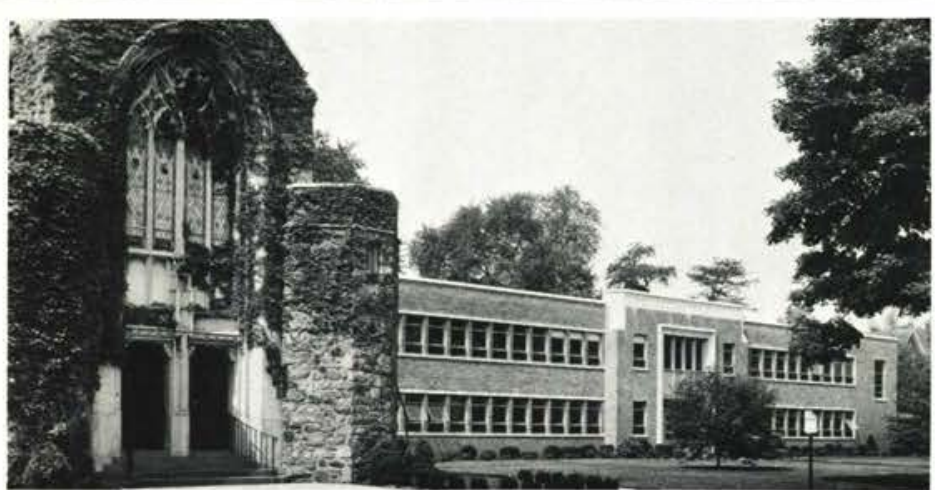

FIRST BAPTIST

\section{CHURCH}

Anderson Park Hackensack, N. J.

JOSEPH M. STOWELL M.A.

D.D., Pastor

CHARLES S. WILKES, M.S.

Minister of Music

WARREN E. SIMMONS, B.R.E.

Christian Ed. Director

LAWRENCE G. FETZER, Th. M.,

Youth Director

"We Believe in Cedarville's Ministry"
SINCERE CHRISTIAN WISHES

to the

Graduating Class of 1965

HUNTSBURG

BAPTIST

CHURCH

Huntsburg, Ohio

Ben H. Garlich, Pastor 


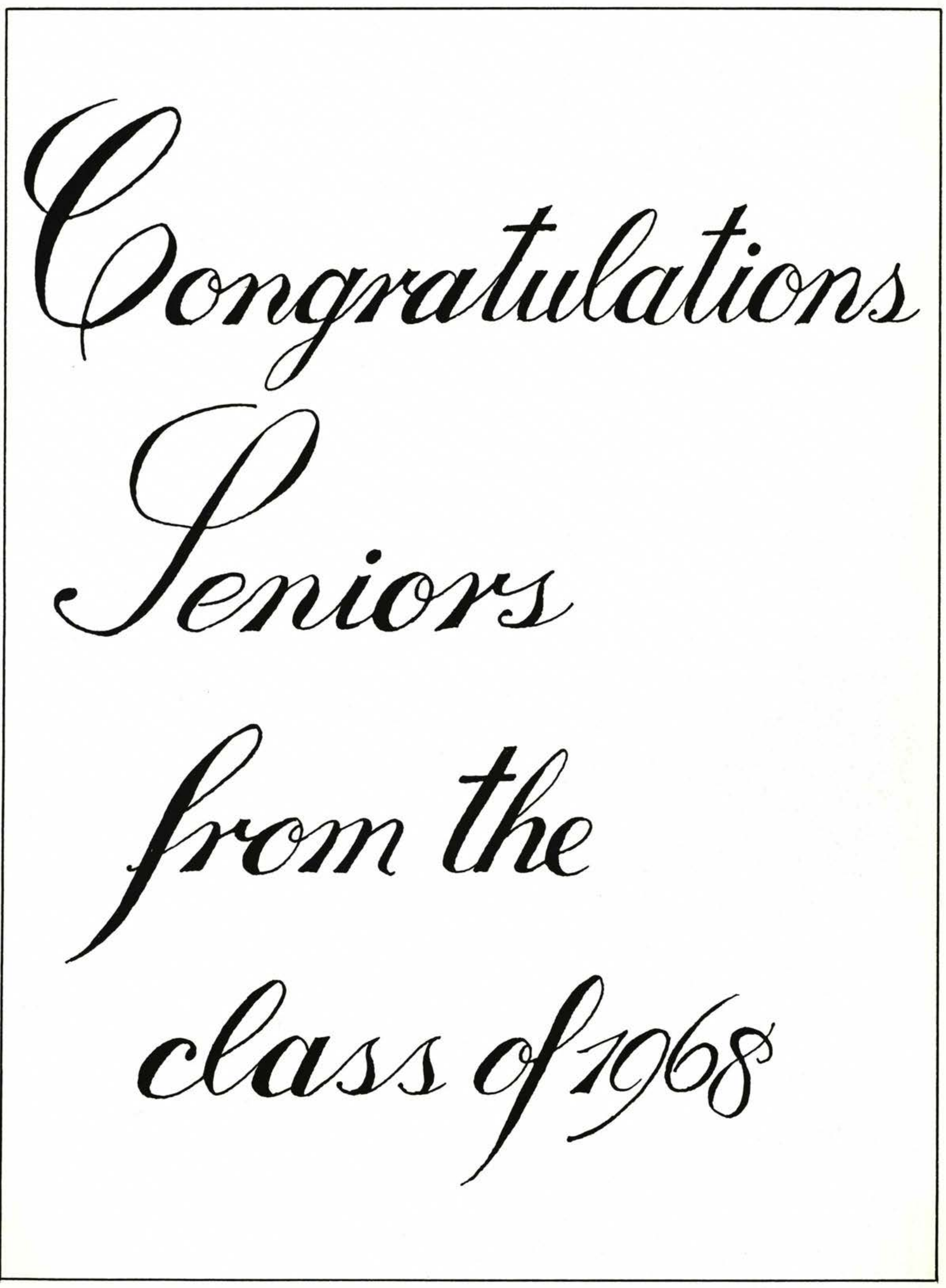




\section{BETHEL \\ BAPTIST CHURCH}

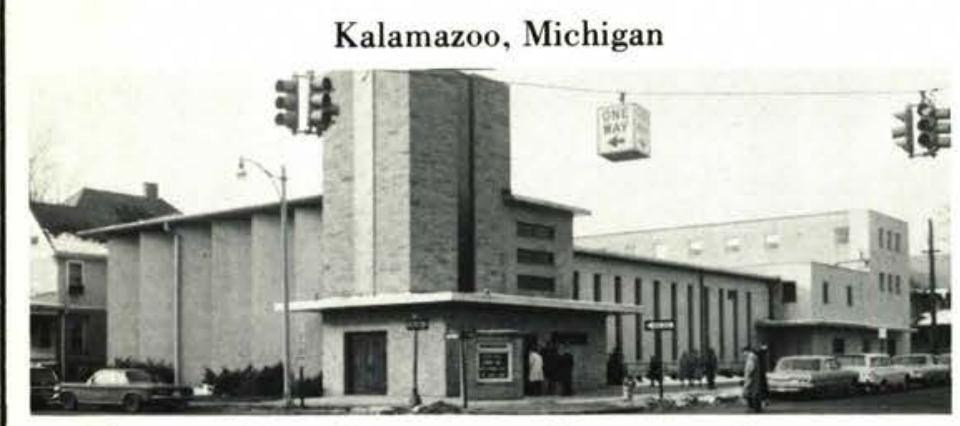

We extend to all a sincere Christian welcome. As you travel in the southwestern Michigan area, be sure to plan your schedule so that you can visit us for a service. We trust you will find here an atmosphere of cordiality and spirituality that will be refreshing.

Worship Services 8:30 \& 11:00 AM Sunday School

9:45 AM

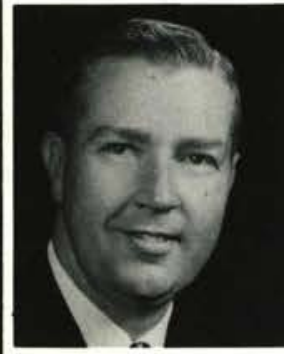

Phi Theta Chi College

Fellowship

4:15 PM

Training Hour for Youth 5:15 PM

Evening Gospel Service

7:00 PM

Thursday Prayer Meeting 7:30 PM

A modern, supervised nursery is

available at all services. Ask an usher.

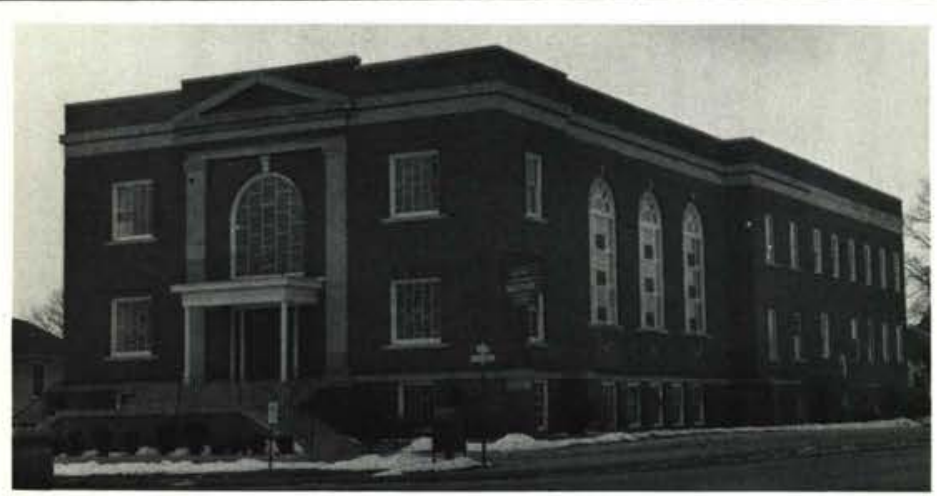

\section{RIVERSIDE}

\section{BAPTIST CHURCH}

S. Jasper \& E. Cleveland Sts. Decatur, Illinois, 62525

M. Donald Currey, Pastor

Sunday Services

9:30 A.M. Sunday School

10:45 A.M. Morning Worship

6:30 P.M. Youth Groups

7:30 P.M. Evening Worship

Wednesday - 7:30 P.M. Prayer Meeting

Awana Youth Program

In Fellowship with

G.A.R.B.C.; A.C.C.C.; I.C.C.C.

\section{IMMANUEL \\ BAPTIST CHURCH}

3417 Palmetto at Derrer Road

Columbus, Ohio

WILliaM A. BROCK, Pastor

\section{FIRST BAPTIST CHURCH}

Of La Salle, Illinois

GREETS AND CONGRATULATES

CEDARVILLE COLLEGE - AND THE

GRADUATING CLASS

CONTINUE "HOLDING FORTH THE

WORD OF LIFE!"

VISIT OUR CHURCH IN THE

HEART OF THE ILLINOIS VALLEY

SERVICES SUNDAY 9:30 A.M.

10:45 A.M., 7:30 P.M. 


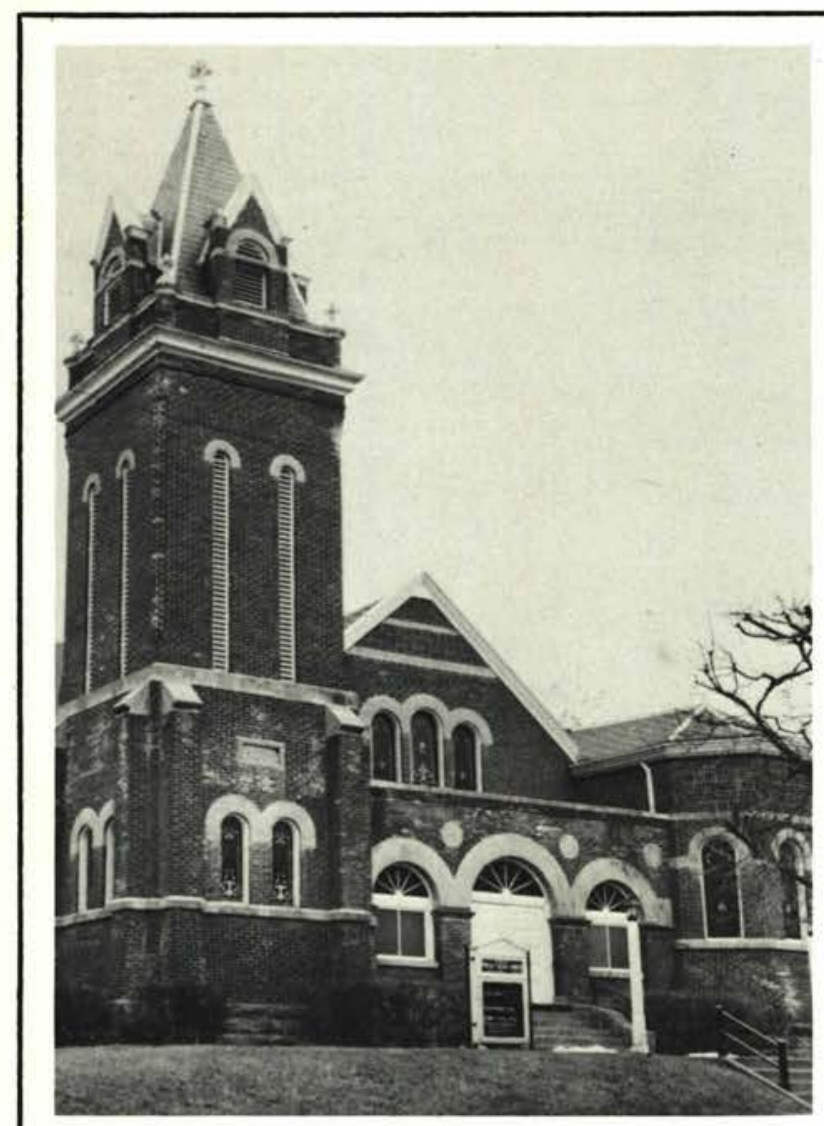

\section{GRACE \\ BAPTIST CHURCH}

Cedarville, Ohio

\begin{tabular}{|c|c|}
\hline \multicolumn{2}{|c|}{ A. Donald Moffat, Pastor } \\
\hline Bible School & 9:45 a.m. \\
\hline Morning Worship & 11:00 a.m. \\
\hline Youth Groups & 6:00 p.m. \\
\hline Evening Service & 7:00 p.m. \\
\hline $\begin{array}{l}\text { WEDNESDAY EVENING } \\
\text { PRAYER SERVIC̈ES }\end{array}$ & $6: 30$ and $7: 30$ p.m. \\
\hline
\end{tabular}

Serving Cedarville College and Community for Christ!

\section{BLESSED HOPE BAPTIST CHURCH}

315 Kensington Place, Springfield, Ohio

9:30 Sunday School

10:45 Morning Worship

6:15 Youth Groups

7:30 Evening Service

Midweek Prayer Hour -

7:30 Wednesday
GOD'S CHOICEST BLESSINGS ON EACH OF YOU FROM THE

\section{EUCLID}

NOTTINGHAM

\section{BAPTIST CHURCH}

18901 Lake Shore Boulevard Euclid 19, Ohio

Rev. George E. Huffman, Pastor 


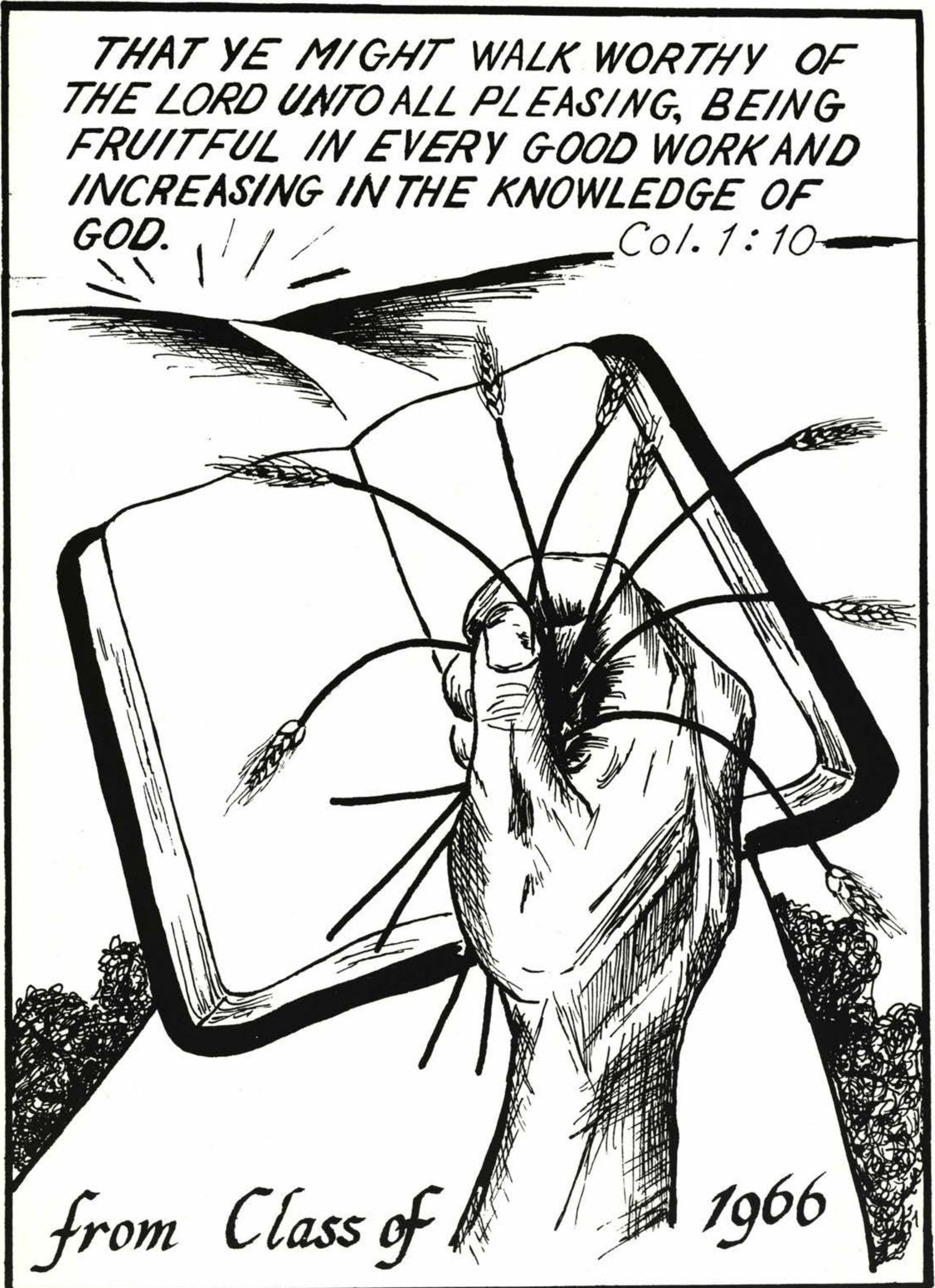




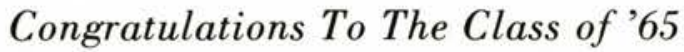 \\ CEDAR HILL BAPTIST CHURCH
}

\author{
12601 Cedar Road
}

Cleveland, Ohio

\author{
Rev. John G. Balyo, Pastor
}

ATTENDING CEDARVILLE FROM CEDAR HILL

Martha Bennett

David Curtis

Sandra Curtis

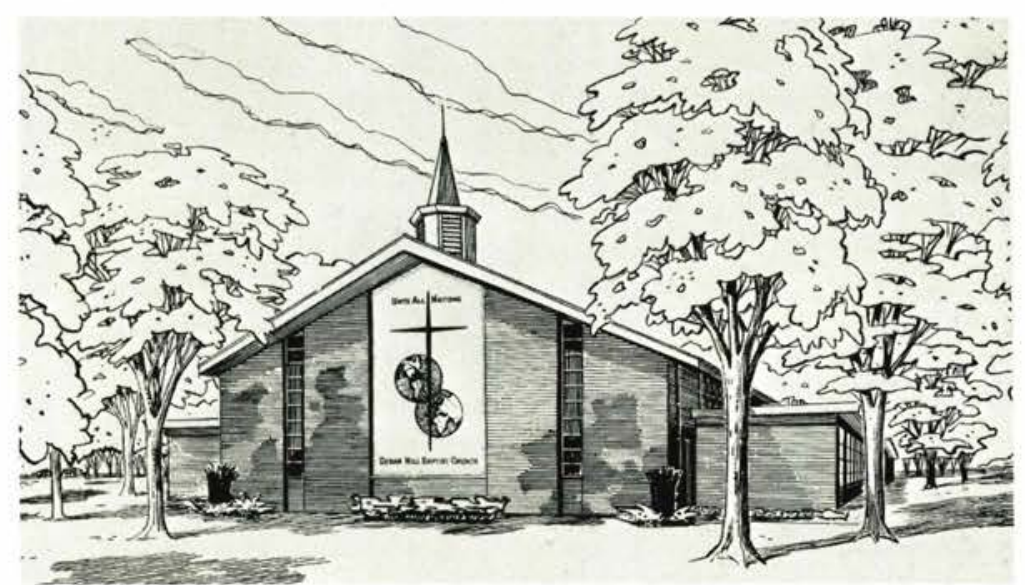

Joyce Johnson

David Vanderground Jon Walborn

\section{We Desire the Lord's Blessing for the Class of 1965}

BETHEL BAPTIST CHURCH

Howell, Michigan

Robert Taylor, Pastor

BIBLE BAPTIST CHURCH

9 Birch Rise Drive

Newton, Connecticut

FIRST BAPTIST CHURCH

Parma, Ohio

Samuel Elliott, Pastor

GRACE BAPTIST CHURCH

Rocky River, Ohio

Rev. Douglas R. Couch, Pastor

FIRST BAPTIST CHURCH

Milford, Michigan

Rev. Clark L. Mann, Pastor

CALVARY BAPTIST CHURCH

Cresaptown, Maryland

Rev. Harold Allem, Pastor

Evangelist and Mrs. P. R. Halvorsen

\section{FIRST BAPTIST CHURCH}

221 New Castle Street

Butler, Pennsylvania

RALPH O. BURNS, PASTOR

WE ARE PROUD TO HAVE SIX

STUDENTS AT CEDARVILLE

\section{CALVARY BAPTIST CHURCH}

700 Burton Street S. E.

GRAND RAPIDS, MICHIGAN 49507 


\section{IRVINE DAIRY ISLE}

$$
\begin{array}{cc}
\text { ICE CREAM } & \text { SOFT DRIN } \\
\text { SANDWICHES } & \text { SUNDAES } \\
\text { SODAS } & \text { CANDY } \\
\multicolumn{2}{c}{\text { SHAKES }}
\end{array}
$$
SANDWICHES SUNDAES
SODAS CANDY

North Main Street

\section{DINNEN'S \\ SOHIO SERVICE}

\section{BATTERIES \\ LUBRICATION \\ WRECKER}

Myron Dinnen, Proprietor

\section{RECO \\ SPORTING GOODS \\ AND \\ GIFT STORE}

\section{Uniforms \\ Jackets \\ Sweaters}

Sports Equipment

Come and Browse Around

113 E. High St. Springfield, Ohio

\section{CEDARVILLE SUPER VALU}

MEATS

PRODUCE GROCERIES

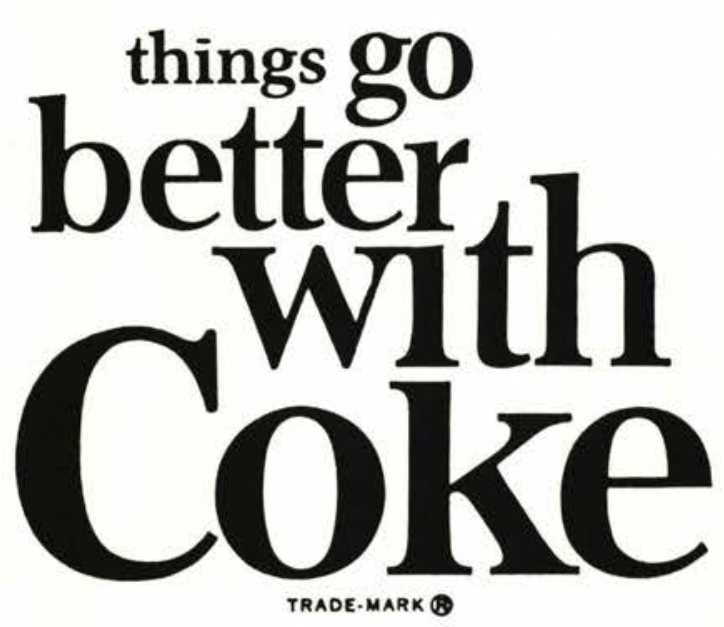

Phone 766-1201

Cedarville, Ohio 


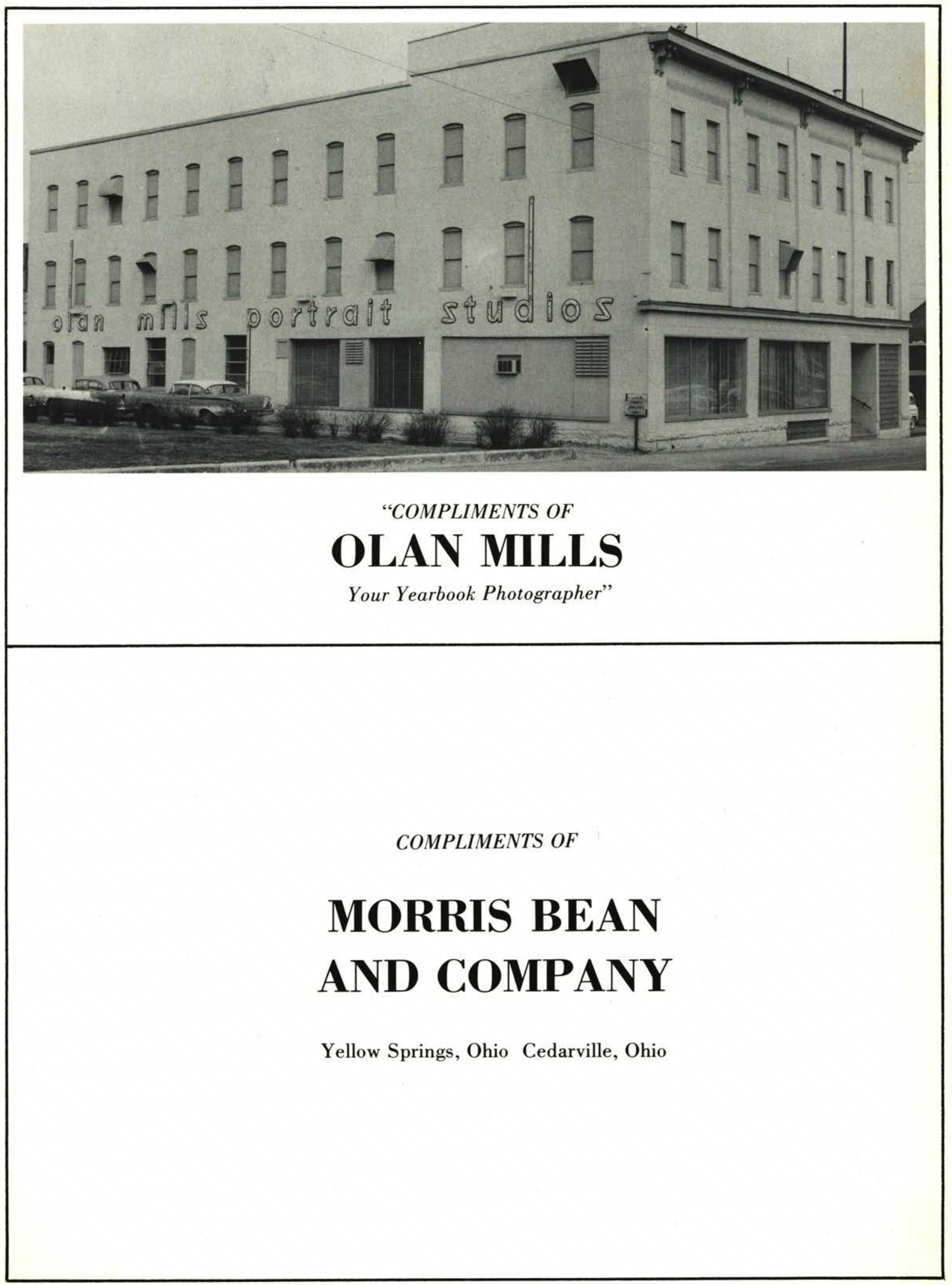




\section{NOW!}

ENJOY THE FINEST

in Dairy Products ... Produced

in Springfield's Most Modern Self-Serve

Home-Owned Dairy!

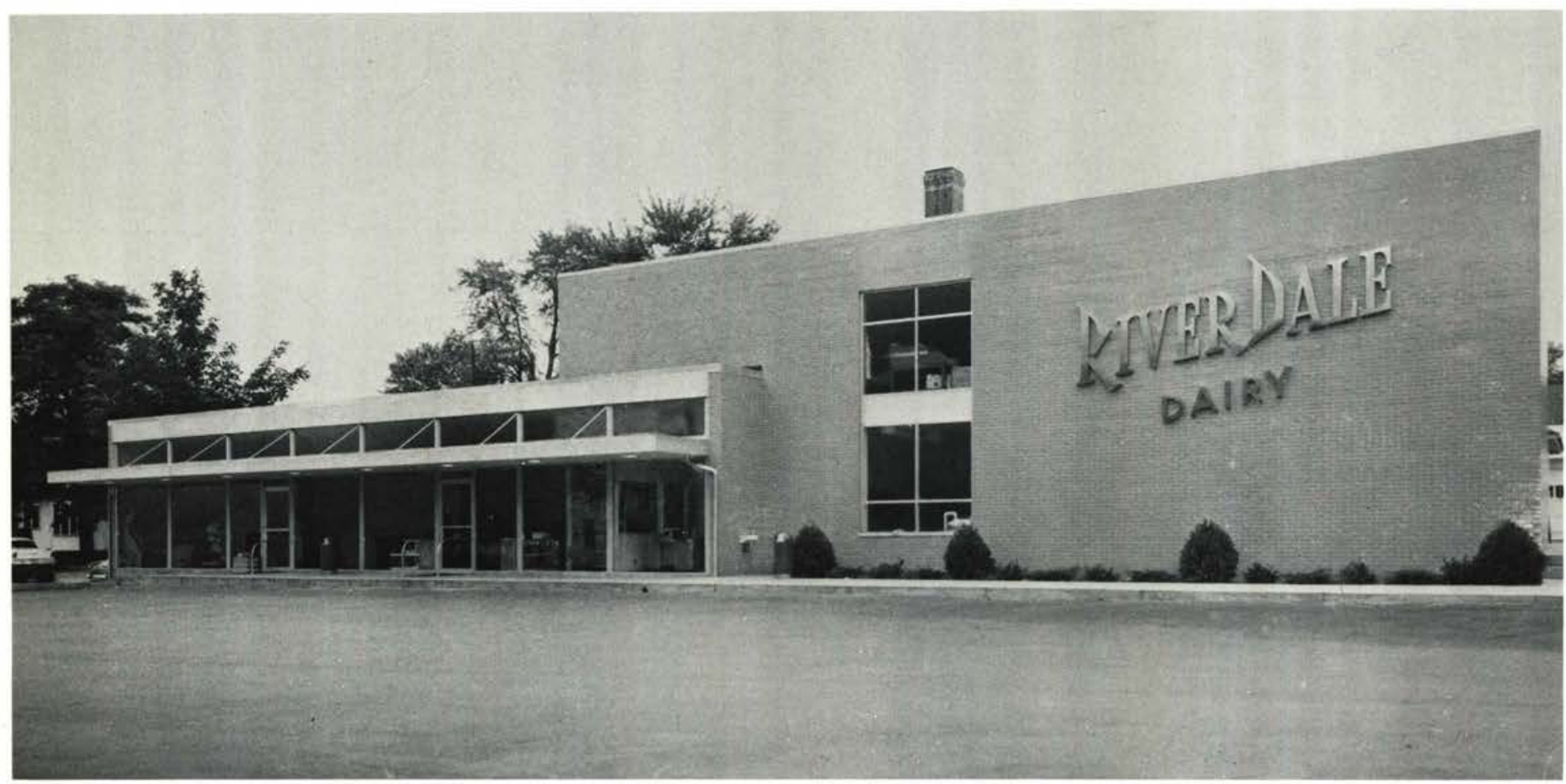

\section{Ice Cream For All \\ Occasions}

Fruit Punch

Orange Drink

Chocolate Milk

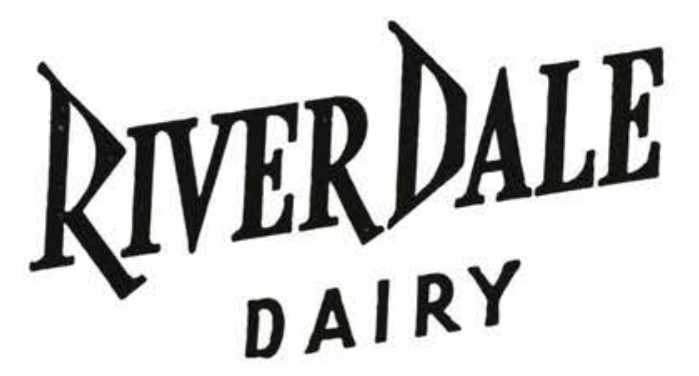

Individual Party Slices

Nut Rolls

Anniversary Cakes

CORNER SELMA ROAD AND OAK STREET

OPEN DAILY 8 A.M. till 9 P.M. PLENTY OF PARKING SPACE 
CEDARVILLE

LUMBER CO.

"One Stop Building Center"

32 East Street

Cedarville, Ohio

Phone 766-2611

\section{LEACH QUICK WASH}

\section{4-Hour Self-Service}

Cedarville, Ohio

E. Xenia Street Behind Firehouse

Phone 766-5186

\section{BEAL'S}

\section{DEPARTMENT STORE}

Gift Items

Clothing and Shoes

S. Main

Cedarville, Ohio

Phone 766-5362

\section{RANDALL \& STORMONT}

\section{INC.}

Purina Chows And Health Aids

Phone 766-1031

Cedarville, Ohio

\section{CEDARVILLE FEED \& GRAIN}

Grain, Feed, Seed, Coal, Salt

Wool, Fertilizer,

Builders' Supplies and Fencing

Cedarville, Ohio

Phone 766-2021

\section{CEDARVILLE HARDWARE}

Progressive Hardware Service

Cedarville, Ohio

Phone 766-1941

General Electric Appliances

For More Than 20 Years

\section{HOAK'S GREENHOUSE}

Greenhouses and Nursery

Fairborn, Ohio

FLOWERS FOR ALL OCCASIONS

Phone $878-8621$ or $878-8572$

\section{"JAMES" REXALL STORE}

Cedarville, Ohio

42 N. Main

Phone 766-1771 


\section{MIAMI DEPOSIT BANK}

FULL BANKING SERVICES

Two Offices Located at

Cedarville, Ohio AND

Yellow Springs, Ohio

Member of

Federal Deposit Insurance

Corporation

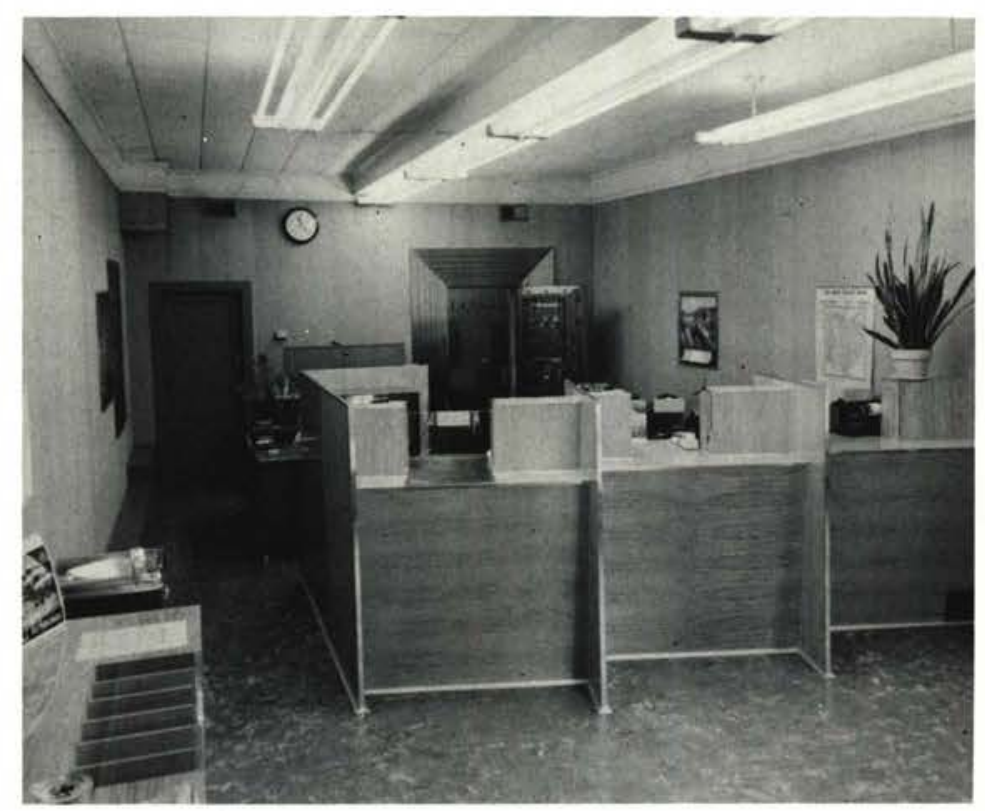

\section{STROBRIDGE AUTO} BODY AND GLASS

25 Church Street

Cedarville, Ohio

Complete Auto Rebuilding and Refinishing

\section{BIG WHEEL}

RESTAURANT

N. Main

Cedarville, Ohio

Phone: 766-5220

"Enjoy Your Food in the Rustic

Atmosphere of the BIG WHEEL

\section{THE CENTRAL OHIO PAPER CO.}

\section{DAYTON \\ COLUMBUS \\ INDIANAPOLIS}

The Finest in Papers for Schools, Printing, and Industry.

The "Copco" Brand 


\section{CONGRATULATIONS TO THE CLASS OF' 65}

XENIA GAZETTE

"Growing With All Green County"

XENIA OFFICE SUPPLY

Your Local Stationer

372-2381

BAND BOX MUSIC STORE

57 West Main Street

Xenia-Fairborn

ROGERS JEWELRY

44 S. Detroit Street

$372-7681$

EICHMANS APPLIANCE

82 West Main Street

Xenia, Ohio

JAMES SUPER MARKET

52 East Market Street

Xenia, Ohio

CEDARCLIFF BEAUTY SALON

Cedarville, Ohio

766-4861

CHICK'S BARBERSHOP

Cedarville, Ohio

CENTRAL BARBER SHOP

57 East Main Street

Gary-Phil

HOME FURNITURE

84. East Main Street

Xenia, Ohio

VILLAGE RESTAURANT

Home Cooking-Steaks - Homemade Pies-Good Place To EatCedarville

CEDARVILLE LOCKER

Bill Everhart

766-1141
MAIN AUTO SUPPLY

44 West Main Street

Xenia, Ohio

CAMERA SHOP

34 West Main Street

372-1469

BLACK'S FURNITURE

"For Furniture New"

Northeast on Rt. 42

DONGES DRUG STORE

58 South Detroit

Xenia, Ohio

NILSON-BONE-FLORISTS

68 West Main Street

$372-4211$

DAUM AND SON

114 S. Detroit Street

372-5231

RAY COX INSURANCE

37 S. Detroit - Gazette Bldg.

Xenia - 372-7666

DEL 'ABAR HARDWARE

Xenia, Ohio

SMITH'S BAKERY

"The Man Who Kneads The Dough" 78 W. Main-Xenia, Ohio

CUMMINGS CHEVROLET SALES

49 Xenia Avenue

Cedarville, Ohio

HITCHCOCK'S JEWELRY STORE

$68 \mathrm{~S}$. Detroit St.

Xenia, Ohio

XENIA NEW CAR DEALERS

Xenia, Ohio
RICH JEWELRY STORE

10 N. Detroit Street

Xenia, Ohio

FAMOUS AUTO SUPPLY

Sporting Goods

372-4071 - 64 East Main

LITT BROTHERS

25 East Main Street

Xenia, Ohio

GEYERS RESTAURANT

37 West Main Street

Xenia, Ohio

TIFFANY JEWELRY STORE

20 South Detroit Street

Xenia, Ohio

ADAIR'S FINE FURNITURE

Xenia, Ohio

"Worth A Trip To Xenia"

THE CARD SHOP

Hallmark Cards and Gifts

17 East Main Street

EHRHART SPORTS SHOP

Fairborn-Xenia, Ohio

DUTCH OVEN BAKERY

60 North Detroit Street

Xenia, Ohio

CHERRY'S FURNISHINGS

56 W. Main Street, Xenia

372-3579

CEDARVILLE FEDERAL

SAVINGS AND LOAN ASSN.

Cedarville, Ohio

CEDARVILLE VARIETY STORE

Clothes and Gifts for the Family

N. Main St., Cedarville, O. 
CONGRATULATIONS CLASS OF 1965

FROM THE

CEDARVILLE COLLEGE FOOD SERVICE

\section{MIAMI VALLEY}

CHRISTIAN

BROADCASTING

\section{ASSOCIATION, INC.}

7333 Manning Road (Box 222) Miamisburg, Ohio

Phone: 866-5929

"Witnessing For Christ Jesus" For Millions

WFCJ-FM $93.7 \mathrm{mc}$

The First Christian Station in the Ohio Valley

L. Morse Weimer Clarence E. Morrison Chairman of the Board

\section{BRAUN'S JEWELERS}

REGISTERED JEWELERS

AMERICAN GEM SOCIETY

22 N. Detroit St.

Xenia, Ohio

"An Education In Diamonds \& Gems Awaits You"

Ask To See Our Collection of

Fluorescent Blue Diamonds

Omega-Rolex-Accutron-Bulova Watches

Fine Repair Work Diamond Setting

\section{LONG's}

FOOD SERVICE EQUIPMENT, INC.

MODERN AIR CONTROL CO.

“A Business Built With Satisfied

Customers"

559-561 Selma Road

Springfield, Ohio
A COMPLETE LINEN AND TOWEL SERVICE

THE INDEPENDENT

TOWEL SUPPLY CO.

212 Willis Ave.

Springfield, Ohio

Phone: 322-9306

\section{CHAPLIN CLEANERS}

Laundry Service

Cleaning

Shoe Repair

Phone 766-3871

Cedarville, Ohio
PIZZA MENU

Stop in and have lunch with Pizza Menu $13 " .9 "$
1.ge. Small $^{\prime \prime}$

Cherese

sausage.

Mushroom

Pepperoni

Anchovy

Olive

Hamburger

Ham IDiced)

Sausage \& Mushroom

Sauxage \& Pepperoni

Pepperoni

1.T Speecial
$1.50 \quad 75$

$1.75 \quad .90$

$\begin{array}{ll}1.75 & .90 \\ 1.90 & 1.90\end{array}$

$1.90 \quad 1.00$

$1.75 \quad 90$

$2.00 \quad 1.00$

$1.75 \quad .90$

$2.00 \quad 1.00$

$2.00 \quad 1.05$

$2.15 \quad 1.15$

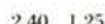

$\begin{array}{ll}2.65 & 1.35\end{array}$

\section{THE I-T PIZZA}

Hours Daily 11-1:00 A.M.

Except Sat. till 2 A.M.,

Sun. 12 to 12

Wed. 5:00 to 1:00 A.M.

Phone: Dr. 2-3587

Broasted Chicken

\section{PAUL'S}

WHOLESALE

SPRINGFIELD, OHIO 
Today's kind of living calls for two kinds of Pepsi-Cola. Drink famous regular Pepsi for the clean, bold taste...plus energy to liven your pace. Enjoy new Diet Pepsi-Cola when you want honest-to-Pepsi taste, lively Pepsi sparkle...with less than a calorie a bottle.Either way, it's the official drink of today's generation !

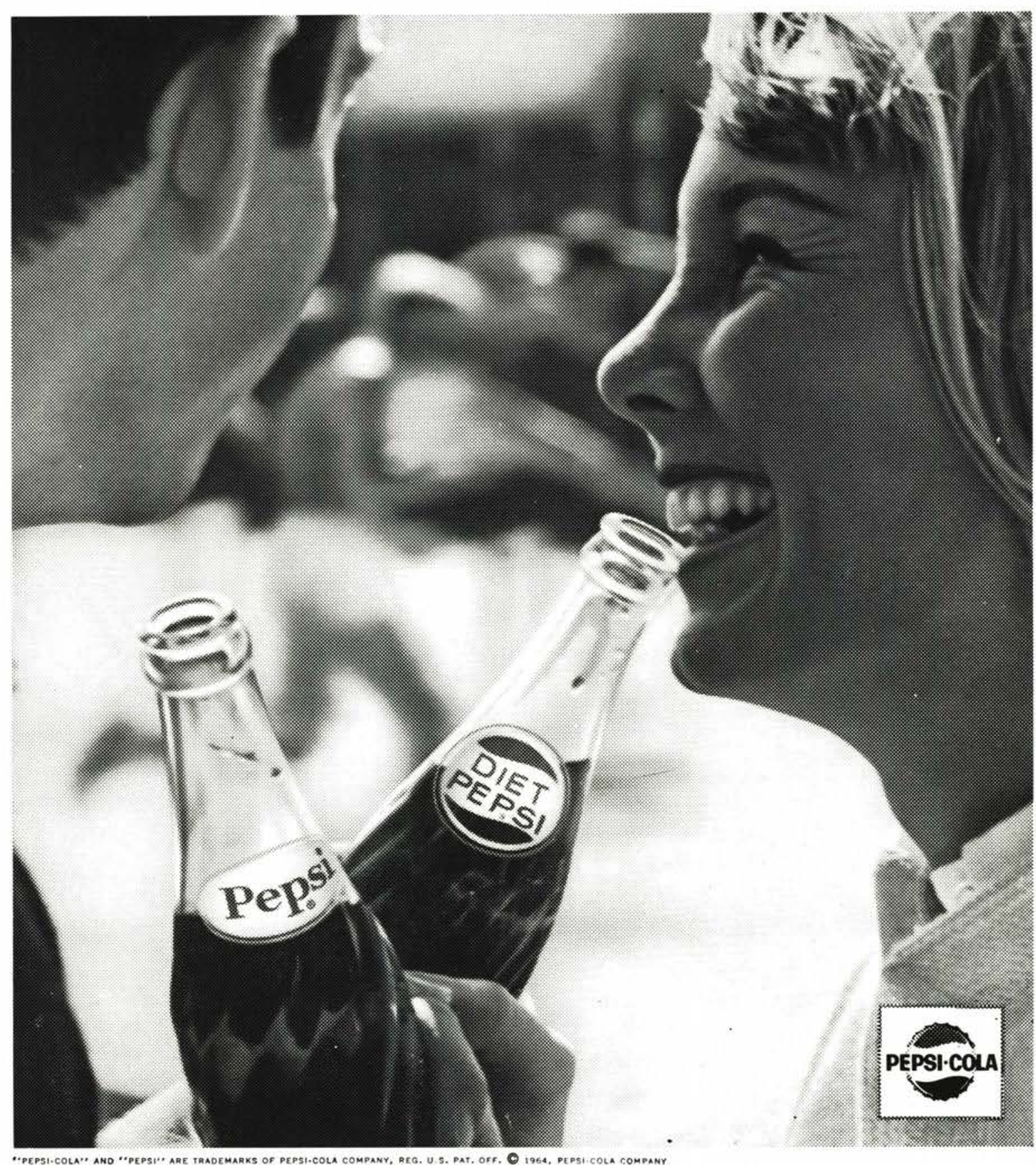


Congratulations To The Class of 1965

Welcome To

\section{THE ALUMNI ASSOCIATION}

President . . . . . . . . . Don Sewell

Executive

Vice-President . . . . . J James Johnson

Secretary . . . . Ray Bartholomew

Treasurer ....... Stuart Chaffe

\section{Sincere Congratulations Seniors FUTURE BUSINESS ASSOCIATES}

Prominent Lecturere In Business

Monthly Business Meetings

Delicious Refreshments

Unusual Field Trips

Interesting Films

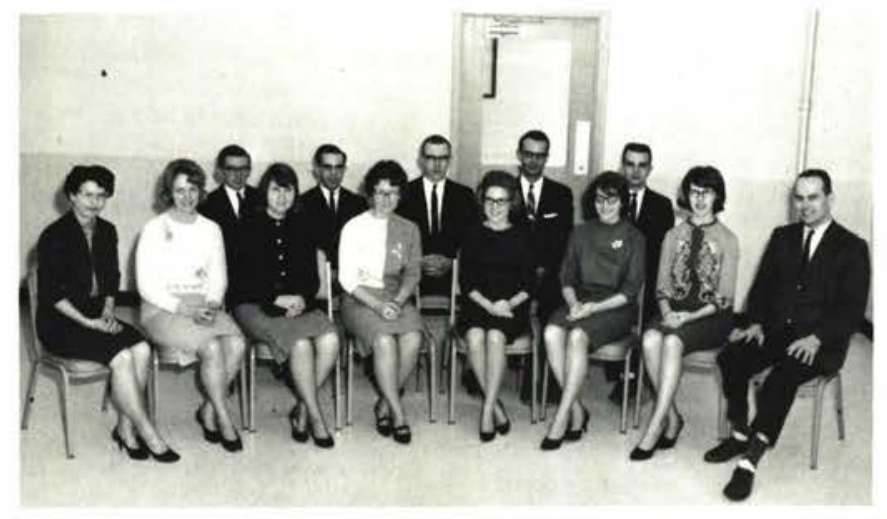

"The Association With A Business Future"

\section{WINDSOR VILLAGE BAPTIST CHURCH}

2151 North Kitley
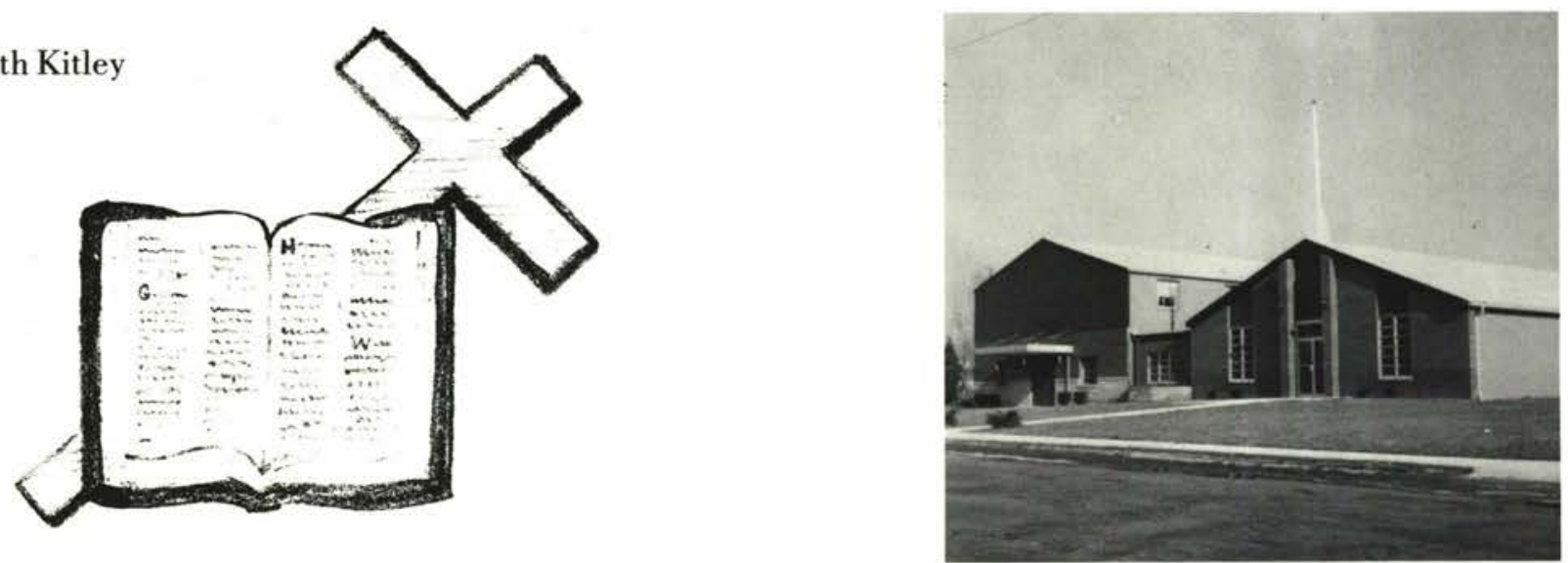


\section{BROWN STREET BAPTIST CHURCH}

Alton, Illinois

Gaylord S. Hamilton, Pastor

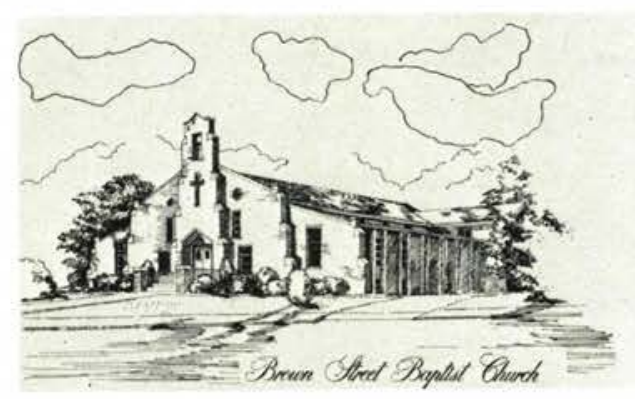

"A friendly church with a warm welcome"

\section{SUNDAY}

9:30 A.M.

10:30 A.M.

6:00 P.M.

7:00 P.M.

WEDNESDAY

7:00 P.M.
Bible School Morning Worship Training Unions Evening Service

\section{GEORGE MEEK CO., INC.}

\author{
36 N. Fountain Ave. \\ Springfield, Ohio \\ 323-4918
}

Your Complete Sporting Goods Store

We Outfit The Yellow Jackets

Good Luck Cedarville Graduates

\section{FIRST \\ BAPTIST \\ CHURCH}

Butler, Pennsylvania

\section{MILNER}

\section{ELECTRIC}

\section{COMPANY}

Sincere Congratulations and

Best Wishes To The

Class of ' 65

"Electrical Supplies of all types"

1320 St. Clair Avenue

Cleveland, Ohio 44114

\section{MEMORIAL BAPTIST CHURCH}

\author{
201 South Main Street \\ Verona, Wisconsin
}

REV. R. D. MATTHEWS, PASTOR

REV. DON MOORE, ASSOCIATE

PASTOR

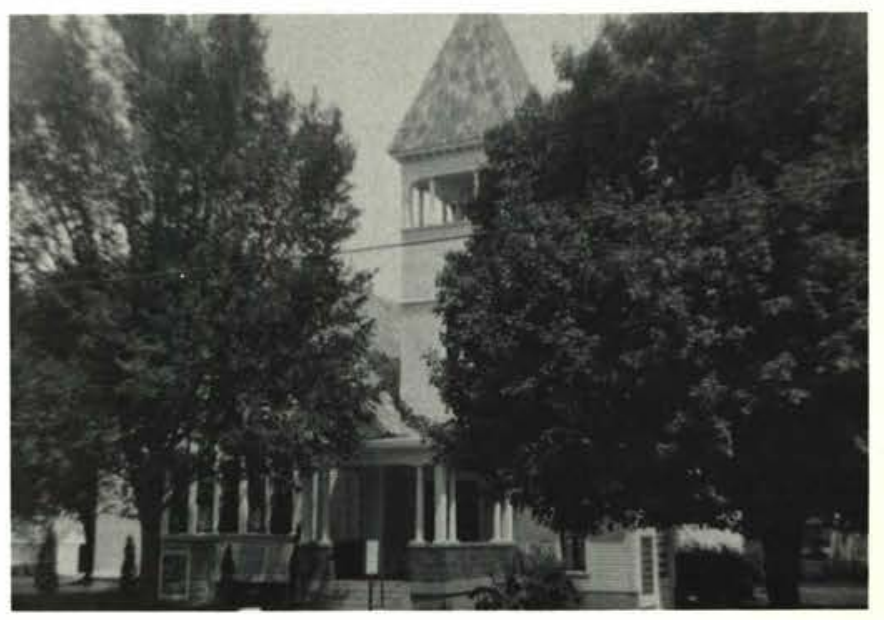




\section{CLARK'S DISCOUNT STORE}

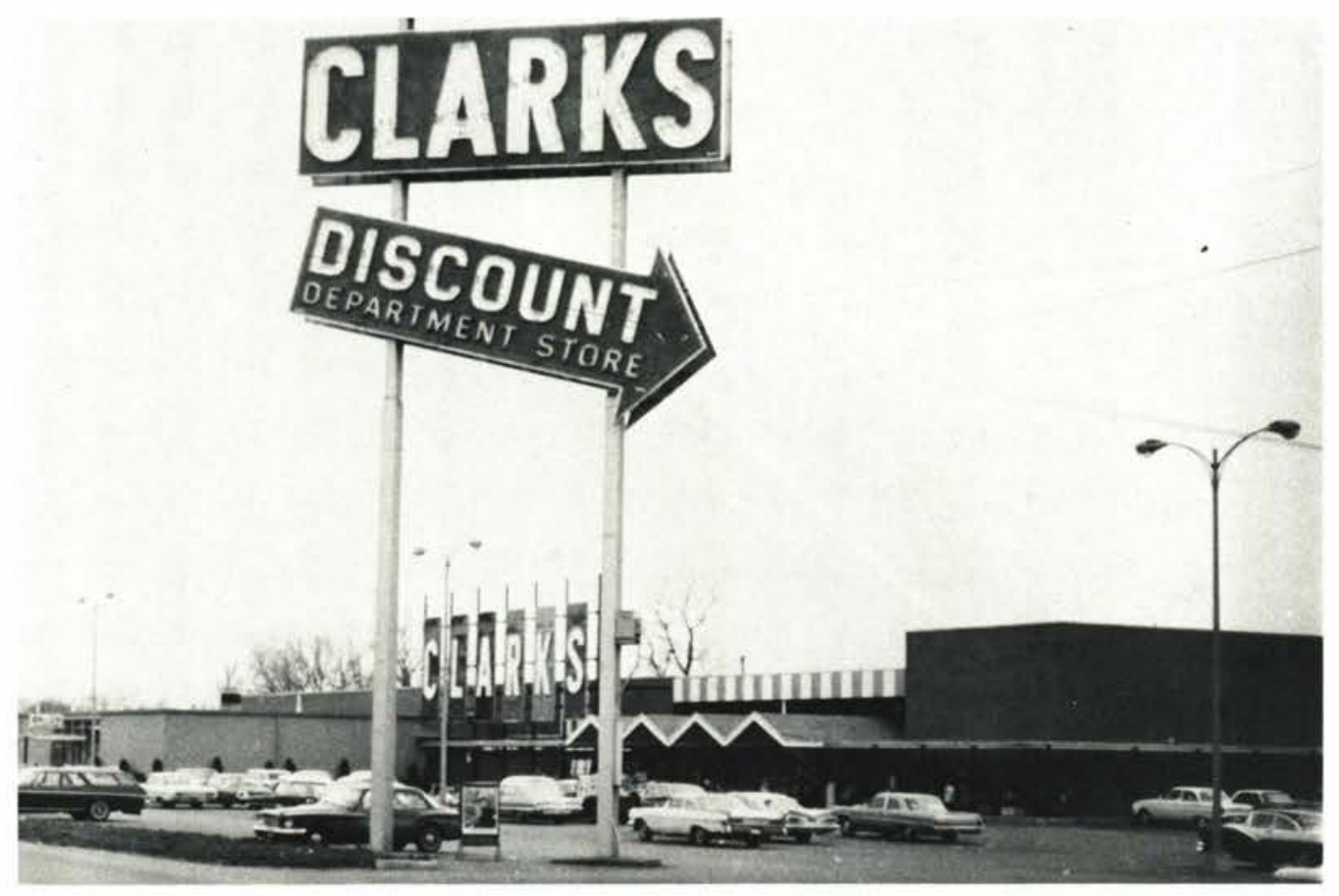

Off Route 72

\section{THE CRITERION}

Xenia, Ohio

MEN'S CLOTHING AND

FURNISHINGS

$$
\begin{gathered}
\text { VARSITY TOWN-ARROW - } \\
\text { MacGREGOR - STETSON- } \\
\text { PALM BEACH }
\end{gathered}
$$

\section{SIMMS TYPEWRITER \\ SALES and SERVICE}

- Smith-Corona - Underwood-Olivetti

- Royal-Olympia • Standard - Electric

- Portable - SALES \& SERVICE

Adding \& Calculating Machines

- REPAIRS • RENTALS

Office Equipment \& Supplies

Phone: 878-5739

Fairborn, Ohio

\section{CAMPUS \\ BICYCLE RENTAL}

Owned and Operated by

Mr. and Mrs. Kenneth H. St. Clair $467 \mathrm{~W}$ alnut Street

Cedarville, Ohio

While in Cedarville try a Bike Built for Two

See the Beautiful Ohio Countryside on a Bicycle 





